Supporting Information

\title{
Nucleobase Stacking at Clay Edges, a Favourable Interaction for RNA/DNA Oligomerization
}

\footnotetext{
Pierre Mignon ${ }^{\star}$, Javier Navarro-Ruiz $₫ \S$, Albert Rimola ${ }^{\ddagger}$, Mariona Sodupe $\ddagger^{\star}$

† Institut Lumière Matière, UMR 5306, Univ-Lyon, Université Claude Bernard Lyon 1, CNRS, F-69622 Villeurbanne, France

‡ Departament de Química, Universitat Autònoma de Barcelona, E-08193 Bellaterra, Spain

$\S$ Institute of Chemical Research of Catalonia, ICIQ, and The Barcelona Institute of Science and Technology, BIST, Av. Països Catalans 16, E-43007 Tarragona, Spain
} 


\section{Energy evaluation}

The adsorption energy $\left(\Delta \mathrm{E}_{\mathrm{ads}}\right)$ of a single nucleobase $(\mathrm{B})$ on the montmorillonite edge surfaces (MNT) was computed as:

$\Delta \mathrm{E}_{\mathrm{ads}}(\mathrm{B} / \mathrm{MNT})=\mathrm{E}(\mathrm{B} / \mathrm{MNT} / / \mathrm{B} / \mathrm{MNT})-\mathrm{E}(\mathrm{B} / / \mathrm{B})-\mathrm{E}(\mathrm{MNT} / / \mathrm{MNT})$

in which $E(B / M N T / / B / M N T)$ is the absolute energy of the optimized $B / M N T$ complex and $E(B / / B)$ and $E(M N T / / M N T)$ are the absolute energies of the isolated nucleobase and montmorillonite, respectively, at their optimized geometries. Note that he symbol following the double slash identifies the geometry at which the energy has been computed.

We define here the interaction energy $\left(\Delta \mathrm{E}_{\text {int }}{ }^{*}\right)$ as:

$\Delta \mathrm{E}_{\text {int }}{ }^{*}(\mathrm{~B} / \mathrm{MNT})=\mathrm{E}(\mathrm{B} / \mathrm{MNT} / / \mathrm{B} / \mathrm{MNT})-\mathrm{E}(\mathrm{B} / / \mathrm{B} / \mathrm{MNT})-\mathrm{E}(\mathrm{MNT} / / \mathrm{B} / \mathrm{MNT})$

in which $E(B / / B / M N T)$ and $E(M N T / / B / M N T)$ are the absolute energies of isolated $B$ and MNT, respectively, at the same geometries as found in the B/MNT complex; namely, the interaction energy between B and MNT when they are deformed. The deformation energies $\left(\Delta E_{\text {def }}\right)$ of these components are computed as:

$\Delta \mathrm{E}_{\text {def }}(\mathrm{B})=\mathrm{E}(\mathrm{B} / / \mathrm{B} / \mathrm{MNT})-\mathrm{E}(\mathrm{B} / / \mathrm{B})$

$\Delta \mathrm{E}_{\text {def }}(\mathrm{MNT})=\mathrm{E}(\mathrm{MNT} / / \mathrm{B} / \mathrm{MNT})-\mathrm{E}(\mathrm{MNT} / / \mathrm{MNT})$

$\Delta \mathrm{E}_{\mathrm{def}}=\Delta \mathrm{E}_{\mathrm{def}}(\mathrm{B})+\Delta \mathrm{E}_{\mathrm{def}}(\mathrm{MNT})$

which allow us to evaluate the energy cost induced by molecular interactions in the complex.

For the stacked nucleobases (B1 and B2) on the clay edge, the total adsorption energy was computed as:

$\Delta \mathrm{E}_{\mathrm{ads}}(\mathrm{B} 1 / \mathrm{B} 2 / \mathrm{MNT})=\mathrm{E}(\mathrm{B} 1 / \mathrm{B} 2 / \mathrm{MNT} / / \mathrm{B} 1 / \mathrm{B} 2 / \mathrm{MNT})-\mathrm{E}(\mathrm{B} 1 / / \mathrm{B} 1)-\mathrm{E}(\mathrm{B} 2 / / \mathrm{B} 2)-$ $\mathrm{E}(\mathrm{MNT} / / \mathrm{MNT})$

This total adsorption energy has different contributions; i.e., the interaction of B1 with the clay, the interaction of B2 with the clay, and the interaction between B1 and B2. Analytic energy decomposition of $\Delta \mathrm{E}_{\mathrm{ads}}(\mathrm{B} 1 / \mathrm{B} 2 / \mathrm{MNT})$ with these terms is not possible 
since it consists of a three-body problem. However, they can be estimated; that is, the interaction energy of B1 or B2 with the clay as

$\Delta \mathrm{E}_{\text {int }}{ }^{*}(\mathrm{~B} 1 / \mathrm{MNT})=\mathrm{E}(\mathrm{B} 1 / \mathrm{MNT} / / \mathrm{B} 1 / \mathrm{B} 2 / \mathrm{MNT})-\mathrm{E}(\mathrm{B} 1 / / \mathrm{B} 1 / \mathrm{B} 2 / \mathrm{MNT}) \quad-$ $\mathrm{E}(\mathrm{MNT} / / \mathrm{B} 1 / \mathrm{B} 2 / \mathrm{MNT})$

$\Delta \mathrm{E}_{\text {int }}{ }^{*}(\mathrm{~B} 2 / \mathrm{MNT})=\mathrm{E}(\mathrm{B} 2 / \mathrm{MNT} / / \mathrm{B} 1 / \mathrm{B} 2 / \mathrm{MNT})-\mathrm{E}(\mathrm{B} 2 / / \mathrm{B} 1 / \mathrm{B} 2 / \mathrm{MNT})-$ $\mathrm{E}(\mathrm{MNT} / / \mathrm{B} 1 / \mathrm{B} 2 / \mathrm{MNT})$

and the interaction energy between B1 and B2 as

$\Delta \mathrm{E}_{\text {int }}{ }^{*}(\mathrm{~B} 1 / \mathrm{B} 2)=\mathrm{E}(\mathrm{B} 1 / \mathrm{B} 2 / / \mathrm{B} 1 / \mathrm{B} 2 / \mathrm{MNT})-\mathrm{E}(\mathrm{B} 1 / / \mathrm{B} 1 / \mathrm{B} 2 / \mathrm{MNT})-\mathrm{E}(\mathrm{B} 2 / / \mathrm{B} 1 / \mathrm{B} 2 / \mathrm{MNT})$

To check if the adsorption brings associated with positive or negative cooperative effects we will compare the sum of the estimated interaction energy contributions (i.e., $\left.\Delta \mathrm{E}_{\text {int }}{ }^{*}(\mathrm{~B} 1 / \mathrm{MNT})+\Delta \mathrm{E}_{\text {int }}{ }^{*}(\mathrm{~B} 2 / \mathrm{MNT})+\Delta \mathrm{E}_{\text {int }}{ }^{*}(\mathrm{~B} 1 / \mathrm{B} 2)\right)$ with the interaction energies of the deformed components $\left(\Delta \mathrm{E}_{\mathrm{int}}{ }^{*}(\mathrm{~B} 1 / \mathrm{B} 2 / \mathrm{MNT})\right)$; that is,

$\Delta \mathrm{E}_{\text {int }}{ }^{*}(\mathrm{~B} 1 / \mathrm{B} 2 / \mathrm{MNT})=\mathrm{E}(\mathrm{B} 1 / \mathrm{B} 2 / \mathrm{MNT} / / \mathrm{B} 1 / \mathrm{B} 2 / \mathrm{MNT})-\mathrm{E}(\mathrm{B} 1 / / \mathrm{B} 1 / \mathrm{B} 2 / \mathrm{MNT})-$ $E(B 2 / / B 1 / B 2 / M N T)-E(M N T / / B 1 / B 2 / M N T)$ 


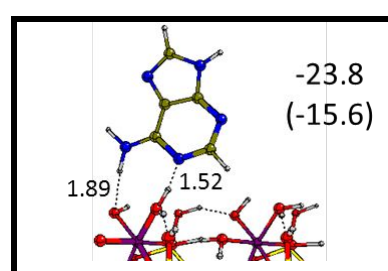

A1: $\mathrm{N} 1\left[\mathrm{Al}(\mathrm{OH})_{2}\right] / \mathrm{N} 6[\mathrm{AlOH}]$

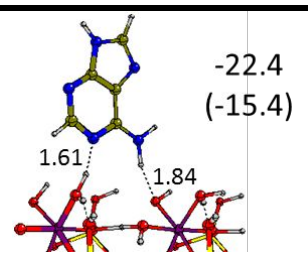

A2: $\mathrm{N} 1\left[\mathrm{Al}(\mathrm{OH})_{2}\right] / \mathrm{N} 6[\mathrm{AlOH}]$

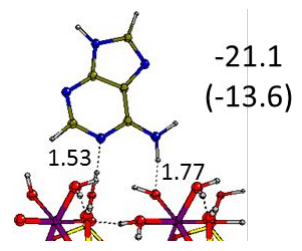

A3: N1[SiOH] / N6[AIOH]

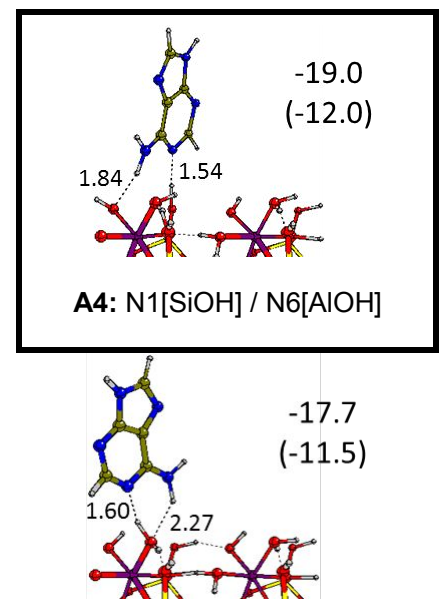

A5: $\mathrm{N} 1\left[\mathrm{Al}(\mathrm{OH})_{2}\right] / \mathrm{N} 6\left[\mathrm{Al}(\mathrm{OH})_{2}\right]$

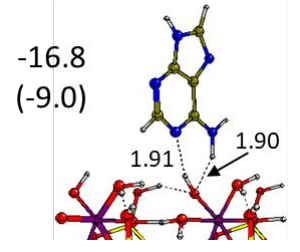

A6: N1[AlOH] / N6[AIOH]

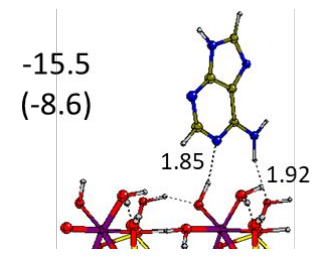

A7: N1[AIOH] / N6[SiOH]

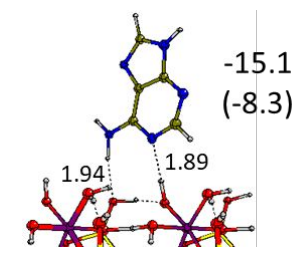

A8: N1[AlOH] / N6[SiOH]

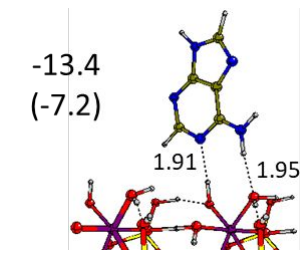

A9: $\mathrm{N} 1[\mathrm{AIOH}] / \mathrm{N} 6\left[\mathrm{Al}(\mathrm{OH})_{2}\right]$

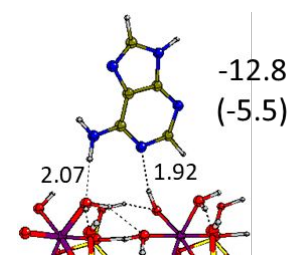

A10: N1[AlOH] / N6[Al(OH $\left.)_{2}\right]$

Figure S1. Adsorption structures of adenine nucleobase. Total (and DFT in parentheses) binding energies are in $\mathrm{kcal} / \mathrm{mol}$ (see Table S1) and $\mathrm{H}$-bonds distances between adenine-edge hydroxyls or water molecules are given in $\AA$. A1 and A4 (highlighted in bold) are involved in A1-A2 and A2-G2 stacked nucleobases. Colour code: Si (light yellow), Al (magenta), O (red), N (blue), C (yellow) and H (white). 


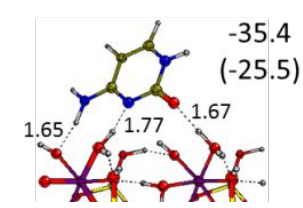

C1: O2[Al(OH) $)_{2} / \mathrm{N} 3[\mathrm{AlOH}]$ / N4[AIOH]

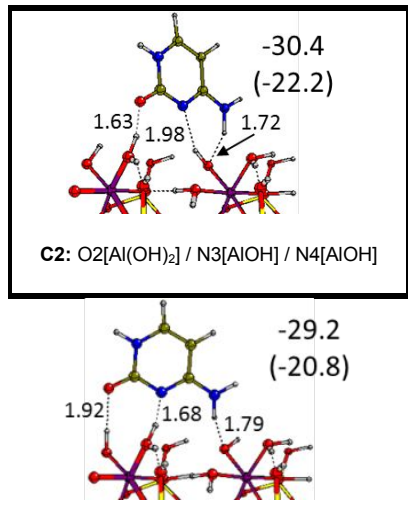

C3: O2[AIOH] / N3[AI(OH) 2] / N4[AIOH]

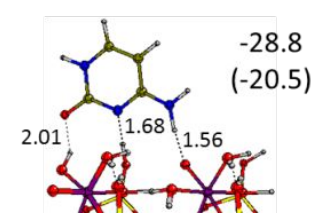

C4: O2[AIOH] / N3[SiOH] / N4[AIOH]
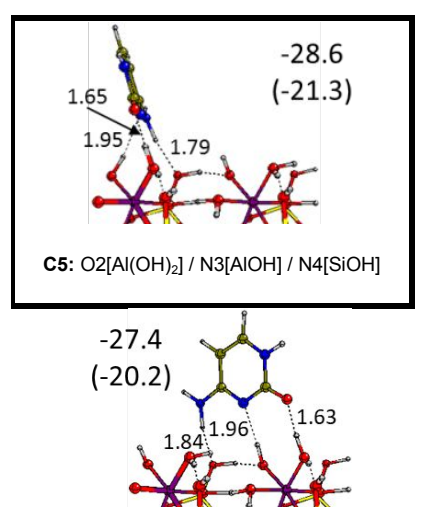

C6: O2[Al $\left.(\mathrm{OH})_{2}\right] / \mathrm{N} 3[\mathrm{AlOH}] / \mathrm{N} 4[\mathrm{SiOH}]$

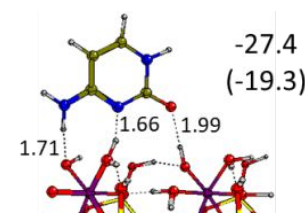

C7: O2[AIOH] / N3[AI(OH) 2] / N4[AIOH]

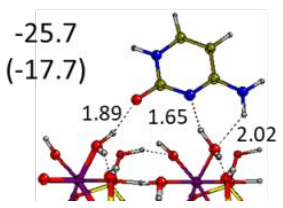

C8: $\mathrm{O} 2\left[\mathrm{Al}(\mathrm{OH})_{2}\right] / \mathrm{N} 3\left[\mathrm{Al}(\mathrm{OH})_{2}\right] / \mathrm{N} 4\left[\mathrm{Al}(\mathrm{OH})_{2}\right]$

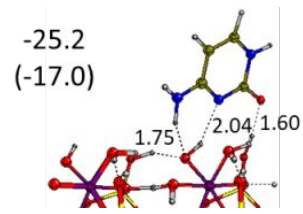

C9: O2[SiOH] / N3[AIOH] / N4[AIOH]

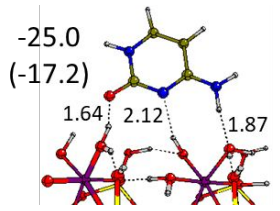

C10: $\mathrm{O} 2\left[\mathrm{Al}(\mathrm{OH})_{2}\right] / \mathrm{N} 3[\mathrm{AIOH}] / \mathrm{N} 4\left[\mathrm{Al}(\mathrm{OH})_{2}\right]$

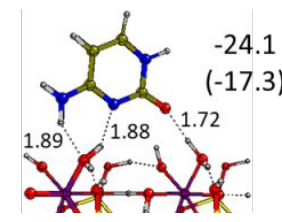

C11: O2[Al(OH $\left.)_{2}\right] / \mathrm{N} 3\left[\mathrm{Al}(\mathrm{OH})_{2}\right] / \mathrm{N} 4\left[\mathrm{Al}(\mathrm{OH})_{2}\right]$

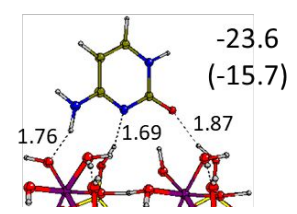

C12: $\mathrm{O} 2[\mathrm{SiOH}] / \mathrm{N} 3[\mathrm{SiOH}] / \mathrm{N} 4[\mathrm{AlOH}]$

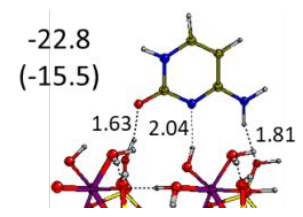

C13: O2[SiOH] / N3[AIOH] / N4[SiOH]

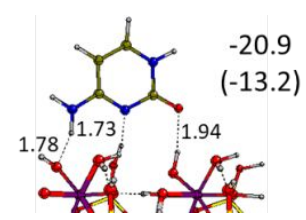

C14: O2[AIOH] / N3[SiOH] / N4[AIOH]

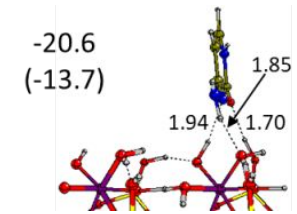

C15: O2[SiOH] / N3[AIOH] / N4[Al(OH $\left.)_{2}\right]$

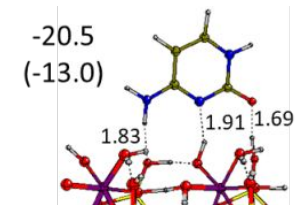

C16: O2[SiOH] / N3[AIOH] / N4[SiOH]

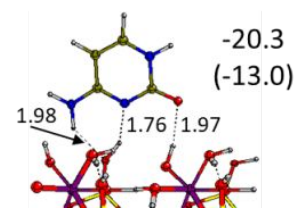

C17: O2[AlOH] / N3[SiOH] / N4[SiOH]

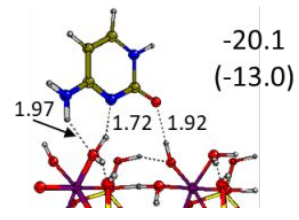

C18: O2[AIOH] / N3[AI (OH $\left.)_{2}\right]$ / N4[AI $\left.(\mathrm{OH})_{2}\right]$

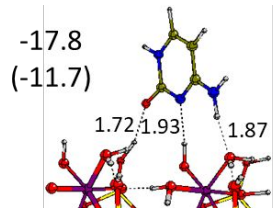

C19: O2[SiOH] / N3[AlOH] / N4[Al(OH $\left.)_{2}\right]$

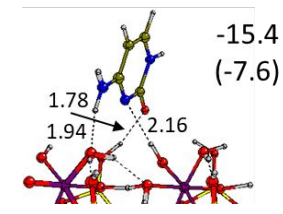

C20: $\mathrm{O} 2[\mathrm{SiOH}] / \mathrm{N} 3[\mathrm{AIOH}] / \mathrm{N} 4\left[\mathrm{Al}(\mathrm{OH})_{2}\right]$

Figure S2. Adsorption structures of cytosine nucleobase. Total (and DFT in parentheses) binding energies are in $\mathrm{kcal} / \mathrm{mol}$ (see Table S2) and $\mathrm{H}$-bonds distances between cytosine-edge hydroxyls or water molecules are given in $\AA$. C2 and C5 (highlighted in bold) are involved in C1-A2, C2-C2, C1-T2 and C1-G2 stacked nucleobases. Colour code: Si (light yellow), Al (magenta), O (red), N (blue), C (yellow) and $\mathrm{H}$ (white). 


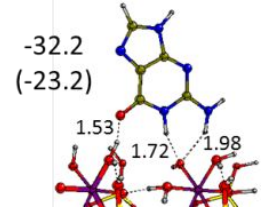

G1: O6[AIOH] / N1[AIOH] / N2[Al(OH) $)_{2}$

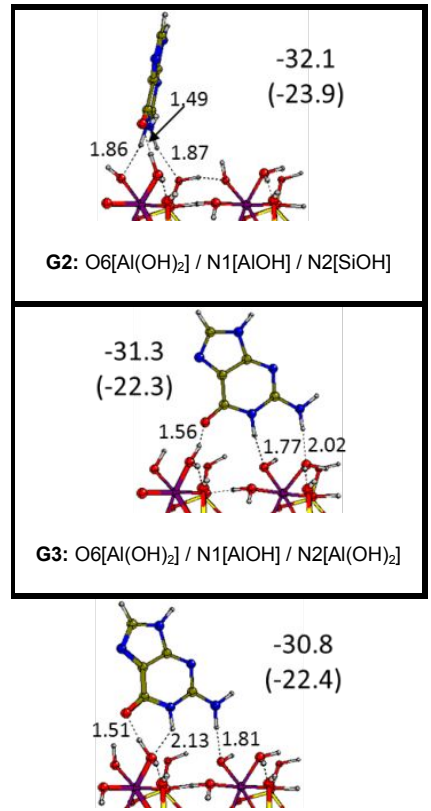

G4: $\mathrm{O} 6\left[\mathrm{Al}(\mathrm{OH})_{2}\right] / \mathrm{N} 1\left[\mathrm{Al}(\mathrm{OH})_{2}\right] / \mathrm{N} 2[\mathrm{AlOH}]$

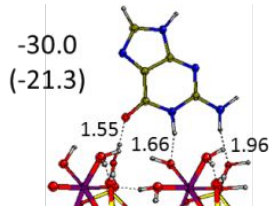

G5: O6[SiOH] / N1[AlOH] / N2[SiOH]

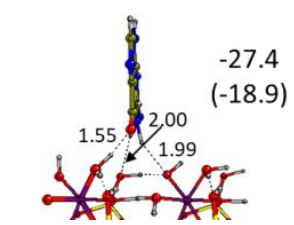

G6: O6[Al(OH $\left.)_{2}\right] / \mathrm{N} 1[\mathrm{AlOH}] / \mathrm{N} 2[\mathrm{SiOH}]$

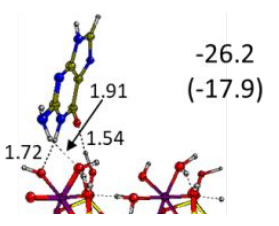

G7: O6[SiOH] / N1 $[\mathrm{AlOH}] /$ N2[Al $\left.(\mathrm{OH})_{2}\right]$

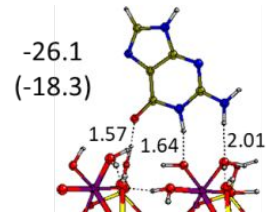

G8: O6[SiOH] / N1[AlOH] / N2[Al(OH $\left.)_{2}\right]$

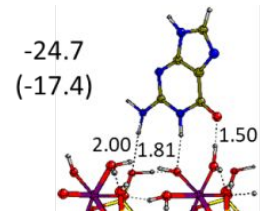

G9: O6[Al $\left.(\mathrm{OH})_{2}\right] / \mathrm{N} 1[\mathrm{AlOH}] / \mathrm{N} 2[\mathrm{SiOH}]$

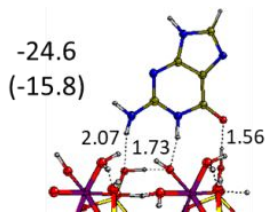

G10: O6[SiOH] / N1[AlOH] / N2[SiOH]

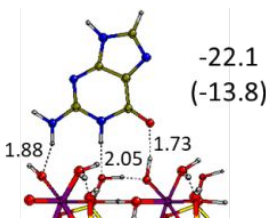

G11: O6[AIOH] / N1[SiOH] / N2[AIOH]

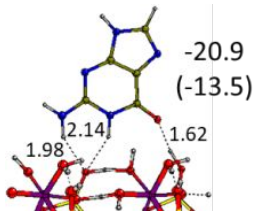

G12: O6[SiOH] / N1[SiOH] / N2[SiOH]

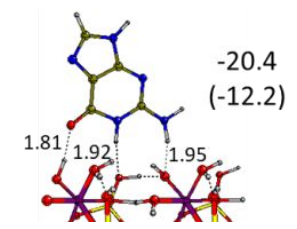

G13: O6[AlOH] / N1[SiOH] / N2[AIOH]

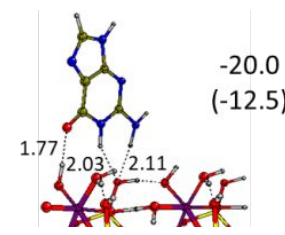

G14: O6[AlOH] / N1[SiOH] / N2[SiOH]

Figure S3. Adsorption structures of guanine nucleobase. Total (and DFT in parentheses) binding energies are in $\mathrm{kcal} / \mathrm{mol}$ (see Table S1) and $\mathrm{H}$-bonds distances between guanine-edge hydroxyls or water molecules are given in $\AA$. G2 and G3 (highlighted in bold) are involved in G2-T2 and G1-G2 stacked nucleobases. Colour code: Si (light yellow), Al (magenta), O (red), N (blue), C (yellow) and H (white). 


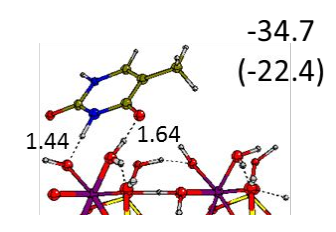

T1: O4[Al(OH) $)_{2} / \mathrm{N} 3[\mathrm{AIOH}]$

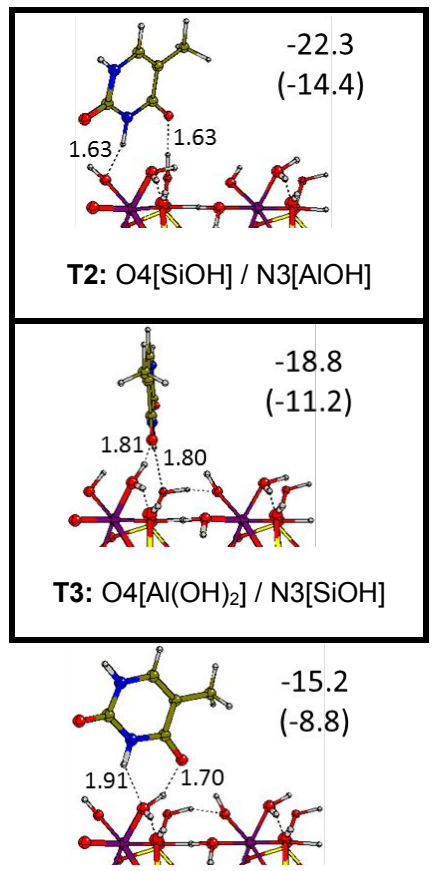

T4: $\mathrm{O} 4\left[\mathrm{Al}(\mathrm{OH})_{2}\right] / \mathrm{N} 3\left[\mathrm{Al}(\mathrm{OH})_{2}\right]$

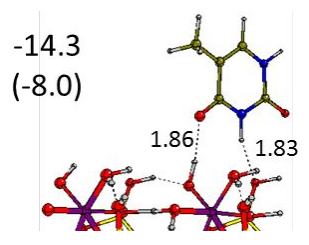

T5: O4[AIOH] / N3[SiOH]

Figure S4. Adsorption structures of thymine nucleobase. Total (and DFT in parentheses) binding energies are in $\mathrm{kcal} / \mathrm{mol}$ (see Table S2) and $\mathrm{H}$-bonds distances between thymine-edge hydroxyls or water molecules are given in $\AA$. T2 and T3 (highlighted in bold) are involved in T2-A2 and T1-T2 stacked nucleobases. Colour code: Si (light yellow), Al (magenta), O (red), N (blue), C (yellow) and H (white). 
Table S1. Computed adsorption energies of all the geometries for the different purines on montmorillonite edge sites (in $\mathrm{kcal} / \mathrm{mol}$ ). DFT adsorption energy (Ads.E $E_{D F T}$ ); contribution of dispersion to the adsorption energy ( $\left.A d s . E_{D 3}\right)$; and total adsorption energy $\left(A d s . E=A d s . E_{D F T}+A d s . E_{D 3}\right)$.

\begin{tabular}{|c|c|c|c|c|c|}
\hline Nucleobase & Mode & Coordination & $A d s . E_{D F T}$ & $A d s . E_{D 3}$ & Ads.E \\
\hline \multirow[t]{10}{*}{ Adenine } & $\mathrm{A} 1$ & $\mathrm{~N} 1\left[\mathrm{Al}(\mathrm{OH})_{2}\right] / \mathrm{N} 6[\mathrm{AlOH}]$ & -15.6 & -8.2 & -23.8 \\
\hline & A2 & $\mathrm{N} 1\left[\mathrm{Al}(\mathrm{OH})_{2}\right] / \mathrm{N} 6[\mathrm{AlOH}]$ & -15.4 & -7.1 & -22.4 \\
\hline & A3 & $\mathrm{N} 1[\mathrm{SiOH}] / \mathrm{N} 6[\mathrm{AlOH}]$ & -13.6 & -7.5 & -21.1 \\
\hline & A4 & $\mathrm{N} 1[\mathrm{SiOH}] / \mathrm{N} 6[\mathrm{AlOH}]$ & -12.0 & -7.0 & -19.0 \\
\hline & A5 & $\mathrm{N} 1\left[\mathrm{Al}(\mathrm{OH})_{2}\right] / \mathrm{N} 6\left[\mathrm{Al}(\mathrm{OH})_{2}\right]$ & -11.5 & -6.2 & -17.7 \\
\hline & A6 & $\mathrm{N} 1[\mathrm{AlOH}] / \mathrm{N} 6[\mathrm{AlOH}]$ & -9.0 & -7.8 & -16.8 \\
\hline & A7 & $\mathrm{N} 1[\mathrm{AlOH}] / \mathrm{N} 6[\mathrm{SiOH}]$ & -8.6 & -6.8 & -15.5 \\
\hline & A8 & $\mathrm{N} 1[\mathrm{AlOH}] / \mathrm{N} 6[\mathrm{SiOH}]$ & -8.3 & -6.8 & -15.1 \\
\hline & A9 & $\mathrm{N} 1[\mathrm{AlOH}] / \mathrm{N} 6\left[\mathrm{Al}(\mathrm{OH})_{2}\right]$ & -7.2 & -6.3 & -13.4 \\
\hline & A10 & $\mathrm{N} 1[\mathrm{AlOH}] / \mathrm{N} 6\left[\mathrm{Al}(\mathrm{OH})_{2}\right]$ & -5.5 & -7.3 & -12.8 \\
\hline \multirow[t]{14}{*}{ Guanine } & G1 & $\mathrm{O} 6[\mathrm{AlOH}] / \mathrm{N} 1[\mathrm{AlOH}] / \mathrm{N} 2\left[\mathrm{Al}(\mathrm{OH})_{2}\right]$ & -23.2 & -9.0 & -32.2 \\
\hline & G2 & $\mathrm{O} 6\left[\mathrm{Al}(\mathrm{OH})_{2}\right] / \mathrm{N} 1[\mathrm{AlOH}] / \mathrm{N} 2[\mathrm{SiOH}]$ & -23.9 & -8.2 & -32.1 \\
\hline & G3 & $\mathrm{O} 6\left[\mathrm{Al}(\mathrm{OH})_{2}\right] / \mathrm{N} 1[\mathrm{AlOH}] / \mathrm{N} 2\left[\mathrm{Al}(\mathrm{OH})_{2}\right]$ & -22.3 & -9.1 & -31.3 \\
\hline & G4 & $\mathrm{O} 6\left[\mathrm{Al}(\mathrm{OH})_{2}\right] / \mathrm{N} 1\left[\mathrm{Al}(\mathrm{OH})_{2}\right] / \mathrm{N} 2[\mathrm{AlOH}]$ & -22.4 & -8.4 & -30.8 \\
\hline & G5 & $\mathrm{O} 6[\mathrm{SiOH}] / \mathrm{N} 1[\mathrm{AlOH}] / \mathrm{N} 2[\mathrm{SiOH}]$ & -21.3 & -8.7 & -30.0 \\
\hline & G6 & $\mathrm{O} 6\left[\mathrm{Al}(\mathrm{OH})_{2}\right] / \mathrm{N} 1[\mathrm{AlOH}] / \mathrm{N} 2[\mathrm{SiOH}]$ & -18.9 & -8.6 & -27.4 \\
\hline & G7 & $\mathrm{O} 6[\mathrm{SiOH}] / \mathrm{N} 1[\mathrm{AlOH}] / \mathrm{N} 2\left[\mathrm{Al}(\mathrm{OH})_{2}\right]$ & -17.9 & -8.3 & -26.2 \\
\hline & G8 & $\mathrm{O} 6[\mathrm{SiOH}] / \mathrm{N} 1[\mathrm{AlOH}] / \mathrm{N} 2\left[\mathrm{Al}(\mathrm{OH})_{2}\right]$ & -18.3 & -7.8 & -26.1 \\
\hline & G9 & $\mathrm{O} 6\left[\mathrm{Al}(\mathrm{OH})_{2}\right] / \mathrm{N} 1[\mathrm{AlOH}] / \mathrm{N} 2[\mathrm{SiOH}]$ & -17.4 & -7.2 & -24.7 \\
\hline & G10 & $\mathrm{O} 6[\mathrm{SiOH}] / \mathrm{N} 1[\mathrm{AlOH}] / \mathrm{N} 2[\mathrm{SiOH}]$ & -15.8 & -8.7 & -24.6 \\
\hline & G11 & $\mathrm{O} 6[\mathrm{AlOH}] / \mathrm{N} 1[\mathrm{SiOH}] / \mathrm{N} 2[\mathrm{AlOH}]$ & -13.8 & -8.3 & -22.1 \\
\hline & G12 & $\mathrm{O} 6[\mathrm{SiOH}] / \mathrm{N} 1[\mathrm{SiOH}] / \mathrm{N} 2[\mathrm{SiOH}]$ & -13.5 & -7.4 & -20.9 \\
\hline & G13 & $\mathrm{O} 6[\mathrm{AlOH}] / \mathrm{N} 1[\mathrm{SiOH}] / \mathrm{N} 2[\mathrm{AlOH}]$ & -12.2 & -8.2 & -20.4 \\
\hline & G14 & $\mathrm{O} 6[\mathrm{AlOH}] / \mathrm{N} 1[\mathrm{SiOH}] / \mathrm{N} 2[\mathrm{SiOH}]$ & -12.5 & -7.4 & -20.0 \\
\hline
\end{tabular}


Table S2. Computed adsorption energies of all the geometries for the different pyrimidines on montmorillonite edge sites (in $\mathrm{kcal} / \mathrm{mol}$ ). DFT adsorption energy ( $\left.A d s . E_{D F T}\right)$; contribution of dispersion to the adsorption energy (Ads. $\left.E_{D 3}\right)$; and total adsorption energy $\left(A d s . E=A d s . E_{D F T}+A d s . E_{D 3}\right)$.

\begin{tabular}{|c|c|c|c|c|c|}
\hline Nucleobase & Mode & Coordination & $A d s . E_{D F T}$ & $A d s . E_{D 3}$ & Ads.E \\
\hline \multirow[t]{20}{*}{ Cytosine } & C1 & $\mathrm{O} 2\left[\mathrm{Al}(\mathrm{OH})_{2}\right] / \mathrm{N} 3[\mathrm{AlOH}] / \mathrm{N} 4[\mathrm{AlOH}]$ & -25.5 & -9.9 & -35.4 \\
\hline & $\mathrm{C} 2$ & $\mathrm{O} 2\left[\mathrm{Al}(\mathrm{OH})_{2}\right] / \mathrm{N} 3[\mathrm{AlOH}] / \mathrm{N} 4[\mathrm{AlOH}]$ & -22.2 & -8.2 & -30.4 \\
\hline & C3 & $\mathrm{O} 2[\mathrm{AlOH}] / \mathrm{N} 3\left[\mathrm{Al}(\mathrm{OH})_{2}\right] / \mathrm{N} 4[\mathrm{AlOH}]$ & -20.8 & -8.4 & -29.2 \\
\hline & $\mathrm{C} 4$ & $\mathrm{O} 2[\mathrm{AIOH}] / \mathrm{N} 3[\mathrm{SiOH}] / \mathrm{N} 4[\mathrm{AlOH}]$ & -20.5 & -8.3 & -28.8 \\
\hline & C5 & $\mathrm{O} 2\left[\mathrm{Al}(\mathrm{OH})_{2}\right] / \mathrm{N} 3[\mathrm{AlOH}] / \mathrm{N} 4[\mathrm{SiOH}]$ & -21.3 & -7.4 & -28.6 \\
\hline & C6 & $\mathrm{O} 2\left[\mathrm{Al}(\mathrm{OH})_{2}\right] / \mathrm{N} 3[\mathrm{AlOH}] / \mathrm{N} 4[\mathrm{SiOH}]$ & -20.2 & -7.2 & -27.4 \\
\hline & $\mathrm{C} 7$ & $\mathrm{O} 2[\mathrm{AlOH}] / \mathrm{N} 3\left[\mathrm{Al}(\mathrm{OH})_{2}\right] / \mathrm{N} 4[\mathrm{AlOH}]$ & -19.3 & -8.1 & -27.4 \\
\hline & $\mathrm{C} 8$ & $\mathrm{O} 2\left[\mathrm{Al}(\mathrm{OH})_{2}\right] / \mathrm{N} 3\left[\mathrm{Al}(\mathrm{OH})_{2}\right] / \mathrm{N} 4\left[\mathrm{Al}(\mathrm{OH})_{2}\right]$ & -17.7 & -8.0 & -25.7 \\
\hline & $\mathrm{C9}$ & $\mathrm{O} 2[\mathrm{SiOH}] / \mathrm{N} 3[\mathrm{AlOH}] / \mathrm{N} 4[\mathrm{AlOH}]$ & -17.0 & -8.2 & -25.2 \\
\hline & C10 & $\mathrm{O} 2\left[\mathrm{Al}(\mathrm{OH})_{2}\right] / \mathrm{N} 3[\mathrm{AlOH}] / \mathrm{N} 4\left[\mathrm{Al}(\mathrm{OH})_{2}\right]$ & -17.2 & -7.7 & -25.0 \\
\hline & C11 & $\mathrm{O} 2\left[\mathrm{Al}(\mathrm{OH})_{2}\right] / \mathrm{N} 3\left[\mathrm{Al}(\mathrm{OH})_{2}\right] / \mathrm{N} 4\left[\mathrm{Al}(\mathrm{OH})_{2}\right]$ & -17.3 & -6.9 & -24.1 \\
\hline & C12 & $\mathrm{O} 2[\mathrm{SiOH}] / \mathrm{N} 3[\mathrm{SiOH}] / \mathrm{N} 4[\mathrm{AlOH}]$ & -15.7 & -7.9 & -23.6 \\
\hline & C13 & $\mathrm{O} 2[\mathrm{SiOH}] / \mathrm{N} 3[\mathrm{AlOH}] / \mathrm{N} 4[\mathrm{SiOH}]$ & -15.5 & -7.3 & -22.8 \\
\hline & C14 & $\mathrm{O} 2[\mathrm{AlOH}] / \mathrm{N} 3[\mathrm{SiOH}] / \mathrm{N} 4[\mathrm{AlOH}]$ & -13.2 & -7.8 & -20.9 \\
\hline & C15 & $\mathrm{O} 2[\mathrm{SiOH}] / \mathrm{N} 3[\mathrm{AlOH}] / \mathrm{N} 4\left[\mathrm{Al}(\mathrm{OH})_{2}\right]$ & -13.7 & -6.9 & -20.6 \\
\hline & C16 & $\mathrm{O} 2[\mathrm{SiOH}] / \mathrm{N} 3[\mathrm{AlOH}] / \mathrm{N} 4[\mathrm{SiOH}]$ & -13.0 & -7.5 & -20.5 \\
\hline & C17 & $\mathrm{O} 2[\mathrm{AlOH}] / \mathrm{N} 3[\mathrm{SiOH}] / \mathrm{N} 4[\mathrm{SiOH}]$ & -13.0 & -7.2 & -20.3 \\
\hline & C18 & $\mathrm{O} 2[\mathrm{AlOH}] / \mathrm{N} 3\left[\mathrm{Al}(\mathrm{OH})_{2}\right] / \mathrm{N} 4\left[\mathrm{Al}(\mathrm{OH})_{2}\right]$ & -13.0 & -7.1 & -20.1 \\
\hline & C19 & $\mathrm{O} 2[\mathrm{SiOH}] / \mathrm{N} 3[\mathrm{AlOH}] / \mathrm{N} 4\left[\mathrm{Al}(\mathrm{OH})_{2}\right]$ & -11.7 & -6.1 & -17.8 \\
\hline & $\mathrm{C} 20$ & $\mathrm{O} 2[\mathrm{SiOH}] / \mathrm{N} 3[\mathrm{AlOH}] / \mathrm{N} 4\left[\mathrm{Al}(\mathrm{OH})_{2}\right]$ & -7.6 & -7.8 & -15.4 \\
\hline \multirow[t]{5}{*}{ Thymine } & $\mathrm{T} 1$ & $\mathrm{O} 4\left[\mathrm{Al}(\mathrm{OH})_{2}\right] / \mathrm{N} 3[\mathrm{AlOH}]$ & -22.4 & -12.2 & -34.7 \\
\hline & $\mathrm{T} 2$ & $\mathrm{O} 4[\mathrm{SiOH}] / \mathrm{N} 3[\mathrm{AlOH}]$ & -14.4 & -7.9 & -22.3 \\
\hline & T3 & $\mathrm{O} 4\left[\mathrm{Al}(\mathrm{OH})_{2}\right] / \mathrm{N} 3[\mathrm{SiOH}]$ & -11.2 & -7.6 & -18.8 \\
\hline & $\mathrm{T} 4$ & $\mathrm{O} 4\left[\mathrm{Al}(\mathrm{OH})_{2}\right] / \mathrm{N} 3\left[\mathrm{Al}(\mathrm{OH})_{2}\right]$ & -8.8 & -6.4 & -15.2 \\
\hline & T5 & $\mathrm{O} 4[\mathrm{AlOH}] / \mathrm{N} 3[\mathrm{SiOH}]$ & -8.0 & -6.4 & -14.3 \\
\hline
\end{tabular}


Table S3. Energetics for all considered configurations of stacked nucleobases (in $\mathrm{kcal} / \mathrm{mol}$ ).

\begin{tabular}{|c|c|c|c|c|c|c|c|c|c|c|c|c|c|c|c|c|}
\hline & \multicolumn{2}{|c|}{ ADENINE } & \multicolumn{2}{|l|}{ AlOHI-SiOHI } & \multirow[b]{2}{*}{ A1_G2 } & \multirow[b]{2}{*}{$\operatorname{VDW}(\mathrm{D} 3)$} & \multirow[b]{2}{*}{ A1_T2 } & \multirow[b]{2}{*}{$\operatorname{VDW}(\mathrm{D} 3)$} & \multirow[b]{2}{*}{ A2_A2 } & \multirow[b]{2}{*}{$\operatorname{VDW}(\mathrm{D} 3)$} & \multirow[b]{2}{*}{ A2_C2 } & \multirow[b]{2}{*}{$\operatorname{VDW}(\mathrm{D} 3)$} & \multirow[b]{2}{*}{ A2_G2 } & \multirow[b]{2}{*}{$\operatorname{VDW}(\mathrm{D} 3)$} & \multirow[b]{2}{*}{ A2_T2 } & \multirow[b]{2}{*}{$\operatorname{VDW}(\mathrm{D} 3)$} \\
\hline & A1_A2 & VDW (D3) & A1_C2 & VDW (D3) & & & & & & & & & & & & \\
\hline \multirow[t]{3}{*}{ DE (kcal/mol) } & 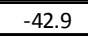 & -21.8 & \begin{tabular}{c|}
-38.7 \\
\end{tabular} & -21.2 & 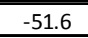 & -23.8 & $\begin{array}{l}-42.8 \\
\end{array}$ & -21.9 & $\begin{array}{l}-39.3 \\
\end{array}$ & -20.5 & $\begin{array}{l}-43.8 \\
\end{array}$ & $\begin{array}{c}-17.4 \\
\end{array}$ & $\begin{array}{ll}-60.3 \\
\end{array}$ & -22.1 & $\begin{array}{l}-50.9 \\
\end{array}$ & -21.3 \\
\hline & \multicolumn{2}{|c|}{ ADENINE } & \multicolumn{2}{|c|}{ AlOHI-AIOH2I } & & & & & & & & & & & & \\
\hline & A1_A2 & VDW (D3) & A1_C2 & VDW (D3) & A1_G2 & $\mathrm{VDW}(\mathrm{D} 3)$ & A1_T2 & VDW (D3) & A2_A2 & VDW (D3) & A2_C2 & VDW (D3) & A2_G2 & VDW (D3) & A2_T2 & VDW (D3) \\
\hline \multirow[t]{3}{*}{$\mathrm{DE}(\mathrm{kcal} / \mathrm{mol})$} & $\begin{array}{l}-43.9 \\
\end{array}$ & -20.3 & -45.3 & 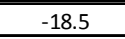 & $\begin{array}{l}-57.8 \\
\end{array}$ & $\begin{array}{l}-22.6 \\
\end{array}$ & \begin{tabular}{c|c|}
-44.1 \\
\end{tabular} & -20.3 & \begin{tabular}{c|}
-39.2 \\
\end{tabular} & \begin{tabular}{c|}
-21.4 \\
\end{tabular} & $\begin{array}{l}-46.5 \\
\end{array}$ & -21.2 & 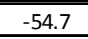 & -22.5 & $\begin{array}{l}-40.5 \\
\end{array}$ & -21.2 \\
\hline & \multicolumn{2}{|c|}{ CYTOSINE } & \multicolumn{2}{|c|}{ Hr-AlOHr-AlOH2I } & & & & & & & & & & & & \\
\hline & C1_A2 & VDW (D3) & C1_C2 & VDW (D3) & C1_G2 & $\operatorname{VDW}(\mathrm{D} 3)$ & C1_T2 & $\operatorname{VDW}(\mathrm{D} 3)$ & C2_A2 & VDW (D3) & $\mathrm{C2}$ & VDW (D3) & C2_G2 & VDW (D3) & C2_T2 & VDW (D3) \\
\hline \multirow[t]{3}{*}{$\mathrm{DE}(\mathrm{kcal} / \mathrm{mol})$} & \begin{tabular}{c|}
-52.4 \\
\end{tabular} & \begin{tabular}{c|}
-20.1 \\
\end{tabular} & \begin{tabular}{c|}
-54.3 \\
\end{tabular} & \begin{tabular}{c|}
-19.9 \\
\end{tabular} & \begin{tabular}{c|}
-58.0 \\
\end{tabular} & -22.2 & $\begin{array}{l}-54.7 \\
\end{array}$ & -19.3 & $\begin{array}{l}-47.7 \\
\end{array}$ & -19.5 & $\begin{array}{ll}-56.3 \\
\end{array}$ & -20.2 & $\begin{array}{ll}-58.7 \\
\end{array}$ & -21.2 & $\begin{array}{ll}-53.0 \\
\end{array}$ & $2-21.0$ \\
\hline & \multicolumn{2}{|c|}{ CYTOSINE } & \multicolumn{2}{|c|}{ HI-AIOHI-AlOH2I } & & & & & & & & & & & & \\
\hline & C1_A2 & VDW (D3) & C1_C2 & VDW (D3) & C1_G2 & $\operatorname{VDW}(\mathrm{D} 3)$ & C1_T2 & $\operatorname{VDW}(\mathrm{D} 3)$ & C2_A2 & VDW (D3) & $\mathrm{C2}_{-} \mathrm{C2}$ & VDW (D3) & C2_G2 & VDW (D3) & $\mathrm{C2}_{-} \mathrm{T} 2$ & VDW (D3) \\
\hline \multirow[t]{3}{*}{$\mathrm{DE}(\mathrm{kcal} / \mathrm{mol})$} & 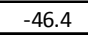 & -17.8 & -49.0 & -16.5 & 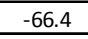 & -22.7 & \begin{tabular}{c|}
-54.6 \\
\end{tabular} & -20.0 & -48.8 & -19.5 & -45.4 & -18.5 & -60.5 & -19.7 & -44.7 & -19.0 \\
\hline & \multicolumn{2}{|c|}{ GUANINE } & \multicolumn{2}{|c|}{ 12r-AlOHr-AlOH2I } & & & & & & & & & & & & \\
\hline & G1_A2 & $\operatorname{VDW}(\mathrm{D} 3)$ & G1_C2 & VDW (D3) & G1_G2 & $\operatorname{VDW}(\mathrm{D} 3)$ & G1_T2 & $\operatorname{VDW}(\mathrm{D} 3)$ & G2_A2 & VDW (D3) & G2_C2 & VDW (D3) & G2_G2 & VDW (D3) & G2_T2 & VDW (D3) \\
\hline \multirow[t]{3}{*}{ DE (kcal/mol) } & -56.5 & -22.8 & -62.7 & -22.5 & \begin{tabular}{c|c|}
-57.3 \\
\end{tabular} & -21.8 & -52.1 & -20.8 & -51.5 & -24.7 & -50.5 & -22.5 & $\begin{array}{l}-48.6 \\
\end{array}$ & -22.8 & -50.3 & -23.6 \\
\hline & \multicolumn{2}{|c|}{ GUANINE } & \multicolumn{2}{|c|}{ HI-AlOHI-AlOH2I } & & & & & & & & & & & & \\
\hline & G1_A2 & VDW (D3) & G1_C2 & VDW (D3) & G1_G2 & $\operatorname{VDW}(\mathrm{D} 3)$ & G1_T2 & $\operatorname{VDW}(\mathrm{D} 3)$ & G2_A2 & VDW (D3) & G2_C2 & VDW (D3) & G2_G2 & $\operatorname{VDW}(\mathrm{D} 3)$ & G2_T2 & VDW (D3) \\
\hline $\mathrm{DE}(\mathrm{kcal} / \mathrm{mol})$ & \begin{tabular}{c|c|}
-39.9 \\
\end{tabular} & -19.8 & \begin{tabular}{c|}
-46.4 \\
\end{tabular} & -20.0 & 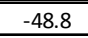 & -22.2 & $\begin{array}{ll}-43.4 \\
\end{array}$ & -21.1 & \begin{tabular}{c|c|}
-36.3 \\
\end{tabular} & $\begin{array}{l}-19.7 \\
\end{array}$ & 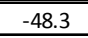 & -22.6 & \begin{tabular}{c|c|}
-33.6 \\
\end{tabular} & -21.2 & $\begin{array}{ll}-60.2 \\
\end{array}$ & -21.7 \\
\hline & & IINE & AlOHI-SiOH & & & & & & & & & & & & & \\
\hline & T1_A2 & VDW (D3) & $\mathrm{T} 1$ C 2 & $\operatorname{VDW}(\mathrm{D} 3)$ & T1_G2 & VDW (D3) & T1_T2 & $\operatorname{VDW}(\mathrm{D} 3)$ & T2_A2 & VDW (D3) & $\mathrm{T} 2 \mathrm{C} 2$ & VDW (D3) & T2_G2 & VDW (D3) & T2_T2 & VDW (D3) \\
\hline $\mathrm{DE}(\mathrm{kcal} / \mathrm{mol})$ & 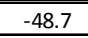 & $\begin{array}{l}-22.7 \\
\end{array}$ & \begin{tabular}{c|}
-46.4 \\
\end{tabular} & -19.5 & $\begin{array}{l}-41.6 \\
\end{array}$ & $\begin{array}{l}-21.6 \\
\end{array}$ & $\begin{array}{l}-41.2 \\
\end{array}$ & -20.3 & $\begin{array}{l}-55.0 \\
\end{array}$ & -21.2 & $\begin{array}{l}-40.7 \\
\end{array}$ & -20.7 & $\begin{array}{l}-47.1 \\
\end{array}$ & -20.6 & $\begin{array}{ll}-39.8 \\
\end{array}$ & -18.3 \\
\hline & & IINE & SiOHI-AlOH2 & & & & & & & & & & & & & \\
\hline & T1_A2 & VDW (D3) & T1_C2 & VDW (D3) & T1_G2 & VDW (D3) & T1_T2 & VDW (D3) & T2_A2 & VDW (D3) & $\mathrm{T} 2$ & VDW (D3) & T2_G2 & $\operatorname{VDW}(\mathrm{D} 3)$ & $\mathrm{T} 2 \mathrm{T2}$ & VDW (D3) \\
\hline$\overline{\mathrm{DE}(\mathrm{kcal} / \mathrm{mol})}$ & -49.2 & $\overline{-21.2}$ & 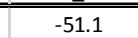 & 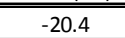 & $\begin{array}{l}-52.7 \\
\end{array}$ & \begin{tabular}{c|}
-24.3 \\
\end{tabular} & $\begin{array}{c}-46.1 \\
\end{array}$ & \begin{tabular}{c|}
-20.4 \\
\end{tabular} & 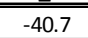 & $\begin{array}{l}-19.7 \\
\end{array}$ & $2-41.6$ & \begin{tabular}{c|c|}
-18.5 \\
\end{tabular} & $=-49.2$ & 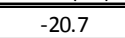 & $\begin{array}{c}-36.5 \\
\end{array}$ & 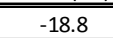 \\
\hline
\end{tabular}


Table S4. Adsorption, interaction and deformation energies' components of considered stacked nucleobases adsorbed complexes (in $\mathrm{kcal} / \mathrm{mol}$ ).

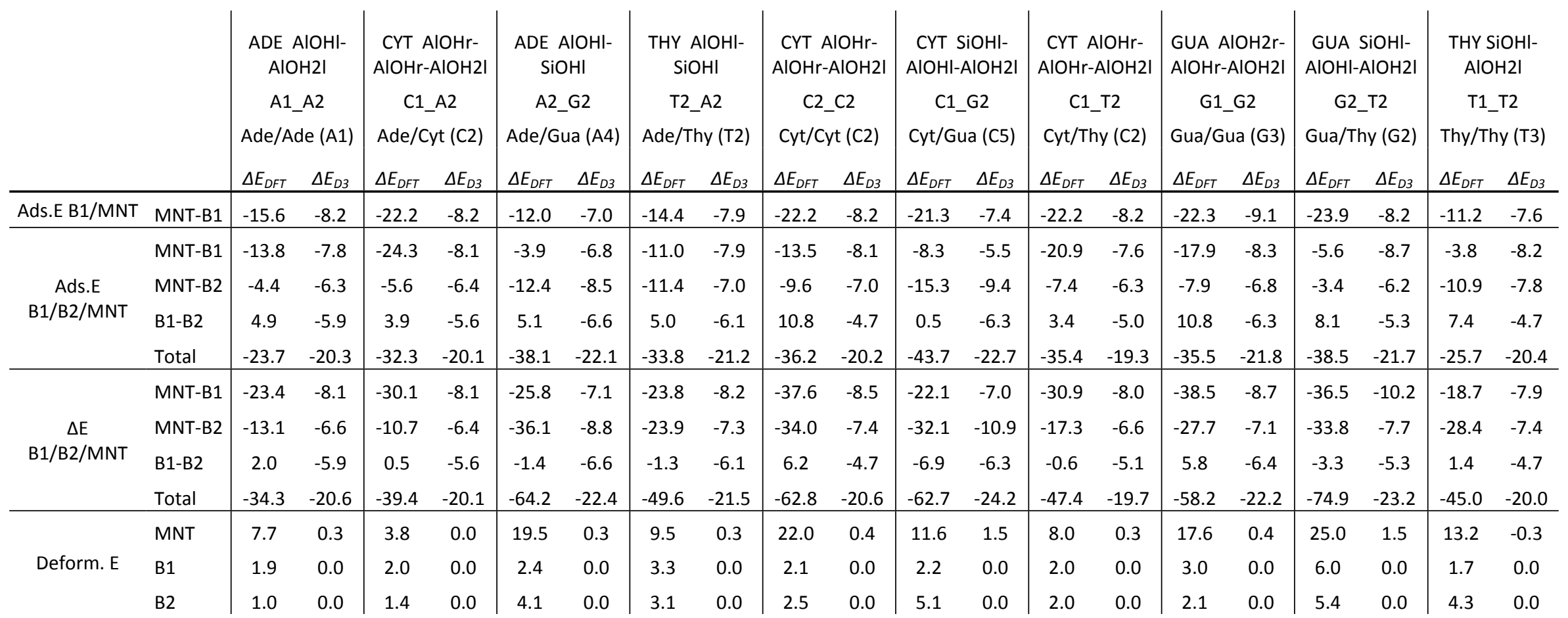


Relaxed structures provided as CONTCAR files:

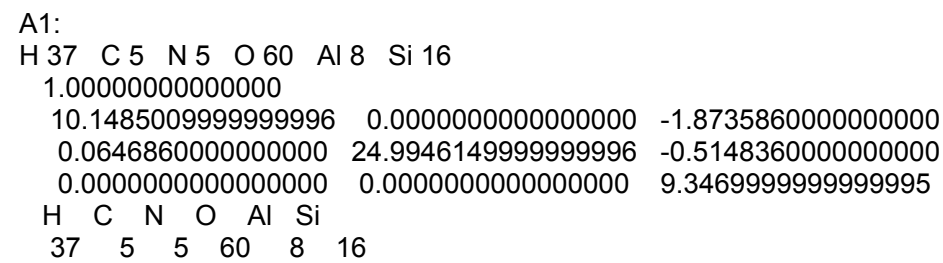


$\begin{array}{llll}0.0417633568107783 & 0.2888793966235328 & 0.6182465039674674\end{array}$ $\begin{array}{lllll}0.2907860185351140 & 0.2470817780258656 & 0.6159125548847931\end{array}$ $\begin{array}{lllll}0.2025629659560213 & 0.3215388546329327 & 0.8622298307370888\end{array}$ $\begin{array}{lllll}0.0315767787119268 & 0.2422800149290067 & 0.8685537421502096\end{array}$ $\begin{array}{llll}0.2411296741736083 & 0.3613408275483889 & 0.6134924237686081\end{array}$ $\begin{array}{llll}0.4589547618345062 & 0.1405802363934665 & 0.8833903186434241\end{array}$ $\begin{array}{lllllll}0.5087092340102043 & 0.1789510026623511 & 0.6323865643436771\end{array}$ $\begin{array}{lllll}0.4499251559636359 & 0.3425877870729688 & 0.8269884859785461\end{array}$ $\begin{array}{lllll}0.5929882851423877 & 0.3498083588695810 & 0.3758341898786531\end{array}$ $\begin{array}{lllll}0.5608789593759084 & 0.2405786328914806 & 0.3913536794729067\end{array}$ $\begin{array}{lllll}0.8118030988332915 & 0.2826916566767407 & 0.3937543400473656\end{array}$ $\begin{array}{llllll}0.9004470318601017 & 0.2096023600392714 & 0.1486148009322127\end{array}$ $\begin{array}{lllll}0.5699548952994741 & 0.2868970280390007 & 0.1399901044672029\end{array}$ $\begin{array}{llll}0.6543150469422826 & 0.1855102911051608 & 0.1828725036501749\end{array}$ $\begin{array}{lllll}0.8630393628039555 & 0.1687529645556049 & 0.3979230089734070\end{array}$ $\begin{array}{lllll}0.8595272851325161 & 0.0559157756174032 & 0.4082099752839648\end{array}$ $\begin{array}{lllll}0.5944509282947542 & 0.1097756264065274 & 0.4380323184155376\end{array}$ $\begin{array}{lllll}0.8229019005175713 & 0.1060329272888896 & 0.1677823265569115\end{array}$ $\begin{array}{lllll}0.7988039416374494 & 0.1154441999851983 & 0.6303356865396808\end{array}$ $\begin{array}{lllll}0.5434866238606213 & 0.0676315361349888 & 0.6706978469627198\end{array}$ $\begin{array}{lllll}0.7793437490761401 & 0.0652959601889324 & 0.8840383139249630\end{array}$ $\begin{array}{lllll}0.7095071814178530 & 0.1667149130792735 & 0.8499276601493190\end{array}$ $\begin{array}{lllll}0.5420861619927040 & 0.2878928298202511 & 0.6172763185492540\end{array}$ $\begin{array}{lllll}0.7909873066516324 & 0.2469073125985108 & 0.6149123887329460\end{array}$ $\begin{array}{lllll}0.7031823867711924 & 0.3207384491278653 & 0.8596457139116357\end{array}$ $\begin{array}{lllll}0.5334673027342794 & 0.2411343703865788 & 0.8682706675583247\end{array}$ $\begin{array}{llll}0.7413459652114346 & 0.3608906203472233 & 0.6097590137362942\end{array}$ $\begin{array}{llll}0.9597467141334859 & 0.1414609471742522 & 0.8829701517702624\end{array}$ $\begin{array}{llll}0.0091591592079354 & 0.1797044477983289 & 0.6323943989599607\end{array}$ 0.94929662705029290 .34431230111893390 .8262167038727539 $\begin{array}{lllll}0.3066672333948948 & 0.4133361188422404 & 0.3796602720195995\end{array}$ $\begin{array}{llllll}0.0661888784333013 & 0.4659083657150949 & 0.3422992722472874\end{array}$ $\begin{array}{lllll}0.1457422943508244 & 0.3903734541035437 & 0.1286473263889793\end{array}$ $\begin{array}{lllll}0.3308610933235616 & 0.4644075156169594 & 0.1303228502014600\end{array}$ $\begin{array}{lllll}0.3942224600746640 & 0.3617218318056498 & 0.1573101143582221\end{array}$ $\begin{array}{lllll}0.2381894980272102 & 0.4723461112029261 & 0.6009715186434242\end{array}$ $\begin{array}{lllll}0.0057241379490962 & 0.4198983790528792 & 0.5671129806965006\end{array}$ $\begin{array}{lllll}0.2898936435351601 & 0.4239073714239945 & 0.8462594542710768\end{array}$ $\begin{array}{llll}0.8045599000200979 & 0.4128006406220465 & 0.3754211415508434\end{array}$ $\begin{array}{llllll}0.5641998104912792 & 0.4617563353124987 & 0.3361883796124471\end{array}$ $\begin{array}{lllll}0.6444375666731686 & 0.3870288032717621 & 0.1235761943233549\end{array}$ $\begin{array}{lllll}0.8182326520529398 & 0.4650717342057165 & 0.1231722023609093\end{array}$ $\begin{array}{lllll}0.8963244328474856 & 0.3641055827433520 & 0.1550734459488776\end{array}$ $\begin{array}{lllll}0.7498386527344444 & 0.4736478521438212 & 0.5969892418106112\end{array}$ $\begin{array}{lllll}0.5135441217407182 & 0.4219246493113445 & 0.5722009433805368\end{array}$ $\begin{array}{lllll}0.7806167960548716 & 0.4241548721300936 & 0.8386689474500841\end{array}$ $\begin{array}{lllll}0.4266154646477462 & 0.2339269682253323 & 0.5081548990804382\end{array}$ $\begin{array}{lllll}0.1763201283146066 & 0.2963170131155050 & 0.5030783451593144\end{array}$ $\begin{array}{lllll}0.4455092343556410 & 0.1128422954417814 & 0.5390447114601289\end{array}$ $\begin{array}{llll}0.9278397414475001 & 0.2345575303269539 & 0.5079926642927900\end{array}$ 0.67525431539095320 .29520268194721650 .5012711896313885 $\begin{array}{lllll}0.9458223536357639 & 0.1135797381424914 & 0.5385330368415699\end{array}$ $\begin{array}{lllll}0.1616005320168412 & 0.4177705395239048 & 0.4748191691277659\end{array}$ $\begin{array}{lllll}0.6583717131260822 & 0.4160953673309149 & 0.4689686123115870\end{array}$ $\begin{array}{lllll}0.0476715433985173 & 0.2325437728204092 & 0.2151345652113784\end{array}$ $\begin{array}{lllll}0.3068237786544263 & 0.1681383475014756 & 0.2223973435499779\end{array}$ $\begin{array}{lllll}0.2960888073893055 & 0.3615395888895690 & 0.7895188796075880\end{array}$ $\begin{array}{lllll}0.3102899816496754 & 0.1214682462550845 & 0.8061811336549517\end{array}$ $\begin{array}{lllll}0.0527485878876831 & 0.1816369797012816 & 0.8100176413201052\end{array}$ $\begin{array}{lllll}0.0556570665332667 & 0.2988433077536927 & 0.7949095973918049\end{array}$ $\begin{array}{lllll}0.0523036307303956 & 0.3497827900283569 & 0.2000614066154855\end{array}$ $\begin{array}{lllll}0.5472523832581128 & 0.2306046395218497 & 0.2148412240361837\end{array}$ $\begin{array}{lllll}0.8096266121724073 & 0.1688447987352961 & 0.2218352072657256\end{array}$ $\begin{array}{lllll}0.7942031992980083 & 0.3612201610641971 & 0.7859817876735953\end{array}$ $\begin{array}{lllll}0.8120759263538584 & 0.1211586482961527 & 0.8054679398477064\end{array}$ $\begin{array}{lllll}0.5528358296231251 & 0.1803313533765252 & 0.8100488238566235\end{array}$ $\begin{array}{llllll}0.5560049983813714 & 0.2976526191896424 & 0.7938759231437762\end{array}$ $\begin{array}{llll}0.5506011613757730 & 0.3477339902980862 & 0.1979318863060790\end{array}$ $\begin{array}{lllll}0.2961358770250710 & 0.4082087910396562 & 0.2054203428010514\end{array}$ $\begin{array}{llll}0.7911512836069980 & 0.4080636818931313 & 0.2004966628221425\end{array}$

A2:

H 37 C 5 N 5 O 60 Al 8 Si 16 
1.00000000000000

$10.1485009999999996 \quad 0.0000000000000000 \quad-1.8735860000000000$

$\begin{array}{llll}0.0646860000000000 & 24.9946149999999996 & -0.5148360000000000\end{array}$

$\begin{array}{llll}0.0000000000000000 & 0.0000000000000000 & 9.3469999999999995\end{array}$

$\mathrm{H} \quad \mathrm{C} \quad \mathrm{N}$ O Al Si

$\begin{array}{llllll}37 & 5 & 5 & 60 & 8 & 16\end{array}$

Direct

0.26270835069830380 .76007936123927330 .5368339706141624

$\begin{array}{llll}0.4612698367231976 & 0.7725831632090284 & 0.4096804617904968\end{array}$

$\begin{array}{llll}0.1440004921012636 & 0.5763399105113080 & 0.6224087009861800\end{array}$

$\begin{array}{lllll}0.5011768965508899 & 0.5247052406843208 & 0.4029818651904519\end{array}$

$\begin{array}{llll}0.5596592784712640 & 0.5877181017221575 & 0.3679386225809878\end{array}$

$\begin{array}{llll}0.1798486223242969 & 0.0572965490509624 & 0.8215046968391821\end{array}$

$\begin{array}{llll}0.6815310214049970 & 0.0574417989525628 & 0.8197175851808639\end{array}$

$\begin{array}{llll}0.1013497341244102 & 0.1373541941425465 & 0.3522940632746294\end{array}$

$\begin{array}{lllll}0.3464429945715641 & 0.3108867487182086 & 0.3289986469672067\end{array}$

$\begin{array}{lllll}0.2610554757276771 & 0.2209659470007116 & 0.6658742943754951\end{array}$

$\begin{array}{lllll}0.5237631827023391 & 0.0306499293495558 & 0.6276505560563845\end{array}$

$\begin{array}{lllll}0.3445639539084133 & 0.0672148687343481 & 0.2913309856776746\end{array}$

$0.6006454249924524 \quad 0.1382295904494356 \quad 0.3544454150151204$

$\begin{array}{lllll}0.8406129433885430 & 0.3085829041976607 & 0.3175266200859360\end{array}$

$\begin{array}{lllll}0.7611364544390042 & 0.2204716002413661 & 0.6628965543371579\end{array}$

$\begin{array}{lllll}0.0179815414669260 & 0.0314415983010145 & 0.6295323049266609\end{array}$

$\begin{array}{lllll}0.8434961013200555 & 0.0682545506090866 & 0.2903103939172378\end{array}$

$\begin{array}{lllll}0.1127668570947858 & 0.4902370777973912 & 0.2964553810137300\end{array}$

$\begin{array}{lllll}0.5007108011623703 & 0.3958485582273907 & 0.6314966236708279\end{array}$

$\begin{array}{llll}0.2635521660741629 & 0.4645406606778208 & 0.6901157162430666\end{array}$

$\begin{array}{lllll}0.6213608596219904 & 0.4786938399740445 & 0.2698577886252510\end{array}$

$\begin{array}{lllll}0.9991706391060978 & 0.3868427937878209 & 0.6184553667348176\end{array}$

$\begin{array}{llll}0.7620338081677904 & 0.4617953430854362 & 0.6931931539658299\end{array}$ $\begin{array}{lllll}0.4002838469134540 & 0.4803864176439159 & 0.1578296228807125\end{array}$ $\begin{array}{lllll}0.9180176146801093 & 0.4724513248996982 & 0.1747158954955145\end{array}$ $\begin{array}{lllll}0.3068045508087113 & 0.4389309677083972 & 0.9308967155047358\end{array}$ $\begin{array}{llll}0.8113367650685794 & 0.4378141815585339 & 0.9308144081914616\end{array}$ $\begin{array}{lllll}0.2922311532467552 & 0.0908259566003275 & 0.0543854367275419\end{array}$ $\begin{array}{lllll}0.7925099630522416 & 0.0918796201037856 & 0.0531388023135183\end{array}$ $\begin{array}{lllll}0.2754040599852692 & 0.5082079678038938 & 0.5562273022073837\end{array}$ $\begin{array}{lllll}0.8399265833756547 & 0.4779032646492360 & 0.5697994357054326\end{array}$ $\begin{array}{lllll}0.2713658856086600 & 0.0485572747136231 & 0.4171105244787132\end{array}$ $\begin{array}{llllll}0.7676678375482276 & 0.0505489431263664 & 0.4144330274971659\end{array}$ $\begin{array}{lllll}0.4258828812873263 & 0.4204474654388671 & 0.4795721099926789\end{array}$ $\begin{array}{lllll}0.9236414572099592 & 0.4138066860507249 & 0.4718497284484746\end{array}$ $\begin{array}{llll}0.6754125577858633 & 0.1143613029197364 & 0.5081464283643411\end{array}$ $\begin{array}{lllll}0.1747071326622955 & 0.1135863952283820 & 0.5067685441421521\end{array}$ $\begin{array}{llll}0.4247835885648745 & 0.7339714686939335 & 0.4359779807180401\end{array}$ $\begin{array}{lllll}0.2157785831330259 & 0.5993695622432997 & 0.5763587054452999\end{array}$ $0.29722148710197780 .6760104353233852 \quad 0.5206583728261102$ $\begin{array}{lllll}0.3963554274635018 & 0.6505344974205591 & 0.4602403115260532\end{array}$ $\begin{array}{lllll}0.3999805739921637 & 0.5938014403037796 & 0.4621656492915583\end{array}$ $\begin{array}{lllll}0.2036982638709762 & 0.6524468694345278 & 0.5807226857376464\end{array}$ $\begin{array}{lllll}0.3172681855215657 & 0.7297915931541437 & 0.5040582979048552\end{array}$ $\begin{array}{llll}0.4751918106827093 & 0.6871400249914922 & 0.4076980929372805\end{array}$ $\begin{array}{lllll}0.3060366211993336 & 0.5692793063625478 & 0.5221591909061274\end{array}$ $\begin{array}{lllll}0.4913160021658604 & 0.5660067322493167 & 0.4070227540254441\end{array}$ $\begin{array}{lllll}0.0959916453393433 & 0.3506437450012662 & 0.3646811617372600\end{array}$ $\begin{array}{lllll}0.0614258581815074 & 0.2417146630434466 & 0.3793228260787950\end{array}$ $\begin{array}{lllll}0.3127156460170404 & 0.2827205417825594 & 0.3820378102070223\end{array}$ $\begin{array}{lllll}0.4000255266006445 & 0.2075940416137684 & 0.1355039923066414\end{array}$ $\begin{array}{lllll}0.0737736102979851 & 0.2877385901252306 & 0.1286110111849177\end{array}$ $\begin{array}{lllll}0.1531391404515695 & 0.1855406950320583 & 0.1711317608457878\end{array}$ $\begin{array}{lllll}0.3611086516480618 & 0.1681965711574030 & 0.3858472720427561\end{array}$ $\begin{array}{lllll}0.3595099953162585 & 0.0548310695725927 & 0.3963516263553937\end{array}$ $\begin{array}{llll}0.0924420890812550 & 0.1095973650280344 & 0.4220333511695816\end{array}$ $\begin{array}{lllll}0.3178472555830309 & 0.1048449292441329 & 0.1563119217501302\end{array}$ $\begin{array}{llll}0.2965231928633087 & 0.1149795255932945 & 0.6162963889556300\end{array}$ $\begin{array}{lllll}0.0373971528273886 & 0.0685243335380615 & 0.6573531528192583\end{array}$ $\begin{array}{lllll}0.2709509377897866 & 0.0659539266685031 & 0.8732861146243764\end{array}$ $\begin{array}{lllll}0.2095799994212531 & 0.1671623019492675 & 0.8350042445144258\end{array}$ $\begin{array}{lllll}0.0420310995071507 & 0.2889735873164015 & 0.6056246138870827\end{array}$ $\begin{array}{lllll}0.2908053901094522 & 0.2469220234532745 & 0.6030712933199215\end{array}$ $\begin{array}{llllll}0.2028104200144258 & 0.3220671716537186 & 0.8485400397168281\end{array}$ $\begin{array}{llll}0.0342516748793529 & 0.2417336682222123 & 0.8561439457493720\end{array}$ $\begin{array}{llll}0.2423375157613177 & 0.3616776186679329 & 0.5989736312027196\end{array}$ 
$\begin{array}{llll}0.4586913887651612 & 0.1397549025174308 & 0.8686573039398640\end{array}$ $\begin{array}{llllll}0.5085053609686944 & 0.1786787212324017 & 0.6202630170954208\end{array}$ $\begin{array}{lllll}0.4498037240887273 & 0.3433364755661785 & 0.8135725740008827\end{array}$ $\begin{array}{llll}0.5916174908835018 & 0.3500546288004852 & 0.3648130091100295\end{array}$ $\begin{array}{llll}0.5607136634357932 & 0.2408283188850462 & 0.3786056176139874\end{array}$ $\begin{array}{lllll}0.8112721799378011 & 0.2827320549882861 & 0.3808906010076426\end{array}$ $\begin{array}{lllll}0.9010829660901647 & 0.2089855854362822 & 0.1356125903543671\end{array}$ $\begin{array}{lllll}0.5656251299967876 & 0.2885551552659493 & 0.1277130583075881\end{array}$ $\begin{array}{llll}0.6536357975053511 & 0.1871510816291536 & 0.1679677843924910\end{array}$ $\begin{array}{lllll}0.8610784133074905 & 0.1689739180211706 & 0.3845273168059277\end{array}$ $\begin{array}{lllll}0.8567390421137590 & 0.0559801181576668 & 0.3952754558829465\end{array}$ $\begin{array}{lllll}0.5931620651402497 & 0.1097841088053148 & 0.4226583304992816\end{array}$ $\begin{array}{llll}0.8181618802817052 & 0.1060846264593149 & 0.1547896093076636\end{array}$ $\begin{array}{lllll}0.7957661520237913 & 0.1167670556047220 & 0.6158986776718668\end{array}$ $0.5399473507572625 \quad 0.06771915663348590 .6579017039655383$ $\begin{array}{llllll}0.7724949280150434 & 0.0663335758471029 & 0.8719091896912252\end{array}$ $\begin{array}{lllll}0.7090239127467494 & 0.1674338226501881 & 0.8367477006837043\end{array}$ $\begin{array}{lllll}0.5421976625905761 & 0.2874884092666581 & 0.6055201095867209\end{array}$ $\begin{array}{llll}0.7913956637679839 & 0.2471089946453944 & 0.6023759427352418\end{array}$ $0.70227684763249250 .3204803024287856 \quad 0.8486141732183523$ $\begin{array}{lllll}0.5306861568742999 & 0.2413127466203127 & 0.8562536442185860\end{array}$ $\begin{array}{lllll}0.7405393731796682 & 0.3607465309361760 & 0.5992753039803540\end{array}$ $\begin{array}{llllll}0.9587034352586349 & 0.1408624059771287 & 0.8680415925237646\end{array}$ $\begin{array}{lllll}0.0090463402689566 & 0.1794438856572212 & 0.6190117080671083\end{array}$ $\begin{array}{lllll}0.9487362794185404 & 0.3429718289499953 & 0.8154289100882786\end{array}$ $\begin{array}{lllll}0.3043977507652747 & 0.4160476987652307 & 0.3656063898797420\end{array}$ $\begin{array}{lllll}0.0581422275591589 & 0.4622104758383513 & 0.3246625310180941\end{array}$ $\begin{array}{lllll}0.1431286393732113 & 0.3888286216907703 & 0.1133202498658166\end{array}$ $\begin{array}{lllll}0.3146063691346224 & 0.4662236303471073 & 0.1084147308148812\end{array}$ $\begin{array}{llllll}0.3935925936005011 & 0.3653308304632997 & 0.1445717184773051\end{array}$ $\begin{array}{lllll}0.2332041553820563 & 0.4737701364976706 & 0.5860949278542371\end{array}$ $\begin{array}{llll}0.0053513922990252 & 0.4174019245321302 & 0.5566851009602060\end{array}$ $\begin{array}{lllll}0.2889483205443905 & 0.4247021007587498 & 0.8293981896145253\end{array}$ $\begin{array}{lllll}0.8029417622695887 & 0.4127630274074202 & 0.3645653244383988\end{array}$ $\begin{array}{lllll}0.5639445164966941 & 0.4628806074362041 & 0.3291315558179648\end{array}$ $\begin{array}{lllll}0.6439916933957101 & 0.3886429000186381 & 0.1139093930133213\end{array}$ $\begin{array}{lllll}0.8297178083276654 & 0.4627764755121736 & 0.1147698796674924\end{array}$ $\begin{array}{lllll}0.8941354503665885 & 0.3604083937328545 & 0.1475542643498636\end{array}$ $\begin{array}{lllll}0.7502965604944976 & 0.4731245837148041 & 0.5880707973318249\end{array}$ $\begin{array}{llll}0.5115157094794481 & 0.4222208740533989 & 0.5590991743245279\end{array}$ $\begin{array}{lllll}0.7829893819154378 & 0.4235214824131860 & 0.8299865738244516\end{array}$ $\begin{array}{lllll}0.4269708468015489 & 0.2338670385058797 & 0.4956426222635827\end{array}$ $\begin{array}{lllll}0.1768889037491838 & 0.2962155725288165 & 0.4902297155399803\end{array}$ $\begin{array}{lllll}0.4440543154870402 & 0.1133318574261501 & 0.5262089889236925\end{array}$ $\begin{array}{llll}0.9275475600458630 & 0.2346733957561593 & 0.4947387281286000\end{array}$ $\begin{array}{lllll}0.6750253836733324 & 0.2951911931509972 & 0.4889224923526298\end{array}$ $\begin{array}{llllll}0.9429923063531946 & 0.1142311696622663 & 0.5248348409076081\end{array}$ $\begin{array}{lllll}0.1581949436358780 & 0.4180182071843712 & 0.4597574885954064\end{array}$ $\begin{array}{lllll}0.6588895103958526 & 0.4157503195385739 & 0.4603373030169879\end{array}$ $\begin{array}{lllll}0.0483635828426603 & 0.2316238181291140 & 0.2029725084012916\end{array}$ $\begin{array}{lllll}0.3078093296667617 & 0.1678908591043564 & 0.2097830769706868\end{array}$ $\begin{array}{lllll}0.2957173414516555 & 0.3620629589966376 & 0.7751054969215216\end{array}$ $\begin{array}{llllll}0.3100140237740619 & 0.1201465742582414 & 0.7914643853266126\end{array}$ $\begin{array}{lllll}0.0529997615301992 & 0.1810505325679422 & 0.7968803354817277\end{array}$ $\begin{array}{lllll}0.0564810314664118 & 0.2984654490469961 & 0.7826479492804170\end{array}$ $\begin{array}{lllll}0.0521274185901137 & 0.3483765624782126 & 0.1874070585696960\end{array}$ $\begin{array}{llll}0.5460158021979667 & 0.2317068204702885 & 0.2016761975042724\end{array}$ $\begin{array}{lllll}0.8079224238675961 & 0.1691101978859331 & 0.2083491553130554\end{array}$ $\begin{array}{lllll}0.7944094922668452 & 0.3607247475896169 & 0.7755602648282436\end{array}$ $\begin{array}{llll}0.8102048233943412 & 0.1209960210562176 & 0.7913905418398406\end{array}$ $\begin{array}{lllll}0.5520697689976749 & 0.1806204096788000 & 0.7982431762621337\end{array}$ $\begin{array}{lllll}0.5550148775582201 & 0.2977409272826251 & 0.7818544757000613\end{array}$ $\begin{array}{llllll}0.5484325843800087 & 0.3494657768324054 & 0.1869625246012628\end{array}$ $\begin{array}{lllll}0.2900413565381766 & 0.4099813156909941 & 0.1912113048793980\end{array}$ $\begin{array}{llll}0.7935444637612016 & 0.4068923407791501 & 0.1902099107480208\end{array}$

A3:

H 37 C 5 N 5 O 60 Al 8 Si 16

1.00000000000000

$\begin{array}{llll}10.1485009999999996 & 0.0000000000000000 & -1.8735860000000000\end{array}$

$0.0646860000000000 \quad 24.9946149999999996 \quad-0.5148360000000000$

$\begin{array}{lll}0.0000000000000000 & 0.0000000000000000 & 9.3469999999999995\end{array}$

$\begin{array}{lllllll}\mathrm{H} & \mathrm{C} & \mathrm{N} & \mathrm{O} & \mathrm{Al} & \mathrm{Si}\end{array}$ 
Direct

$\begin{array}{lllll}5 & 5 & 60 & 8 & 16\end{array}$

$\begin{array}{llll}0.2344511475255351 & 0.7440940475912919 & 0.1264270754569161\end{array}$

$\begin{array}{lllll}0.4757991648384139 & 0.7665532053249910 & 0.1180801006607546\end{array}$ $\begin{array}{llllll}0.1300390991910866 & 0.5565830807541441 & 0.1818099755747651\end{array}$ $\begin{array}{llll}0.5671040120236865 & 0.5216079141674289 & 0.2244586580343720\end{array}$ $\begin{array}{llllll}0.6378097273971844 & 0.5828393664287368 & 0.1825396185886418\end{array}$ $\begin{array}{llllll}0.1805309344521695 & 0.0559203234584617 & 0.8119545890051071\end{array}$ $\begin{array}{lllll}0.6759983942220187 & 0.0579363662547094 & 0.8102914237140002\end{array}$ $\begin{array}{lllll}0.0940297763797515 & 0.1403195634132497 & 0.3574002582998183\end{array}$ $\begin{array}{lllll}0.3287512124130894 & 0.3083639668321501 & 0.3094536811049216\end{array}$ $\begin{array}{llll}0.2523945575027180 & 0.2226142551842962 & 0.6613825958118941\end{array}$ $\begin{array}{lllll}0.4784421699010368 & 0.0406870332224567 & 0.6811374527511206\end{array}$ $\begin{array}{llll}0.3370530838237618 & 0.0684400850226800 & 0.2864172120611532\end{array}$ $\begin{array}{llllll}0.5944109517779950 & 0.1425134897893065 & 0.3616528339804798\end{array}$ $\begin{array}{lllll}0.8263694352850064 & 0.3073228396658472 & 0.3053115210160759\end{array}$ $\begin{array}{llllll}0.7530388456235223 & 0.2236415956183701 & 0.6639248935066482\end{array}$ $\begin{array}{llll}-0.0223680384307616 & 0.0423392829037025 & 0.6866457808009552\end{array}$ $\begin{array}{llll}0.8314360345884423 & 0.0680808284199626 & 0.2885503567670514\end{array}$ $\begin{array}{llllll}0.0268888386455931 & 0.4951289456252806 & 0.3677541277665314\end{array}$ $\begin{array}{llll}0.4869063983541697 & 0.3925760480667733 & 0.6307483412223279\end{array}$ $\begin{array}{llll}0.2448509945478202 & 0.4627284469876650 & 0.6830743426143107\end{array}$ $\begin{array}{lllll}0.4712887018533875 & 0.4754714388043700 & 0.3493226362820018\end{array}$ $\begin{array}{lllll}0.9909140508000392 & 0.3927336274199408 & 0.6218171857419842\end{array}$ $\begin{array}{llllll}0.7500974110459143 & 0.4618730073501588 & 0.6955082166312043\end{array}$ $\begin{array}{lllll}0.3142434341023493 & 0.5002313084368121 & 0.1275635588618962\end{array}$ $\begin{array}{lllll}0.9170334037160420 & 0.4697514778312011 & 0.1582883063289102\end{array}$ $\begin{array}{lllll}0.2854596602246595 & 0.4419260317669552 & 0.9184716606783401\end{array}$ $\begin{array}{lllll}0.8027699541292292 & 0.4382598405851675 & 0.9292468514353028\end{array}$ $\begin{array}{lllll}0.2868484825307913 & 0.0917347990126482 & 0.0496720677273209\end{array}$ $\begin{array}{lllll}0.7782090482157292 & 0.0914016202769337 & 0.0505163823124877\end{array}$ $\begin{array}{lllll}0.3086254950587026 & 0.4873454899301038 & 0.5546285206975651\end{array}$ $\begin{array}{lllll}0.8088475752773020 & 0.4870266325452163 & 0.5646632837895342\end{array}$ $\begin{array}{lllll}0.2644230253107068 & 0.0485063992186099 & 0.4114734501061051\end{array}$ $\begin{array}{llllll}0.7640314473361494 & 0.0476551143868366 & 0.4169597775463233\end{array}$ $\begin{array}{llll}0.4130899259620527 & 0.4139470024698982 & 0.4749041990368082\end{array}$ $\begin{array}{lllll}0.9133982248126277 & 0.4173425547203347 & 0.4704575585625210\end{array}$ $\begin{array}{llllll}0.6693961818840747 & 0.1141546385864524 & 0.5071861989100337\end{array}$ $\begin{array}{llll}0.1683884355015222 & 0.1134985549324870 & 0.5057887460031083\end{array}$ $\begin{array}{llll}0.4399314442559402 & 0.7264744431596453 & 0.1311977306252823\end{array}$ $\begin{array}{lllll}0.2126666596307427 & 0.5831969139690222 & 0.1759064953534876\end{array}$ $\begin{array}{llll}0.2967006513762392 & 0.6627408505028555 & 0.1520401200893956\end{array}$ $\begin{array}{lllll}0.4245251546487043 & 0.6423792898733037 & 0.1576403166950270\end{array}$ $\begin{array}{lllll}0.4411221888878349 & 0.5864070367291827 & 0.1760517924666918\end{array}$ $\begin{array}{lllll}0.1868240987685631 & 0.6352844342037143 & 0.1617119587782942\end{array}$ $\begin{array}{lllll}0.3090061697822061 & 0.7169483116161735 & 0.1350875832528433\end{array}$ $\begin{array}{llll}0.5127554840221594 & 0.6826734721319481 & 0.1447846548275342\end{array}$ $\begin{array}{lllll}0.3299996043615385 & 0.5580839572672197 & 0.1829947153177376\end{array}$ $\begin{array}{lllll}0.5563217739668606 & 0.5606236253586365 & 0.1851689734178389\end{array}$ $\begin{array}{lllll}0.0846793126094555 & 0.3505870542597370 & 0.3586642239036074\end{array}$ $\begin{array}{lllll}0.0506351713226748 & 0.2410561958154861 & 0.3742161458677975\end{array}$ $\begin{array}{lllll}0.3010087552270579 & 0.2826901660229568 & 0.3744245692685860\end{array}$ $\begin{array}{lllll}0.3900190027099960 & 0.2095876248269086 & 0.1308388913540533\end{array}$ $\begin{array}{llll}0.0560784938978504 & 0.2869929900753478 & 0.1224863664738525\end{array}$ $\begin{array}{lllll}0.1446366141609760 & 0.1858875151599102 & 0.1657541096375710\end{array}$ $\begin{array}{lllll}0.3527228781050898 & 0.1689284394044108 & 0.3809561719089658\end{array}$ $\begin{array}{llll}0.3520629738395402 & 0.0560949517872156 & 0.3912186447807391\end{array}$ $\begin{array}{llll}0.0850792157796399 & 0.1107985191297817 & 0.4218086666417270\end{array}$ $\begin{array}{llll}0.3123467686361969 & 0.1060925385691759 & 0.1509024564855165\end{array}$ $\begin{array}{lllll}0.2901329006749832 & 0.1150404283339389 & 0.6134370179725531\end{array}$ $\begin{array}{lllll}0.0337746771776824 & 0.0689860838805552 & 0.6548960580892966\end{array}$ $\begin{array}{lllll}0.2691433690700292 & 0.0661157290494548 & 0.8678623868810170\end{array}$ 0.20046576148179630 .16760706918303590 .8309401999681555 $\begin{array}{lllll}0.0320497002379194 & 0.2895033395987930 & 0.5989538474546526\end{array}$ $\begin{array}{lllll}0.2813295549871081 & 0.2482815308554713 & 0.5973407107009340\end{array}$ $\begin{array}{llll}0.1927910928482335 & 0.3236272044753451 & 0.8398378742449921\end{array}$ $\begin{array}{lllll}0.0270814864433910 & 0.2427002209157815 & 0.8507880280882619\end{array}$ $\begin{array}{lllll}0.2328823132487121 & 0.3624787599369131 & 0.5883814037309867\end{array}$ $\begin{array}{lllll}0.4505065426656907 & 0.1419571149088875 & 0.8658161254366207\end{array}$ $\begin{array}{lllll}0.4984052834586455 & 0.1804078988380505 & 0.6152879338168588\end{array}$ $\begin{array}{lllll}0.4390573988205406 & 0.3457073254131490 & 0.8052830094366926\end{array}$ $\begin{array}{lllll}0.5821985150379485 & 0.3514905747868576 & 0.3601900641163980\end{array}$ $\begin{array}{lllll}0.5509249802031468 & 0.2420362862244962 & 0.3737120011391599\end{array}$ 
$\begin{array}{llll}0.8008167806362403 & 0.2830676915266052 & 0.3753740263298838\end{array}$ $\begin{array}{llll}0.8910828992778911 & 0.2067021111692592 & 0.1311581761867176\end{array}$ $\begin{array}{lllll}0.5563736582886680 & 0.2904749262386199 & 0.1235064189527839\end{array}$ $\begin{array}{llll}0.6435090842389801 & 0.1886598762957702 & 0.1633076212693730\end{array}$ $\begin{array}{lllll}0.8496188146493003 & 0.1687659537886397 & 0.3817620165238472\end{array}$ $\begin{array}{lllll}0.8501753374590949 & 0.0558610686204183 & 0.3935270078448584\end{array}$ $\begin{array}{lllll}0.5863867477559577 & 0.1118030486290614 & 0.4224271831425639\end{array}$ $\begin{array}{lllll}0.8017226622639708 & 0.1052852081562475 & 0.1526500909126240\end{array}$ $\begin{array}{llll}0.7895922208003875 & 0.1155087133505592 & 0.6154135625415450\end{array}$ $\begin{array}{lllll}0.5340984106698786 & 0.0684452138888086 & 0.6537106941386487\end{array}$ $\begin{array}{lllll}0.7639603019172805 & 0.0679440689797912 & 0.8691731567676155\end{array}$ $\begin{array}{llllll}0.7000505751833751 & 0.1698809787178974 & 0.8309320024451996\end{array}$ $\begin{array}{lllll}0.5316931981866959 & 0.2891082183180536 & 0.5998028023773612\end{array}$ $\begin{array}{lllll}0.7815590753569409 & 0.2487968977496037 & 0.5982277441283134\end{array}$ $\begin{array}{llll}0.6917108697414958 & 0.3218520216998305 & 0.8434468224187450\end{array}$ $\begin{array}{lllll}0.5199396360509411 & 0.2431776737789042 & 0.8508676428293198\end{array}$ $\begin{array}{llll}0.7304830701432398 & 0.3623350677297632 & 0.5943497514369341\end{array}$ $\begin{array}{lllll}0.9496819829529011 & 0.1425601218607830 & 0.8667025510761818\end{array}$ $\begin{array}{llll}-0.0014989709324283 & 0.1803495775293595 & 0.6148941765910144\end{array}$ $\begin{array}{lllll}0.9387490357668252 & 0.3435361838668445 & 0.8091867263397954\end{array}$ $\begin{array}{lllll}0.2909408243857823 & 0.4189836837989088 & 0.3525057688590764\end{array}$ $\begin{array}{lllll}0.0486153675245954 & 0.4633269478634254 & 0.3153244340487371\end{array}$ $\begin{array}{lllll}0.1253775926724518 & 0.3874920603556538 & 0.1078407781848412\end{array}$ $\begin{array}{lllll}0.2938749804341567 & 0.4621617739881198 & 0.0795826413993746\end{array}$ $\begin{array}{lllll}0.3791576946406741 & 0.3645431665460703 & 0.1403409010828938\end{array}$ $\begin{array}{llll}0.2246972197870615 & 0.4761000907209432 & 0.5779674487308052\end{array}$ $\begin{array}{llll}-0.0018519562124521 & 0.4206280829591715 & 0.5521151508946651\end{array}$ $\begin{array}{llll}0.2724744887506205 & 0.4265872855282505 & 0.8148786688603414\end{array}$ $\begin{array}{lllll}0.7908590249628854 & 0.4175904306424882 & 0.3630589689875068\end{array}$ $\begin{array}{llll}0.5555123616525350 & 0.4636549524320654 & 0.3276664308375888\end{array}$ $\begin{array}{lllll}0.6309727300885404 & 0.3913451419473274 & 0.1110910461351304\end{array}$ $\begin{array}{llll}0.8248738043395442 & 0.4621848041722839 & 0.1049293324674035\end{array}$ $\begin{array}{lllll}0.8773599379412503 & 0.3600889270164577 & 0.1510262541589151\end{array}$ $\begin{array}{llll}0.7269707438550135 & 0.4749470126654439 & 0.5912749129805976\end{array}$ $\begin{array}{llll}0.4939375781822955 & 0.4191857115734308 & 0.5572861290254492\end{array}$ $\begin{array}{lllll}0.7761802201218840 & 0.4242979893104077 & 0.8269345332163580\end{array}$ $\begin{array}{llll}0.4172958836403922 & 0.2350401901069966 & 0.4896653772411201\end{array}$ $\begin{array}{lllll}0.1658802431697225 & 0.2963231079012523 & 0.4830891789259975\end{array}$ $\begin{array}{lllll}0.4371004444782875 & 0.1139808526477309 & 0.5217967743049835\end{array}$ $\begin{array}{llllll}0.9165171500275037 & 0.2351163044211141 & 0.4898855282367164\end{array}$ $\begin{array}{lllll}0.6650222359333915 & 0.2966940878142842 & 0.4832172920670856\end{array}$ $\begin{array}{lllll}0.9359230164010804 & 0.1139607636529126 & 0.5223536180687993\end{array}$ $\begin{array}{lllll}0.1465673368474433 & 0.4174684217803070 & 0.4474318949408208\end{array}$ $\begin{array}{lllll}0.6465695796850566 & 0.4175750805424961 & 0.4571165119583691\end{array}$ $\begin{array}{lllll}0.0368806292190850 & 0.2305177299454594 & 0.1979109135143297\end{array}$ $\begin{array}{lllll}0.2997229401989095 & 0.1689188743954043 & 0.2048457923718517\end{array}$ $\begin{array}{lllll}0.2842042641718627 & 0.3636866454208591 & 0.7650147179811343\end{array}$ $\begin{array}{lllll}0.3028288251688125 & 0.1216382315452502 & 0.7882873939747390\end{array}$ $\begin{array}{lllll}0.0440359373159590 & 0.1817541292127185 & 0.7923134398030434\end{array}$ $\begin{array}{llll}0.0467614621121442 & 0.2993056353168793 & 0.7756379012956879\end{array}$ $\begin{array}{lllll}0.0350782887217860 & 0.3473800211503759 & 0.1820459474787869\end{array}$ $\begin{array}{lllll}0.5365027957992325 & 0.2334828993420393 & 0.1964859366759300\end{array}$ $\begin{array}{lllll}0.7958121434787060 & 0.1684985401111496 & 0.2056759345124049\end{array}$ $\begin{array}{lllll}0.7846945721100693 & 0.3617624348216386 & 0.7703731969295993\end{array}$ $\begin{array}{llllll}0.8012771600411569 & 0.1229813048160371 & 0.7899770126926242\end{array}$ $\begin{array}{llll}0.5425791281491733 & 0.1825404566322960 & 0.7928018086455140\end{array}$ $\begin{array}{lllll}0.5443191937815085 & 0.2994861742450314 & 0.7760569478332158\end{array}$ $\begin{array}{lllll}0.5359492475023983 & 0.3514959242080535 & 0.1824286611850731\end{array}$ $\begin{array}{lllll}0.2736089782887394 & 0.4106851907640318 & 0.1767612163357534\end{array}$ $\begin{array}{lllll}0.7801820546540762 & 0.4094949259158460 & 0.1887632229686064\end{array}$

A4 :

H 37 C 5 N 5 O 60 Al 8 Si 16

1.00000000000000

$\begin{array}{llll}10.1485009999999996 & 0.0000000000000000 & -1.8735860000000000\end{array}$

$0.0646860000000000 \quad 24.9946149999999996 \quad-0.5148360000000000$

$\begin{array}{lll}0.0000000000000000 & 0.0000000000000000 & 9.3469999999999995\end{array}$

$\mathrm{H} \mathrm{C} \mathrm{N} \mathrm{O}$ Al Si

$\begin{array}{llllll}37 & 5 & 5 & 60 & 8 & 16\end{array}$

Direct

$\begin{array}{llll}0.4046806716737507 & 0.7511198752192082 & 0.1738917236625133\end{array}$

$\begin{array}{llll}0.2874963598790013 & 0.7676849204469787 & 0.3911674824063073\end{array}$

$0.40706956591283430 .5677860675368058-0.0146277485400818$ 
$\begin{array}{llll}0.1566558305284729 & 0.5245415598459824 & 0.3323143277847083\end{array}$ $\begin{array}{lllll}0.1569389623212138 & 0.5853458846663645 & 0.4296989549101310\end{array}$ $\begin{array}{lllll}0.1865191024482764 & 0.0567441036936530 & 0.8207578522305697\end{array}$ $\begin{array}{llll}0.6923978992146403 & 0.0549640450740628 & 0.8230937812384206\end{array}$ $\begin{array}{lllll}0.1057741668407214 & 0.1398361450913171 & 0.3681110773753104\end{array}$ $\begin{array}{lllll}0.3399901410588926 & 0.3077030601882190 & 0.3224692123772207\end{array}$ $\begin{array}{lllll}0.2651406098354151 & 0.2215178329194878 & 0.6754864834048849\end{array}$ $\begin{array}{lllll}0.4905086456373078 & 0.0408394367355462 & 0.6965592649900993\end{array}$ $\begin{array}{llll}0.3480666520214670 & 0.0674797405365740 & 0.2988409644833411\end{array}$ $\begin{array}{llll}0.6061017395142818 & 0.1418600813273963 & 0.3739997827676560\end{array}$ $\begin{array}{lllll}0.8386753289549398 & 0.3059992711618334 & 0.3165895162341195\end{array}$ $\begin{array}{llll}0.7636795573570810 & 0.2218637004946639 & 0.6733603886086887\end{array}$ $\begin{array}{llllll}-0.0103134405655813 & 0.0393711130733184 & 0.6904798491359126\end{array}$ $\begin{array}{llll}0.8435093876984407 & 0.0680175794668105 & 0.2978431355940069\end{array}$ $\begin{array}{llll}-0.0108306183524796 & 0.4802964560152517 & 0.3830790367054389\end{array}$ $\begin{array}{lllll}0.4889064101347753 & 0.3825515384023990 & 0.6099764168097742\end{array}$ $\begin{array}{llllll}0.2619723348008658 & 0.4606085109436417 & 0.7024223776608389\end{array}$ $\begin{array}{lllll}0.5838315275784430 & 0.4800834850970598 & 0.2699699274486062\end{array}$ $\begin{array}{lllll}1.0030226713642514 & 0.3922257923654948 & 0.6467101269749624\end{array}$ $\begin{array}{lllll}0.7526237617641415 & 0.4611626462152694 & 0.7036656844169097\end{array}$ $\begin{array}{lllll}0.3009820067722025 & 0.5038810750522043 & 0.1244114306522433\end{array}$ $\begin{array}{lllll}0.9239553655783935 & 0.4701380560102503 & 0.1562571666584670\end{array}$ $\begin{array}{lllll}0.2967331357824312 & 0.4405323846985776 & 0.9350827062333222\end{array}$ $\begin{array}{llll}0.8087571312927253 & 0.4376518785760315 & 0.9380964916356040\end{array}$ 0.29461008785236690 .09102288240458640 .0618991124938161 $\begin{array}{lllll}0.7917328205263755 & 0.0913716327853783 & 0.0610212287124881\end{array}$ $\begin{array}{lllll}0.3145167901961997 & 0.4881065865817605 & 0.5680946025148744\end{array}$ $\begin{array}{llll}0.8161527545241657 & 0.4857195761823022 & 0.5767079976898928\end{array}$ $\begin{array}{lllll}0.2772540289022374 & 0.0471464512568860 & 0.4249976506631438\end{array}$ $0.77435229696289790 .0477732291563640 \quad 0.4248805130602743$ $\begin{array}{lllll}0.4236608040204595 & 0.4196614062005707 & 0.4796835561377534\end{array}$ $\begin{array}{lllll}0.9241179197461020 & 0.4129319678571437 & 0.4909304912373834\end{array}$ $\begin{array}{lllll}0.6810460804158265 & 0.1130160288094456 & 0.5185460241110396\end{array}$ $\begin{array}{llll}0.1799137135165119 & 0.1128245238351678 & 0.5163182871204520\end{array}$ $\begin{array}{lllll}0.2956626769909024 & 0.7287825273823597 & 0.3393287583437469\end{array}$ $\begin{array}{lllll}0.3718841062751219 & 0.5919346624321671 & 0.0695772491328991\end{array}$ $\begin{array}{lllll}0.3522918151634608 & 0.6689338010746405 & 0.1835377656674461\end{array}$ $\begin{array}{lllll}0.2820838259947544 & 0.6458586162414638 & 0.2815283975129760\end{array}$ $\begin{array}{lllll}0.2574151967798285 & 0.5901795203802882 & 0.2647815132807146\end{array}$ $\begin{array}{llll}0.4000773001741355 & 0.6439671667891125 & 0.0751527156542516\end{array}$ $\begin{array}{lllll}0.3599890982298261 & 0.7223911768262261 & 0.2227969512531282\end{array}$ $\begin{array}{lllll}0.2472753257966971 & 0.6836743626549209 & 0.3779724280313292\end{array}$ $\begin{array}{llllll}0.3048928694315288 & 0.5644785481207084 & 0.1556539652368032\end{array}$ $\begin{array}{lllll}0.1916549638841252 & 0.5634524161322975 & 0.3519516265703707\end{array}$ $\begin{array}{llllll}0.0965892031632157 & 0.3498161338633969 & 0.3746210906471878\end{array}$ $\begin{array}{lllll}0.0632168807399637 & 0.2403901392351360 & 0.3868659077922334\end{array}$ $\begin{array}{lllll}0.3133440144283595 & 0.2821234370902024 & 0.3883682899449311\end{array}$ $\begin{array}{lllll}0.4014827190271006 & 0.2083623458775763 & 0.1431812821196291\end{array}$ $\begin{array}{lllll}0.0724214193111198 & 0.2871305285254930 & 0.1369343580704912\end{array}$ $\begin{array}{llll}0.1561638610392989 & 0.1852359242803862 & 0.1782901652284292\end{array}$ $\begin{array}{lllll}0.3647084903915052 & 0.1680246831252272 & 0.3935620351845117\end{array}$ $\begin{array}{lllll}0.3642980801539863 & 0.0551015996958306 & 0.4036375676505873\end{array}$ $\begin{array}{lllll}0.0968021540606793 & 0.1102552663034475 & 0.4323633063053142\end{array}$ $\begin{array}{lllll}0.3225222983329920 & 0.1051214166900558 & 0.1631504152653520\end{array}$ $\begin{array}{lllll}0.3018784748580843 & 0.1142570683994436 & 0.6254834968334338\end{array}$ $\begin{array}{lllll}0.0451283656808535 & 0.0673806285746050 & 0.6639373622036904\end{array}$ $\begin{array}{lllll}0.2749854806843110 & 0.0663689256723834 & 0.8791025891288158\end{array}$ $\begin{array}{lllll}0.2123578516191821 & 0.1683211743310789 & 0.8412129392661933\end{array}$ $\begin{array}{lllll}0.0443434973063682 & 0.2881215463591484 & 0.6122442406889166\end{array}$ $\begin{array}{lllll}0.2935807588242699 & 0.2469343608056250 & 0.6103892534577307\end{array}$ $\begin{array}{lllll}0.2033772934950051 & 0.3222423734273784 & 0.8545621467004891\end{array}$ $\begin{array}{lllll}0.0344809429649995 & 0.2419183172918890 & 0.8629455989875864\end{array}$ 0.24642557501888130 .36112922055518650 .6045610748321424 $\begin{array}{llll}0.4616779601810055 & 0.1405599292843350 & 0.8779254213806585\end{array}$ $\begin{array}{llllll}0.5113805533228909 & 0.1793538122505071 & 0.6278151277803686\end{array}$ $\begin{array}{lllll}0.4503881389873873 & 0.3422635449303626 & 0.8241258475902187\end{array}$ $\begin{array}{lllll}0.5952310515289934 & 0.3496145412339467 & 0.3719395680021532\end{array}$ $\begin{array}{lllll}0.5626323759815446 & 0.2410454587025721 & 0.3861445338968440\end{array}$ $\begin{array}{llllll}0.8130360928922191 & 0.2819626454833574 & 0.3870487707235074\end{array}$ $\begin{array}{llllll}0.9034782503154363 & 0.2073126800355548 & 0.1428927506642628\end{array}$ $\begin{array}{lllll}0.5626759701018443 & 0.2902020163689423 & 0.1346690879204356\end{array}$ $\begin{array}{llll}0.6556625653999508 & 0.1889661201107553 & 0.1745318014422323\end{array}$ $\begin{array}{llll}0.8617594609646253 & 0.1682041596504422 & 0.3925109449377690\end{array}$ 
$\begin{array}{llll}0.8612208644890017 & 0.0554135400053184 & 0.4025058541584529\end{array}$ $\begin{array}{llll}0.5979966007074971 & 0.1108857435256908 & 0.4338340081165815\end{array}$ $\begin{array}{llll}0.8137950631486095 & 0.1055852631236465 & 0.1627248470988420\end{array}$ $\begin{array}{llll}0.8017884618942176 & 0.1142683515358032 & 0.6260901392560563\end{array}$ $\begin{array}{llll}0.5467540854105405 & 0.0676221331336335 & 0.6655733333062543\end{array}$ $\begin{array}{lllll}0.7804942196984048 & 0.0652638055430713 & 0.8802509738582148\end{array}$ $\begin{array}{lllll}0.7125572280776723 & 0.1667549096176396 & 0.8441342828803058\end{array}$ $\begin{array}{lllll}0.5435205510358420 & 0.2885787680410713 & 0.6132266742993082\end{array}$ $\begin{array}{lllll}0.7930078750235411 & 0.2475784036497788 & 0.6097148240784666\end{array}$ $\begin{array}{llllll}0.7047668589597907 & 0.3211460462267686 & 0.8557589724506713\end{array}$ $\begin{array}{llllll}0.5368029284952848 & 0.2411830892710112 & 0.8646200282824668\end{array}$ $\begin{array}{lllll}0.7422100109585065 & 0.3613311556803787 & 0.6046131604448883\end{array}$ $\begin{array}{lllll}0.9629340875839807 & 0.1408926959746860 & 0.8777063211652927\end{array}$ $\begin{array}{lllll}0.0097940717507677 & 0.1792756477355596 & 0.6264587832279442\end{array}$ $\begin{array}{lllll}0.9504316457307898 & 0.3437100076674192 & 0.8192420397009121\end{array}$ $\begin{array}{lllll}0.2960897099652790 & 0.4210061074425488 & 0.3677430081587996\end{array}$ $\begin{array}{lllll}0.0581437692534166 & 0.4639584881305749 & 0.3383943077377703\end{array}$ $\begin{array}{llllll}0.1382540933156197 & 0.3886658383822091 & 0.1241955579474233\end{array}$ $\begin{array}{lllll}0.3040333740982644 & 0.4635120242432145 & 0.0918859578910893\end{array}$ $\begin{array}{lllll}0.3902947227963215 & 0.3661760211429110 & 0.1559297617503231\end{array}$ $\begin{array}{lllll}0.2352971009797442 & 0.4746782989606093 & 0.5983917955897606\end{array}$ $\begin{array}{lllll}0.0082348605550398 & 0.4180587379933743 & 0.5708696763699853\end{array}$ $\begin{array}{lllll}0.2879378170129694 & 0.4246873009590763 & 0.8321190908314788\end{array}$ $\begin{array}{llll}0.8035848803036945 & 0.4176788096790408 & 0.3746214189978028\end{array}$ $\begin{array}{lllll}0.5499747677336154 & 0.4576917561601212 & 0.3376560635655061\end{array}$ $\begin{array}{lllll}0.6438562615999333 & 0.3897885109395400 & 0.1227023571230462\end{array}$ $\begin{array}{llllll}0.8316481576526984 & 0.4607393212334617 & 0.1128660548166362\end{array}$ $\begin{array}{lllll}0.8915783313398699 & 0.3590583432481782 & 0.1641841195641467\end{array}$ $\begin{array}{llll}0.7320979570165469 & 0.4744268453040990 & 0.5993448443721869\end{array}$ $\begin{array}{llll}0.5036236574545221 & 0.4163258011708699 & 0.5646856196863909\end{array}$ $\begin{array}{lllll}0.7860752124667686 & 0.4239546638972573 & 0.8353282540179393\end{array}$ $\begin{array}{llll}0.4295037792917324 & 0.2341232905572026 & 0.5027960857299156\end{array}$ $\begin{array}{llll}0.1782874616756370 & 0.2952506937530225 & 0.4973582975212163\end{array}$ $\begin{array}{llll}0.4490576944442414 & 0.1130677965354889 & 0.5340557831861522\end{array}$ $\begin{array}{llll}0.9286527740971323 & 0.2341385340576864 & 0.5016285941643024\end{array}$ $\begin{array}{lllll}0.6772642282479316 & 0.2955584120367707 & 0.4951011386294993\end{array}$ $\begin{array}{llll}0.9476598562454950 & 0.1130771549903719 & 0.5326100481543441\end{array}$ $\begin{array}{lllll}0.1580191523280120 & 0.4163124249439757 & 0.4670998991541159\end{array}$ $\begin{array}{lllll}0.6541122710411569 & 0.4165946811788441 & 0.4656222600625527\end{array}$ $\begin{array}{lllll}0.0498595478171757 & 0.2305221482792133 & 0.2103827536551510\end{array}$ $\begin{array}{lllll}0.3110767022264270 & 0.1679674751835788 & 0.2175770595826717\end{array}$ $\begin{array}{llll}0.2976329785487675 & 0.3618202009939332 & 0.7816583619070256\end{array}$ $\begin{array}{lllll}0.3132048103988105 & 0.1212905465024754 & 0.8001534111841192\end{array}$ $\begin{array}{lllll}0.0550340745334616 & 0.1812542532162440 & 0.8039183373623837\end{array}$ $\begin{array}{llllll}0.0574223143510811 & 0.2984443742544621 & 0.7885065245690728\end{array}$ $\begin{array}{lllll}0.0502970885936056 & 0.3474445987712530 & 0.1974174904465248\end{array}$ $\begin{array}{lllll}0.5471462749667512 & 0.2330154832686760 & 0.2084580388622088\end{array}$ $\begin{array}{lllll}0.8079310693430358 & 0.1687652532971411 & 0.2163801588298070\end{array}$ $\begin{array}{lllll}0.7954530945595788 & 0.3612513482938188 & 0.7804963136418663\end{array}$ $\begin{array}{lllll}0.8148310330118766 & 0.1207697890073118 & 0.8010182971450429\end{array}$ $\begin{array}{llll}0.5558323439111731 & 0.1805045432288660 & 0.8054264697816903\end{array}$ $\begin{array}{llll}0.5575975974218172 & 0.2979588505483267 & 0.7905209050853633\end{array}$ $\begin{array}{lllll}0.5460502472272843 & 0.3513502632190788 & 0.1952312381048757\end{array}$ $\begin{array}{lllll}0.2868401974080627 & 0.4129409128913468 & 0.1936240691523049\end{array}$ $\begin{array}{llll}0.7929183524606390 & 0.4076138969856431 & 0.2006907879341682\end{array}$

A5 :

H $37 \quad$ C 5 N 5 O 60 Al 8 Si 16

1.00000000000000

$10.1485009999999996 \quad 0.0000000000000000 \quad-1.8735860000000000$

$\begin{array}{llll}0.0646860000000000 & 24.9946149999999996 & -0.5148360000000000\end{array}$ $\begin{array}{llll}0.0000000000000000 & 0.0000000000000000 & 9.3469999999999995\end{array}$ $\mathrm{H} \quad \mathrm{C} \quad \mathrm{N} \quad \mathrm{O} \quad \mathrm{Al} \mathrm{Si}$ $\begin{array}{llllll}37 & 5 & 5 & 60 & 8 & 16\end{array}$

Direct

$0.04618442704975650 .7482496958264373 \quad 0.7591061364698583$ $\begin{array}{llll}0.2249605120089764 & 0.7842002340662719 & 0.6276072148593103\end{array}$ $\begin{array}{llll}0.0154820846528136 & 0.5550281087839881 & 0.7490588068132651\end{array}$ $\begin{array}{lllll}0.3236768470325432 & 0.5430629787489631 & 0.5024394297858903\end{array}$ $\begin{array}{llll}0.3684279183466614 & 0.6090080567945239 & 0.4741580029539564\end{array}$ $\begin{array}{lllll}0.1781630279650255 & 0.0573495276187361 & 0.8250568596571467\end{array}$ $\begin{array}{lllll}0.6824286821137020 & 0.0562817046308293 & 0.8260490975608605\end{array}$ $\begin{array}{lllll}0.1016657515264489 & 0.1375073186088726 & 0.3576347907759088\end{array}$ 
$\begin{array}{llll}0.3416097013799437 & 0.3083651903021619 & 0.3247785684972100\end{array}$ $\begin{array}{lllll}0.2611774434851862 & 0.2207647138455783 & 0.6712548950459313\end{array}$ $\begin{array}{lllll}0.5307092053273575 & 0.0305825802369154 & 0.6258866901967830\end{array}$ $\begin{array}{lllll}0.3430584882107878 & 0.0669583359955380 & 0.2952886115403779\end{array}$ $\begin{array}{llll}0.6012051667669573 & 0.1371740083218229 & 0.3568956462051525\end{array}$ $\begin{array}{lllll}0.8405949504042513 & 0.3085755874876924 & 0.3244230161446023\end{array}$ $\begin{array}{llllll}0.7608965882353709 & 0.2196389110978911 & 0.6677717913150257\end{array}$ $\begin{array}{lllll}0.0281496449770888 & 0.0309496696190815 & 0.6258217111765288\end{array}$ $\begin{array}{lllll}0.8459379123621921 & 0.0678676061173906 & 0.2940494237197184\end{array}$ $\begin{array}{lllll}0.1132943519022363 & 0.4867467073569162 & 0.2952351656162753\end{array}$ $\begin{array}{lllll}0.4982539441548028 & 0.3900880425541622 & 0.6265563312541687\end{array}$ $\begin{array}{lllll}0.2642827104246427 & 0.4599555302867800 & 0.6988113400316777\end{array}$ $\begin{array}{lllll}0.6194989756048107 & 0.4877335576152609 & 0.2992647758554395\end{array}$ $\begin{array}{lllll}0.9950883409171043 & 0.3867160471816389 & 0.6253596871079632\end{array}$ $\begin{array}{lllll}0.7586046658131584 & 0.4614595753196733 & 0.6966689030224841\end{array}$ $\begin{array}{lllll}0.4189255298901054 & 0.4730329675403196 & 0.1798698108063022\end{array}$ $\begin{array}{lllll}0.9099173648432605 & 0.4742535621968837 & 0.1777478738153022\end{array}$ $\begin{array}{lllll}0.3179125983818533 & 0.4372783366134030 & 0.9395667789939824\end{array}$ $\begin{array}{llll}0.8079770693198629 & 0.4377645729767522 & 0.9353172660058280\end{array}$ $\begin{array}{lllll}0.2891380107131045 & 0.0906465320535116 & 0.0583229088478597\end{array}$ $\begin{array}{lllll}0.7950467791505171 & 0.0914723766544230 & 0.0577666362094778\end{array}$ $\begin{array}{lllll}0.1763348710196725 & 0.5068359016505595 & 0.6085165376026349\end{array}$ $\begin{array}{lllll}0.8379340985046795 & 0.4762607191797201 & 0.5735569810855894\end{array}$ $\begin{array}{lllll}0.2717757873317943 & 0.0479800090189389 & 0.4221571534944396\end{array}$ $\begin{array}{llllll}0.7707469037174297 & 0.0492364849841711 & 0.4181114386553216\end{array}$ $\begin{array}{lllll}0.4244819329567003 & 0.4211666208701408 & 0.4859923820358841\end{array}$ $\begin{array}{lllll}0.9199781464387189 & 0.4130006524826139 & 0.4764266413840282\end{array}$ $\begin{array}{llll}0.6753855787750722 & 0.1135960119546493 & 0.5112157100797619\end{array}$ $\begin{array}{llll}0.1758101414904857 & 0.1134904243195383 & 0.5116932104472376\end{array}$ 0.20073325415239490 .74208339420844390 .6323986121376189 $\begin{array}{lllll}0.0433539177027416 & 0.5857918340287458 & 0.7094128393922330\end{array}$ $\begin{array}{llll}0.0977966757384658 & 0.6702974231909765 & 0.6899659211470933\end{array}$ $\begin{array}{lllll}0.1952028338041140 & 0.6571791587912690 & 0.6078764746836239\end{array}$ $\begin{array}{lllll}0.2130609662566261 & 0.6021001414546159 & 0.5797761689805518\end{array}$ $\begin{array}{llll}0.0187786186369756 & 0.6364500133049165 & 0.7435849303155192\end{array}$ $\begin{array}{lllll}0.1031529410430648 & 0.7252281968331370 & 0.7043032648633936\end{array}$ $\begin{array}{llll}0.2585604045408897 & 0.7023942225231610 & 0.5728653469412445\end{array}$ $\begin{array}{lllll}0.1334469742928855 & 0.5673920485155730 & 0.6322475303237413\end{array}$ $\begin{array}{lllll}0.3036837384523184 & 0.5830616330550947 & 0.5042934591372884\end{array}$ $\begin{array}{llll}0.0939797482131266 & 0.3503808815836579 & 0.3705759462943355\end{array}$ $\begin{array}{lllll}0.0607960020997129 & 0.2415134198021167 & 0.3840617674880272\end{array}$ $\begin{array}{llll}0.3117303586651887 & 0.2821814376920420 & 0.3869438162845076\end{array}$ $\begin{array}{lllll}0.4004067008863688 & 0.2067123497972373 & 0.1403645739531983\end{array}$ $\begin{array}{llll}0.0714911227411966 & 0.2885564466969205 & 0.1337346482901850\end{array}$ $\begin{array}{lllll}0.1527019996349235 & 0.1863290345448914 & 0.1748679590074269\end{array}$ $\begin{array}{lllll}0.3601191237528529 & 0.1676896863784695 & 0.3903067443751771\end{array}$ $\begin{array}{lllll}0.3594088379122411 & 0.0544536469147361 & 0.4002613657582958\end{array}$ $\begin{array}{lllll}0.0933591829419852 & 0.1094583327174519 & 0.4267287915959139\end{array}$ $\begin{array}{lllll}0.3140812500070487 & 0.1045328946557821 & 0.1604802479131914\end{array}$ $\begin{array}{lllll}0.2965434215573194 & 0.1146074164569831 & 0.6207194385840324\end{array}$ $\begin{array}{lllll}0.0381534422206570 & 0.0676507712587232 & 0.6617019436129583\end{array}$ $\begin{array}{lllll}0.2693290477634582 & 0.0657881163481076 & 0.8775451486719846\end{array}$ $\begin{array}{lllll}0.2099544444927373 & 0.1671345974320709 & 0.8394089729006146\end{array}$ $\begin{array}{lllll}0.0416042689811492 & 0.2881750477855269 & 0.6111343245803847\end{array}$ $\begin{array}{lllll}0.2906925829596824 & 0.2466320359452748 & 0.6080259423434174\end{array}$ $\begin{array}{lllll}0.2016253221101061 & 0.3208804358404778 & 0.8548208890194797\end{array}$ $\begin{array}{lllll}0.0321234085463758 & 0.2409679571374012 & 0.8614572226894406\end{array}$ $\begin{array}{lllll}0.2413404197605178 & 0.3611292949158793 & 0.6058502356035654\end{array}$ $\begin{array}{lllll}0.4589817606795826 & 0.1388568933158344 & 0.8726681110878863\end{array}$ $\begin{array}{lllll}0.5084851737602011 & 0.1782025033048084 & 0.6244709317637819\end{array}$ $\begin{array}{lllll}0.4488320324872584 & 0.3413082591023013 & 0.8209360993730193\end{array}$ $\begin{array}{llll}0.5920466822299431 & 0.3493821228952920 & 0.3701815093948121\end{array}$ $\begin{array}{lllll}0.5603333423348231 & 0.2402107687827074 & 0.3839767541413912\end{array}$ $\begin{array}{lllll}0.8110125209705272 & 0.2822290932711142 & 0.3862390889650727\end{array}$ $\begin{array}{llllll}0.9003326735951540 & 0.2091479634448552 & 0.1402201314176700\end{array}$ $\begin{array}{lllll}0.5669824291798597 & 0.2872483026110775 & 0.1330567858287311\end{array}$ $\begin{array}{lllll}0.6537694413644890 & 0.1857684684636284 & 0.1742645977047271\end{array}$ $\begin{array}{lllll}0.8620395890751245 & 0.1684189164675459 & 0.3892949231974340\end{array}$ $\begin{array}{lllll}0.8594848367309985 & 0.0552912480626528 & 0.3988199471005033\end{array}$ $\begin{array}{lllll}0.5930906061711614 & 0.1091833838726139 & 0.4262306916999934\end{array}$ $\begin{array}{lllll}0.8210652310390585 & 0.1057656895831105 & 0.1592045176567395\end{array}$ 0.79663837092478930 .11557295280025950 .6197011070198277 $\begin{array}{llll}0.5396672825491478 & 0.0673156007580874 & 0.6616785248813758\end{array}$ 
$\begin{array}{llll}0.7738409281277255 & 0.0650521104498561 & 0.8765399377767497\end{array}$ $\begin{array}{llll}0.7096563893601731 & 0.1660015730985853 & 0.8405200853851376\end{array}$ $\begin{array}{lllll}0.5419878206659848 & 0.2871213597629673 & 0.6111183490939526\end{array}$ $\begin{array}{llll}0.7909724431681360 & 0.2463730764076661 & 0.6073849309758497\end{array}$ $\begin{array}{llll}0.7022473183506571 & 0.3202324845964560 & 0.8541549801864535\end{array}$ $\begin{array}{lllll}0.5332612406538950 & 0.2401037626313645 & 0.8619050858772206\end{array}$ $\begin{array}{lllll}0.7400584822294473 & 0.3604001514109793 & 0.6042218558368020\end{array}$ $\begin{array}{lllll}0.9595708822086366 & 0.1394987206499513 & 0.8719732395442702\end{array}$ $\begin{array}{lllll}0.0091004278866983 & 0.1788889451399907 & 0.6240019275166855\end{array}$ $\begin{array}{llll}0.9481209845721038 & 0.3426572682843585 & 0.8200727054927458\end{array}$ $\begin{array}{lllll}0.3014792471272180 & 0.4160519782706492 & 0.3730156769569307\end{array}$ $\begin{array}{lllll}0.0559591875587159 & 0.4629685058321070 & 0.3360020492576273\end{array}$ $\begin{array}{lllll}0.1421713634404079 & 0.3899386671062935 & 0.1205099450301850\end{array}$ $\begin{array}{llll}0.3305047859293413 & 0.4637218115006791 & 0.1204766934565814\end{array}$ $\begin{array}{lllll}0.3905707428910392 & 0.3615503450389179 & 0.1559772533336974\end{array}$ $\begin{array}{llll}0.2322357562401810 & 0.4728924585501755 & 0.5971727624258610\end{array}$ $\begin{array}{lllll}0.0015334902748441 & 0.4168871882250205 & 0.5623196541921699\end{array}$ $\begin{array}{lllll}0.2904463757348786 & 0.4230637536122683 & 0.8385502670673016\end{array}$ $\begin{array}{llll}0.8009367055308723 & 0.4127929238150085 & 0.3696050018028844\end{array}$ $\begin{array}{lllll}0.5609018609348307 & 0.4621151841092633 & 0.3312736315730903\end{array}$ $\begin{array}{lllll}0.6409507860256762 & 0.3874436725559315 & 0.1180144816027831\end{array}$ $\begin{array}{llll}0.8229645537736474 & 0.4634733544421505 & 0.1185514217887556\end{array}$ $\begin{array}{lllll}0.8924957551275772 & 0.3618013772941874 & 0.1520987513710046\end{array}$ $\begin{array}{llll}0.7478050055656732 & 0.4730729583145100 & 0.5918276990979303\end{array}$ $0.5099565854439629 \quad 0.42022835518791450 .5654352214534305$ $\begin{array}{lllll}0.7829730802425067 & 0.4234502159133409 & 0.8341397294494209\end{array}$ $\begin{array}{lllll}0.4264870436884614 & 0.2335441448291129 & 0.5003424637395134\end{array}$ $\begin{array}{llll}0.1761014092406488 & 0.2957540353462952 & 0.4951528513236358\end{array}$ $\begin{array}{lllll}0.4438661604495313 & 0.1129939782046382 & 0.5300984573962146\end{array}$ $\begin{array}{lllll}0.9272801383778193 & 0.2341227564570367 & 0.4998536102903581\end{array}$ $\begin{array}{lllll}0.6750524069743949 & 0.2948533445252299 & 0.4944982650912337\end{array}$ $\begin{array}{lllll}0.9440583174436018 & 0.1136680275806651 & 0.5294364643764047\end{array}$ $\begin{array}{lllll}0.1557720908267582 & 0.4173044772872501 & 0.4666202045929677\end{array}$ $\begin{array}{lllll}0.6553254028006086 & 0.4159228903487913 & 0.4632984243805139\end{array}$ $\begin{array}{llllll}0.0474635138174231 & 0.2320928847211406 & 0.2074477879823202\end{array}$ $\begin{array}{lllll}0.3065268463354090 & 0.1676469251342805 & 0.2142891212688761\end{array}$ $\begin{array}{lllll}0.2955336202995226 & 0.3605569778741624 & 0.7819720265578697\end{array}$ $\begin{array}{lllll}0.3097703445682085 & 0.1197716512446232 & 0.7958625588886055\end{array}$ $\begin{array}{lllll}0.0529219950034714 & 0.1804707277923397 & 0.8018596199227244\end{array}$ $\begin{array}{lllll}0.0551144217876945 & 0.2977060154841074 & 0.7880318692796536\end{array}$ $\begin{array}{lllll}0.0499273559006130 & 0.3492436891054307 & 0.1930160571120905\end{array}$ $\begin{array}{lllll}0.5464323236303558 & 0.2306023524960620 & 0.2073284736895057\end{array}$ $\begin{array}{lllll}0.8088132603675271 & 0.1686618253513798 & 0.2132130433024434\end{array}$ $\begin{array}{lllll}0.7936715023831892 & 0.3605329952781327 & 0.7803142529538332\end{array}$ $\begin{array}{lllll}0.8109322034479198 & 0.1196461058606253 & 0.7951342372230445\end{array}$ $\begin{array}{lllll}0.5529022612406336 & 0.1795530711701398 & 0.8023365286428902\end{array}$ $\begin{array}{lllll}0.5557037671036034 & 0.2967610217153119 & 0.7879049145802024\end{array}$ $\begin{array}{llll}0.5474220320639036 & 0.3479337116988307 & 0.1926223715033753\end{array}$ $\begin{array}{llll}0.2910556705326728 & 0.4088834268349963 & 0.1990752713099160\end{array}$ $\begin{array}{llll}0.7890665949711515 & 0.4073456431889809 & 0.1949238152470693\end{array}$

C1:

$\begin{array}{lllllll}\mathrm{H} 37 & \mathrm{C} 4 & \mathrm{~N} 3 & \mathrm{O} 61 & \text { Al } 8 & \text { Si } 16\end{array}$

1.00000000000000

$\begin{array}{llll}10.1485009999999996 & 0.0000000000000000 & -1.8735860000000000\end{array}$

$\begin{array}{llll}0.0646860000000000 & 24.9946149999999996 & -0.5148360000000000\end{array}$ $\begin{array}{lll}0.0000000000000000 & 0.0000000000000000 & 9.3469999999999995\end{array}$ $\begin{array}{llllll}\mathrm{H} & \mathrm{C} & \mathrm{N} & \mathrm{O} & \mathrm{Al} & \mathrm{Si}\end{array}$

\section{Direct}

$0.4921957756595874 \quad 0.6893897460714541 \quad 0.3488063209872322$ $\begin{array}{lllll}0.2808454389265932 & 0.6501188477682673 & 0.2101656692857276\end{array}$ $\begin{array}{llll}0.1489863694957731 & 0.5198081362427233 & 0.3128003036304129\end{array}$ $\begin{array}{llll}0.1351821528712211 & 0.5748766808778953 & 0.1936109769392721\end{array}$ $\begin{array}{llll}0.6107989903450844 & 0.6393987539434275 & 0.5460454008280516\end{array}$ $\begin{array}{lllll}0.2014415504206807 & 0.0582619697842493 & 0.8201511145659267\end{array}$ $\begin{array}{llll}0.7081016908844938 & 0.0561735101230066 & 0.8235545649143075\end{array}$ $\begin{array}{lllll}0.1207206732971749 & 0.1441015109167608 & 0.3740418412736272\end{array}$ $\begin{array}{llll}0.3523717378202028 & 0.3087464171003425 & 0.3184585676012234\end{array}$ $\begin{array}{lllll}0.2799051625036365 & 0.2241922541761179 & 0.6770106307561607\end{array}$ $\begin{array}{lllll}0.5042432925447801 & 0.0434191219451733 & 0.6972118175353017\end{array}$ $\begin{array}{llll}0.3587307282841089 & 0.0701330894472919 & 0.2976538628085530\end{array}$ $\begin{array}{llll}0.6208306361839671 & 0.1430948172258192 & 0.3712440299302393\end{array}$ 
$\begin{array}{llll}0.8554165067633621 & 0.3096916475417673 & 0.3239611563136841\end{array}$ $\begin{array}{lllll}0.7791363627253143 & 0.2235417769194845 & 0.6761381245701723\end{array}$ $\begin{array}{lllll}0.0042712210995573 & 0.0416191045869070 & 0.6921519896307976\end{array}$ $\begin{array}{llll}0.8611655834378904 & 0.0698819409854003 & 0.2978267421537439\end{array}$ $\begin{array}{llll}0.0007381847608375 & 0.4820087060045785 & 0.3939970128783514\end{array}$ $\begin{array}{lllll}0.5065471798141664 & 0.3730776317693295 & 0.5946037843684975\end{array}$ $\begin{array}{lllll}0.2832527783615577 & 0.4666230258489410 & 0.7001047238469038\end{array}$ $\begin{array}{lllll}0.5006915922321388 & 0.4837611091504669 & 0.3844552678078593\end{array}$ $\begin{array}{llll}1.0120920048104283 & 0.3931124182606388 & 0.6494290202739801\end{array}$ $\begin{array}{lllll}0.7629119443372042 & 0.4629166001596922 & 0.7042695526642089\end{array}$ $\begin{array}{lllll}0.4380235616894623 & 0.4718196242076479 & 0.1646513632670274\end{array}$ $\begin{array}{llll}0.9288922345047128 & 0.4742377425439234 & 0.1593204035915250\end{array}$ $\begin{array}{lllll}0.3304224544509505 & 0.4399999926219277 & 0.9398545633973978\end{array}$ $\begin{array}{llll}0.8221312424916483 & 0.4396356657654000 & 0.9409285269685743\end{array}$ $\begin{array}{llll}0.3047568817223262 & 0.0935352339772229 & 0.0612526915382743\end{array}$ $\begin{array}{lllll}0.8098908277270132 & 0.0932929912411447 & 0.0617482005849263\end{array}$ $\begin{array}{llllll}0.3019178103949208 & 0.5027753055206707 & 0.5563185055261686\end{array}$ $\begin{array}{lllll}0.6641110319521293 & 0.5045967941220603 & 0.6122510406675559\end{array}$ $\begin{array}{lllll}0.2903139704121345 & 0.0494184932710377 & 0.4250821365815760\end{array}$ $\begin{array}{lllll}0.7908258174589360 & 0.0495020883965521 & 0.4240845807771838\end{array}$ $\begin{array}{lllll}0.4312104678969869 & 0.4157452292739897 & 0.4887653801068353\end{array}$ $\begin{array}{lllll}0.9338467485848382 & 0.4163754426710699 & 0.4974590090830198\end{array}$ $\begin{array}{lllll}0.6948509439238680 & 0.1147730947861857 & 0.5171021633227816\end{array}$ $\begin{array}{lllll}0.1955032666050751 & 0.1149331066776437 & 0.5181056399366283\end{array}$ $\begin{array}{lllll}0.4526386908370247 & 0.6513768703405736 & 0.3762877288538957\end{array}$ $\begin{array}{lllll}0.3388118659816560 & 0.6298648837434327 & 0.3023745177877357\end{array}$ $\begin{array}{lllll}0.2971602943131798 & 0.5795337083011202 & 0.3510688275566066\end{array}$ $\begin{array}{lllll}0.4848911557741217 & 0.5753036414563700 & 0.5362838311748108\end{array}$ $\begin{array}{lllll}0.5233142639507263 & 0.6251328301791551 & 0.4910882170342454\end{array}$ $\begin{array}{lllll}0.1850273981056516 & 0.5571869384635937 & 0.2840376242017711\end{array}$ $\begin{array}{lllll}0.3694783686976758 & 0.5541299676497955 & 0.4653554584274942\end{array}$ $\begin{array}{lllll}0.5588703282928155 & 0.5532454992747661 & 0.6389463932002047\end{array}$ $\begin{array}{lllll}0.1089477783381916 & 0.3526743157515401 & 0.3750257864578019\end{array}$ $\begin{array}{lllll}0.0773172525470905 & 0.2431185676666960 & 0.3867505793703633\end{array}$ $\begin{array}{lllll}0.3276892376067188 & 0.2847268418854701 & 0.3895910396902181\end{array}$ $\begin{array}{lllll}0.4174489038848705 & 0.2097959233129033 & 0.1437076958980223\end{array}$ $\begin{array}{lllll}0.0810535055528098 & 0.2917572405708408 & 0.1366552720168527\end{array}$ $\begin{array}{llllll}0.1704442142829423 & 0.1902557713598517 & 0.1755817115600237\end{array}$ $\begin{array}{llll}0.3766787898511057 & 0.1702712815254864 & 0.3933027467686192\end{array}$ $\begin{array}{lllll}0.3767878562011301 & 0.0574199248060699 & 0.4022327386800311\end{array}$ $\begin{array}{lllll}0.1123172367233776 & 0.1130644694336288 & 0.4335470849376502\end{array}$ $\begin{array}{lllll}0.3303721447114107 & 0.1076311813886245 & 0.1627537628190583\end{array}$ $\begin{array}{lllll}0.3164201511097547 & 0.1158115363811570 & 0.6259849938993144\end{array}$ $\begin{array}{llll}0.0605759494599297 & 0.0690192932747358 & 0.6641376503574637\end{array}$ $\begin{array}{lllll}0.2896818851554436 & 0.0678289350934125 & 0.8792631468171795\end{array}$ $\begin{array}{lllll}0.2276955731152686 & 0.1698605226881449 & 0.8423998611724183\end{array}$ $\begin{array}{lllll}0.0588305714720815 & 0.2897594548842048 & 0.6140921999562783\end{array}$ $\begin{array}{lllll}0.3078875115908084 & 0.2492866874461580 & 0.6108326665321746\end{array}$ $\begin{array}{lllll}0.2169078894221372 & 0.3243880874312591 & 0.8572765290278919\end{array}$ $\begin{array}{lllll}0.0499906210758665 & 0.2434624627665568 & 0.8650522288920917\end{array}$ $\begin{array}{lllll}0.2582669101173177 & 0.3630744852774395 & 0.6077619937175748\end{array}$ $\begin{array}{llll}0.4769253159777538 & 0.1417858015617693 & 0.8777918578430454\end{array}$ $\begin{array}{lllll}0.5254008772752713 & 0.1811836633790988 & 0.6271507521273798\end{array}$ $\begin{array}{lllll}0.4633471558596261 & 0.3422389233530028 & 0.8292012414179413\end{array}$ $\begin{array}{llll}0.6113555323795133 & 0.3512317233794378 & 0.3732638795277869\end{array}$ $\begin{array}{lllll}0.5770900834762398 & 0.2428770968254302 & 0.3875569274742360\end{array}$ $\begin{array}{lllll}0.8282021520180104 & 0.2840232965384241 & 0.3893758047782433\end{array}$ $\begin{array}{lllll}0.9167948254147992 & 0.2102790396926705 & 0.1433714136451949\end{array}$ $\begin{array}{lllll}0.5827004363801378 & 0.2904772644552864 & 0.1356879189684849\end{array}$ $\begin{array}{llll}0.6706977402216612 & 0.1889654515664620 & 0.1777747592214443\end{array}$ $\begin{array}{lllll}0.8781618249643403 & 0.1701193627572035 & 0.3935351288829672\end{array}$ $\begin{array}{llll}0.8779048388951955 & 0.0571877554816118 & 0.4024044777296366\end{array}$ $\begin{array}{llll}0.6119213170887230 & 0.1126772467383940 & 0.4327641003301290\end{array}$ $\begin{array}{lllll}0.8338739071788969 & 0.1075343783734575 & 0.1631359805615667\end{array}$ $\begin{array}{lllll}0.8168828962876519 & 0.1155752373569934 & 0.6255682936356632\end{array}$ $\begin{array}{lllll}0.5608622875999745 & 0.0695218061384171 & 0.6639433998408530\end{array}$ $\begin{array}{lllll}0.7962396686852070 & 0.0665486896126593 & 0.8800038031200477\end{array}$ $\begin{array}{lllll}0.7275208795206248 & 0.1679455492958871 & 0.8432426213336670\end{array}$ $\begin{array}{lllll}0.5572410435118431 & 0.2910957960240652 & 0.6145773842692828\end{array}$ $\begin{array}{lllll}0.8075624903596295 & 0.2490238972195646 & 0.6112660379276682\end{array}$ $\begin{array}{lllll}0.7186955548753727 & 0.3228152887314458 & 0.8579881803911902\end{array}$ $\begin{array}{llllll}0.5527322825771047 & 0.2421430769493060 & 0.8650360744009837\end{array}$ $\begin{array}{lllll}0.7572727558028227 & 0.3631837305311575 & 0.6071802123032848\end{array}$ 
$\begin{array}{llll}0.9778200531280745 & 0.1423593872374390 & 0.8778588647061834\end{array}$ $\begin{array}{lllll}0.0251266545465637 & 0.1811764888803323 & 0.6277811108516018\end{array}$ $\begin{array}{llll}0.9641907857075458 & 0.3448573712455102 & 0.8216327590801861\end{array}$ $\begin{array}{llll}0.3081171696431935 & 0.4212978440074699 & 0.3725024673406286\end{array}$ $\begin{array}{lllll}0.0676215123140022 & 0.4662068684626669 & 0.3446045846495639\end{array}$ $\begin{array}{lllll}0.1511677244200955 & 0.3934015777925452 & 0.1245666777445299\end{array}$ $\begin{array}{lllll}0.3458881907865515 & 0.4637470371044332 & 0.1140124079571139\end{array}$ $\begin{array}{llllll}0.4017037066068294 & 0.3622834912861040 & 0.1663236628850426\end{array}$ $\begin{array}{llll}0.2446393014835273 & 0.4769835490548549 & 0.5981700703716606\end{array}$ $0.01940952752904320 .4187056085162723 \quad 0.5738759251241280$ $\begin{array}{lllll}0.3069090454407558 & 0.4261892639940481 & 0.8374779262660800\end{array}$ $\begin{array}{lllll}0.8103254479258802 & 0.4205576169459845 & 0.3759430233210616\end{array}$ $\begin{array}{lllll}0.5636440281855503 & 0.4636033696529852 & 0.3385287999517966\end{array}$ $\begin{array}{llll}0.6503552732819881 & 0.3925588106366825 & 0.1248839183686974\end{array}$ $\begin{array}{llllll}0.8370493422103686 & 0.4652288061436871 & 0.1144513073948790\end{array}$ $\begin{array}{lllll}0.9025102835931679 & 0.3648839334733409 & 0.1645402538638117\end{array}$ $\begin{array}{lllll}0.7310574035931720 & 0.4762547358175264 & 0.6026367718949788\end{array}$ $\begin{array}{lllll}0.5138872150606593 & 0.4104843903249127 & 0.5677477603688086\end{array}$ $\begin{array}{lllll}0.8029712067661315 & 0.4254843269742031 & 0.8388144145614669\end{array}$ $\begin{array}{lllll}0.4430141085233645 & 0.2362750828725901 & 0.5029722354874513\end{array}$ $\begin{array}{lllll}0.1919419926559682 & 0.2978806826361542 & 0.4979208605307409\end{array}$ $\begin{array}{llll}0.4627297474969284 & 0.1150086671196539 & 0.5329227102413933\end{array}$ $\begin{array}{llll}0.9439470414518641 & 0.2361605692665202 & 0.5034475053869684\end{array}$ $\begin{array}{lllll}0.6932135002595055 & 0.2976299553690807 & 0.4978382513794943\end{array}$ $0.9632588049726103 \quad 0.11496524760154850 .5332855980653999$ $\begin{array}{lllll}0.1698887511443089 & 0.4187989039974851 & 0.4741988984655919\end{array}$ $\begin{array}{lllll}0.6654410072937478 & 0.4191578436483024 & 0.4691497459304240\end{array}$ $\begin{array}{lllll}0.0626226986318547 & 0.2345098347030544 & 0.2096136045559971\end{array}$ $\begin{array}{lllll}0.3232656697957100 & 0.1706910742641296 & 0.2171514311951435\end{array}$ $\begin{array}{llll}0.3125107500195726 & 0.3635002355230009 & 0.7842480939614899\end{array}$ $\begin{array}{lllll}0.3281237240948056 & 0.1228173706793086 & 0.8007174653278518\end{array}$ $\begin{array}{llll}0.0703640769347821 & 0.1829553983764863 & 0.8052278053404158\end{array}$ $\begin{array}{lllll}0.0718167998533232 & 0.3001708874750051 & 0.7904828037488837\end{array}$ $\begin{array}{llll}0.0602926961732623 & 0.3521604705105463 & 0.1982052798570453\end{array}$ $\begin{array}{llll}0.5634065135102920 & 0.2337111002302608 & 0.2104484908542288\end{array}$ $\begin{array}{llll}0.8245562324779055 & 0.1705157665021785 & 0.2174994998645590\end{array}$ $\begin{array}{llll}0.8097016583886185 & 0.3630992053573208 & 0.7832834154479705\end{array}$ $\begin{array}{lllll}0.8300205462241573 & 0.1221011688519899 & 0.8004538191175462\end{array}$ $\begin{array}{llll}0.5708057892097971 & 0.1816759148435010 & 0.8046568541931495\end{array}$ $\begin{array}{llll}0.5720081626071871 & 0.2994110988612649 & 0.7928943934455658\end{array}$ $\begin{array}{llll}0.5604251210412280 & 0.3507205469148613 & 0.1973505194887042\end{array}$ $\begin{array}{llll}0.3021498873874791 & 0.4113599487403714 & 0.2003845495898570\end{array}$ $\begin{array}{lll}0.7993039714777472 & 0.4119721765683722 & 0.2025656980446906\end{array}$

C2:

H 37 C 4 N 3 O 61 Al 8 Si 16

1.00000000000000

$10.1485009999999996 \quad 0.0000000000000000 \quad-1.8735860000000000$

$\begin{array}{llll}0.0646860000000000 & 24.9946149999999996 & -0.5148360000000000\end{array}$

$\begin{array}{llll}0.0000000000000000 & 0.0000000000000000 & 9.3469999999999995\end{array}$

$\mathrm{H} \quad \mathrm{C} N \mathrm{~N}$ Al Si

$\begin{array}{llllll}37 & 4 & 3 & 61 & 8 & 16\end{array}$

Direct

$0.4163685846420793 \quad 0.7138765370319573 \quad 0.3199590637788594$

$\begin{array}{lllll}0.5956058737336841 & 0.6640972909078557 & 0.2217615497908876\end{array}$ $\begin{array}{lllll}0.6268363867183561 & 0.5154281501400930 & 0.2724237120427112\end{array}$ $\begin{array}{lllll}0.6916611710884036 & 0.5727672537271197 & 0.1961940081720268\end{array}$ $\begin{array}{llll}0.2751372673283407 & 0.6637746540827335 & 0.4426180191544934\end{array}$ $\begin{array}{lllll}0.1833872087781878 & 0.0543084286150698 & 0.8172322563515069\end{array}$ $\begin{array}{llll}0.6828442468222455 & 0.0559293878493903 & 0.8139265380628337\end{array}$ $\begin{array}{lllll}0.0996693839660367 & 0.1391947078697734 & 0.3637221804110077\end{array}$ $\begin{array}{llll}0.3385662161803794 & 0.3085046340488512 & 0.3217388648782716\end{array}$ $\begin{array}{lllll}0.2575819867669191 & 0.2217282759267407 & 0.6690936585442663\end{array}$ $\begin{array}{llll}0.4842441078425440 & 0.0388238424060815 & 0.6859310994301927\end{array}$ $\begin{array}{llll}0.3395040322829730 & 0.0672663441734499 & 0.2921349431380063\end{array}$ $\begin{array}{lllll}0.5986149574320745 & 0.1406464948462660 & 0.3647981187454680\end{array}$ $\begin{array}{lllll}0.8373661699801254 & 0.3086107761474943 & 0.3233638373059923\end{array}$ $\begin{array}{lllll}0.7594921123442648 & 0.2203447087917282 & 0.6694868622857593\end{array}$ $\begin{array}{lllll}0.0196344095503602 & 0.0406628771678378 & 0.6899509870606262\end{array}$ $\begin{array}{llll}0.8390701531759801 & 0.0678922362213895 & 0.2925770074868216\end{array}$ $\begin{array}{lllll}0.1046771803331659 & 0.4926516838361094 & 0.3088551011514334\end{array}$ $\begin{array}{lllll}0.4957407991735191 & 0.3933942522679963 & 0.6419828901858933\end{array}$ $\begin{array}{llll}0.2583544483416557 & 0.4666080770401863 & 0.6914865208341672\end{array}$ 
$\begin{array}{llll}0.5062838701329861 & 0.4854893774221029 & 0.3773409961273546\end{array}$ $\begin{array}{lllll}0.9935615180604339 & 0.3847492183695580 & 0.6172202394964899\end{array}$ $\begin{array}{lllll}0.7567984497433751 & 0.4605141410956604 & 0.6974106270282143\end{array}$ $\begin{array}{llll}0.4087963431080678 & 0.4746373330996402 & 0.1517376313864101\end{array}$ $\begin{array}{llll}0.9140591917814224 & 0.4718399176681344 & 0.1797767492400498\end{array}$ $\begin{array}{llll}0.3042771259998310 & 0.4384634905807372 & 0.9326586743475088\end{array}$ $\begin{array}{lllll}0.8077144214292065 & 0.4367715841704321 & 0.9349197208624924\end{array}$ $\begin{array}{lllll}0.2870682458998411 & 0.0905678941570399 & 0.0561748659163093\end{array}$ $\begin{array}{lllll}0.7885098888736167 & 0.0914127324217724 & 0.0556485679240995\end{array}$ $\begin{array}{lllll}0.2563295147655614 & 0.5094899063737836 & 0.5536672816453532\end{array}$ $\begin{array}{lllll}0.8293324356081572 & 0.4787153482055196 & 0.5708734931739027\end{array}$ $\begin{array}{lllll}0.2693001262266074 & 0.0470369675338725 & 0.4186282302536796\end{array}$ $\begin{array}{lllll}0.7651333450228145 & 0.0490755824304070 & 0.4173433609695135\end{array}$ $\begin{array}{llll}0.4213149747372253 & 0.4174771215485205 & 0.4889173267918830\end{array}$ $\begin{array}{llll}0.9175634239871289 & 0.4137365709505320 & 0.4746632653711151\end{array}$ $\begin{array}{lllll}0.6740066625094172 & 0.1135394112607879 & 0.5121585306354952\end{array}$ $0.1736548571650705 \quad 0.11187801673246230 .5116357470053536$ $\begin{array}{lllll}0.4317126678552966 & 0.6708794070251639 & 0.3316087431629389\end{array}$ $\begin{array}{llll}0.5281631690970212 & 0.6436603731747720 & 0.2791092918520937\end{array}$ $\begin{array}{llll}0.5364723110277800 & 0.5869019510214140 & 0.3023923766169745\end{array}$ $\begin{array}{lllll}0.3572391207773918 & 0.5887802822947104 & 0.4250206532239389\end{array}$ $\begin{array}{llllll}0.3483797772264217 & 0.6444253997291191 & 0.4027530186580439\end{array}$ $\begin{array}{llll}0.6275520913641320 & 0.5567835612316923 & 0.2535766380528722\end{array}$ $\begin{array}{lllll}0.4528650436222681 & 0.5615429496823567 & 0.3737866302199923\end{array}$ $0.2742968552994558 \quad 0.5688058411300156 \quad 0.4908532034155348$ $\begin{array}{lllll}0.0902156524494408 & 0.3506162149379610 & 0.3662042961791151\end{array}$ $\begin{array}{llll}0.0575286586181532 & 0.2414609843611450 & 0.3812651459419316\end{array}$ $\begin{array}{lllll}0.3084163343868613 & 0.2822381086869749 & 0.3834798547530580\end{array}$ $\begin{array}{llll}0.3967894637599013 & 0.2070704981797423 & 0.1378725285099484\end{array}$ $\begin{array}{llll}0.0664703287016056 & 0.2883048310035245 & 0.1301723222894312\end{array}$ $\begin{array}{lllll}0.1495086318253733 & 0.1863585541953626 & 0.1720396032567248\end{array}$ $\begin{array}{lllll}0.3562547432974147 & 0.1675695756899766 & 0.3879489435351524\end{array}$ $\begin{array}{lllll}0.3564029560274726 & 0.0546086980470738 & 0.3968111658573700\end{array}$ $\begin{array}{llll}0.0906481406559630 & 0.1094195865327831 & 0.4273238102451526\end{array}$ $\begin{array}{llllll}0.3116805466309914 & 0.1047460370145801 & 0.1576181847986865\end{array}$ $\begin{array}{lllll}0.2951831820828347 & 0.1127987511261610 & 0.6201146665713464\end{array}$ $\begin{array}{lllll}0.0372927523481043 & 0.0672724951165876 & 0.6593792423834531\end{array}$ $\begin{array}{lllll}0.2719500792049819 & 0.0641372843060495 & 0.8740292214777421\end{array}$ $\begin{array}{lllll}0.2070148635336155 & 0.1658671201924017 & 0.8384371209498743\end{array}$ $\begin{array}{lllll}0.0381807080179407 & 0.2888108022397275 & 0.6081805262779155\end{array}$ $\begin{array}{llll}0.2868837051081520 & 0.2472196746595243 & 0.6048585684388023\end{array}$ $\begin{array}{llll}0.1992196125272982 & 0.3224096212931099 & 0.8504071854615589\end{array}$ $\begin{array}{llllll}0.0341190027514958 & 0.2410441415516974 & 0.8589756557155496\end{array}$ $\begin{array}{llll}0.2372697897129016 & 0.3618679142658983 & 0.5999442999965808\end{array}$ $\begin{array}{lllll}0.4562758564634841 & 0.1390704806115218 & 0.8723439836164373\end{array}$ $\begin{array}{lllll}0.5032135367887345 & 0.1782993072216705 & 0.6222604596592084\end{array}$ $\begin{array}{llll}0.4454544345634495 & 0.3430303224405106 & 0.8137017317254924\end{array}$ $\begin{array}{lllll}0.5894531835881053 & 0.3485011520848845 & 0.3690474666090122\end{array}$ $\begin{array}{lllll}0.5572475780491546 & 0.2396136642827635 & 0.3813150402585050\end{array}$ $\begin{array}{lllll}0.8079638181322008 & 0.2818605874709428 & 0.3842007246284379\end{array}$ $\begin{array}{lllll}0.8969285391828494 & 0.2086101162676038 & 0.1377760624685244\end{array}$ $\begin{array}{lllll}0.5632189931998016 & 0.2878089730347297 & 0.1307381827028185\end{array}$ $\begin{array}{lllll}0.6505073410178968 & 0.1863590219375247 & 0.1707359283949370\end{array}$ $\begin{array}{lllll}0.8586330759196206 & 0.1681928205835274 & 0.3872779943370814\end{array}$ $\begin{array}{lllll}0.8534311996503270 & 0.0555601801693124 & 0.3973361426331397\end{array}$ $\begin{array}{lllll}0.5910756110878035 & 0.1104084772338368 & 0.4273984883374858\end{array}$ $\begin{array}{lllll}0.8153026356167753 & 0.1055879590617348 & 0.1568188218297953\end{array}$ $\begin{array}{lllll}0.7947175450312087 & 0.1154040202415579 & 0.6198954449755842\end{array}$ $\begin{array}{lllll}0.5399429056393124 & 0.0664635825411867 & 0.6580057046038206\end{array}$ $\begin{array}{llll}0.7711360467210291 & 0.0657864504879249 & 0.8722426028616699\end{array}$ $\begin{array}{lllll}0.7054367406707985 & 0.1675969643572394 & 0.8382007179780795\end{array}$ $\begin{array}{lllll}0.5388250719831714 & 0.2864670151620286 & 0.6081258432185602\end{array}$ $\begin{array}{lllll}0.7884876386819446 & 0.2461036080143199 & 0.6057070572260459\end{array}$ $\begin{array}{lllll}0.6977141608445473 & 0.3194503982515681 & 0.8521180742013048\end{array}$ $\begin{array}{lllll}0.5253552695776136 & 0.2406299803124288 & 0.8585835210663721\end{array}$ $\begin{array}{lllll}0.7378254236612370 & 0.3596215963004082 & 0.6035466456897076\end{array}$ $\begin{array}{llllll}0.9554411825384292 & 0.1410040238466671 & 0.8721188849920751\end{array}$ $\begin{array}{lllll}0.0067176148848374 & 0.1790499675971501 & 0.6215589081063849\end{array}$ $\begin{array}{lllll}0.9447442549680058 & 0.3416528122177528 & 0.8205394721193805\end{array}$ $\begin{array}{lllll}0.2977647093195420 & 0.4167823410751881 & 0.3687402754106275\end{array}$ $\begin{array}{lllll}0.0511290911799486 & 0.4625821238699106 & 0.3272865320444681\end{array}$ $\begin{array}{lllll}0.1384966325474890 & 0.3892647010267417 & 0.1160519898330615\end{array}$ $\begin{array}{lllll}0.3184081832050604 & 0.4635849880660248 & 0.1079926766905455\end{array}$ 
0.38773107385801650 .36238024293437330 .1535316489278105 $0.2239362548537244 \quad 0.47494293255096890 .5877815709086465$ $\begin{array}{lllll}-0.0001639857728387 & 0.4164341516291084 & 0.5595647507474248\end{array}$ $\begin{array}{lllll}0.2866680288806198 & 0.4249930163946480 & 0.8299280376634244\end{array}$ $\begin{array}{llll}0.7973316656951159 & 0.4123276886762976 & 0.3688222343583610\end{array}$ $\begin{array}{lllll}0.5593066159597311 & 0.4593733935831030 & 0.3321585794349937\end{array}$ $\begin{array}{lllll}0.6367783037959965 & 0.3887103580769851 & 0.1181614822079638\end{array}$ $\begin{array}{lllll}0.8241590230760344 & 0.4638532535441985 & 0.1197439037805472\end{array}$ $\begin{array}{llll}0.8880600708699292 & 0.3620066840068862 & 0.1496328988182016\end{array}$ $\begin{array}{llll}0.7415235335026810 & 0.4723453334323988 & 0.5925402005560287\end{array}$ $\begin{array}{lllll}0.5064510345501880 & 0.4189403369183041 & 0.5674063422846489\end{array}$ $\begin{array}{llll}0.7791428197023719 & 0.4224498689225646 & 0.8342773952330649\end{array}$ $\begin{array}{lllll}0.4225993637420155 & 0.2335053995241959 & 0.4973585069087463\end{array}$ $\begin{array}{lllll}0.1724790848512474 & 0.2963483846080371 & 0.4912440143974218\end{array}$ $\begin{array}{lllll}0.4418298951333176 & 0.1123112478815436 & 0.5277271387933791\end{array}$ $\begin{array}{lllll}0.9245736151112423 & 0.2340701986892733 & 0.4977104883101165\end{array}$ $\begin{array}{lllll}0.6719880313577882 & 0.2940863429641971 & 0.4925664661544663\end{array}$ $\begin{array}{lllll}0.9414730230744510 & 0.1130612442822215 & 0.5275377399769599\end{array}$ $\begin{array}{llll}0.1515167478631097 & 0.4180590145780276 & 0.4615972622863380\end{array}$ $\begin{array}{llll}0.6538374039967669 & 0.4140910642613452 & 0.4648763988107691\end{array}$ $\begin{array}{lllll}0.0437255225686112 & 0.2318781648319507 & 0.2044696882313082\end{array}$ $\begin{array}{llllll}0.3032668607922747 & 0.1678169811411827 & 0.2117717167253096\end{array}$ $\begin{array}{lllll}0.2914494639315879 & 0.3623281839742799 & 0.7758479540288696\end{array}$ $\begin{array}{llll}0.3079134919955405 & 0.1194688167674783 & 0.7949805952857234\end{array}$ $0.0507121960082560 \quad 0.18029292626093390 .7992326268287215$ $\begin{array}{llll}0.0534969721441521 & 0.2980570809960698 & 0.7854985901650210\end{array}$ $\begin{array}{lllll}0.0455330273093466 & 0.3491082695192852 & 0.1888503290376537\end{array}$ $\begin{array}{llllll}0.5429901072250033 & 0.2309392310290827 & 0.2042490233389713\end{array}$ $\begin{array}{llll}0.8048444156246776 & 0.1685345541876430 & 0.2111975563409380\end{array}$ $\begin{array}{llll}0.7907605361595386 & 0.3596169862332412 & 0.7798894333042384\end{array}$ $\begin{array}{lllll}0.8072473251766400 & 0.1213960274867926 & 0.7949237799695819\end{array}$ $\begin{array}{llll}0.5478785908712337 & 0.1801000506797583 & 0.7998217698204086\end{array}$ $\begin{array}{lllll}0.5504996198819936 & 0.2969950635425518 & 0.7841592381118012\end{array}$ $\begin{array}{llll}0.5441171312399310 & 0.3484049435139180 & 0.1916345257894163\end{array}$ $\begin{array}{llll}0.2864575034531232 & 0.4092206182087683 & 0.1951260522136333\end{array}$ $\begin{array}{lllll}0.7857813527540213 & 0.4079559044523458 & 0.1941279023481169\end{array}$

C3:

$$
\begin{array}{lllll}
\text { H } 37 \text { C } 4 \text { N } 3 \text { O } 61 \text { Al } 8 \text { Si } 16 & \\
1.00000000000000 & & \\
10.1485009999999996 & 0.0000000000000000 & -1.8735860000000000 \\
0.0646860000000000 & 24.9946149999999996 & -0.5148360000000000 \\
0.0000000000000000 & 0.0000000000000000 & 9.3469999999999995 \\
\text { H C N O Al Si } & & \\
37 \quad 4 \quad 3 \quad 61 \quad 8 \quad 16 &
\end{array}
$$

Direct

$\begin{array}{llll}0.6065114193507368 & 0.5762561311239055 & 0.5124766685217064\end{array}$ $\begin{array}{lllll}0.5174759983066096 & 0.5184681389122503 & 0.4540769659734632\end{array}$ $\begin{array}{lllll}0.1871827791143846 & 0.0537638575480584 & 0.8252494485035013\end{array}$ $\begin{array}{lllll}0.6856189628343102 & 0.0551731883956460 & 0.8218848197827978\end{array}$ $\begin{array}{lllll}0.1024091752483449 & 0.1380242873746707 & 0.3703178885128368\end{array}$ $\begin{array}{lllll}0.3464786988854922 & 0.3109183377735374 & 0.3440053138206152\end{array}$ $\begin{array}{lllll}0.2610399702408465 & 0.2197799582719345 & 0.6771185278758529\end{array}$ $\begin{array}{lllll}0.4868670228042891 & 0.0389191400706640 & 0.6967866948597954\end{array}$ $\begin{array}{lllllll}0.3448050891397431 & 0.0661426708883744 & 0.3002943477502122\end{array}$ $\begin{array}{lllll}0.6019530979896499 & 0.1394141474675819 & 0.3726001391377707\end{array}$ $\begin{array}{lllll}0.8378964578559830 & 0.3060741124864391 & 0.3224074429823679\end{array}$ $\begin{array}{lllll}0.7609517217262325 & 0.2203857770531723 & 0.6750763278213048\end{array}$ $\begin{array}{lllll}0.9839292934406659 & 0.0403407514160308 & 0.6993241417697713\end{array}$ $\begin{array}{lllll}0.8398908977864394 & 0.0668548666879481 & 0.3004169604475120\end{array}$ $\begin{array}{lllll}0.0577290365933712 & 0.4954930338502195 & 0.3636084083835455\end{array}$ $\begin{array}{lllll}0.4990973233930964 & 0.3941919017936205 & 0.6419111494404178\end{array}$ $\begin{array}{llll}0.2662809720683725 & 0.5042731633447318 & 0.5448150869590193\end{array}$ $\begin{array}{lllll}0.6181928323536741 & 0.4802963116076690 & 0.2865726694295810\end{array}$ $\begin{array}{llll}0.9952947178879016 & 0.3848227496490186 & 0.6297154213964614\end{array}$ $\begin{array}{lllll}0.7615337618791289 & 0.4599989003758030 & 0.7048497097967172\end{array}$ $\begin{array}{lllll}0.3966268094985788 & 0.4789816395377958 & 0.1693210852050458\end{array}$ $\begin{array}{lllll}0.9268009746914572 & 0.4674248208906132 & 0.1776908170075892\end{array}$ $\begin{array}{lllll}0.3056972719365669 & 0.4380750573247131 & 0.9370624056393637\end{array}$ $\begin{array}{llllll}0.8119053511506996 & 0.4360530167906134 & 0.9411835552015603\end{array}$ $\begin{array}{lllll}0.2938766359496145 & 0.0894028103234460 & 0.0639177022078009\end{array}$ $\begin{array}{llllll}0.7882717677200819 & 0.0901429977481249 & 0.0629711726242531\end{array}$ $\begin{array}{llll}0.2640495234321911 & 0.4652377236123109 & 0.6924606185679437\end{array}$ 
$\begin{array}{llll}0.8395008637153816 & 0.4757205181762179 & 0.5811682636701119\end{array}$ $\begin{array}{lllll}0.2723667195852092 & 0.0464671024962058 & 0.4257266154973338\end{array}$ $\begin{array}{llllll}0.7689297422147823 & 0.0474437161835448 & 0.4269312978546081\end{array}$ $\begin{array}{lllll}0.4231766801644374 & 0.4173354206227832 & 0.4876174194508576\end{array}$ $\begin{array}{llll}0.9226986017371138 & 0.4123336247853760 & 0.4825652941866606\end{array}$ $\begin{array}{lllll}0.67743392050202871 & 0.1123392520884353 & 0.5201860035390909\end{array}$ $\begin{array}{lllll}0.1763074547977911 & 0.1111858950785690 & 0.5188324333831901\end{array}$ $\begin{array}{lllll}0.2973790131226632 & 0.7164900432808117 & 0.4909906410205354\end{array}$ $\begin{array}{llll}0.0972849620625459 & 0.6666473500844753 & 0.4420166639346762\end{array}$ 0.51139865095090690 .66548960103242800 .5208205057914141 $\begin{array}{lllll}0.4083177659571794 & 0.5875792001286821 & 0.4869604103123849\end{array}$ $\begin{array}{llll}0.3018217608450117 & 0.6729359378144130 & 0.4844900461068091\end{array}$ $\begin{array}{lllll}0.1756760931412969 & 0.5902705267001621 & 0.4470958876769300\end{array}$ $\begin{array}{llll}0.4169259389123062 & 0.6450137837327019 & 0.5011244317150920\end{array}$ $\begin{array}{lllll}0.1846713029524087 & 0.6466627228448149 & 0.4564308252871145\end{array}$ $\begin{array}{lllll}0.5175249716266577 & 0.5578703757967797 & 0.4944192808389647\end{array}$ $\begin{array}{lllll}0.2913662152190393 & 0.5622633537861549 & 0.4669004492030716\end{array}$ $\begin{array}{lllll}0.0645478091717551 & 0.5696635382532498 & 0.4230222164924378\end{array}$ $\begin{array}{lllll}0.0936267228096298 & 0.3480777255948371 & 0.3733939834436109\end{array}$ $\begin{array}{lllll}0.0605134192697853 & 0.2393496189623217 & 0.3883999055961536\end{array}$ $\begin{array}{lllll}0.3116207916117467 & 0.2813911288665049 & 0.3913224697704897\end{array}$ $\begin{array}{lllll}0.3987691234380092 & 0.2068109140481595 & 0.1445692920044313\end{array}$ $\begin{array}{lllll}0.0711887840807118 & 0.2854068034816706 & 0.1369039293114976\end{array}$ $\begin{array}{lllll}0.1527327795939198 & 0.1835642078033980 & 0.1800876779141534\end{array}$ $\begin{array}{lllll}0.3610452312859572 & 0.1667476422126766 & 0.3950711270379722\end{array}$ $\begin{array}{lllll}0.3600573052000848 & 0.0537261002443241 & 0.4051075040643005\end{array}$ $\begin{array}{lllll}0.0932818425782873 & 0.1085333372930532 & 0.4348247399036539\end{array}$ $\begin{array}{lllll}0.3198440364987812 & 0.1036582942625947 & 0.1651588125305904\end{array}$ $\begin{array}{lllll}0.2986499460339989 & 0.1123759686406305 & 0.6273789349781358\end{array}$ $\begin{array}{lllll}0.0407371423032112 & 0.0665578662127440 & 0.6668531927141470\end{array}$ $\begin{array}{lllll}0.2757762044680082 & 0.0637687636462355 & 0.8815511509040144\end{array}$ $\begin{array}{lllll}0.2092862251459416 & 0.1654587766031947 & 0.8445576573547904\end{array}$ $\begin{array}{lllll}0.0411374870304446 & 0.2872536780415134 & 0.6144953108621026\end{array}$ $\begin{array}{lllll}0.2897085529378949 & 0.2452148072642152 & 0.6122719158118556\end{array}$ $\begin{array}{lllll}0.2013539863784758 & 0.3215562656280777 & 0.8570605137769177\end{array}$ $\begin{array}{lllll}0.0352328802635638 & 0.2402945895987650 & 0.8652581431241612\end{array}$ $\begin{array}{llll}0.2408225096089075 & 0.3600234946063908 & 0.6068489456464004\end{array}$ $\begin{array}{lllll}0.4590241628145783 & 0.1389647236795065 & 0.8795853941368899\end{array}$ $\begin{array}{lllll}0.5077521049376208 & 0.1776785992379096 & 0.6293297277503996\end{array}$ $\begin{array}{llll}0.4483146222145942 & 0.3423449360786250 & 0.8216467750380689\end{array}$ $\begin{array}{lllll}0.5897126097860554 & 0.3491811920243538 & 0.3730818305494910\end{array}$ $\begin{array}{lllll}0.5596357764307268 & 0.2397319519384373 & 0.3874995540035940\end{array}$ $\begin{array}{lllll}0.8102162726623074 & 0.2812791734274557 & 0.3897572671136719\end{array}$ $\begin{array}{lllll}0.9002267522154976 & 0.2063520837370025 & 0.1449108087762133\end{array}$ $\begin{array}{llllll}0.5644976027715524 & 0.2878748308340421 & 0.1364991777580503\end{array}$ $\begin{array}{lllll}0.6524362674870136 & 0.1864678323814271 & 0.1770646874782260\end{array}$ $\begin{array}{llll}0.8594229877955765 & 0.1670966288262816 & 0.3944841370322264\end{array}$ $\begin{array}{lllll}0.8563778065617307 & 0.0544771890314120 & 0.4052546944013975\end{array}$ $\begin{array}{llll}0.5944577302349850 & 0.1092019952895535 & 0.4352794843204355\end{array}$ $\begin{array}{llll}0.8129699499908288 & 0.1042229848436325 & 0.1645773112113810\end{array}$ $\begin{array}{lllll}0.7974829090420208 & 0.1141617604205050 & 0.6279840262214593\end{array}$ $\begin{array}{llll}0.5432294359573080 & 0.0658814311517131 & 0.6668131385997914\end{array}$ $\begin{array}{lllll}0.7737798335335229 & 0.0650165090638512 & 0.8806155951815894\end{array}$ $\begin{array}{lllll}0.7087498153023652 & 0.1668879487651027 & 0.8462068768945377\end{array}$ $\begin{array}{lllll}0.5408719035855315 & 0.2863372996413113 & 0.6141402165843035\end{array}$ $\begin{array}{lllll}0.7901749276278468 & 0.2460610682516579 & 0.6112653483271620\end{array}$ $\begin{array}{llllll}0.7008264347888898 & 0.3190634847929917 & 0.8574341979404141\end{array}$ $\begin{array}{lllll}0.5291820916946663 & 0.2402465851730169 & 0.8649775541508958\end{array}$ $\begin{array}{llllll}0.7389803550390784 & 0.3595325699136334 & 0.6080788186831687\end{array}$ $\begin{array}{llll}0.9586080013880796 & 0.1399614604696154 & 0.8796358115392501\end{array}$ $\begin{array}{lllll}0.0077974774226652 & 0.1781441676470241 & 0.6284078098528210\end{array}$ $\begin{array}{lllll}0.9470928915203894 & 0.3412008601430523 & 0.8240050563863109\end{array}$ $\begin{array}{lllll}0.3035174101496158 & 0.4120130939949101 & 0.3744592465937937\end{array}$ $\begin{array}{lllll}0.0565018668594769 & 0.4579944914600005 & 0.3295590689209230\end{array}$ $\begin{array}{llll}0.1393267650794070 & 0.3867034714244302 & 0.1224674243135958\end{array}$ $\begin{array}{llll}0.3121217965547838 & 0.4647073027451997 & 0.1170204615848961\end{array}$ $\begin{array}{llll}0.3914563062018550 & 0.3645988006432965 & 0.1513181490945556\end{array}$ $\begin{array}{lllll}0.2281695566268553 & 0.4724269616072033 & 0.5885147339538400\end{array}$ $\begin{array}{lllll}0.0034194995104899 & 0.4151246226159576 & 0.5677107504818011\end{array}$ $\begin{array}{lllll}0.2887219033246475 & 0.4243019720930557 & 0.8350369922723546\end{array}$ $\begin{array}{lllll}0.7993869099456248 & 0.4129987898347663 & 0.3738505386934144\end{array}$ $\begin{array}{lllll}0.5609483302496906 & 0.4637197152343621 & 0.3447467114054012\end{array}$ $\begin{array}{lllll}0.6422069616996121 & 0.3886031746402974 & 0.1239088065779803\end{array}$ 
$\begin{array}{llll}0.8351035310224598 & 0.4598072957459861 & 0.1204233403068878\end{array}$ $\begin{array}{lllll}0.8905239147024820 & 0.3573742418811556 & 0.1604283368529585\end{array}$ $\begin{array}{lllll}0.7495987236436636 & 0.4721226935026359 & 0.6000074241535174\end{array}$ $\begin{array}{llll}0.5087427965156384 & 0.4202943316343093 & 0.5684011150337906\end{array}$ $\begin{array}{lllll}0.7821868717296387 & 0.4219529566597107 & 0.8396890858908124\end{array}$ $\begin{array}{lllll}0.4260012520427951 & 0.2326254327449802 & 0.5047660435601308\end{array}$ $\begin{array}{llll}0.1755593140604209 & 0.2943452158814822 & 0.4992844386838544\end{array}$ $\begin{array}{llllll}0.4455225602786693 & 0.1115543666286430 & 0.5355731959028571\end{array}$ $\begin{array}{llll}0.9261418812127366 & 0.2331441737619276 & 0.5038538782599614\end{array}$ $\begin{array}{lllll}0.6737129274704416 & 0.2941318590468528 & 0.4971658748496300\end{array}$ $\begin{array}{lllll}0.9439000489523303 & 0.1120995419650495 & 0.5349645991645680\end{array}$ $\begin{array}{llllll}0.1552459013382861 & 0.4151574917344381 & 0.4655937345155484\end{array}$ $\begin{array}{lllll}0.6567416402951153 & 0.4145603283332085 & 0.4701017043068962\end{array}$ $\begin{array}{lllll}0.0471769793115190 & 0.2293035566181921 & 0.2119024568507281\end{array}$ $\begin{array}{lllll}0.3078012801953961 & 0.1666013920871019 & 0.2189293317180686\end{array}$ $\begin{array}{llllll}0.2942378750062947 & 0.3613262467828541 & 0.7827335332968233\end{array}$ $\begin{array}{llll}0.3108953136575294 & 0.1191545932819078 & 0.8021491465063229\end{array}$ $\begin{array}{lllll}0.0526925154751579 & 0.1795303623902479 & 0.8059117565576945\end{array}$ $\begin{array}{llll}0.0558611500556952 & 0.2971246588782899 & 0.7913945375225353\end{array}$ $\begin{array}{lllll}0.0491059396019609 & 0.3458707267752457 & 0.1962679055061495\end{array}$ $\begin{array}{lllll}0.5447585313437486 & 0.2310198283022973 & 0.2103869194195880\end{array}$ $\begin{array}{llll}0.8057008224291040 & 0.1673724001233363 & 0.2183711673867761\end{array}$ $\begin{array}{lllll}0.7930178581713216 & 0.3592949491836165 & 0.7844431260175484\end{array}$ $\begin{array}{lllll}0.8100879589977227 & 0.1205523871734288 & 0.8029602629915826\end{array}$ $\begin{array}{lllll}0.5513889634738158 & 0.1796404458627492 & 0.8069916799504919\end{array}$ $\begin{array}{lllll}0.5533642372497976 & 0.2966661718677813 & 0.7903994456801560\end{array}$ $\begin{array}{lllll}0.5460887724595810 & 0.3489080464009494 & 0.1949047789567788\end{array}$ $\begin{array}{lllll}0.2860689533597930 & 0.4086290705058208 & 0.1993802048207254\end{array}$ $\begin{array}{llll}0.7930189968977653 & 0.4058559379438365 & 0.2002282734399175\end{array}$

C4 :

H 37 C 4 N 3 O 61 Al 8 Si 16

1.00000000000000

$10.1485009999999996 \quad 0.0000000000000000 \quad-1.8735860000000000$

$\begin{array}{llll}0.0646860000000000 & 24.9946149999999996 & -0.5148360000000000\end{array}$

$\begin{array}{llll}0.0000000000000000 & 0.0000000000000000 & 9.3469999999999995\end{array}$

$\mathrm{H} C \mathrm{~N} O \mathrm{Al} \mathrm{Si}$

$\begin{array}{llllll}37 & 4 & 3 & 61 & 8 & 16\end{array}$

Direct

$\begin{array}{llll}0.2263151877791865 & 0.7087805914549555 & 0.2603050384479652\end{array}$ $\begin{array}{lllll}0.4518889607333118 & 0.6717491242726992 & 0.3546054599475532\end{array}$ $\begin{array}{llll}0.5232559362904311 & 0.5233751735319924 & 0.3440800062514220\end{array}$ $\begin{array}{llll}0.5790175803331470 & 0.5899733207946807 & 0.3935253768773889\end{array}$ $\begin{array}{llll}0.0545007503843116 & 0.6470413601429322 & 0.1664876483735188\end{array}$ $\begin{array}{lllll}0.1951695825441778 & 0.0558674589346331 & 0.8140083954313230\end{array}$ $\begin{array}{lllll}0.6906142140174056 & 0.0573547269635465 & 0.8138755255409859\end{array}$ $\begin{array}{llll}0.1085666874032585 & 0.1414074936646736 & 0.3644172894035743\end{array}$ $\begin{array}{lllll}0.3473371871531940 & 0.3097991669178116 & 0.3201250515953755\end{array}$ $\begin{array}{lllll}0.2671162392497959 & 0.2217237375930624 & 0.6645369731549842\end{array}$ $\begin{array}{lllll}0.4916248424254713 & 0.0412564354656271 & 0.6871885346993145\end{array}$ $\begin{array}{lllll}0.3510355565842835 & 0.0689644296860003 & 0.2898646375552894\end{array}$ $\begin{array}{lllll}0.6094821025640574 & 0.1410234579967670 & 0.3631084167196484\end{array}$ $\begin{array}{llll}0.8491064178045152 & 0.3098955242516162 & 0.3221296837624270\end{array}$ $\begin{array}{llllll}0.7676837979891076 & 0.2227588279497150 & 0.6681936425865335\end{array}$ $\begin{array}{llll}-0.0075461200213665 & 0.0420514365911898 & 0.6917133940609972\end{array}$ $\begin{array}{lllll}0.8480426418182643 & 0.0677124521083813 & 0.2926259475704773\end{array}$ $\begin{array}{lllll}0.0868731106027691 & 0.4841616522567349 & 0.2697118584850333\end{array}$ $\begin{array}{lllll}0.5019537358468840 & 0.3936914000279677 & 0.6240691864068575\end{array}$ $\begin{array}{lllll}0.2612149668920378 & 0.4633632435713375 & 0.6837043500368112\end{array}$ $\begin{array}{lllll}0.5771904174850939 & 0.4616376665103388 & 0.2303866903169291\end{array}$ $\begin{array}{llll}1.0052518707781239 & 0.3901564901730957 & 0.6251967531925764\end{array}$ $\begin{array}{llll}0.7659442648300958 & 0.4623811039424459 & 0.6927592167863397\end{array}$ $\begin{array}{lllll}0.3068611191391543 & 0.4990513690900784 & 0.1460233528805834\end{array}$ $\begin{array}{lllll}0.8985859642474034 & 0.4858042540423573 & 0.1335591938071764\end{array}$ $\begin{array}{lllll}0.3013837373017498 & 0.4414787671832478 & 0.9216209623768459\end{array}$ $\begin{array}{llll}0.8031769881341807 & 0.4396420317865078 & 0.9276495149202247\end{array}$ $\begin{array}{lllll}0.3007227228299745 & 0.0920686258274677 & 0.0535793967285638\end{array}$ $\begin{array}{lllll}0.7941355792661237 & 0.0909343870235548 & 0.0550335409127322\end{array}$ $\begin{array}{lllll}0.3275399994056307 & 0.4845629722411332 & 0.5540197522677570\end{array}$ $\begin{array}{lllll}0.8342733419496283 & 0.4827269959729288 & 0.5638689418529408\end{array}$ $\begin{array}{lllll}0.2775825103923887 & 0.0492976453942223 & 0.4143834130992792\end{array}$ $\begin{array}{lllll}0.7800486782600889 & 0.0474365530206518 & 0.4208206731988287\end{array}$ $\begin{array}{llll}0.4300668734367722 & 0.4180642120345355 & 0.4696259583796896\end{array}$ 
$\begin{array}{llll}0.9317959446991317 & 0.4171211855610635 & 0.4760214056104913\end{array}$ $\begin{array}{lllll}0.6841669346885411 & 0.1135555140760148 & 0.5104902654447316\end{array}$ $\begin{array}{lllll}0.1838756523782086 & 0.1137169811228681 & 0.5107538569217351\end{array}$ $\begin{array}{lllll}0.2485847756813952 & 0.6661088876466204 & 0.2578334187970502\end{array}$ $\begin{array}{lllll}0.3702567215877449 & 0.6456924624193694 & 0.3083074405141515\end{array}$ $\begin{array}{lllll}0.3871013335003680 & 0.5884863474664138 & 0.2975804795807998\end{array}$ $\begin{array}{llll}0.1626241595282588 & 0.5768992621326243 & 0.1918761237821439\end{array}$ $\begin{array}{lllll}0.1471779866641477 & 0.6329104067999524 & 0.2015556397784216\end{array}$ $\begin{array}{lllll}0.5030569094886422 & 0.5657943725561831 & 0.3490265688635698\end{array}$ $0.2860338043599593 \quad 0.55672504599029040 .2355058018161552$ $\begin{array}{llll}0.0639971953395639 & 0.5491497079264722 & 0.1444713389930244\end{array}$ $\begin{array}{llll}0.0984252541001605 & 0.3489961346455075 & 0.3630332850389871\end{array}$ $\begin{array}{lllll}0.0665431936370291 & 0.2401842318500074 & 0.3785490607459100\end{array}$ $\begin{array}{lllll}0.3168244386534839 & 0.2825505030279868 & 0.3786367939279296\end{array}$ $\begin{array}{llll}0.4063190856648310 & 0.2096995552322030 & 0.1345857226464544\end{array}$ $\begin{array}{lllll}0.0681905680012984 & 0.2878266905271016 & 0.1263431862939029\end{array}$ $\begin{array}{lllll}0.1600894063611317 & 0.1869354861809476 & 0.1678966365144242\end{array}$ $\begin{array}{lllll}0.3679971106063796 & 0.1690521273229785 & 0.3843432518292836\end{array}$ $\begin{array}{lllll}0.3656421939608456 & 0.0563707049655462 & 0.3945293305714821\end{array}$ $\begin{array}{llllll}0.1005943727908201 & 0.1110126300085387 & 0.4263613743920846\end{array}$ $\begin{array}{lllll}0.3268508424331401 & 0.1064874052250582 & 0.1545545421840068\end{array}$ $\begin{array}{llllll}0.3050504714041261 & 0.1153872771813397 & 0.6176959541852948\end{array}$ $\begin{array}{lllll}0.0493975144498046 & 0.0680796700414367 & 0.6586569463063240\end{array}$ $\begin{array}{lllll}0.2836957795699328 & 0.0659060010136933 & 0.8709035286020429\end{array}$ $\begin{array}{lllll}0.2153208383846176 & 0.1674990673867000 & 0.8362202297508762\end{array}$ $\begin{array}{lllll}0.0472972220459283 & 0.2887311042565436 & 0.6038422192036634\end{array}$ $\begin{array}{llllll}0.2963752505920884 & 0.2474860549145631 & 0.6009933696515737\end{array}$ $\begin{array}{lllll}0.2071848624799944 & 0.3229959178456512 & 0.8448189152658494\end{array}$ $\begin{array}{lllll}0.0401736772288932 & 0.2423360662069354 & 0.8553928259600662\end{array}$ $\begin{array}{lllll}0.2480140275532151 & 0.3616555104925129 & 0.5937772742744961\end{array}$ $\begin{array}{lllll}0.4656763857608031 & 0.1417343447430643 & 0.8697129164724771\end{array}$ $\begin{array}{lllll}0.5140753601142652 & 0.1801030727932137 & 0.6188587513373536\end{array}$ $\begin{array}{lllll}0.4534863248589324 & 0.3450025971682963 & 0.8113668790960183\end{array}$ $\begin{array}{lllll}0.5996395367594011 & 0.3507862138234885 & 0.3639640269988817\end{array}$ $\begin{array}{lllll}0.5670648053576214 & 0.2419256390616564 & 0.3777707658292217\end{array}$ $\begin{array}{lllll}0.8174778402335350 & 0.2828312186013066 & 0.3803719659061380\end{array}$ $\begin{array}{lllll}0.9062918028301187 & 0.2064831930664760 & 0.1353787065657958\end{array}$ $\begin{array}{lllll}0.5769367975874699 & 0.2893735352794777 & 0.1270388378782615\end{array}$ $\begin{array}{lllll}0.6588615276498789 & 0.1872105337457338 & 0.1687645592590089\end{array}$ $\begin{array}{llll}0.8654674391670057 & 0.1682742880070063 & 0.3863114540756785\end{array}$ $\begin{array}{lllll}0.8664353207438582 & 0.0553602433891503 & 0.3975803897853822\end{array}$ $\begin{array}{llllll}0.6011438976429943 & 0.1109945696398434 & 0.4260960095614292\end{array}$ $\begin{array}{lllll}0.8187829347971489 & 0.1047571747748540 & 0.1570303145584274\end{array}$ $\begin{array}{lllll}0.8049684978570082 & 0.1147062239654317 & 0.6193493797907922\end{array}$ $\begin{array}{llllll}0.5483203357619215 & 0.0680971138624711 & 0.6572333712809952\end{array}$ $\begin{array}{lllll}0.7786485931827345 & 0.0672156581479906 & 0.8727903673885876\end{array}$ $\begin{array}{llllll}0.7152671938026492 & 0.1692246519379047 & 0.8347983280223266\end{array}$ $\begin{array}{lllll}0.5468137528602953 & 0.2891099621740602 & 0.6038176435310730\end{array}$ $\begin{array}{lllll}0.7962191239194659 & 0.2478088617978987 & 0.6022304217026067\end{array}$ $\begin{array}{lllll}0.7063730447951212 & 0.3220950991584822 & 0.8474156411433534\end{array}$ $\begin{array}{lllll}0.5358783498923193 & 0.2428214973124985 & 0.8544410145959176\end{array}$ $\begin{array}{lllll}0.7463112233723189 & 0.3618894589584768 & 0.5975262606745981\end{array}$ $\begin{array}{lllll}0.9648248714639049 & 0.1416858141974021 & 0.8708799430589105\end{array}$ $\begin{array}{lllll}0.0137356631185654 & 0.1797602985111176 & 0.6199453979930144\end{array}$ $\begin{array}{lllll}0.9531269731069579 & 0.3435344405083601 & 0.8125479166130347\end{array}$ $\begin{array}{lllll}0.3078281719316627 & 0.4166733463658849 & 0.3598837000577122\end{array}$ $\begin{array}{llll}0.0529050708753783 & 0.4547703219001622 & 0.3188452005287512\end{array}$ $\begin{array}{lllll}0.1518511419276148 & 0.3858904812502165 & 0.1104120509474007\end{array}$ $\begin{array}{lllll}0.3210779560022000 & 0.4637058165682606 & 0.0951382050655101\end{array}$ $\begin{array}{lllll}0.4025855464298260 & 0.3654382481520734 & 0.1425467834238092\end{array}$ $\begin{array}{lllll}0.2423600845111648 & 0.4756694715939950 & 0.5787367500141669\end{array}$ $\begin{array}{lllll}0.0145881032397836 & 0.4193655701726238 & 0.5601199184161149\end{array}$ $\begin{array}{lllll}0.2875847076820571 & 0.4261646989018516 & 0.8199755868225161\end{array}$ $\begin{array}{lllll}0.8104011146843744 & 0.4159439411429913 & 0.3668434511562453\end{array}$ $\begin{array}{llll}0.5687263626861632 & 0.4639795178469817 & 0.3320870296325125\end{array}$ $\begin{array}{lllll}0.6516866783827299 & 0.3916749500527845 & 0.1189141030477467\end{array}$ $\begin{array}{lllll}0.8155955409444418 & 0.4667006611486977 & 0.0995987209165421\end{array}$ $\begin{array}{lllll}0.8993204254511219 & 0.3650413837018279 & 0.1436468397812586\end{array}$ $\begin{array}{lllll}0.7480303482726534 & 0.4751945592221593 & 0.5880803829735817\end{array}$ $\begin{array}{lllll}0.5122531974037444 & 0.4214400995218698 & 0.5549972330712688\end{array}$ $\begin{array}{lllll}0.7883271793543152 & 0.4248666623455411 & 0.8258577576955910\end{array}$ $\begin{array}{lllll}0.4327822331662252 & 0.2348583739053713 & 0.4936156002937226\end{array}$ $\begin{array}{llll}0.1810163977644610 & 0.2956169951568018 & 0.4872294690215859\end{array}$ 
$\begin{array}{llll}0.4517687300559922 & 0.1138821614819936 & 0.5254155285247688\end{array}$ $\begin{array}{lllll}0.9323795180116785 & 0.2344783889965666 & 0.4947384357464676\end{array}$ $\begin{array}{lllll}0.6811406232231700 & 0.2962165138392893 & 0.4882705078436013\end{array}$ $\begin{array}{lllll}0.9515709312034334 & 0.1134717278211667 & 0.5268911738503039\end{array}$ $\begin{array}{llll}0.1605110373129967 & 0.4162205318186349 & 0.4525483280286581\end{array}$ $\begin{array}{lllll}0.6642492647697706 & 0.4169750153574125 & 0.4585671998290408\end{array}$ $\begin{array}{lllll}0.0515507625885904 & 0.2311968971079563 & 0.2013699269024566\end{array}$ $\begin{array}{lllll}0.3145486790632666 & 0.1694518155353697 & 0.2082148195806396\end{array}$ $\begin{array}{lllll}0.2988832947110628 & 0.3630258886210210 & 0.7702777706177709\end{array}$ $\begin{array}{llll}0.3176587477822883 & 0.1216783596730470 & 0.7927014727774286\end{array}$ $\begin{array}{lllll}0.0587055760932803 & 0.1814440600989233 & 0.7974566810538598\end{array}$ $\begin{array}{llll}0.0609463446210896 & 0.2989533371949222 & 0.7804135888181466\end{array}$ $\begin{array}{lllll}0.0548028485171558 & 0.3491696642031519 & 0.1853980799366810\end{array}$ $\begin{array}{llll}0.5532854505653801 & 0.2328093651690658 & 0.2008668744563988\end{array}$ $\begin{array}{lllll}0.8120765221339766 & 0.1680010423951908 & 0.2101692299538969\end{array}$ $\begin{array}{lllll}0.7991543710100547 & 0.3619172064920254 & 0.7738867316448801\end{array}$ $\begin{array}{lllll}0.8163097983493446 & 0.1222906846973766 & 0.7938504456873091\end{array}$ $\begin{array}{lllll}0.5579606637045359 & 0.1821797708585092 & 0.7964327329786708\end{array}$ $\begin{array}{llll}0.5591752580522427 & 0.2993856537624666 & 0.7801709101315198\end{array}$ $\begin{array}{llll}0.5577296067988953 & 0.3502023730779553 & 0.1860893325255683\end{array}$ $\begin{array}{lllll}0.2969618683215303 & 0.4100756831072194 & 0.1849413794304139\end{array}$ $\begin{array}{llll}0.8029387821175218 & 0.4117688276083458 & 0.1926393639129897\end{array}$

C5 :

$\begin{array}{llllll}\mathrm{H} 37 & \mathrm{C} 4 & \mathrm{~N} 3 & \mathrm{O} 61 & \mathrm{Al} 8 & \mathrm{Si} 16\end{array}$

1.00000000000000

$\begin{array}{llll}10.1485009999999996 & 0.0000000000000000 & -1.8735860000000000\end{array}$

$0.0646860000000000 \quad 24.9946149999999996-0.5148360000000000$

$\begin{array}{llll}0.0000000000000000 & 0.0000000000000000 & 9.3469999999999995\end{array}$

$\mathrm{H} \quad \mathrm{C} \quad \mathrm{N}$ O $\mathrm{Al} \mathrm{Si}$

$\begin{array}{llllll}37 & 4 & 3 & 61 & 8 & 16\end{array}$

Direct

$\begin{array}{llll}0.0743258365951588 & 0.7140701636977491 & 0.2899422300409275\end{array}$

$\begin{array}{lllll}0.1278704380505592 & 0.6638877399887170 & 0.0714048208448158\end{array}$

$0.2466881951725295 \quad 0.52241960999238580 .1053381412625805$ $0.20232625033396280 .5764742499559581-0.0120825541391561$ $\begin{array}{llll}0.0927367124025246 & 0.6673014920604764 & 0.5124345540213608\end{array}$ $\begin{array}{lllll}0.1908793317296698 & 0.0553693242152036 & 0.8209274379941708\end{array}$ $\begin{array}{lllll}0.6883098018219762 & 0.0558297023455682 & 0.8213591578103199\end{array}$ $\begin{array}{lllll}0.1061122563499486 & 0.1407822900616119 & 0.3718183747865733\end{array}$ $\begin{array}{lllll}0.3444358495546831 & 0.3092165220295021 & 0.3296604186280220\end{array}$ $\begin{array}{lllll}0.2645753881621858 & 0.2218175246396698 & 0.6757952035843304\end{array}$ $\begin{array}{lllll}0.4886642496825244 & 0.0405314658154702 & 0.6949948050309636\end{array}$ $\begin{array}{lllll}0.3457589448085437 & 0.0679466755925400 & 0.2985722741341676\end{array}$ $\begin{array}{lllll}0.6064220221677153 & 0.1396410202471668 & 0.3691789949812488\end{array}$ $\begin{array}{lllll}0.8437975956497583 & 0.3085358780704157 & 0.3275685847788792\end{array}$ $\begin{array}{llll}0.7644212229984847 & 0.2212145550417802 & 0.6751196145572664\end{array}$ $\begin{array}{lllll}0.0099287121166549 & 0.0406019434248731 & 0.6965523311720783\end{array}$ $\begin{array}{lllll}0.8472339340884468 & 0.0675983545523926 & 0.2984852700190930\end{array}$ $\begin{array}{lllll}0.1105551502442660 & 0.4938180887464342 & 0.3238262799809925\end{array}$ $\begin{array}{lllll}0.5046119471933119 & 0.3946286529404495 & 0.6460840315344497\end{array}$ $\begin{array}{llll}0.2582420170005815 & 0.4641127609848162 & 0.6995194688322844\end{array}$ $\begin{array}{lllll}0.5318365909040208 & 0.4920447847920891 & 0.3887268919281310\end{array}$ $\begin{array}{lllll}0.9962418580921965 & 0.3859181365833952 & 0.6245308751976287\end{array}$ $\begin{array}{lllll}0.7617999778118606 & 0.4621855751079209 & 0.6979276209369208\end{array}$ $\begin{array}{llll}0.4333268202161292 & 0.4678188491056696 & 0.1881048930110527\end{array}$ $\begin{array}{lllll}0.9205218954341620 & 0.4724797416665549 & 0.1761042873400026\end{array}$ $\begin{array}{lllll}0.3226681337747791 & 0.4359186868179923 & 0.9419867666770406\end{array}$ $\begin{array}{lllll}0.8139036531406326 & 0.4385965624502730 & 0.9367789920045277\end{array}$ $\begin{array}{lllll}0.2941532587629158 & 0.0913644182377877 & 0.0618446524500100\end{array}$ $\begin{array}{llll}0.7945566590499494 & 0.0909749384376915 & 0.0620096994385535\end{array}$ 0.20304616091186340 .51133846620810910 .5822379287976083 $\begin{array}{llll}0.8294431921252210 & 0.4817460382310805 & 0.5681266572909389\end{array}$ $\begin{array}{lllll}0.2738059490782903 & 0.0485797664016120 & 0.4243667835221080\end{array}$ $\begin{array}{lllll}0.7752808209025701 & 0.0478158016036064 & 0.4240495687811502\end{array}$ $\begin{array}{lllll}0.4266811552994707 & 0.4173631801821866 & 0.4922269080481377\end{array}$ $\begin{array}{lllll}0.9235770722571339 & 0.4151021370753155 & 0.4805072027809807\end{array}$ $\begin{array}{lllll}0.6806953047359249 & 0.1127030198172761 & 0.5176058591603351\end{array}$ $\begin{array}{lllll}0.1810035180713577 & 0.1131084460279592 & 0.5185192277342310\end{array}$ $\begin{array}{lllll}0.1052757838486396 & 0.6722650201937118 & 0.2933535412300354\end{array}$ $\begin{array}{lllll}0.1342876059115058 & 0.6448056045732191 & 0.1760274650987776\end{array}$ $\begin{array}{llll}0.1745694189379205 & 0.5898602590026278 & 0.1971367025935950\end{array}$ 0.15268440767492630 .59374884418026890 .4422693422710561 
$0.11479360091291990 .6476981324454796 \quad 0.4234653520064373$ $\begin{array}{lllll}0.2061146121140329 & 0.5603138808970922 & 0.0871634468897360\end{array}$ $\begin{array}{lllll}0.1816136197762359 & 0.5661306746909364 & 0.3261198174798738\end{array}$ $\begin{array}{lllll}0.1568977891867398 & 0.5746438330723957 & 0.5659589275993714\end{array}$ $\begin{array}{lllll}0.0939226446876829 & 0.3499271281394647 & 0.3728182176275733\end{array}$ $\begin{array}{lllll}0.0635548511048250 & 0.2408211573179874 & 0.3871871537185198\end{array}$ $\begin{array}{lllll}0.3135222236040051 & 0.2824127094041928 & 0.3893138544159294\end{array}$ $\begin{array}{lllll}0.4036811717388609 & 0.2080935882335057 & 0.1431180278092125\end{array}$ $\begin{array}{lllll}0.0694544444871990 & 0.2884873395786334 & 0.1357218542320156\end{array}$ $\begin{array}{lllll}0.1565117364689321 & 0.1870174200368892 & 0.1768437952112071\end{array}$ $\begin{array}{lllll}0.3639433562931683 & 0.1683038403557142 & 0.3930977735202016\end{array}$ $\begin{array}{lllll}0.3615407769880836 & 0.0554690904922624 & 0.4032981112056720\end{array}$ $\begin{array}{llllll}0.0980406669272786 & 0.1104093146834971 & 0.4337750919548412\end{array}$ $\begin{array}{lllll}0.3196556444144893 & 0.1055808630730748 & 0.1631117762814796\end{array}$ $0.30189360280725390 .1145977236624826 \quad 0.6261285624626634$ $\begin{array}{lllll}0.0466311889068649 & 0.0671070120724157 & 0.6648717500702284\end{array}$ $\begin{array}{llll}0.2790545806897944 & 0.0653217694690843 & 0.8789961319189672\end{array}$ $\begin{array}{lllll}0.2124458009038696 & 0.1670326140141299 & 0.8443984060292189\end{array}$ $\begin{array}{lllll}0.0439668394613760 & 0.2880309721830319 & 0.6140970022823976\end{array}$ $\begin{array}{llll}0.2932873616167821 & 0.2472171526792592 & 0.6109004569725209\end{array}$ $\begin{array}{lllll}0.2049476809619162 & 0.3207284501723897 & 0.8571362012983885\end{array}$ $\begin{array}{lllll}0.0352125757257199 & 0.2409957707325392 & 0.8646328431632512\end{array}$ $\begin{array}{lllll}0.2412733151480201 & 0.3614754298898405 & 0.6079403647673463\end{array}$ $\begin{array}{llll}0.4626415797790419 & 0.1406902899097664 & 0.8781370197680595\end{array}$ $\begin{array}{lllll}0.5110329308228602 & 0.1791738908039686 & 0.6272420960753473\end{array}$ $\begin{array}{lllll}0.4509207365700529 & 0.3430166902898982 & 0.8212335049576902\end{array}$ $\begin{array}{lllll}0.5957357057854743 & 0.3496937533987554 & 0.3720727659725591\end{array}$ $\begin{array}{lllll}0.5634687084648907 & 0.2408447141444494 & 0.3865477698555342\end{array}$ $\begin{array}{lllll}0.8140863624294055 & 0.2821020851910989 & 0.3891615671904477\end{array}$ $\begin{array}{llll}0.9031211031403956 & 0.2079374694049952 & 0.1438203913638822\end{array}$ $\begin{array}{lllll}0.5748098853302778 & 0.2874700493579923 & 0.1356187663037235\end{array}$ $\begin{array}{lllll}0.6565191775543989 & 0.1856391407026254 & 0.1780951767082189\end{array}$ $\begin{array}{lllll}0.8644798421881997 & 0.1678828007199791 & 0.3937593013046418\end{array}$ $\begin{array}{lllll}0.8628993074892412 & 0.0551036591027908 & 0.4032443871227485\end{array}$ $\begin{array}{llllll}0.5976222331144377 & 0.1100053391394171 & 0.4333222232299344\end{array}$ $\begin{array}{lllll}0.8216315413363342 & 0.1050925673306357 & 0.1632737249811338\end{array}$ $\begin{array}{llll}0.8020737727501558 & 0.1139207326833805 & 0.6261369662049255\end{array}$ $\begin{array}{llll}0.5453866142419920 & 0.0673309578828329 & 0.6648987633340693\end{array}$ $\begin{array}{lllll}0.7767231514510904 & 0.0655076196808581 & 0.8794750476182924\end{array}$ $\begin{array}{llll}0.7126244320725873 & 0.1673804010066412 & 0.8430661640550449\end{array}$ $\begin{array}{llll}0.5447158104181524 & 0.2879276578800729 & 0.6128331428156519\end{array}$ $\begin{array}{lllll}0.7933498649609670 & 0.2467009324753451 & 0.6105889896309866\end{array}$ $\begin{array}{llll}0.7039869476567719 & 0.3217824843390139 & 0.8558403735602224\end{array}$ $\begin{array}{lllll}0.5356259535287640 & 0.2415028288405030 & 0.8636080488560914\end{array}$ $\begin{array}{lllll}0.7447451824833082 & 0.3608570160297040 & 0.6059635986320386\end{array}$ $\begin{array}{llll}0.9622628140767423 & 0.1400707284438364 & 0.8782511817651893\end{array}$ $\begin{array}{llll}0.0111262160698809 & 0.1789466177500598 & 0.6280094557136553\end{array}$ $\begin{array}{lllll}0.9510440126221105 & 0.3424874601779485 & 0.8237208879005388\end{array}$ $\begin{array}{lllll}0.3057526884608306 & 0.4121993763239284 & 0.3773135854679743\end{array}$ $\begin{array}{llll}0.0613349553758341 & 0.4609559540777936 & 0.3331489908660799\end{array}$ $\begin{array}{lllll}0.1455635342286689 & 0.3887658053297123 & 0.1226360723736798\end{array}$ $\begin{array}{llll}0.3408582202109436 & 0.4616854159185351 & 0.1279358405149505\end{array}$ $\begin{array}{lllll}0.3938827613267074 & 0.3600177422606078 & 0.1569490227909489\end{array}$ $\begin{array}{lllll}0.2372145525312875 & 0.4734999728910667 & 0.5946325273510656\end{array}$ $\begin{array}{llll}0.0049796644734711 & 0.4170564313814050 & 0.5656781922184506\end{array}$ $\begin{array}{lllll}0.2889040137203124 & 0.4236731573480564 & 0.8407185252343429\end{array}$ $\begin{array}{lllll}0.8020256150908711 & 0.4147539018404655 & 0.3719834883083031\end{array}$ $\begin{array}{llll}0.5600213736808002 & 0.4621688238304605 & 0.3333577373547680\end{array}$ $\begin{array}{lllll}0.6417220665439396 & 0.3896519266133239 & 0.1223922765570156\end{array}$ $\begin{array}{llll}0.8310541253356749 & 0.4638644784312206 & 0.1178852451093888\end{array}$ $\begin{array}{lllll}0.8928836655604366 & 0.3626263712373217 & 0.1559198108060031\end{array}$ $0.74340724306180340 .4744164868119864 \quad 0.5931941732875905$ $\begin{array}{lllll}0.5129774695135941 & 0.4200060408342096 & 0.5704324892553603\end{array}$ $\begin{array}{lllll}0.7883055323873887 & 0.4243793163300761 & 0.8351255692554697\end{array}$ $\begin{array}{lllll}0.4292128604406310 & 0.2342390380071717 & 0.5028974862975149\end{array}$ $\begin{array}{lllll}0.1776164488585392 & 0.2958790687863114 & 0.4973619757279644\end{array}$ $\begin{array}{llll}0.4484027787087758 & 0.1131566472402779 & 0.5334434709646761\end{array}$ $\begin{array}{lllll}0.9297304947379527 & 0.2339820764931853 & 0.5034138332420726\end{array}$ $\begin{array}{lllll}0.6783047573914611 & 0.2953099062021618 & 0.4972260238367123\end{array}$ $\begin{array}{llll}0.9488643646084328 & 0.1128465876232417 & 0.5339110564337569\end{array}$ $0.15784547044720220 .4169638135791593 \quad 0.4675594678551753$ $\begin{array}{lllll}0.6576811914250411 & 0.4158678897856382 & 0.4652132051589233\end{array}$ $\begin{array}{lllll}0.0493770698726936 & 0.2318462558775743 & 0.2101160996070559\end{array}$ 
$\begin{array}{llll}0.3103686421489735 & 0.1686501496459061 & 0.2170065536832014\end{array}$ $\begin{array}{lllll}0.2964125980664445 & 0.3611883473804691 & 0.7833142542864922\end{array}$ $\begin{array}{lllll}0.3143842405856017 & 0.1209617587472200 & 0.8010789922879966\end{array}$ $\begin{array}{llll}0.0554448211375801 & 0.1803986815426046 & 0.8055789936114681\end{array}$ $\begin{array}{lllll}0.0580147209464028 & 0.2977070053084411 & 0.7910546138927022\end{array}$ $\begin{array}{llll}0.0499613940055278 & 0.3493072400758722 & 0.1953346449390740\end{array}$ $\begin{array}{lllll}0.5505290205587099 & 0.2311433048843977 & 0.2100493196460582\end{array}$ $\begin{array}{lllll}0.8111319388189957 & 0.1680313537067269 & 0.2176972989524311\end{array}$ $\begin{array}{llll}0.7975396102194512 & 0.3613154647626393 & 0.7822216005201730\end{array}$ $\begin{array}{lllll}0.8137414231221434 & 0.1207420019143763 & 0.8008860265293998\end{array}$ $\begin{array}{lllll}0.5556318633625622 & 0.1808451530804879 & 0.8047637499614383\end{array}$ $\begin{array}{lllll}0.5577997735526798 & 0.2981157219788854 & 0.7891101127898184\end{array}$ $\begin{array}{lllll}0.5515470899901227 & 0.3478860364558010 & 0.1944908147221302\end{array}$ $\begin{array}{lllll}0.2942940907421795 & 0.4067353556564665 & 0.2035114797966957\end{array}$ $\begin{array}{lllll}0.7910380108440059 & 0.4094927848836272 & 0.1976205130960960\end{array}$

G1 :

H 37 C 5 N 5 O 61 Al 8 Si 16

1.00000000000000

$10.1485009999999996 \quad 0.0000000000000000 \quad-1.8735860000000000$

$\begin{array}{lll}0.0646860000000000 & 24.9946149999999996 & -0.5148360000000000\end{array}$ $\begin{array}{llll}0.0000000000000000 & 0.0000000000000000 & 9.3469999999999995\end{array}$ $\begin{array}{llllll}\mathrm{H} & \mathrm{C} & \mathrm{N} & \mathrm{O} & \mathrm{Al} & \mathrm{Si}\end{array}$

$\begin{array}{llllll}37 & 5 & 5 & 61 & 8 & 16\end{array}$

Direct

0.53400833023138430 .75406602231909440 .4077610170953331 $\begin{array}{lllll}0.7756712034688704 & 0.5865686522310724 & 0.2984276101597689\end{array}$ $\begin{array}{lllll}0.6933551916417322 & 0.5263049843236686 & 0.3203026615295451\end{array}$ $\begin{array}{llll}0.3040628908643971 & 0.7672974543185329 & 0.4748904609033539\end{array}$ $\begin{array}{llll}0.5078914561437963 & 0.5242864027994658 & 0.3903094483245588\end{array}$ $\begin{array}{llll}0.1870751922500196 & 0.0541341097740725 & 0.8157838275861653\end{array}$ $\begin{array}{llll}0.6817720062495735 & 0.0555748018505478 & 0.8144281360015070\end{array}$ $\begin{array}{lllll}0.0992448199187770 & 0.1372610051500949 & 0.3558846594802607\end{array}$ $\begin{array}{lllll}0.3504814410096603 & 0.3161243251052445 & 0.3557822579968631\end{array}$ $\begin{array}{lllll}0.2593098994523694 & 0.2192782532577513 & 0.6658104700225574\end{array}$ $\begin{array}{lllll}0.4842496784850995 & 0.0385029570778776 & 0.6828180245425951\end{array}$ $\begin{array}{llllll}0.3436091645568117 & 0.0666191143786181 & 0.2906756028281862\end{array}$ $\begin{array}{lllll}0.6001170924754450 & 0.1400992040812241 & 0.3637483381898478\end{array}$ $\begin{array}{lllll}0.8396392757564402 & 0.3078796190525932 & 0.3182046567032157\end{array}$ $\begin{array}{lllll}0.7582573625311382 & 0.2205888812772018 & 0.6647230348903935\end{array}$ $\begin{array}{lllll}-0.0138562203419582 & 0.0378593279090300 & 0.6811447006923533\end{array}$ $\begin{array}{llll}0.8367826923608990 & 0.0669440033470265 & 0.2906530252459762\end{array}$ $\begin{array}{lllll}0.0797450441407040 & 0.4974439899126437 & 0.3556681266029159\end{array}$ $\begin{array}{lllll}0.5018638731680076 & 0.3954992663169744 & 0.6358510146763419\end{array}$ $\begin{array}{llll}0.2663196672858765 & 0.4669392402599245 & 0.6815620879477883\end{array}$ $\begin{array}{lllll}0.5439116908124098 & 0.4593093098193229 & 0.2346359642434210\end{array}$ $\begin{array}{lllll}1.0039866272004347 & 0.3920860631239788 & 0.6295663726663726\end{array}$ $\begin{array}{llll}0.7639603137986535 & 0.4609263318453305 & 0.6945673592183853\end{array}$ $\begin{array}{llll}0.2444060049245733 & 0.4947013364245852 & 0.1302060088763529\end{array}$ $\begin{array}{lllll}0.9336015795794316 & 0.4678380740526102 & 0.1731625680954830\end{array}$ $\begin{array}{llll}0.2942772562863081 & 0.4384397560609473 & 0.9251765500507434\end{array}$ $\begin{array}{lllll}0.8161088352569604 & 0.4364853729735592 & 0.9310139271763680\end{array}$ $\begin{array}{lllll}0.2951442443833984 & 0.0899702772282349 & 0.0538025338656107\end{array}$ $\begin{array}{lllll}0.7839129987176374 & 0.0904178172814416 & 0.0536076501702179\end{array}$ $\begin{array}{lllll}0.2756799421669372 & 0.5059016313097104 & 0.5357913547146652\end{array}$ $\begin{array}{lllll}0.8297788180001298 & 0.4840589103316407 & 0.5665793379976894\end{array}$ $\begin{array}{llll}0.2689933288997711 & 0.0474279256076185 & 0.4149779269100369\end{array}$ $\begin{array}{lllll}0.7678010135128807 & 0.0467065588085927 & 0.4179640518251619\end{array}$ $\begin{array}{lllll}0.4294077122761051 & 0.4183747578985870 & 0.4817953656604796\end{array}$ $\begin{array}{llll}0.9265572978648774 & 0.4138823877895756 & 0.4751811997190223\end{array}$ $\begin{array}{llll}0.6749442445183709 & 0.1122599660424860 & 0.5104122526481909\end{array}$ $\begin{array}{llll}0.1733106041970744 & 0.1123341370473553 & 0.5073978149402506\end{array}$ $\begin{array}{lllll}0.3480384394821346 & 0.7285075739294479 & 0.4588335833958553\end{array}$ $\begin{array}{lllll}0.6045786684465992 & 0.5952651898567229 & 0.3738662439921397\end{array}$ $\begin{array}{lllll}0.4984503013255103 & 0.6703579551605168 & 0.4108974408664569\end{array}$ $\begin{array}{lllll}0.3855556137216412 & 0.6448214327643470 & 0.4410049536344455\end{array}$ $\begin{array}{lllll}0.3836518912867717 & 0.5877874649892220 & 0.4365591452618177\end{array}$ $\begin{array}{lllll}0.7082112754632049 & 0.5663997575318467 & 0.3426760920218149\end{array}$ $\begin{array}{llllll}0.6086189951987464 & 0.6484158158368387 & 0.3768204273788093\end{array}$ $\begin{array}{lllll}0.4723551179498517 & 0.7240431623558117 & 0.4229628938908033\end{array}$ $\begin{array}{lllll}0.2930371134124137 & 0.6819002494052366 & 0.4704856036869058\end{array}$ $\begin{array}{lllll}0.4997523719995781 & 0.5660878036532111 & 0.4030670648920401\end{array}$ $\begin{array}{llll}0.2911418941800341 & 0.5578632634288845 & 0.4567314377794912\end{array}$ 
$\begin{array}{llll}0.0912936544245897 & 0.3486694103898038 & 0.3646921629643494\end{array}$ $\begin{array}{lllll}0.0589887989957829 & 0.2392727860380046 & 0.3790403687923720\end{array}$ $\begin{array}{lllll}0.3110238000032416 & 0.2833920610649614 & 0.3827297170408889\end{array}$ $\begin{array}{lllll}0.3957202227215906 & 0.2082099692320451 & 0.1340870435702866\end{array}$ $\begin{array}{lllll}0.0703474555284932 & 0.28444447748897382 & 0.1279984996489142\end{array}$ $\begin{array}{lllll}0.1508441132453439 & 0.1827840135932118 & 0.1713924352798469\end{array}$ $\begin{array}{lllll}0.3602716563393435 & 0.1679130302002927 & 0.3846750964081446\end{array}$ $\begin{array}{lllll}0.3571357973299965 & 0.0547491818241598 & 0.3957452928218032\end{array}$ $\begin{array}{llll}0.0905105517079592 & 0.1087832583947679 & 0.4236072974530914\end{array}$ $\begin{array}{llll}0.3214093967443088 & 0.1043473717409493 & 0.1549222976637143\end{array}$ $\begin{array}{lllll}0.2958271967277725 & 0.1144559991717215 & 0.6166941826526402\end{array}$ $\begin{array}{lllll}0.0396598126708895 & 0.0668649139005149 & 0.6564286415432999\end{array}$ $\begin{array}{lllll}0.2758725127305744 & 0.0644408975177810 & 0.8707360051340761\end{array}$ $\begin{array}{llll}0.2066000496776153 & 0.1658139500051349 & 0.8359097884610754\end{array}$ $\begin{array}{llll}0.0396032529264357 & 0.2870023410861242 & 0.6047294037042743\end{array}$ $\begin{array}{lllll}0.2882105165667623 & 0.2452900708399641 & 0.6026064810859258\end{array}$ $\begin{array}{lllll}0.2010874323996307 & 0.3207189123563988 & 0.8470487866765954\end{array}$ $\begin{array}{lllll}0.0318076981496730 & 0.2403841316698772 & 0.8553018163790991\end{array}$ $\begin{array}{llll}0.2399735823640315 & 0.3599993213398645 & 0.5975599058760941\end{array}$ $\begin{array}{lllll}0.4566963772150754 & 0.1405580689554835 & 0.8692832446128000\end{array}$ $\begin{array}{lllll}0.5069734078782148 & 0.1787200758406726 & 0.6189903368967276\end{array}$ $\begin{array}{llll}0.4475696985403660 & 0.3436404358781632 & 0.8123189110917631\end{array}$ $\begin{array}{lllll}0.5886576491393777 & 0.3504454179899107 & 0.3615129839302934\end{array}$ $\begin{array}{llll}0.5576536361551450 & 0.2412086958968022 & 0.3767476600010184\end{array}$ $\begin{array}{lllll}0.8088360622451559 & 0.2816829312737076 & 0.3797369205739810\end{array}$ $\begin{array}{lllll}0.8983650418745073 & 0.2059059544264151 & 0.1353541093925400\end{array}$ $\begin{array}{llll}0.5600618296085657 & 0.2893361961561369 & 0.1245879890903471\end{array}$ $\begin{array}{lllll}0.6498769586684561 & 0.1880325414584179 & 0.1663773565115041\end{array}$ $\begin{array}{llll}0.8558063964755197 & 0.1675016970741028 & 0.3850101372130705\end{array}$ $\begin{array}{llll}0.8545167123174623 & 0.0545424564097295 & 0.3954717733985964\end{array}$ $\begin{array}{llll}0.5919346166232001 & 0.1096945942294970 & 0.4254870011584515\end{array}$ $\begin{array}{lllll}0.8077201298777748 & 0.1045267340427573 & 0.1554238523832907\end{array}$ $\begin{array}{lllll}0.7951688510529128 & 0.1137386154383810 & 0.6183824580543879\end{array}$ $\begin{array}{lllll}0.5393004411236646 & 0.0669078680231091 & 0.6576426664864423\end{array}$ $\begin{array}{llll}0.7700744561890808 & 0.0654362070861568 & 0.8724561620307297\end{array}$ $\begin{array}{lllll}0.7072540424112530 & 0.1673233397812123 & 0.8358113113795692\end{array}$ $\begin{array}{lllll}0.5393320682801628 & 0.2877557325816958 & 0.6038091711259808\end{array}$ $\begin{array}{lllll}0.7878875561633105 & 0.2463915258040586 & 0.6014575541833572\end{array}$ $\begin{array}{lllll}0.6998908430602712 & 0.3206451262977031 & 0.8472184707572743\end{array}$ $\begin{array}{lllll}0.5294699470946795 & 0.2414304433347499 & 0.8546530586148419\end{array}$ $\begin{array}{llll}0.7398514385931361 & 0.3599927859635401 & 0.5977931179554428\end{array}$ $\begin{array}{llllll}0.9562294403603068 & 0.1398331760996334 & 0.8700965585150838\end{array}$ $\begin{array}{lllll}0.0051700122717762 & 0.1780356589789664 & 0.6188812608052841\end{array}$ $\begin{array}{lllll}0.9477040886278536 & 0.3417388031474425 & 0.8142248717000978\end{array}$ $\begin{array}{llll}0.3064197816204909 & 0.4092184504206763 & 0.3634775678391076\end{array}$ $\begin{array}{lllll}0.0700822441927455 & 0.4609608139021708 & 0.3168252506936073\end{array}$ $\begin{array}{lllll}0.1397621654442744 & 0.3847897949662756 & 0.1131079117605374\end{array}$ $\begin{array}{lllll}0.3029902504081818 & 0.4662856790949252 & 0.1077361680276647\end{array}$ $\begin{array}{lllll}0.3967131603056634 & 0.3712165610937604 & 0.1317761087645147\end{array}$ $\begin{array}{lllll}0.2392557892824420 & 0.4725659990530688 & 0.5758254871529673\end{array}$ $\begin{array}{lllll}0.0096996712922749 & 0.4192565798629879 & 0.5575243260923229\end{array}$ $\begin{array}{lllll}0.2810783463280309 & 0.4242796868312810 & 0.8242288458181047\end{array}$ $\begin{array}{lllll}0.8067825691918356 & 0.4112375665618589 & 0.3673205426103825\end{array}$ $\begin{array}{lllll}0.5747686218549597 & 0.4655559430902519 & 0.3378638770293483\end{array}$ $\begin{array}{lllll}0.6456218468416562 & 0.3903331842086863 & 0.1159651906665644\end{array}$ $\begin{array}{lllll}0.8418851478651470 & 0.4605333580961318 & 0.1161416219608446\end{array}$ $\begin{array}{lllll}0.8909191955885144 & 0.3573966117172841 & 0.1486687555037265\end{array}$ $\begin{array}{llll}0.7457197287774860 & 0.4727255931049053 & 0.5896016396158206\end{array}$ $\begin{array}{lllll}0.5129267863698511 & 0.4210779557880059 & 0.5615577288067829\end{array}$ $\begin{array}{lllll}0.7861172573574482 & 0.4228494812133561 & 0.8295927294782917\end{array}$ $\begin{array}{lllll}0.4245401205197211 & 0.2335313537571540 & 0.4947747100487174\end{array}$ $\begin{array}{llll}0.1733606097186358 & 0.2942775746881677 & 0.4902407023499509\end{array}$ $\begin{array}{llll}0.4430757120766873 & 0.1125092640894508 & 0.5258088986211819\end{array}$ $\begin{array}{lllll}0.9238861709413166 & 0.2331018642900672 & 0.4943683517841615\end{array}$ $\begin{array}{llll}0.6726095253740157 & 0.2948723789994862 & 0.4870412163297448\end{array}$ $\begin{array}{lllll}0.9415453838629438 & 0.1120389776263379 & 0.5252091064522592\end{array}$ $\begin{array}{llll}0.1609730143493723 & 0.4151921796004201 & 0.4574784517469224\end{array}$ $\begin{array}{llll}0.6609118096488974 & 0.4152388874834105 & 0.4604596821848361\end{array}$ $\begin{array}{lllll}0.0452105458073854 & 0.2285301441937261 & 0.2027771887118378\end{array}$ $\begin{array}{lllll}0.3069222989593102 & 0.1670935020751943 & 0.2086143325083769\end{array}$ $\begin{array}{lllll}0.2925825561884708 & 0.3613862147541989 & 0.7736893877278019\end{array}$ $\begin{array}{lllll}0.3090963194793797 & 0.1201462672503125 & 0.7917705181090700\end{array}$ $\begin{array}{lllll}0.0499519373358317 & 0.1795379446013511 & 0.7965244844599231\end{array}$ 
$\begin{array}{llll}0.0546901212301011 & 0.2969470093926012 & 0.7814081673659752\end{array}$ $\begin{array}{llll}0.0486490791107282 & 0.3447360035507204 & 0.1874018455017424\end{array}$ $\begin{array}{llll}0.5417942710483319 & 0.2324028894973969 & 0.1995661338668652\end{array}$ $\begin{array}{llll}0.8021150683681519 & 0.1677234702890040 & 0.2088326765928223\end{array}$ $\begin{array}{llll}0.7938851564570855 & 0.3600035533078305 & 0.7740853314569138\end{array}$ $\begin{array}{lllll}0.8077382010547676 & 0.1203854501517830 & 0.7933029025178074\end{array}$ $\begin{array}{llll}0.5502543211302192 & 0.1806340542440685 & 0.7967217424004854\end{array}$ $\begin{array}{lllll}0.5526533639351828 & 0.2979316305837415 & 0.7802450853534312\end{array}$ $\begin{array}{lllll}0.5470457572248715 & 0.3504761314788337 & 0.1831308107450159\end{array}$ $\begin{array}{lllll}0.2846429414674324 & 0.4089209988697090 & 0.1885388171644390\end{array}$ $\begin{array}{lllll}0.7969115821020686 & 0.4064342121927766 & 0.1928047791405188\end{array}$

G2 :

H $37 \quad$ C 5 N 5 O 61 Al 8 Si 16

1.00000000000000

$10.1485009999999996 \quad 0.0000000000000000 \quad-1.8735860000000000$

$\begin{array}{llll}0.0646860000000000 & 24.9946149999999996 & -0.5148360000000000\end{array}$

$\begin{array}{lll}0.0000000000000000 & 0.0000000000000000 & 9.3469999999999995\end{array}$

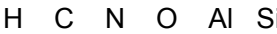

$\begin{array}{llllll}37 & 5 & 5 & 61 & 8 & 16\end{array}$

Direct

0.29509539047123590 .74835459212498460 .2635819396478681 0.24130298501109390 .57880833013828140 .9755307386272886 $\begin{array}{llll}0.2484124101401682 & 0.5202722616010521 & 0.0723707642247520\end{array}$ $\begin{array}{llll}0.2842105192386334 & 0.7695779669976922 & 0.5324816474663965\end{array}$ $\begin{array}{lllll}0.1722824610270127 & 0.5253310951478556 & 0.3018633371674040\end{array}$ $\begin{array}{llllll}0.1958536295548441 & 0.0538783910194311 & 0.8071823849082325\end{array}$ $\begin{array}{llll}0.7013735431352689 & 0.0513576066964778 & 0.8107451725703522\end{array}$ $\begin{array}{llll}0.1143571113414602 & 0.1383799874863850 & 0.3572528713957158\end{array}$ $\begin{array}{lllll}0.3520612222956113 & 0.3063949820569499 & 0.3134101870066053\end{array}$ $\begin{array}{lllll}0.2730552104066538 & 0.2192625822870917 & 0.6613548745703588\end{array}$ $\begin{array}{llll}0.4974823503407252 & 0.0384122248501095 & 0.6816385523027337\end{array}$ $\begin{array}{llllll}0.3530194063305853 & 0.0654497549017880 & 0.2838129987300106\end{array}$ $\begin{array}{lllll}0.6144613321187070 & 0.1367507924938484 & 0.3536047411182054\end{array}$ $\begin{array}{lllll}0.8525032499060774 & 0.3059017537684304 & 0.3120281039284820\end{array}$ $\begin{array}{lllll}0.7711143205139886 & 0.2178982419823656 & 0.6565776417422037\end{array}$ $\begin{array}{lllll}-0.0005274935523900 & 0.0363865722215768 & 0.6765622379916404\end{array}$ $\begin{array}{lllll}0.8563491951590197 & 0.0647482989735911 & 0.2837662266048427\end{array}$ $\begin{array}{lllll}0.0195579829860285 & 0.4832623025248208 & 0.3721213503981610\end{array}$ $\begin{array}{lllll}0.5131227926344256 & 0.3902354061339237 & 0.6265994815466185\end{array}$ $\begin{array}{lllll}0.2731384315138485 & 0.4606062255430868 & 0.6896490610724727\end{array}$ $\begin{array}{lllll}0.5518321025744524 & 0.4923255978323636 & 0.3685679157396221\end{array}$ $\begin{array}{lllll}1.0089538929555537 & 0.3904119304171013 & 0.6271591069860085\end{array}$ $\begin{array}{lllll}0.7629839584689290 & 0.4598669257626128 & 0.6829082159897411\end{array}$ $\begin{array}{lllll}0.4429447597887890 & 0.4653833304297477 & 0.1726655223642070\end{array}$ $\begin{array}{llll}0.9207570201035674 & 0.4729621277325245 & 0.1296514511845275\end{array}$ $\begin{array}{llll}0.3327543103724124 & 0.4327983713588052 & 0.9302049644788004\end{array}$ $\begin{array}{lllll}0.8106398790643132 & 0.4362993023386919 & 0.9181306603089014\end{array}$ $\begin{array}{llll}0.3010389126337132 & 0.0888138971013465 & 0.0468670835230351\end{array}$ $\begin{array}{lllll}0.8064774126808624 & 0.0880335335681750 & 0.0472722673464553\end{array}$ $\begin{array}{lllll}0.2194373378672586 & 0.5081820013350119 & 0.5687817159362187\end{array}$ $\begin{array}{lllll}0.8342005241314464 & 0.4817396870099938 & 0.5587814166841117\end{array}$ $\begin{array}{lllll}0.2822587122953292 & 0.0453618703985877 & 0.4100289468926533\end{array}$ $\begin{array}{lllll}0.7849752184131596 & 0.0442718039686565 & 0.4093935791770529\end{array}$ $\begin{array}{lllll}0.4365884795467799 & 0.4156894830256602 & 0.4769488161978944\end{array}$ $\begin{array}{lllll}0.9334297086088933 & 0.4107534636215937 & 0.4703326868918241\end{array}$ $\begin{array}{lllll}0.6883801602668737 & 0.1098550312113592 & 0.5022277771874355\end{array}$ $\begin{array}{llllll}0.1893962552043324 & 0.1106867940582164 & 0.5040648613597094\end{array}$ $\begin{array}{lllll}0.2681066220648740 & 0.7301068700427220 & 0.4815491647916046\end{array}$ $\begin{array}{llll}0.2250516367676338 & 0.5902587084519014 & 0.1837339883355809\end{array}$ $\begin{array}{llllll}0.2504749592023277 & 0.6679049813206457 & 0.3066457268102177\end{array}$ $\begin{array}{llll}0.2298393306967115 & 0.6470141391990528 & 0.4391357916320665\end{array}$ $\begin{array}{lllll}0.2027815219945768 & 0.5913527996405846 & 0.4423517155627336\end{array}$ $\begin{array}{lllll}0.2164143533049228 & 0.5592576824466977 & 0.0610075735374425\end{array}$ $\begin{array}{llllll}0.2498564600455684 & 0.6421921986682861 & 0.1786855791993066\end{array}$ $\begin{array}{lllll}0.2747843562200328 & 0.7213069891275714 & 0.3360545609685499\end{array}$ $0.2412509713247774 \quad 0.68653189575699490 .5467937933381813$ $\begin{array}{lllll}0.2029008573751502 & 0.5653962523908450 & 0.3076737690719321\end{array}$ $\begin{array}{lllll}0.1791294530512454 & 0.5654292783892066 & 0.5488611475009917\end{array}$ $\begin{array}{lllll}0.1030005251404361 & 0.3483088612397069 & 0.3597348915197942\end{array}$ $\begin{array}{lllll}0.0718032470634063 & 0.2386005787191658 & 0.3719192534439605\end{array}$ $\begin{array}{lllll}0.3216794152318083 & 0.2800895977377600 & 0.3748519998240046\end{array}$ 0.41210519871540590 .20540626919776590 .1279324596737106 
$\begin{array}{llll}0.0773636266076692 & 0.2867006511877674 & 0.1216793730685645\end{array}$ $\begin{array}{lllll}0.1650147224131267 & 0.1852099665977950 & 0.1609155837869193\end{array}$ $\begin{array}{lllll}0.3714815074623021 & 0.1659580728144410 & 0.3780446427770397\end{array}$ $\begin{array}{lllll}0.3694314380949458 & 0.0530665367036370 & 0.3885902132398080\end{array}$ $\begin{array}{llll}0.1063161685935089 & 0.1080042245879742 & 0.4191443829945412\end{array}$ $\begin{array}{llll}0.3269591100802530 & 0.1030984944342095 & 0.1482285715002456\end{array}$ $\begin{array}{lllll}0.3093993530007285 & 0.1123213393412673 & 0.6114923936615230\end{array}$ $\begin{array}{lllll}0.0542308604307830 & 0.0647769644934047 & 0.6507480002619045\end{array}$ $\begin{array}{lllll}0.2841550404718515 & 0.0638520011209566 & 0.8652008412955352\end{array}$ $\begin{array}{lllll}0.2199958977888743 & 0.1657578361886700 & 0.8285521199866498\end{array}$ $\begin{array}{lllll}0.0519419059455605 & 0.2852402010758318 & 0.5986908735525820\end{array}$ $\begin{array}{lllll}0.3017554056478420 & 0.2446172169277638 & 0.5962766086085418\end{array}$ $\begin{array}{llll}0.2125417968675356 & 0.3173401004254066 & 0.8428186461843683\end{array}$ $\begin{array}{lllll}0.0397887843950844 & 0.2389879458857168 & 0.8491631067332170\end{array}$ $\begin{array}{llll}0.2507210237971751 & 0.3584445444510421 & 0.5951377905045232\end{array}$ $\begin{array}{lllll}0.4694825963988145 & 0.1383252990419686 & 0.8633437595329030\end{array}$ $\begin{array}{llllll}0.5194602914958190 & 0.1766275758878344 & 0.6119994946123986\end{array}$ $\begin{array}{lllll}0.4598105792336625 & 0.3393780890845189 & 0.8086265403050570\end{array}$ $\begin{array}{lllll}0.6059223147544046 & 0.3475108710343327 & 0.3555199312990338\end{array}$ $\begin{array}{llll}0.5713206745707297 & 0.2385504389758500 & 0.3708990455137612\end{array}$ $\begin{array}{lllll}0.8225695014364749 & 0.2795377706661070 & 0.3735013557530183\end{array}$ $\begin{array}{llllll}0.9112534417047001 & 0.2057417976648580 & 0.1288842255207247\end{array}$ $\begin{array}{lllll}0.5838918423697588 & 0.2845845365701923 & 0.1199177140333674\end{array}$ $\begin{array}{llll}0.6650479674533883 & 0.1827882205997922 & 0.1632132422672098\end{array}$ $\begin{array}{llll}0.8727348445757225 & 0.1653935487253935 & 0.3785063473158362\end{array}$ $\begin{array}{lllll}0.8720899511735665 & 0.0524518779884374 & 0.3885839871847470\end{array}$ $\begin{array}{llll}0.6053057358650995 & 0.1073026390966055 & 0.4181599468613454\end{array}$ $\begin{array}{llll}0.8313234976451722 & 0.1024947298273920 & 0.1484557597798539\end{array}$ $\begin{array}{llll}0.8101032489622196 & 0.1112104687496013 & 0.6108087453940546\end{array}$ $\begin{array}{llllll}0.5532696154678819 & 0.0653726751539566 & 0.6506773496388800\end{array}$ $\begin{array}{lllll}0.7898195191540632 & 0.0619158897285789 & 0.8657117484981904\end{array}$ $\begin{array}{llll}0.7204091872509071 & 0.1632693452455392 & 0.8289436383140070\end{array}$ $\begin{array}{lllll}0.5525963064675818 & 0.2856056199670757 & 0.5972383766593005\end{array}$ $\begin{array}{llll}0.8011419101777733 & 0.2441105014747890 & 0.5947683554036404\end{array}$ $\begin{array}{lllll}0.7133790213564557 & 0.3188597266820603 & 0.8392780283627124\end{array}$ $\begin{array}{lllll}0.5467784324223465 & 0.2383835537682621 & 0.8486989245042604\end{array}$ $\begin{array}{lllll}0.7533345282734136 & 0.3582768871850870 & 0.5883562487448120\end{array}$ $\begin{array}{lllll}0.9705043212935425 & 0.1377762957790848 & 0.8633453368900804\end{array}$ $\begin{array}{llll}0.0186640315722143 & 0.1765275458466649 & 0.6129218863830115\end{array}$ $\begin{array}{lllll}0.9598403920562811 & 0.3415119220487848 & 0.8056906856036268\end{array}$ $\begin{array}{lllll}0.3144140703218413 & 0.4098540360270659 & 0.3616745814579567\end{array}$ $\begin{array}{llll}0.0817406486314315 & 0.4630564104094909 & 0.3253389992020629\end{array}$ $\begin{array}{lllll}0.1531350653471446 & 0.3877106943620159 & 0.1103983290013199\end{array}$ $\begin{array}{lllll}0.3510668158422975 & 0.4585194027387761 & 0.1121455298600122\end{array}$ $\begin{array}{lllll}0.4018223904191907 & 0.3565896746637466 & 0.1425810082150471\end{array}$ $\begin{array}{lllll}0.2525171484933714 & 0.4692367711846238 & 0.5845239392244450\end{array}$ $\begin{array}{lllll}0.0148670453742534 & 0.4166123081330664 & 0.5525103488484285\end{array}$ $\begin{array}{lllll}0.2973646377608014 & 0.4198379176903472 & 0.8297957402185896\end{array}$ $\begin{array}{lllll}0.8122623205110100 & 0.4142790556917669 & 0.3555834563166753\end{array}$ $\begin{array}{llll}0.5690617797326150 & 0.4596790016758170 & 0.3167330802701404\end{array}$ $\begin{array}{llll}0.6499605923509609 & 0.3868379178005999 & 0.1064225585458331\end{array}$ $\begin{array}{llll}0.8295116122149491 & 0.4614817117662910 & 0.0926645569025025\end{array}$ $\begin{array}{lllll}0.9025431568274627 & 0.3615766818783399 & 0.1408772141558955\end{array}$ $\begin{array}{lllll}0.7474911528522898 & 0.4722690634190704 & 0.5780587319605133\end{array}$ $\begin{array}{llll}0.5225453915295054 & 0.4169883579078688 & 0.5547745382221720\end{array}$ $\begin{array}{lllll}0.7917334228965612 & 0.4217574722721742 & 0.8162066567504780\end{array}$ $\begin{array}{lllll}0.4371380188196911 & 0.2316107761084356 & 0.4877474038576619\end{array}$ $\begin{array}{lllll}0.1858780489717760 & 0.2932654884512857 & 0.4831853899188462\end{array}$ $\begin{array}{lllll}0.4560953309967494 & 0.1105007717647209 & 0.5186184187109462\end{array}$ $\begin{array}{llll}0.9378608742481959 & 0.2313020636523803 & 0.4878888049374283\end{array}$ $\begin{array}{llll}0.6864313755063398 & 0.2927210218303393 & 0.4811155446629323\end{array}$ $\begin{array}{lllll}0.9570177133704733 & 0.1102085228877224 & 0.5189779893631323\end{array}$ $\begin{array}{lllll}0.1725330055865457 & 0.4144171811120520 & 0.4578484628519100\end{array}$ $\begin{array}{lllll}0.6664544256438667 & 0.4137186314215646 & 0.4488106587620464\end{array}$ $\begin{array}{lllll}0.0575580435439937 & 0.2296662023329315 & 0.1948977872648194\end{array}$ $\begin{array}{lllll}0.3183315528317707 & 0.1661302958570253 & 0.2018395693259770\end{array}$ $\begin{array}{lllll}0.3055785145127914 & 0.3577721323783056 & 0.7709774990757052\end{array}$ $\begin{array}{lllll}0.3210070774864568 & 0.1189728834575636 & 0.7862990673596518\end{array}$ $\begin{array}{lllll}0.0625208767715533 & 0.1784276614697679 & 0.7905273072019821\end{array}$ $\begin{array}{lllll}0.0650032285351228 & 0.2953464422212161 & 0.7750881398958926\end{array}$ $\begin{array}{lllll}0.0589846004728054 & 0.3473863295998244 & 0.1822420763168406\end{array}$ $\begin{array}{llll}0.5590307268340255 & 0.2282991583525005 & 0.1946224942950242\end{array}$ $\begin{array}{llll}0.8198353274267519 & 0.1653559509034506 & 0.2023625463551310\end{array}$ 
$\begin{array}{llll}0.8048409422752510 & 0.3588474892876699 & 0.7646474417478081\end{array}$ $\begin{array}{llll}0.8228327132360678 & 0.1174319213691781 & 0.7857122602435223\end{array}$ $\begin{array}{lllll}0.5641494084687346 & 0.1775360317237372 & 0.7895762606315546\end{array}$ $\begin{array}{llll}0.5669387704069028 & 0.2950105100505370 & 0.7739718888309781\end{array}$ $\begin{array}{llll}0.5599649101224831 & 0.3449261665499938 & 0.1783139756806478\end{array}$ $\begin{array}{lllll}0.3039875419383464 & 0.4042315455440702 & 0.1882305565000101\end{array}$ $\begin{array}{lllll}0.7985213288997498 & 0.4074388621562075 & 0.1811600718518777\end{array}$

G4 :

H 37 C 5 N 5 O 61 Al 8 Si 16

1.00000000000000

$\begin{array}{llll}10.1485009999999996 & 0.0000000000000000 & -1.8735860000000000\end{array}$

$\begin{array}{llll}0.0646860000000000 & 24.9946149999999996 & -0.5148360000000000\end{array}$

$\begin{array}{llll}0.0000000000000000 & 0.0000000000000000 & 9.3469999999999995\end{array}$

$\mathrm{H} \quad \mathrm{C} \quad \mathrm{N} O \mathrm{Al} \mathrm{Si}$

$\begin{array}{llllll}37 & 5 & 5 & 61 & 8 & 16\end{array}$

Direct

$\begin{array}{llll}0.3276888190289270 & 0.7551902458237185 & 0.4383424950618927\end{array}$

$\begin{array}{llll}0.6112125975075544 & 0.5939640778817156 & 0.4142042016268386\end{array}$ $\begin{array}{lllll}0.5406022213149012 & 0.5299078387653160 & 0.4166856146927406\end{array}$ $\begin{array}{lllll}0.0894537200803870 & 0.7656272551072849 & 0.4836522188336829\end{array}$ $\begin{array}{lllll}0.3334820039710530 & 0.5274607696566344 & 0.4904102933476071\end{array}$ $\begin{array}{lllll}0.1906327948932774 & 0.0527668544492779 & 0.8248699786691995\end{array}$ $\begin{array}{lllll}0.6861265171932522 & 0.0537427606936706 & 0.8237139114465265\end{array}$ $\begin{array}{lllll}0.1041140661824596 & 0.1378192091718768 & 0.3697164780584558\end{array}$ $\begin{array}{lllll}0.3478443086044041 & 0.3103880581620391 & 0.3455886766040508\end{array}$ $\begin{array}{lllll}0.2630517766859124 & 0.2182344763075896 & 0.6769948175519045\end{array}$ $\begin{array}{lllll}0.4884432781326195 & 0.0375575686219836 & 0.6934896459827837\end{array}$ $\begin{array}{lllll}0.3470131401945115 & 0.0655447028682009 & 0.2998648667739853\end{array}$ $\begin{array}{llllll}0.6047415180260916 & 0.1391633170714382 & 0.3739841173796410\end{array}$ $\begin{array}{lllll}0.8394415447235924 & 0.3051504277399776 & 0.3220391587121596\end{array}$ $\begin{array}{lllll}0.7621843731077113 & 0.2200430328546927 & 0.6760919319159946\end{array}$ $\begin{array}{lllll}0.9892522340203886 & 0.0383978690133778 & 0.6965712691691260\end{array}$ $\begin{array}{llll}0.8420273327132687 & 0.0660061695870483 & 0.2992455102176966\end{array}$ $\begin{array}{llll}0.0535170209073342 & 0.4936726505094033 & 0.3747518898892936\end{array}$ $\begin{array}{lllll}0.5013616138023247 & 0.3941914368605012 & 0.6420015492343905\end{array}$ $\begin{array}{lllll}0.2530727519036287 & 0.4600866415156099 & 0.7020589885358983\end{array}$ $\begin{array}{llll}0.6146683703828140 & 0.4752768420943853 & 0.2720000232035503\end{array}$ $\begin{array}{lllll}-0.0037506502499026 & 0.3850864484520972 & 0.6357182259062505\end{array}$ $\begin{array}{lllll}0.7636789081610456 & 0.4601520777072091 & 0.7054147125656534\end{array}$ $\begin{array}{lllll}0.3958299289881524 & 0.4793654233517084 & 0.1696112654213674\end{array}$ $\begin{array}{lllll}0.9285024218553927 & 0.4675109644561069 & 0.1741993101870930\end{array}$ $\begin{array}{lllll}0.3038029087186155 & 0.4361797922112173 & 0.9409106049110225\end{array}$ $\begin{array}{lllll}0.8153261091819392 & 0.4357172517504280 & 0.9414594768500482\end{array}$ $\begin{array}{lllll}0.2969287099989681 & 0.0889861483973507 & 0.0633485476571708\end{array}$ $\begin{array}{lllll}0.7885313714712003 & 0.0892950679496607 & 0.0628362878348039\end{array}$ $\begin{array}{llll}0.1759828900423265 & 0.5047107215176645 & 0.5822473487522273\end{array}$ $\begin{array}{llll}0.8328846199330516 & 0.4810269798024005 & 0.5785053112304500\end{array}$ $\begin{array}{llll}0.2735411793358776 & 0.0459687616690705 & 0.4245732806469500\end{array}$ $\begin{array}{llll}0.7748140342240611 & 0.0445569488663915 & 0.4268798386312310\end{array}$ $\begin{array}{lllll}0.4250523948744363 & 0.4175562540805123 & 0.4887992525236917\end{array}$ $\begin{array}{llll}0.9239593415207390 & 0.4119535195882513 & 0.4866995013627720\end{array}$ $\begin{array}{lllll}0.6791912939627087 & 0.1105219612818917 & 0.5192803278777165\end{array}$ $\begin{array}{lllll}0.1783650878692004 & 0.1110172513908172 & 0.5181798107937402\end{array}$ $\begin{array}{llll}0.1401843224440368 & 0.7274950857846552 & 0.4804134110465365\end{array}$ $\begin{array}{lllll}0.4249765615990832 & 0.5978056193254592 & 0.4482014436945856\end{array}$ $\begin{array}{lllll}0.3030625607544080 & 0.6713536430121628 & 0.4576991313421107\end{array}$ $\begin{array}{lllll}0.1904427625358428 & 0.6446442576808274 & 0.4849256261236263\end{array}$ $\begin{array}{lllll}0.1983452521329071 & 0.5883036745194247 & 0.4981837388445670\end{array}$ $\begin{array}{lllll}0.5355858898506213 & 0.5711416071222483 & 0.4336608121073264\end{array}$ $\begin{array}{lllll}0.4194749271779822 & 0.6508862338366003 & 0.4384363820880829\end{array}$ $0.2685382787421973 \quad 0.7245051849562887 \quad 0.4552107354291994$ $\begin{array}{lllll}0.0902826164245264 & 0.6804804329496283 & 0.4989265040469798\end{array}$ $\begin{array}{lllll}0.3203962330859629 & 0.5678536618565840 & 0.4760098636935444\end{array}$ $\begin{array}{llll}0.1118844405093533 & 0.5568726708525960 & 0.5284483972364024\end{array}$ $\begin{array}{lllll}0.0957390442416349 & 0.3470139098745363 & 0.3757906034585541\end{array}$ $\begin{array}{lllll}0.0619596077850388 & 0.2382742034787700 & 0.3887142064209931\end{array}$ $\begin{array}{lllll}0.3130661566590385 & 0.2807013385917221 & 0.3922955195713884\end{array}$ $\begin{array}{lllll}0.4006198461769496 & 0.2068224997658542 & 0.1444008277353502\end{array}$ $\begin{array}{lllll}0.0726138335934561 & 0.2847092620563647 & 0.1378284043366586\end{array}$ $\begin{array}{lllll}0.1552114924162865 & 0.1829919782242473 & 0.1801030904876845\end{array}$ $\begin{array}{lllll}0.3638379093781755 & 0.1663294678868131 & 0.3946276121706745\end{array}$ $\begin{array}{llll}0.3614549661543782 & 0.0533074476593090 & 0.4047044614990004\end{array}$ 
$\begin{array}{llll}0.0953737910704876 & 0.1082443461171396 & 0.4340571175933387\end{array}$ $\begin{array}{lllll}0.3231030913230159 & 0.1033564518567618 & 0.1644516614279818\end{array}$ $\begin{array}{lllll}0.3003548656849577 & 0.1124787305023510 & 0.6267275814334515\end{array}$ $\begin{array}{llll}0.0447591203710245 & 0.0654931526034968 & 0.6657219225924957\end{array}$ $\begin{array}{llll}0.2789724163675851 & 0.0631441933023904 & 0.8809959705410848\end{array}$ $\begin{array}{lllll}0.2102388965361909 & 0.1646199147419969 & 0.8444291048786026\end{array}$ $\begin{array}{llll}0.0428743918756499 & 0.2855639280458225 & 0.6154447137304568\end{array}$ $\begin{array}{lllll}0.2917105324883733 & 0.2439472320533316 & 0.6128390617133173\end{array}$ $\begin{array}{llll}0.2033339579175798 & 0.3183013231752702 & 0.8589678498396541\end{array}$ $\begin{array}{lllll}0.0332226002887939 & 0.2385995504330751 & 0.8657440595731533\end{array}$ $\begin{array}{lllll}0.2427367025679630 & 0.3584908191547527 & 0.6099229541890052\end{array}$ $\begin{array}{llllll}0.4606167989107037 & 0.1387770463813744 & 0.8792646483231409\end{array}$ $\begin{array}{lllll}0.5102962531018226 & 0.1772356646546464 & 0.6290092106377189\end{array}$ $\begin{array}{lllll}0.4500122443339994 & 0.3412737885677342 & 0.8248627626361607\end{array}$ $\begin{array}{llll}0.5917206396684765 & 0.3493245486910510 & 0.3739945611055180\end{array}$ $\begin{array}{lllll}0.5613047319100732 & 0.2398288513433719 & 0.3875232752240751\end{array}$ $\begin{array}{lllll}0.8118698742975157 & 0.2807309117339007 & 0.3903594824840764\end{array}$ $\begin{array}{lllll}0.9023595927147021 & 0.2052994388514413 & 0.1450043593804751\end{array}$ $\begin{array}{lllll}0.5660682238272746 & 0.2880199261365108 & 0.1368131712038635\end{array}$ $\begin{array}{llll}0.6542806399534818 & 0.1865823745246509 & 0.1767292293205569\end{array}$ $\begin{array}{lllll}0.8601365036857254 & 0.1662562682958797 & 0.3945093602266860\end{array}$ $\begin{array}{lllll}0.8607796715937968 & 0.0532805708385338 & 0.4038086920752517\end{array}$ $\begin{array}{lllll}0.5960772014006126 & 0.1085238968055250 & 0.4347642970230632\end{array}$ $\begin{array}{llll}0.8127953511448782 & 0.1035311946619790 & 0.1644055001056220\end{array}$ $\begin{array}{llll}0.7999118648286215 & 0.1116035148279233 & 0.6276697439809713\end{array}$ $\begin{array}{lllll}0.5440792126848332 & 0.0654432111990932 & 0.6666610129266174\end{array}$ $\begin{array}{llllll}0.7743720889369047 & 0.0636067804597931 & 0.8818180506882461\end{array}$ $\begin{array}{lllll}0.7111820404369229 & 0.1654661111428745 & 0.8446920930622734\end{array}$ $\begin{array}{lllll}0.5424102365508241 & 0.2862642756453844 & 0.6149575972932779\end{array}$ $\begin{array}{lllll}0.7911529868254406 & 0.2455219765898252 & 0.6115445013295907\end{array}$ $\begin{array}{lllll}0.7029973422125610 & 0.3192686355698934 & 0.8581054595241715\end{array}$ $\begin{array}{lllll}0.5339898061878184 & 0.2395155582580998 & 0.8657567349912326\end{array}$ $\begin{array}{lllll}0.7411107483087382 & 0.3594161795540667 & 0.6082699287688108\end{array}$ $\begin{array}{lllll}0.9603535329766787 & 0.1378502970834854 & 0.8795482816628883\end{array}$ $\begin{array}{lllll}0.0080716186532091 & 0.1768979210093532 & 0.6285927305525532\end{array}$ $\begin{array}{lllll}0.9495675124689481 & 0.3403149158738273 & 0.8236044085343639\end{array}$ $\begin{array}{lllll}0.3049740628418057 & 0.4115234397690417 & 0.3771970416425509\end{array}$ $\begin{array}{lllll}0.0609775662710999 & 0.4576135595732166 & 0.3350828344555609\end{array}$ $\begin{array}{lllll}0.1405619168916097 & 0.3862659630789582 & 0.1258477208551768\end{array}$ $\begin{array}{llll}0.3111774677622900 & 0.4645651259512688 & 0.1195211828547563\end{array}$ $\begin{array}{lllll}0.3931537228174137 & 0.3647329714636380 & 0.1526404296472338\end{array}$ 0.23320367973452930 .47041706978163550 .5970178890726684 $\begin{array}{lllll}0.0054732138445193 & 0.4142729183219214 & 0.5705035229249700\end{array}$ $\begin{array}{lllll}0.2840817825993984 & 0.4215078025076363 & 0.8398991752967770\end{array}$ $\begin{array}{lllll}0.8015690087261594 & 0.4135952228635943 & 0.3753001397253440\end{array}$ $\begin{array}{lllll}0.5622434770038844 & 0.4638153590039175 & 0.3422742640438129\end{array}$ $\begin{array}{llll}0.6444234824517076 & 0.3887365204529225 & 0.1254838755796154\end{array}$ $\begin{array}{lllll}0.8372559283724036 & 0.4590452999699519 & 0.1200657930148891\end{array}$ $\begin{array}{lllll}0.8923387252560006 & 0.3566126047749151 & 0.1634249963762658\end{array}$ $\begin{array}{llll}0.7465682447199379 & 0.4723638447730897 & 0.6006452892241669\end{array}$ $\begin{array}{llll}0.5105501234840205 & 0.4205680883763308 & 0.5690936150171158\end{array}$ $\begin{array}{llll}0.7874898500353457 & 0.4217338575647500 & 0.8396438445544255\end{array}$ $\begin{array}{lllll}0.4282875423048339 & 0.2320352500690011 & 0.5050784441914977\end{array}$ $\begin{array}{lllll}0.1770317117531352 & 0.2927550926542073 & 0.5006907877752538\end{array}$ $\begin{array}{lllll}0.4472564751287991 & 0.1110393436380015 & 0.5350546753563207\end{array}$ $\begin{array}{llll}0.9268534851382644 & 0.2319901102513229 & 0.5040387019328711\end{array}$ $\begin{array}{llll}0.6754914691419812 & 0.2941047090960323 & 0.4977761397952679\end{array}$ $\begin{array}{lllll}0.9461862181793786 & 0.1107470060456198 & 0.5345720070834686\end{array}$ $\begin{array}{lllll}0.1583534406308079 & 0.4132294449179592 & 0.4701360051841186\end{array}$ $\begin{array}{lllll}0.6579448112360506 & 0.4153364280061813 & 0.4704746568035409\end{array}$ $\begin{array}{llll}0.0490439809032803 & 0.2283422599413263 & 0.2121796981760554\end{array}$ $\begin{array}{lllll}0.3104620069762038 & 0.1661599152950403 & 0.2185549511541473\end{array}$ $\begin{array}{llll}0.2952134653545278 & 0.3589035102571409 & 0.7861714339044952\end{array}$ $\begin{array}{lllll}0.3128224338471631 & 0.1186589369089699 & 0.8016316798402685\end{array}$ $\begin{array}{lllll}0.0532506636658057 & 0.1779775719074313 & 0.8060849807149074\end{array}$ $\begin{array}{lllll}0.0565515314061078 & 0.2952129719965317 & 0.7922263941203720\end{array}$ $\begin{array}{lllll}0.0508683101542047 & 0.3449056597716992 & 0.1986861052174163\end{array}$ $\begin{array}{lllll}0.5465827264807801 & 0.2310078979426387 & 0.2103727544711535\end{array}$ $\begin{array}{lllll}0.8068402812002928 & 0.1666573507018576 & 0.2182905959304289\end{array}$ $\begin{array}{lllll}0.7956931267540222 & 0.3590335875642141 & 0.7843377514905929\end{array}$ $\begin{array}{lllll}0.8117594088465941 & 0.1185611895315081 & 0.8024234729790305\end{array}$ $\begin{array}{lllll}0.5541667654622424 & 0.1788502313545982 & 0.8066349490583605\end{array}$ $\begin{array}{llll}0.5560828933770958 & 0.2961863291118872 & 0.7915468140938824\end{array}$ 
$\begin{array}{llll}0.5479148778753736 & 0.3489301823497287 & 0.1960221592714439\end{array}$ 0.28748440170592820 .40827751020768190 .2019851161713310 $\begin{array}{lll}0.7956029634354522 & 0.4054481471405635 & 0.2021683921972976\end{array}$

G5 :

$\begin{array}{lllllll}\mathrm{H} 37 & \mathrm{C} 5 & \mathrm{~N} 5 & \mathrm{O} 61 & \mathrm{Al} 8 & \mathrm{Si} 16\end{array}$

1.00000000000000

$10.1485009999999996 \quad 0.0000000000000000 \quad-1.8735860000000000$

$0.0646860000000000 \quad 24.9946149999999996-0.5148360000000000$

$\begin{array}{lll}0.0000000000000000 & 0.0000000000000000 & 9.3469999999999995\end{array}$

H C N O Al Si

$\begin{array}{llllll}37 & 5 & 5 & 61 & 8 & 16\end{array}$

Direct

0.59418873037444410 .75125275442598350 .2103669729591582

$\begin{array}{llll}0.8819599769175922 & 0.5902130721279025 & 0.2402012248070613\end{array}$

$\begin{array}{llll}0.8054166126936910 & 0.5286103018705127 & 0.2129716795925571\end{array}$

$\begin{array}{lllll}0.3432780877308620 & 0.7616932533595885 & 0.1968855681994388\end{array}$

$\begin{array}{lllll}0.5876140172647671 & 0.5221261259947586 & 0.2630935499631691\end{array}$

$\begin{array}{llll}0.1840857075504427 & 0.0574175798851678 & 0.8250980750194677\end{array}$ $\begin{array}{lllll}0.6800610907925810 & 0.0591275117689886 & 0.8200453812961294\end{array}$ $\begin{array}{lllll}0.1017867286395597 & 0.1368727650690745 & 0.3505078521676837\end{array}$ $\begin{array}{lllll}0.3414650039652453 & 0.3091433384441432 & 0.3185921326222954\end{array}$ $\begin{array}{llllll}0.2616891638936388 & 0.2212972305767762 & 0.6637568054128050\end{array}$ $\begin{array}{lllll}0.5208305473971184 & 0.0315568468700351 & 0.6331843490567848\end{array}$ $\begin{array}{lllll}0.3485761061108346 & 0.0676971790768176 & 0.2921226765457887\end{array}$ $\begin{array}{lllll}0.6018853459200704 & 0.1398572350272912 & 0.3580175428081933\end{array}$ $\begin{array}{lllll}0.8376076076498263 & 0.3073159864980894 & 0.3105756514602522\end{array}$ $\begin{array}{lllll}0.7620898359169480 & 0.2222042224390434 & 0.6651287222049176\end{array}$ $\begin{array}{llllll}0.0262509994431465 & 0.0323939566897700 & 0.6276566988847031\end{array}$ $\begin{array}{lllll}0.8408880249333056 & 0.0681197618895743 & 0.2928094718672659\end{array}$ $\begin{array}{lllll}0.1114658227783138 & 0.4950460035279918 & 0.3083894181011990\end{array}$ $\begin{array}{llll}0.4991229413163808 & 0.3928827853145165 & 0.6377042237305128\end{array}$ $\begin{array}{lllll}0.2567926477556173 & 0.4631816675442785 & 0.6873151469786918\end{array}$ 0.48329069163263850 .47366510081337350 .3499169641292390 $\begin{array}{llllll}1.0033643328156165 & 0.3892514096857644 & 0.6172904625508051\end{array}$ $\begin{array}{lllll}0.7683343039448574 & 0.4627443724652263 & 0.6985853419415395\end{array}$ $\begin{array}{lllll}0.3265090506666958 & 0.5000497032631350 & 0.1402299386959523\end{array}$ $\begin{array}{lllll}0.9387966769795770 & 0.4658868845761727 & 0.1829120034416824\end{array}$ $\begin{array}{llll}0.2951710304237871 & 0.4424575924589905 & 0.9221019843275827\end{array}$ $\begin{array}{llll}0.8215472092508022 & 0.4375507346520722 & 0.9342456746189498\end{array}$ $\begin{array}{lllll}0.2982527780760438 & 0.0913491687571068 & 0.0548462308722569\end{array}$ $\begin{array}{lllll}0.7876058644374379 & 0.0916391898425911 & 0.0546371471722305\end{array}$ $\begin{array}{lllll}0.3213652226293269 & 0.4880515856111530 & 0.5599750051723080\end{array}$ $\begin{array}{lllll}0.8162216568866690 & 0.4906730763889246 & 0.5628743648394834\end{array}$ $\begin{array}{llll}0.2734590279923332 & 0.0489909096093529 & 0.4163999388954349\end{array}$ $\begin{array}{llll}0.7704658990416812 & 0.0492469542558492 & 0.4203830607294107\end{array}$ $\begin{array}{lllll}0.4262415526653822 & 0.4140632630737403 & 0.4817398435178364\end{array}$ $\begin{array}{llll}0.9259734567273089 & 0.4183717259413622 & 0.4750028507538239\end{array}$ $\begin{array}{lllll}0.6770044919517879 & 0.1149106278039878 & 0.5100841829977000\end{array}$ 0.17481757881809190 .11414312689864690 .5063604752797095 $\begin{array}{llll}0.3951224421382045 & 0.7235672118742601 & 0.2054757520742849\end{array}$ $\begin{array}{lllll}0.6874640499195919 & 0.5938425497694245 & 0.2378780224283925\end{array}$ $\begin{array}{lllll}0.5641992355085333 & 0.6674457194335744 & 0.2220515170196111\end{array}$ $\begin{array}{lllll}0.4444742419316141 & 0.6405635861993010 & 0.2202211713406217\end{array}$ $\begin{array}{llll}0.4492200552553179 & 0.5837331710723200 & 0.2290098741393999\end{array}$ $\begin{array}{lllll}0.8043498959753631 & 0.5671050035387432 & 0.2530202205492895\end{array}$ $\begin{array}{llll}0.6859562990058533 & 0.6468938054910304 & 0.2295465943133103\end{array}$ $\begin{array}{lllll}0.5303639725488942 & 0.7206376837560777 & 0.2124950346687265\end{array}$ $\begin{array}{lllll}0.3403558430707393 & 0.6764428221472852 & 0.2098765598783132\end{array}$ $\begin{array}{lllll}0.5769017812893734 & 0.5634115227926226 & 0.2371204489493938\end{array}$ $\begin{array}{lllll}0.3546164601364646 & 0.5522047768697682 & 0.2321338518149548\end{array}$ $\begin{array}{llllll}0.0969148248573267 & 0.3506109237194894 & 0.3634898908104716\end{array}$ $\begin{array}{lllll}0.0610577843050590 & 0.2412050369785911 & 0.3796568705424460\end{array}$ $\begin{array}{lllll}0.3121429128424454 & 0.2826432204527409 & 0.3801007791098440\end{array}$ $\begin{array}{lllll}0.3989537560515548 & 0.2094907420975493 & 0.1358358234103152\end{array}$ $\begin{array}{lllll}0.0709157390282261 & 0.2861035287757538 & 0.1283741429865898\end{array}$ $\begin{array}{lllll}0.1538093195216897 & 0.1843733418287524 & 0.1726502729872068\end{array}$ $\begin{array}{lllll}0.3633251939047127 & 0.1688025390327109 & 0.3860205397213652\end{array}$ $\begin{array}{llll}0.3619860404488320 & 0.0555064401148723 & 0.3971299893966690\end{array}$ $\begin{array}{llll}0.0925049819722075 & 0.1098994561950276 & 0.4222584524710078\end{array}$ $\begin{array}{lllll}0.3239358693223929 & 0.1055845384198337 & 0.1565467288690062\end{array}$ $\begin{array}{lllll}0.2978455113852497 & 0.1158405893044141 & 0.6162956261209224\end{array}$ $\begin{array}{llll}0.0391900095450139 & 0.0695123501448210 & 0.6590231667674822\end{array}$ 
$\begin{array}{llll}0.2756232939155321 & 0.0663429757992702 & 0.8742617717782523\end{array}$ $\begin{array}{lllll}0.2101674038990384 & 0.1671796117417547 & 0.8353957818343011\end{array}$ $\begin{array}{lllll}0.0428476447160007 & 0.2895792347912086 & 0.6044517215727978\end{array}$ $\begin{array}{llll}0.2917173004653532 & 0.2476832532259344 & 0.6023310375434815\end{array}$ $\begin{array}{lllll}0.2042551868235725 & 0.3232735958277317 & 0.8453774691876371\end{array}$ $\begin{array}{lllll}0.0373846248012233 & 0.2423191510506826 & 0.8558627934793666\end{array}$ $\begin{array}{lllll}0.2446097883111047 & 0.3622994548407540 & 0.5941447667711048\end{array}$ $\begin{array}{lllll}0.4597365485267003 & 0.1412970920942385 & 0.8690184518054797\end{array}$ $\begin{array}{llll}0.5094443893945201 & 0.1799168843473531 & 0.6206798101330144\end{array}$ $\begin{array}{llll}0.4504714292399760 & 0.3456132305254097 & 0.8108091782543699\end{array}$ $\begin{array}{lllll}0.5924237947056976 & 0.3513123810929539 & 0.3652371665305587\end{array}$ $\begin{array}{lllll}0.5610648514878894 & 0.2419656814767173 & 0.3784886318512461\end{array}$ $\begin{array}{lllll}0.8113303664103207 & 0.2831240293914523 & 0.3803308584589729\end{array}$ $\begin{array}{lllll}0.9012585604324774 & 0.2071097516677398 & 0.1360654261488929\end{array}$ $\begin{array}{lllll}0.5644350342863842 & 0.2905881618315763 & 0.1283050864526276\end{array}$ $\begin{array}{lllll}0.6527530155765910 & 0.1888249910994593 & 0.1673237781522442\end{array}$ $\begin{array}{lllll}0.8590236399739561 & 0.1691030818284934 & 0.3857995155124984\end{array}$ $\begin{array}{lllll}0.8578456837386731 & 0.0559366293118883 & 0.3980028588339551\end{array}$ $\begin{array}{llll}0.5945415648809921 & 0.1108537839012535 & 0.4245485378425752\end{array}$ $\begin{array}{llll}0.8113385660460506 & 0.1055718519869335 & 0.1570249810278840\end{array}$ $\begin{array}{lllll}0.7966033676420921 & 0.1170546955208827 & 0.6180855370503666\end{array}$ $\begin{array}{lllll}0.5410199595200753 & 0.0686945563909430 & 0.6596196474151688\end{array}$ $\begin{array}{lllll}0.7704709920561522 & 0.0680404910125728 & 0.87444412507194342\end{array}$ $\begin{array}{lllll}0.7100120879004382 & 0.1694138593858037 & 0.8368038894902592\end{array}$ $\begin{array}{llll}0.5426450809870783 & 0.2887707589220909 & 0.6052149372447291\end{array}$ $\begin{array}{lllll}0.7918543361548264 & 0.2483773896105292 & 0.6028883591021038\end{array}$ $\begin{array}{lllll}0.7025819829686755 & 0.3218074150546516 & 0.8488047821435002\end{array}$ $\begin{array}{lllll}0.5301999205204484 & 0.2429762921686697 & 0.8562082935632322\end{array}$ $\begin{array}{lllll}0.7421723993436978 & 0.3619542414069328 & 0.6002038112764644\end{array}$ $\begin{array}{lllll}0.9592571779323033 & 0.1417905678808724 & 0.8693172299475195\end{array}$ $\begin{array}{lllll}0.0089509876973906 & 0.1800034985728990 & 0.6193225876902478\end{array}$ $\begin{array}{llll}0.9500068956896732 & 0.3428330287802904 & 0.8160080607407115\end{array}$ $\begin{array}{lllll}0.3030523184844391 & 0.4192239472594358 & 0.3576204622549933\end{array}$ $\begin{array}{llll}0.0602202971439825 & 0.4634171528985408 & 0.3198959268576286\end{array}$ $\begin{array}{lllll}0.1383291061139831 & 0.3863746720540930 & 0.1107725727115288\end{array}$ $\begin{array}{lllll}0.2981208546299618 & 0.4646691795711645 & 0.0873777248746613\end{array}$ $\begin{array}{lllll}0.3920666495021379 & 0.3670055180732541 & 0.1425662641437945\end{array}$ $\begin{array}{llll}0.2376750760503847 & 0.4760200515809653 & 0.5822646051110324\end{array}$ $\begin{array}{llll}0.0112242936919971 & 0.4198487689085318 & 0.5562191710332824\end{array}$ $\begin{array}{lllll}0.2843565253925237 & 0.4265053468293469 & 0.8205137408769978\end{array}$ $\begin{array}{lllll}0.8038769208774396 & 0.4156541126433350 & 0.3686233681339781\end{array}$ $\begin{array}{llll}0.5700572814533291 & 0.4628013934958597 & 0.3332745168765701\end{array}$ $\begin{array}{llll}0.6444034037988293 & 0.3906526460910303 & 0.1155805294236448\end{array}$ $\begin{array}{lllll}0.8457670866116200 & 0.4610235801859696 & 0.1197464070944931\end{array}$ $\begin{array}{llll}0.8902601777125970 & 0.3583885120466676 & 0.1554170703918011\end{array}$ $\begin{array}{lllll}0.7403829026755689 & 0.4747937472184420 & 0.5945710112104174\end{array}$ $\begin{array}{lllll}0.5071634273605771 & 0.4190746724750885 & 0.5634777827969342\end{array}$ $\begin{array}{llll}0.7897649628157928 & 0.4241935127692422 & 0.8329488680922059\end{array}$ $\begin{array}{lllll}0.4280714516055342 & 0.2346860195328787 & 0.4950693355644785\end{array}$ $\begin{array}{lllll}0.1770209596734670 & 0.2961388007733208 & 0.4886451341630085\end{array}$ $\begin{array}{lllll}0.4456566616539556 & 0.1142138340104815 & 0.5270191395694607\end{array}$ $\begin{array}{lllll}0.9269258003158440 & 0.2350666483627148 & 0.4946813073194344\end{array}$ $\begin{array}{lllll}0.6754646568894074 & 0.2962528964358214 & 0.4880960819868515\end{array}$ $\begin{array}{llll}0.9432705041871218 & 0.1145768217579523 & 0.5261868286791440\end{array}$ $\begin{array}{lllll}0.1582120488253885 & 0.4176836752716880 & 0.4532970888844079\end{array}$ $\begin{array}{lllll}0.6602110368203447 & 0.4166844742185870 & 0.4637744036386850\end{array}$ $\begin{array}{lllll}0.0477915438743837 & 0.2300336957607823 & 0.2037549950702408\end{array}$ $\begin{array}{lllll}0.3098697522557152 & 0.1683653223559021 & 0.2100660945544523\end{array}$ $\begin{array}{lllll}0.2955590711407621 & 0.3634829924633094 & 0.7708854224001276\end{array}$ $\begin{array}{llll}0.3118010960018200 & 0.1208071001274711 & 0.7914972445485754\end{array}$ $\begin{array}{lllll}0.0540007688073292 & 0.1814355965032223 & 0.7970638678296661\end{array}$ $\begin{array}{lllll}0.0580906252976081 & 0.2989440089655333 & 0.7814872117703022\end{array}$ $\begin{array}{lllll}0.0486833052199814 & 0.3465499404790628 & 0.1867614222390690\end{array}$ $\begin{array}{llll}0.5455054288435572 & 0.2334762764586825 & 0.2011823019070718\end{array}$ $\begin{array}{lllll}0.8053349563309712 & 0.1688360043271239 & 0.2096743362544128\end{array}$ $\begin{array}{lllll}0.7965772582773638 & 0.3614594687728419 & 0.7763297838294890\end{array}$ $\begin{array}{llll}0.8103808868387683 & 0.1221956893114143 & 0.7933441514466772\end{array}$ $\begin{array}{llll}0.5527804983621558 & 0.1822450921822260 & 0.7985990908906700\end{array}$ $\begin{array}{lllll}0.5553516885245647 & 0.2992221782996494 & 0.7814805332986969\end{array}$ $\begin{array}{lllll}0.5471946436069330 & 0.3518068871890118 & 0.1872991329714408\end{array}$ $0.28401049552356630 .4114038017174254 \quad 0.1815446197303534$ $\begin{array}{llll}0.7935254808605303 & 0.4076629944686305 & 0.1944901677104889\end{array}$ 
T1:

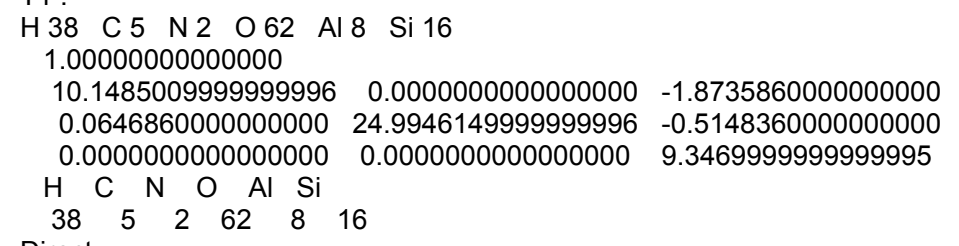
Direct

$\begin{array}{lll}0.3542695980654962 & 0.6384300355634505 & 0.0313688751612452\end{array}$ $\begin{array}{llll}0.1343068825863220 & 0.5150731029950347 & 0.3094878424266805\end{array}$ $\begin{array}{llll}0.1406807973664793 & 0.6032568380617483 & 0.9455155971392254\end{array}$ $\begin{array}{llll}0.5175258599218678 & 0.6350594646676088 & 0.4097082371233191\end{array}$ $\begin{array}{lllll}0.5474470989991154 & 0.6470909045698117 & 0.2304296921734436\end{array}$ $\begin{array}{lllll}0.5809951463175942 & 0.5831952443418877 & 0.3108295617259576\end{array}$ $\begin{array}{llll}0.2033813349412873 & 0.0639480762824012 & 0.8150462897414306\end{array}$ $\begin{array}{llll}0.7130895053263385 & 0.0599333650332075 & 0.8224900641205641\end{array}$ $\begin{array}{lllll}0.1234598780677448 & 0.1506276049098894 & 0.3726240723248537\end{array}$ $\begin{array}{llll}0.3563974944518347 & 0.3140932148542452 & 0.3168232631884091\end{array}$ $\begin{array}{llll}0.2834164620319239 & 0.2283469362570639 & 0.6728158205752590\end{array}$ $\begin{array}{llll}0.5071571167063365 & 0.0481270634775206 & 0.6916772606544198\end{array}$ $\begin{array}{llll}0.3601388943113271 & 0.0757517084740069 & 0.2930551355895505\end{array}$ $\begin{array}{lllll}0.6241529380538003 & 0.1465888486597631 & 0.3637007386498092\end{array}$ $\begin{array}{lllll}0.8598450061221516 & 0.3152261336508072 & 0.3215507325827842\end{array}$ $\begin{array}{llll}0.7804420945847123 & 0.2275657583998216 & 0.6683144849014455\end{array}$ $\begin{array}{llll}0.0105859158894626 & 0.0450918352569906 & 0.6840152575610511\end{array}$ $\begin{array}{llll}0.8666382381558122 & 0.0743708521428199 & 0.2943814030813398\end{array}$ $\begin{array}{lllll}0.9883500150820834 & 0.4757082186798510 & 0.3736728774676871\end{array}$ $\begin{array}{lllll}0.5169361114104996 & 0.3896364752544175 & 0.6179113221836798\end{array}$ $\begin{array}{llll}0.2922632383950211 & 0.4717852736077483 & 0.6995176091007080\end{array}$ $\begin{array}{llll}0.5260953150065515 & 0.4962546766009194 & 0.3876683273006900\end{array}$ $\begin{array}{llll}0.0168358034630833 & 0.3974680593285921 & 0.6588858916672802\end{array}$ $\begin{array}{lllll}0.7692950666735568 & 0.4681588781605541 & 0.6997734667960493\end{array}$ $\begin{array}{llll}0.4485329736352225 & 0.4750785577106009 & 0.1735336826515247\end{array}$ $\begin{array}{llll}0.8973371049675809 & 0.4958061257448121 & 0.1064221960184182\end{array}$ $\begin{array}{llll}0.3388119591650853 & 0.4435747576901262 & 0.9417514491404413\end{array}$ $\begin{array}{llll}0.8083680115675296 & 0.4464057489138477 & 0.9311263129760288\end{array}$ $\begin{array}{lllll}0.3061621353373760 & 0.0992171304540042 & 0.0566527735174038\end{array}$ $\begin{array}{lllll}0.8153068751352347 & 0.0978013708465765 & 0.0584666206336746\end{array}$ $\begin{array}{llll}0.2985943169751080 & 0.5072591693825668 & 0.5487766012667880\end{array}$ $0.82937227499639330 .4949031907788244 \quad 0.5714319293975287$ $\begin{array}{llll}0.2918054209735313 & 0.0548678674491674 & 0.4202808742390819\end{array}$ $\begin{array}{lllll}0.7972089041377155 & 0.0531880875437347 & 0.4207470595252895\end{array}$ $\begin{array}{llll}0.4402305427291209 & 0.4240493257885463 & 0.4868323457885522\end{array}$ $\begin{array}{llll}0.9420476176495484 & 0.4163250803901963 & 0.5021037245145638\end{array}$ $\begin{array}{llll}0.6973534428048861 & 0.1191362214646845 & 0.5115494315158803\end{array}$ $\begin{array}{llll}0.1990898373419074 & 0.1206395664271530 & 0.5146626665793796\end{array}$ $\begin{array}{lllll}0.5125605177595304 & 0.6175151648274312 & 0.2999553348480115\end{array}$ $\begin{array}{llll}0.3106766107679158 & 0.6129428355039437 & 0.1032214003498479\end{array}$ $\begin{array}{llll}0.3753849946746278 & 0.5997415975840298 & 0.2379070404106724\end{array}$ $\begin{array}{llll}0.3077339941488518 & 0.5644995175113137 & 0.3221368435985166\end{array}$ $\begin{array}{lllll}0.1179446307663214 & 0.5607214789194407 & 0.1235498687449046\end{array}$ $\begin{array}{lllll}0.1861880074127334 & 0.5945903511744318 & 0.0482796450705370\end{array}$ $\begin{array}{llll}0.1817020649571967 & 0.5485721955648102 & 0.2606085177753312\end{array}$ $\begin{array}{llll}0.3615615098301135 & 0.5485966193773886 & 0.4457679248988974\end{array}$ $0.0067464383175717 \quad 0.5438305402564476 \quad 0.0676398168064605$ $\begin{array}{llll}0.1104055728561137 & 0.3570794823297712 & 0.3744454511919864\end{array}$ $\begin{array}{llll}0.0807941074489067 & 0.2476048632893599 & 0.3836649480183052\end{array}$ $\begin{array}{llll}0.3306882221834763 & 0.2896160095110237 & 0.3863568512788406\end{array}$ $\begin{array}{llll}0.4215258944162231 & 0.2152655924552604 & 0.1392874724771547\end{array}$ $0.0792908802175466 \quad 0.2979453126276796 \quad 0.1343226867417605$ $\begin{array}{llll}0.1741738786417096 & 0.1968609236287886 & 0.1702461572588300\end{array}$ $\begin{array}{lllll}0.3803745874234932 & 0.1757679700784822 & 0.3886468975539722\end{array}$ $\begin{array}{lllll}0.3782463662530476 & 0.0630181784930370 & 0.3975811052995832\end{array}$ $0.11575881998573150 .1189175083203822 \quad 0.4298913884711695$ $\begin{array}{llll}0.3318241488161521 & 0.1134136743210140 & 0.1580752568859276\end{array}$ $\begin{array}{lllll}0.3191775839097976 & 0.1219105573486244 & 0.6218980175206383\end{array}$ $\begin{array}{lllll}0.0650326263708054 & 0.0739963767701934 & 0.6604584235668157\end{array}$ $0.2915379443055188 \quad 0.0735022346907743 \quad 0.8748297395231391$ $\begin{array}{llll}0.2302988291024882 & 0.1755813402176564 & 0.8387747006545219\end{array}$ $\begin{array}{llll}0.0629735637110845 & 0.2941952091089446 & 0.6118093949941302\end{array}$ $\begin{array}{llll}0.3117591447900525 & 0.2538310940028314 & 0.6078228514126430\end{array}$ 
$\begin{array}{llll}0.2212854544724468 & 0.3283042952299567 & 0.8559303392055293\end{array}$ $\begin{array}{lllll}0.0505922991894450 & 0.2485924039257476 & 0.8624020742480845\end{array}$ $\begin{array}{llllll}0.2632105653128707 & 0.3674221457225015 & 0.6076465921138469\end{array}$ $\begin{array}{lllll}0.4795045247907952 & 0.1471314844530787 & 0.8739232717567921\end{array}$ $\begin{array}{llll}0.5295128179261744 & 0.1861337402278840 & 0.6229701464773407\end{array}$ $\begin{array}{lllll}0.4692151297183412 & 0.3472527914431180 & 0.8247125745326540\end{array}$ $\begin{array}{lllll}0.6172328088200514 & 0.3573663788754574 & 0.3697158862470746\end{array}$ $\begin{array}{lllll}0.5806693563465430 & 0.2485317025775927 & 0.3833941849993041\end{array}$ $\begin{array}{lllll}0.8322100331177518 & 0.2893109852667182 & 0.3858701498883979\end{array}$ $\begin{array}{lllll}0.9197031506627187 & 0.2151805243570217 & 0.1400234524433041\end{array}$ $\begin{array}{lllll}0.5906599338987100 & 0.2949683503652218 & 0.1326809474198489\end{array}$ $\begin{array}{lllll}0.6739184818960178 & 0.1929069521505956 & 0.1755187941517475\end{array}$ $\begin{array}{lllll}0.8815663771415014 & 0.1749521028445720 & 0.3901991591370523\end{array}$ $\begin{array}{lllll}0.8837368748884226 & 0.0618199887684857 & 0.3990513338308211\end{array}$ $\begin{array}{lllll}0.6145012213880774 & 0.1170540522504041 & 0.4278312729656682\end{array}$ $\begin{array}{lllll}0.8387455460947365 & 0.1121391571460723 & 0.1598278658171124\end{array}$ $\begin{array}{llll}0.8204294982115883 & 0.1199256362622921 & 0.6215672751341297\end{array}$ $\begin{array}{lllll}0.5630124542648842 & 0.0747942037608554 & 0.6595537923835887\end{array}$ $\begin{array}{lllll}0.8012421158203018 & 0.0707857937738564 & 0.8770899303044057\end{array}$ $0.7307002513016495 \quad 0.17191631646049300 .8396084140018540$ $\begin{array}{lllll}0.5622158834884630 & 0.2955939490863320 & 0.6108128387334407\end{array}$ $\begin{array}{lllll}0.8107086907919568 & 0.2539004287739858 & 0.6070103831765413\end{array}$ $\begin{array}{llll}0.7238935118175298 & 0.3280949714581236 & 0.8529566691152436\end{array}$ $\begin{array}{llll}0.5585241646565680 & 0.2469799117977155 & 0.8616944027325950\end{array}$ $\begin{array}{lllll}0.7626533415984825 & 0.3682793913456670 & 0.6011449248823789\end{array}$ $\begin{array}{lllll}0.9810204267461089 & 0.1469591087097115 & 0.8743881098503297\end{array}$ $\begin{array}{lllll}0.0275885680010473 & 0.1861154879343031 & 0.6249136244813642\end{array}$ $\begin{array}{lllll}0.9691075794760867 & 0.3511918412939422 & 0.8161656679578040\end{array}$ $\begin{array}{lllll}0.3154490927609287 & 0.4239455763349441 & 0.3745427595765376\end{array}$ $\begin{array}{llll}0.0743247783010602 & 0.4672649438730229 & 0.3503024230779460\end{array}$ $\begin{array}{lllll}0.1594932356991560 & 0.3980345921214929 & 0.1243732823707898\end{array}$ $\begin{array}{llll}0.3567797861306740 & 0.4674113384171261 & 0.1173613759146413\end{array}$ $\begin{array}{lllll}0.4073579789248189 & 0.3656894897478558 & 0.1650841544874487\end{array}$ $\begin{array}{llll}0.2485407932753374 & 0.4809704723266245 & 0.5988901363265916\end{array}$ $\begin{array}{lllll}0.0233997670824468 & 0.4218058568061820 & 0.5797059612194545\end{array}$ $\begin{array}{lllll}0.3128140190689103 & 0.4301789545728751 & 0.8391036593175404\end{array}$ $\begin{array}{llll}0.8169123257574249 & 0.4289697672840015 & 0.3671985057725911\end{array}$ $\begin{array}{llll}0.5703646158261970 & 0.4703291057537025 & 0.3333693138211832\end{array}$ $\begin{array}{lllll}0.6538656765143318 & 0.3972295634886261 & 0.1205949324319533\end{array}$ $\begin{array}{llll}0.8171758445064395 & 0.4727577680475952 & 0.0932917409932250\end{array}$ $\begin{array}{lllll}0.9083380416221890 & 0.3744988473830140 & 0.1566294522628763\end{array}$ $\begin{array}{llll}0.7471642100392689 & 0.4821595436142455 & 0.5956546471870936\end{array}$ $\begin{array}{llll}0.5252682607882823 & 0.4226415699016955 & 0.5663437561316060\end{array}$ $\begin{array}{llll}0.8015968396612325 & 0.4313165927317286 & 0.8291166128950722\end{array}$ $\begin{array}{llll}0.4469367621118775 & 0.2413213112996909 & 0.4992926449565607\end{array}$ $\begin{array}{lllll}0.1953610948575120 & 0.3019577799637826 & 0.4949905943600524\end{array}$ $\begin{array}{lllll}0.4653921137547800 & 0.1201532943091247 & 0.5284563792685325\end{array}$ $\begin{array}{lllll}0.9470729716875588 & 0.2408818985298718 & 0.4998842388021802\end{array}$ $\begin{array}{lllll}0.6965536315049963 & 0.3028352033750585 & 0.4940157359092850\end{array}$ $\begin{array}{lllll}0.9670324015223866 & 0.1198303925026000 & 0.5299468176927632\end{array}$ $\begin{array}{lllll}0.1769106080356051 & 0.4217619523035898 & 0.4774424705580567\end{array}$ $\begin{array}{llll}0.6715183002787320 & 0.4248591997013422 & 0.4610592378015059\end{array}$ $\begin{array}{lllll}0.0648979541664635 & 0.2401585498657737 & 0.2058435984612425\end{array}$ $\begin{array}{lllll}0.3262814217173611 & 0.1764906254779152 & 0.2125210397794450\end{array}$ $\begin{array}{llllll}0.3171014986779925 & 0.3676241120773062 & 0.7842309778179788\end{array}$ $\begin{array}{lllll}0.3305956355113977 & 0.1284008525079848 & 0.7967505198932660\end{array}$ $\begin{array}{lllll}0.0725739241887065 & 0.1880866072381173 & 0.8023698280975469\end{array}$ $\begin{array}{lllll}0.0752045159118730 & 0.3049817548732490 & 0.7878927715134454\end{array}$ $\begin{array}{lllll}0.0638521409529552 & 0.3587748339158497 & 0.1968604491300733\end{array}$ $\begin{array}{lllll}0.5678906950415282 & 0.2384067301158894 & 0.2068796765660930\end{array}$ $\begin{array}{lllll}0.8283904547699970 & 0.1750358353206496 & 0.2141099362594055\end{array}$ $\begin{array}{lllll}0.8135368831349432 & 0.3686188716227499 & 0.7775936364533312\end{array}$ $\begin{array}{lllll}0.8335626462573761 & 0.1263013795538665 & 0.7964154461859272\end{array}$ $\begin{array}{lllll}0.5745553763474034 & 0.1863492173838833 & 0.8005708821660551\end{array}$ $\begin{array}{llllll}0.5775096326038288 & 0.3039833070783411 & 0.7884424727846173\end{array}$ $\begin{array}{lllll}0.5663241913147726 & 0.3550459707850240 & 0.1935145965909842\end{array}$ $\begin{array}{lllll}0.3098055998143293 & 0.4152659258947339 & 0.2016970684875681\end{array}$ $\begin{array}{llll}0.8016728637399526 & 0.4203095567902638 & 0.1921709621158384\end{array}$

T2 :

$\begin{array}{lllllll}\mathrm{H} 38 & \mathrm{C} & 5 & \mathrm{~N} 2 & \mathrm{O} 62 & \mathrm{Al} 8 & \mathrm{Si} 16\end{array}$

1.00000000000000

$10.1485009999999996 \quad 0.0000000000000000 \quad-1.8735860000000000$ 
$0.0646860000000000 \quad 24.9946149999999996-0.5148360000000000$ $\begin{array}{llll}0.0000000000000000 & 0.0000000000000000 & 9.3469999999999995\end{array}$ $\mathrm{H} \quad \mathrm{C} \quad \mathrm{N} \quad \mathrm{O} \quad \mathrm{Al} \mathrm{Si}$ $\begin{array}{llllll}38 & 5 & 2 & 62 & 8 & 16\end{array}$ Direct

$\begin{array}{llll}0.2067468582039655 & 0.7084296417615132 & 0.4507454709884150\end{array}$ $\begin{array}{lllll}0.1349855898181500 & 0.5205099899074986 & 0.3164647529834071\end{array}$ $\begin{array}{llll}0.0461738054845493 & 0.6549770443347955 & 0.5322384685664774\end{array}$ $\begin{array}{lllll}0.4697386394307211 & 0.6551362104093766 & 0.2890419508193923\end{array}$ $\begin{array}{lllll}0.3820897753345087 & 0.7164405982847472 & 0.3035095394697290\end{array}$ $\begin{array}{lllll}0.3495376856055144 & 0.6756950529632728 & 0.1416478907578660\end{array}$ $\begin{array}{llll}0.1988731610180285 & 0.0569382780853854 & 0.8210724616557710\end{array}$ $\begin{array}{lllll}0.6958329378527829 & 0.0570526085430590 & 0.8209943039347199\end{array}$ $\begin{array}{lllll}0.1131149934941068 & 0.1408135560647491 & 0.3672758232897244\end{array}$ $\begin{array}{llll}0.3494440085377720 & 0.3093445494213458 & 0.3242721466064593\end{array}$ $\begin{array}{lllll}0.2721728893029247 & 0.2221545456322178 & 0.6729252188482711\end{array}$ $\begin{array}{lllll}0.4956987686219246 & 0.0421001761748899 & 0.6953805336375710\end{array}$ $\begin{array}{lllll}0.3555822825608372 & 0.0684706419996207 & 0.2985887451406237\end{array}$ $\begin{array}{llll}0.6137020522541092 & 0.1430205041274185 & 0.3740746752975148\end{array}$ $\begin{array}{llll}0.8480391040860920 & 0.3075478927585119 & 0.3171276224210970\end{array}$ $\begin{array}{llll}0.7716831489222952 & 0.2239167063969242 & 0.6741148447191105\end{array}$ $\begin{array}{llll}-0.0032732278329658 & 0.0420715489045540 & 0.6956836227986882\end{array}$ $\begin{array}{lllll}0.8506612905509382 & 0.0692101138809772 & 0.2966018287946766\end{array}$ $\begin{array}{llll}0.0074602045313128 & 0.4835095540714221 & 0.3874668790765202\end{array}$ $\begin{array}{llll}0.5005051753669345 & 0.3862805188349265 & 0.6153835512354418\end{array}$ $0.26618692641461590 .4620595792784754 \quad 0.6985064321912240$ $\begin{array}{llllll}0.5925135095799540 & 0.4820981051589489 & 0.2685926819443308\end{array}$ $\begin{array}{lllll}1.0113124847565016 & 0.3933666494371864 & 0.6431458996108718\end{array}$ $\begin{array}{lllll}0.7703218274666910 & 0.4645173516742780 & 0.6972430354050623\end{array}$ $\begin{array}{lllll}0.3113970716840647 & 0.5033813454381164 & 0.1359159721014153\end{array}$ $\begin{array}{lllll}0.9317568549025976 & 0.4714830209643983 & 0.1575168778911791\end{array}$ $\begin{array}{llll}0.3015061973024096 & 0.4418705847922650 & 0.9310585955283154\end{array}$ $\begin{array}{lllll}0.8190058083923756 & 0.4396372631078167 & 0.9346713155386381\end{array}$ $\begin{array}{llll}0.3053709931691155 & 0.0918928250478518 & 0.0611814421669330\end{array}$ $\begin{array}{lllll}0.7971031204197613 & 0.0923382116268752 & 0.0601579940315995\end{array}$ $\begin{array}{llll}0.3362712715382461 & 0.4823861754516032 & 0.5708073284403126\end{array}$ $\begin{array}{lllll}0.8169806559367380 & 0.4945290333266738 & 0.5637201702526067\end{array}$ $\begin{array}{lllll}0.2822928308615205 & 0.0492981692889126 & 0.4237243836524640\end{array}$ $\begin{array}{lllll}0.7830559204102722 & 0.0482073963694796 & 0.4242176929636080\end{array}$ $\begin{array}{lllll}0.4333340789244060 & 0.4194958742052122 & 0.4769210744316847\end{array}$ $\begin{array}{lllll}0.9332023178404698 & 0.4153070550545157 & 0.4883192556399408\end{array}$ $\begin{array}{llll}0.6883166954945505 & 0.1137402431654770 & 0.5179520765054196\end{array}$ $\begin{array}{lllll}0.1876792338072423 & 0.1145394700799768 & 0.5164692705682380\end{array}$ $\begin{array}{lllll}0.3732747424578894 & 0.6750389481801472 & 0.2613003105572604\end{array}$ $\begin{array}{lllll}0.1960545494573636 & 0.6668383006539681 & 0.4128546040665605\end{array}$ $\begin{array}{lllll}0.2705738225458358 & 0.6450151939086761 & 0.3207290356569914\end{array}$ $\begin{array}{lllll}0.2488811595196893 & 0.5888149931131851 & 0.2782301513491799\end{array}$ $\begin{array}{lllll}0.0741356030959912 & 0.5846887703943224 & 0.4264180777320364\end{array}$ $\begin{array}{lllll}0.1017593629795370 & 0.6379661734059110 & 0.4641520681506463\end{array}$ $\begin{array}{llllll}0.1525892267749135 & 0.5625793235487874 & 0.3357388906508877\end{array}$ $\begin{array}{lllll}0.3130403467759883 & 0.5653625476981651 & 0.1945992548450811\end{array}$ $\begin{array}{llll}-0.0138901656419779 & 0.5600555062534348 & 0.4710134135541131\end{array}$ $\begin{array}{lllll}0.1051246408135786 & 0.3502012893993510 & 0.3720572096440680\end{array}$ $\begin{array}{lllll}0.0708923908799815 & 0.2410578210347408 & 0.3854618978895668\end{array}$ $\begin{array}{lllll}0.3211767741594727 & 0.2829617607900428 & 0.3868523901504005\end{array}$ $\begin{array}{lllll}0.4091243793973853 & 0.2093984858115513 & 0.1420384186033898\end{array}$ $\begin{array}{lllll}0.0805137289486024 & 0.2876897865602608 & 0.1348746756606939\end{array}$ $\begin{array}{lllll}0.1639173116942473 & 0.18587777085019573 & 0.1769795274652148\end{array}$ $\begin{array}{llll}0.3721987819760763 & 0.1691569052738092 & 0.3925274665187539\end{array}$ $\begin{array}{llll}0.3702514554263047 & 0.0563586521201595 & 0.4035653201008710\end{array}$ $\begin{array}{lllll}0.1047657674059854 & 0.1113722933004073 & 0.4321949106762142\end{array}$ $\begin{array}{lllll}0.3312108549349314 & 0.1060208584394995 & 0.1625999950261598\end{array}$ $\begin{array}{lllll}0.3092147022383129 & 0.1162095452158455 & 0.6249349278346268\end{array}$ $\begin{array}{lllll}0.0531514818374503 & 0.0684801017978939 & 0.6633765356913814\end{array}$ $\begin{array}{lllll}0.2870739887572932 & 0.0671748662803224 & 0.8782875726394306\end{array}$ $\begin{array}{lllll}0.2189347930535461 & 0.1688549125138815 & 0.8417605643964146\end{array}$ $\begin{array}{lllll}0.0515991232140930 & 0.2888221371000320 & 0.6106326379754397\end{array}$ $\begin{array}{lllll}0.3014240157744194 & 0.2479117102863339 & 0.6093231782519192\end{array}$ $\begin{array}{lllll}0.2106584361656813 & 0.3223501977810369 & 0.8532789842230554\end{array}$ $\begin{array}{lllll}0.0407415643582696 & 0.2425829686505038 & 0.8614490497866234\end{array}$ $\begin{array}{llll}0.2529901941355251 & 0.3616940570882075 & 0.6034259925881190\end{array}$ $\begin{array}{llll}0.4694381096628846 & 0.1428515548193220 & 0.8771079151664611\end{array}$ $\begin{array}{llll}0.5193422245949245 & 0.1808210145153891 & 0.6264895387836602\end{array}$ 
$\begin{array}{llll}0.4572110935067720 & 0.3444007681290512 & 0.8220298581686878\end{array}$ $\begin{array}{lllll}0.6025577788205693 & 0.3510241997562366 & 0.3698126751590295\end{array}$ $\begin{array}{lllll}0.5704330143691529 & 0.2422272495369302 & 0.3848132011657957\end{array}$ $\begin{array}{lllll}0.8208891047660084 & 0.2830518945947088 & 0.3855539589079331\end{array}$ $\begin{array}{lllll}0.9111474588430837 & 0.2080902312279878 & 0.1417765078730337\end{array}$ $\begin{array}{lllll}0.5703110783482956 & 0.2912346803014527 & 0.1330910630892875\end{array}$ $\begin{array}{llll}0.6632333139657758 & 0.1900732815291538 & 0.1730983971679864\end{array}$ $\begin{array}{lllll}0.8688673842611078 & 0.1691688975006171 & 0.3915798285191713\end{array}$ $\begin{array}{lllll}0.8693678845387804 & 0.0563474578692170 & 0.4011296373467470\end{array}$ $\begin{array}{lllll}0.6051830457216537 & 0.1118977963599323 & 0.4332554170549364\end{array}$ $\begin{array}{llll}0.8208976935943250 & 0.1065376257988279 & 0.1617867447661012\end{array}$ $\begin{array}{lllll}0.8089981225996780 & 0.1147359305590547 & 0.6253904367896594\end{array}$ $\begin{array}{lllll}0.5524932660658533 & 0.0688920493459653 & 0.6653833079352958\end{array}$ $\begin{array}{llll}0.7841516300513326 & 0.0668138681075161 & 0.8792261450244161\end{array}$ $\begin{array}{lllll}0.7203391969113613 & 0.1686895603200298 & 0.8423482873747428\end{array}$ $\begin{array}{lllll}0.5508202957437571 & 0.2902365087063533 & 0.6112909108606844\end{array}$ $\begin{array}{lllll}0.8003327909492760 & 0.2490226117252360 & 0.6084155838625185\end{array}$ $\begin{array}{lllll}0.7113424043282874 & 0.3238716014041216 & 0.8533827483249801\end{array}$ $\begin{array}{lllll}0.5450678678810216 & 0.2433118584479186 & 0.8628004754916183\end{array}$ $\begin{array}{lllll}0.7503004848373711 & 0.3630102013900195 & 0.6022668314127129\end{array}$ $\begin{array}{lllll}0.9695997795037018 & 0.1414769521380156 & 0.8769328082358993\end{array}$ $\begin{array}{llll}0.0165400535003400 & 0.1801356980755998 & 0.6256795599621445\end{array}$ $\begin{array}{llll}0.9578492924934875 & 0.3445111254426900 & 0.8174788310997316\end{array}$ $\begin{array}{lllll}0.3066644824384783 & 0.4200012096014535 & 0.3670491044542908\end{array}$ $\begin{array}{lllll}0.0661185678889965 & 0.4630649752712536 & 0.3349692355441011\end{array}$ $\begin{array}{lllll}0.1484324777764407 & 0.3891624301726592 & 0.1221962756848429\end{array}$ $\begin{array}{lllll}0.3103753198763921 & 0.4659298967218327 & 0.0947229031386895\end{array}$ $\begin{array}{lllll}0.4001646935228943 & 0.3682356373164354 & 0.1500475678221012\end{array}$ $\begin{array}{lllll}0.2493706255715486 & 0.4750473327517643 & 0.5934725733793301\end{array}$ $\begin{array}{lllll}0.0181649198366511 & 0.4192044159428470 & 0.5679521424447531\end{array}$ $\begin{array}{llll}0.2890786026308832 & 0.4254467241454652 & 0.8300966771081945\end{array}$ $\begin{array}{lllll}0.8125822023246491 & 0.4178180685805850 & 0.3720942191080755\end{array}$ $\begin{array}{llllll}0.5589836210980672 & 0.4586202448399384 & 0.3341236449939718\end{array}$ $\begin{array}{lllll}0.6536093275797339 & 0.3904821343658474 & 0.1198245156210597\end{array}$ $\begin{array}{llll}0.8402366715590053 & 0.4620633577208772 & 0.1112172117946400\end{array}$ $\begin{array}{llll}0.9011510415530406 & 0.3603275075545284 & 0.1604710430171211\end{array}$ $\begin{array}{llll}0.7423348444202830 & 0.4768604169837700 & 0.5934285516399747\end{array}$ $\begin{array}{lllll}0.5131670948544479 & 0.4187292569894497 & 0.5631630890858641\end{array}$ $0.79751282378292590 .4262054557403943 \quad 0.8316344745812556$ $\begin{array}{lllll}0.4374887346757400 & 0.2353717601957668 & 0.5014610397401067\end{array}$ $\begin{array}{lllll}0.1856928783117850 & 0.2958594364170815 & 0.4957316907426308\end{array}$ $\begin{array}{llll}0.4560699567398855 & 0.1144828720663002 & 0.5331456250243418\end{array}$ $\begin{array}{llll}0.9358494370841673 & 0.2350793186878948 & 0.5004442858753611\end{array}$ $\begin{array}{lllll}0.6849333257867055 & 0.2971084650123465 & 0.4933697489132538\end{array}$ $\begin{array}{llll}0.9550808114503025 & 0.1139086606803115 & 0.5319312631581011\end{array}$ $\begin{array}{lllll}0.1669831617236588 & 0.4162305475177494 & 0.4652781381480866\end{array}$ $\begin{array}{lllll}0.6643482927048220 & 0.4182515670352887 & 0.4631935862799049\end{array}$ $\begin{array}{lllll}0.0576802255569566 & 0.2312425734110726 & 0.2089201345186002\end{array}$ $\begin{array}{lllll}0.3190132949103638 & 0.1689117918401303 & 0.2164494188316597\end{array}$ $\begin{array}{lllll}0.3032873675771016 & 0.3625867037524692 & 0.7805456420884344\end{array}$ $\begin{array}{lllll}0.3215748570835079 & 0.1228041129768779 & 0.7997567804111942\end{array}$ $\begin{array}{llll}0.0617240910943289 & 0.1818617059894762 & 0.8031367201689801\end{array}$ $\begin{array}{lllll}0.0641475956707853 & 0.2990386674158825 & 0.7868817345499379\end{array}$ $\begin{array}{lllll}0.0592549898159167 & 0.3481530908948671 & 0.1947825550842231\end{array}$ $\begin{array}{lllll}0.5547752257194200 & 0.2341449253703517 & 0.2071318536069466\end{array}$ $\begin{array}{lllll}0.8152775174382410 & 0.1697617591130272 & 0.2153659280062427\end{array}$ $\begin{array}{lllll}0.8037726638140081 & 0.3632804947034224 & 0.7781465824764433\end{array}$ $\begin{array}{lllll}0.8210842980184931 & 0.1219575338995363 & 0.8001199024760959\end{array}$ $\begin{array}{lllll}0.5636165489638499 & 0.1825675878226790 & 0.8040547828920934\end{array}$ $\begin{array}{lllll}0.5648599988078731 & 0.3001263613347716 & 0.7883175157738442\end{array}$ $\begin{array}{llllll}0.5553325246156871 & 0.3526188099605274 & 0.1927709206137662\end{array}$ $\begin{array}{llll}0.2962601672625539 & 0.4135543734925791 & 0.1926662197880871\end{array}$ $\begin{array}{llll}0.8023170204390465 & 0.4086454014883338 & 0.1982558023271919\end{array}$

T3:

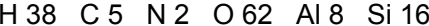

1.00000000000000

$10.1485009999999996 \quad 0.0000000000000000 \quad-1.8735860000000000$

$\begin{array}{llll}0.0646860000000000 & 24.9946149999999996 & -0.5148360000000000\end{array}$

$\begin{array}{llll}0.0000000000000000 & 0.0000000000000000 & 9.3469999999999995\end{array}$

$\mathrm{H} \quad \mathrm{C} \quad \mathrm{N} \quad \mathrm{O}$ Al $\mathrm{Si}$

$\begin{array}{llllll}38 & 5 & 2 & 62 & 8 & 16\end{array}$

Direct 
$\begin{array}{llll}0.2787506329506446 & 0.7171498130688980 & 0.2748790076086736\end{array}$ $\begin{array}{lllll}0.3050989351803973 & 0.5305540583277002 & 0.1363307812963403\end{array}$ $\begin{array}{lllll}0.3001279145251900 & 0.6892091033343690 & 0.0364935015768121\end{array}$ $\begin{array}{llll}0.1745664497441246 & 0.6331733008593233 & 0.5316781881855950\end{array}$ $\begin{array}{llll}0.2645744194251302 & 0.6936760584039510 & 0.5273391009978373\end{array}$ $\begin{array}{lllll}0.3468228092729426 & 0.6327685566076569 & 0.5873323736163889\end{array}$ $\begin{array}{llllll}0.1838486529437472 & 0.0553762636785812 & 0.8160008832315671\end{array}$ $\begin{array}{lllll}0.6862248379915661 & 0.0553129907336978 & 0.8154427400504581\end{array}$ $\begin{array}{lllll}0.1015340242889386 & 0.1404159547423899 & 0.3660582023438524\end{array}$ $\begin{array}{lllll}0.3371969249693493 & 0.3073697026925127 & 0.3175938217507974\end{array}$ $\begin{array}{lllll}0.2600964235856435 & 0.2221899430543843 & 0.6709674305768190\end{array}$ $\begin{array}{lllll}0.4854740721280573 & 0.0399901352796246 & 0.6888799746839137\end{array}$ $\begin{array}{llllll}0.3402734993718823 & 0.0675936878850967 & 0.2921428869311011\end{array}$ $\begin{array}{lllll}0.6010994950114623 & 0.1396551885143858 & 0.3629249508096241\end{array}$ $\begin{array}{lllll}0.8381517957500022 & 0.3082147254032647 & 0.3211000133084256\end{array}$ $\begin{array}{lllll}0.7599080500668649 & 0.2206342318452490 & 0.6686350525122875\end{array}$ $\begin{array}{llll}-0.0162988027103348 & 0.0401975583822339 & 0.6889994224554312\end{array}$ $\begin{array}{lllll}0.8425858622031787 & 0.0674240878236301 & 0.2931152642889827\end{array}$ $\begin{array}{lllll}0.1077585886500191 & 0.4912202652849890 & 0.3125613305276139\end{array}$ $\begin{array}{llll}0.4972199435483823 & 0.3929016567734360 & 0.6394187414407100\end{array}$ $\begin{array}{lllll}0.2602255180137354 & 0.4666600252814979 & 0.6920305495271615\end{array}$ $\begin{array}{lllll}0.5124046688657966 & 0.4888652241058167 & 0.3820623952682030\end{array}$ $\begin{array}{lllll}0.9888397530132528 & 0.3829392910634406 & 0.6116101321929561\end{array}$ $\begin{array}{lllll}0.7547746895220251 & 0.4616205000196087 & 0.6937839487098728\end{array}$ $\begin{array}{lllll}0.4349667751139918 & 0.4645241945956667 & 0.1815023190818878\end{array}$ $\begin{array}{lllll}0.9099944313730655 & 0.4734315709962251 & 0.1678022242014837\end{array}$ $\begin{array}{llllll}0.3196950610334113 & 0.4374629376024701 & 0.9335254563303318\end{array}$ $\begin{array}{llll}0.8059144284213998 & 0.4384471426919755 & 0.9315274605430424\end{array}$ $\begin{array}{lllll}0.2871276452207866 & 0.0910392306069625 & 0.0558179272773993\end{array}$ $\begin{array}{lllll}0.7919986714261198 & 0.0908902100521548 & 0.0561240584685108\end{array}$ $\begin{array}{llll}0.2669014975041253 & 0.5035403192055583 & 0.5472440906866117\end{array}$ $\begin{array}{llll}0.8182674027850941 & 0.4835297009368993 & 0.5627988513251040\end{array}$ $\begin{array}{llll}0.2708188871619627 & 0.0471737733453518 & 0.4189458267063257\end{array}$ $\begin{array}{llll}0.7695640619197132 & 0.0481068519973828 & 0.4182914325657210\end{array}$ $\begin{array}{lllll}0.4190093632465273 & 0.4160296881997751 & 0.4857637311948736\end{array}$ $\begin{array}{lllll}0.9163216414923466 & 0.4154358522924000 & 0.4744370541720705\end{array}$ $\begin{array}{lllll}0.6757684550467213 & 0.1131170281502649 & 0.5116967087162558\end{array}$ $\begin{array}{lllll}0.1761056139018825 & 0.1123355783974246 & 0.5122896396453970\end{array}$ $\begin{array}{llll}0.2664425946672070 & 0.6501913175334819 & 0.5082458218695736\end{array}$ $\begin{array}{llll}0.2850121834899231 & 0.6743228946230779 & 0.2518529498091642\end{array}$ $\begin{array}{llll}0.2815523477648400 & 0.6370612024494799 & 0.3551606516564446\end{array}$ $\begin{array}{llll}0.2925778678884274 & 0.5811556715163707 & 0.3122343560780295\end{array}$ $\begin{array}{lllll}0.3032463389810386 & 0.6080895552530697 & 0.0602462404805091\end{array}$ $\begin{array}{llll}0.2964255712733785 & 0.6606999645850850 & 0.1116243710606214\end{array}$ $\begin{array}{lllll}0.2985844861234794 & 0.5707698979475597 & 0.1666548317790555\end{array}$ $\begin{array}{llll}0.2965735451180090 & 0.5429898052432590 & 0.3968795684192956\end{array}$ $0.31256161306384870 .5971150839266052-0.0661154884158618$ $\begin{array}{lllll}0.0893534932365592 & 0.3499247073334722 & 0.3664202157225724\end{array}$ $\begin{array}{lllll}0.0584997972698044 & 0.2411561235469950 & 0.3812752441698395\end{array}$ $\begin{array}{lllll}0.3089337280342430 & 0.2821020866035301 & 0.3834131206999727\end{array}$ $\begin{array}{lllll}0.3988376859364637 & 0.2072645246295134 & 0.1378462985424187\end{array}$ $\begin{array}{lllll}0.0641995432639277 & 0.2888921443040303 & 0.1296764583549869\end{array}$ $\begin{array}{lllll}0.1513086075678835 & 0.1873476440761772 & 0.1710406425450137\end{array}$ $\begin{array}{llllll}0.3579652895543984 & 0.1677973449761684 & 0.3877271761122434\end{array}$ $\begin{array}{lllll}0.3576963512754010 & 0.0549013600610827 & 0.3967469142185950\end{array}$ $\begin{array}{llll}0.0929164002067050 & 0.1100755294209498 & 0.4279197433094628\end{array}$ $\begin{array}{lllll}0.3119800755713215 & 0.1051494541036740 & 0.1573569870557892\end{array}$ $\begin{array}{lllll}0.2971814308005830 & 0.1133228461678833 & 0.6203373247669315\end{array}$ $\begin{array}{lllll}0.0402474319315744 & 0.0672423689643450 & 0.6597253078558134\end{array}$ $\begin{array}{lllll}0.2723384749494687 & 0.0650008021311932 & 0.8739188760355434\end{array}$ $\begin{array}{lllll}0.2085983532737463 & 0.1668092290583492 & 0.8378482216297751\end{array}$ $\begin{array}{llllll}0.0390620461176048 & 0.2886333969431604 & 0.6083689602769073\end{array}$ $\begin{array}{lllll}0.2884983634068655 & 0.2473434442425969 & 0.6052339964463289\end{array}$ $\begin{array}{lllll}0.1993595761962375 & 0.3223690700035700 & 0.8513275867020684\end{array}$ $\begin{array}{lllll}0.0335817507182273 & 0.2412635867359567 & 0.8591268220734811\end{array}$ $0.2364682267589574 \quad 0.3617376758464344 \quad 0.6011253007400080$ $\begin{array}{lllll}0.4578852237198145 & 0.1396298079573431 & 0.8723979511270478\end{array}$ $\begin{array}{lllll}0.5054767456756173 & 0.1786228186286873 & 0.6218346742333219\end{array}$ $\begin{array}{lllll}0.4455732815338947 & 0.3424741693557006 & 0.8147372765948073\end{array}$ $\begin{array}{lllll}0.5910707300396676 & 0.3491094670111344 & 0.3677265417582422\end{array}$ $\begin{array}{lllll}0.5586125446881077 & 0.2401609579084897 & 0.3812565661804615\end{array}$ $\begin{array}{llll}0.8092693703042919 & 0.2820331529346920 & 0.3839363138267613\end{array}$ $\begin{array}{llll}0.8979255603174814 & 0.2082958521705898 & 0.1379852906126899\end{array}$ 
$\begin{array}{llll}0.5688142605403165 & 0.2870086939667766 & 0.1306084805917996\end{array}$ $\begin{array}{lllll}0.6518671612005968 & 0.1852442617872216 & 0.1724211316090566\end{array}$ $\begin{array}{lllll}0.8601621922873075 & 0.1679277090125607 & 0.3877871742573327\end{array}$ $\begin{array}{llll}0.8574573264333677 & 0.0551754913225529 & 0.3979626667701913\end{array}$ $\begin{array}{llll}0.5927987569236294 & 0.1100375731932407 & 0.4272855668130816\end{array}$ $\begin{array}{lllll}0.8181517654642649 & 0.1050772016052670 & 0.1573908149238321\end{array}$ 0.79697495787166120 .11480204035176490 .6199708593827834 $\begin{array}{llll}0.5416783597340303 & 0.0669051792273524 & 0.6584300931618967\end{array}$ 0.77449034962925340 .06536645853434890 .8729764392437300 $\begin{array}{llll}0.7075166966865295 & 0.1670864919290569 & 0.8380962774796639\end{array}$ $\begin{array}{lllll}0.5399025952939601 & 0.2871682445109082 & 0.6075727687876444\end{array}$ $\begin{array}{llllll}0.7891994305018082 & 0.2464903156312274 & 0.6053401320652385\end{array}$ $\begin{array}{llllll}0.6983843219549383 & 0.3210328382120370 & 0.8508718639168367\end{array}$ $\begin{array}{lllll}0.5294308826803645 & 0.2408052117646310 & 0.8582527997314736\end{array}$ $\begin{array}{lllll}0.7388860139099951 & 0.3605387791222096 & 0.6011858071919127\end{array}$ $\begin{array}{lllll}0.9576544751621653 & 0.1407922422221097 & 0.8723200176547803\end{array}$ $\begin{array}{lllll}0.0074320621429267 & 0.1791633692256710 & 0.6219521300798155\end{array}$ $\begin{array}{lllll}0.9449989697311000 & 0.3421107103441058 & 0.8192845642400907\end{array}$ $\begin{array}{lllll}0.2975987963535247 & 0.4142776490770704 & 0.3713879796419919\end{array}$ $\begin{array}{lllll}0.0523532841435099 & 0.4612409517991303 & 0.3274187089882767\end{array}$ $\begin{array}{lllll}0.1397269977599676 & 0.3895599871854519 & 0.1169234966560080\end{array}$ $\begin{array}{llll}0.3411637746240620 & 0.4604971792829538 & 0.1203595508309574\end{array}$ $\begin{array}{lllll}0.3870078563938191 & 0.3587741613657545 & 0.1563590793318799\end{array}$ $\begin{array}{llll}0.2202341874064417 & 0.4753297651309795 & 0.5895782675252088\end{array}$ $\begin{array}{lllll}0.0016544813533921 & 0.4156387413146557 & 0.5591075345891173\end{array}$ $\begin{array}{llll}0.2891604835652610 & 0.4248453046459383 & 0.8316958425526582\end{array}$ $\begin{array}{llll}0.7947904448907643 & 0.4153534735029838 & 0.3663576793447539\end{array}$ $\begin{array}{llll}0.5524409379090039 & 0.4613996897658736 & 0.3285902085903914\end{array}$ $\begin{array}{lllll}0.6344557869335465 & 0.3891456887551749 & 0.1175277200633168\end{array}$ $\begin{array}{lllll}0.8211794434431564 & 0.4638267032122188 & 0.1107201826180036\end{array}$ $\begin{array}{lllll}0.8871750858691279 & 0.3630299391125053 & 0.1511891655051608\end{array}$ $\begin{array}{llll}0.7341109898237890 & 0.4740152839246717 & 0.5891474159520238\end{array}$ $\begin{array}{lllll}0.5049330715953430 & 0.4188098197331383 & 0.5647667280128189\end{array}$ 0.78104012995200120 .42386377815698850 .8301369901285733 $\begin{array}{lllll}0.4241331842181364 & 0.2337939833792690 & 0.4972031913039960\end{array}$ $\begin{array}{lllll}0.1731580047240527 & 0.2959201971423887 & 0.4912534105637602\end{array}$ $\begin{array}{lllll}0.4436510466985139 & 0.1125450319269414 & 0.5275696360327478\end{array}$ $\begin{array}{lllll}0.9252409819458793 & 0.2340480154989805 & 0.4976861054394870\end{array}$ $\begin{array}{lllll}0.6734689621813438 & 0.2949145382166896 & 0.4922925861606809\end{array}$ $\begin{array}{lllll}0.9438665456058083 & 0.1130077226781725 & 0.5280132514264441\end{array}$ $\begin{array}{lllll}0.1498009168870723 & 0.4167029989903931 & 0.4621329280869700\end{array}$ $\begin{array}{lllll}0.6511811676777352 & 0.4155870036478299 & 0.4606785790645264\end{array}$ $\begin{array}{lllll}0.0442658465524186 & 0.2321891683052294 & 0.2041467743394710\end{array}$ $\begin{array}{lllll}0.3045511248881524 & 0.1682226201117033 & 0.2116120023363675\end{array}$ $\begin{array}{llll}0.2920305390791522 & 0.3620234252365959 & 0.7766249208905007\end{array}$ $\begin{array}{lllll}0.3094192440724052 & 0.1201843457315871 & 0.7951343215166640\end{array}$ $\begin{array}{lllll}0.0518055521434298 & 0.1806130625135505 & 0.7995354938884711\end{array}$ $\begin{array}{lllll}0.0534894428714030 & 0.2982436789134048 & 0.7855652863776017\end{array}$ $\begin{array}{lllll}0.0444119830331412 & 0.3497322324135046 & 0.1890751729929054\end{array}$ $\begin{array}{lllll}0.5454955137382073 & 0.2305029166678935 & 0.2047563298641698\end{array}$ $\begin{array}{lllll}0.8067503667931214 & 0.1679608156747541 & 0.2117288204555278\end{array}$ $\begin{array}{lllll}0.7915542395196976 & 0.3608796190048281 & 0.7775590507726984\end{array}$ $\begin{array}{llll}0.8095779321744749 & 0.1210000048577552 & 0.7949259159923899\end{array}$ $\begin{array}{lllll}0.5503161411450971 & 0.1801667512254647 & 0.7993832047885362\end{array}$ $\begin{array}{llll}0.5522751762367791 & 0.2973798773274682 & 0.7837758072597836\end{array}$ $\begin{array}{lllll}0.5452214069338867 & 0.3472486794153347 & 0.1905230568892307\end{array}$ $\begin{array}{lllll}0.2889105316975565 & 0.4071356592900771 & 0.1978494658877907\end{array}$ $\begin{array}{llll}0.7836982468367919 & 0.4094273477036311 & 0.1920591853423233\end{array}$

T4 :

H 38 C 5 N 2 O 62 Al 8 Si 16

1.00000000000000

$10.1485009999999996 \quad 0.0000000000000000 \quad-1.8735860000000000$

$\begin{array}{llll}0.0646860000000000 & 24.9946149999999996 & -0.5148360000000000\end{array}$

$\begin{array}{llll}0.0000000000000000 & 0.0000000000000000 & 9.3469999999999995\end{array}$

$\mathrm{H} \quad \mathrm{C} \quad \mathrm{N} \quad \mathrm{O}$ Al $\mathrm{Si}$

$\begin{array}{llllll}38 & 5 & 2 & 62 & 8 & 16\end{array}$

Direct

$0.29809136593662110 .7251170079942550 \quad 0.5713992034375136$

$\begin{array}{lllll}0.1617509045678811 & 0.5417736028076304 & 0.5797515623871198\end{array}$

$\begin{array}{llll}0.0856484801652213 & 0.7013834741016202 & 0.6104183413188357\end{array}$

$\begin{array}{lllll}0.5705646368893375 & 0.6347700551742254 & 0.6015602894060986\end{array}$

$\begin{array}{llll}0.5170825981907385 & 0.6976613740513619 & 0.5235938232755204\end{array}$ 
$\begin{array}{llll}0.5098533364010368 & 0.6381045954008072 & 0.4133245410125373\end{array}$ $\begin{array}{llllll}0.1817986949138095 & 0.0574675275958737 & 0.8208233907062036\end{array}$ $\begin{array}{lllll}0.6836321082005689 & 0.0560185151898314 & 0.8220600170245798\end{array}$ $\begin{array}{llll}0.1042681521420537 & 0.1384634193286725 & 0.3557039317297675\end{array}$ $\begin{array}{llll}0.3423402713977856 & 0.3084162262212061 & 0.3204418760179871\end{array}$ $\begin{array}{llll}0.2646337389895488 & 0.2197529540926266 & 0.6665721397294819\end{array}$ $\begin{array}{lllll}0.5256107044441071 & 0.0307171404182096 & 0.6255216320399877\end{array}$ $\begin{array}{llll}0.3472439574006780 & 0.0682180232324525 & 0.2908405583251288\end{array}$ $\begin{array}{lllll}0.6042647415787266 & 0.1377830593467098 & 0.3545892760955370\end{array}$ $\begin{array}{lllll}0.8422300779393607 & 0.3076340283338875 & 0.3171271593398771\end{array}$ $\begin{array}{llllll}0.7629077237510943 & 0.2202017768311944 & 0.6648424221638687\end{array}$ $\begin{array}{llll}0.0344106871733561 & 0.0307438131581370 & 0.6208894514613893\end{array}$ $\begin{array}{lllll}0.8469744955177655 & 0.0675743303325524 & 0.2911583059593359\end{array}$ $\begin{array}{lllll}0.0085506765291733 & 0.4857681125020784 & 0.3808821537918028\end{array}$ $\begin{array}{lllll}0.4993362097275956 & 0.3856566931382803 & 0.6260525499705181\end{array}$ $\begin{array}{lllll}0.2646429426538785 & 0.4586908050656012 & 0.7018816371837970\end{array}$ $\begin{array}{llllll}0.5214386295560266 & 0.4917766357060662 & 0.3846599433435656\end{array}$ $\begin{array}{lllll}0.9999076732522789 & 0.3908817080934980 & 0.6400104194121652\end{array}$ $\begin{array}{lllll}0.7556775505576497 & 0.4621675318665150 & 0.6997135733595364\end{array}$ $\begin{array}{lllll}0.4231730189859633 & 0.4714921586123844 & 0.1648841591000552\end{array}$ $\begin{array}{lllll}0.9248368150030276 & 0.4713927039472429 & 0.1544935090376313\end{array}$ $\begin{array}{lllll}0.3141968812340224 & 0.4368683688435967 & 0.9372107348571233\end{array}$ $\begin{array}{lllll}0.8116680678526154 & 0.4380206793986878 & 0.9342690713632695\end{array}$ $\begin{array}{llll}0.2948009287484287 & 0.0919318124450830 & 0.0546021403211870\end{array}$ 0.79366103505640820 .09112570770358540 .0549455263657068 $\begin{array}{lllll}0.3117965846270923 & 0.4923047331585337 & 0.5689216643529000\end{array}$ $\begin{array}{lllll}0.8056466245591445 & 0.4925547400810167 & 0.5697083038117564\end{array}$ $\begin{array}{lllll}0.2709194612186627 & 0.0505838456658093 & 0.4145368061369780\end{array}$ $\begin{array}{lllll}0.7760474379639332 & 0.0479272342248372 & 0.4178158641296378\end{array}$ $\begin{array}{lllll}0.4241544812829202 & 0.4155271990627744 & 0.4836282824547782\end{array}$ $\begin{array}{llll}0.9243007210951610 & 0.4147884614990645 & 0.4870795902687106\end{array}$ $\begin{array}{llllll}0.6779920735618938 & 0.1131775099795441 & 0.5076770436145668\end{array}$ $\begin{array}{lllll}0.1787808451451333 & 0.1142610149477102 & 0.5091835146069935\end{array}$ $\begin{array}{lllll}0.4964898816193076 & 0.6545523755513031 & 0.5198239202239494\end{array}$ $\begin{array}{lllll}0.2733478799211387 & 0.6827030616406985 & 0.5695112146349074\end{array}$ $\begin{array}{lllll}0.3602396248964306 & 0.6438317407497303 & 0.5456045503424164\end{array}$ $\begin{array}{llll}0.3174059854264700 & 0.5885435768871530 & 0.5477274145887290\end{array}$ $\begin{array}{lllll}0.0991984103218781 & 0.6199418710525137 & 0.5954071105936167\end{array}$ $0.1487366030920407 \quad 0.6715460229688095 \quad 0.5932144394247980$ 0.18963936616966720 .58109136644035190 .5727629667528958 $\begin{array}{lllll}0.3881353437552580 & 0.5490678541348246 & 0.5302725646783298\end{array}$ $\begin{array}{llll}-0.0127497218024450 & 0.6104222838947516 & 0.6154846155945080\end{array}$ $\begin{array}{lllll}0.0957746923091652 & 0.3493932458351010 & 0.3705613992118708\end{array}$ $\begin{array}{lllll}0.0638488398183599 & 0.2404706206506754 & 0.3812064548669861\end{array}$ $\begin{array}{lllll}0.3142331538612270 & 0.2823506921241782 & 0.3842436935631370\end{array}$ $\begin{array}{lllll}0.4039070020734399 & 0.2090325760054173 & 0.1371475928253123\end{array}$ $\begin{array}{llll}0.0693841124514251 & 0.2888241879930285 & 0.1311149159259362\end{array}$ $\begin{array}{lllll}0.1570861055296528 & 0.1870549202513318 & 0.1702666103983055\end{array}$ $\begin{array}{lllll}0.3646203997316519 & 0.1686342057016990 & 0.3864231770563883\end{array}$ $\begin{array}{llll}0.3602064672923402 & 0.0556162216009449 & 0.3955952633870891\end{array}$ $\begin{array}{lllll}0.0965369878103068 & 0.1099324132895387 & 0.4236129489854338\end{array}$ $\begin{array}{lllll}0.3218781074712561 & 0.1060888788892110 & 0.1560766489299327\end{array}$ $\begin{array}{lllll}0.2992522120882704 & 0.1157933761429004 & 0.6170841056444420\end{array}$ $\begin{array}{lllll}0.0422854792832282 & 0.0673182551951067 & 0.6580515455474766\end{array}$ $\begin{array}{llllll}0.2730760342343278 & 0.0659063477952971 & 0.8731240679009888\end{array}$ $\begin{array}{lllll}0.2119526057609627 & 0.1671463187488197 & 0.8367311989237349\end{array}$ $\begin{array}{llll}0.0451680347302320 & 0.2868259849502954 & 0.6092599695865422\end{array}$ $\begin{array}{lllll}0.2945217704783609 & 0.2462821780833691 & 0.6054552089972669\end{array}$ $\begin{array}{lllll}0.2043511595439395 & 0.3197123949128817 & 0.8534076535793944\end{array}$ $\begin{array}{llll}0.0326258893597035 & 0.2404355653931882 & 0.8594483702185733\end{array}$ $\begin{array}{lllll}0.2441161272965652 & 0.3599097245837067 & 0.6043397934394116\end{array}$ $\begin{array}{lllll}0.4615493970823163 & 0.1394890289644462 & 0.8694726660746384\end{array}$ $\begin{array}{lllll}0.5123421285159813 & 0.1787106568259418 & 0.6213407165788150\end{array}$ $\begin{array}{lllll}0.4514064387214458 & 0.3411765554242319 & 0.8204736588155218\end{array}$ $\begin{array}{llllll}0.5971426299734068 & 0.3504261136470641 & 0.3680247412834663\end{array}$ $\begin{array}{llll}0.5638430779009956 & 0.2416402641792505 & 0.3808885160415921\end{array}$ $\begin{array}{lllll}0.8146793105083694 & 0.2823221772688369 & 0.3833100398469308\end{array}$ $\begin{array}{llll}0.9038660121073836 & 0.2077318576700892 & 0.1371751605775600\end{array}$ $\begin{array}{lllll}0.5728105594156494 & 0.2891243001291024 & 0.1303714679274302\end{array}$ $\begin{array}{llll}0.6564335084625864 & 0.1869695811906529 & 0.1711903354274479\end{array}$ $\begin{array}{lllll}0.8635085325094179 & 0.1680020819676342 & 0.3866743626244942\end{array}$ $\begin{array}{lllll}0.8635063183793065 & 0.0547551777505446 & 0.3958833997353423\end{array}$ $\begin{array}{llll}0.5956266295639252 & 0.1094745576478046 & 0.4228348101816979\end{array}$ 
$\begin{array}{llll}0.8186411432525806 & 0.1052399374149458 & 0.1566902909627687\end{array}$ $\begin{array}{lllll}0.7995293367999627 & 0.1144646196196424 & 0.6169644338862432\end{array}$ $\begin{array}{llll}0.5411411018902375 & 0.0676327829694307 & 0.6576588990857818\end{array}$ $\begin{array}{lllll}0.7748310806014691 & 0.0645743930591346 & 0.8736387267033434\end{array}$ $\begin{array}{lllll}0.7128039616407301 & 0.1656169491310453 & 0.8372569077305168\end{array}$ $\begin{array}{lllll}0.5453001915277714 & 0.2879690552088986 & 0.6091465320251090\end{array}$ $\begin{array}{llllll}0.7935879108090056 & 0.2467980554693848 & 0.6045290175536634\end{array}$ $\begin{array}{lllll}0.7054890057678112 & 0.3212245227881430 & 0.8520308140492874\end{array}$ $\begin{array}{llll}0.5385208354033022 & 0.2403280978686533 & 0.8599320202280354\end{array}$ $\begin{array}{llll}0.7443287518068220 & 0.3611995480827835 & 0.6015642119469503\end{array}$ $\begin{array}{lllll}0.9623170327264305 & 0.1385343582345996 & 0.8693707132440236\end{array}$ $\begin{array}{lllll}0.0104338216851323 & 0.1784330620604558 & 0.6216413970464092\end{array}$ $\begin{array}{lllll}0.9517364052408884 & 0.3426904238189767 & 0.8164371229514984\end{array}$ $\begin{array}{llll}0.3022921490992408 & 0.4150338927715905 & 0.3715498879188152\end{array}$ $\begin{array}{llll}0.0620215727455720 & 0.4613437319451826 & 0.3339753546357580\end{array}$ $\begin{array}{lllll}0.1418724747376846 & 0.3900980198498220 & 0.1215748979825163\end{array}$ $\begin{array}{lllll}0.3318699450338026 & 0.4626332171605485 & 0.1127689620609170\end{array}$ $\begin{array}{llllll}0.3921916148754403 & 0.3615614696887713 & 0.1556443590755697\end{array}$ $\begin{array}{llll}0.2373906038973095 & 0.4717636744366814 & 0.5980954755161544\end{array}$ $\begin{array}{lllll}0.0081831599130828 & 0.4177418519509296 & 0.5677997199674590\end{array}$ $\begin{array}{lllll}0.2888326688358679 & 0.4223278988426968 & 0.8354837021546534\end{array}$ $\begin{array}{lllll}0.8020293730417172 & 0.4182318382316935 & 0.3717773463082401\end{array}$ $\begin{array}{lllll}0.5564452973987689 & 0.4626146844291655 & 0.3321475300039337\end{array}$ $\begin{array}{lllll}0.6422627283068755 & 0.3912138816820117 & 0.1209473147897379\end{array}$ $\begin{array}{lllll}0.8321354538100945 & 0.4628694546724913 & 0.1096274190270938\end{array}$ $\begin{array}{lllll}0.8915782369808323 & 0.3619434723855432 & 0.1589765694782438\end{array}$ $\begin{array}{llll}0.7291267380137727 & 0.4753940086237208 & 0.5961490406193672\end{array}$ $\begin{array}{lllll}0.5077706074286965 & 0.4164385479524587 & 0.5659425626100637\end{array}$ $\begin{array}{lllll}0.7893142535184614 & 0.4239714633876890 & 0.8317938938668831\end{array}$ $\begin{array}{lllll}0.4304404497511375 & 0.2342453908966551 & 0.4972590207078546\end{array}$ $\begin{array}{lllll}0.1786820995359363 & 0.2948406939253386 & 0.4931568131045069\end{array}$ $\begin{array}{lllll}0.4465747840131202 & 0.1137180273153772 & 0.5262592133122930\end{array}$ $\begin{array}{lllll}0.9296165450353073 & 0.2338399367819248 & 0.4969398046652720\end{array}$ $\begin{array}{lllll}0.6791638086867018 & 0.2958703380506547 & 0.4917606763206853\end{array}$ $\begin{array}{llllll}0.9468585885891364 & 0.1133051453425961 & 0.5266047107790317\end{array}$ $\begin{array}{lllll}0.1583669935238468 & 0.4154637693812975 & 0.4650383627536336\end{array}$ $\begin{array}{llll}0.6548668965192768 & 0.4173062647784804 & 0.4621189452085471\end{array}$ $\begin{array}{lllll}0.0498317927219394 & 0.2317664019740093 & 0.2041874399591924\end{array}$ $\begin{array}{lllll}0.3113876331887088 & 0.1690629139399013 & 0.2102829746488290\end{array}$ $\begin{array}{llll}0.2975721715701388 & 0.3596079700330802 & 0.7804113894736612\end{array}$ $\begin{array}{lllll}0.3126016941014736 & 0.1202434552016075 & 0.7924398447188676\end{array}$ $\begin{array}{lllll}0.0546590418291210 & 0.1800427707465737 & 0.7995024438653088\end{array}$ $\begin{array}{lllll}0.0574743227056283 & 0.2969417621871449 & 0.7856007378806867\end{array}$ $\begin{array}{lllll}0.0493304586403197 & 0.3492095801849346 & 0.1933617723459273\end{array}$ $\begin{array}{lllll}0.5505109294254398 & 0.2324079668837355 & 0.2040920698675027\end{array}$ $\begin{array}{lllll}0.8102944318363199 & 0.1683578879436983 & 0.2105848078182043\end{array}$ $\begin{array}{lllll}0.7974078103220966 & 0.3611548238570148 & 0.7774875549702969\end{array}$ $\begin{array}{llll}0.8134609987600707 & 0.1189624829829544 & 0.7923070108431041\end{array}$ 0.55643461662478630 .17979171249900590 .7992821448672280 $\begin{array}{llll}0.5594341909745729 & 0.2971649592635243 & 0.7862368139291834\end{array}$ $\begin{array}{llll}0.5501265182330370 & 0.3495436043480741 & 0.1908926928393516\end{array}$ $\begin{array}{llllll}0.2918730748037651 & 0.4092992573166981 & 0.1973963749810043\end{array}$ $\begin{array}{llll}0.7919252611203220 & 0.4102693953647796 & 0.1977821578344034\end{array}$

T5 :

$\begin{array}{llllll}\mathrm{H} 38 & \mathrm{C} 5 & \mathrm{~N} 2 & \mathrm{O} 62 & \text { Al } 8 & \text { Si } 16\end{array}$

1.00000000000000

$10.1485009999999996 \quad 0.0000000000000000 \quad-1.8735860000000000$

$\begin{array}{llll}0.0646860000000000 & 24.9946149999999996 & -0.5148360000000000\end{array}$

$\begin{array}{lll}0.0000000000000000 & 0.0000000000000000 & 9.3469999999999995\end{array}$

$\mathrm{H} \quad \mathrm{C} \quad \mathrm{N} \quad \mathrm{O}$ Al $\mathrm{Si}$

$\begin{array}{llllll}38 & 5 & 2 & 62 & 8 & 16\end{array}$

Direct

$\begin{array}{llll}0.7806646356948983 & 0.7241542118053923 & 0.1893344254279473\end{array}$

$\begin{array}{lllll}0.7719707045671323 & 0.5309899509876436 & 0.1627077249465923\end{array}$

$\begin{array}{lllll}0.9262627523633394 & 0.6727617186053813 & 0.0716484702830205\end{array}$

$\begin{array}{lllll}0.4883098783276726 & 0.6729090620922755 & 0.2890916170572950\end{array}$

$\begin{array}{lllll}0.6012554321391956 & 0.7273733332779568 & 0.3312370483542897\end{array}$

$\begin{array}{lllll}0.6069414234377275 & 0.6719958855377596 & 0.4503015346007110\end{array}$

$\begin{array}{lllll}0.1775127196681589 & 0.0574478293193476 & 0.8238401826711983\end{array}$

$\begin{array}{lllll}0.6804595831348216 & 0.0573908685472021 & 0.8231481124085317\end{array}$

$\begin{array}{llll}0.1003590759752755 & 0.1371283957950803 & 0.3551057071040813\end{array}$

$\begin{array}{lllll}0.3395440239455821 & 0.3074768598031229 & 0.3175921885158444\end{array}$ 
$\begin{array}{llll}0.2593612941975436 & 0.2211024057334915 & 0.6667392917168741\end{array}$ $\begin{array}{lllll}0.5300996307681106 & 0.0308823506926276 & 0.6242257410522889\end{array}$ $\begin{array}{lllll}0.3417583586088391 & 0.0669808860265566 & 0.2937544371228025\end{array}$ $\begin{array}{llll}0.5996197032346513 & 0.1369289718849000 & 0.3543205194764567\end{array}$ $\begin{array}{llll}0.8434881432978719 & 0.3107019375420801 & 0.3312420323827405\end{array}$ $\begin{array}{llll}0.7605547086400452 & 0.2200167948628900 & 0.6674372156691185\end{array}$ $\begin{array}{llll}0.0239968347248840 & 0.0312901552456165 & 0.6264670478331533\end{array}$ $\begin{array}{lllll}0.8444805358526751 & 0.0678859213122840 & 0.2927947988159765\end{array}$ $\begin{array}{llll}0.1100174242657213 & 0.4894128908109619 & 0.2921339138661178\end{array}$ $\begin{array}{llll}0.4974173984357621 & 0.3909288766328099 & 0.6278008662239396\end{array}$ $\begin{array}{lllll}0.2577256267055870 & 0.4625776034997530 & 0.6932486092520169\end{array}$ $\begin{array}{lllll}0.5871960488293044 & 0.4963889541615686 & 0.3300833880342294\end{array}$ $\begin{array}{llllll}1.0030318965919283 & 0.3923107685031180 & 0.6308723758391750\end{array}$ $\begin{array}{lllll}0.7588725238949646 & 0.4618135790963775 & 0.6927101827293680\end{array}$ $\begin{array}{llll}0.4226918909534328 & 0.4715393177401409 & 0.1639950011400655\end{array}$ $\begin{array}{lllll}0.9201632922712100 & 0.4702353869919862 & 0.1782005166108640\end{array}$ $\begin{array}{llll}0.3108643649308884 & 0.4387845386988300 & 0.9307440273229753\end{array}$ $\begin{array}{lllll}0.8101440276414341 & 0.4379199196560589 & 0.9316325861128943\end{array}$ $\begin{array}{lllll}0.2884422119829174 & 0.0905314516162754 & 0.0564968733268746\end{array}$ $\begin{array}{llll}0.7944193837709003 & 0.0915023131692750 & 0.0557323245503675\end{array}$ $\begin{array}{lllll}0.3265101277235468 & 0.4836011292829084 & 0.5660963921980845\end{array}$ $\begin{array}{llll}0.8302870421241030 & 0.4817536571560533 & 0.5666474423601549\end{array}$ $\begin{array}{lllll}0.2708826912840885 & 0.0478246873255099 & 0.4209391826171063\end{array}$ $\begin{array}{lllll}0.7675867447460105 & 0.0503191654277291 & 0.4163436601600717\end{array}$ $\begin{array}{llll}0.4259486295624108 & 0.4175022648411779 & 0.4776094478848919\end{array}$ $\begin{array}{lllll}0.9242350994995360 & 0.4144680422560624 & 0.4767398336843392\end{array}$ $\begin{array}{lllll}0.6740955852003155 & 0.1141829169947571 & 0.5096883927951382\end{array}$ $\begin{array}{lllll}0.1742116648212315 & 0.1134330600560936 & 0.5096907196122564\end{array}$ $\begin{array}{lllll}0.5907147001931519 & 0.6837596186297696 & 0.3355633626314781\end{array}$ $\begin{array}{llll}0.7730762137500106 & 0.6806253503066565 & 0.1895641661699543\end{array}$ $\begin{array}{llll}0.6848479267147564 & 0.6552705919199345 & 0.2560010282776347\end{array}$ $\begin{array}{lllll}0.6825994024919164 & 0.5967878773510652 & 0.2497312197203458\end{array}$ $\begin{array}{lllll}0.8647036107243867 & 0.5976006012520695 & 0.1077817455599107\end{array}$ $\begin{array}{llll}0.8591584903962892 & 0.6532309314922966 & 0.1184614406365295\end{array}$ $\begin{array}{lllll}0.7714491098167297 & 0.5724794791756325 & 0.1723996382926918\end{array}$ $\begin{array}{lllll}0.6074004861309684 & 0.5695752689053111 & 0.3083798618962301\end{array}$ $\begin{array}{llllll}0.9451334897045804 & 0.5741153252148691 & 0.0467760751382018\end{array}$ $\begin{array}{lllll}0.0928460428140078 & 0.3507469263144144 & 0.3650619898053557\end{array}$ $\begin{array}{lllll}0.0596109328350879 & 0.2417843794825026 & 0.3807173789936165\end{array}$ $\begin{array}{lllll}0.3107226742206168 & 0.2821416978704432 & 0.3827655548254038\end{array}$ $\begin{array}{lllll}0.3994505422489616 & 0.2066823613681257 & 0.1383261666702402\end{array}$ $\begin{array}{lllll}0.0712723221294768 & 0.2882644691386449 & 0.1298418430242736\end{array}$ $\begin{array}{lllll}0.1515037231199184 & 0.1861425621257783 & 0.1723212655854326\end{array}$ $\begin{array}{llllll}0.3588138498092741 & 0.1677318926964374 & 0.3881158452077835\end{array}$ $\begin{array}{lllll}0.3583215272422522 & 0.0545151747676099 & 0.3987727856189450\end{array}$ $\begin{array}{lllll}0.0918487803788859 & 0.1093093390274016 & 0.4247836080508320\end{array}$ $\begin{array}{llll}0.3133332277293730 & 0.1044492146249962 & 0.1586623726589615\end{array}$ $\begin{array}{lllll}0.2951408729554173 & 0.1148110728537498 & 0.6189431085506341\end{array}$ $\begin{array}{llllll}0.0362534621913233 & 0.0681610589253346 & 0.6603536080883750\end{array}$ $\begin{array}{llllll}0.2687660161463427 & 0.0659046669588831 & 0.8758607567736243\end{array}$ $\begin{array}{llll}0.2088112978825561 & 0.1672053110109009 & 0.8379656336445388\end{array}$ $\begin{array}{llll}0.0404775461294385 & 0.2887365857652385 & 0.6070137452042494\end{array}$ $\begin{array}{lllll}0.2894439601228483 & 0.2471587827138993 & 0.6044505257697791\end{array}$ $\begin{array}{lllll}0.2008242100745382 & 0.3221061229756439 & 0.8492798812594119\end{array}$ $\begin{array}{lllll}0.0326727729139540 & 0.2417953153641513 & 0.8579390634135081\end{array}$ $\begin{array}{lllll}0.2407465158235082 & 0.3614588071422190 & 0.5990893916549912\end{array}$ $\begin{array}{llll}0.4578074484653491 & 0.1393286075538758 & 0.8706758061681434\end{array}$ $\begin{array}{lllll}0.5067264547908777 & 0.1784839048822487 & 0.6221692689495071\end{array}$ $\begin{array}{llll}0.4478390277408859 & 0.3429387415154285 & 0.8157193640747888\end{array}$ $\begin{array}{llll}0.5921444406837412 & 0.3490153697219880 & 0.3675634680455827\end{array}$ $\begin{array}{lllll}0.5598011723342724 & 0.2400438935178049 & 0.3816753936583492\end{array}$ $\begin{array}{llll}0.8103503973395798 & 0.2823107614641515 & 0.3840505260757470\end{array}$ $\begin{array}{lllll}0.8991034459332076 & 0.2091175751914063 & 0.1373771427854606\end{array}$ $\begin{array}{lllll}0.5673942180783702 & 0.2867069742717210 & 0.1304245583292841\end{array}$ $\begin{array}{lllll}0.6527109447609625 & 0.1852602357381546 & 0.1720612745475960\end{array}$ $\begin{array}{lllll}0.8612225260433995 & 0.1686555976897192 & 0.3870296239422043\end{array}$ $\begin{array}{llll}0.8568849832858578 & 0.0556137687992456 & 0.3977424973016446\end{array}$ $\begin{array}{lllll}0.5919154389203007 & 0.1092240128854970 & 0.4246046837412090\end{array}$ $\begin{array}{lllll}0.8209766930633804 & 0.1056828895945814 & 0.1573044936922819\end{array}$ $\begin{array}{llll}0.7950465251539404 & 0.1165445228593897 & 0.6178721590247440\end{array}$ $\begin{array}{lllll}0.5383538726832633 & 0.0675933181129053 & 0.6602875925547843\end{array}$ $\begin{array}{llll}0.7717902138917359 & 0.0661604332873885 & 0.8743075049579843\end{array}$ $\begin{array}{lllll}0.7079019176874147 & 0.1672180999060493 & 0.8383450534241265\end{array}$ 
$\begin{array}{llll}0.5405750337036029 & 0.2873298029763748 & 0.6079636528706213\end{array}$ $\begin{array}{llllll}0.7901716349964538 & 0.2463594927177131 & 0.6055880768650542\end{array}$ $\begin{array}{lllll}0.7007302928528636 & 0.3203609363880320 & 0.8513227389833675\end{array}$ $\begin{array}{llll}0.5294102391191038 & 0.2409370210066749 & 0.8584134498113950\end{array}$ $\begin{array}{lllll}0.7389065721458150 & 0.3603908537243296 & 0.6021529132104227\end{array}$ $\begin{array}{lllll}0.9577392020549597 & 0.1407023446481004 & 0.8700083235595453\end{array}$ $\begin{array}{lllll}0.0087500049181116 & 0.1792349628601156 & 0.6215682678715529\end{array}$ $\begin{array}{lllll}0.9474329248177540 & 0.3432592977854573 & 0.8171365076956665\end{array}$ $\begin{array}{llll}0.3019091129990599 & 0.4164924109434292 & 0.3668988209203421\end{array}$ $\begin{array}{lllll}0.0559323592831145 & 0.4622519687251189 & 0.3252277687671301\end{array}$ $\begin{array}{lllll}0.1441655622890357 & 0.3892906136818716 & 0.1149836041605610\end{array}$ $\begin{array}{lllll}0.3320981642528073 & 0.4626628570006616 & 0.1101860793509561\end{array}$ $\begin{array}{lllll}0.3916866048439171 & 0.3608706407524806 & 0.1526814838144045\end{array}$ $\begin{array}{llll}0.2402448355353387 & 0.4749680171927512 & 0.58849598774477350\end{array}$ $\begin{array}{llll}0.0080842378609751 & 0.4201594429195245 & 0.5603803400941944\end{array}$ $\begin{array}{lllll}0.2857241268540996 & 0.4246110308611187 & 0.8287707433189275\end{array}$ $\begin{array}{lllll}0.8063673451302741 & 0.4098039511542370 & 0.3703555073650405\end{array}$ $\begin{array}{llll}0.5646365636679332 & 0.4582204009978445 & 0.3226531765167706\end{array}$ $\begin{array}{llll}0.6429696436037117 & 0.3865648988498168 & 0.1158914182203355\end{array}$ $\begin{array}{lllll}0.8275309034801982 & 0.4634591561008421 & 0.1226463875390699\end{array}$ $\begin{array}{lllll}0.8934881656523223 & 0.3625047878600035 & 0.1450956754515965\end{array}$ $\begin{array}{lllll}0.7434080088286816 & 0.4732050399440746 & 0.5875577028080791\end{array}$ $\begin{array}{lllll}0.5080626581003495 & 0.4197124672030905 & 0.5617545302228805\end{array}$ $\begin{array}{lllll}0.7812418141545473 & 0.4237151020052852 & 0.8312805636496833\end{array}$ $\begin{array}{llllll}0.4253683203877947 & 0.2337260180677471 & 0.4972598234922523\end{array}$ $\begin{array}{lllll}0.1747550609857524 & 0.2959745163203981 & 0.4904271469682524\end{array}$ $\begin{array}{lllll}0.4424590766513389 & 0.1131837063140936 & 0.5283570459818369\end{array}$ $\begin{array}{lllll}0.9263960834661853 & 0.2343070727426690 & 0.4974549552749183\end{array}$ $\begin{array}{lllll}0.6743614029389964 & 0.2947427561044690 & 0.4925043015669327\end{array}$ $\begin{array}{lllll}0.9425752265103456 & 0.1140228241610708 & 0.5276254792810522\end{array}$ $\begin{array}{lllll}0.1553416772552888 & 0.4173503256401072 & 0.4580249771451748\end{array}$ $\begin{array}{llll}0.6571600825599758 & 0.4152819525921603 & 0.4594287968171823\end{array}$ $\begin{array}{lllll}0.0462807574725516 & 0.2320616186724614 & 0.2042101051234695\end{array}$ $\begin{array}{llll}0.3054202438106702 & 0.1676025782941765 & 0.2120690341986492\end{array}$ $\begin{array}{llll}0.2939933488429982 & 0.3616471664364007 & 0.7752728248109684\end{array}$ $\begin{array}{lllll}0.3087093751277372 & 0.1199807170749304 & 0.7941343624339454\end{array}$ $\begin{array}{lllll}0.0521899930529037 & 0.1810508847880092 & 0.7994953278841603\end{array}$ $\begin{array}{lllll}0.0543876274949032 & 0.2984787464670282 & 0.7836981004448349\end{array}$ $\begin{array}{lllll}0.0501907603305965 & 0.3492442207451681 & 0.1870107286679988\end{array}$ $\begin{array}{llll}0.5458529326780057 & 0.2302978685318622 & 0.2050934800429395\end{array}$ $\begin{array}{lllll}0.8080765258653345 & 0.1685874888473675 & 0.2109061251411585\end{array}$ $\begin{array}{lllll}0.7925880829779279 & 0.3606993517467691 & 0.7780105455242943\end{array}$ $\begin{array}{lllll}0.8092427405009002 & 0.1207664513081495 & 0.7932975485230442\end{array}$ $\begin{array}{lllll}0.5508340078992336 & 0.1803061448982670 & 0.8000169689440969\end{array}$ $\begin{array}{llll}0.5537262538677393 & 0.2974135717257823 & 0.7844428130987715\end{array}$ $\begin{array}{lllll}0.5485177087991099 & 0.3474116165603452 & 0.1900227842963884\end{array}$ $\begin{array}{llll}0.2927257610925669 & 0.4088246030165405 & 0.1928000277275677\end{array}$ $\begin{array}{llll}0.7898955889804846 & 0.4067563543432963 & 0.1947694451188625\end{array}$

\section{Ade/Ade :}

$\begin{array}{lllllll}\mathrm{H} 138 & \mathrm{C} 10 & \mathrm{~N} 10 & \mathrm{O} 240 & \mathrm{Al} 32 & \mathrm{Si}\end{array}$

1.00000000000000

$20.2970019999999991 \quad 0.0000000000000000 \quad-3.7471719999999999$

$0.0646860000000000 \quad 24.9946149999999996-0.5148360000000000$

$\begin{array}{llll}0.0000000000000000 & 0.0000000000000000 & 18.6939999999999991\end{array}$ $\begin{array}{lllllll}\mathrm{H} & \mathrm{C} & \mathrm{N} & \mathrm{O} & \mathrm{Al} & \mathrm{Si} & \\ 138 & 10 & 10 & 240 & 32\end{array}$ $\begin{array}{llllll}138 & 10 & 10 & 240 & 32 & 64\end{array}$

Direct

$0.7865422172971255 \quad 0.7397093386786925 \quad 0.5544808046784261$ $\begin{array}{llllll}0.6683780033713603 & 0.7773362258677137 & 0.5358908551840971\end{array}$ $\begin{array}{llll}0.8111838818340198 & 0.5463973409840841 & 0.5752092906484221\end{array}$ $\begin{array}{llll}0.5887167673640896 & 0.5384776413401259 & 0.5445097756765218\end{array}$ $\begin{array}{llll}0.5603275785296397 & 0.6054613622288633 & 0.5385587923022606\end{array}$ $\begin{array}{lllll}0.0931070134209767 & 0.0546517073319734 & 0.4145745638357354\end{array}$ $\begin{array}{lllll}0.3447291262249358 & 0.0542831251319699 & 0.4153687754129578\end{array}$ $\begin{array}{lllll}0.0513563100788952 & 0.1394508368740013 & 0.1880899586078834\end{array}$ $\begin{array}{lllll}0.1702924226108767 & 0.3070323851969073 & 0.1654648319905002\end{array}$ $\begin{array}{lllll}0.1302748585563872 & 0.2203413629624168 & 0.3396388578965994\end{array}$ $\begin{array}{lllll}0.2443164092098529 & 0.0388705989548683 & 0.3499587262449639\end{array}$ $\begin{array}{lllll}0.1714424775882567 & 0.0664579548903599 & 0.1524749841573682\end{array}$ $\begin{array}{llll}0.3016511597904221 & 0.1388214792112630 & 0.1875886876790148\end{array}$ $\begin{array}{llll}0.4208183919093020 & 0.3082449949885009 & 0.1674851711204514\end{array}$ $\begin{array}{llll}0.3805914021079730 & 0.2202108202777293 & 0.3398684933462185\end{array}$ 
$\begin{array}{llll}0.4943799277274441 & 0.0394757707540645 & 0.3504455014148269\end{array}$ $\begin{array}{lllll}0.4228827722163128 & 0.0667808217844855 & 0.1531087524678348\end{array}$ $\begin{array}{lllll}0.0574793585636242 & 0.4889132532748063 & 0.1560432369123054\end{array}$ $\begin{array}{llll}0.2492792071201149 & 0.3887126744143212 & 0.3178070091459125\end{array}$ $\begin{array}{lllll}0.1294625761688654 & 0.4613784968172471 & 0.3530107757349691\end{array}$ $\begin{array}{lllll}0.3071188688791498 & 0.4891074760467021 & 0.1570231630184893\end{array}$ $\begin{array}{lllll}0.9995811144188766 & 0.3885250645593903 & 0.3173466565891374\end{array}$ $\begin{array}{llllll}0.3784981698470042 & 0.4613299506297191 & 0.3525738807298822\end{array}$ $\begin{array}{lllll}0.2083175535860467 & 0.4724348034617444 & 0.0912444436031140\end{array}$ $\begin{array}{lllll}0.4551802931881300 & 0.4741705392355984 & 0.0903251194749035\end{array}$ $\begin{array}{lllll}0.1557281887028673 & 0.4380618374919447 & 0.4716576422297155\end{array}$ $\begin{array}{llll}0.4035259987972569 & 0.4378098429016903 & 0.4714911558359431\end{array}$ $\begin{array}{lllll}0.1450699861246892 & 0.0900147536181354 & 0.0338341036231779\end{array}$ $\begin{array}{lllll}0.3983771521489340 & 0.0901435219398203 & 0.0344964314513086\end{array}$ $\begin{array}{lllll}0.1649497235176749 & 0.4814917898015514 & 0.2899395576832816\end{array}$ $\begin{array}{lllll}0.4152117944713849 & 0.4803360391291596 & 0.2900061878008609\end{array}$ $\begin{array}{lllll}0.1369830729380277 & 0.0455512300592307 & 0.2160191647346792\end{array}$ $\begin{array}{lllll}0.3869916598475416 & 0.0466085214767820 & 0.2158522090201523\end{array}$ $\begin{array}{lllll}0.2120900661549950 & 0.4156908045579347 & 0.2435709514998467\end{array}$ $\begin{array}{llllll}0.4618371771341387 & 0.4155254284847181 & 0.2437686889693680\end{array}$ $\begin{array}{llll}0.3389124945898790 & 0.1122348690061645 & 0.2619292420110489\end{array}$ $\begin{array}{llllll}0.0885832059364197 & 0.1119822105394662 & 0.2617903594142048\end{array}$ $\begin{array}{lllll}0.0916225617487757 & 0.0552541030495775 & 0.9146322076564433\end{array}$ $\begin{array}{llll}0.3467671042497798 & 0.0533077094846379 & 0.9152612385088750\end{array}$ 0.05129626637550990 .14130584442189350 .6877814841951461 $\begin{array}{lllll}0.1712162821450073 & 0.3074866144627753 & 0.6664452184393668\end{array}$ $\begin{array}{lllll}0.1307571605979107 & 0.2212302338867788 & 0.8410268001121841\end{array}$ $\begin{array}{lllll}0.2449148844299421 & 0.0398191023781412 & 0.8518436094499311\end{array}$ $\begin{array}{lllll}0.1715085406088552 & 0.0664816575117434 & 0.6522727171227348\end{array}$ $0.3017988516404427 \quad 0.1392991965029506 \quad 0.6878501276615812$ $\begin{array}{lllll}0.4218451973832005 & 0.3101692914574057 & 0.6702312821204840\end{array}$ $\begin{array}{lllll}0.3798994585312200 & 0.2188331138812221 & 0.8374687627914552\end{array}$ $\begin{array}{lllll}0.4947020583981658 & 0.0400013751914081 & 0.8500760807247796\end{array}$ $\begin{array}{lllll}0.4229940979486915 & 0.0673870781437950 & 0.6531797281562136\end{array}$ $\begin{array}{llll}0.0582949064454813 & 0.4908603595501227 & 0.6587557711971134\end{array}$ $\begin{array}{lllll}0.2499697894909399 & 0.3899957122988766 & 0.8190160370723278\end{array}$ $\begin{array}{lllll}0.1306111765977567 & 0.4618178627000131 & 0.8535342963747250\end{array}$ $\begin{array}{lllll}0.3083116490424135 & 0.4896586078502548 & 0.6586977752474235\end{array}$ $\begin{array}{lllll}0.0006819288258242 & 0.3896520866016699 & 0.8183521133897800\end{array}$ $\begin{array}{lllll}0.3776823299094419 & 0.4611201621868634 & 0.8524833207037179\end{array}$ $\begin{array}{lllll}0.2092682286402031 & 0.4730885588165558 & 0.5907577755224903\end{array}$ $\begin{array}{llll}0.4533416670082493 & 0.4755032829552649 & 0.5945647887176179\end{array}$ $\begin{array}{lllll}0.1561966441781923 & 0.4380166172846561 & 0.9721250218065278\end{array}$ $\begin{array}{lllll}0.4021059204583194 & 0.4376130088723698 & 0.9715991329426410\end{array}$ $\begin{array}{lllll}0.1451994579438868 & 0.0899557686776472 & 0.5339691333673471\end{array}$ $\begin{array}{lllll}0.3977885499251118 & 0.0906227169548680 & 0.5346660091997816\end{array}$ $\begin{array}{lllll}0.1665113549840805 & 0.4816894351134874 & 0.7907460284737947\end{array}$ $\begin{array}{lllll}0.4204964683780190 & 0.4740483154273528 & 0.7936166902717627\end{array}$ $\begin{array}{lllll}0.1385392496170114 & 0.0445296779137469 & 0.7165302903136022\end{array}$ $\begin{array}{lllll}0.3868450851305996 & 0.0475533165101727 & 0.7158567428975744\end{array}$ $\begin{array}{lllll}0.2128049382337280 & 0.4163120510790630 & 0.7439385689850206\end{array}$ $\begin{array}{lllll}0.4601608267720737 & 0.4126892707468316 & 0.7418641278922529\end{array}$ $\begin{array}{lllll}0.3393065167327464 & 0.1128214593858216 & 0.7621006675940996\end{array}$ $\begin{array}{lllll}0.0877826902863269 & 0.1122106562411278 & 0.7602398770931827\end{array}$ $\begin{array}{lllll}0.5943549439949352 & 0.0556650645639354 & 0.4148101167496673\end{array}$ $\begin{array}{lllll}0.8416423696473752 & 0.0567769604649950 & 0.4135223960076886\end{array}$ $\begin{array}{lllll}0.5516046143450838 & 0.1392416569016680 & 0.1878147588746761\end{array}$ $\begin{array}{lllll}0.6702147672482730 & 0.3084821613798378 & 0.1661228643318858\end{array}$ $\begin{array}{lllll}0.6304149311060554 & 0.2210513906136238 & 0.3394143326393132\end{array}$ $\begin{array}{lllll}0.7433886450362847 & 0.0391874737140427 & 0.3479680995443631\end{array}$ $\begin{array}{llll}0.6722432499540235 & 0.0676184414867538 & 0.1528802946391440\end{array}$ $\begin{array}{llll}0.8013171534619014 & 0.1399141919526610 & 0.1877761962231235\end{array}$ $\begin{array}{lllll}0.9201023748107490 & 0.3076672785933439 & 0.1654085001299072\end{array}$ $\begin{array}{lllll}0.8807625466950818 & 0.2216806684803933 & 0.3407296343668665\end{array}$ $\begin{array}{lllll}0.9929845482640995 & 0.0398371330031647 & 0.3502683017202836\end{array}$ $\begin{array}{lllll}0.9213531216207298 & 0.0671966393669204 & 0.1524344217796535\end{array}$ $\begin{array}{llll}0.5562493125848024 & 0.4887282201199725 & 0.1548287582601402\end{array}$ $\begin{array}{lllll}0.7496905968226896 & 0.3906546727719457 & 0.3197364414104188\end{array}$ $\begin{array}{lllll}0.6281945884552278 & 0.4617736947477480 & 0.3524346839944246\end{array}$ $\begin{array}{lllll}0.8073765686240405 & 0.4895923243922486 & 0.1562965537440728\end{array}$ $\begin{array}{llll}0.4994214290358981 & 0.3901402306291222 & 0.3190133526150220\end{array}$ $\begin{array}{llll}0.8799564212965171 & 0.4622456639521358 & 0.3536006487678990\end{array}$ $\begin{array}{llll}0.7076402546713231 & 0.4740172205602137 & 0.0939781078048018\end{array}$ 
$\begin{array}{llll}0.9577699457287645 & 0.4737851173629256 & 0.0913313434906839\end{array}$ $\begin{array}{lllll}0.6437004638654317 & 0.4394193551454401 & 0.4701313808666601\end{array}$ $\begin{array}{lllll}0.9059385791065848 & 0.4387363443752583 & 0.4722774259416985\end{array}$ $\begin{array}{llll}0.6473322670847349 & 0.0911729990120330 & 0.0340853248835300\end{array}$ $\begin{array}{lllll}0.8957345272723647 & 0.0909944923467874 & 0.0336377884620690\end{array}$ $\begin{array}{llll}0.6638267040685795 & 0.4824335660941633 & 0.2894725765131574\end{array}$ $\begin{array}{lllll}0.9147823513595404 & 0.4825917096522234 & 0.2900964676405794\end{array}$ $\begin{array}{lllll}0.6353608140095461 & 0.0484041291063571 & 0.2151400416048799\end{array}$ $\begin{array}{lllll}0.8858864309292038 & 0.0469926896231919 & 0.2154867069076873\end{array}$ $\begin{array}{llll}0.7121901696857794 & 0.4162512857298675 & 0.2446378735281069\end{array}$ $\begin{array}{lllll}0.9623725692880805 & 0.4162996043675890 & 0.2439234438543004\end{array}$ $\begin{array}{lllll}0.8386572734124919 & 0.1130080684027368 & 0.2619772223828621\end{array}$ $\begin{array}{lllll}0.5892430274721440 & 0.1132134553319381 & 0.2625937821491273\end{array}$ $\begin{array}{lllll}0.7346676944198626 & 0.7372552837853712 & 0.7407764677700902\end{array}$ $\begin{array}{llll}0.6169468152205519 & 0.7756374137002655 & 0.7163401473044755\end{array}$ $\begin{array}{lllll}0.7569063170313378 & 0.5438196023260812 & 0.7678499407136115\end{array}$ $\begin{array}{llll}0.5334715574065866 & 0.5363759728447360 & 0.7171046900533188\end{array}$ $\begin{array}{lllll}0.5070501850510449 & 0.6040100049010619 & 0.7139571573890092\end{array}$ $\begin{array}{llll}0.5949473172742091 & 0.0558626777972058 & 0.9145063715127428\end{array}$ $\begin{array}{lllll}0.8426145303881195 & 0.0566809279383587 & 0.9137822176690337\end{array}$ $\begin{array}{lllll}0.5516332135265924 & 0.1394176150802056 & 0.6869435633004191\end{array}$ $\begin{array}{lllll}0.6741187762952703 & 0.3150428459657936 & 0.6758268723764147\end{array}$ $\begin{array}{lllll}0.6297499402048757 & 0.2211391871223005 & 0.8380769983243126\end{array}$ $\begin{array}{llll}0.7427420399419704 & 0.0413968682704223 & 0.8493296609760227\end{array}$ 0.67220648841680790 .06924600928249830 .6522506472170344 $\begin{array}{lllll}0.8007568530999469 & 0.1432891690717274 & 0.6895464656677009\end{array}$ $\begin{array}{lllll}0.9201817345736434 & 0.3094151776371886 & 0.6651850009179681\end{array}$ $\begin{array}{lllll}0.8804677967107218 & 0.2223579669310146 & 0.8392174953169194\end{array}$ $\begin{array}{lllll}0.9923321556479383 & 0.0401038621279497 & 0.8472370895122762\end{array}$ $\begin{array}{lllll}0.9195439213114663 & 0.0693677894501509 & 0.6510316969047951\end{array}$ $\begin{array}{llll}0.5577316336595918 & 0.4791493212079035 & 0.6403822377891301\end{array}$ $\begin{array}{lllll}0.7471535887303151 & 0.3919448447363919 & 0.8200117963639956\end{array}$ $\begin{array}{lllll}0.6310705387525570 & 0.4651127752105420 & 0.8530961295142976\end{array}$ $\begin{array}{lllll}0.7980531404489748 & 0.4870033776706874 & 0.6502080519889374\end{array}$ $\begin{array}{lllll}0.4988338426062699 & 0.3908826262828982 & 0.8192871285769737\end{array}$ $\begin{array}{lllll}0.8801356711813336 & 0.4636080532921255 & 0.8533876839789030\end{array}$ $\begin{array}{llllll}0.6812444698563942 & 0.4968811603011681 & 0.5658550228496400\end{array}$ $\begin{array}{lllll}0.9594592424688028 & 0.4750382748827827 & 0.5920887741282731\end{array}$ $\begin{array}{lllll}0.6563511889500316 & 0.4386346710108867 & 0.9739535732642073\end{array}$ $\begin{array}{lllll}0.9053559875047441 & 0.4391093159637928 & 0.9720508039576850\end{array}$ $\begin{array}{lllll}0.6469881885272478 & 0.0920430507591784 & 0.5341702853723244\end{array}$ $\begin{array}{lllll}0.8930437780080740 & 0.0924987747981287 & 0.5330769468798566\end{array}$ $\begin{array}{lllll}0.63774772237327398 & 0.5060628559460346 & 0.7812621618130718\end{array}$ $\begin{array}{lllll}0.9133328613013086 & 0.4853699871688941 & 0.7890953495774053\end{array}$ $\begin{array}{lllll}0.6348866161791402 & 0.0501839381210420 & 0.7142410862200997\end{array}$ $\begin{array}{lllll}0.8847696378076874 & 0.0488981619698737 & 0.7144100509123529\end{array}$ $\begin{array}{lllll}0.7135634071492069 & 0.4232652272478508 & 0.7487446668419523\end{array}$ $\begin{array}{lllll}0.9632344514655758 & 0.4172802967560828 & 0.7448060467199156\end{array}$ $\begin{array}{lllll}0.8382403938138878 & 0.1142323203095509 & 0.7616182484640164\end{array}$ $\begin{array}{llllll}0.5893098201289304 & 0.1140494352874620 & 0.7623230436974000\end{array}$ $\begin{array}{lllll}0.6808097107776155 & 0.7351858339727689 & 0.5427321526317661\end{array}$ $\begin{array}{llll}0.7731475221230006 & 0.5775367395637324 & 0.5695006101783987\end{array}$ $\begin{array}{lllll}0.7436166041309211 & 0.6625566620029971 & 0.5589975800145671\end{array}$ $\begin{array}{llll}0.6764118810070958 & 0.6505468493525760 & 0.5517817070499135\end{array}$ $\begin{array}{llll}0.6602989005687155 & 0.5958985343723628 & 0.5544705606793048\end{array}$ $\begin{array}{llll}0.6286117836940988 & 0.7334123477084729 & 0.7236889672991312\end{array}$ $\begin{array}{lllll}0.7198311008548955 & 0.5755773500666572 & 0.7582411083722584\end{array}$ $\begin{array}{lllll}0.6905412632360299 & 0.6604494428249433 & 0.7428531962851606\end{array}$ $\begin{array}{lllll}0.6230299707940555 & 0.6489184402333118 & 0.7334587675383339\end{array}$ $\begin{array}{llll}0.6061005364104602 & 0.5944468106190816 & 0.7366125472855016\end{array}$ $\begin{array}{lllll}0.7941555725803709 & 0.6278129799889928 & 0.5678897776472980\end{array}$ $\begin{array}{llll}0.7453614541143364 & 0.7174512499420699 & 0.5534909164511687\end{array}$ $\begin{array}{lll}0.6378599184988565 & 0.6961960075761814 & 0.5415729118829966\end{array}$ $\begin{array}{lllll}0.7105751638966588 & 0.5600125706032764 & 0.5637603976077969\end{array}$ $\begin{array}{llll}0.5977040959680352 & 0.5785793918057003 & 0.5482800477567223\end{array}$ $\begin{array}{llll}0.7410950447861275 & 0.6255993611408608 & 0.7547039687294089\end{array}$ $\begin{array}{llll}0.6929401901927406 & 0.7151748286903591 & 0.7362713965054575\end{array}$ $\begin{array}{lllll}0.5850628393891905 & 0.6947771582785884 & 0.7214367768438226\end{array}$ $\begin{array}{lllll}0.6567102631599127 & 0.5589377681108926 & 0.7506004679961574\end{array}$ $\begin{array}{lllll}0.5436132236010902 & 0.5766846244357834 & 0.7270571865933372\end{array}$ $\begin{array}{llllll}0.0470618637183883 & 0.3494700837491934 & 0.1889966613446482\end{array}$ $\begin{array}{lllll}0.0302403977935185 & 0.2404975710550582 & 0.1963514638637629\end{array}$ $\begin{array}{lllll}0.1556653615769215 & 0.2813276093122707 & 0.1973780609776335\end{array}$ 
$\begin{array}{llll}0.2004532267344908 & 0.2065097359482314 & 0.0748842986154511\end{array}$ $\begin{array}{lllll}0.0343964983524987 & 0.2874874072586023 & 0.0707402792247119\end{array}$ $\begin{array}{lllll}0.0768440860732811 & 0.1859783639033446 & 0.0914830722942660\end{array}$ $\begin{array}{lllll}0.1803367929607858 & 0.1670778620715220 & 0.1997706802990464\end{array}$ $\begin{array}{lllll}0.1801431128437976 & 0.0542177493387251 & 0.2049158000585612\end{array}$ $\begin{array}{lllll}0.0469404477782300 & 0.1096150505121146 & 0.2197914358231684\end{array}$ $\begin{array}{lllll}0.1579786566096727 & 0.1041476486667405 & 0.0846238806368143\end{array}$ $\begin{array}{lllll}0.1492988019322773 & 0.1134355228743224 & 0.3160440860963594\end{array}$ $\begin{array}{llll}0.0208324710495049 & 0.0673491980910240 & 0.3359771163110257\end{array}$ $\begin{array}{llll}0.1373015039540813 & 0.0646879898337930 & 0.4430949321247195\end{array}$ $\begin{array}{lllll}0.1046268399792307 & 0.1664098987283244 & 0.4248049101000052\end{array}$ $\begin{array}{lllll}0.0206799129079017 & 0.2880697670218157 & 0.3095016187892466\end{array}$ $\begin{array}{lllll}0.1452166200087247 & 0.2462646601273444 & 0.3082552817392660\end{array}$ $\begin{array}{lllll}0.1006008002480114 & 0.3212270489583581 & 0.4309946496597092\end{array}$ $\begin{array}{lllll}0.0166783572315357 & 0.2409716044064922 & 0.4350237932023201\end{array}$ $\begin{array}{lllll}0.1207540693363449 & 0.3606082347233096 & 0.3060207819764538\end{array}$ $\begin{array}{lllll}0.2293320647961503 & 0.1396961657957156 & 0.4422485251529226\end{array}$ $\begin{array}{lllll}0.2540452048715591 & 0.1782407233409640 & 0.3168243365702613\end{array}$ $\begin{array}{lllll}0.2240781026865182 & 0.3420168371478133 & 0.4142057847582263\end{array}$ $\begin{array}{lllll}0.2963965408482861 & 0.3489297573939779 & 0.1890149488051981\end{array}$ $\begin{array}{lllll}0.2804137106498212 & 0.2397721781210312 & 0.1964685973736190\end{array}$ $\begin{array}{lllll}0.4055630547666242 & 0.2816154729217761 & 0.1976555500251793\end{array}$ $\begin{array}{llllll}0.4503071448087945 & 0.2076229392371311 & 0.0750905599817926\end{array}$ $\begin{array}{lllll}0.2845395356802276 & 0.2865840034910240 & 0.0708940243444250\end{array}$ $\begin{array}{lllll}0.3271368907949335 & 0.1850579186372072 & 0.0919429730776443\end{array}$ $\begin{array}{lllll}0.4309812487668565 & 0.1675451365030405 & 0.2000397852490371\end{array}$ $\begin{array}{llll}0.4306251958664756 & 0.0547063896479795 & 0.2055853203181444\end{array}$ $\begin{array}{lllll}0.2974451004675779 & 0.1092623109469662 & 0.2198264455817752\end{array}$ $\begin{array}{lllll}0.4103570877527056 & 0.1045174513974622 & 0.0851545062600859\end{array}$ $\begin{array}{llll}0.3996751298234142 & 0.1142260105202706 & 0.3163282295107107\end{array}$ $\begin{array}{lllll}0.2716177030771908 & 0.0669194170045451 & 0.3360201481207283\end{array}$ $\begin{array}{llllll}0.3888825253751056 & 0.0647516801109000 & 0.4433922560026816\end{array}$ $\begin{array}{lllll}0.3545155498392676 & 0.1661890155255252 & 0.4253880771725159\end{array}$ $\begin{array}{lllll}0.2705089768519797 & 0.2873217171112834 & 0.3094594115636071\end{array}$ $\begin{array}{lllll}0.3952905277836029 & 0.2462376461498904 & 0.3084629457492011\end{array}$ $\begin{array}{lllll}0.3506052465180835 & 0.3204608475146382 & 0.4310015427529476\end{array}$ $\begin{array}{lllll}0.2661197091068708 & 0.2403964115695040 & 0.4348737249520762\end{array}$ $\begin{array}{lllll}0.3700035921390781 & 0.3604269417971954 & 0.3061497368733295\end{array}$ $\begin{array}{lllll}0.4795467696950984 & 0.1406539725662711 & 0.4426097549644806\end{array}$ $\begin{array}{lllll}0.0040343358951625 & 0.1789530917970872 & 0.3167555176372451\end{array}$ $\begin{array}{lllll}0.4739046796852039 & 0.3429151769458814 & 0.4139745461092850\end{array}$ $\begin{array}{lllll}0.1518233212912451 & 0.4140300743172489 & 0.1895243487317215\end{array}$ $\begin{array}{lllll}0.0299119313673171 & 0.4609731538783750 & 0.1697730881004672\end{array}$ $\begin{array}{lllll}0.0714267884534464 & 0.3880325868463179 & 0.0634627332898508\end{array}$ $\begin{array}{lllll}0.1640009247776290 & 0.4626808516878180 & 0.0626070700844153\end{array}$ $\begin{array}{lllll}0.1960343298378056 & 0.3608304759337804 & 0.0807482450152719\end{array}$ $\begin{array}{lllll}0.1214079750443903 & 0.4734944509792131 & 0.3005261033023847\end{array}$ $\begin{array}{lllll}0.0040716473642408 & 0.4185539783548494 & 0.2859975504015609\end{array}$ $\begin{array}{lllll}0.1427618436250843 & 0.4237451667105037 & 0.4209819517855808\end{array}$ $\begin{array}{lllll}0.4014620773758444 & 0.4130849070195526 & 0.1895811080719366\end{array}$ $\begin{array}{lllll}0.2798114476912718 & 0.4605037763723258 & 0.1694869882943329\end{array}$ $\begin{array}{lllll}0.3210902698524265 & 0.3871895917336868 & 0.0634373796606107\end{array}$ $\begin{array}{lllll}0.4108803278665749 & 0.4636730284901686 & 0.0627933976189995\end{array}$ $\begin{array}{lllll}0.4462467890778041 & 0.3622755359596720 & 0.0794832953161235\end{array}$ $\begin{array}{llll}0.3712368459204445 & 0.4732175976802627 & 0.3001148135114284\end{array}$ $\begin{array}{lllll}0.2536403162606022 & 0.4184128025120488 & 0.2858618961384867\end{array}$ $\begin{array}{llll}0.3910406120738815 & 0.4235434138426237 & 0.4209893401539790\end{array}$ $\begin{array}{lllll}0.0481938856005607 & 0.3501850256805318 & 0.6890685243310475\end{array}$ $\begin{array}{lllll}0.0302510323829957 & 0.2413012746222360 & 0.6959026325548393\end{array}$ $\begin{array}{lllll}0.1560313223285794 & 0.2813686177445839 & 0.6974099697284212\end{array}$ $0.2006415467218106 \quad 0.2062321683827254 \quad 0.5747798480057494$ $\begin{array}{lllll}0.0353259498129322 & 0.2883406059019188 & 0.5706173988532138\end{array}$ $\begin{array}{lllll}0.0770081329394565 & 0.1864567233945716 & 0.5915694259808076\end{array}$ $\begin{array}{lllll}0.1803901896123362 & 0.1670603948811888 & 0.6998093427335745\end{array}$ $\begin{array}{lllll}0.1811295380533445 & 0.0539675535337665 & 0.7046061982535877\end{array}$ $\begin{array}{lllll}0.0462311756680758 & 0.1110056733168622 & 0.7184935912279813\end{array}$ $\begin{array}{llll}0.1575454711586880 & 0.1041309545772690 & 0.5847684065247705\end{array}$ $\begin{array}{lllll}0.1493321655172310 & 0.1125015711775661 & 0.8154261993763418\end{array}$ $\begin{array}{lllll}0.0200465255615697 & 0.0682076248795334 & 0.8341966207861199\end{array}$ $\begin{array}{lllll}0.1358582087225214 & 0.0652108928887373 & 0.9429734012278477\end{array}$ $\begin{array}{lllll}0.1043948883397763 & 0.1671457712602621 & 0.9229146689603880\end{array}$ $\begin{array}{lllll}0.0207874784226362 & 0.2889602985973475 & 0.8089613731368543\end{array}$ $\begin{array}{llll}0.1451497239750727 & 0.2463546249320125 & 0.8081950631465142\end{array}$ 
$\begin{array}{llll}0.1008458865024463 & 0.3215642055197055 & 0.9307675324359311\end{array}$ $\begin{array}{lllll}0.0165551263714282 & 0.2416350219766933 & 0.9342555217429892\end{array}$ $\begin{array}{lllll}0.1217965999001946 & 0.3609417863450775 & 0.8061051804352478\end{array}$ $\begin{array}{llll}0.2286541907347996 & 0.1392355948259541 & 0.9419390770412290\end{array}$ $\begin{array}{llll}0.2540594971060648 & 0.1780268055125629 & 0.8169413082757405\end{array}$ $\begin{array}{lllll}0.2246694398270332 & 0.3417491993139727 & 0.9146154168333955\end{array}$ $\begin{array}{lllll}0.2958644682287778 & 0.3488935327222352 & 0.6889929780868410\end{array}$ $\begin{array}{lllll}0.2805794790153838 & 0.2395972068818330 & 0.6964508076421416\end{array}$ $\begin{array}{llll}0.4057233460736911 & 0.2822101343759896 & 0.6977156421647311\end{array}$ $\begin{array}{llll}0.4503371194041055 & 0.2084312533180823 & 0.5751323231629190\end{array}$ $\begin{array}{lllll}0.2841494951842962 & 0.2866069001480780 & 0.5708029011658661\end{array}$ $\begin{array}{lllll}0.3273427912157953 & 0.1852816749646139 & 0.5917062307431042\end{array}$ $\begin{array}{lllll}0.4314188244448749 & 0.1682258327968660 & 0.6999787036435831\end{array}$ $\begin{array}{llll}0.4306020757593729 & 0.0553407571341253 & 0.7056755719277816\end{array}$ $\begin{array}{lllll}0.2978806039201186 & 0.1095749879107067 & 0.7199174117793472\end{array}$ $\begin{array}{lllll}0.4108450897783568 & 0.1051012228798069 & 0.5851712611494734\end{array}$ $\begin{array}{llll}0.3999217273766250 & 0.1152467222724884 & 0.8163180212124320\end{array}$ $\begin{array}{lllll}0.2724069916365719 & 0.0668972101707189 & 0.8359437389042639\end{array}$ $\begin{array}{lllll}0.3907468214888696 & 0.0642882688010287 & 0.9429957116552666\end{array}$ $\begin{array}{lllll}0.3541087863580724 & 0.1653621212886316 & 0.9262851838281175\end{array}$ $\begin{array}{lllll}0.2706166998701641 & 0.2868629566275746 & 0.8096201600597818\end{array}$ $\begin{array}{llllll}0.3953408689686398 & 0.2459695336780467 & 0.8082099404136615\end{array}$ $\begin{array}{lllll}0.3510593514283118 & 0.3194092565660624 & 0.9311595333164077\end{array}$ $\begin{array}{llll}0.2658640801257643 & 0.2398924934028381 & 0.9350021672571130\end{array}$ $\begin{array}{lllll}0.3697858646686271 & 0.3598857217402327 & 0.8062998887415604\end{array}$ $\begin{array}{lllll}0.4796010165821845 & 0.1414474391009247 & 0.9425988641777374\end{array}$ $\begin{array}{lllll}0.0032892873718845 & 0.1798872234413785 & 0.8156456595639658\end{array}$ $\begin{array}{lllll}0.4739158966360650 & 0.3435111657969735 & 0.9144047211767512\end{array}$ $\begin{array}{llll}0.1528481343030458 & 0.4147473528752436 & 0.6897734400103913\end{array}$ $\begin{array}{lllll}0.0311692692808345 & 0.4618411305270486 & 0.6703797771400603\end{array}$ $\begin{array}{lllll}0.0720601265248955 & 0.3890973357919418 & 0.5635467287526756\end{array}$ $\begin{array}{lllll}0.1648235064347720 & 0.4634072379319587 & 0.5625477552467801\end{array}$ $\begin{array}{lllll}0.1960280360791253 & 0.3613382057370113 & 0.5804495338857928\end{array}$ $\begin{array}{llll}0.1228011393431236 & 0.4739941029174808 & 0.8010976064640433\end{array}$ $\begin{array}{lllll}0.0052967442793584 & 0.4193850855624963 & 0.7865894685451037\end{array}$ $\begin{array}{lllll}0.1439217093310617 & 0.4238433115912636 & 0.9212740724334152\end{array}$ $\begin{array}{lllll}0.4011797162609062 & 0.4113188534487061 & 0.6888107624496569\end{array}$ $\begin{array}{lllll}0.2809292720310722 & 0.4606547172466852 & 0.6698299881222834\end{array}$ $\begin{array}{llll}0.3207833126570619 & 0.3869548645795349 & 0.5627041908705148\end{array}$ $\begin{array}{llll}0.4103387666657008 & 0.4643589534003096 & 0.5644097703732559\end{array}$ $\begin{array}{lllll}0.4466851781385037 & 0.3628293834696867 & 0.5784789077642591\end{array}$ $\begin{array}{lllll}0.3743957554325886 & 0.4720232546949028 & 0.7999703825416566\end{array}$ $\begin{array}{llll}0.2544147416751622 & 0.4191945021829152 & 0.7863084716460007\end{array}$ $\begin{array}{lllll}0.3890426335484046 & 0.4229993345531585 & 0.9211795134740092\end{array}$ $\begin{array}{lllll}0.5458630943491897 & 0.3498653047055478 & 0.1889362179848820\end{array}$ $\begin{array}{lllll}0.5303983205532870 & 0.2407069018786284 & 0.1965514802397321\end{array}$ $\begin{array}{llllll}0.6554625456718204 & 0.2823976211524539 & 0.1974376225130808\end{array}$ $\begin{array}{lllll}0.7002673652998869 & 0.2083374536662281 & 0.0751021669116317\end{array}$ $\begin{array}{lllll}0.5345812375182166 & 0.2877702168782584 & 0.0710292983641199\end{array}$ $\begin{array}{llllll}0.5769115306740517 & 0.1862071331431284 & 0.0916785244116047\end{array}$ $\begin{array}{lllll}0.6805541574388346 & 0.1683482100256558 & 0.1998848936002398\end{array}$ $\begin{array}{llll}0.6794193565971537 & 0.0556128362982456 & 0.2053679909178921\end{array}$ $\begin{array}{lllll}0.5477550089095896 & 0.1097490771503205 & 0.2202773956894671\end{array}$ $\begin{array}{lllll}0.6597319119147558 & 0.1054048823448754 & 0.0848203190635655\end{array}$ $\begin{array}{lllll}0.6493883102507038 & 0.1154123998756410 & 0.3164909936176488\end{array}$ $\begin{array}{lllll}0.5218693693877482 & 0.0674867498400210 & 0.3367396207598732\end{array}$ $\begin{array}{llll}0.6386310653852975 & 0.0656947584715226 & 0.4432413946236915\end{array}$ $\begin{array}{lllll}0.6048165381989753 & 0.1671662928247184 & 0.4260355753586521\end{array}$ $\begin{array}{lllll}0.5204579839654461 & 0.2879936468223029 & 0.3096120361417991\end{array}$ $\begin{array}{llll}0.6453105111338130 & 0.2472543957850766 & 0.3084033494246959\end{array}$ $\begin{array}{lllll}0.6005247693504125 & 0.3213662084274194 & 0.4311395671280899\end{array}$ $\begin{array}{llll}0.5161451153332715 & 0.2413291289674412 & 0.4352120552421935\end{array}$ $\begin{array}{lllll}0.6196414142813390 & 0.3610267346159375 & 0.3057559428356720\end{array}$ $\begin{array}{lllll}0.7298930296883954 & 0.1414575122623444 & 0.4425297194296160\end{array}$ $\begin{array}{llll}0.7542046961190108 & 0.1796739845014456 & 0.3169658778854774\end{array}$ $\begin{array}{lllll}0.7234913067876969 & 0.3441127954831941 & 0.4133070311623639\end{array}$ $\begin{array}{llll}0.7969972411618500 & 0.3502964853231055 & 0.1894034466033629\end{array}$ $\begin{array}{lllll}0.7804308598473922 & 0.2411751815351202 & 0.1966297280133056\end{array}$ $\begin{array}{lllll}0.9056233041082518 & 0.2821245760019948 & 0.1976303040866996\end{array}$ $\begin{array}{lllll}0.9501879949158686 & 0.2075149626284557 & 0.0748337568279399\end{array}$ $\begin{array}{lllll}0.7850442936053613 & 0.2881342439649222 & 0.0712675806130752\end{array}$ $\begin{array}{lllll}0.8267357058895032 & 0.1864035537625846 & 0.0917803753977912\end{array}$ $\begin{array}{llll}0.9303223980143340 & 0.1678147652407169 & 0.1997585731763863\end{array}$ 
$\begin{array}{llll}0.9294128562349054 & 0.0550520104253453 & 0.2048978625763185\end{array}$ $\begin{array}{lllll}0.7970319915378319 & 0.1102803042177295 & 0.2198623441192341\end{array}$ $\begin{array}{llll}0.9084291204582711 & 0.1049649009926900 & 0.0845066867468141\end{array}$ $\begin{array}{lllll}0.8990538531789619 & 0.1146085589576054 & 0.3160966292253385\end{array}$ $\begin{array}{llll}0.7708660490796431 & 0.0678976197643320 & 0.3361359239452795\end{array}$ $\begin{array}{llll}0.8859094696001160 & 0.0662955996260600 & 0.4427732937004252\end{array}$ $\begin{array}{lllll}0.8548669465346304 & 0.1682684282843268 & 0.4246981224846578\end{array}$ $\begin{array}{lllll}0.7705967357807676 & 0.2887667960195421 & 0.3095697991037814\end{array}$ $\begin{array}{lllll}0.8953790920227815 & 0.2472054069398677 & 0.3085747441548247\end{array}$ $\begin{array}{lllll}0.8501923386823288 & 0.3221844314438259 & 0.4312684899299993\end{array}$ $\begin{array}{lllll}0.7659000934652344 & 0.2422641240108704 & 0.4350300608962941\end{array}$ $\begin{array}{lllll}0.8707029861321050 & 0.3615134479869613 & 0.3064899279417501\end{array}$ $\begin{array}{lllll}0.9793238751408937 & 0.1404944497251151 & 0.4422472238116931\end{array}$ $\begin{array}{lllll}0.5046118177866358 & 0.1788940359763897 & 0.3172294856593029\end{array}$ $\begin{array}{lllll}0.9739490562474975 & 0.3423903735828734 & 0.4145596996736126\end{array}$ $\begin{array}{lllll}0.6514994147944755 & 0.4139775337328063 & 0.1902962845265846\end{array}$ $\begin{array}{lllll}0.5288930811308031 & 0.4609253620563822 & 0.1692909834312439\end{array}$ $\begin{array}{lllll}0.5717144640674034 & 0.3883685135396953 & 0.0637904935960493\end{array}$ $\begin{array}{lllll}0.6634838691677407 & 0.4642500444410099 & 0.0649152017573834\end{array}$ $\begin{array}{lllll}0.6964879802288906 & 0.3622533862496270 & 0.0809758562473294\end{array}$ $\begin{array}{lllll}0.6202950694488248 & 0.4742864537088535 & 0.2999697093980814\end{array}$ $\begin{array}{lllll}0.5031592301649062 & 0.4193459825742396 & 0.2860664480052120\end{array}$ $\begin{array}{llll}0.6394604200618582 & 0.4245730556737800 & 0.4194139917658130\end{array}$ $\begin{array}{lllll}0.9017707886502605 & 0.4149827326422763 & 0.1900274267387868\end{array}$ $\begin{array}{lllll}0.7797974652914061 & 0.4618404973430307 & 0.1704774632572141\end{array}$ $\begin{array}{lllll}0.8216110743978285 & 0.38877779082235518 & 0.0639325126946068\end{array}$ $\begin{array}{lllll}0.9134906007598808 & 0.4638014418107682 & 0.0630135415439729\end{array}$ $\begin{array}{lllll}0.9462607578520700 & 0.3619555944011780 & 0.0811539629863188\end{array}$ $\begin{array}{lllll}0.8714690036233068 & 0.4743635886866627 & 0.3011415250243085\end{array}$ $\begin{array}{lllll}0.7538062594869164 & 0.4196215123944323 & 0.2865511967780518\end{array}$ $\begin{array}{llll}0.8930974761665319 & 0.4244922785322900 & 0.4216285558184766\end{array}$ $\begin{array}{lllll}0.5458533418556749 & 0.3514477658084061 & 0.6895595343555624\end{array}$ $\begin{array}{lllll}0.5308614308414801 & 0.2417997775820774 & 0.6965916412768532\end{array}$ $\begin{array}{lllll}0.6560809019624562 & 0.2851686054849221 & 0.6981843351108424\end{array}$ $\begin{array}{llll}0.6994800770785609 & 0.2097665317879423 & 0.5741271289779297\end{array}$ $\begin{array}{llll}0.5348883907630301 & 0.2884571708464242 & 0.5711258130460041\end{array}$ $\begin{array}{llll}0.5765452006999835 & 0.1867600900045793 & 0.5914991144087184\end{array}$ $\begin{array}{lllll}0.6802255352795914 & 0.1699406048534448 & 0.6994503005141863\end{array}$ $\begin{array}{llll}0.6791360219705094 & 0.0569903253559982 & 0.7047124342184192\end{array}$ $\begin{array}{llll}0.5478976296476046 & 0.1102055907246034 & 0.7198995429038602\end{array}$ $\begin{array}{lllll}0.6600340141022545 & 0.1066378509721067 & 0.5846229866801386\end{array}$ $\begin{array}{llll}0.6492774666421824 & 0.1165023058405413 & 0.8162298125490899\end{array}$ $\begin{array}{lllll}0.5220420437913008 & 0.0681921519380997 & 0.8365616525303146\end{array}$ $\begin{array}{lllll}0.6392467230960869 & 0.0660242513591223 & 0.9426683417400489\end{array}$ $\begin{array}{lllll}0.6048509957010111 & 0.1675458506973153 & 0.9264828728328919\end{array}$ $\begin{array}{llll}0.5202518496246362 & 0.2889142616858864 & 0.8096226081149873\end{array}$ $\begin{array}{lllll}0.6450205177860169 & 0.2479006348627299 & 0.8081318813344941\end{array}$ $\begin{array}{lllll}0.6006928306721913 & 0.3219984798727754 & 0.9311172849123269\end{array}$ $\begin{array}{lllll}0.5163338321948290 & 0.2419795109912947 & 0.9350167154399969\end{array}$ $\begin{array}{llll}0.6198037261877818 & 0.3623016013452063 & 0.8067601253055622\end{array}$ $\begin{array}{llll}0.7299599891210294 & 0.1420324229685821 & 0.9421339410143604\end{array}$ $\begin{array}{lllll}0.7541874122693736 & 0.1806518460883847 & 0.8165407229815518\end{array}$ $\begin{array}{llll}0.7240174443262242 & 0.3438456548092627 & 0.9140010383402115\end{array}$ $\begin{array}{llll}0.7959891241207642 & 0.3510084521000388 & 0.6891814421662716\end{array}$ $\begin{array}{lllll}0.7800402740420459 & 0.2422369948100649 & 0.6956899262811357\end{array}$ $\begin{array}{lllll}0.9054596635490723 & 0.2834147707797813 & 0.6966472179057446\end{array}$ $\begin{array}{lllll}0.9506208209602865 & 0.2085457186059673 & 0.5739266876019288\end{array}$ $\begin{array}{lllll}0.7808294356792672 & 0.2915288816746878 & 0.5701133340801406\end{array}$ $\begin{array}{lllll}0.8265004745170623 & 0.1904567427229961 & 0.5893884221188208\end{array}$ $\begin{array}{llll}0.9291624461013419 & 0.1694517672462287 & 0.6986249263830465\end{array}$ $\begin{array}{llll}0.9282262502466756 & 0.0565810958979509 & 0.7033262070544288\end{array}$ $\begin{array}{lllll}0.7966610912323792 & 0.1121971822583846 & 0.7192497886242084\end{array}$ $\begin{array}{lllll}0.9055397950630936 & 0.1069215963881458 & 0.5837134075892031\end{array}$ $\begin{array}{lllll}0.8985723562080115 & 0.1154193495919041 & 0.8153159679726572\end{array}$ $\begin{array}{llll}0.7708021606948550 & 0.0688353986545921 & 0.8352322590282033\end{array}$ $\begin{array}{lllll}0.8869471905454962 & 0.0664616572418693 & 0.9422861939548395\end{array}$ $\begin{array}{llll}0.8548937119952205 & 0.1683524892967239 & 0.9245180003617575\end{array}$ $\begin{array}{lllll}0.7707333651663906 & 0.2894291812141467 & 0.8092586507895890\end{array}$ $\begin{array}{lllll}0.8952013759189529 & 0.2482500719891656 & 0.8076543955206213\end{array}$ $\begin{array}{lllll}0.8507801423816069 & 0.3225903908623732 & 0.9308297725401148\end{array}$ $\begin{array}{lllll}0.7666587774247110 & 0.2426287846764868 & 0.9348814803843160\end{array}$ $\begin{array}{llllll}0.8708391422490358 & 0.3621042324896271 & 0.8059851286280735\end{array}$ $\begin{array}{llll}0.9794315971123796 & 0.1411005971110849 & 0.9412767004837530\end{array}$ 
$\begin{array}{llll}0.5052267875910356 & 0.1795022626656216 & 0.8171164615602754\end{array}$ $\begin{array}{lllll}0.9742405007295161 & 0.3432343350280547 & 0.9140432971767513\end{array}$ $\begin{array}{lllll}0.6496430157947651 & 0.4181303516327485 & 0.6886939985363975\end{array}$ $\begin{array}{lllll}0.5283864744082146 & 0.4666346026204914 & 0.6717570945236671\end{array}$ $\begin{array}{lllll}0.5713900759785076 & 0.3893485838292216 & 0.5647652674052941\end{array}$ $\begin{array}{lllll}0.6454806101709386 & 0.4681966768132801 & 0.5545447449761352\end{array}$ $\begin{array}{lllll}0.6976364854631063 & 0.3702738396376856 & 0.5774045177640823\end{array}$ $\begin{array}{lllll}0.6151614495179748 & 0.4733675676172202 & 0.8010708583461313\end{array}$ $\begin{array}{lllll}0.5007361688140807 & 0.4192343842934818 & 0.7845857993741884\end{array}$ $\begin{array}{lllll}0.6428988973839559 & 0.4247008059683383 & 0.9233525712133890\end{array}$ $\begin{array}{lllll}0.9027892862240892 & 0.4155455079413026 & 0.6910674838200244\end{array}$ $\begin{array}{llll}0.7775056739100420 & 0.4581413855040351 & 0.6714058862204934\end{array}$ $\begin{array}{lllll}0.8237030773257515 & 0.3903694963803066 & 0.5639613679174497\end{array}$ $\begin{array}{llll}0.9150513481560045 & 0.4651146121280107 & 0.5640459454973482\end{array}$ 0.94742713999232990 .36307921768725090 .5815942365887733 $\begin{array}{lllll}0.8708233654901235 & 0.4756978527736049 & 0.8010177289861986\end{array}$ $\begin{array}{lllll}0.7549250182198434 & 0.4211916379634559 & 0.7888727856320737\end{array}$ $\begin{array}{lllll}0.8932997604449913 & 0.4250614386860728 & 0.9211706850149665\end{array}$ $\begin{array}{lllll}0.2132240929581466 & 0.2329417524531027 & 0.2543981062870848\end{array}$ $\begin{array}{lllll}0.0877659344486817 & 0.2948201966981629 & 0.2514085177680208\end{array}$ $\begin{array}{lllll}0.2227585758352078 & 0.1119358031115260 & 0.2699893565031994\end{array}$ $\begin{array}{lllll}0.4635289640261391 & 0.2334563425386095 & 0.2547121409891984\end{array}$ $\begin{array}{lllll}0.3374664204996272 & 0.2944078107577733 & 0.2514903595502126\end{array}$ $\begin{array}{llll}0.4731869203695440 & 0.1125638467344040 & 0.2704754228086600\end{array}$ $\begin{array}{llll}0.0784846065623490 & 0.4157764824731393 & 0.2357558108093928\end{array}$ $\begin{array}{lllll}0.3280820592306155 & 0.4152278601894097 & 0.2356260138643258\end{array}$ $\begin{array}{lllll}0.2132567645147779 & 0.2328152550660939 & 0.7545477988928648\end{array}$ $\begin{array}{lllll}0.0881921070015242 & 0.2952728796299416 & 0.7513627814594744\end{array}$ $\begin{array}{lllll}0.2230893564737851 & 0.1116902448670564 & 0.7699551118318610\end{array}$ $\begin{array}{lllll}0.4639477482807565 & 0.2341481598200798 & 0.7547085061483592\end{array}$ $\begin{array}{lllll}0.3374115217995826 & 0.2942653047415210 & 0.7515488504736971\end{array}$ $\begin{array}{lllll}0.4734382726962029 & 0.1132833921483055 & 0.7703510576296646\end{array}$ $\begin{array}{lllll}0.0794568421737265 & 0.4164026960613988 & 0.7361823711372986\end{array}$ $\begin{array}{lllll}0.3284980906545362 & 0.4149589128183486 & 0.7358237524045648\end{array}$ $\begin{array}{llllll}0.7133430165847717 & 0.2343367640005501 & 0.2545203426723387\end{array}$ $\begin{array}{lllll}0.5873939509691746 & 0.2954276284592937 & 0.2513954421347716\end{array}$ $\begin{array}{lllll}0.7225950893673716 & 0.1134573715304114 & 0.2701293141749813\end{array}$ $\begin{array}{llllll}0.9631814937631493 & 0.2337180314198697 & 0.2544777632200165\end{array}$ $\begin{array}{lllll}0.8377405949988576 & 0.2956388195911254 & 0.2517277451166408\end{array}$ $\begin{array}{lllll}0.9724093941869467 & 0.1126836555676381 & 0.2699359176934705\end{array}$ $\begin{array}{llll}0.5777717973684532 & 0.4161957830638522 & 0.2356334259219620\end{array}$ $\begin{array}{lllll}0.8284274427524301 & 0.4166088123385043 & 0.2363514533608662\end{array}$ $\begin{array}{lllll}0.7131509913095646 & 0.2356107365911667 & 0.7542676078433842\end{array}$ $\begin{array}{lllll}0.5874609099332658 & 0.2969775109536806 & 0.7519536418542404\end{array}$ $\begin{array}{lllll}0.7224100523638863 & 0.1146195341631693 & 0.7697109685687236\end{array}$ $\begin{array}{llll}0.9628539882962448 & 0.2349209612624261 & 0.7535216604407219\end{array}$ $\begin{array}{lllll}0.8374027063590652 & 0.2965286336320101 & 0.7506891147914281\end{array}$ $\begin{array}{lllll}0.9716246640476387 & 0.1138808582297277 & 0.7685753952901043\end{array}$ $\begin{array}{lllll}0.5783215577548279 & 0.4184744776189852 & 0.7376004441535915\end{array}$ $\begin{array}{llllll}0.8285973661073747 & 0.4169227631790544 & 0.7370927410604451\end{array}$ $\begin{array}{lllll}0.0234853874620238 & 0.2309274671378423 & 0.1079435293958690\end{array}$ $\begin{array}{llll}0.1536576474007480 & 0.1671139158741352 & 0.1116710689918357\end{array}$ $\begin{array}{lllll}0.1471980274789828 & 0.3607487518660026 & 0.3941746351949220\end{array}$ $\begin{array}{lllll}0.1552763528760788 & 0.1199039822338463 & 0.4034731473688803\end{array}$ $\begin{array}{lllll}0.0262626003416681 & 0.1801972543970747 & 0.4055822061000439\end{array}$ $\begin{array}{lllll}0.0273882650748055 & 0.2976349557170676 & 0.3979366937338304\end{array}$ $\begin{array}{lllll}0.0246421136976320 & 0.3482599669216705 & 0.1001905971686942\end{array}$ $\begin{array}{lllll}0.2736632736619333 & 0.2300301021602059 & 0.1080941103886695\end{array}$ $\begin{array}{lllll}0.4044637263067582 & 0.1674395634924205 & 0.1119013455996861\end{array}$ $\begin{array}{lllll}0.3966412743099736 & 0.3605115070126170 & 0.3942343365223818\end{array}$ $\begin{array}{llll}0.4057594656640940 & 0.1203097350481118 & 0.4037850454738834\end{array}$ $\begin{array}{lllll}0.2760863713759644 & 0.1795959623373335 & 0.4056760332590007\end{array}$ $\begin{array}{lllll}0.2772913522542250 & 0.2969805122162179 & 0.3978609776715278\end{array}$ $\begin{array}{lllll}0.2744826276551109 & 0.3473369266392070 & 0.1001392939158050\end{array}$ $\begin{array}{llll}0.1456118137922230 & 0.4075454958717733 & 0.1020641331281429\end{array}$ $\begin{array}{lllll}0.3948963596179576 & 0.4076964255094263 & 0.1019775867665922\end{array}$ $\begin{array}{llll}0.0238403403115890 & 0.2317527992100415 & 0.6075338039326423\end{array}$ $\begin{array}{lllll}0.1536423951791293 & 0.1671650538383079 & 0.6117288675268916\end{array}$ $\begin{array}{lllll}0.1479387509553296 & 0.3608790494635647 & 0.8942720463993481\end{array}$ $\begin{array}{llllll}0.1545676303996363 & 0.1198854399346002 & 0.9026079498442231\end{array}$ $\begin{array}{lllll}0.0259786139282802 & 0.1809765166776684 & 0.9043728021990094\end{array}$ $\begin{array}{lllll}0.0274842448713410 & 0.2983607893248988 & 0.8973970738259563\end{array}$ $\begin{array}{llll}0.0256346654115090 & 0.3490772771845517 & 0.6003011076428922\end{array}$ 
$\begin{array}{llll}0.2736728505999926 & 0.2300119863104182 & 0.6080388371184020\end{array}$ $\begin{array}{lllll}0.4047534259764169 & 0.1680162456748960 & 0.6118749876581543\end{array}$ $\begin{array}{lllll}0.3963058906998158 & 0.3602569690736211 & 0.8945058734916216\end{array}$ $\begin{array}{lllll}0.4061051672001207 & 0.1205042243292578 & 0.9038735285248625\end{array}$ $\begin{array}{llll}0.2758426149695607 & 0.1790596273457120 & 0.9058358666054338\end{array}$ $\begin{array}{lllll}0.2775227160822173 & 0.2964263450317844 & 0.8980542208252903\end{array}$ $\begin{array}{lllll}0.2742683396538237 & 0.3473534120993320 & 0.6000574726433501\end{array}$ $\begin{array}{lllll}0.1462704435415167 & 0.4084186813709538 & 0.6022410456527911\end{array}$ $\begin{array}{llll}0.3945466403341625 & 0.4072156282153028 & 0.6012233955879652\end{array}$ $\begin{array}{lllll}0.5235763601897473 & 0.2312142901762843 & 0.1081320938947357\end{array}$ $\begin{array}{lllll}0.6541019706292567 & 0.1683074533497106 & 0.1117365273894984\end{array}$ $\begin{array}{lllll}0.6460708006578052 & 0.3616107688972592 & 0.3939513315582179\end{array}$ $\begin{array}{lllll}0.6559409980532640 & 0.1213003305072218 & 0.4040482677940234\end{array}$ $\begin{array}{llll}0.5263713973068068 & 0.1805454545575595 & 0.4061433130059988\end{array}$ $\begin{array}{lllll}0.5273086257367982 & 0.2978282350790159 & 0.3979839924853436\end{array}$ $\begin{array}{llllll}0.5245731909474458 & 0.3486504798041761 & 0.0999909444617798\end{array}$ $\begin{array}{lllll}0.7736459901651265 & 0.2316023202086043 & 0.1082272302724860\end{array}$ $\begin{array}{lllll}0.9036923999980624 & 0.1678812150914180 & 0.1116536717386530\end{array}$ $\begin{array}{llll}0.8972050552632735 & 0.3614867774075549 & 0.3946502929859536\end{array}$ 0.90504237160080780 .12126554467799290 .4035135825823369 $\begin{array}{lllll}0.7762967530101157 & 0.1815698242298011 & 0.4058078440407326\end{array}$ $\begin{array}{lllll}0.7769049272604728 & 0.2988747008434700 & 0.3978787288167676\end{array}$ $\begin{array}{lllll}0.7748985700931851 & 0.3488979871829557 & 0.1005277551027825\end{array}$ $\begin{array}{lllll}0.6455835327784530 & 0.4083829095845204 & 0.1027868802164435\end{array}$ $\begin{array}{lllll}0.8956767498762812 & 0.4085365935086478 & 0.1025675450935858\end{array}$ $\begin{array}{lllll}0.5236014899498685 & 0.2320395751624577 & 0.6081937198731923\end{array}$ $\begin{array}{llll}0.6539942693956975 & 0.1696056215919665 & 0.6112491171994170\end{array}$ $\begin{array}{lllll}0.6469538504640027 & 0.3624830363552460 & 0.8949143012196482\end{array}$ $\begin{array}{lllll}0.6559326188150664 & 0.1219982705867364 & 0.9038782048560212\end{array}$ $\begin{array}{llll}0.5265124292590301 & 0.1812000776278892 & 0.9060868978106227\end{array}$ $\begin{array}{lllll}0.5275057309215058 & 0.2986099835128701 & 0.8980632197692076\end{array}$ $\begin{array}{lllll}0.5251621893552048 & 0.3492291279952555 & 0.6005722638475884\end{array}$ $\begin{array}{lllll}0.7722891800941740 & 0.2344049661007351 & 0.6068324294518477\end{array}$ $\begin{array}{lllll}0.9025524115657831 & 0.1701219203773144 & 0.6104538078211974\end{array}$ $\begin{array}{llll}0.8973756376973285 & 0.3621664801621821 & 0.8940605281685314\end{array}$ $\begin{array}{llll}0.9051278855005040 & 0.1216920517881138 & 0.9028452794340189\end{array}$ $\begin{array}{llll}0.7764663081324316 & 0.1820078896333564 & 0.9053330128684073\end{array}$ $\begin{array}{lllll}0.77744934799432622 & 0.2992096539681945 & 0.8976031893273759\end{array}$ $\begin{array}{llll}0.7743519577013275 & 0.3528515787710203 & 0.6002734520064773\end{array}$ $\begin{array}{llll}0.6449973280135972 & 0.4131936218374986 & 0.6017691767910254\end{array}$ $\begin{array}{llll}0.8972786736439468 & 0.4098155047121004 & 0.6036914407063886\end{array}$

Ade/Cyt :

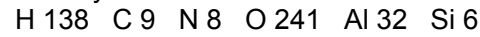

1.00000000000000

$20.2970019999999991 \quad 0.0000000000000000 \quad-3.7471719999999999$

$\begin{array}{lll}0.0646860000000000 & 24.9946149999999996 & -0.5148360000000000\end{array}$

$\begin{array}{llll}0.0000000000000000 & 0.0000000000000000 & 18.6939999999999991\end{array}$ $\begin{array}{llllll}\mathrm{H} & \mathrm{C} & \mathrm{N} & \mathrm{O} & \mathrm{Al} & \mathrm{Si}\end{array}$ $\begin{array}{llllll}138 & 9 & 8 & 241 & 32 & 64\end{array}$

Direct

$\begin{array}{llll}0.5340211003250170 & 0.7615033320496050 & 0.5850842612382665\end{array}$ $\begin{array}{lllll}0.5912587495833126 & 0.7631470957570748 & 0.4739442729341130\end{array}$ $\begin{array}{lllll}0.5131493959724229 & 0.5865661002887266 & 0.6915054530242802\end{array}$ $\begin{array}{lllll}0.6246747719438160 & 0.5195095977661774 & 0.5212086192428412\end{array}$ $\begin{array}{lllll}0.6313186984826226 & 0.5735050719398121 & 0.4641861566029321\end{array}$ $\begin{array}{lllll}0.0936266329382773 & 0.0549368546989798 & 0.4088967011992550\end{array}$ $\begin{array}{lllll}0.3425775577651008 & 0.0553661880887764 & 0.4084321280817750\end{array}$ $\begin{array}{lllll}0.0508913859299691 & 0.1389077805097985 & 0.1812405144987399\end{array}$ $\begin{array}{lllll}0.1700769178565468 & 0.3082977988277684 & 0.1605875368920351\end{array}$ $\begin{array}{lllll}0.1298601586283465 & 0.2204593012096523 & 0.3331893687055089\end{array}$ $\begin{array}{lllll}0.2430973466166217 & 0.0392539501381166 & 0.3430028921291299\end{array}$ $\begin{array}{lllll}0.1715252703823333 & 0.0669435348868860 & 0.1466339232357460\end{array}$ $\begin{array}{lllll}0.3007259385052986 & 0.1393219708839736 & 0.1813725081604300\end{array}$ $\begin{array}{lllll}0.4198671884192606 & 0.3079344551154328 & 0.1597444171418488\end{array}$ $\begin{array}{llll}0.3798322736105856 & 0.2210884705123083 & 0.3336739613131211\end{array}$ $\begin{array}{lllll}0.4922338615684385 & 0.0391131493567834 & 0.3427509819228360\end{array}$ $\begin{array}{lllll}0.4210080758581441 & 0.0670946695162003 & 0.1459138273954620\end{array}$ $\begin{array}{lllll}0.0563368318185841 & 0.4887593750211613 & 0.1492453156948007\end{array}$ $\begin{array}{llllll}0.2488396747954374 & 0.3895276425217862 & 0.3115612635404455\end{array}$ $\begin{array}{lllll}0.1284082801375219 & 0.4615901255337245 & 0.3464928074206553\end{array}$ $\begin{array}{lllll}0.3058929815304988 & 0.4888030159744493 & 0.1482505068954303\end{array}$ $\begin{array}{llll}0.4986257714181825 & 0.3889757225948730 & 0.3119145087269110\end{array}$ 
$\begin{array}{llll}0.3784740430926388 & 0.4620352827204180 & 0.3461169509523442\end{array}$ $\begin{array}{llllll}0.2054888028571674 & 0.4739482712653031 & 0.0839724444582344\end{array}$ $\begin{array}{lllll}0.4559176959559748 & 0.4743524599961583 & 0.0836646059003013\end{array}$ $\begin{array}{lllll}0.1532116306034696 & 0.4384587385125747 & 0.4651778674919618\end{array}$ $\begin{array}{llll}0.4032437336832920 & 0.4395212051776855 & 0.4643737627653302\end{array}$ $\begin{array}{llll}0.1473681625867594 & 0.0904060286059107 & 0.0278905824918634\end{array}$ $\begin{array}{lllll}0.3961487565866944 & 0.0909454983058219 & 0.0270466582773327\end{array}$ $\begin{array}{lllll}0.1640649119714144 & 0.4816344953593680 & 0.2835106153365112\end{array}$ $\begin{array}{lllll}0.4127622017688899 & 0.4831948987042604 & 0.2824801638714991\end{array}$ $\begin{array}{lllll}0.1352728590534466 & 0.0472510555138607 & 0.2092871890256461\end{array}$ $\begin{array}{lllll}0.3848385713570266 & 0.0472525774390156 & 0.2085255748177721\end{array}$ $\begin{array}{lllll}0.2113428992696703 & 0.4159727406528936 & 0.2371508091964057\end{array}$ $\begin{array}{lllll}0.4616377937691876 & 0.4161374442494956 & 0.2378414226741776\end{array}$ $\begin{array}{llll}0.3380355907484949 & 0.1125555689771889 & 0.2556721971330509\end{array}$ $\begin{array}{lllll}0.0882372885635475 & 0.1125858051394071 & 0.2558386562083470\end{array}$ $\begin{array}{llllll}0.0959689959778281 & 0.0541615558271590 & 0.9087082849683961\end{array}$ $\begin{array}{lllll}0.3438476661622474 & 0.0569954918572811 & 0.9073482273659997\end{array}$ $0.0508205331941548 \quad 0.13861119050076390 .6814265138172394$ $\begin{array}{llll}0.1699665112791779 & 0.3096210448652599 & 0.6606659213842589\end{array}$ $\begin{array}{llll}0.1290778960468037 & 0.2199991682445249 & 0.8305376014769715\end{array}$ $\begin{array}{lllll}0.2441000795615205 & 0.0403966900887729 & 0.8431778262683081\end{array}$ $\begin{array}{llllll}0.1718511883928674 & 0.0678872573255849 & 0.6466579271678575\end{array}$ $\begin{array}{lllll}0.3007439272607583 & 0.1400010093376802 & 0.6802261905088625\end{array}$ $\begin{array}{lllll}0.4200279360793768 & 0.3106216629125237 & 0.6598860980231213\end{array}$ $\begin{array}{llll}0.3796321433607662 & 0.2219385361027613 & 0.8313428987237608\end{array}$ $\begin{array}{lllll}0.4919305134515803 & 0.0416055375261588 & 0.8427716789696202\end{array}$ $\begin{array}{lllll}0.4215547421052138 & 0.0691247244514948 & 0.6463065941050182\end{array}$ $\begin{array}{lllll}0.0562032357256620 & 0.4884025505757271 & 0.6472283207628305\end{array}$ $\begin{array}{lllll}0.2491237123159616 & 0.3917140717653241 & 0.8124633876633635\end{array}$ $\begin{array}{lllll}0.1273248336677758 & 0.4623156753375798 & 0.8455329607131185\end{array}$ $\begin{array}{lllll}0.3046883879704326 & 0.4903719285397650 & 0.6479673197221689\end{array}$ $\begin{array}{lllll}0.4980635185314624 & 0.3868383780331300 & 0.8049884977530747\end{array}$ $\begin{array}{lllll}0.3777023330730456 & 0.4632233895448478 & 0.8450436622119486\end{array}$ $\begin{array}{lllll}0.2047094399956611 & 0.4744640611661020 & 0.5846206594089965\end{array}$ $\begin{array}{lllll}0.4546587341562334 & 0.4762222657917752 & 0.5810057456183683\end{array}$ $\begin{array}{llll}0.1523068222179213 & 0.4387248066729393 & 0.9645537323056622\end{array}$ $\begin{array}{llll}0.4027910679634392 & 0.4394986843139438 & 0.9642549080565470\end{array}$ $\begin{array}{lllll}0.1462677888768809 & 0.0909539496970929 & 0.5280331934282201\end{array}$ $\begin{array}{llll}0.3958421363925347 & 0.0919749612640315 & 0.5279450514658699\end{array}$ $\begin{array}{llllll}0.1636345267206674 & 0.4818879931826324 & 0.7828727796039712\end{array}$ $\begin{array}{lllll}0.4150654949308993 & 0.4810695407748061 & 0.7827189681491526\end{array}$ $\begin{array}{lllll}0.1359253671563244 & 0.0480407263749798 & 0.7094622817004606\end{array}$ $\begin{array}{lllll}0.3846386417447666 & 0.0499118562088058 & 0.7085787486392349\end{array}$ $\begin{array}{lllll}0.2110619149285906 & 0.4165280612517651 & 0.7370892375738559\end{array}$ $\begin{array}{llll}0.4606440221896640 & 0.4168491613632230 & 0.7345081224950356\end{array}$ $\begin{array}{lllll}0.3384418967128774 & 0.1147019292033619 & 0.7555306420635921\end{array}$ $\begin{array}{lllll}0.0887367716671424 & 0.1128896929682950 & 0.7561784954085764\end{array}$ $\begin{array}{lllll}0.5911573064612784 & 0.0555940731800871 & 0.4072630669807501\end{array}$ $\begin{array}{lllll}0.8444292120456738 & 0.0535797886077041 & 0.4089322802137387\end{array}$ $\begin{array}{lllll}0.5505525391925955 & 0.1395667386996526 & 0.1817284329098210\end{array}$ $\begin{array}{lllll}0.6697134667211500 & 0.3073246454522694 & 0.1597014188369391\end{array}$ $\begin{array}{llll}0.6301022475497138 & 0.2205918292264051 & 0.3341008234330771\end{array}$ $\begin{array}{lllll}0.7430393658501149 & 0.0394909507356080 & 0.3443176136909893\end{array}$ $\begin{array}{lllll}0.6708318798948393 & 0.0668882724869892 & 0.1460761662195837\end{array}$ $\begin{array}{llllll}0.8007005729871411 & 0.1387583537791397 & 0.1811378278715871\end{array}$ $\begin{array}{lllll}0.9198869189635446 & 0.3077376354526407 & 0.1602008255717253\end{array}$ $\begin{array}{llll}0.8795304777731860 & 0.2196112277039115 & 0.3324339118194134\end{array}$ $\begin{array}{lllll}0.9936749078534488 & 0.0387884324588498 & 0.3431344803398177\end{array}$ $\begin{array}{lllll}0.9214244058133368 & 0.0666798297232116 & 0.1465719511613672\end{array}$ $\begin{array}{lllll}0.5569074197719888 & 0.4881267024219161 & 0.1487064659543687\end{array}$ $\begin{array}{lllll}0.7490296291904510 & 0.3881372296174079 & 0.3112988025379041\end{array}$ $\begin{array}{lllll}0.6296707365557547 & 0.4604523480390586 & 0.3472230933514101\end{array}$ $\begin{array}{lllll}0.8070880747726552 & 0.4884575949052791 & 0.1494845090045832\end{array}$ $\begin{array}{lllll}0.9989813492849825 & 0.3892703785408189 & 0.3120085048311104\end{array}$ $\begin{array}{lllll}0.8787205233540558 & 0.4609966580640210 & 0.3473522827350888\end{array}$ $\begin{array}{llllll}0.7069881373783532 & 0.4737536520528197 & 0.0869594108279440\end{array}$ $\begin{array}{llll}0.9563858579965070 & 0.4734660503710494 & 0.0851163607035288\end{array}$ $\begin{array}{llllll}0.6595483986823281 & 0.4352970043394724 & 0.4657995906155974\end{array}$ $\begin{array}{llll}0.9037884546658436 & 0.4375353771549100 & 0.4659992281332898\end{array}$ $\begin{array}{lllll}0.6449406258297410 & 0.0906027971677254 & 0.0274873169973207\end{array}$ $\begin{array}{lllll}0.8952684068981764 & 0.0902360834375531 & 0.0279093125248964\end{array}$ $\begin{array}{llllll}0.6643738786986759 & 0.4807013317241884 & 0.2836227785629057\end{array}$ $\begin{array}{llll}0.9143219169467431 & 0.4811251133571158 & 0.2843765817284951\end{array}$ 
$\begin{array}{llll}0.6354786838099773 & 0.0464994332769306 & 0.2091517417334690\end{array}$ $\begin{array}{llllll}0.8857936695048547 & 0.0466084697358160 & 0.2095345796374639\end{array}$ $\begin{array}{lllll}0.7114095684502685 & 0.4154760854177508 & 0.2375435064296144\end{array}$ $\begin{array}{llll}0.9614630941175556 & 0.4158000243935790 & 0.2376087473569904\end{array}$ $\begin{array}{lllll}0.8380532750956295 & 0.1122559320770921 & 0.2555420255977571\end{array}$ $\begin{array}{lllll}0.5878714977944919 & 0.1122813731761447 & 0.2555210634660620\end{array}$ $\begin{array}{lllll}0.7052886812950243 & 0.7130327760586250 & 0.6589319485883134\end{array}$ $\begin{array}{lllll}0.7889886831606853 & 0.6652683621748650 & 0.5978090819121723\end{array}$ $\begin{array}{llll}0.8164219560051028 & 0.5183864825949882 & 0.6260886513318435\end{array}$ $\begin{array}{llll}0.8399830899960040 & 0.5757069500781655 & 0.5819977875942965\end{array}$ $\begin{array}{llll}0.6471961890519968 & 0.6620541249450295 & 0.7330257869687600\end{array}$ $\begin{array}{llll}0.5913036520697020 & 0.0569845570532566 & 0.9083234711472240\end{array}$ $\begin{array}{lllll}0.8419303448839284 & 0.0557637254623175 & 0.9082264443767675\end{array}$ $\begin{array}{llll}0.5509836440900251 & 0.1420481453974345 & 0.6824990842795855\end{array}$ $\begin{array}{llll}0.6696436848001144 & 0.3072122567906806 & 0.6565596856584499\end{array}$ $\begin{array}{lllll}0.6298535111358509 & 0.2238620949594014 & 0.8343523019879812\end{array}$ $\begin{array}{llllll}0.7422086948353821 & 0.0405081992728807 & 0.8443464416018858\end{array}$ $\begin{array}{lllll}0.6697568579715865 & 0.0679963763539158 & 0.6460926576675518\end{array}$ $\begin{array}{lllll}0.8007752376361520 & 0.1400752203523617 & 0.6819829615128477\end{array}$ $\begin{array}{lllll}0.9216666438116770 & 0.3090040720463624 & 0.6641945103611807\end{array}$ $\begin{array}{lllll}0.8806633654737769 & 0.2204406286127822 & 0.8352364966036953\end{array}$ $\begin{array}{llllll}0.9940233517613378 & 0.0402212277360812 & 0.8464095116130352\end{array}$ $\begin{array}{lllll}0.9218268699097816 & 0.0661200781804278 & 0.6473850377170570\end{array}$ $\begin{array}{lllll}0.5486154476312362 & 0.4943013448784010 & 0.6440988085438651\end{array}$ $\begin{array}{llllll}0.7492944012562769 & 0.3933689630581114 & 0.8178499331386271\end{array}$ $\begin{array}{lllll}0.6294626711870424 & 0.4670067286990116 & 0.8442103136126190\end{array}$ $\begin{array}{lllll}0.7599960640637231 & 0.4890251736976106 & 0.6871481675644560\end{array}$ $\begin{array}{lllll}0.9991661429955461 & 0.3908076824144043 & 0.8127859182945850\end{array}$ $\begin{array}{llll}0.8790176745435795 & 0.4619018268776129 & 0.8462517158900416\end{array}$ $\begin{array}{llllll}0.7170754749973587 & 0.4664860439238341 & 0.5835245577527656\end{array}$ $\begin{array}{llll}0.9554659648134199 & 0.4734213417563810 & 0.5864339420428026\end{array}$ $\begin{array}{llll}0.6562452613169673 & 0.4395464919800162 & 0.9656977481589200\end{array}$ $\begin{array}{lllll}0.9039996384765505 & 0.4381091410106743 & 0.9655723587288914\end{array}$ $\begin{array}{llll}0.6431636432462059 & 0.0912032513303638 & 0.5276550570900260\end{array}$ $\begin{array}{llll}0.8970211120942866 & 0.0895057927276858 & 0.5285349541240093\end{array}$ $\begin{array}{lllll}0.6365601205339978 & 0.5099329484696080 & 0.7792232887717533\end{array}$ $\begin{array}{lllll}0.9130793904530424 & 0.4826333855467776 & 0.7821106803127903\end{array}$ $\begin{array}{lllll}0.6351713620379240 & 0.0477239183373765 & 0.7097409676583352\end{array}$ $\begin{array}{lllll}0.8863595796474939 & 0.0463036692833682 & 0.7107226059833380\end{array}$ $\begin{array}{lllll}0.7120895958132880 & 0.4183046444860782 & 0.7418681621195723\end{array}$ $\begin{array}{lllll}0.9610216146145729 & 0.4157707585311768 & 0.7374386460775689\end{array}$ $\begin{array}{lllll}0.8378892267612248 & 0.1126811075455035 & 0.7556310213567260\end{array}$ $\begin{array}{lllll}0.5878774535369987 & 0.1136790979433598 & 0.7555666418995094\end{array}$ $\begin{array}{lllll}0.5825199109175007 & 0.7270329755466867 & 0.5021978606478797\end{array}$ $\begin{array}{llllll}0.5310569010959145 & 0.6049660440950561 & 0.6462528030249368\end{array}$ $\begin{array}{lllll}0.5484480346840173 & 0.6765832012721480 & 0.5844213200157887\end{array}$ $\begin{array}{llll}0.5773945590608039 & 0.6461533100326006 & 0.5348239439871360\end{array}$ $\begin{array}{llllll}0.5821250996874689 & 0.5903489319997184 & 0.5463798452084466\end{array}$ $\begin{array}{llll}0.7170109271807140 & 0.6707263264818020 & 0.6666636206869302\end{array}$ $\begin{array}{lllll}0.7620474808811026 & 0.6445582776707867 & 0.6337854997950615\end{array}$ $\begin{array}{lllll}0.7707681779491007 & 0.5884199915544196 & 0.6473649053262294\end{array}$ $\begin{array}{llll}0.6928868946918654 & 0.5890711526832482 & 0.7243857791113129\end{array}$ $\begin{array}{lllll}0.5247574372359149 & 0.6583828807437127 & 0.6417667708027253\end{array}$ $\begin{array}{lllll}0.5523657028846346 & 0.7287402165287694 & 0.5625148028718882\end{array}$ $\begin{array}{llll}0.5987148251350289 & 0.6781369760492002 & 0.4840799156619121\end{array}$ $\begin{array}{lllll}0.5576779925212615 & 0.5706868796575792 & 0.6030862366679409\end{array}$ $\begin{array}{llll}0.6099329338000101 & 0.5569904494669551 & 0.5032437533788880\end{array}$ $\begin{array}{lllll}0.6839170238923515 & 0.6441169601279281 & 0.7113941140688490\end{array}$ $\begin{array}{lllll}0.8133424328316817 & 0.5591562571216157 & 0.6162385811050919\end{array}$ $\begin{array}{lllll}0.7366812613329777 & 0.5625141167729174 & 0.6910162953975872\end{array}$ $\begin{array}{lllll}0.0458858520670927 & 0.3494433050547445 & 0.1827681363004152\end{array}$ $\begin{array}{lllll}0.0298939144767226 & 0.2402628814735952 & 0.1903416958405637\end{array}$ $\begin{array}{lllll}0.1550064157034223 & 0.2819334395257965 & 0.1912681768589161\end{array}$ $\begin{array}{lllll}0.1995642061039718 & 0.2076861599299169 & 0.0686656726865852\end{array}$ $\begin{array}{llll}0.0339355402099253 & 0.2871535352595255 & 0.0648070395463917\end{array}$ $\begin{array}{lllll}0.0762986067003625 & 0.1855406442595675 & 0.0856029585881281\end{array}$ $\begin{array}{lllll}0.1800151606697198 & 0.1677653828354849 & 0.1935752158676582\end{array}$ $\begin{array}{lllll}0.1790638919269306 & 0.0549482388952149 & 0.1991347635680198\end{array}$ $\begin{array}{lllll}0.0467666934977387 & 0.1094252569456341 & 0.2136262975898494\end{array}$ $\begin{array}{llllll}0.1591172305522846 & 0.1047193256804007 & 0.0786325993078663\end{array}$ $\begin{array}{lllll}0.1487406966133576 & 0.1146589613187198 & 0.3100295269089968\end{array}$ $\begin{array}{lllll}0.0208937251338711 & 0.0671983880455878 & 0.3299757933729172\end{array}$ $\begin{array}{llll}0.1378977331551565 & 0.0651390364293182 & 0.4370087785188228\end{array}$ 
$\begin{array}{llll}0.1041721364127756 & 0.1666504270874124 & 0.4193948904974427\end{array}$ $\begin{array}{lllll}0.0200048995713970 & 0.2877925443952002 & 0.3033264479636928\end{array}$ $\begin{array}{lllll}0.1447711089429070 & 0.2466437088892436 & 0.3021502466506111\end{array}$ $\begin{array}{llll}0.1000283603916916 & 0.3211244841361661 & 0.4247076481993830\end{array}$ $\begin{array}{lllll}0.0158241286346313 & 0.2408874744915813 & 0.4287968175988375\end{array}$ $\begin{array}{lllll}0.1196542148531446 & 0.3608194428543910 & 0.2997572490336254\end{array}$ $\begin{array}{llllll}0.2291033099612574 & 0.1407819897216397 & 0.4360884473702058\end{array}$ $\begin{array}{lllll}0.2537852564995832 & 0.1790259174263863 & 0.3105659854619370\end{array}$ $\begin{array}{llll}0.2233246015157065 & 0.3428315424307724 & 0.4078192693230744\end{array}$ $\begin{array}{lllll}0.2959641925088146 & 0.3498189000867498 & 0.1824675099891061\end{array}$ $\begin{array}{lllll}0.2797623495086342 & 0.2407077188831845 & 0.1901291177728464\end{array}$ $\begin{array}{lllll}0.4050034098477123 & 0.2819615691600438 & 0.1911252913202333\end{array}$ $\begin{array}{llllll}0.4495791639048738 & 0.2076318912508321 & 0.0685798265642528\end{array}$ $\begin{array}{lllll}0.2839824965843104 & 0.2876183473968339 & 0.0645581020271385\end{array}$ $\begin{array}{lllll}0.3261357326202782 & 0.1860533692173322 & 0.0852841012883337\end{array}$ $\begin{array}{lllll}0.4296343044005576 & 0.1676945055801961 & 0.1933471455533103\end{array}$ $\begin{array}{lllll}0.4286269094345429 & 0.0549670420697427 & 0.1983799119783062\end{array}$ $\begin{array}{lllll}0.2964972808642606 & 0.1096841369111847 & 0.2134718200421087\end{array}$ $\begin{array}{lllll}0.4083070052543838 & 0.1049112787590423 & 0.0780107631622508\end{array}$ $\begin{array}{llll}0.3984596494955424 & 0.1142556186321955 & 0.3097885218358692\end{array}$ $\begin{array}{lllll}0.2705928991913082 & 0.0674931683798407 & 0.3299030100059478\end{array}$ $\begin{array}{llllll}0.3869120002564959 & 0.0652599960514139 & 0.4368095050926083\end{array}$ $\begin{array}{lllll}0.3542929198862602 & 0.1670116632520639 & 0.4188872347046059\end{array}$ $\begin{array}{lllll}0.2699330309339151 & 0.2882873051768273 & 0.3030696228995173\end{array}$ $\begin{array}{llll}0.3945610711937008 & 0.2468840843223789 & 0.3019763259121425\end{array}$ $\begin{array}{lllll}0.3500391176626889 & 0.3217503941554297 & 0.4243640610262815\end{array}$ $\begin{array}{lllll}0.2660963668365703 & 0.2413987355879284 & 0.4286065455970833\end{array}$ $\begin{array}{lllll}0.3697055916210706 & 0.3612032512997511 & 0.2993714912079414\end{array}$ $\begin{array}{lllll}0.4789800168582425 & 0.1401691319685741 & 0.4358831941645398\end{array}$ $\begin{array}{lllll}0.0038399192879578 & 0.1785934816665194 & 0.3107438890330861\end{array}$ $\begin{array}{lllll}0.4733704248476308 & 0.3429505121753650 & 0.4073068666987150\end{array}$ $\begin{array}{lllll}0.1508498848617822 & 0.4138574450843526 & 0.1830908175050995\end{array}$ $\begin{array}{lllll}0.0288770209354127 & 0.4608255345312973 & 0.1631984692518049\end{array}$ $\begin{array}{lllll}0.0706822723498110 & 0.3876869365904204 & 0.0570956887782939\end{array}$ $\begin{array}{llll}0.1612238272374300 & 0.4635973320646620 & 0.0560617888766354\end{array}$ $\begin{array}{lllll}0.1957461701456364 & 0.3620926991478536 & 0.0736741640674245\end{array}$ $\begin{array}{lllll}0.1204724291927340 & 0.4736364466166122 & 0.2939936387303067\end{array}$ $\begin{array}{lllll}0.5032182978093799 & 0.4185516625928970 & 0.2798506629592249\end{array}$ $\begin{array}{llll}0.1409922181309781 & 0.4239776111555860 & 0.4145355974027775\end{array}$ $\begin{array}{llll}0.4009233020080971 & 0.4146844488475669 & 0.1831867347120390\end{array}$ $\begin{array}{llll}0.2784742867100560 & 0.4610136230952605 & 0.1626270740427674\end{array}$ $\begin{array}{llll}0.3210369459721374 & 0.3881392983201662 & 0.0570050508411717\end{array}$ $\begin{array}{lllll}0.4117958663430412 & 0.4637467801666897 & 0.0559308630484138\end{array}$ $\begin{array}{lllll}0.4458328376606385 & 0.3620475060703049 & 0.0744258402821940\end{array}$ $\begin{array}{lllll}0.3696947701125156 & 0.4742982852688347 & 0.2936269668531497\end{array}$ $\begin{array}{lllll}0.2528628709192565 & 0.4190984045219799 & 0.2792985739917347\end{array}$ $\begin{array}{lllll}0.3915894785723875 & 0.4245559689734998 & 0.4137792679284984\end{array}$ $\begin{array}{lllll}0.0443623277783291 & 0.3497977293614319 & 0.6820584827980442\end{array}$ $\begin{array}{lllll}0.0299644878134732 & 0.2403491026978308 & 0.6901485607490402\end{array}$ $\begin{array}{lllll}0.1546103195291230 & 0.2828169811693373 & 0.6905085464709121\end{array}$ $\begin{array}{llll}0.1998164123507561 & 0.2083072756657840 & 0.5681142397117916\end{array}$ $\begin{array}{lllll}0.0324017858643032 & 0.2875586710533589 & 0.5642607798346959\end{array}$ $\begin{array}{lllll}0.0765070787545465 & 0.1864088689675175 & 0.5849029545044426\end{array}$ $\begin{array}{lllll}0.1802170019716144 & 0.1687127247787757 & 0.6932210746696812\end{array}$ $\begin{array}{llll}0.1796391838833524 & 0.0558682561201234 & 0.6991783744280604\end{array}$ $0.0472020384772645 \quad 0.1091666918413164 \quad 0.7140308141417888$ $\begin{array}{llll}0.1591093344225606 & 0.1054702980450428 & 0.5785703182693414\end{array}$ $\begin{array}{lllll}0.1491429421468643 & 0.1157812939237194 & 0.8099993120068887\end{array}$ $\begin{array}{lllll}0.0216044097428063 & 0.0673466522795204 & 0.8307619614313336\end{array}$ $\begin{array}{lllll}0.1401323862253812 & 0.0647874124081842 & 0.9365253421971187\end{array}$ $\begin{array}{lllll}0.1040681995440234 & 0.1658996510044214 & 0.9206182941919304\end{array}$ $\begin{array}{llll}0.0200284344301075 & 0.2877421633777648 & 0.8033005017875257\end{array}$ $\begin{array}{llllll}0.1446728779221143 & 0.2471718239146944 & 0.8014569769687104\end{array}$ $\begin{array}{lllll}0.1003678984545703 & 0.3212515955036634 & 0.9243073432583387\end{array}$ $\begin{array}{lllll}0.0164507663415586 & 0.2408525500296366 & 0.9289672588054932\end{array}$ $\begin{array}{lllll}0.1187973211791417 & 0.3612382549899769 & 0.7990066365479748\end{array}$ $\begin{array}{llll}0.2293820372375515 & 0.1419514402703472 & 0.9359435826910179\end{array}$ $\begin{array}{llllll}0.2541431069364145 & 0.1801255706380144 & 0.8102139720160988\end{array}$ $\begin{array}{lllll}0.2231759101656545 & 0.3443228135965268 & 0.9067057411104029\end{array}$ $\begin{array}{lllll}0.2951385105384860 & 0.3510887534212152 & 0.6815311390777646\end{array}$ $\begin{array}{lllll}0.2797944939336776 & 0.2418227033704994 & 0.6894175720581672\end{array}$ $\begin{array}{llllll}0.4048979079094484 & 0.2840520084901171 & 0.6902455070303618\end{array}$ $\begin{array}{llll}0.4497383946192444 & 0.2097349372065072 & 0.5679174085542410\end{array}$ 
$\begin{array}{llll}0.2838009082351079 & 0.2886122595462595 & 0.5637564254085924\end{array}$ $\begin{array}{lllll}0.3264740776530081 & 0.1871855270157732 & 0.5847246593185528\end{array}$ $\begin{array}{lllll}0.4302634795511054 & 0.1698974586769953 & 0.6929177196947586\end{array}$ $\begin{array}{llll}0.4287092949417177 & 0.0570534395780678 & 0.6987909945263859\end{array}$ $\begin{array}{lllll}0.2969878195491136 & 0.1108211443073317 & 0.7132078774172651\end{array}$ $\begin{array}{lllll}0.4094928194806768 & 0.1066592163666183 & 0.5782396073789209\end{array}$ $\begin{array}{lllll}0.3985386534656603 & 0.1173681504045392 & 0.8095102117686569\end{array}$ $\begin{array}{lllll}0.2712733023778757 & 0.0687762991278126 & 0.8298407126580519\end{array}$ $\begin{array}{llll}0.3881071186283953 & 0.0670796207442934 & 0.9358172351392380\end{array}$ $\begin{array}{lllll}0.3542368174939192 & 0.1687370203392882 & 0.9194060338303638\end{array}$ $\begin{array}{llllll}0.2695577414657745 & 0.2893495277174743 & 0.8021543279687183\end{array}$ $\begin{array}{lllll}0.3947133454092243 & 0.2485944997241777 & 0.8011022102849945\end{array}$ $\begin{array}{llll}0.3496890401090300 & 0.3225843147295335 & 0.9234919832534170\end{array}$ $\begin{array}{llll}0.2652143513412636 & 0.2427458788996605 & 0.9278857857847843\end{array}$ $\begin{array}{llll}0.3692904000774258 & 0.3623190100203599 & 0.7985550447205148\end{array}$ $\begin{array}{lllll}0.4792340613974537 & 0.1430192411499626 & 0.9355193160667943\end{array}$ $\begin{array}{lllll}0.0044895606848270 & 0.1784718222475973 & 0.8110817946908134\end{array}$ $\begin{array}{lllll}0.4729057187175915 & 0.3443289376318965 & 0.9077847970594151\end{array}$ $\begin{array}{llll}0.1501086355213419 & 0.4137129424103093 & 0.6825624644698505\end{array}$ $\begin{array}{llllll}0.0281356416974333 & 0.4612858497455647 & 0.6620928193832673\end{array}$ $\begin{array}{lllll}0.0700782788749294 & 0.3878679340686833 & 0.5565084240361430\end{array}$ $\begin{array}{lllll}0.1604779780781083 & 0.4642172237186963 & 0.5562048586169738\end{array}$ $\begin{array}{llll}0.1953264275727712 & 0.3628587857939959 & 0.5724905432429136\end{array}$ $\begin{array}{lllll}0.1198419110364552 & 0.4741376252302633 & 0.7929928182057752\end{array}$ $\begin{array}{llll}0.5013722353462428 & 0.4189234220553218 & 0.7769857842237919\end{array}$ $\begin{array}{lllll}0.1397138924259085 & 0.4244841913607132 & 0.9138328993737296\end{array}$ $\begin{array}{lllll}0.3993308344098332 & 0.4158214278286839 & 0.6809515821238782\end{array}$ $\begin{array}{lllll}0.2772935130886234 & 0.4624316864807456 & 0.6620302517333447\end{array}$ $\begin{array}{lllll}0.3205147416194647 & 0.3890429667816742 & 0.5554548044122680\end{array}$ $\begin{array}{lllll}0.4102295314487523 & 0.4655710218566561 & 0.5535413003917089\end{array}$ $\begin{array}{lllll}0.4462110181833953 & 0.3646193496781140 & 0.5723295930468761\end{array}$ $\begin{array}{lllll}0.3707244611031921 & 0.4749885385700177 & 0.7925196206743963\end{array}$ $\begin{array}{lllll}0.2525622579167775 & 0.4205539265688386 & 0.7788697513194646\end{array}$ $\begin{array}{lllll}0.3899527417748553 & 0.4253868496836639 & 0.9135234048995872\end{array}$ $\begin{array}{llll}0.5461359094272218 & 0.3493613543742315 & 0.1828547317456696\end{array}$ $\begin{array}{lllll}0.5297290075474772 & 0.2402727619472394 & 0.1901746584484159\end{array}$ $\begin{array}{lllll}0.6551297790369383 & 0.2814193777123813 & 0.1913590997276591\end{array}$ $\begin{array}{lllll}0.6997302437120116 & 0.2072434442150017 & 0.0688277721690130\end{array}$ $\begin{array}{lllll}0.5340317413069147 & 0.2875464690469721 & 0.0648119038343603\end{array}$ $\begin{array}{llll}0.5762520751879961 & 0.1859643527408585 & 0.0853289258994865\end{array}$ $\begin{array}{lllll}0.6798714381730648 & 0.1673804478934948 & 0.1936161613114170\end{array}$ $\begin{array}{llll}0.6789773700558710 & 0.0546037883109199 & 0.1985198655086677\end{array}$ $\begin{array}{lllll}0.5462826975905316 & 0.1096877642598559 & 0.2134221073358760\end{array}$ $\begin{array}{lllll}0.6580546555127010 & 0.1046332210170950 & 0.0783540367814601\end{array}$ $\begin{array}{llllll}0.6484752766806430 & 0.1138511386541411 & 0.3097573661178512\end{array}$ $\begin{array}{lllll}0.5201119860092894 & 0.0669572608428461 & 0.3293372722400816\end{array}$ $\begin{array}{llll}0.6353848267400438 & 0.0652117034964770 & 0.4364093937231552\end{array}$ $\begin{array}{lllll}0.6039481816257219 & 0.1671492583083919 & 0.4183760693886411\end{array}$ $\begin{array}{lllll}0.5199439973508723 & 0.2877121873263894 & 0.3031664269786343\end{array}$ $\begin{array}{lllll}0.6447506444655184 & 0.2462985850547160 & 0.3022188793420429\end{array}$ $\begin{array}{lllll}0.5998628939335889 & 0.3206397259950606 & 0.4245884224147082\end{array}$ $\begin{array}{llll}0.5149972021942024 & 0.2410595244187180 & 0.4286167572955987\end{array}$ $\begin{array}{lllll}0.6200469276628375 & 0.3601553357634307 & 0.2996467089838199\end{array}$ $\begin{array}{lllll}0.7284860564970617 & 0.1395583380779081 & 0.4360199959309489\end{array}$ $\begin{array}{lllll}0.7536769879665348 & 0.1782480922585852 & 0.3107222843610258\end{array}$ $\begin{array}{lllll}0.7235380722237541 & 0.3412344734751304 & 0.4085641181427065\end{array}$ $\begin{array}{lllll}0.7962202030973649 & 0.3492772002686114 & 0.1830528940094820\end{array}$ $\begin{array}{lllll}0.7798985241125492 & 0.2401143131398962 & 0.1903927219622573\end{array}$ $\begin{array}{lllll}0.9051666709467006 & 0.2816399986913438 & 0.1915066096653558\end{array}$ $\begin{array}{llllll}0.9496986815683660 & 0.2071593253816004 & 0.0688285062106500\end{array}$ $\begin{array}{llllll}0.7841166066678964 & 0.2872137312864768 & 0.0649754118202409\end{array}$ $\begin{array}{llll}0.8263209905935852 & 0.1855886169966802 & 0.0855617014913772\end{array}$ $\begin{array}{lllll}0.9300351545656431 & 0.1674161684309564 & 0.1937112338484203\end{array}$ $\begin{array}{lllll}0.9293901327184664 & 0.0545765870717092 & 0.1990529114038761\end{array}$ $\begin{array}{lllll}0.7965798808353890 & 0.1092301575087811 & 0.2134464310212920\end{array}$ $\begin{array}{lllll}0.9084306345666230 & 0.1044332449201886 & 0.0786355095502328\end{array}$ $\begin{array}{lllll}0.8987841228449370 & 0.1141496196636774 & 0.3099458593922776\end{array}$ $\begin{array}{lllll}0.7707215564999180 & 0.0669312247144473 & 0.3295729872778547\end{array}$ $\begin{array}{lllll}0.8886187715360298 & 0.0640042704986768 & 0.4368057692300664\end{array}$ $\begin{array}{lllll}0.8539126188400638 & 0.1653948342653146 & 0.4196300723680232\end{array}$ $\begin{array}{lllll}0.7702153625976786 & 0.2873306716880545 & 0.3035594670132635\end{array}$ $\begin{array}{lllll}0.8948248894775189 & 0.2462166184188542 & 0.3022670513087573\end{array}$ $\begin{array}{llll}0.8502006667188118 & 0.3204744068330815 & 0.4251575968651713\end{array}$ 
$\begin{array}{llll}0.7662039554162187 & 0.2400105626255047 & 0.4290378108955336\end{array}$ $\begin{array}{lllll}0.8697320400607285 & 0.3604675685326463 & 0.3002359314317648\end{array}$ $\begin{array}{lllll}0.9790011400695195 & 0.1401868019171242 & 0.4361520652760703\end{array}$ $\begin{array}{lllll}0.5031859837780137 & 0.1787536198135801 & 0.3104726901523960\end{array}$ $\begin{array}{llll}0.9734672317874454 & 0.3423979050479808 & 0.4081190595439012\end{array}$ $\begin{array}{lllll}0.6512313889533499 & 0.4137856363296757 & 0.1838768985105445\end{array}$ $\begin{array}{llllll}0.5292297424102504 & 0.4608364447904076 & 0.1638227421669637\end{array}$ $\begin{array}{llllll}0.5710553417635196 & 0.3882051586255167 & 0.0576068918963629\end{array}$ $\begin{array}{lllll}0.6629359068941384 & 0.4637493420626704 & 0.0580426133289599\end{array}$ $\begin{array}{lllll}0.6957430738153326 & 0.3615800666711132 & 0.0747121327987372\end{array}$ $\begin{array}{lllll}0.6209916058923238 & 0.4731403485000126 & 0.2950066493411683\end{array}$ $\begin{array}{llllll}0.0030682735394464 & 0.4188535713367084 & 0.2797794251264302\end{array}$ $\begin{array}{lllll}0.6430312201640115 & 0.4227621444451281 & 0.4148341246124944\end{array}$ $\begin{array}{lllll}0.9010768521801161 & 0.4138102553463921 & 0.1837074267960451\end{array}$ $\begin{array}{lllll}0.7793389690145143 & 0.4610466799453807 & 0.1641855775129465\end{array}$ $\begin{array}{lllll}0.8208016073735503 & 0.3878928216234084 & 0.0576225660244556\end{array}$ $\begin{array}{lllll}0.9121354852293002 & 0.4633137652636855 & 0.0568777893773849\end{array}$ $\begin{array}{lllll}0.9455564635759841 & 0.3615554951351807 & 0.0743675591737854\end{array}$ $\begin{array}{lllll}0.8707292156250300 & 0.4732679712646207 & 0.2949807885054704\end{array}$ $\begin{array}{lllll}0.7530803933685913 & 0.4182452200110380 & 0.2799123707137461\end{array}$ $\begin{array}{lllll}0.8910085115857395 & 0.4233487952259215 & 0.4153757114168907\end{array}$ $\begin{array}{lllll}0.6592895781813741 & 0.5687568677176840 & 0.7660718914487816\end{array}$ $\begin{array}{lllll}0.5469362941479685 & 0.3522251909936542 & 0.6814470696534566\end{array}$ $\begin{array}{lllll}0.5298289851831508 & 0.2430287901251221 & 0.6894333443245549\end{array}$ $\begin{array}{lllll}0.6554572411904289 & 0.2827889332733854 & 0.6904764919548800\end{array}$ $\begin{array}{lllll}0.7003121668231158 & 0.2071748064255846 & 0.5684128398627943\end{array}$ $\begin{array}{lllll}0.5338499878598845 & 0.2899273586737570 & 0.5635856564781857\end{array}$ $\begin{array}{llll}0.5762285949221527 & 0.1883355626159591 & 0.5846384251140911\end{array}$ $\begin{array}{lllll}0.6790510415358908 & 0.1682757754109428 & 0.6933269667919045\end{array}$ $\begin{array}{llll}0.6785311542800070 & 0.0554757877837858 & 0.6984503806688055\end{array}$ $\begin{array}{lllll}0.5463722517100298 & 0.1116846388447375 & 0.7132763356010599\end{array}$ $\begin{array}{llll}0.6559458466915092 & 0.1054514784003966 & 0.5783101681761412\end{array}$ $\begin{array}{lllll}0.6483072538429356 & 0.1144181684449694 & 0.8098056051059909\end{array}$ $\begin{array}{lllll}0.5198735626956754 & 0.0694762201535336 & 0.8295344358283493\end{array}$ $\begin{array}{llll}0.6356818858574221 & 0.0666035321377520 & 0.9368794803827407\end{array}$ $\begin{array}{llll}0.6043746702948272 & 0.1686565390510239 & 0.9181340164371856\end{array}$ $\begin{array}{lllll}0.5198802758084309 & 0.2907836344513378 & 0.8023573379304864\end{array}$ $\begin{array}{lllll}0.6445041285298065 & 0.2488445278536964 & 0.8015575468168057\end{array}$ $\begin{array}{lllll}0.6001117900482670 & 0.3239590543938364 & 0.9238897104441507\end{array}$ $\begin{array}{lllll}0.5167191783429710 & 0.2434719129679818 & 0.9279759702590933\end{array}$ $\begin{array}{lllll}0.6199919335710677 & 0.3633877453955759 & 0.7988997068378771\end{array}$ $\begin{array}{llll}0.7287092544827629 & 0.1407663023531142 & 0.9358689915757955\end{array}$ $\begin{array}{lllll}0.7525319330332955 & 0.1793235041358164 & 0.8104116140467381\end{array}$ $\begin{array}{lllll}0.7236406049684823 & 0.3436781428539595 & 0.9065209768219262\end{array}$ $\begin{array}{lllll}0.7973877648747948 & 0.3483653123396174 & 0.6835881956757998\end{array}$ $\begin{array}{lllll}0.7801603473338284 & 0.2397841910886371 & 0.6903134257395617\end{array}$ $\begin{array}{lllll}0.9055534196981193 & 0.2811549670927710 & 0.6918317887011465\end{array}$ $\begin{array}{llll}0.9497423644014383 & 0.2066723445639234 & 0.5687937983257634\end{array}$ $\begin{array}{llll}0.7859525578097007 & 0.2863618996498229 & 0.5649011045435108\end{array}$ $\begin{array}{lllll}0.8266770674761414 & 0.1844666010464658 & 0.5860985274656554\end{array}$ $\begin{array}{lllll}0.9306757909908724 & 0.1670493641037280 & 0.6940226384185385\end{array}$ $\begin{array}{llll}0.9298582387293578 & 0.0541735111104581 & 0.6999151930861223\end{array}$ $\begin{array}{lllll}0.7963187381269701 & 0.1102437275985593 & 0.7137029445139218\end{array}$ $\begin{array}{lllll}0.9095152797329702 & 0.1037518781086424 & 0.5792141259717543\end{array}$ $\begin{array}{llll}0.8989652294869163 & 0.1140456458392573 & 0.8100907632143802\end{array}$ $\begin{array}{lllll}0.7702626541480698 & 0.0675978698349542 & 0.8294223985606771\end{array}$ $\begin{array}{lllll}0.8861140368966546 & 0.0657311903345854 & 0.9369654389761117\end{array}$ $\begin{array}{lllll}0.8536401099278681 & 0.1675393272274039 & 0.9181088869379392\end{array}$ $\begin{array}{lllll}0.7706381868225656 & 0.2875912238569610 & 0.8031666877276515\end{array}$ $\begin{array}{lllll}0.8951596603305464 & 0.2457454267003063 & 0.8027247887001994\end{array}$ $\begin{array}{llll}0.8500173285847721 & 0.3210925195055305 & 0.9249203060468654\end{array}$ $\begin{array}{llll}0.7649549443983163 & 0.2416491041575360 & 0.9286013088287243\end{array}$ $\begin{array}{lllll}0.8709451375163894 & 0.3602380668540900 & 0.8003233071074242\end{array}$ $\begin{array}{lllll}0.9783639307152888 & 0.1405638711797756 & 0.9363464117009416\end{array}$ $\begin{array}{llll}0.5039021514740735 & 0.1812635292072470 & 0.8099049683194421\end{array}$ $\begin{array}{lllll}0.9738295464706842 & 0.3421793666760459 & 0.9088177996139576\end{array}$ $\begin{array}{lllll}0.6514840838542343 & 0.4168627556833387 & 0.6830660219704745\end{array}$ $\begin{array}{lllll}0.5272511031038148 & 0.4626765391887034 & 0.6596340901476229\end{array}$ $\begin{array}{lllll}0.5724350777843378 & 0.3899245925518474 & 0.5558457164824611\end{array}$ $\begin{array}{llll}0.6715154432826242 & 0.4593388532196989 & 0.5546161717977979\end{array}$ $\begin{array}{lllll}0.6953356733536055 & 0.3581260315376512 & 0.5776187342852201\end{array}$ $\begin{array}{lllll}0.6144780996238023 & 0.4763267865253840 & 0.7923034218427452\end{array}$ $0.00279642052355490 .4197031878925652 \quad 0.7793623874124088$ 
$\begin{array}{llll}0.6442124183404234 & 0.4260548886901093 & 0.9147675581839104\end{array}$ $\begin{array}{lllll}0.9011666856577127 & 0.4124933184056386 & 0.6836065337096037\end{array}$ $\begin{array}{llll}0.7813010252197350 & 0.4599040891847092 & 0.6628114470803760\end{array}$ $\begin{array}{lllll}0.8190519633303306 & 0.3883590465661327 & 0.5584494092057815\end{array}$ $\begin{array}{llll}0.9107664912233046 & 0.4643257552408159 & 0.5578487170871903\end{array}$ $\begin{array}{llll}0.9445797909099346 & 0.3627605780168904 & 0.5722427073902104\end{array}$ 0.87028915004530770 .47336626514533850 .7936510292267016 $\begin{array}{lllll}0.7549016552038990 & 0.4193569844827697 & 0.7812401531433373\end{array}$ $\begin{array}{lllll}0.8920962200798138 & 0.4237368353959323 & 0.9148768947899854\end{array}$ $\begin{array}{lllll}0.2128188149600535 & 0.2336920721341118 & 0.2481971659106138\end{array}$ $\begin{array}{lllll}0.0869902672889858 & 0.2949208132104075 & 0.2452214847188476\end{array}$ $\begin{array}{llllll}0.2220589871565213 & 0.1127515708006477 & 0.2638375382749895\end{array}$ $\begin{array}{lllll}0.4625143336395715 & 0.2335337306232626 & 0.2480835405862219\end{array}$ $\begin{array}{lllll}0.3369809914247193 & 0.2953188288362517 & 0.2450094977542104\end{array}$ $\begin{array}{lllll}0.4716902311915802 & 0.1125443564343111 & 0.2635114486916670\end{array}$ $\begin{array}{lllll}0.0775486824835894 & 0.4158051816940829 & 0.2293597707498675\end{array}$ $\begin{array}{lllll}0.3274163792047717 & 0.4162901698619398 & 0.2290527275718426\end{array}$ $\begin{array}{lllll}0.2128362700949602 & 0.2347049061327107 & 0.7475205166445797\end{array}$ $\begin{array}{lllll}0.0865170541105534 & 0.2953354703860830 & 0.7446037838737798\end{array}$ $\begin{array}{llll}0.2225383944616233 & 0.1138370523880999 & 0.7636872240878800\end{array}$ $\begin{array}{lllll}0.4631056010939625 & 0.2360981900555245 & 0.7474223828131300\end{array}$ $\begin{array}{llllll}0.3367450465068913 & 0.2967300067517238 & 0.7441459968043884\end{array}$ $\begin{array}{lllll}0.4718836996049607 & 0.1150407993157924 & 0.7633503076984481\end{array}$ $\begin{array}{lllll}0.0767349341938121 & 0.4161588125441293 & 0.7286027924305882\end{array}$ $\begin{array}{llll}0.3268916210345688 & 0.4175690406434406 & 0.7278750099398844\end{array}$ $\begin{array}{lllll}0.7127689707169901 & 0.2330831129085196 & 0.2483670967292829\end{array}$ $\begin{array}{lllll}0.5869948019486231 & 0.2946032004497199 & 0.2450750262632221\end{array}$ $\begin{array}{lllll}0.7219462710104346 & 0.1120928270522250 & 0.2636783259286061\end{array}$ $\begin{array}{lllll}0.9628878001597762 & 0.2332756214069166 & 0.2483307102649396\end{array}$ $\begin{array}{lllll}0.8370834468516215 & 0.2945910431473215 & 0.2453277288409655\end{array}$ $\begin{array}{lllll}0.9722279755124309 & 0.1123339338330683 & 0.2639249034104135\end{array}$ $\begin{array}{llll}0.5776348290323101 & 0.4155954765149488 & 0.2296302115656951\end{array}$ $\begin{array}{lllll}0.8276805969572238 & 0.4155869187422632 & 0.2298408436365428\end{array}$ $\begin{array}{lllll}0.7122217420849812 & 0.2343178958203737 & 0.7478733943793077\end{array}$ $\begin{array}{lllll}0.5877032760671435 & 0.2981702125291372 & 0.7443524012686594\end{array}$ $\begin{array}{lllll}0.7215944105365760 & 0.1131152303850287 & 0.7636337059823038\end{array}$ $\begin{array}{lllll}0.9631957559680959 & 0.2329863844200542 & 0.7487599438882147\end{array}$ $\begin{array}{llllll}0.8376441008558693 & 0.2941295972446544 & 0.7460234118464465\end{array}$ $\begin{array}{lllll}0.9726901507662109 & 0.1121270285079749 & 0.7644981966080371\end{array}$ $\begin{array}{lllll}0.5773856128414734 & 0.4209302412203424 & 0.7278112431805556\end{array}$ $\begin{array}{lllll}0.8282665171533223 & 0.4144924355248552 & 0.7303995071591993\end{array}$ $\begin{array}{llll}0.0229927596706719 & 0.2306002699055063 & 0.1019282323017204\end{array}$ $\begin{array}{lllll}0.1535193013728279 & 0.1676451666508235 & 0.1054383989626618\end{array}$ $\begin{array}{lllll}0.1462654380708796 & 0.3610040780327081 & 0.3878928429885272\end{array}$ $\begin{array}{lllll}0.1551587358410113 & 0.1206469014500736 & 0.3975473936046875\end{array}$ $\begin{array}{lllll}0.0257512687211489 & 0.1800906156590861 & 0.3996260088992593\end{array}$ $\begin{array}{lllll}0.0268555654387534 & 0.2974635195150314 & 0.3917093513003824\end{array}$ $\begin{array}{lllll}0.0239469804305276 & 0.3479734991791496 & 0.0938900342314569\end{array}$ $\begin{array}{lllll}0.2728977573442418 & 0.2311153179398888 & 0.1017221954529410\end{array}$ $\begin{array}{llllll}0.4031872352553383 & 0.1677761410989244 & 0.1051915907564935\end{array}$ $\begin{array}{lllll}0.3963811535055997 & 0.3615219183176518 & 0.3875167141250967\end{array}$ $\begin{array}{llll}0.4048162889116028 & 0.1205108234933085 & 0.3972785536368705\end{array}$ $\begin{array}{lllll}0.2758700944319538 & 0.1805952958001677 & 0.3994204657945140\end{array}$ $\begin{array}{lllll}0.2768746207058629 & 0.2980373621322147 & 0.3914771602775353\end{array}$ $\begin{array}{llll}0.2741134076062418 & 0.3484761243579599 & 0.0935959346380109\end{array}$ $\begin{array}{lllll}0.1446024049418986 & 0.4079251167611067 & 0.0955610005963970\end{array}$ $\begin{array}{lllll}0.3949480127394367 & 0.4082843382223138 & 0.0957528920848717\end{array}$ $\begin{array}{lllll}0.0226840609713214 & 0.2308733371654379 & 0.6016737010343177\end{array}$ $\begin{array}{lllll}0.1536586591786454 & 0.1684908118970342 & 0.6050894820225204\end{array}$ $\begin{array}{lllll}0.1457451966484103 & 0.3615959889069457 & 0.8870937967260383\end{array}$ $\begin{array}{llll}0.1557734326509325 & 0.1210304236390369 & 0.8976493200961139\end{array}$ $\begin{array}{lllll}0.0259287438597803 & 0.1799173961738924 & 0.9000396454132970\end{array}$ $\begin{array}{lllll}0.0271315945446870 & 0.2974475162672712 & 0.8917142858286556\end{array}$ $\begin{array}{lllll}0.0227607935343513 & 0.3484936593654557 & 0.5930717682359664\end{array}$ $\begin{array}{llll}0.2729956340828188 & 0.2321135379803830 & 0.6010612793006624\end{array}$ $\begin{array}{lllll}0.4038321040287162 & 0.1696767127662942 & 0.6048073362580799\end{array}$ $\begin{array}{lllll}0.3960060420103592 & 0.3625424961464790 & 0.8867300696738390\end{array}$ $\begin{array}{llll}0.4052630796883556 & 0.1229655742448705 & 0.8971352960028991\end{array}$ $\begin{array}{lllll}0.2757919829419060 & 0.1819614047884194 & 0.8991422085467553\end{array}$ $\begin{array}{llll}0.2764766110198247 & 0.2991755375164441 & 0.8905196616598433\end{array}$ $\begin{array}{lllll}0.2738252079759019 & 0.3495364320718231 & 0.5925704919758006\end{array}$ $\begin{array}{llllll}0.1439491433284667 & 0.4082063767596177 & 0.5950008901868546\end{array}$ $\begin{array}{llll}0.3943436413587756 & 0.4100403974881535 & 0.5937718396354029\end{array}$ 
$\begin{array}{llll}0.5229099868587346 & 0.2309581329155466 & 0.1017063958290390\end{array}$ $\begin{array}{llll}0.6532204897816460 & 0.1675213520045221 & 0.1054953874155087\end{array}$ $\begin{array}{llll}0.6467110439807260 & 0.3597668972894628 & 0.3875940194957875\end{array}$ $\begin{array}{llll}0.6542692814191140 & 0.1202921813572383 & 0.3971791251883158\end{array}$ $\begin{array}{llll}0.5253766281433107 & 0.1803770217521642 & 0.3993056090979686\end{array}$ $\begin{array}{llll}0.5264041313643083 & 0.2975810321314327 & 0.3914895838903234\end{array}$ $\begin{array}{llll}0.5241754759770720 & 0.3483662916507361 & 0.0939854236500559\end{array}$ $\begin{array}{llll}0.7730159783650284 & 0.2306318045555938 & 0.1019436851974971\end{array}$ $\begin{array}{llll}0.9033838341084458 & 0.1673478734207826 & 0.1056110524711991\end{array}$ $\begin{array}{llll}0.8963460526374233 & 0.3603841689931459 & 0.3883291363040533\end{array}$ $\begin{array}{llll}0.9051942166939881 & 0.1198013614082087 & 0.3974865297673340\end{array}$ $\begin{array}{llll}0.7756486710343963 & 0.1792043909248519 & 0.3995960932372475\end{array}$ $\begin{array}{llll}0.7771320988134806 & 0.2966173352131954 & 0.3920182714993325\end{array}$ $\begin{array}{lllll}0.7740786320023693 & 0.3479986724367087 & 0.0941628510911195\end{array}$ $\begin{array}{llll}0.6451025752463848 & 0.4080085590337842 & 0.0962324669877710\end{array}$ $\begin{array}{llll}0.8948136041875661 & 0.4077957027000063 & 0.0961481810301194\end{array}$ $\begin{array}{llll}0.5229959907511215 & 0.2334862885345253 & 0.6010546977100093\end{array}$ $\begin{array}{llll}0.6525892381976434 & 0.1685935720848225 & 0.6052120025261526\end{array}$ $\begin{array}{lllll}0.6468145010793218 & 0.3635516487120310 & 0.8868087059538539\end{array}$ $\begin{array}{lllll}0.6543626934294472 & 0.1215825986664978 & 0.8971440077254597\end{array}$ $\begin{array}{llll}0.5260584199354098 & 0.1827241588558000 & 0.8987011943650113\end{array}$ $\begin{array}{llll}0.5270596627857065 & 0.3002713685018921 & 0.8909063873088056\end{array}$ $\begin{array}{llll}0.5245860629186176 & 0.3510305745821525 & 0.5928739421746495\end{array}$ $\begin{array}{llll}0.7737757056767568 & 0.2299794370366433 & 0.6020688866006366\end{array}$ $\begin{array}{llll}0.9039320184123896 & 0.1667192619955130 & 0.6059456327665796\end{array}$ $\begin{array}{llll}0.8968559886063144 & 0.3606863617687506 & 0.8884873861019540\end{array}$ $\begin{array}{llll}0.9043612288924645 & 0.1208766512191032 & 0.8973750652733707\end{array}$ $0.77507797389572990 .1809597616142805 \quad 0.8991490459108576$ $\begin{array}{lllll}0.7765041063743822 & 0.2981335003820412 & 0.8912098480372939\end{array}$ $\begin{array}{llll}0.7743871096642391 & 0.3465322984044165 & 0.5951145716577693\end{array}$ $\begin{array}{llll}0.6467530835708257 & 0.4071824463311191 & 0.5969564737881988\end{array}$ $\begin{array}{llll}0.8936275069041786 & 0.4080782364386288 & 0.5960035325938935\end{array}$

Ade/Gua :

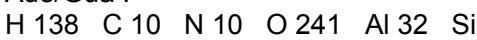

1.00000000000000

$20.2970019999999991 \quad 0.0000000000000000-3.7471719999999999$

$\begin{array}{llll}0.0646860000000000 & 24.9946149999999996 & -0.5148360000000000\end{array}$

$\begin{array}{llll}0.0000000000000000 & 0.0000000000000000 & 18.6939999999999991\end{array}$ $\mathrm{H} \mathrm{C} \mathrm{N} \mathrm{O} \mathrm{Al} \mathrm{Si}$

$\begin{array}{llllll}138 & 10 & 10 & 241 & 32 & 64\end{array}$

Direct

$\begin{array}{llll}0.8212258367691191 & 0.7494713979202459 & 0.7795413077226842\end{array}$

$\begin{array}{llll}0.8836548318925538 & 0.5864840673068803 & 0.6412609681701952\end{array}$

$\begin{array}{llll}0.8404541755238398 & 0.5272580043552058 & 0.6547445344204705\end{array}$ $\begin{array}{llll}0.7329007764514146 & 0.7626683920308411 & 0.8601131661226292\end{array}$ $\begin{array}{lllll}0.7640614707329967 & 0.5269186387047806 & 0.7177845606775364\end{array}$ $0.0937146434594190 \quad 0.0557788094196395 \quad 0.4119053735817727$ $\begin{array}{llll}0.3441207933982291 & 0.0559278309217332 & 0.4119348314801770\end{array}$ $\begin{array}{llll}0.0511016505760149 & 0.1397865915504644 & 0.1844984831175999\end{array}$ $\begin{array}{llll}0.1704073908071124 & 0.3088486352809481 & 0.1633538413573455\end{array}$ $\begin{array}{llll}0.1298791074809548 & 0.2210246176960352 & 0.3357370359825584\end{array}$ $\begin{array}{llll}0.2440224328959806 & 0.0400364780118035 & 0.3466081662162481\end{array}$ $\begin{array}{llll}0.1714051638739223 & 0.0674182689576180 & 0.1498733907212658\end{array}$ $\begin{array}{lllll}0.3011346148225379 & 0.1397899487224807 & 0.1842272928297328\end{array}$ 0.42058169925475650 .30929710429083750 .1635390166042838 $\begin{array}{lllll}0.3797203636896594 & 0.2211072844522885 & 0.3352223215670594\end{array}$ $\begin{array}{llll}0.4936828414298513 & 0.0401701290251878 & 0.3460768263288480\end{array}$ $\begin{array}{lllll}0.4220819090999636 & 0.0679173220637809 & 0.1495873333963771\end{array}$ $\begin{array}{lllll}0.5562082228603832 & 0.4876839932605390 & 0.1470840048329439\end{array}$ $\begin{array}{llll}0.2494947886317770 & 0.3903425805070289 & 0.3140802762365395\end{array}$ $\begin{array}{llll}0.1294574240350803 & 0.4624754217372253 & 0.3494603327919933\end{array}$ $\begin{array}{llll}0.3062989347682173 & 0.4901375252388666 & 0.1518839492858828\end{array}$ $\begin{array}{llll}-0.0003135446376033 & 0.3903243890607116 & 0.3148182270783383\end{array}$ $\begin{array}{lllll}0.3784096374172955 & 0.4624272377843362 & 0.3478952593123428\end{array}$ $\begin{array}{lllll}0.2067346538671604 & 0.4741079959671408 & 0.0868950770572189\end{array}$ $\begin{array}{lllll}0.4548824260977011 & 0.4750767667179903 & 0.0858274740943839\end{array}$ $\begin{array}{llll}0.1540406618886746 & 0.4392416577683781 & 0.4682844591948931\end{array}$ $0.4021483996086320 \quad 0.43892797837148680 .4666083246274972$ $\begin{array}{lllll}0.1463348543678741 & 0.0909535462471771 & 0.0308594741041409\end{array}$ $\begin{array}{llll}0.3972272286744176 & 0.0913739017044808 & 0.0307546981717569\end{array}$ $\begin{array}{lllll}0.1653834044488857 & 0.4822852294045765 & 0.2865858289916477\end{array}$ $0.4146780359808496 \quad 0.4821273511184373 \quad 0.2851720380360410$ 
$0.13631683625277790 .0471695469183444 \quad 0.2132298589003860$ $\begin{array}{llllll}0.3860528608586497 & 0.0478996263420859 & 0.2122693417718390\end{array}$ $\begin{array}{lllll}0.2119463927363579 & 0.4167943447369514 & 0.2396653641609658\end{array}$ $\begin{array}{llll}0.4613473734006672 & 0.4165289540398751 & 0.2391089991396684\end{array}$ $\begin{array}{lllll}0.3385430223541453 & 0.1134539094865985 & 0.2587614854094764\end{array}$ $\begin{array}{lllll}0.0884693161530816 & 0.1132408359196442 & 0.2588815268320648\end{array}$ $\begin{array}{lllll}0.0941081924460111 & 0.0556785555114149 & 0.9116559115641304\end{array}$ $\begin{array}{lllll}0.3443938678392396 & 0.0561322279421750 & 0.9119372663218621\end{array}$ $0.05086940913266990 .1406013668048273 \quad 0.6842771615657726$ $\begin{array}{lllll}0.1695661732202149 & 0.3092626111447889 & 0.6624256709496300\end{array}$ $\begin{array}{lllll}0.1302478489009205 & 0.2216436414116746 & 0.8357536982977053\end{array}$ $\begin{array}{lllll}0.2441146356566028 & 0.0407496999067481 & 0.8464234798214137\end{array}$ $\begin{array}{lllll}0.1715412363092022 & 0.0682774776318399 & 0.6491790345304270\end{array}$ $\begin{array}{lllll}0.3012694602752674 & 0.1404808454616269 & 0.6839080551024457\end{array}$ $\begin{array}{lllll}0.4183006881814542 & 0.3071926331273628 & 0.6582559465174243\end{array}$ $\begin{array}{lllll}0.3804626903522552 & 0.2224142135955223 & 0.8355426033295962\end{array}$ $\begin{array}{llllll}0.4932761841336654 & 0.0407071281951604 & 0.8459186909217765\end{array}$ $\begin{array}{llllll}0.4217782105945844 & 0.0684311578313100 & 0.6493279782408498\end{array}$ $\begin{array}{llll}0.4974649746550831 & 0.4828587001402939 & 0.6923594449526801\end{array}$ $\begin{array}{lllll}0.2488528721984947 & 0.3898924245184267 & 0.8141507459019494\end{array}$ $\begin{array}{lllll}0.1292637452527214 & 0.4629644735645491 & 0.8491639724240925\end{array}$ $\begin{array}{llllll}0.3044327443406894 & 0.4918143613095971 & 0.6538502010682082\end{array}$ $\begin{array}{lllll}0.0013720399024296 & 0.3924690590961503 & 0.8187322229405899\end{array}$ $\begin{array}{llll}0.3750317489187125 & 0.4626607061954263 & 0.8501566469821178\end{array}$ $\begin{array}{llllll}0.2059588520125064 & 0.4747050833891814 & 0.5876094037882842\end{array}$ $\begin{array}{lllll}0.4592673970581141 & 0.4723268928615389 & 0.5769777411754720\end{array}$ $\begin{array}{llll}0.1539208923414432 & 0.4390935194168998 & 0.9679959698191768\end{array}$ $\begin{array}{lllll}0.4028397870487752 & 0.4394716621528840 & 0.9679106062218814\end{array}$ $\begin{array}{lllll}0.1460314723693240 & 0.0915261192314579 & 0.5306744922738842\end{array}$ 0.39668785838498930 .09179905294369220 .5307052858499304 $\begin{array}{lllll}0.1661450881045433 & 0.4817306993729275 & 0.7866784518896308\end{array}$ $\begin{array}{lllll}0.4140997659400782 & 0.4808842960382276 & 0.7900085830318717\end{array}$ $\begin{array}{lllll}0.1366377132341426 & 0.0475538958688084 & 0.7124930272710648\end{array}$ $\begin{array}{lllll}0.3861388425823897 & 0.0481042422754311 & 0.7121917230854152\end{array}$ $\begin{array}{llll}0.2110037363841970 & 0.4164204858650638 & 0.7397580683104629\end{array}$ $\begin{array}{lllll}0.4618875824808260 & 0.4127736691941342 & 0.7433874057204616\end{array}$ $\begin{array}{lllll}0.3385532607229075 & 0.1139378174800073 & 0.7583573726691498\end{array}$ $\begin{array}{lllll}0.0883058841647367 & 0.1136619148635825 & 0.7582961055360546\end{array}$ $\begin{array}{lllll}0.7188270538811204 & 0.5583427037870660 & 0.4990063666357870\end{array}$ $\begin{array}{lllll}0.5931362202714828 & 0.0566797995367097 & 0.4121150140885046\end{array}$ $\begin{array}{llll}0.8427274234564722 & 0.0566634952679596 & 0.4116562249436361\end{array}$ $\begin{array}{lllll}0.5512816635175901 & 0.1404257138642180 & 0.1847410085670035\end{array}$ $\begin{array}{lllll}0.6701793808135812 & 0.3087389090871905 & 0.1624234438572074\end{array}$ $\begin{array}{llll}0.6300906535257798 & 0.2219220374248423 & 0.3362494913740866\end{array}$ $\begin{array}{lllll}0.7435904134478427 & 0.0401277023305809 & 0.3459688668665668\end{array}$ $\begin{array}{lllll}0.6715423264481080 & 0.0680501583117138 & 0.1498150000769362\end{array}$ $\begin{array}{lllll}0.8011626871816078 & 0.1403657022554033 & 0.1847108984454613\end{array}$ $\begin{array}{lllll}0.9200029757522417 & 0.3083625686847041 & 0.1626223117252652\end{array}$ $\begin{array}{lllll}0.8805164802006030 & 0.2220107902618813 & 0.3373187604358496\end{array}$ $\begin{array}{lllll}0.9935556539881324 & 0.0403450320285630 & 0.3470134441261644\end{array}$ $\begin{array}{lllll}0.9214418232549461 & 0.0676918591340993 & 0.1499640916496587\end{array}$ $\begin{array}{lllll}0.0572947278083137 & 0.4899445231349189 & 0.1525252057434544\end{array}$ $\begin{array}{lllll}0.7492633304871796 & 0.3907251679965597 & 0.3154597298456235\end{array}$ $\begin{array}{lllll}0.6282580541768528 & 0.4622316275109670 & 0.3484075728221639\end{array}$ $\begin{array}{llllll}0.8069462644908159 & 0.4903213320769841 & 0.1530264798639283\end{array}$ $\begin{array}{lllll}0.4989278785053212 & 0.3909115264841372 & 0.3141679657824394\end{array}$ $\begin{array}{lllll}0.8794100963176668 & 0.4629913120634363 & 0.3503334122142724\end{array}$ $\begin{array}{lllll}0.7072549286702181 & 0.4748285469439239 & 0.0886492629031725\end{array}$ $\begin{array}{lllll}0.9576455993936994 & 0.4741977655639301 & 0.0880763788055293\end{array}$ $\begin{array}{llll}0.6506902496426200 & 0.4400396470068197 & 0.4670863173760821\end{array}$ $\begin{array}{llll}0.9050422076041099 & 0.4390417133295949 & 0.4694762012899126\end{array}$ $\begin{array}{lllll}0.6456473155462191 & 0.0915624470665750 & 0.0310500824387552\end{array}$ $\begin{array}{lllll}0.8964641963974961 & 0.0911645269559986 & 0.0312496484346445\end{array}$ $\begin{array}{lllll}0.6614985904949688 & 0.4848964694813220 & 0.2844043821444212\end{array}$ $\begin{array}{lllll}0.9146854182556544 & 0.4831421656451754 & 0.2871069903204328\end{array}$ $\begin{array}{lllll}0.6358607475271170 & 0.0480115701548677 & 0.2127618196322731\end{array}$ $\begin{array}{llll}0.8858155108106311 & 0.0476138393795985 & 0.2129691006222076\end{array}$ $\begin{array}{lllll}0.7120844516941139 & 0.4173436380194221 & 0.2411449191980328\end{array}$ $\begin{array}{lllll}0.9623089218459893 & 0.4172020637481204 & 0.2406931821987091\end{array}$ $\begin{array}{lllll}0.8384275441013125 & 0.1136205062971098 & 0.2589961016928662\end{array}$ $\begin{array}{lllll}0.5885779652213629 & 0.1137136989796270 & 0.2590402244547401\end{array}$ $\begin{array}{lllll}0.7153813878679167 & 0.7455569648060927 & 0.5770018234268929\end{array}$ $\begin{array}{lllll}0.6363267949521637 & 0.7706106447128914 & 0.6644350931830517\end{array}$ 
$\begin{array}{llll}0.5675613166748235 & 0.5282672847261261 & 0.6491442375622596\end{array}$ $\begin{array}{lllll}0.5596091037936048 & 0.5923831449594523 & 0.6863628033308843\end{array}$ $\begin{array}{lllll}0.5921927547640314 & 0.0570930234612300 & 0.9114599386899681\end{array}$ $\begin{array}{lllll}0.8438463291892100 & 0.0555342722726014 & 0.9116592446415087\end{array}$ $\begin{array}{llll}0.5514737122559943 & 0.1404332076783239 & 0.6843224288502761\end{array}$ $\begin{array}{lllll}0.6703330225465752 & 0.3094808632204573 & 0.6630012861912994\end{array}$ $\begin{array}{lllll}0.6305886363645608 & 0.2229876411649271 & 0.8376059977601182\end{array}$ $\begin{array}{lllll}0.7428165296170315 & 0.0416957218021196 & 0.8487560077925191\end{array}$ $\begin{array}{llll}0.6715728809878424 & 0.0682878736851061 & 0.6496826980330360\end{array}$ $\begin{array}{lllll}0.8010594935291921 & 0.1409911070461428 & 0.6856103171831610\end{array}$ $\begin{array}{lllll}0.9218826523576784 & 0.3118004780980013 & 0.6685864527052184\end{array}$ $\begin{array}{lllll}0.8802815422292835 & 0.2213600608800346 & 0.8370480665389660\end{array}$ $\begin{array}{lllll}0.9936781460318533 & 0.0403872146204253 & 0.8463778973671983\end{array}$ $\begin{array}{lllll}0.9209346062292304 & 0.0684302433501412 & 0.6493364877704771\end{array}$ $\begin{array}{llll}0.0564481893597981 & 0.4918502028226275 & 0.6559107344775763\end{array}$ $\begin{array}{lllll}0.7467685945270273 & 0.3900043994505327 & 0.8124948832829525\end{array}$ $\begin{array}{lllll}0.6275931583882716 & 0.4649365984391898 & 0.8507847065937697\end{array}$ $\begin{array}{lllll}0.7601279644796943 & 0.4619149890356333 & 0.6267199859246498\end{array}$ $\begin{array}{llll}0.5006919480131991 & 0.3930152233610698 & 0.8214118974844252\end{array}$ $\begin{array}{llllll}0.8818210568784571 & 0.4623358535117154 & 0.8512037022521137\end{array}$ $\begin{array}{lllll}0.6516096372854577 & 0.5039542133323990 & 0.5597828941196273\end{array}$ $\begin{array}{lllll}0.9580925364124037 & 0.4740656378271337 & 0.5914252198321107\end{array}$ $\begin{array}{lllll}0.6561147877809854 & 0.4396769617155502 & 0.9702150959149480\end{array}$ $\begin{array}{llll}0.9052454459428387 & 0.4391259165936321 & 0.9695225516514716\end{array}$ $\begin{array}{llll}0.6462284463650675 & 0.0917640573100525 & 0.5311233767835173\end{array}$ $\begin{array}{lllll}0.8950785468756919 & 0.0917314460405046 & 0.5308337058866539\end{array}$ $\begin{array}{llll}0.6450694349925540 & 0.5061504018254177 & 0.7902332369654228\end{array}$ $\begin{array}{lllll}0.9122779264252919 & 0.4870460558943223 & 0.7857150746584312\end{array}$ $\begin{array}{llll}0.6358653441802767 & 0.0482320071042538 & 0.7126507951942936\end{array}$ $\begin{array}{llll}0.8856193258003859 & 0.0483444184221526 & 0.7125122713050542\end{array}$ $\begin{array}{lllll}0.7138865759723576 & 0.4233109768128098 & 0.7433359543174616\end{array}$ $\begin{array}{llll}0.9628816990014555 & 0.4165352282355807 & 0.7427677113281234\end{array}$ $\begin{array}{lllll}0.8385426579381202 & 0.1135743003640608 & 0.7592214167954975\end{array}$ $\begin{array}{llll}0.5886056680729040 & 0.1137816526841543 & 0.7588332243788626\end{array}$ $\begin{array}{lllll}0.7419563767536467 & 0.7251181022962779 & 0.8331888551353720\end{array}$ $\begin{array}{lllll}0.8165437267087556 & 0.5952569223384095 & 0.7079303630940004\end{array}$ $\begin{array}{llll}0.7890540414254636 & 0.6685244252360178 & 0.7649353579435183\end{array}$ $\begin{array}{lllll}0.7395245916514251 & 0.6439226450876189 & 0.7951634858684953\end{array}$ $\begin{array}{lllll}0.7260327071242058 & 0.5887123901895018 & 0.7775071724768037\end{array}$ $\begin{array}{llllll}0.6416762736746234 & 0.7305426477053397 & 0.6424886010247963\end{array}$ $\begin{array}{llll}0.6948414560733885 & 0.5856129012566402 & 0.5326612064431470\end{array}$ $\begin{array}{llll}0.6797474230767795 & 0.6657593870650981 & 0.5786890745384506\end{array}$ $\begin{array}{lllll}0.6326943441758135 & 0.6471574886838455 & 0.6175943574064923\end{array}$ $\begin{array}{lllll}0.6183095952563191 & 0.5917536557582002 & 0.6118438047329126\end{array}$ $\begin{array}{llll}0.8536580930263743 & 0.5662886852838385 & 0.6684322951845046\end{array}$ $\begin{array}{lllll}0.8283259436693533 & 0.6470957894109491 & 0.7215454639112263\end{array}$ $\begin{array}{lllll}0.7895868775296844 & 0.7207896760834526 & 0.7895484524124771\end{array}$ $\begin{array}{lllll}0.7108910905644941 & 0.6799180050592737 & 0.8374680516029480\end{array}$ $\begin{array}{llll}0.7679745814138865 & 0.5674452291997709 & 0.7330844041253652\end{array}$ $\begin{array}{llll}0.7123814303529619 & 0.6367945146969718 & 0.5349873919791814\end{array}$ $\begin{array}{llll}0.6848528891771491 & 0.7195903975860941 & 0.5955181107158900\end{array}$ $\begin{array}{lllll}0.6092563996379278 & 0.6879957252231843 & 0.6569131086813351\end{array}$ $\begin{array}{lllll}0.6509232343431915 & 0.5621397567728579 & 0.5677727269747822\end{array}$ $\begin{array}{lllll}0.5750419901126967 & 0.5692301987906792 & 0.6473172041083343\end{array}$ $\begin{array}{llll}0.6823747450846233 & 0.5606709629642124 & 0.7966178828738965\end{array}$ $\begin{array}{llll}0.0466784038894225 & 0.3504179592789404 & 0.1857787590083454\end{array}$ $\begin{array}{lllll}0.0301763762527412 & 0.2413149012914139 & 0.1933526888765065\end{array}$ $\begin{array}{lllll}0.1553107914862062 & 0.2824910842493825 & 0.1940276048948788\end{array}$ $\begin{array}{lllll}0.1999707194600463 & 0.2076559541290832 & 0.0714696726396649\end{array}$ $\begin{array}{llll}0.0344157034532203 & 0.2881083760051225 & 0.0677402010598781\end{array}$ $0.07653429810102750 .1864868968234574 \quad 0.0886055067353532$ $\begin{array}{lllll}0.1802689858233940 & 0.1682800952706218 & 0.1965854175463540\end{array}$ $\begin{array}{lllll}0.1796967642236128 & 0.0554286626784708 & 0.2023817704877191\end{array}$ $\begin{array}{lllll}0.0468990718642662 & 0.1102986550581841 & 0.2168423135822258\end{array}$ $\begin{array}{llllll}0.1583979622768297 & 0.1051138603256616 & 0.0816959379170365\end{array}$ $\begin{array}{lllll}0.1491246596485958 & 0.1152275506602014 & 0.3131116768517759\end{array}$ $\begin{array}{lllll}0.0210532134151191 & 0.0683515475545621 & 0.3333183754563457\end{array}$ $\begin{array}{lllll}0.1379828389783543 & 0.0659140947650473 & 0.4401056269850811\end{array}$ $\begin{array}{lllll}0.1044245650218939 & 0.1674476006157512 & 0.4223460612588744\end{array}$ $\begin{array}{llllll}0.0204470106959794 & 0.2889645586067123 & 0.3063553262479185\end{array}$ $\begin{array}{lllll}0.1450319129354626 & 0.2473377351376321 & 0.3050461008265248\end{array}$ $\begin{array}{lllll}0.1004078499611857 & 0.3222104241739461 & 0.4277616396836670\end{array}$ $\begin{array}{llll}0.0163863191661492 & 0.2419946011885420 & 0.4319110699700462\end{array}$ 
$\begin{array}{llll}0.1204496765523267 & 0.3616224940926614 & 0.3027241228738623\end{array}$ $\begin{array}{lllll}0.2292789099816789 & 0.1414678992987949 & 0.4392253695382983\end{array}$ $\begin{array}{lllll}0.2541205082481037 & 0.1796610194225768 & 0.3135870936130399\end{array}$ $\begin{array}{lllll}0.2238491765920442 & 0.3434639522533806 & 0.4109605614642212\end{array}$ $\begin{array}{lllll}0.2958507022432107 & 0.3502053597311333 & 0.1847723325032825\end{array}$ $\begin{array}{lllll}0.2800348752990341 & 0.2410861963465585 & 0.1928676876007477\end{array}$ $\begin{array}{lllll}0.4051745302654334 & 0.2826367050411743 & 0.1935520607037046\end{array}$ $\begin{array}{lllll}0.4500288384753829 & 0.2084844506769424 & 0.0714258953436328\end{array}$ $\begin{array}{lllll}0.2838820981835516 & 0.2877505565678737 & 0.0670249895557564\end{array}$ $\begin{array}{llll}0.3267052610480000 & 0.1863744498390952 & 0.0882713862712818\end{array}$ $\begin{array}{lllll}0.4304549012288005 & 0.1686845792567536 & 0.1964464300898048\end{array}$ $\begin{array}{lllll}0.4297489334200399 & 0.0558939011731738 & 0.2020933878771421\end{array}$ $\begin{array}{llllll}0.2970248449053394 & 0.1103176711652601 & 0.2166306176757584\end{array}$ $\begin{array}{lllll}0.4095330946946777 & 0.1056311883410615 & 0.0815267771506703\end{array}$ $\begin{array}{llll}0.3991101280081406 & 0.1156462163699250 & 0.3129666360880546\end{array}$ $\begin{array}{lllll}0.2712420282573015 & 0.0683705191369099 & 0.3332450177005541\end{array}$ $\begin{array}{lllll}0.3883776312047307 & 0.0661847430145340 & 0.4400114270588115\end{array}$ $\begin{array}{lllll}0.3544397394150371 & 0.1676432105484929 & 0.4222520087202861\end{array}$ $\begin{array}{llll}0.2700649162163075 & 0.2888466228162940 & 0.3057635540668204\end{array}$ $\begin{array}{llll}0.3948077270358711 & 0.2475155598739225 & 0.3046166047926655\end{array}$ $\begin{array}{lllll}0.3503586858853424 & 0.3220562647214137 & 0.4269273679104215\end{array}$ $\begin{array}{lllll}0.2662004279328887 & 0.2419996750191810 & 0.4315181880640542\end{array}$ $\begin{array}{lllll}0.3698784062416869 & 0.3613742529880209 & 0.3014164597372828\end{array}$ $\begin{array}{lllll}0.4793486560182176 & 0.1419716513160917 & 0.4391518620804908\end{array}$ $\begin{array}{llllll}0.0040842892483731 & 0.1797622060666789 & 0.3138791771862626\end{array}$ $\begin{array}{lllll}0.4734581554907307 & 0.3440698535120645 & 0.4101717275150609\end{array}$ $\begin{array}{llll}0.1516202903834150 & 0.4145899134854805 & 0.1858125489278423\end{array}$ $\begin{array}{lllll}0.0299091190732942 & 0.4618586629094090 & 0.1662262623651431\end{array}$ $\begin{array}{lllll}0.0709670898498070 & 0.3886681548864248 & 0.0599260715708626\end{array}$ $\begin{array}{lllll}0.1623688571986286 & 0.4640556906470826 & 0.05877775651743963\end{array}$ $\begin{array}{lllll}0.1957011297330592 & 0.3623738215777149 & 0.0762029165691438\end{array}$ $\begin{array}{lllll}0.1217055844302986 & 0.4744335076060699 & 0.2969325277543428\end{array}$ $\begin{array}{lllll}0.0040102371852621 & 0.4199654251713110 & 0.2827730780722842\end{array}$ $\begin{array}{lllll}0.1419184699957219 & 0.4248445960071296 & 0.4175756255032501\end{array}$ $\begin{array}{llll}0.4010653647330792 & 0.4142315242416468 & 0.1849259779018620\end{array}$ $\begin{array}{lllll}0.2791894901488831 & 0.4615157287314490 & 0.1646933967076356\end{array}$ $\begin{array}{lllll}0.3209274025306443 & 0.3880600393887335 & 0.0589614557814903\end{array}$ $\begin{array}{lllll}0.4107609154965864 & 0.4643921353115197 & 0.0579506412471547\end{array}$ $\begin{array}{lllll}0.4461656712587833 & 0.3630180830684968 & 0.0753203548088715\end{array}$ $\begin{array}{lllll}0.3708901006081142 & 0.4744283095267687 & 0.2953476056308829\end{array}$ $\begin{array}{lllll}0.2535904749764070 & 0.4198836938002929 & 0.2817976299688357\end{array}$ $\begin{array}{lllll}0.3904846539653201 & 0.4247859751574637 & 0.4155897482859284\end{array}$ $\begin{array}{llllll}0.0452038819656153 & 0.3509082383671011 & 0.6854067160411303\end{array}$ $\begin{array}{lllll}0.0298082053245413 & 0.2415800382136755 & 0.6930357716650564\end{array}$ $\begin{array}{lllll}0.1548743966481523 & 0.2830825222785071 & 0.6936252962266222\end{array}$ $\begin{array}{lllll}0.2000126743498135 & 0.2086704665300892 & 0.5711303797765824\end{array}$ $\begin{array}{lllll}0.0334898409335419 & 0.2887091139229442 & 0.5674371570058999\end{array}$ $\begin{array}{lllll}0.0765972121364099 & 0.1873575464391151 & 0.5881602193828508\end{array}$ $\begin{array}{lllll}0.1803133762860163 & 0.1690129975221273 & 0.6961230381667237\end{array}$ $\begin{array}{llllll}0.1799387208609028 & 0.0560391778531064 & 0.7016241946277898\end{array}$ $\begin{array}{lllll}0.0466881998676282 & 0.1108953819702778 & 0.7162841680248045\end{array}$ $\begin{array}{lllll}0.1585409428332534 & 0.1059966867657736 & 0.5813239995637075\end{array}$ $\begin{array}{llll}0.1490661382327118 & 0.1155016347844565 & 0.8125192065657753\end{array}$ $\begin{array}{lllll}0.0210684144055797 & 0.0684429416821654 & 0.8326156595431387\end{array}$ $\begin{array}{lllll}0.1383553983703082 & 0.0659785866856135 & 0.9396183794789286\end{array}$ $\begin{array}{llll}0.1043261267549405 & 0.1674494735979550 & 0.9219355704789709\end{array}$ $\begin{array}{lllll}0.0202884418310095 & 0.2888823990767443 & 0.8061822545962660\end{array}$ $\begin{array}{lllll}0.1449884262086531 & 0.2478332493095263 & 0.8046049143401108\end{array}$ $\begin{array}{lllll}0.1008925596939966 & 0.3218375483495948 & 0.9273465767831633\end{array}$ $\begin{array}{llll}0.0163825744194615 & 0.2419155201977236 & 0.9317697707058376\end{array}$ $\begin{array}{llll}0.1201859439445060 & 0.3616723481609156 & 0.8023262107021234\end{array}$ $\begin{array}{llll}0.2292054798055915 & 0.1417959531336619 & 0.9386201222752262\end{array}$ $\begin{array}{llllll}0.2540282300123884 & 0.1801954780509077 & 0.8130731213109968\end{array}$ $\begin{array}{lllll}0.2241249636242174 & 0.3439787132994313 & 0.9103420848075425\end{array}$ $\begin{array}{lllll}0.2964661356765941 & 0.3512762333106249 & 0.6847630421907863\end{array}$ $\begin{array}{lllll}0.2799442597770361 & 0.2419914691241691 & 0.6925487147444901\end{array}$ $\begin{array}{lllll}0.4052998430924547 & 0.2831585675945775 & 0.6933390625272835\end{array}$ $\begin{array}{llll}0.4501812296487783 & 0.2092558319855461 & 0.5716125398218079\end{array}$ $\begin{array}{llll}0.2843214743002480 & 0.2887999322433034 & 0.5670912153391666\end{array}$ $\begin{array}{llllll}0.3267924025750546 & 0.1872417496786834 & 0.5881227653783277\end{array}$ $\begin{array}{lllll}0.4305856207884415 & 0.1691646124149344 & 0.6962490105115298\end{array}$ $\begin{array}{lllll}0.4296987792353898 & 0.0562714324529138 & 0.7017662361743057\end{array}$ $\begin{array}{llll}0.2970698803314644 & 0.1109437488711280 & 0.7161709178178089\end{array}$ 
$\begin{array}{llll}0.4093236152237258 & 0.1062676670191621 & 0.5813219698051794\end{array}$ $\begin{array}{lllll}0.3990642496761204 & 0.1159755673687449 & 0.8126317282000833\end{array}$ $\begin{array}{lllll}0.2713248697939127 & 0.0689429048968449 & 0.8326805520198252\end{array}$ $\begin{array}{llll}0.3886226220256555 & 0.0665513848659749 & 0.9398082331503835\end{array}$ $\begin{array}{llll}0.3543724017536429 & 0.1680341419346761 & 0.9218376657133722\end{array}$ $\begin{array}{llllll}0.2702295493636320 & 0.2894166382505701 & 0.8053810282480789\end{array}$ $\begin{array}{lllll}0.3953113689234752 & 0.2486122157724762 & 0.8044783466698231\end{array}$ $\begin{array}{llllll}0.3508039220128025 & 0.3219619507401187 & 0.9266340803566949\end{array}$ $\begin{array}{lllll}0.2660365012896938 & 0.2423678281206777 & 0.9309793731808375\end{array}$ $\begin{array}{lllll}0.3699284269480367 & 0.3624251570067263 & 0.8017590920284463\end{array}$ $\begin{array}{lllll}0.4793141681693284 & 0.1424075679485274 & 0.9387517574857367\end{array}$ $\begin{array}{lllll}0.0038490132131002 & 0.1798071548821964 & 0.8135620806525248\end{array}$ $\begin{array}{lllll}0.4740766066355305 & 0.3449998059281665 & 0.9096564513085555\end{array}$ $\begin{array}{llll}0.1510002143424357 & 0.4144606348736976 & 0.6858992537563650\end{array}$ $\begin{array}{llll}0.0295376247294802 & 0.4623789123529592 & 0.6665994457622081\end{array}$ $\begin{array}{lllll}0.0705002686482571 & 0.3890192319374452 & 0.5598229330810890\end{array}$ $\begin{array}{lllll}0.1617748855915139 & 0.4645533989928404 & 0.5591099368892574\end{array}$ $\begin{array}{lllll}0.1956230814813535 & 0.3630482430696507 & 0.5764077756509364\end{array}$ $\begin{array}{lllll}0.1220943396012271 & 0.4747790333223832 & 0.7966417489380128\end{array}$ $\begin{array}{lllll}0.0048365863497801 & 0.4207153224835104 & 0.7843178439676208\end{array}$ $\begin{array}{lllll}0.1414388477333868 & 0.4247824075883723 & 0.9172429899727608\end{array}$ $\begin{array}{lllll}0.4002270279682882 & 0.4181526039231914 & 0.6854261195750834\end{array}$ $\begin{array}{llllll}0.2775439930107833 & 0.4628336581498077 & 0.6660277598644456\end{array}$ $\begin{array}{lllll}0.3209110439069001 & 0.3895351343192631 & 0.5595186092349052\end{array}$ $\begin{array}{llll}0.4133317406616344 & 0.4624945117899070 & 0.5551077274647188\end{array}$ $\begin{array}{lllll}0.4454172456059622 & 0.3611202019317685 & 0.5806489924708808\end{array}$ $\begin{array}{lllll}0.3691172938458614 & 0.4751044377266087 & 0.7977614460938091\end{array}$ $\begin{array}{lllll}0.2526075516817685 & 0.4196525278464328 & 0.7820786024221702\end{array}$ $\begin{array}{llll}0.3904878134217812 & 0.4250333182224886 & 0.9171631545985632\end{array}$ $\begin{array}{llllll}0.5458345910060877 & 0.3505204600880054 & 0.1849001403525598\end{array}$ $\begin{array}{lllll}0.5301469721478170 & 0.2415093004276860 & 0.1929004262574824\end{array}$ $\begin{array}{lllll}0.6553416700750548 & 0.2827724974249960 & 0.1938337860065147\end{array}$ $\begin{array}{lllll}0.7002172337262381 & 0.2084917644301524 & 0.0719086815494434\end{array}$ $\begin{array}{lllll}0.5343641949901238 & 0.2884013714776381 & 0.0671645502093268\end{array}$ $\begin{array}{llllll}0.5766888435869354 & 0.1869081534102864 & 0.0882568138117227\end{array}$ $\begin{array}{lllll}0.6802494718989747 & 0.1687693342783624 & 0.1967452298113254\end{array}$ $\begin{array}{lllll}0.6794707336470843 & 0.0559521315871538 & 0.2022938701621367\end{array}$ $\begin{array}{lllll}0.5471097487234859 & 0.1107564933949778 & 0.2168127989459529\end{array}$ $\begin{array}{llll}0.6588844374705100 & 0.1057889575379429 & 0.0817438125055404\end{array}$ $\begin{array}{llll}0.6490435602074968 & 0.1155314558394738 & 0.3133096339773461\end{array}$ $\begin{array}{lllll}0.5210237900034854 & 0.0686113027138910 & 0.3332226798106466\end{array}$ $\begin{array}{lllll}0.6374497088146586 & 0.0666899202350846 & 0.4404317909431376\end{array}$ $\begin{array}{lllll}0.6044662595667676 & 0.1683188714926774 & 0.4221082273480996\end{array}$ $\begin{array}{llll}0.5200466865306382 & 0.2891421106225333 & 0.3057679685374790\end{array}$ $\begin{array}{llll}0.6449321023020018 & 0.2479351760062602 & 0.3049250584203336\end{array}$ $\begin{array}{lllll}0.6001220235883665 & 0.3227870973524126 & 0.4271236879725206\end{array}$ $\begin{array}{llll}0.5160774062246688 & 0.2426173233150760 & 0.4316279665884227\end{array}$ $\begin{array}{lllll}0.6196810325229193 & 0.3616570091041600 & 0.3015244246983131\end{array}$ $\begin{array}{lllll}0.7292726007129476 & 0.1418663257644271 & 0.4394151986546633\end{array}$ $\begin{array}{llllll}0.7538301071524066 & 0.1800945132066981 & 0.3138492681693544\end{array}$ $\begin{array}{lllll}0.7233072753507983 & 0.3441117142359134 & 0.4103021120515825\end{array}$ $\begin{array}{lllll}0.7969674136311153 & 0.3506345240261398 & 0.1861387662040211\end{array}$ $\begin{array}{lllll}0.7802868819608054 & 0.2414636906216728 & 0.1935076199978244\end{array}$ $\begin{array}{llll}0.9054571338534790 & 0.2826303425225291 & 0.1945454797267357\end{array}$ $\begin{array}{lllll}0.9499819807631295 & 0.2082767318909234 & 0.0718958592391993\end{array}$ $\begin{array}{lllll}0.7850409918869188 & 0.2883077278987104 & 0.0681332953255251\end{array}$ $\begin{array}{lllll}0.8266736179804336 & 0.1865245194851345 & 0.0888398088639894\end{array}$ $\begin{array}{lllll}0.9304006768945051 & 0.1684960532955009 & 0.1968726363270225\end{array}$ $\begin{array}{llll}0.9293768637648422 & 0.0556386925819963 & 0.2024418201732903\end{array}$ $\begin{array}{lllll}0.7968964155726768 & 0.1107812899156547 & 0.2168854947222806\end{array}$ $\begin{array}{lllll}0.9091026961465255 & 0.1054859250732244 & 0.0819028548004195\end{array}$ $\begin{array}{llll}0.8990271712743885 & 0.1153671460205419 & 0.3132847230912917\end{array}$ $\begin{array}{llll}0.7709650169955043 & 0.0685875407564924 & 0.3332393430649440\end{array}$ $\begin{array}{lllll}0.8869796906368007 & 0.0666029451001504 & 0.4402619349768960\end{array}$ $\begin{array}{lllll}0.8544008574638925 & 0.1683073413034967 & 0.4220318792388061\end{array}$ $\begin{array}{lllll}0.7704180516514073 & 0.2891732446625428 & 0.3063293788009363\end{array}$ $\begin{array}{lllll}0.8952456647028076 & 0.2477916172272506 & 0.3055809386063061\end{array}$ $\begin{array}{lllll}0.8499863040301214 & 0.3226063934900052 & 0.4282064808760689\end{array}$ $\begin{array}{lllll}0.7658772567203443 & 0.2425109992299658 & 0.4318800500687038\end{array}$ $\begin{array}{lllll}0.8703276723359258 & 0.3620779479825454 & 0.3034222328711071\end{array}$ 0.97913475084269820 .14154701238025050 .4394109129224463 $\begin{array}{lllll}0.5040456386347978 & 0.1800853273959043 & 0.3136060698324369\end{array}$ $\begin{array}{llll}0.9737458128456681 & 0.3434204942218159 & 0.4113185388866633\end{array}$ 
$\begin{array}{llll}0.6512235071815476 & 0.4151307902346258 & 0.1862174407986851\end{array}$ $\begin{array}{lllll}0.5284440921853926 & 0.4613522161589407 & 0.1641646733664362\end{array}$ $\begin{array}{lllll}0.5718219356848261 & 0.3887635190874292 & 0.0596305823037242\end{array}$ $\begin{array}{llll}0.6632992183908794 & 0.4643567387782055 & 0.0600715669983602\end{array}$ $\begin{array}{llll}0.6964499653575713 & 0.3623524032813363 & 0.0777853657564773\end{array}$ $\begin{array}{lllll}0.6188861253458925 & 0.4750943073209916 & 0.2959398402001288\end{array}$ $\begin{array}{lllll}0.5027482687200480 & 0.4201430162666952 & 0.2813193576286730\end{array}$ $\begin{array}{lllll}0.6410411846160844 & 0.4254199278015169 & 0.4151916771127634\end{array}$ $\begin{array}{lllll}0.9017752008007960 & 0.4152574777097913 & 0.1868441110212158\end{array}$ $\begin{array}{lllll}0.7798267483208059 & 0.4620819505794391 & 0.1668165421717555\end{array}$ $\begin{array}{lllll}0.8215165599904796 & 0.3889917217154400 & 0.0606524381890276\end{array}$ $\begin{array}{llll}0.9133752415164672 & 0.4641119675545245 & 0.0597535855971023\end{array}$ $\begin{array}{lllll}0.9460106076996957 & 0.3623339338935251 & 0.0777995186728814\end{array}$ $\begin{array}{lllll}0.8712758841880697 & 0.4748575730398102 & 0.2978527792576965\end{array}$ $\begin{array}{llllll}0.7536610978296199 & 0.4200800805966974 & 0.2829880571559666\end{array}$ $\begin{array}{lllll}0.8922857475627667 & 0.4250483164626940 & 0.4188007139268800\end{array}$ $\begin{array}{lllll}0.5486941713670758 & 0.3520761455851067 & 0.6868389843434243\end{array}$ $\begin{array}{lllll}0.5305112609254062 & 0.2422836015691153 & 0.6932167909390390\end{array}$ $\begin{array}{lllll}0.6558962355747479 & 0.2834036644346958 & 0.6945679092493885\end{array}$ $\begin{array}{llllll}0.6997375503574196 & 0.2089375283821384 & 0.5718970777901653\end{array}$ $\begin{array}{lllll}0.5351992770060452 & 0.2889002251901156 & 0.5682708500557111\end{array}$ $\begin{array}{lllll}0.5765893099417131 & 0.1868872762035223 & 0.5886974058276793\end{array}$ $\begin{array}{lllll}0.6803389331406468 & 0.1689964277742889 & 0.6968795084963573\end{array}$ $\begin{array}{lllll}0.6794994151388333 & 0.0559914298695586 & 0.7021004919323504\end{array}$ $\begin{array}{lllll}0.5471260937623259 & 0.1109397474214371 & 0.7166118706923390\end{array}$ $\begin{array}{lllll}0.6591466373692684 & 0.1060252101066918 & 0.5818428445853273\end{array}$ $\begin{array}{llll}0.6490402396797333 & 0.1153308435971697 & 0.8131685775154004\end{array}$ $\begin{array}{lllll}0.5208140263448738 & 0.0689508443174459 & 0.8328987296171799\end{array}$ $\begin{array}{lllll}0.6364639146864454 & 0.0669848972448844 & 0.9400912793305410\end{array}$ $\begin{array}{llllll}0.6044094166294041 & 0.1689215568047744 & 0.9215361933259471\end{array}$ $\begin{array}{lllll}0.5207098900002917 & 0.2898087089015491 & 0.8057094368534093\end{array}$ $\begin{array}{lllll}0.6452953515340946 & 0.2484080495346172 & 0.8054000398022265\end{array}$ $\begin{array}{lllll}0.6007268320230815 & 0.3226625094139166 & 0.9270239490275984\end{array}$ $\begin{array}{lllll}0.5157018049849715 & 0.2431208676452806 & 0.9310599721863283\end{array}$ $\begin{array}{lllll}0.6221092267000105 & 0.3632304522642664 & 0.8031794783024949\end{array}$ $\begin{array}{lllll}0.7290012540383626 & 0.1415045611296186 & 0.9393296010325911\end{array}$ $\begin{array}{lllll}0.7538786574304633 & 0.1800092466262950 & 0.8139637501250837\end{array}$ $\begin{array}{lllll}0.7244840410951758 & 0.3435905409367355 & 0.9120208622098543\end{array}$ $\begin{array}{lllll}0.7959072411998042 & 0.3508718855177232 & 0.6866174510642967\end{array}$ $\begin{array}{llll}0.7802279780364406 & 0.2417435000669493 & 0.6933551422450465\end{array}$ $\begin{array}{llll}0.9052200211136507 & 0.2832969797903063 & 0.6947467600314224\end{array}$ $\begin{array}{llll}0.9499461262202763 & 0.2083010336832244 & 0.5714069473096414\end{array}$ $\begin{array}{lllll}0.7828701958350923 & 0.2893906420142488 & 0.5675914199407268\end{array}$ $\begin{array}{lllll}0.8264459188263394 & 0.1879562177433294 & 0.5879353952953692\end{array}$ $\begin{array}{lllll}0.9297383956402787 & 0.1690284230581066 & 0.6965145905967709\end{array}$ $\begin{array}{lllll}0.9291828028554480 & 0.0560885294054087 & 0.7017695159611548\end{array}$ $\begin{array}{lllll}0.7969292681216295 & 0.1109021007088803 & 0.7170039940534255\end{array}$ $\begin{array}{lllll}0.9075188643569956 & 0.1060113251244630 & 0.5816007333799215\end{array}$ $\begin{array}{lllll}0.8989846024990289 & 0.1152340170880412 & 0.8130539934515550\end{array}$ $\begin{array}{llll}0.7710315772218739 & 0.0683246460008190 & 0.8330665624477667\end{array}$ $\begin{array}{lllll}0.8880371022933908 & 0.0657572369685607 & 0.9399378943801399\end{array}$ $\begin{array}{lllll}0.8543058222618705 & 0.1673718992812386 & 0.9223726399993968\end{array}$ $\begin{array}{lllll}0.7706141991574499 & 0.2889912720262515 & 0.8069156711449655\end{array}$ $\begin{array}{llll}0.8949139416863429 & 0.2473265426652940 & 0.8055088282997656\end{array}$ $\begin{array}{llll}0.8510648701746807 & 0.3217484881361363 & 0.9286078429521515\end{array}$ $\begin{array}{lllll}0.7664063544673964 & 0.2419957888905405 & 0.9322647526683944\end{array}$ $\begin{array}{llll}0.8703530040464660 & 0.3614683756152082 & 0.8038996105277980\end{array}$ $\begin{array}{llll}0.9791665479607173 & 0.1414046372765036 & 0.9391087452791621\end{array}$ $\begin{array}{lllll}0.5039434314482356 & 0.1805758480464727 & 0.8132377978449605\end{array}$ $\begin{array}{llll}0.9744037563947741 & 0.3434678802777133 & 0.9113884396894376\end{array}$ $\begin{array}{lllll}0.6500546224475855 & 0.4213824756916058 & 0.6832442500531497\end{array}$ $\begin{array}{llll}0.5291606515875454 & 0.4648168616910635 & 0.6674780971965079\end{array}$ $\begin{array}{lllll}0.5685951484090203 & 0.3903403413015937 & 0.5607460175304168\end{array}$ $\begin{array}{lllll}0.6613414323210103 & 0.4620943846736127 & 0.5494519142736817\end{array}$ $\begin{array}{lllll}0.6949335716582404 & 0.3644356114711436 & 0.5786552695363343\end{array}$ $\begin{array}{lllll}0.6154309549758128 & 0.4754478793918053 & 0.7987177476091413\end{array}$ $\begin{array}{llll}0.5026586995186596 & 0.4192354652985235 & 0.7838315811994223\end{array}$ $\begin{array}{llll}0.6427431583417358 & 0.4250888055643492 & 0.9198148325830262\end{array}$ $\begin{array}{lllll}0.9033243994311668 & 0.4120563819189310 & 0.6884377695726774\end{array}$ $\begin{array}{lllll}0.7888076475560936 & 0.4677568293694275 & 0.6734710520248273\end{array}$ $\begin{array}{lllll}0.8197307079314758 & 0.3911667581903085 & 0.5631624077130787\end{array}$ $\begin{array}{llll}0.9131293663077213 & 0.4654133242287375 & 0.5630568021017147\end{array}$ $\begin{array}{llll}0.9450493676055620 & 0.3635337856678701 & 0.5757894251296615\end{array}$ 
$\begin{array}{llll}0.8715081639186909 & 0.4741075385460996 & 0.7986793892353046\end{array}$ $\begin{array}{llll}0.7549388060286474 & 0.4205567430943905 & 0.7836911781501767\end{array}$ $0.8931381190061384 \quad 0.4246433378756305 \quad 0.9188149435513413$ $\begin{array}{lllll}0.2130179588222581 & 0.2342207364684907 & 0.2510383188438991\end{array}$ $\begin{array}{llll}0.0874627710594814 & 0.2958056062458982 & 0.2481992734408485\end{array}$ $\begin{array}{llll}0.2225376217666284 & 0.1133241482913995 & 0.2669960148806307\end{array}$ $\begin{array}{lllll}0.4630832602331855 & 0.2346107198036418 & 0.2509369331586843\end{array}$ $\begin{array}{llll}0.3370902582606862 & 0.2957157786663061 & 0.2474450308918333\end{array}$ $\begin{array}{llll}0.4725287380423080 & 0.1137216035651208 & 0.2669235488622247\end{array}$ $\begin{array}{lllll}0.0783979674529836 & 0.4167431973669019 & 0.2323253613031846\end{array}$ $\begin{array}{llll}0.3277845450326054 & 0.4165940766900098 & 0.2310514789877451\end{array}$ $\begin{array}{llll}0.2129397643754370 & 0.2348684268597579 & 0.7506218412165778\end{array}$ $\begin{array}{llll}0.0869530013488937 & 0.2961087144239385 & 0.7477497788581937\end{array}$ $\begin{array}{lllll}0.2225429460611271 & 0.1138667858645517 & 0.7664466032498670\end{array}$ $\begin{array}{llll}0.4634769209523349 & 0.2353432008974428 & 0.7506988117065412\end{array}$ $\begin{array}{llll}0.3373434891259200 & 0.2965039953383692 & 0.7472227327947828\end{array}$ $\begin{array}{lllll}0.4724499203481676 & 0.1141956801956022 & 0.7665964926935863\end{array}$ $0.0779384023700934 \quad 0.41695273831793990 .7325588422835104$ $\begin{array}{llll}0.3268853121327908 & 0.4177409664346259 & 0.7316728463121323\end{array}$ $\begin{array}{lllll}0.7130342644571575 & 0.2347445237372159 & 0.2512517212493737\end{array}$ $\begin{array}{llll}0.5872243255126584 & 0.2961872691609569 & 0.2476183495021464\end{array}$ $\begin{array}{llll}0.7223623596343958 & 0.1137981654944305 & 0.2671018945595761\end{array}$ $\begin{array}{llll}0.9631538472936775 & 0.2344348607010026 & 0.2514984963130623\end{array}$ $\begin{array}{lllll}0.8375376025871023 & 0.2960724029860207 & 0.2485661894494712\end{array}$ $\begin{array}{llll}0.9724036812463662 & 0.1134352805724240 & 0.2671662553388854\end{array}$ $\begin{array}{llll}0.5773888590648555 & 0.4171114681939767 & 0.2313103314144853\end{array}$ $\begin{array}{lllll}0.8282818569956599 & 0.4170778001276077 & 0.2329147158803468\end{array}$ $\begin{array}{llll}0.7131421989520327 & 0.2348968456913327 & 0.7516363048453464\end{array}$ $\begin{array}{lllll}0.5882252883932011 & 0.2972878945794611 & 0.7487135779977291\end{array}$ $\begin{array}{llll}0.7224352440533055 & 0.1137201880493000 & 0.7671895792512222\end{array}$ $\begin{array}{llll}0.9628117131224158 & 0.2345985833459097 & 0.7513947152788293\end{array}$ $\begin{array}{llll}0.8372965186025880 & 0.2959644526926525 & 0.7489360243669302\end{array}$ $\begin{array}{llll}0.9723010725854192 & 0.1136407656869731 & 0.7667378312873353\end{array}$ $\begin{array}{llll}0.5808210569657881 & 0.4194446538867000 & 0.7338867198139336\end{array}$ $\begin{array}{lllll}0.8300215152865673 & 0.4163118251652124 & 0.7344824161086166\end{array}$ $\begin{array}{llll}0.0233386199286730 & 0.2316136509509412 & 0.1049739751752524\end{array}$ $\begin{array}{llll}0.1535672743727818 & 0.1680855463831895 & 0.1084826237728359\end{array}$ $\begin{array}{llll}0.1468452797983295 & 0.3618607889439698 & 0.3908755378491596\end{array}$ $\begin{array}{lllll}0.1553653095840010 & 0.1213547369552407 & 0.4005952348155756\end{array}$ $\begin{array}{llll}0.0260934149844156 & 0.1811848616582880 & 0.4027348449422366\end{array}$ $\begin{array}{llll}0.0271841472753489 & 0.2986126026970125 & 0.3947475399487730\end{array}$ $0.02443632503971590 .3489092841241404 \quad 0.0969521432044588$ $\begin{array}{llll}0.2731846558750011 & 0.2312768939305632 & 0.1044749645510023\end{array}$ $\begin{array}{llll}0.4039463061737770 & 0.1685262707538806 & 0.1083080930847844\end{array}$ $\begin{array}{lllll}0.3962828869854830 & 0.3618302401652277 & 0.3895129452935481\end{array}$ 0.40552271437671050 .12168762181700920 .4004707385780041 $\begin{array}{lllll}0.2760741096120314 & 0.1811724796744136 & 0.4024620110445778\end{array}$ $0.2770615478252054 \quad 0.2985150771585765 \quad 0.3941799795105385$ $\begin{array}{llll}0.2740250356157824 & 0.3486219829249581 & 0.0959134108652477\end{array}$ $\begin{array}{lllll}0.1450537386748904 & 0.4085982709854361 & 0.0982420099695229\end{array}$ $0.3947436103587824 \quad 0.4085126982504375 \quad 0.0974234064919595$ $\begin{array}{lllll}0.0229682994779339 & 0.2320887295890844 & 0.6046121077874260\end{array}$ $\begin{array}{llll}0.1536021649113140 & 0.1689750363111221 & 0.6080326551953020\end{array}$ $\begin{array}{llll}0.1468928499109200 & 0.3618844689041438 & 0.8904565028189400\end{array}$ $\begin{array}{lllll}0.1553583655546660 & 0.1215219622830318 & 0.9000359252639148\end{array}$ $\begin{array}{llll}0.0259982554555029 & 0.1811331824071801 & 0.9024093743210800\end{array}$ $\begin{array}{llll}0.0274854178222372 & 0.2984580231067688 & 0.8946038984696536\end{array}$ $\begin{array}{llll}0.0232926242185831 & 0.3495197842111120 & 0.5963906158340855\end{array}$ $\begin{array}{llll}0.2732655301045023 & 0.2321794255970522 & 0.6042098148411743\end{array}$ $\begin{array}{llll}0.4039588807645027 & 0.1691698323246283 & 0.6081695052919381\end{array}$ $0.3965606961492572 \quad 0.36228747790309210 .8899182131252462$ $\begin{array}{lllll}0.4054611118147853 & 0.1220872076381628 & 0.9001252196024307\end{array}$ $\begin{array}{llll}0.2759980073359881 & 0.1815367050294497 & 0.9019290026810155\end{array}$ $0.2772725803890553 \quad 0.29885017347710090 .8938447074095169$ $0.2740022641206361 \quad 0.34962221789849850 .5959554203515566$ $0.1444234358087394 \quad 0.4090037149500506 \quad 0.5983080125695758$ $\begin{array}{llll}0.3950480769128841 & 0.4088728812667574 & 0.5985335524263157\end{array}$ $\begin{array}{llll}0.5233275428662527 & 0.2319631560560905 & 0.1044977259985261\end{array}$ 0.65375847882889520 .16871843926795090 .1086137843718445 $\begin{array}{lllll}0.6461977903654824 & 0.3625312337440902 & 0.3896646346570145\end{array}$ $\begin{array}{llll}0.6552562380051047 & 0.1219536207366347 & 0.4007521822865884\end{array}$ $\begin{array}{lllll}0.5260300630604599 & 0.1818243930592261 & 0.4024523206143553\end{array}$ $\begin{array}{llll}0.5269966797606818 & 0.2991181778849996 & 0.3941310161645103\end{array}$ 
$\begin{array}{llll}0.5244762668637988 & 0.3493496771914537 & 0.0960167753477191\end{array}$ $\begin{array}{lllll}0.7735498736056032 & 0.2317941342508287 & 0.1051239333889486\end{array}$ $\begin{array}{lllll}0.9037842728206913 & 0.1684064391280317 & 0.1087655326236448\end{array}$ $\begin{array}{llll}0.8968140432829745 & 0.3620854618606270 & 0.3915491333241456\end{array}$ $\begin{array}{lllll}0.9050726781344097 & 0.1218030711309787 & 0.4007059799981321\end{array}$ $\begin{array}{lllll}0.7759198061934994 & 0.1817560096654331 & 0.4026763868326304\end{array}$ $\begin{array}{llllll}0.7767804508453238 & 0.2991143454557874 & 0.3946295928186266\end{array}$ $\begin{array}{lllll}0.7748714461993798 & 0.3491108391854021 & 0.0972809492041463\end{array}$ $\begin{array}{lllll}0.6456389578275158 & 0.4087992567710851 & 0.0988628296518854\end{array}$ $\begin{array}{llllll}0.8954778851140921 & 0.4089162188109691 & 0.0993534256404130\end{array}$ $\begin{array}{lllll}0.5236833062103347 & 0.2322859474901746 & 0.6049925812105464\end{array}$ $\begin{array}{lllll}0.6537636072648074 & 0.1689133788983924 & 0.6087775873519582\end{array}$ $\begin{array}{llll}0.6478272011700906 & 0.3629617512395515 & 0.8916480695546275\end{array}$ $\begin{array}{llll}0.6548064616795120 & 0.1221095798458178 & 0.9005500957723733\end{array}$ $\begin{array}{llll}0.5259037896277410 & 0.1823258946228129 & 0.9020618505344952\end{array}$ $\begin{array}{lllll}0.5274353420650703 & 0.2995931127582839 & 0.8939787937433994\end{array}$ $\begin{array}{llll}0.5245834364679807 & 0.3493170829548362 & 0.5984550939894091\end{array}$ $\begin{array}{lllll}0.7729540095796610 & 0.2325640401253069 & 0.6048175708913196\end{array}$ $\begin{array}{llll}0.9032089593563611 & 0.1690468115005164 & 0.6083634981371500\end{array}$ $\begin{array}{lllll}0.8972748121123967 & 0.3617233103813712 & 0.8919772783161529\end{array}$ $\begin{array}{lllll}0.9052244291163605 & 0.1213179813549493 & 0.9005590429659456\end{array}$ $\begin{array}{llll}0.7759827242665479 & 0.1812571935122243 & 0.9028139210136983\end{array}$ $\begin{array}{lllll}0.7775585174738424 & 0.2986887828348260 & 0.8953901054416405\end{array}$ $\begin{array}{lllll}0.7730308171124545 & 0.3497872819764080 & 0.5978828725740379\end{array}$ $0.6432796673296555 \quad 0.41182822722088050 .5963414138114497$ $\begin{array}{llll}0.8950674123736347 & 0.4093837963832491 & 0.6005928101534878\end{array}$

Ade/Thy :

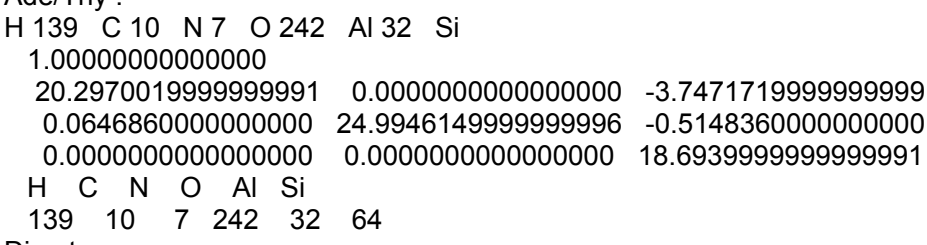

0.72696003113080850 .74211232564830600 .4055530112947302 $\begin{array}{lllll}0.8255939428481321 & 0.7542208532877240 & 0.3408896105014804\end{array}$ $\begin{array}{lllll}0.6089203192124488 & 0.5724205656124346 & 0.3614436966372325\end{array}$ $\begin{array}{lllll}0.7638953913558567 & 0.5247921400303286 & 0.2144540229498097\end{array}$ $\begin{array}{lllll}0.8095123520022355 & 0.5839520799209440 & 0.2232066314813662\end{array}$ $\begin{array}{lllll}0.0977897433039313 & 0.0580013445283374 & 0.4108924238544837\end{array}$ $\begin{array}{llll}0.3478439887270103 & 0.0569541877765478 & 0.4124656332166650\end{array}$ $\begin{array}{llll}0.0551642999858901 & 0.1410877984670870 & 0.1829720931076396\end{array}$ $\begin{array}{llll}0.1750123774917123 & 0.3111484193352086 & 0.1645633374154539\end{array}$ $\begin{array}{lllll}0.1350949272480833 & 0.2226506355438811 & 0.3364497957384229\end{array}$ $\begin{array}{lllll}0.2471307030238631 & 0.0422991420837608 & 0.3475237512701047\end{array}$ $\begin{array}{lllll}0.1756394657679609 & 0.0688177572235154 & 0.1503483570660572\end{array}$ $\begin{array}{lllll}0.3055853144663110 & 0.1409231327954078 & 0.1847964798698888\end{array}$ $\begin{array}{lllll}0.4234835552639442 & 0.3082947767371767 & 0.1588852734204280\end{array}$ $\begin{array}{lllll}0.3841104019917201 & 0.2232937852871659 & 0.3350844458248350\end{array}$ $\begin{array}{lllll}0.4973587714225129 & 0.0416806504880669 & 0.3469087090168818\end{array}$ $\begin{array}{lllll}0.4255761760672561 & 0.0691126712143168 & 0.1490963772809717\end{array}$ $\begin{array}{lllll}0.0541117742181949 & 0.4984231570352817 & 0.1707681291961975\end{array}$ $\begin{array}{lllll}0.2540328429089321 & 0.3908893200786143 & 0.3128886161162607\end{array}$ $\begin{array}{lllll}0.1336197573205244 & 0.4639407320324094 & 0.3490290438112341\end{array}$ $\begin{array}{lllll}0.3095161626079236 & 0.4909225430665001 & 0.1484255974162786\end{array}$ $\begin{array}{lllll}0.0047454646820392 & 0.3927587758501143 & 0.3181880824114616\end{array}$ $\begin{array}{lllll}0.3858241572865974 & 0.4661835071994201 & 0.3472217618515191\end{array}$ $\begin{array}{lllll}0.2095883406637647 & 0.4758612143146678 & 0.0874767878194806\end{array}$ $\begin{array}{lllll}0.4625912662813764 & 0.4723649289872748 & 0.0760329854576880\end{array}$ $\begin{array}{llll}0.1575757370225728 & 0.4402535043443616 & 0.4680515489587539\end{array}$ $\begin{array}{lllll}0.4103372689465354 & 0.4411267699301975 & 0.4668915289028090\end{array}$ $\begin{array}{lllll}0.1514311294871320 & 0.0919802343578703 & 0.0313793507784926\end{array}$ $\begin{array}{lllll}0.3999389168125579 & 0.0925674173025393 & 0.0305898236693560\end{array}$ $\begin{array}{lllll}0.1715599893201628 & 0.4815000545486970 & 0.2868763508537574\end{array}$ $\begin{array}{lllll}0.4091648681592282 & 0.4959959533632666 & 0.2803724552365849\end{array}$ $\begin{array}{lllll}0.1394006768449236 & 0.04977744020903275 & 0.2133584874267546\end{array}$ 0.39031250285679840 .04860571448533520 .2121037568937736 $\begin{array}{llll}0.2154432737363376 & 0.4170706507222064 & 0.2385534079746814\end{array}$ $\begin{array}{lllll}0.4654243362849825 & 0.4136395489592560 & 0.2446750494720438\end{array}$ $\begin{array}{lllll}0.3427216790688316 & 0.1139310046748588 & 0.2589677375550983\end{array}$ $\begin{array}{lllll}0.0924981160822958 & 0.1156812646935642 & 0.2583185583265415\end{array}$ 
$\begin{array}{llll}0.0984208391059567 & 0.0560976479855769 & 0.9129279871736454\end{array}$ $\begin{array}{lllll}0.3476864597099817 & 0.0570632580455403 & 0.9114556690354584\end{array}$ $\begin{array}{lllll}0.0548487155065663 & 0.1403995526294350 & 0.6841186573510832\end{array}$ $\begin{array}{llll}0.1747155883385172 & 0.3104561949362541 & 0.6643644392005534\end{array}$ $\begin{array}{llll}0.1335173024789545 & 0.2218485891917035 & 0.8353861973168171\end{array}$ $\begin{array}{llll}0.2476184712478633 & 0.0413639502431535 & 0.8467994235996914\end{array}$ $\begin{array}{lllll}0.1751468497448064 & 0.0684561849521288 & 0.6497219681885500\end{array}$ $\begin{array}{llllll}0.3047828676651733 & 0.1406873867779591 & 0.6838719474781975\end{array}$ $\begin{array}{lllll}0.4240690655521524 & 0.3101197175802201 & 0.6625915673924584\end{array}$ $\begin{array}{lllll}0.3834986543271267 & 0.2222681616257703 & 0.8349253912803515\end{array}$ $\begin{array}{lllll}0.4974711661123616 & 0.0416436016453996 & 0.8463889147798606\end{array}$ $\begin{array}{lllll}0.4253631588265980 & 0.0690962070186298 & 0.6496507876434875\end{array}$ $\begin{array}{llllll}0.0612890679386236 & 0.4925169066269141 & 0.6555399318736496\end{array}$ $\begin{array}{lllll}0.2535475727598278 & 0.3917760781356592 & 0.8142697240161003\end{array}$ $\begin{array}{llll}0.1330586820837248 & 0.4634849597445855 & 0.8495685110359494\end{array}$ $\begin{array}{lllll}0.3103726947637241 & 0.4907065525391891 & 0.6506281077976008\end{array}$ $\begin{array}{lllll}0.0037577603305225 & 0.3910290788567236 & 0.8146753630897944\end{array}$ $\begin{array}{lllll}0.3824871125637830 & 0.4632875534966512 & 0.8477551453947287\end{array}$ $\begin{array}{lllll}0.2100019556529205 & 0.4754697842836372 & 0.5869737449176201\end{array}$ $\begin{array}{lllll}0.4609523245233916 & 0.4751696999219785 & 0.5877316464349457\end{array}$ $\begin{array}{lllll}0.1575209141399091 & 0.4402563943513325 & 0.9683430638180945\end{array}$ $\begin{array}{llll}0.4056978163777585 & 0.4397986219809245 & 0.9663652658470422\end{array}$ $\begin{array}{lllll}0.1503729169641141 & 0.0921533025849720 & 0.5306440637902733\end{array}$ $\begin{array}{lllll}0.4003604846672096 & 0.0924229911171177 & 0.5308701119111047\end{array}$ $\begin{array}{llll}0.1704562852607058 & 0.4819793001924899 & 0.7873677516790919\end{array}$ $\begin{array}{lllll}0.4184809197630087 & 0.4832398362755292 & 0.7848845603341931\end{array}$ $\begin{array}{lllll}0.1396280371069821 & 0.0486844866180627 & 0.71297373334688550\end{array}$ $\begin{array}{lllll}0.3895818063674633 & 0.0492107695169332 & 0.7126110325518963\end{array}$ $\begin{array}{lllll}0.2157651858858509 & 0.4175250695249516 & 0.7392896101675537\end{array}$ $\begin{array}{lllll}0.4654868547405587 & 0.4176756627817773 & 0.7391816438839237\end{array}$ $\begin{array}{lllll}0.3422816281128651 & 0.1146147643819670 & 0.7586210740446433\end{array}$ $\begin{array}{llll}0.0921597042524023 & 0.1142262693459217 & 0.7588879829127115\end{array}$ $\begin{array}{lllll}0.5795702366233362 & 0.7092149948067884 & 0.2141410482600418\end{array}$ $\begin{array}{lllll}0.5545996395902703 & 0.5206966460897815 & 0.1505435855988635\end{array}$ $\begin{array}{lllll}0.5015292743763010 & 0.6537992906323371 & 0.2545176232657321\end{array}$ $\begin{array}{lllll}0.7154801620753696 & 0.6598303218242552 & 0.1412154280988703\end{array}$ $\begin{array}{lllll}0.6683677852240204 & 0.7197511141069660 & 0.1430664858576431\end{array}$ $\begin{array}{lllll}0.6586062944670730 & 0.6778547051438670 & 0.0630644343898925\end{array}$ $\begin{array}{lllll}0.5972746404630443 & 0.0572027303910764 & 0.4109484597933225\end{array}$ $\begin{array}{llll}0.8484595641184193 & 0.0564705109141835 & 0.4114478269042809\end{array}$ $\begin{array}{lllll}0.5555233003593534 & 0.1410187034169633 & 0.1841562455973230\end{array}$ $\begin{array}{llll}0.6759303489931346 & 0.3122036167542605 & 0.1669267807373128\end{array}$ $\begin{array}{lllll}0.6351916965333085 & 0.2224094928925663 & 0.3369894700241038\end{array}$ $\begin{array}{lllll}0.7475354061296718 & 0.0421375496864765 & 0.3472714700495254\end{array}$ $\begin{array}{llllll}0.6766675637826133 & 0.0694768188899514 & 0.1490588772320518\end{array}$ $\begin{array}{lllll}0.8053528059020961 & 0.1429589412105254 & 0.1859185697973921\end{array}$ $\begin{array}{lllll}0.9238881370177894 & 0.3100465542072701 & 0.1618496215002066\end{array}$ $\begin{array}{lllll}0.8843639401651517 & 0.2230620818433141 & 0.3360852174297405\end{array}$ $\begin{array}{lllll}0.9982001896591316 & 0.0414499898973109 & 0.3457839904419080\end{array}$ $\begin{array}{lllll}0.9246136026501829 & 0.0700337834083156 & 0.1488489084717525\end{array}$ $\begin{array}{lllll}0.4990846345118711 & 0.4811154219800036 & 0.1930501827152161\end{array}$ $\begin{array}{lllll}0.7511751860033286 & 0.3932162541871392 & 0.3155913656282093\end{array}$ $\begin{array}{llllll}0.6302887786533315 & 0.4638776741106774 & 0.3504438903646134\end{array}$ $\begin{array}{lllll}0.7938063455765948 & 0.4736221435026931 & 0.1271807189260023\end{array}$ $\begin{array}{llll}0.5038104503327829 & 0.3897149412704456 & 0.3199987502737727\end{array}$ $\begin{array}{lllll}0.8851414649586993 & 0.4634172236783505 & 0.3525074881702434\end{array}$ $\begin{array}{llll}0.6476259520380753 & 0.5044962518961470 & 0.0649770939189321\end{array}$ $\begin{array}{lllll}0.9659267800713429 & 0.4733897190035321 & 0.0886031108576831\end{array}$ $\begin{array}{lllll}0.6602033004462061 & 0.4396531347748064 & 0.4700603969821602\end{array}$ $\begin{array}{llll}0.9099980474327087 & 0.4396295284047812 & 0.4702504098296599\end{array}$ $\begin{array}{lllll}0.6515062034833683 & 0.0927026055603021 & 0.0308773944991558\end{array}$ $\begin{array}{llll}0.8974958286097833 & 0.0934321493990593 & 0.0305322643957022\end{array}$ $\begin{array}{lllll}0.6479719706569018 & 0.5060125982415553 & 0.2903275351161373\end{array}$ $\begin{array}{lllll}0.9201596880432288 & 0.4837057353526701 & 0.2888450438446699\end{array}$ $\begin{array}{lllll}0.6400688328547471 & 0.0497868269887862 & 0.2114718915731885\end{array}$ $\begin{array}{llll}0.8902640364022127 & 0.0491703500534159 & 0.2123387076136545\end{array}$ $\begin{array}{llll}0.7172860232026620 & 0.4241093980590648 & 0.2439691783062685\end{array}$ $\begin{array}{lllll}0.9674473820363569 & 0.4181384037014299 & 0.2425962246584260\end{array}$ $\begin{array}{lllll}0.8429150789794710 & 0.1147569118162274 & 0.2587420545912055\end{array}$ $\begin{array}{llll}0.5927687039951078 & 0.1144793396273273 & 0.2585827851016886\end{array}$ $\begin{array}{llll}0.5975884096862353 & 0.0575345518300800 & 0.9121874274096821\end{array}$ $\begin{array}{lllll}0.8454729472177537 & 0.0582769918624738 & 0.9112132425024415\end{array}$ $\begin{array}{llll}0.5549386522391387 & 0.1408735908231530 & 0.6837768944114313\end{array}$ 
$\begin{array}{llll}0.6740635718923379 & 0.3102399659872091 & 0.6624253889054761\end{array}$ $\begin{array}{llll}0.6334981872954331 & 0.2227088461687983 & 0.8351784824951995\end{array}$ $\begin{array}{lllll}0.7467443918731761 & 0.0414278558642409 & 0.8455872146761194\end{array}$ $\begin{array}{llll}0.6754487171193536 & 0.0692284709975774 & 0.6496764066327059\end{array}$ $\begin{array}{lllll}0.8048399893088757 & 0.1413630878245797 & 0.6844387246520294\end{array}$ $\begin{array}{llllll}0.9236037221584741 & 0.3090813560796434 & 0.6618269484813376\end{array}$ $\begin{array}{lllll}0.8842215065219471 & 0.2233688153856119 & 0.8373838230096305\end{array}$ $\begin{array}{llll}0.9970365796682589 & 0.0421841693767152 & 0.8482976543295356\end{array}$ $\begin{array}{llll}0.9248967174387085 & 0.0687907809022521 & 0.6497936453884298\end{array}$ $\begin{array}{lllll}0.5606863855022355 & 0.4915125815139447 & 0.6517584907012550\end{array}$ $\begin{array}{lllll}0.7529066708535723 & 0.3921725591856100 & 0.8153739316684800\end{array}$ $\begin{array}{lllll}0.6318973777117735 & 0.4635238734115413 & 0.8485902264715445\end{array}$ $\begin{array}{lllll}0.8112864042359699 & 0.4910430266238032 & 0.6522055340375771\end{array}$ $\begin{array}{lllll}0.5030533911795523 & 0.3919087994954182 & 0.8141138092053601\end{array}$ $\begin{array}{lllll}0.8834550519718137 & 0.4638484573332348 & 0.8502451087095420\end{array}$ $\begin{array}{lllll}0.7113067003954338 & 0.4756561224046131 & 0.5891281694588260\end{array}$ $\begin{array}{llll}0.9629955922847385 & 0.4744409919162625 & 0.5895430853615923\end{array}$ $\begin{array}{lllll}0.6500753991377163 & 0.4426284578995842 & 0.9660659126783386\end{array}$ $\begin{array}{llll}0.9103262391168123 & 0.4400857560204274 & 0.9691859476804597\end{array}$ $\begin{array}{llll}0.6502058712608462 & 0.0925918776306923 & 0.5308208505481578\end{array}$ $\begin{array}{llll}0.9002442382426077 & 0.0924142365611205 & 0.5309192875132103\end{array}$ $\begin{array}{lllll}0.6678548765429089 & 0.4841640385033557 & 0.7858540944135517\end{array}$ $\begin{array}{lllll}0.9182762754875013 & 0.4842426776949206 & 0.7867737607973327\end{array}$ $\begin{array}{lllll}0.6388676441703978 & 0.0500431730496277 & 0.7122415694202666\end{array}$ $\begin{array}{lllll}0.8895362078815220 & 0.0487025433890686 & 0.7130170501887244\end{array}$ $\begin{array}{lllll}0.7157634741317197 & 0.4181437306293034 & 0.7403951662082866\end{array}$ $\begin{array}{lllll}0.9662677575667201 & 0.4181654510103742 & 0.7409046669448510\end{array}$ $\begin{array}{lllll}0.8420990658151984 & 0.1146555337376017 & 0.7588119597566414\end{array}$ $\begin{array}{lllll}0.5922278101217701 & 0.1149398651503546 & 0.7587790669097889\end{array}$ $\begin{array}{lllll}0.7948570440950844 & 0.7186817028812907 & 0.3355782647985259\end{array}$ $\begin{array}{lllll}0.6506373983973758 & 0.5937417367121768 & 0.3467202783670224\end{array}$ $\begin{array}{lllll}0.7145337486819848 & 0.6649043997886822 & 0.3535184840611306\end{array}$ $\begin{array}{lllll}0.7532373930155652 & 0.6415686160813723 & 0.3071888709007058\end{array}$ $\begin{array}{lllll}0.7360628114202327 & 0.5891713845361367 & 0.2794688664841730\end{array}$ $\begin{array}{llll}0.6668021738981732 & 0.6781194478171580 & 0.1228968420406530\end{array}$ $\begin{array}{lllll}0.5754560128974561 & 0.6677985196395257 & 0.1944140785115814\end{array}$ $\begin{array}{lllll}0.6145133994704660 & 0.6470546361960591 & 0.1494329317275808\end{array}$ $\begin{array}{lllll}0.6058715347643916 & 0.5907170858127742 & 0.1283421647875335\end{array}$ $\begin{array}{lllll}0.5184763660812631 & 0.5840414897626280 & 0.2020514318975199\end{array}$ $\begin{array}{llll}0.6618655712850392 & 0.6430296162258469 & 0.3750063636088301\end{array}$ $\begin{array}{lllll}0.7420421423566360 & 0.7146654333256860 & 0.3711971606025075\end{array}$ $\begin{array}{lllll}0.8030297964651710 & 0.6755558824874217 & 0.2962528592485365\end{array}$ $\begin{array}{lllll}0.6834262686238877 & 0.5660894333027703 & 0.3014346036741397\end{array}$ $\begin{array}{lllll}0.7690777479625328 & 0.5645654861500676 & 0.2332899809012258\end{array}$ $\begin{array}{llll}0.5284226993800243 & 0.6379057816295232 & 0.2187664872810350\end{array}$ $\begin{array}{llllll}0.5580658910765633 & 0.5634106113756637 & 0.1562844703953787\end{array}$ $\begin{array}{lllll}0.4773236380899084 & 0.5572479547431007 & 0.2266551910120083\end{array}$ $\begin{array}{lllll}0.0506181396106081 & 0.3516411428065009 & 0.1859012535532585\end{array}$ $\begin{array}{lllll}0.0344733391218034 & 0.2424289841202970 & 0.1931975262130536\end{array}$ $\begin{array}{llllll}0.1596624170342131 & 0.2840905999539631 & 0.1940619144202418\end{array}$ $\begin{array}{lllll}0.2042047128974019 & 0.2093282315960680 & 0.0714092737243853\end{array}$ $\begin{array}{lllll}0.0395375080321997 & 0.2888992361783570 & 0.0677689884156422\end{array}$ $\begin{array}{lllll}0.0810562389118942 & 0.1871198337338210 & 0.0889493820408166\end{array}$ $\begin{array}{lllll}0.1850580885045445 & 0.1699031360109123 & 0.1966262807767559\end{array}$ $\begin{array}{llllll}0.1832061206421962 & 0.0570643084339870 & 0.2029365298899688\end{array}$ $\begin{array}{lllll}0.0510875526283403 & 0.1121244174162482 & 0.2161828002924757\end{array}$ $\begin{array}{llllll}0.1637786269649029 & 0.1064127519009796 & 0.0820390352753451\end{array}$ $\begin{array}{lllll}0.1531529839551484 & 0.1176942003150877 & 0.3128624179432007\end{array}$ $\begin{array}{lllll}0.0254545286608838 & 0.0697145873387039 & 0.3323050089974612\end{array}$ $\begin{array}{lllll}0.1420407085603442 & 0.0679855296855676 & 0.4394238645207871\end{array}$ $0.1085314490845563 \quad 0.16955604527881320 .4220416662014905$ $\begin{array}{llll}0.0247846778205322 & 0.2899547167495726 & 0.3061397095323696\end{array}$ $\begin{array}{llllll}0.1497460103539514 & 0.2487534859926576 & 0.3051067820652682\end{array}$ $\begin{array}{lllll}0.1049880636591334 & 0.3228445826055671 & 0.4275353163235964\end{array}$ $\begin{array}{lllll}0.0197805096161180 & 0.2432585674258362 & 0.4315591631794848\end{array}$ $\begin{array}{lllll}0.1248760543811932 & 0.3626032294025802 & 0.3027416609969199\end{array}$ $\begin{array}{lllll}0.2335067262546427 & 0.1437123778111201 & 0.4389935439574201\end{array}$ $\begin{array}{lllll}0.2589597342442236 & 0.1814750023921424 & 0.3134155169039799\end{array}$ $\begin{array}{lllll}0.2283210690106393 & 0.3449741125590236 & 0.4110789425979906\end{array}$ $\begin{array}{lllll}0.3003762866207533 & 0.3523274352909349 & 0.1845679833813562\end{array}$ $\begin{array}{lllll}0.2844214857365504 & 0.2430213910689791 & 0.1927924123227337\end{array}$ $\begin{array}{lllll}0.4099784203339897 & 0.2841079957416197 & 0.1935144975054065\end{array}$ $\begin{array}{llll}0.4545909560462478 & 0.2093635483461900 & 0.0717953514486614\end{array}$ 
$\begin{array}{llll}0.28777754275481311 & 0.2897073504049888 & 0.0670010301994677\end{array}$ $\begin{array}{lllll}0.3309133257014654 & 0.1883715789235553 & 0.0881261264592065\end{array}$ $\begin{array}{lllll}0.4342603932392484 & 0.1696171122413388 & 0.1964892004361713\end{array}$ $\begin{array}{llllll}0.4338161155394880 & 0.0567454034717901 & 0.2015038935745178\end{array}$ $\begin{array}{lllll}0.3011403426258805 & 0.1112841155242718 & 0.2168162843097085\end{array}$ $\begin{array}{lllll}0.4124633169356117 & 0.1068168474544968 & 0.0813279127009830\end{array}$ $\begin{array}{llllll}0.4033035335823073 & 0.1157311069169535 & 0.3129915758408257\end{array}$ $\begin{array}{lllll}0.2748211443587321 & 0.0701175625175222 & 0.3336931132566463\end{array}$ $\begin{array}{lllll}0.3922250674015130 & 0.0670202380924211 & 0.4403254113585772\end{array}$ $0.3590424994203066 \quad 0.16866988169550590 .4220384739800103$ $\begin{array}{llllll}0.2748243418704296 & 0.2909637739265654 & 0.3055685438993413\end{array}$ $\begin{array}{lllll}0.3994747109906036 & 0.2495657011711144 & 0.3045011835004763\end{array}$ $\begin{array}{lllll}0.3550613935946124 & 0.3249087931044830 & 0.4265632967316219\end{array}$ $\begin{array}{lllll}0.2720158922548075 & 0.2439140663532846 & 0.4312834777238320\end{array}$ $\begin{array}{lllll}0.3745712757046737 & 0.3640833494880431 & 0.3012770950270203\end{array}$ $\begin{array}{lllll}0.4837530283533381 & 0.1421753360742185 & 0.4389933998423014\end{array}$ $\begin{array}{lllll}0.0078680954504620 & 0.1810535740911589 & 0.3133525242245036\end{array}$ $\begin{array}{llllll}0.4785066977123581 & 0.3449166261802554 & 0.4093713678308803\end{array}$ $\begin{array}{lllll}0.1558908416099007 & 0.4146515184697773 & 0.1858817628296579\end{array}$ $\begin{array}{lllll}0.0350048288164712 & 0.4628939656605310 & 0.1669865397268435\end{array}$ $\begin{array}{lllll}0.0741413983460592 & 0.3899126260328211 & 0.0601774247852699\end{array}$ $\begin{array}{lllll}0.1653756801352278 & 0.4657522732099060 & 0.0588759488259816\end{array}$ $\begin{array}{llll}0.1995134574942229 & 0.3644255483648590 & 0.0756545671820735\end{array}$ $\begin{array}{lllll}0.1270852630543848 & 0.4755902957413531 & 0.2964261791697000\end{array}$ $\begin{array}{llllll}0.0091477055462220 & 0.4212969240135793 & 0.2844566147218888\end{array}$ $\begin{array}{lllll}0.1453165429005556 & 0.4258615239649576 & 0.4173439301557943\end{array}$ $\begin{array}{lllll}0.4040828340625540 & 0.4186241254865847 & 0.1847329812178041\end{array}$ $\begin{array}{lllll}0.2817107277369557 & 0.4641068875164626 & 0.1644123504051194\end{array}$ $\begin{array}{lllll}0.3252047077205086 & 0.3898338755155342 & 0.0589091125538189\end{array}$ $\begin{array}{lllll}0.4169106269572592 & 0.4624323177637359 & 0.0537548210895492\end{array}$ $\begin{array}{lllll}0.4498699459809707 & 0.3611508349529266 & 0.0805483376720784\end{array}$ $\begin{array}{lllll}0.3718736661087329 & 0.4781899228808295 & 0.2953413376526953\end{array}$ $\begin{array}{lllll}0.2570546919291285 & 0.42097774472095614 & 0.2811455491701082\end{array}$ $\begin{array}{lllll}0.3984409535819694 & 0.4272874261816964 & 0.4159070258970552\end{array}$ $\begin{array}{llllll}0.0504421480760249 & 0.3515056288718691 & 0.6858233769142119\end{array}$ $\begin{array}{lllll}0.0339672108535618 & 0.2422977118209995 & 0.6933341146590869\end{array}$ $\begin{array}{lllll}0.1591153287505425 & 0.2836665430847475 & 0.6940481811788535\end{array}$ $\begin{array}{lllll}0.2036582414329680 & 0.2089210611796406 & 0.5713981329135178\end{array}$ $\begin{array}{lllll}0.0386376348423967 & 0.2889764468935331 & 0.5678319689789566\end{array}$ $\begin{array}{lllll}0.0803254747584107 & 0.1872681564956172 & 0.5886501387748666\end{array}$ $\begin{array}{lllll}0.1839922556909760 & 0.1693817197289508 & 0.6965357833989612\end{array}$ $\begin{array}{lllll}0.1831747411415980 & 0.0565332062276780 & 0.7022536448212614\end{array}$ $\begin{array}{llll}0.0506088904917419 & 0.1111471024972266 & 0.7168157219938337\end{array}$ $\begin{array}{lllll}0.1625762260232725 & 0.1062305542687688 & 0.5815348085794011\end{array}$ $\begin{array}{llll}0.1528269268562066 & 0.1161264823570409 & 0.8130842848325021\end{array}$ $\begin{array}{lllll}0.0247227044293697 & 0.0695336710271093 & 0.8333321484018433\end{array}$ $\begin{array}{lllll}0.1427147265486718 & 0.0666046588613806 & 0.9404259888698323\end{array}$ $\begin{array}{lllll}0.1079688284537078 & 0.1679596184965164 & 0.9224928593879800\end{array}$ $\begin{array}{lllll}0.0241773537580877 & 0.2899429678579077 & 0.8063485189981378\end{array}$ $\begin{array}{lllll}0.1487680289174181 & 0.2483208128358980 & 0.8049975319949690\end{array}$ $\begin{array}{lllll}0.1042824248699517 & 0.3229726582704043 & 0.9277172043509158\end{array}$ $\begin{array}{llll}0.0202826516835334 & 0.2427877248483223 & 0.9318881563024037\end{array}$ $\begin{array}{lllll}0.1241981767527621 & 0.3625769939871528 & 0.8027748559619198\end{array}$ $\begin{array}{lllll}0.2329998897898707 & 0.1425812310611977 & 0.9390430527484274\end{array}$ $\begin{array}{llll}0.2578353373551359 & 0.1807532132597621 & 0.8134482130021473\end{array}$ $\begin{array}{lllll}0.2276945621608787 & 0.3447122056614422 & 0.9107512363153576\end{array}$ $\begin{array}{lllll}0.2995637862896064 & 0.3514657210759317 & 0.6844940815687399\end{array}$ $\begin{array}{lllll}0.2837341447129154 & 0.2422712622893915 & 0.6927225358453812\end{array}$ $\begin{array}{lllll}0.4089754933083714 & 0.2837683532934848 & 0.6932884463675472\end{array}$ $\begin{array}{llll}0.4539328023759729 & 0.2095300577093283 & 0.5712817490067192\end{array}$ $\begin{array}{lllll}0.2874421090072930 & 0.2891496768532467 & 0.5669113839023588\end{array}$ $\begin{array}{llll}0.3304770702056001 & 0.1878626190391740 & 0.5880901359217910\end{array}$ $\begin{array}{lllll}0.4341841053658997 & 0.1698587641297950 & 0.6963171169846571\end{array}$ $\begin{array}{llll}0.4332338587734620 & 0.0570263894762435 & 0.7021477317565912\end{array}$ $\begin{array}{llll}0.3007263875447554 & 0.1113435171736875 & 0.7164953403605530\end{array}$ $\begin{array}{lllll}0.4127734660100191 & 0.1067856460727297 & 0.5815649628448940\end{array}$ $\begin{array}{llll}0.4027644424996801 & 0.1168133145009608 & 0.8128004932491675\end{array}$ $\begin{array}{lllll}0.2749798755526946 & 0.0694533961798621 & 0.8330673042361674\end{array}$ $\begin{array}{lllll}0.3919421991974784 & 0.0672242048387144 & 0.9396726860490547\end{array}$ $\begin{array}{lllll}0.3581307721722580 & 0.1687704836445895 & 0.9221350786754844\end{array}$ $\begin{array}{lllll}0.2737854349425657 & 0.2899552915897156 & 0.8055688224482664\end{array}$ $\begin{array}{lllll}0.3986077937683767 & 0.2486706442241901 & 0.8043343872705625\end{array}$ $\begin{array}{llll}0.3542011528315910 & 0.3230159196429696 & 0.9265804576365533\end{array}$ 
$0.2698138887331707 \quad 0.24314770036138750 .9313131938194033$ $\begin{array}{lllll}0.3736887441153340 & 0.3624008652972519 & 0.8010805145591263\end{array}$ $\begin{array}{llll}0.4830566206229960 & 0.1429488218172169 & 0.9389196578155605\end{array}$ $\begin{array}{llll}0.0077533314060357 & 0.1807884334751429 & 0.8137606345441118\end{array}$ $\begin{array}{llll}0.4772407906478812 & 0.3450769780993824 & 0.9099717770912028\end{array}$ $\begin{array}{lllll}0.1554805779369364 & 0.4152909622657287 & 0.6858192428553496\end{array}$ $\begin{array}{llllll}0.0341899362040674 & 0.4633178869500358 & 0.6666875776630531\end{array}$ $\begin{array}{lllll}0.0745063920684260 & 0.3897179271595991 & 0.5600203973406979\end{array}$ $0.1655446641115065 \quad 0.46551400649122790 .5590251545901369$ $\begin{array}{llll}0.1995512887394625 & 0.3640299642791854 & 0.5756599883897424\end{array}$ $\begin{array}{llll}0.1262050599439453 & 0.4753203435168470 & 0.7970004749674928\end{array}$ $\begin{array}{lllll}0.0079739844964452 & 0.4208614902782414 & 0.7829023145885656\end{array}$ $\begin{array}{llllll}0.1452255194290725 & 0.4257644109082961 & 0.9176584282813887\end{array}$ $\begin{array}{lllll}0.4050735852753912 & 0.4153769823545931 & 0.6848096886035417\end{array}$ $\begin{array}{llllll}0.2829953685100253 & 0.4626858489522184 & 0.6644956936828398\end{array}$ $\begin{array}{lllll}0.3249743324348861 & 0.3895075910073895 & 0.5587801066762266\end{array}$ $\begin{array}{lllll}0.4168678491174260 & 0.4651943501741938 & 0.5586678935390011\end{array}$ $\begin{array}{lllll}0.4499405411412069 & 0.3634629033105706 & 0.5756186603107302\end{array}$ $\begin{array}{lllll}0.3747551215446246 & 0.4755396958818671 & 0.7952304099662315\end{array}$ $\begin{array}{llll}0.2573773756964728 & 0.4210779926870543 & 0.7815064452429227\end{array}$ $\begin{array}{lllll}0.3941710138020657 & 0.4257902050088129 & 0.9151338869822124\end{array}$ $\begin{array}{llll}0.6394535827319678 & 0.5674201260710779 & 0.0878854056783841\end{array}$ $\begin{array}{lllll}0.5525989298212429 & 0.3511159083000067 & 0.1871859012160345\end{array}$ $\begin{array}{llll}0.5349733561813971 & 0.2419368561891865 & 0.1934271058494534\end{array}$ $\begin{array}{llll}0.6604070204632659 & 0.2842065970886235 & 0.1948114683776739\end{array}$ $\begin{array}{lllll}0.7038331711119197 & 0.2104694849316126 & 0.0714918060003193\end{array}$ $\begin{array}{lllll}0.5396068196178406 & 0.2889389264826496 & 0.0682622129157423\end{array}$ $\begin{array}{lllll}0.5810116066072355 & 0.1870659265708634 & 0.0885785353251418\end{array}$ $\begin{array}{lllll}0.6850839851536624 & 0.1700154673392295 & 0.1965497705383026\end{array}$ $\begin{array}{llllll}0.6839989326695856 & 0.0571598902800881 & 0.2014937180821371\end{array}$ $\begin{array}{lllll}0.5512422556914156 & 0.1115142225266261 & 0.2164731538024807\end{array}$ $\begin{array}{llll}0.6646628824950235 & 0.1071005795594956 & 0.0814763064543183\end{array}$ $\begin{array}{lllll}0.6534770802525494 & 0.1162460749621016 & 0.3127516030859721\end{array}$ $\begin{array}{llll}0.5252600094454940 & 0.0691427609327593 & 0.3325841209333776\end{array}$ $\begin{array}{llll}0.6415468291172818 & 0.0671346842826164 & 0.4395094302125226\end{array}$ $\begin{array}{lllll}0.6086564905015701 & 0.1689262671184120 & 0.4215974162206645\end{array}$ $\begin{array}{lllll}0.5252761578459882 & 0.2894731358914404 & 0.3061931549960478\end{array}$ $\begin{array}{llllll}0.6499177338907747 & 0.2483516780018014 & 0.3054938555622414\end{array}$ $\begin{array}{llll}0.6052460979866999 & 0.3223554176624550 & 0.4276237270080654\end{array}$ $\begin{array}{llll}0.5199778796642965 & 0.2430019532052669 & 0.4316211407909443\end{array}$ $\begin{array}{lllll}0.6262179444789263 & 0.3628228775068658 & 0.3035713793693599\end{array}$ $\begin{array}{llllll}0.7334706122996534 & 0.1421867754916811 & 0.4389937431046750\end{array}$ $\begin{array}{lllll}0.7586460121805954 & 0.1808211091701173 & 0.3137669996532311\end{array}$ $\begin{array}{lllll}0.7289558861056847 & 0.3442867471845322 & 0.4121977562357277\end{array}$ $\begin{array}{llll}0.7999571258668912 & 0.3523250167748122 & 0.1867141227116105\end{array}$ $\begin{array}{llll}0.7844759546634869 & 0.2431455814035062 & 0.1929735460155461\end{array}$ $\begin{array}{lllll}0.9095102644050643 & 0.2844334140496482 & 0.1940889489640141\end{array}$ $\begin{array}{lllll}0.9547253261633404 & 0.2093949057368431 & 0.0714151709007457\end{array}$ $\begin{array}{lllll}0.7849454898310038 & 0.2924442286652136 & 0.0675305541114350\end{array}$ $\begin{array}{llllll}0.8308169395623878 & 0.1911568031791888 & 0.0868575824632943\end{array}$ $\begin{array}{llll}0.9335829704079001 & 0.1703380202749601 & 0.1962241590964383\end{array}$ $\begin{array}{llll}0.9335422930623423 & 0.0574485716173365 & 0.2012020122067845\end{array}$ $\begin{array}{llll}0.8014235115220960 & 0.1123262577719775 & 0.2164597708376050\end{array}$ $\begin{array}{llll}0.9101365563372635 & 0.1077187457053623 & 0.0812612203774468\end{array}$ $\begin{array}{llll}0.9033380611734513 & 0.1161749462781927 & 0.3128635198769738\end{array}$ $\begin{array}{lllll}0.7755707903811160 & 0.0692979144814651 & 0.3324878050886482\end{array}$ $\begin{array}{lllll}0.8926229415774487 & 0.0666735079895028 & 0.4397666493879264\end{array}$ $\begin{array}{llll}0.8587940061859921 & 0.1681990949398009 & 0.4224169587410632\end{array}$ $\begin{array}{lllll}0.7750026290916066 & 0.2898095880987451 & 0.3067975565871622\end{array}$ $\begin{array}{lllll}0.8993574290486043 & 0.2491394027104415 & 0.3049747099051738\end{array}$ $\begin{array}{llll}0.8555370894888785 & 0.3222663712649960 & 0.4283882701074179\end{array}$ $\begin{array}{llll}0.7707808266663999 & 0.2426918224195436 & 0.4321728913798785\end{array}$ $\begin{array}{lllll}0.8743042740964672 & 0.3629421266886658 & 0.3037461813210700\end{array}$ $\begin{array}{lllll}0.9836578324661835 & 0.1424358695945560 & 0.4389207079806243\end{array}$ $\begin{array}{lllll}0.5077645650083475 & 0.1807390898534782 & 0.3134753727750689\end{array}$ $\begin{array}{llll}0.9785921857251674 & 0.3449417556324121 & 0.4109428395315798\end{array}$ $\begin{array}{llll}0.6529969010392332 & 0.4217895744292489 & 0.1849172440728386\end{array}$ $\begin{array}{lllll}0.5298323257549757 & 0.4618436928745272 & 0.1673475549596405\end{array}$ $\begin{array}{lllll}0.5734688213326522 & 0.3904926116128052 & 0.0619780551843964\end{array}$ $\begin{array}{lllll}0.6571923883822566 & 0.4669516631473978 & 0.0493194782039276\end{array}$ $\begin{array}{llll}0.6998748020236000 & 0.3700919446863631 & 0.0765846897056536\end{array}$ $\begin{array}{llll}0.6177449439396505 & 0.4751856662291963 & 0.2986459585065505\end{array}$ $\begin{array}{llll}0.5068857865534631 & 0.4172799706258249 & 0.2845679279979164\end{array}$ 
$\begin{array}{llll}0.6466424929924058 & 0.4250944701620290 & 0.4197276288746169\end{array}$ $\begin{array}{lllll}0.9065705079284088 & 0.4159403349813153 & 0.1881523364534468\end{array}$ $\begin{array}{lllll}0.7837726421858867 & 0.4646052407431578 & 0.1740540631889753\end{array}$ $\begin{array}{lllll}0.8254858697966407 & 0.3931593188466084 & 0.0631370770298797\end{array}$ $\begin{array}{llll}0.9210544325765043 & 0.4641721831986432 & 0.0606436333244339\end{array}$ $\begin{array}{lllll}0.9495896388586328 & 0.3619229457152377 & 0.0789162911619830\end{array}$ $\begin{array}{llll}0.8769527567059712 & 0.4754929282648805 & 0.3000908775980584\end{array}$ $\begin{array}{lllll}0.7585317670034930 & 0.4226599052085918 & 0.2846187955964087\end{array}$ $\begin{array}{lllll}0.8963177893812914 & 0.4253203299681494 & 0.4196518554320460\end{array}$ $\begin{array}{lllll}0.5498172813420041 & 0.3518973571110126 & 0.6848531224943452\end{array}$ $\begin{array}{lllll}0.5339572055726961 & 0.2426875491876547 & 0.6927223260953728\end{array}$ $\begin{array}{llll}0.6591561001248509 & 0.2841856540111018 & 0.6936647833475249\end{array}$ $\begin{array}{lllll}0.7039219807291852 & 0.2097561636644294 & 0.5715755079233301\end{array}$ $\begin{array}{lllll}0.5387391608177041 & 0.2893619555163846 & 0.5671835104990236\end{array}$ $\begin{array}{llll}0.5804624504422606 & 0.1877177988976369 & 0.5881713784232085\end{array}$ $\begin{array}{llllll}0.6840093012521377 & 0.1699862891089924 & 0.6964389502289150\end{array}$ $\begin{array}{llllll}0.6828168514062287 & 0.0572656433909917 & 0.7021738521809759\end{array}$ $\begin{array}{lllll}0.5507915229954145 & 0.1116006477535957 & 0.7164872872144049\end{array}$ $\begin{array}{llll}0.6631059606307280 & 0.1069010493594928 & 0.5814491845245754\end{array}$ $\begin{array}{llllll}0.6526135879393481 & 0.1170460981691407 & 0.8129890915714439\end{array}$ $\begin{array}{lllll}0.5248324451246361 & 0.0698947937231070 & 0.8330699465549473\end{array}$ $\begin{array}{lllll}0.6419606447753454 & 0.0676787080314021 & 0.9400709803891893\end{array}$ $\begin{array}{lllll}0.6081683856416229 & 0.1692688659567822 & 0.9221708690532839\end{array}$ $\begin{array}{llll}0.5238903559021399 & 0.2902472173850354 & 0.8056482797009202\end{array}$ $\begin{array}{lllll}0.6486539005585958 & 0.2490959471975769 & 0.8046021643783907\end{array}$ $\begin{array}{lllll}0.6039565591912215 & 0.3236706437007293 & 0.9271794384730706\end{array}$ $\begin{array}{lllll}0.5197218683143693 & 0.2435978459448155 & 0.9313571872532727\end{array}$ $\begin{array}{lllll}0.6234633508374470 & 0.3629592558545022 & 0.8017773444703007\end{array}$ $\begin{array}{lllll}0.7331052871498882 & 0.1432520442960984 & 0.9390379704351103\end{array}$ $\begin{array}{llll}0.7575174487390606 & 0.1815020218807605 & 0.8135540447546028\end{array}$ $\begin{array}{lllll}0.7269652236450513 & 0.3458855757527109 & 0.9100292673014757\end{array}$ $\begin{array}{lllll}0.8006215619619590 & 0.3518780296573390 & 0.6858212317894777\end{array}$ $\begin{array}{lllll}0.7839555996776782 & 0.2427383456548271 & 0.6932295475500291\end{array}$ $\begin{array}{lllll}0.9092358051271400 & 0.2836980076343617 & 0.6943397685265552\end{array}$ $\begin{array}{lllll}0.9538679704360756 & 0.2092922658591430 & 0.5718153535319751\end{array}$ $\begin{array}{lllll}0.7886671293325955 & 0.2896456488909478 & 0.5678461644521489\end{array}$ $\begin{array}{llll}0.8304417656743930 & 0.1879341522767942 & 0.5886306824108273\end{array}$ $\begin{array}{lllll}0.9340253540915822 & 0.1695457532164139 & 0.6967371721161529\end{array}$ $\begin{array}{lllll}0.9330279223473043 & 0.0566723991643991 & 0.7022490215729486\end{array}$ $\begin{array}{llll}0.8005044628586027 & 0.1118429578472453 & 0.7166904229589318\end{array}$ $\begin{array}{lllll}0.9123709132805901 & 0.1066103231006262 & 0.5816900113602449\end{array}$ $\begin{array}{lllll}0.9025586179531101 & 0.1163276589297188 & 0.8130601226330381\end{array}$ $\begin{array}{lllll}0.7742831214741565 & 0.0698383789533589 & 0.8330259057842229\end{array}$ $\begin{array}{lllll}0.8897315652207470 & 0.0680669818962075 & 0.9400396203506927\end{array}$ $\begin{array}{lllll}0.8580831304575884 & 0.1699759623460987 & 0.9214593475112737\end{array}$ $\begin{array}{lllll}0.7740675494012837 & 0.2905050757072444 & 0.8061491404182572\end{array}$ $\begin{array}{lllll}0.8989334779184172 & 0.2489592224044038 & 0.8053803603024579\end{array}$ $\begin{array}{lllll}0.8535889574054073 & 0.3239868035694664 & 0.9279730489508794\end{array}$ $\begin{array}{lllll}0.7693124530361797 & 0.2440644873961961 & 0.9317157854398197\end{array}$ $\begin{array}{lllll}0.8742059516792945 & 0.3631106756219723 & 0.8030893719815078\end{array}$ $\begin{array}{lllll}0.9825740441833607 & 0.1424584213499817 & 0.9392133798773642\end{array}$ $\begin{array}{lllll}0.5079049232935871 & 0.1811980851631334 & 0.8134036031044951\end{array}$ $\begin{array}{lllll}0.9774598359443817 & 0.3440919629072195 & 0.9115109544952347\end{array}$ $\begin{array}{lllll}0.6552435446215961 & 0.4159708615520682 & 0.6860923494076107\end{array}$ $\begin{array}{llllll}0.5331982730709577 & 0.4633087334547411 & 0.6649757369112197\end{array}$ $\begin{array}{llll}0.5753753766344741 & 0.3900022542135526 & 0.5595861686984140\end{array}$ $\begin{array}{llll}0.6671960050712048 & 0.4657308261544435 & 0.5601751693453925\end{array}$ $\begin{array}{lllll}0.7002013309406483 & 0.3638867260439537 & 0.5772070855049155\end{array}$ $\begin{array}{llllll}0.6241737895261512 & 0.4761023584745610 & 0.7959935003745757\end{array}$ $\begin{array}{llll}0.5068306514615678 & 0.4212712509117693 & 0.7814399388013249\end{array}$ $\begin{array}{lllll}0.6433848016432198 & 0.4267637382197704 & 0.9152860788981163\end{array}$ $\begin{array}{llll}0.9056317182999363 & 0.4163189375503566 & 0.6868685625160122\end{array}$ $\begin{array}{lllll}0.7836783454283066 & 0.4633758926140389 & 0.6665208175423343\end{array}$ $\begin{array}{lllll}0.8254583162387827 & 0.3903378003344224 & 0.5604945191121334\end{array}$ $\begin{array}{lllll}0.9186909122331174 & 0.4648578367942994 & 0.5604525186123201\end{array}$ $\begin{array}{lllll}0.9497042684279344 & 0.3628086854549102 & 0.5780989497231691\end{array}$ $\begin{array}{lllll}0.8749672992022961 & 0.4759158127685257 & 0.7977503655585941\end{array}$ $\begin{array}{lllll}0.7572949383200955 & 0.4212804976858860 & 0.7824982629321892\end{array}$ $\begin{array}{lllll}0.8966713410086919 & 0.4261049639126587 & 0.9183906099355678\end{array}$ $\begin{array}{llll}0.2177724561255360 & 0.2360562775973668 & 0.2510468969198094\end{array}$ $\begin{array}{lllll}0.0917751999194190 & 0.2968796223324865 & 0.2482965013000804\end{array}$ $\begin{array}{lllll}0.2266492132831245 & 0.1151251496115232 & 0.2670523942612132\end{array}$ $\begin{array}{llll}0.4675249592878461 & 0.2357586775554035 & 0.2507994223318611\end{array}$ 
$\begin{array}{llll}0.3418826100642623 & 0.2979832092110949 & 0.2470745180866277\end{array}$ $\begin{array}{lllll}0.4765653155953066 & 0.1145386506484435 & 0.2666167319487216\end{array}$ $\begin{array}{lllll}0.0830846417115762 & 0.4173567292837332 & 0.2327803980115879\end{array}$ $\begin{array}{lllll}0.3313729825806753 & 0.4196217744389137 & 0.2308920655258567\end{array}$ $\begin{array}{llll}0.2168122490114791 & 0.2353581562531522 & 0.7509947493693324\end{array}$ $\begin{array}{lllll}0.0912286017700682 & 0.2968159153254933 & 0.7482703399400996\end{array}$ $\begin{array}{llllll}0.2262338683472339 & 0.1144328153134828 & 0.7668707725546378\end{array}$ $\begin{array}{llll}0.4668796119859536 & 0.2357901531797945 & 0.7507505456004709\end{array}$ $\begin{array}{llll}0.3408486039009225 & 0.2968957072625662 & 0.7471497044039354\end{array}$ $\begin{array}{llll}0.4762336772988366 & 0.1149217800944465 & 0.7667733685084580\end{array}$ $\begin{array}{lllll}0.0823837366190985 & 0.4177091326013643 & 0.7325029651107764\end{array}$ $\begin{array}{llll}0.3317105633755711 & 0.4177663498209903 & 0.7308518859088809\end{array}$ $\begin{array}{lllll}0.7178791669196652 & 0.2357462099268350 & 0.2515528339410779\end{array}$ $\begin{array}{lllll}0.5924804158142541 & 0.2969202837538204 & 0.2489417781442810\end{array}$ $\begin{array}{lllll}0.7269363230814385 & 0.1147385232488854 & 0.2667248841917441\end{array}$ $\begin{array}{lllll}0.9670408396350892 & 0.2359262379831823 & 0.2509316697116489\end{array}$ $\begin{array}{lllll}0.8414508761920763 & 0.2975212648788389 & 0.2481312252450820\end{array}$ $\begin{array}{lllll}0.9764941711942801 & 0.1149324625490162 & 0.2664027495940327\end{array}$ $\begin{array}{lllll}0.5833122192602259 & 0.4181636303062314 & 0.2344300323243577\end{array}$ $\begin{array}{llll}0.8333365841973261 & 0.4182003461744820 & 0.2347850531079502\end{array}$ $\begin{array}{lllll}0.7167759749799292 & 0.2360918917830643 & 0.7509701249627084\end{array}$ $\begin{array}{llllll}0.5910275323768879 & 0.2974559792182347 & 0.7475829678973834\end{array}$ $\begin{array}{lllll}0.7259755190544536 & 0.1151713974566857 & 0.7668753613293954\end{array}$ $\begin{array}{lllll}0.9668295722653356 & 0.2354951006002269 & 0.7514012505502166\end{array}$ $\begin{array}{llllll}0.8412838116247231 & 0.2972780724242001 & 0.7483136109096370\end{array}$ $\begin{array}{lllll}0.9760562766808778 & 0.1144786125961851 & 0.7670737344583661\end{array}$ $\begin{array}{llll}0.5816249443393720 & 0.4183254546009594 & 0.7316046591131794\end{array}$ $\begin{array}{lllll}0.8320637289109313 & 0.4182262522174923 & 0.7327986226632380\end{array}$ $\begin{array}{lllll}0.0279861210109184 & 0.2324735086970154 & 0.1049352646182396\end{array}$ $\begin{array}{lllll}0.1583651566656321 & 0.1694024836504109 & 0.1085641157519399\end{array}$ $\begin{array}{llll}0.1512170783147761 & 0.3628868936405032 & 0.3909261962750725\end{array}$ $\begin{array}{llll}0.1596124204042405 & 0.1235919977852603 & 0.4003769694389309\end{array}$ $\begin{array}{lllll}0.0300040158059060 & 0.1825794035757297 & 0.4022015706077854\end{array}$ $\begin{array}{llll}0.0315444284557002 & 0.2996862889970913 & 0.3944735331716340\end{array}$ $\begin{array}{llll}0.0284793345860249 & 0.3493854043141579 & 0.0970608052101484\end{array}$ $\begin{array}{lllll}0.2773286487484118 & 0.2331898794620731 & 0.1044257170658247\end{array}$ $\begin{array}{lllll}0.4078321956296356 & 0.1697974286884954 & 0.1083427309776632\end{array}$ $\begin{array}{lllll}0.4016750876871102 & 0.3642022414210499 & 0.3893124142462343\end{array}$ $\begin{array}{lllll}0.4096525109892555 & 0.1223383340585458 & 0.4004621935541399\end{array}$ $\begin{array}{lllll}0.2808889218923310 & 0.1829529365989188 & 0.4022501386061217\end{array}$ $\begin{array}{lllll}0.2821338077400076 & 0.3006208497631040 & 0.3940275939684866\end{array}$ $\begin{array}{llllll}0.2778089470981999 & 0.3506751877982711 & 0.0957585574245297\end{array}$ $\begin{array}{lllll}0.1482674364017709 & 0.4102379694328098 & 0.0981081190947126\end{array}$ $\begin{array}{lllll}0.3994228706251859 & 0.4089331289430869 & 0.0981563683032570\end{array}$ $\begin{array}{lllll}0.0272619755527811 & 0.2325118088354766 & 0.6049948184191899\end{array}$ $\begin{array}{lllll}0.1574639015714818 & 0.1691718255928037 & 0.6084087112041818\end{array}$ $\begin{array}{llll}0.1505966716370599 & 0.3628344134565663 & 0.8909636045200839\end{array}$ $\begin{array}{lllll}0.1591622706799666 & 0.1222168366775228 & 0.9005763696253698\end{array}$ $\begin{array}{lllll}0.0297342960380994 & 0.1819603904078330 & 0.9026176900038282\end{array}$ $\begin{array}{llll}0.0310180506493518 & 0.2994322791478365 & 0.8947855454141150\end{array}$ $\begin{array}{lllll}0.0282773249610513 & 0.3496845284881295 & 0.5970262684736785\end{array}$ $\begin{array}{llll}0.2768486400093441 & 0.2326662705230501 & 0.6043250041593000\end{array}$ $\begin{array}{lllll}0.4076126903261065 & 0.1697845504101159 & 0.6081975684157085\end{array}$ $\begin{array}{lllll}0.4000769856229503 & 0.3628482754953863 & 0.8891955272598847\end{array}$ $\begin{array}{llll}0.4091540009442475 & 0.1228110221747029 & 0.9003339860695958\end{array}$ $0.2797796218202692 \quad 0.18232987574698890 .9023354680091523$ $\begin{array}{lllll}0.2808199098975587 & 0.2996545806333668 & 0.8940026180160506\end{array}$ $\begin{array}{lllll}0.2778323752514917 & 0.3501328681431737 & 0.5956050438306959\end{array}$ $\begin{array}{lllll}0.1486044358155370 & 0.4098047128408092 & 0.5981603435871368\end{array}$ $\begin{array}{llll}0.3989703840557580 & 0.4095463946040101 & 0.5972791414073924\end{array}$ $\begin{array}{lllll}0.5280931622058525 & 0.2324286988157270 & 0.1050641576628467\end{array}$ $\begin{array}{lllll}0.6585124615864926 & 0.1699581247225492 & 0.1084192276052426\end{array}$ $\begin{array}{lllll}0.6519453000280022 & 0.3627574938786784 & 0.3919001474146254\end{array}$ $\begin{array}{lllll}0.6594061108376973 & 0.1225402756493098 & 0.4002002935494849\end{array}$ $\begin{array}{lllll}0.5301337066029028 & 0.1822947143743817 & 0.4022517576649160\end{array}$ $\begin{array}{llll}0.5318287728590294 & 0.2994062752032620 & 0.3944113512587500\end{array}$ $\begin{array}{llll}0.5291440637961741 & 0.3493613147030712 & 0.0987059314160959\end{array}$ $\begin{array}{lllll}0.7766289220921347 & 0.2350930746103193 & 0.1041969666189228\end{array}$ $\begin{array}{llll}0.9069012249771707 & 0.1708501238592480 & 0.1080606621101129\end{array}$ $\begin{array}{llllll}0.9012499920180087 & 0.3626977494585151 & 0.3918064916443180\end{array}$ $\begin{array}{lllll}0.9097200004951616 & 0.1222833195782773 & 0.4003825692394828\end{array}$ $\begin{array}{llllll}0.7804869561346204 & 0.1820110865393371 & 0.4026296261732871\end{array}$ $\begin{array}{llll}0.7819626534924408 & 0.2993516876844889 & 0.3952212289506317\end{array}$ 
$\begin{array}{llll}0.7769559400321796 & 0.3533542921512481 & 0.0980046933125886\end{array}$ $\begin{array}{llll}0.6470893123114430 & 0.4148568476443035 & 0.0978836922168324\end{array}$ $\begin{array}{lllll}0.9010655984345199 & 0.4099648574440811 & 0.1008313239279799\end{array}$ $\begin{array}{llll}0.5272691433235895 & 0.2329351645064326 & 0.6043936634505184\end{array}$ $\begin{array}{lllll}0.6576568706151764 & 0.1698483658888455 & 0.6082930470897465\end{array}$ $\begin{array}{lllll}0.6496669277872826 & 0.3638824814878738 & 0.89007777256945243\end{array}$ $\begin{array}{llll}0.6591139446486434 & 0.1231955769795375 & 0.9005037974822370\end{array}$ $\begin{array}{lllll}0.5297820375835233 & 0.1828243357054078 & 0.9022967820678774\end{array}$ $\begin{array}{llll}0.5307707350350471 & 0.3001196310952278 & 0.8940540765715800\end{array}$ $\begin{array}{llll}0.5283920604659446 & 0.3502279672613363 & 0.5959945620161818\end{array}$ $\begin{array}{lllll}0.7772419436795313 & 0.2331233941175776 & 0.6048513644851458\end{array}$ $\begin{array}{lllll}0.9074511814357864 & 0.1695761344261570 & 0.6086162699680451\end{array}$ $\begin{array}{lllll}0.9007020288681227 & 0.3631646418247373 & 0.8912194392845048\end{array}$ $\begin{array}{lllll}0.9083841774542696 & 0.1230625795631257 & 0.9004524909826002\end{array}$ $\begin{array}{llllll}0.7795556495484194 & 0.1833475858183941 & 0.9023996766033476\end{array}$ $\begin{array}{lllll}0.7802921116277237 & 0.3006685203005846 & 0.8944405787671934\end{array}$ $\begin{array}{llll}0.7785908447073239 & 0.3504789847973523 & 0.5969663959269216\end{array}$ $\begin{array}{llll}0.6492020797269519 & 0.4100878725015206 & 0.5986621945622075\end{array}$ $\begin{array}{lll}0.8995622844923731 & 0.4097906115597185 & 0.5994474295375163\end{array}$

Cyt/Cyt :

H 138 C 8 N 6 O 242 Al 32 Si 6

1.00000000000000

$20.2970019999999991 \quad 0.0000000000000000 \quad-3.7471719999999999$

$\begin{array}{llll}0.0646860000000000 & 24.9946149999999996 & -0.5148360000000000\end{array}$

$\begin{array}{llll}0.0000000000000000 & 0.0000000000000000 & 18.6939999999999991\end{array}$

$\mathrm{H} \quad \mathrm{C} \quad \mathrm{N} \quad \mathrm{O} \quad \mathrm{Al} \mathrm{Si}$

$\begin{array}{llllll}138 & 8 & 6 & 242 & 32 & 64\end{array}$

Direct

$0.7985901275809130 \quad 0.68848120243821140 .8995966505929460$ $\begin{array}{lllll}0.8782172505102442 & 0.6493097844250978 & 0.8250438666042491\end{array}$ $\begin{array}{llll}0.8290540337902754 & 0.5293702213324329 & 0.7061371324344740\end{array}$ $\begin{array}{lllll}0.8922802403677083 & 0.5754590045228332 & 0.7399845903204377\end{array}$ $\begin{array}{lllll}0.6915054280011499 & 0.6495111391626311 & 0.8849219657935922\end{array}$ $\begin{array}{llll}0.0936911948471240 & 0.0552435264726352 & 0.4116741502907823\end{array}$ $\begin{array}{llll}0.3433128190325738 & 0.0554439266422122 & 0.4114524613384539\end{array}$ $\begin{array}{lllll}0.0509461860019692 & 0.1392912102393273 & 0.1842958248409668\end{array}$ $\begin{array}{lllll}0.1700926879720935 & 0.3083730621682217 & 0.1632569162170563\end{array}$ $\begin{array}{lllll}0.1299490504752407 & 0.2208136924881674 & 0.3362298486727836\end{array}$ $\begin{array}{llll}0.2434466281494232 & 0.0394935842804282 & 0.3459645491585232\end{array}$ $\begin{array}{lllll}0.1715560258963353 & 0.0671666731203143 & 0.1496142421503505\end{array}$ $\begin{array}{llllll}0.3010600201921463 & 0.1396792921646511 & 0.1844505478754066\end{array}$ $\begin{array}{lllll}0.4203115683272947 & 0.3088255831154756 & 0.1635615044185955\end{array}$ $\begin{array}{lllll}0.3799856762909361 & 0.2211843145096760 & 0.3363674281836041\end{array}$ $\begin{array}{lllll}0.4933016566118676 & 0.0396331617099327 & 0.3458073136573721\end{array}$ $\begin{array}{lllll}0.4214186731966596 & 0.0675329634030587 & 0.1489065817767190\end{array}$ $\begin{array}{lllll}0.0571107011913609 & 0.4897141024215158 & 0.1537233112349970\end{array}$ $\begin{array}{lllll}0.2491420395191018 & 0.3897635159816015 & 0.3144227704261924\end{array}$ $\begin{array}{lllll}0.1291358495524022 & 0.4620617736385017 & 0.3501011113844009\end{array}$ $\begin{array}{llll}0.3065844309112680 & 0.4889427449200229 & 0.1512688086754373\end{array}$ $\begin{array}{llll}0.4988276569297325 & 0.3902850207736491 & 0.3151593456308715\end{array}$ $\begin{array}{lllll}0.3787804566651183 & 0.4618574874861039 & 0.3492282775489244\end{array}$ $\begin{array}{lllll}0.2062767365305555 & 0.4739512702765723 & 0.0875375652169986\end{array}$ $\begin{array}{lllll}0.4559286040620362 & 0.4741780718147058 & 0.0871749687690211\end{array}$ $\begin{array}{lllll}0.1545852867077998 & 0.4388553289545407 & 0.4686394834642380\end{array}$ $\begin{array}{llll}0.4054654795756279 & 0.4390508864742089 & 0.4678951686107894\end{array}$ $\begin{array}{llllll}0.1475557993729956 & 0.0906463068366874 & 0.0308035285168961\end{array}$ $\begin{array}{llll}0.3962623818579143 & 0.0911652909135965 & 0.0300799420228575\end{array}$ $\begin{array}{lllll}0.1649132986182835 & 0.4818554123891119 & 0.2871332789069113\end{array}$ $\begin{array}{llll}0.4142980706126810 & 0.4819951829279168 & 0.2861506612134945\end{array}$ $\begin{array}{lllll}0.1355446905625739 & 0.0474790147855429 & 0.2124575237456124\end{array}$ $\begin{array}{llll}0.3857760153753144 & 0.0473572606119757 & 0.2118187840551432\end{array}$ $\begin{array}{lllll}0.2117380018315286 & 0.4165267252950441 & 0.2403142016732328\end{array}$ $\begin{array}{lllll}0.4613338224844908 & 0.4159929227728056 & 0.2400550472525674\end{array}$ $\begin{array}{lllll}0.3383706532675925 & 0.1128008195882943 & 0.2586195331920458\end{array}$ $\begin{array}{lllll}0.0883476605516810 & 0.1129698570658147 & 0.2589041598514689\end{array}$ $\begin{array}{llll}0.0966948998016780 & 0.0535213891134642 & 0.9120143042840874\end{array}$ $\begin{array}{lllll}0.3442087731652478 & 0.0567053505973168 & 0.9105918276385722\end{array}$ $\begin{array}{lllll}0.0512845775113700 & 0.1396827899147505 & 0.6838456524423590\end{array}$ $\begin{array}{lllll}0.1702677888914278 & 0.3095170325117771 & 0.6632290716708479\end{array}$ $\begin{array}{lllll}0.1297619890307816 & 0.2205981242863309 & 0.8343313632028360\end{array}$ $\begin{array}{llll}0.2447202445172458 & 0.0400357379031339 & 0.8451765433624429\end{array}$ $\begin{array}{lllll}0.1721808438201616 & 0.0682038198627714 & 0.6488920781886593\end{array}$ 
$\begin{array}{llll}0.3014076624076043 & 0.1406740579749682 & 0.6834989262819350\end{array}$ $\begin{array}{llll}0.4188050971151844 & 0.3078429627282692 & 0.6589627576513397\end{array}$ $\begin{array}{llll}0.3806536137325473 & 0.2220578835790391 & 0.8351673735032061\end{array}$ $\begin{array}{llll}0.4922587071602713 & 0.0410958575632456 & 0.8455850325335723\end{array}$ $\begin{array}{lllll}0.4212773741337248 & 0.0680583362478672 & 0.6494602582861854\end{array}$ $\begin{array}{lll}0.0585556106472980 & 0.4882064575351165 & 0.6494877336834084\end{array}$ $\begin{array}{llll}0.2499205812014823 & 0.3906445316536359 & 0.8151283361444844\end{array}$ $\begin{array}{llll}0.1291254890683982 & 0.4620591454847197 & 0.8500082290631334\end{array}$ $\begin{array}{llll}0.3057610970146586 & 0.4893559173763327 & 0.6502910469951175\end{array}$ $\begin{array}{llll}0.4990930692131805 & 0.3818754707647484 & 0.8020496335470529\end{array}$ $\begin{array}{llll}0.3792767959364103 & 0.4626766497336276 & 0.8487503658978149\end{array}$ $\begin{array}{llll}0.2063777417213855 & 0.4746036660992173 & 0.5889357863134256\end{array}$ $\begin{array}{llll}0.4594926825796612 & 0.4721874145645832 & 0.5861807080563242\end{array}$ $\begin{array}{llll}0.1538543053354867 & 0.4387489504076890 & 0.9683849626942481\end{array}$ $\begin{array}{llll}0.4039615973908885 & 0.4392082261423613 & 0.9674842492665648\end{array}$ $\begin{array}{llll}0.1459260091227311 & 0.0914739859104013 & 0.5306627793526484\end{array}$ $\begin{array}{llll}0.3957447400237169 & 0.0912705907555813 & 0.5307433166505191\end{array}$ $\begin{array}{llll}0.1648341784231813 & 0.4822095596373992 & 0.7870760729333414\end{array}$ $\begin{array}{llll}0.4143199709730964 & 0.4825631751432812 & 0.7851890406227992\end{array}$ $\begin{array}{llll}0.1372345718254640 & 0.0474574626631980 & 0.7120438349512600\end{array}$ $\begin{array}{llll}0.3853373735895746 & 0.0487910949310840 & 0.7124865163545044\end{array}$ $\begin{array}{llll}0.2119298351475039 & 0.4167078269190944 & 0.7407292685132560\end{array}$ $\begin{array}{llll}0.4617335115511715 & 0.4169395659781129 & 0.7376702396719003\end{array}$ $\begin{array}{lllll}0.3389671005586946 & 0.1145860273848786 & 0.7582972122054552\end{array}$ $\begin{array}{lllll}0.0887403912581676 & 0.1130384937467496 & 0.7580751001085846\end{array}$ $\begin{array}{llll}0.5929298080297625 & 0.0557087227186147 & 0.4112231287357901\end{array}$ $\begin{array}{llll}0.8421462708338527 & 0.0563237848903693 & 0.4108915491370768\end{array}$ $\begin{array}{llll}0.5509679425884126 & 0.1402717719066281 & 0.1845952373971152\end{array}$ $\begin{array}{llll}0.6698254101230137 & 0.3081875108539870 & 0.1624172393625122\end{array}$ $\begin{array}{llll}0.6298682567390740 & 0.2213073549541940 & 0.3363716192742390\end{array}$ $\begin{array}{llll}0.7430934027974432 & 0.0396611167866778 & 0.3460892055119397\end{array}$ $\begin{array}{llll}0.6713008013900508 & 0.0675292653605612 & 0.1491928648360785\end{array}$ $\begin{array}{llll}0.8009947734053642 & 0.1400859927895186 & 0.1848040541521534\end{array}$ $\begin{array}{lllll}0.9195736713815355 & 0.3077768127036040 & 0.1625558769618963\end{array}$ $\begin{array}{llll}0.8803551487344069 & 0.2215661912564163 & 0.3376331546097554\end{array}$ $\begin{array}{llll}0.9931357455057711 & 0.0402086268160798 & 0.3473843979420154\end{array}$ $\begin{array}{llll}0.9213060879208222 & 0.0673447825858062 & 0.1497463888331852\end{array}$ $\begin{array}{llll}0.5560798683473105 & 0.4891165633488552 & 0.1515231858448289\end{array}$ $\begin{array}{lllll}0.7483940953639970 & 0.3910982160553987 & 0.3168005184265555\end{array}$ $\begin{array}{llll}0.6275508658359147 & 0.4609061354555378 & 0.3488818754212268\end{array}$ $0.80824529916083790 .4887567240363344 \quad 0.1528015428071611$ $\begin{array}{llll}0.9998352431314221 & 0.3900436191317745 & 0.3162539609148451\end{array}$ $\begin{array}{llll}0.8788546523574327 & 0.4620638091141074 & 0.3511181740519348\end{array}$ $\begin{array}{llll}0.7078276093280160 & 0.4736728966046561 & 0.0906015890093155\end{array}$ $\begin{array}{lllll}0.9585723340331260 & 0.4726552888344217 & 0.0917973345830533\end{array}$ $\begin{array}{llll}0.6479654626704845 & 0.4399714820271372 & 0.4665475197864803\end{array}$ $\begin{array}{lllll}0.9053796709403817 & 0.4378671528813974 & 0.4699768678866369\end{array}$ $\begin{array}{llll}0.6449632680601980 & 0.0908642201046436 & 0.0305722821462616\end{array}$ $\begin{array}{lllll}0.8955913448875232 & 0.0908759557085866 & 0.0309309802360768\end{array}$ $\begin{array}{lllll}0.6626793455357212 & 0.4826168842021113 & 0.2857919728256220\end{array}$ $\begin{array}{llll}0.9147957805931600 & 0.4813412102980821 & 0.2881596105062674\end{array}$ $\begin{array}{llll}0.6358526396627127 & 0.0473604402739005 & 0.2122355018318021\end{array}$ $0.88532074248259160 .0477352526628095 \quad 0.2126180830455826$ $\begin{array}{lllll}0.7112222698424873 & 0.4163808910872914 & 0.2410416673084211\end{array}$ $\begin{array}{llll}0.9618471120517750 & 0.4161303518161171 & 0.2417400605682011\end{array}$ $\begin{array}{llll}0.8383594153603097 & 0.1132408145421478 & 0.2589461492444952\end{array}$ $\begin{array}{lllll}0.5882378128922738 & 0.1130183515705888 & 0.2583762263718530\end{array}$ $\begin{array}{llll}0.7004938044693056 & 0.7078245297885907 & 0.6294288766052933\end{array}$ $\begin{array}{llll}0.7892864321302726 & 0.6549472147254368 & 0.5834304639522853\end{array}$ $\begin{array}{llll}0.7756106417292101 & 0.5074449105554574 & 0.5676632434920145\end{array}$ $\begin{array}{llll}0.8241249344824384 & 0.5616276111821864 & 0.5585593143527418\end{array}$ $0.6048392502199234 \quad 0.66142551201350250 .6491427383076469$ $\begin{array}{llll}0.5913006425059741 & 0.0560111578959530 & 0.9109930491390895\end{array}$ $\begin{array}{llll}0.8428488259076586 & 0.0554922743271210 & 0.9102622363561335\end{array}$ $\begin{array}{lllll}0.5518234058794812 & 0.1402435263346984 & 0.6841652501272356\end{array}$ $\begin{array}{llll}0.6703317942918335 & 0.3082362370064648 & 0.6616027934847588\end{array}$ $\begin{array}{llll}0.6303108052510578 & 0.2229204422568195 & 0.8374231724836743\end{array}$ $\begin{array}{llll}0.7435336689913358 & 0.0394949573979944 & 0.8455629084853857\end{array}$ $\begin{array}{llll}0.6721344677251366 & 0.0681117999774806 & 0.6481184547244486\end{array}$ $\begin{array}{llll}0.8011065180883840 & 0.1414560258160441 & 0.6854459351311821\end{array}$ $\begin{array}{llll}0.9212386448022246 & 0.3102840831897608 & 0.6674879160433331\end{array}$ $\begin{array}{lllll}0.8806011709096437 & 0.2200733937267446 & 0.8372676723711008\end{array}$ $\begin{array}{llll}0.9937899167459632 & 0.0408889579561665 & 0.8480305638309343\end{array}$ 
$\begin{array}{llll}0.9210303741632098 & 0.0679830866885772 & 0.6491617708644438\end{array}$ $\begin{array}{lllll}0.5485630780612976 & 0.4962852013461585 & 0.6523969159733041\end{array}$ $\begin{array}{lllll}0.7496473161362719 & 0.3934031930626794 & 0.8227715457442711\end{array}$ $\begin{array}{llll}0.6270772320205302 & 0.4651214512122278 & 0.8485625732011302\end{array}$ $\begin{array}{llll}0.7504094956909656 & 0.4797346706788401 & 0.68744355820721224\end{array}$ $\begin{array}{lllll}0.0007893508932360 & 0.3887954216963062 & 0.8137501213562516\end{array}$ $\begin{array}{llllll}0.8837035607618386 & 0.4575312551288346 & 0.8582875906427233\end{array}$ $\begin{array}{lllll}0.6621523577222413 & 0.4998869399879981 & 0.5654227211577169\end{array}$ $\begin{array}{llll}0.9574262191501213 & 0.4749558210000028 & 0.5939274567312998\end{array}$ $\begin{array}{llll}0.6574925845693431 & 0.4391621369393499 & 0.9693673517680235\end{array}$ $\begin{array}{lllll}0.9053172796631721 & 0.4353304138806581 & 0.9739600271056993\end{array}$ $\begin{array}{llll}0.6460800485963755 & 0.0915737452250655 & 0.5303464789311927\end{array}$ $\begin{array}{lllll}0.8947863890152680 & 0.0914012835920142 & 0.5307701701636404\end{array}$ $\begin{array}{llll}0.6252247458056712 & 0.5141847004153367 & 0.7907104259757253\end{array}$ $\begin{array}{lllll}0.9292921375873212 & 0.4674474508860979 & 0.8009771703708476\end{array}$ $\begin{array}{lllll}0.6360396783441489 & 0.0477842533440190 & 0.7106179868014388\end{array}$ $\begin{array}{llllll}0.8858885471637634 & 0.0478225969141871 & 0.7124649319573175\end{array}$ $\begin{array}{lllll}0.7144860464542973 & 0.4200104645958178 & 0.7481450938068588\end{array}$ $\begin{array}{lllll}0.9611948185556817 & 0.4145712511774203 & 0.7383377834546107\end{array}$ $\begin{array}{llll}0.8386404967425132 & 0.1134456272189005 & 0.7583792130392130\end{array}$ $\begin{array}{lllll}0.5884764206749704 & 0.1126662580252508 & 0.7581546746518200\end{array}$ $\begin{array}{lllll}0.7858164839413029 & 0.6554010406790943 & 0.8607454230068486\end{array}$ $\begin{array}{lllll}0.8283523550656728 & 0.6339504208350694 & 0.8202062079086332\end{array}$ $\begin{array}{lllll}0.8047655699203664 & 0.5897997488288291 & 0.7719943773625243\end{array}$ $\begin{array}{llll}0.7018348405903463 & 0.5915672021864349 & 0.8070967298148309\end{array}$ $\begin{array}{lllll}0.6962695007177212 & 0.6650038440484866 & 0.6172154521699753\end{array}$ $\begin{array}{llll}0.7439644614924875 & 0.6362040903000441 & 0.5919312154371331\end{array}$ $\begin{array}{lllll}0.7327544186685420 & 0.5798872793502019 & 0.5801309401645195\end{array}$ $\begin{array}{lllll}0.6281560325933117 & 0.5857298181381611 & 0.6154994251093960\end{array}$ $\begin{array}{lllll}0.7238778947869499 & 0.6352650875021416 & 0.8540173666177207\end{array}$ $\begin{array}{lllll}0.8433562499883031 & 0.5671898944423956 & 0.7289523472907442\end{array}$ $\begin{array}{llll}0.7436139232757495 & 0.5700426514923553 & 0.7665554364436874\end{array}$ $\begin{array}{lllll}0.6405152031929946 & 0.6408183229664184 & 0.6291044928362273\end{array}$ $\begin{array}{lllll}0.7780416055653232 & 0.5475415892521707 & 0.5579737846936539\end{array}$ $\begin{array}{lllll}0.6763905267792697 & 0.5573857322781106 & 0.5902208047155753\end{array}$ $\begin{array}{lllll}0.6441282885397785 & 0.5759087765721618 & 0.8062229501852002\end{array}$ $\begin{array}{lllll}0.0464679561911998 & 0.3500670809607043 & 0.1862138379489816\end{array}$ $\begin{array}{lllll}0.0299480801972754 & 0.2409297148326168 & 0.1934615096172017\end{array}$ $\begin{array}{lllll}0.1551210819607548 & 0.2822167903186754 & 0.1943070217853869\end{array}$ $\begin{array}{lllll}0.1996923271676994 & 0.2077283252539625 & 0.0714756319447645\end{array}$ $\begin{array}{lllll}0.0345076632358225 & 0.2876845888987143 & 0.0680322991131733\end{array}$ $\begin{array}{lllll}0.0764384675694851 & 0.1859387881168899 & 0.08879377999971511\end{array}$ $\begin{array}{llll}0.1801618984422215 & 0.1679752318075178 & 0.1965272896222902\end{array}$ $\begin{array}{lllll}0.1792665934214586 & 0.0551701300350426 & 0.2021142686130603\end{array}$ $\begin{array}{lllll}0.0467622819927470 & 0.1098954596033873 & 0.2167931957268063\end{array}$ $\begin{array}{lllll}0.1588377186397794 & 0.1049055137394254 & 0.0815684373535598\end{array}$ $\begin{array}{llll}0.1488673439070278 & 0.1149138777606425 & 0.3129526857833335\end{array}$ $\begin{array}{lllll}0.0208156911381454 & 0.0678546574297828 & 0.3331242287149532\end{array}$ $\begin{array}{lllll}0.1379324905762202 & 0.0654064146004006 & 0.4398842226957482\end{array}$ $\begin{array}{llll}0.1042280353549508 & 0.1669210135603082 & 0.4223517487218514\end{array}$ $\begin{array}{lllll}0.0202401300411941 & 0.2884855555660394 & 0.3065428304440337\end{array}$ $\begin{array}{llll}0.1449118269261559 & 0.2470044918366228 & 0.3052370871340230\end{array}$ $\begin{array}{lllll}0.1000761525664039 & 0.3218353658786118 & 0.4279954876694223\end{array}$ $\begin{array}{llll}0.0162255703195336 & 0.2414401950647420 & 0.4320429616201233\end{array}$ $\begin{array}{lllll}0.1201846669681197 & 0.3613233738234690 & 0.3031083844339417\end{array}$ $\begin{array}{lllll}0.2291646810915459 & 0.1410066253960697 & 0.4390701073765439\end{array}$ $\begin{array}{llll}0.2538545876999130 & 0.1792870657650296 & 0.3135567799526256\end{array}$ $\begin{array}{lllll}0.2236327327135842 & 0.3429553706134795 & 0.4111622030761909\end{array}$ $\begin{array}{lllll}0.2957687714760882 & 0.3500093608515685 & 0.1853729401937440\end{array}$ $\begin{array}{lllll}0.2797801624191164 & 0.2409130314775073 & 0.1929665358532546\end{array}$ $\begin{array}{lllll}0.4049181643311325 & 0.2822705396847640 & 0.1937502844920782\end{array}$ $\begin{array}{lllll}0.4497298521488143 & 0.2078879072834281 & 0.0711358045971204\end{array}$ $\begin{array}{lllll}0.2835990573868690 & 0.2878605620608296 & 0.0672799493303191\end{array}$ $\begin{array}{lllll}0.3263404105964132 & 0.1863957099835687 & 0.0881208411140969\end{array}$ $\begin{array}{llll}0.4299515160749052 & 0.1681079127474963 & 0.1961505295688294\end{array}$ $\begin{array}{llll}0.4293797331827239 & 0.0553282589785860 & 0.2013628048754835\end{array}$ $\begin{array}{lllll}0.2967709086154534 & 0.1100117760283361 & 0.2164813953911103\end{array}$ $\begin{array}{lllll}0.4084063302602783 & 0.1052279548126607 & 0.0809838232393542\end{array}$ $\begin{array}{llllll}0.3988771347403975 & 0.1146104797682044 & 0.3126623756466888\end{array}$ $\begin{array}{lllll}0.2708226054925289 & 0.0678109974583678 & 0.3328599645010795\end{array}$ $\begin{array}{lllll}0.3875959769947351 & 0.0655049157652046 & 0.4397139198621073\end{array}$ $\begin{array}{llll}0.3543766133220614 & 0.1671189202641252 & 0.4218139656584301\end{array}$ $\begin{array}{lllll}0.2699862793104551 & 0.2884972627742111 & 0.3060676020038864\end{array}$ 
$\begin{array}{llll}0.3946118051895707 & 0.2470107983770822 & 0.3046494813445196\end{array}$ $\begin{array}{llllll}0.3503103902780404 & 0.3217608396100534 & 0.4272987078800769\end{array}$ $\begin{array}{lllll}0.2662226391286374 & 0.2415507711830171 & 0.4316506823862249\end{array}$ $\begin{array}{lllll}0.3696844345203992 & 0.3612559856949052 & 0.3022299392688823\end{array}$ $\begin{array}{lllll}0.4792037249657145 & 0.1408082383562884 & 0.4387407868828224\end{array}$ $\begin{array}{lllll}0.0038777300621619 & 0.1793165975628291 & 0.3138696280521006\end{array}$ $\begin{array}{lllll}0.4735194949642295 & 0.3431470318171382 & 0.4101829114398189\end{array}$ $\begin{array}{lllll}0.1510057884691713 & 0.4147118434949320 & 0.1862949056960947\end{array}$ $\begin{array}{lllll}0.0293128723667769 & 0.4618270264477038 & 0.1671840202160567\end{array}$ $\begin{array}{llllll}0.0704764075683137 & 0.3885194710330387 & 0.0605325962663433\end{array}$ $\begin{array}{lllll}0.1619539367139795 & 0.4639128434405643 & 0.0592788316156248\end{array}$ $\begin{array}{lllll}0.1954124758639002 & 0.3622772443909123 & 0.0769806759121280\end{array}$ $\begin{array}{llll}0.1212305499296999 & 0.4741557687692502 & 0.2976031825196008\end{array}$ $\begin{array}{lllll}0.5027902132374236 & 0.4193317802252127 & 0.2820612419166899\end{array}$ $\begin{array}{lllll}0.1417419378240982 & 0.4244088998702175 & 0.4180516863551869\end{array}$ $\begin{array}{lllll}0.4008140537043092 & 0.4141920742451455 & 0.1855485827421349\end{array}$ $\begin{array}{lllll}0.2788759328847897 & 0.4614018266845940 & 0.1657546665295971\end{array}$ $\begin{array}{llllll}0.3205530415526998 & 0.3883497985258710 & 0.0596308279044577\end{array}$ $\begin{array}{llll}0.4116083978840182 & 0.4641442785014935 & 0.0589817848480358\end{array}$ $\begin{array}{lllll}0.4456268230043129 & 0.3625181379781662 & 0.0760170959163658\end{array}$ $\begin{array}{lllll}0.3707062982815387 & 0.4740183102324232 & 0.2966519846757052\end{array}$ $\begin{array}{lllll}0.2532387142043895 & 0.4194725137388863 & 0.2824002426698898\end{array}$ $\begin{array}{lllll}0.3917120670283167 & 0.4244921176358340 & 0.4170902296330768\end{array}$ $\begin{array}{lllll}0.0453058682366042 & 0.3507941117263600 & 0.6855037740323292\end{array}$ $\begin{array}{llllll}0.0300318471182273 & 0.2415325472572724 & 0.6932460609932102\end{array}$ $\begin{array}{lllll}0.1550750752369481 & 0.2831294009204875 & 0.6938089762247058\end{array}$ $\begin{array}{llllll}0.2000556542853696 & 0.2086277543660031 & 0.5711290333390098\end{array}$ $\begin{array}{lllll}0.0331577760504027 & 0.2887565084052477 & 0.5674856170750384\end{array}$ $\begin{array}{llll}0.0767074899317880 & 0.1873588454689823 & 0.5882490815011762\end{array}$ $\begin{array}{llll}0.1804093083805020 & 0.1688778310947955 & 0.6961377356256598\end{array}$ $\begin{array}{lllll}0.1806108990430357 & 0.0558372178192595 & 0.7012864662063867\end{array}$ $\begin{array}{lllll}0.0471814112941263 & 0.1101148830831054 & 0.7160926259421337\end{array}$ $\begin{array}{lllll}0.1585367734158190 & 0.1059588832866422 & 0.5812351169177938\end{array}$ $\begin{array}{lllll}0.1496286688692280 & 0.1149898125688625 & 0.8123784468126101\end{array}$ $\begin{array}{lllll}0.0215261021474071 & 0.0679093882277099 & 0.8324114556470352\end{array}$ $\begin{array}{lllll}0.1408166700889485 & 0.0643385055757140 & 0.9394061992800252\end{array}$ $\begin{array}{lllll}0.1046250026664213 & 0.1653598222861304 & 0.9227457167248831\end{array}$ $\begin{array}{lllll}0.0203511800082562 & 0.2886726702999796 & 0.8068473793923874\end{array}$ $\begin{array}{lllll}0.1450187373106570 & 0.2475348315883767 & 0.8046303911444423\end{array}$ $\begin{array}{llll}0.1011915238443373 & 0.3214834197986969 & 0.9278533738889464\end{array}$ $\begin{array}{lllll}0.0180442432466326 & 0.2405329304714028 & 0.9323546148975107\end{array}$ $\begin{array}{llll}0.1196820176166009 & 0.3616242709375725 & 0.8025461303950941\end{array}$ $\begin{array}{lllll}0.2298430408448406 & 0.1414587357050470 & 0.9385593792555306\end{array}$ $\begin{array}{llllll}0.2542207641032851 & 0.1800633575749311 & 0.8131353703025153\end{array}$ $\begin{array}{lllll}0.2239788840588056 & 0.3440352578639621 & 0.9101559668153563\end{array}$ $\begin{array}{lllll}0.2960502088287637 & 0.3509821142404012 & 0.6849296671655070\end{array}$ $\begin{array}{llll}0.2800964670852349 & 0.2417888256489459 & 0.6926148537815117\end{array}$ $\begin{array}{lllll}0.4054003434878403 & 0.2832269122548066 & 0.6931656446738618\end{array}$ $\begin{array}{lllll}0.4502422955421130 & 0.2081713179136995 & 0.5713690979765378\end{array}$ $\begin{array}{lllll}0.2843444086628288 & 0.2886346128516492 & 0.5670448344934703\end{array}$ $\begin{array}{lllll}0.3266494971616654 & 0.1869983230076104 & 0.5880675425664592\end{array}$ $\begin{array}{lllll}0.4304312161025529 & 0.1689409369345347 & 0.6961860342709347\end{array}$ $\begin{array}{lllll}0.4291146271647331 & 0.0561275355742363 & 0.7020141279213815\end{array}$ $\begin{array}{llll}0.2974802601664081 & 0.1112115696806462 & 0.7159868933513633\end{array}$ $\begin{array}{llll}0.4084073666775508 & 0.1056225147412662 & 0.5813769523549885\end{array}$ $\begin{array}{lllll}0.3990940506756964 & 0.1166017118713977 & 0.8124988039607473\end{array}$ $\begin{array}{lllll}0.2718734047625430 & 0.0685629562830361 & 0.8322127677890289\end{array}$ $\begin{array}{lllll}0.3884083998243739 & 0.0668215136407313 & 0.9391010198444649\end{array}$ $\begin{array}{lllll}0.3546863184129074 & 0.1684392970107031 & 0.9219369931602004\end{array}$ $\begin{array}{llll}0.2702607903161372 & 0.2892260444982516 & 0.8054542597542905\end{array}$ $\begin{array}{lllll}0.3955281588219621 & 0.2484033328134835 & 0.8043408868383696\end{array}$ $\begin{array}{lllll}0.3503802821800541 & 0.3223783426876110 & 0.9268124017670402\end{array}$ $\begin{array}{lllll}0.2657926011550422 & 0.2424095031213477 & 0.9310184746415109\end{array}$ $\begin{array}{lllll}0.3703212496239555 & 0.3619558637580999 & 0.8018954674972730\end{array}$ $\begin{array}{llll}0.4797036294733609 & 0.1425639325323464 & 0.9384574790395870\end{array}$ $\begin{array}{lllll}0.0047957694163138 & 0.1791218028627377 & 0.8136211390586549\end{array}$ $\begin{array}{lllll}0.4736155451978880 & 0.3431917041680057 & 0.9118546354474563\end{array}$ $\begin{array}{lllll}0.1508142751566290 & 0.4147748396234195 & 0.6860920303366349\end{array}$ $\begin{array}{lllll}0.0294547415691417 & 0.4632426633464848 & 0.6671277657109851\end{array}$ $\begin{array}{lllll}0.0708126831654644 & 0.3892111381272104 & 0.5601845655046996\end{array}$ $\begin{array}{lllll}0.1622238801844501 & 0.4646059188827814 & 0.5598121776132241\end{array}$ $\begin{array}{llll}0.1957646069950729 & 0.3628081581776420 & 0.5765052448571101\end{array}$ $\begin{array}{llll}0.1211753876207871 & 0.4744641153632447 & 0.7976005626666590\end{array}$ 
$\begin{array}{llll}0.5025851979098503 & 0.4164524598166512 & 0.7796136230741675\end{array}$ $\begin{array}{lllll}0.1410573273961843 & 0.4245242348835479 & 0.9176430014939345\end{array}$ $\begin{array}{lllll}0.3993783252146829 & 0.4167966284397616 & 0.6842757701446690\end{array}$ $\begin{array}{lllll}0.2774755977086463 & 0.4627040334194504 & 0.6658622174869324\end{array}$ $\begin{array}{llll}0.3208416729311651 & 0.3891787936565406 & 0.5588322240761832\end{array}$ $\begin{array}{lllll}0.4149471261179030 & 0.4625962004027854 & 0.5565533706517803\end{array}$ $\begin{array}{lllll}0.4455776714244675 & 0.3607140604283483 & 0.5795388630204897\end{array}$ $\begin{array}{llll}0.3708610592701588 & 0.4749495552740535 & 0.7963609325697190\end{array}$ $\begin{array}{llll}0.2533932307674898 & 0.4201057411789306 & 0.7824920337398370\end{array}$ $\begin{array}{llll}0.3913384406756729 & 0.4249828380431555 & 0.9168062470272674\end{array}$ $\begin{array}{lllll}0.5455551348884312 & 0.3499788832548475 & 0.1848317375002209\end{array}$ $\begin{array}{lllll}0.5297382793645214 & 0.2408625765321639 & 0.1926219436388621\end{array}$ $\begin{array}{lllll}0.6550166799401981 & 0.2820506844225345 & 0.1936188653508232\end{array}$ $\begin{array}{llll}0.7000066296058970 & 0.2077331499822012 & 0.0715320119917071\end{array}$ $\begin{array}{llllll}0.5339325983804145 & 0.2879660937929344 & 0.0670276809653742\end{array}$ $\begin{array}{lllll}0.5764448558504894 & 0.1864667340207632 & 0.0879427263846258\end{array}$ $\begin{array}{llll}0.6800279029405918 & 0.1679969058419332 & 0.1963214158289502\end{array}$ $\begin{array}{llll}0.6794243768487299 & 0.0552706145684562 & 0.2016374138201070\end{array}$ $\begin{array}{lllll}0.5467736500490710 & 0.1103201307031642 & 0.2162063791039425\end{array}$ $\begin{array}{llll}0.6582933330830142 & 0.1051208225446902 & 0.0812143750900342\end{array}$ $\begin{array}{lllll}0.6488569301054538 & 0.1146210932577066 & 0.3127395809790555\end{array}$ $\begin{array}{lllll}0.5208738608492399 & 0.0676664642293053 & 0.3323212763819878\end{array}$ $\begin{array}{lllll}0.6372074964782206 & 0.0656823777511974 & 0.4396530160459445\end{array}$ $\begin{array}{lllll}0.6042277471173603 & 0.1673622466597462 & 0.4215969185899736\end{array}$ $\begin{array}{lllll}0.5198130798598684 & 0.2882659958007130 & 0.3056243130013667\end{array}$ $\begin{array}{lllll}0.6445256166838609 & 0.2470124092040128 & 0.3045173213017277\end{array}$ $\begin{array}{lllll}0.6002376077569066 & 0.3214116835622274 & 0.4266934002025008\end{array}$ $\begin{array}{llllll}0.5157028833484891 & 0.2415753305800119 & 0.4312330239685756\end{array}$ $\begin{array}{lllll}0.6194876076859076 & 0.3607271417119849 & 0.3012560283276319\end{array}$ $\begin{array}{llll}0.7290391897158145 & 0.1408746156764620 & 0.4389772434744364\end{array}$ $\begin{array}{lllll}0.7534709701298409 & 0.1793560558783711 & 0.3136614389518773\end{array}$ $\begin{array}{lllll}0.7230932819468556 & 0.3438174192478319 & 0.4097956755240815\end{array}$ $\begin{array}{lllll}0.7961879615034274 & 0.3499261593568880 & 0.1864664038043916\end{array}$ $\begin{array}{lllll}0.7799762296358561 & 0.2407346043883327 & 0.1933090923684614\end{array}$ $\begin{array}{llll}0.9051477734126679 & 0.2822042617786563 & 0.1947686648570757\end{array}$ $\begin{array}{lllll}0.9499450191457589 & 0.2078358385445737 & 0.0718612007636640\end{array}$ $\begin{array}{lllll}0.7845156905366376 & 0.2878693244321965 & 0.0680841048125359\end{array}$ $\begin{array}{lllll}0.8265562350039580 & 0.1862246674988289 & 0.0884929129965135\end{array}$ $\begin{array}{lllll}0.9301926938904846 & 0.1680535365597580 & 0.1967267973164534\end{array}$ $\begin{array}{lllll}0.9290650089899029 & 0.0552860765506564 & 0.2022216421268257\end{array}$ $\begin{array}{lllll}0.7968798115247905 & 0.1103027966145445 & 0.2167097122166339\end{array}$ $\begin{array}{lllll}0.9087166854190123 & 0.1050761559591784 & 0.0815864043154811\end{array}$ $\begin{array}{lllll}0.8988148354899784 & 0.1149918088105459 & 0.3131421320365376\end{array}$ $\begin{array}{lllll}0.7707933707354347 & 0.0676209777005009 & 0.3326450335651647\end{array}$ $\begin{array}{llllll}0.8863339501380103 & 0.0662022004451357 & 0.4398360595402674\end{array}$ $\begin{array}{lllll}0.8540617260673231 & 0.1680238841714464 & 0.4218008498003207\end{array}$ $\begin{array}{lllll}0.7701282257715288 & 0.2881496562471705 & 0.3064266334527950\end{array}$ $\begin{array}{llllll}0.8950031387183454 & 0.2472037560968244 & 0.3056503405462853\end{array}$ $\begin{array}{lllll}0.8495930923316346 & 0.3215093355317221 & 0.4285397297015197\end{array}$ $\begin{array}{llll}0.7649318137625272 & 0.2418271990772666 & 0.4319710211374855\end{array}$ $\begin{array}{lllll}0.8695121488814107 & 0.3614092843995329 & 0.3039497490534246\end{array}$ 0.97882281136490330 .14101048958253050 .4393156520717736 $\begin{array}{llll}0.5036305837282464 & 0.1792339070190923 & 0.3132503000528130\end{array}$ $\begin{array}{llll}0.9733376954849329 & 0.3427674821878155 & 0.4114774666551531\end{array}$ $\begin{array}{llll}0.6510950627543615 & 0.4143070724173984 & 0.1863872161440402\end{array}$ $\begin{array}{lllll}0.5285808493561064 & 0.4610710310216207 & 0.1651161366550908\end{array}$ $\begin{array}{lllll}0.5712308683722719 & 0.3885498126955066 & 0.0597756993278611\end{array}$ $\begin{array}{lllll}0.6638060151385421 & 0.4640978804285019 & 0.0610604577278653\end{array}$ $\begin{array}{lllll}0.6959496277879630 & 0.3619851908456221 & 0.0776848413315465\end{array}$ $\begin{array}{llll}0.6192601627628367 & 0.4741414620563076 & 0.2962789738844244\end{array}$ $\begin{array}{llllll}0.0036981537094256 & 0.4194307485773667 & 0.2836409270364908\end{array}$ $\begin{array}{lllll}0.6397631393724629 & 0.4245618235606731 & 0.4145168406539173\end{array}$ $\begin{array}{llll}0.9014441053276171 & 0.4140573619043497 & 0.1881950381790476\end{array}$ $\begin{array}{lllll}0.77999756372990994 & 0.4617611109129867 & 0.1675481572565119\end{array}$ $\begin{array}{llll}0.8212712770151852 & 0.3885725428024119 & 0.0615397938658646\end{array}$ $\begin{array}{llll}0.9141116457264660 & 0.4634870323903025 & 0.0624519006312442\end{array}$ $\begin{array}{lllll}0.9456563613226318 & 0.3615061920931020 & 0.0786810485256233\end{array}$ $\begin{array}{lllll}0.8710314545402544 & 0.4740945654424329 & 0.2987332586768593\end{array}$ $\begin{array}{lllll}0.7528584683624276 & 0.4196971031148993 & 0.2831718950515180\end{array}$ $\begin{array}{llll}0.8916975083044629 & 0.4240773967706593 & 0.4194963768767477\end{array}$ $\begin{array}{llll}0.5758664484804404 & 0.5665759554779369 & 0.6264260577728701\end{array}$ $\begin{array}{llll}0.5493501307227489 & 0.3515918472484375 & 0.6851984006967839\end{array}$ $\begin{array}{llll}0.5304428443528059 & 0.2420735440064682 & 0.6930945199193042\end{array}$ 
$\begin{array}{llll}0.6561773071273378 & 0.2825554927471446 & 0.6939371519854821\end{array}$ $\begin{array}{lllll}0.7000905870652778 & 0.2090214909152579 & 0.5717980297535472\end{array}$ $\begin{array}{lllll}0.5344117734750293 & 0.2878145790515569 & 0.5672535754769805\end{array}$ $\begin{array}{llll}0.5766782350101507 & 0.1863709834088688 & 0.5886426194055234\end{array}$ $\begin{array}{llllll}0.6803583252357941 & 0.1682469471023131 & 0.6963790979388339\end{array}$ $\begin{array}{lllll}0.6798260206289982 & 0.0553956612440510 & 0.7003970431949667\end{array}$ $\begin{array}{lllll}0.5469117745690444 & 0.1105839297293827 & 0.7160074179139549\end{array}$ $\begin{array}{lllll}0.6596092318727830 & 0.1059693157688182 & 0.5808598394103317\end{array}$ $\begin{array}{llll}0.6491461431378449 & 0.1132810936597163 & 0.8123595939469895\end{array}$ $\begin{array}{lllll}0.5203380915139985 & 0.0689325392197026 & 0.8325245606291465\end{array}$ $\begin{array}{lllll}0.6357889153003400 & 0.0653452136565706 & 0.9395538887905984\end{array}$ $\begin{array}{lllll}0.6050772565122657 & 0.1674149870789996 & 0.9206937586609080\end{array}$ $\begin{array}{lllll}0.5205707723124643 & 0.2908080848840708 & 0.8055299610609585\end{array}$ $\begin{array}{lllll}0.6449800518562339 & 0.2480710445390451 & 0.8048569799640757\end{array}$ $\begin{array}{llll}0.6011148160714254 & 0.3235076249271494 & 0.9268212221742063\end{array}$ $\begin{array}{lllll}0.5182807971536713 & 0.2427105682896521 & 0.9309907714470415\end{array}$ $\begin{array}{llllll}0.6224949734793735 & 0.3631575312706181 & 0.8020154374583235\end{array}$ $\begin{array}{llllll}0.7293002907973783 & 0.1389031071105711 & 0.9386920283445767\end{array}$ $\begin{array}{lllll}0.7531505300474425 & 0.1787145789273078 & 0.8136971170904391\end{array}$ $\begin{array}{llllll}0.7249356291045378 & 0.3432553098626854 & 0.9108906778143053\end{array}$ $\begin{array}{lllll}0.7972949560510548 & 0.3505868568064174 & 0.6884991561994726\end{array}$ $\begin{array}{lllll}0.7805273230410381 & 0.2408372396890154 & 0.6935757496220393\end{array}$ $\begin{array}{lllll}0.9055165263506625 & 0.2824360602343705 & 0.6954228775784664\end{array}$ $\begin{array}{lllll}0.9500801848442987 & 0.2081781507626845 & 0.5715851352464718\end{array}$ $\begin{array}{lllll}0.7845708287775548 & 0.2895594360981583 & 0.5691278824238480\end{array}$ $\begin{array}{lllll}0.8267026277862575 & 0.1875228148387435 & 0.5880148114463526\end{array}$ $\begin{array}{llllll}0.9304573358380208 & 0.1684038109670411 & 0.6965005922894242\end{array}$ $\begin{array}{lllll}0.9293938100848146 & 0.0555528874747187 & 0.7015415586813265\end{array}$ $\begin{array}{lllll}0.7972143457962417 & 0.1109024902489483 & 0.7161584269287414\end{array}$ $\begin{array}{llllll}0.9079633828673903 & 0.1056870475777161 & 0.5814137076528668\end{array}$ $\begin{array}{lllll}0.8992673500665264 & 0.1149734507223180 & 0.8127606207604287\end{array}$ $\begin{array}{llll}0.7715207721057619 & 0.0669231376907900 & 0.8313212568021470\end{array}$ $\begin{array}{lllll}0.8869272403546233 & 0.0655455570981230 & 0.9392380634368078\end{array}$ $\begin{array}{llll}0.8539041721891297 & 0.1672818260856665 & 0.9217458784351023\end{array}$ $\begin{array}{lllll}0.7707513274590293 & 0.2869639947413932 & 0.8072397959577933\end{array}$ $\begin{array}{lllll}0.8953608108118764 & 0.2460379453444234 & 0.8058157811140657\end{array}$ $\begin{array}{lllll}0.8508544139009614 & 0.3173563205135396 & 0.9299059582172470\end{array}$ $\begin{array}{lllll}0.7632769097684947 & 0.2402578139331299 & 0.9321329175777644\end{array}$ $\begin{array}{lllll}0.8711673107605651 & 0.3592281979219754 & 0.8065043555892567\end{array}$ $\begin{array}{llll}0.9788867517734859 & 0.1406213287442351 & 0.9390032090381689\end{array}$ $\begin{array}{lllll}0.5044559129218615 & 0.1806850818166562 & 0.8128164574051118\end{array}$ $\begin{array}{lllll}0.9745074937965824 & 0.3412769866337616 & 0.9141930436612314\end{array}$ $\begin{array}{lllll}0.6520918090534874 & 0.4211207500532164 & 0.6831665375689306\end{array}$ $\begin{array}{lllll}0.5271347304856233 & 0.4631253494022841 & 0.6627381841323422\end{array}$ $\begin{array}{llll}0.5704131625483952 & 0.3875564294860693 & 0.5588892670969184\end{array}$ $\begin{array}{lllll}0.6579831900307177 & 0.4602210265222220 & 0.5458826148518253\end{array}$ $\begin{array}{lllll}0.6958029273173428 & 0.3630911174290272 & 0.5794315035400435\end{array}$ $\begin{array}{lllll}0.6149362383803916 & 0.4751412916174303 & 0.7964819845030340\end{array}$ $\begin{array}{lllll}0.0033574687540363 & 0.4194164707528772 & 0.7826864293281788\end{array}$ $\begin{array}{llll}0.6452898003506434 & 0.4255156187808299 & 0.9185261457155929\end{array}$ $\begin{array}{llll}0.9036354045656230 & 0.4116327719494343 & 0.6891036888830185\end{array}$ $\begin{array}{llll}0.7924148570181072 & 0.4664781139253262 & 0.6773898206240152\end{array}$ $\begin{array}{lllll}0.8202976622610503 & 0.3910870883084310 & 0.5638079385282890\end{array}$ $\begin{array}{llll}0.9126272321832258 & 0.4664050802392603 & 0.5648513287655630\end{array}$ $\begin{array}{lllll}0.9454834216500634 & 0.3643685774705651 & 0.5757381080328225\end{array}$ $\begin{array}{lllll}0.8827090643178159 & 0.4688992579520464 & 0.8061658513154125\end{array}$ $\begin{array}{llll}0.7564394365133004 & 0.4201741921421170 & 0.7875333663918378\end{array}$ $\begin{array}{lllll}0.8903364865390029 & 0.4203398539033475 & 0.9239882849226446\end{array}$ $\begin{array}{lllll}0.2129471679647282 & 0.2339699399698224 & 0.2512035627522074\end{array}$ $\begin{array}{lllll}0.0872807205321315 & 0.2953853113274366 & 0.2484882027807324\end{array}$ $\begin{array}{lllll}0.2223101059454394 & 0.1130240887875339 & 0.2668054377884502\end{array}$ $\begin{array}{llll}0.4627457211742096 & 0.2340169661253735 & 0.2508404197952888\end{array}$ $\begin{array}{lllll}0.3370635747422414 & 0.2954944296944139 & 0.2478973395959035\end{array}$ $\begin{array}{lllll}0.4722164215372554 & 0.1130332915344111 & 0.2664359928315759\end{array}$ $\begin{array}{llllll}0.0780469786540081 & 0.4163323189944974 & 0.2330187082397758\end{array}$ $\begin{array}{lllll}0.3277423267585260 & 0.4163658554065476 & 0.2319597891000829\end{array}$ $\begin{array}{llll}0.2132527731654521 & 0.2348200457012241 & 0.7506916892604976\end{array}$ $\begin{array}{lllll}0.0870810404129549 & 0.2959112586861603 & 0.7479082904200487\end{array}$ $\begin{array}{lllll}0.2230669196484886 & 0.1137722210447884 & 0.7662640317276602\end{array}$ $\begin{array}{lllll}0.4636771088220777 & 0.2355790748970256 & 0.7505174825005649\end{array}$ $\begin{array}{lllll}0.3376037601589252 & 0.2965657690574172 & 0.7473297738462014\end{array}$ $\begin{array}{lllll}0.4723456654655194 & 0.1144154691645445 & 0.7661578045593832\end{array}$ $\begin{array}{llll}0.0778984381014805 & 0.4170088746760425 & 0.7324523068323179\end{array}$ 
$\begin{array}{llll}0.3276166864599038 & 0.4176233041218798 & 0.7313787356551562\end{array}$ $\begin{array}{lllll}0.7126085629937612 & 0.2339664489961636 & 0.2510335177904106\end{array}$ $\begin{array}{lllll}0.5868701691798462 & 0.2954837789419462 & 0.2474788355334561\end{array}$ $\begin{array}{llll}0.7221940973360956 & 0.1130511152053709 & 0.2667095350067108\end{array}$ $\begin{array}{llll}0.9629007390704899 & 0.2339796542042923 & 0.2515412677287013\end{array}$ $\begin{array}{lllll}0.8370064535853480 & 0.2952880589898110 & 0.2486435497483231\end{array}$ $\begin{array}{lllll}0.9722376847858603 & 0.1130306480322234 & 0.2670723273577458\end{array}$ $\begin{array}{llll}0.5772984764302479 & 0.4162944938074007 & 0.2318058043804086\end{array}$ $\begin{array}{lllll}0.8275816195472929 & 0.4161603318284683 & 0.2337578538597950\end{array}$ $\begin{array}{lllll}0.7128586471090355 & 0.2340037378888617 & 0.7512914091933479\end{array}$ $\begin{array}{llll}0.5888109211097028 & 0.2977587492340025 & 0.7480022555828950\end{array}$ $\begin{array}{llll}0.7224829274233717 & 0.1127441262661234 & 0.7662222483153157\end{array}$ $\begin{array}{lllll}0.9632288188140584 & 0.2339190440296967 & 0.7517571359032496\end{array}$ $\begin{array}{lllll}0.8373585327954531 & 0.2946917000283731 & 0.7496146562281968\end{array}$ $\begin{array}{llll}0.9726985975936630 & 0.1130256079404470 & 0.7665589567824893\end{array}$ $\begin{array}{lllll}0.5784917068441554 & 0.4212132813256187 & 0.7296251223305651\end{array}$ $\begin{array}{lllll}0.8320041763737446 & 0.4152453210674463 & 0.7387933359194137\end{array}$ $\begin{array}{lllll}0.0232859179941821 & 0.2311150315787278 & 0.1050976979392610\end{array}$ $\begin{array}{llll}0.1536082470693360 & 0.1678640181972956 & 0.1084180098626257\end{array}$ $\begin{array}{llllll}0.1467178065881348 & 0.3614134730704451 & 0.3912619645063228\end{array}$ $\begin{array}{lllll}0.1552298162659622 & 0.1209208798515096 & 0.4004631810581207\end{array}$ $\begin{array}{lllll}0.0258817681800318 & 0.1806338595045510 & 0.4027349328058384\end{array}$ $\begin{array}{llllll}0.0269653870046358 & 0.2980616905218891 & 0.3949314979780237\end{array}$ $\begin{array}{llll}0.0242452260974375 & 0.3483651664427395 & 0.0973991134802529\end{array}$ $\begin{array}{llllll}0.2729608958664118 & 0.2313112998854281 & 0.1045634746761017\end{array}$ $\begin{array}{lllll}0.4034326125198227 & 0.1681524285945559 & 0.1080250047471159\end{array}$ $\begin{array}{lllll}0.3965730213286293 & 0.3616241951749595 & 0.3903783927006048\end{array}$ $\begin{array}{lllll}0.4051911459114140 & 0.1208691959991432 & 0.4001662244431448\end{array}$ $\begin{array}{lllll}0.2760032749444972 & 0.1807704029951810 & 0.4024041792193532\end{array}$ $\begin{array}{lllll}0.2771205708690867 & 0.2981695604929281 & 0.3945247783349752\end{array}$ $\begin{array}{lllll}0.2738687837882674 & 0.3486713021192370 & 0.0965259078782495\end{array}$ $\begin{array}{llllll}0.1446547892490565 & 0.4084314662173667 & 0.0988168067218256\end{array}$ $\begin{array}{lllll}0.3946328499402549 & 0.4083883614618744 & 0.0980266411297145\end{array}$ $\begin{array}{lllll}0.0230547316220826 & 0.2320433779846680 & 0.6047646394785997\end{array}$ $\begin{array}{llll}0.1537174765588729 & 0.1689544929821720 & 0.6080600824167377\end{array}$ $\begin{array}{lllll}0.1467516774880346 & 0.3616349650232289 & 0.8906283414844530\end{array}$ $\begin{array}{lllll}0.1563137932983243 & 0.1204393659214846 & 0.8999786581905019\end{array}$ $\begin{array}{lllll}0.0265926793740501 & 0.1797329790806063 & 0.9025295203510220\end{array}$ $\begin{array}{llll}0.0281085087986572 & 0.2973541381601863 & 0.8955675891272479\end{array}$ $\begin{array}{llll}0.0235303176928760 & 0.3496241186268736 & 0.5965026845779324\end{array}$ $\begin{array}{llll}0.2733648241738870 & 0.2321156399100135 & 0.6042704031448483\end{array}$ $\begin{array}{lllll}0.4037281742872925 & 0.1686548970384971 & 0.6081541651846474\end{array}$ $\begin{array}{lllll}0.3971171598468237 & 0.3620868680331937 & 0.8900738693741994\end{array}$ $\begin{array}{lllll}0.4057366835578720 & 0.1225199522582807 & 0.9000459632596867\end{array}$ $\begin{array}{llllll}0.2762287670524611 & 0.1816697928467947 & 0.9020013687789626\end{array}$ $\begin{array}{lllll}0.2772884090243620 & 0.2989129864546066 & 0.8938619819973596\end{array}$ $\begin{array}{lllll}0.2743734878152008 & 0.3495390746562348 & 0.5960261380597173\end{array}$ $\begin{array}{lllll}0.1450311271676076 & 0.4088227598451691 & 0.5986042766330999\end{array}$ $\begin{array}{lllll}0.3952981369483660 & 0.4087367507966250 & 0.5975142791082743\end{array}$ $\begin{array}{llllll}0.5229920853021260 & 0.2314485304064011 & 0.1042029499766493\end{array}$ $\begin{array}{llllll}0.6534118015010475 & 0.1680761637344601 & 0.1082042020494333\end{array}$ $\begin{array}{lllll}0.6457136540862047 & 0.3618144483715786 & 0.3895334238874428\end{array}$ $\begin{array}{lllll}0.6550004643650010 & 0.1210274049612959 & 0.4001802003892007\end{array}$ $\begin{array}{lllll}0.5257643162612331 & 0.1808476565336172 & 0.4020960600601719\end{array}$ $\begin{array}{lllll}0.5268727248378995 & 0.2981417700638464 & 0.3940636190051389\end{array}$ $\begin{array}{lllll}0.5239832331744916 & 0.3488578854716566 & 0.0959701173810001\end{array}$ $\begin{array}{lllll}0.7731907121873148 & 0.2312314238972430 & 0.1048778316952366\end{array}$ $\begin{array}{lllll}0.9035882684818785 & 0.1680117930163334 & 0.1086046248706345\end{array}$ $\begin{array}{lllll}0.8962859836749664 & 0.3610626448573999 & 0.3919826026781305\end{array}$ $\begin{array}{llll}0.9046841489432969 & 0.1214370988585982 & 0.4005617086892534\end{array}$ $\begin{array}{lllll}0.7754445164091166 & 0.1811614543111271 & 0.4025139110188342\end{array}$ $\begin{array}{lllll}0.7761855062332003 & 0.2983652255574673 & 0.3946699669925278\end{array}$ $\begin{array}{lllll}0.7742467969252459 & 0.3485549271184391 & 0.0975473428275477\end{array}$ $\begin{array}{lllll}0.6450876162968228 & 0.4084042458282344 & 0.0988967504235275\end{array}$ $\begin{array}{llllll}0.8952320550441378 & 0.4080032200562847 & 0.1007322374274833\end{array}$ $\begin{array}{lllll}0.5235156837353668 & 0.2316739671731561 & 0.6048842953734490\end{array}$ $\begin{array}{llll}0.6539664742654726 & 0.16877444636846792 & 0.6082523094265625\end{array}$ $\begin{array}{lllll}0.6480350230666803 & 0.3631288766130060 & 0.8901423886974952\end{array}$ $\begin{array}{llllll}0.6548275821687862 & 0.1201378998813314 & 0.8997297506049799\end{array}$ $\begin{array}{llll}0.5268741466092206 & 0.1819327504027996 & 0.9015698426154106\end{array}$ $\begin{array}{llll}0.5280273395056200 & 0.2997678181103763 & 0.8942953197513400\end{array}$ $\begin{array}{llll}0.5247204901545552 & 0.3486043675196779 & 0.5969671001062155\end{array}$ $\begin{array}{llll}0.7734480459356212 & 0.2324785639342000 & 0.6049351328181680\end{array}$ 
$\begin{array}{llll}0.9034720637047375 & 0.1686799082858808 & 0.6084111783717224\end{array}$ $0.89707132397433590 .3581796516480604 \quad 0.8948470664357213$ $\begin{array}{lllll}0.9048193314898434 & 0.1210275824925504 & 0.9001814968678269\end{array}$ $\begin{array}{llll}0.7751155916125692 & 0.1798968355316380 & 0.9025119287596031\end{array}$ $\begin{array}{llll}0.7764532239924176 & 0.2966458686889465 & 0.8954141617518747\end{array}$ $\begin{array}{lllll}0.7739513217791261 & 0.3500833161800923 & 0.5996855331547978\end{array}$ $\begin{array}{llll}0.6438559463439480 & 0.4105686388672378 & 0.5961724831021838\end{array}$ $\begin{array}{lllll}0.8950278521254025 & 0.4095625911819991 & 0.6010076248948297\end{array}$

Cyt/Gua :

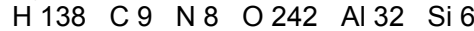

1.00000000000000

$20.2970019999999991 \quad 0.0000000000000000 \quad-3.7471719999999999$

$0.0646860000000000 \quad 24.9946149999999996-0.5148360000000000$

$\begin{array}{llll}0.0000000000000000 & 0.0000000000000000 & 18.6939999999999991\end{array}$

$\begin{array}{llllll}\mathrm{H} & \mathrm{C} & \mathrm{N} & \mathrm{O} & \mathrm{Al} & \mathrm{Si}\end{array}$

$\begin{array}{llllll}138 & 9 & 8 & 242 & 32 & 64\end{array}$

Direct

0.38258862993853290 .72527836295308730 .7313081615054470

$\begin{array}{lllll}0.4763541026882229 & 0.5581520502607097 & 0.8528685539357819\end{array}$

$\begin{array}{lllll}0.5030508199116415 & 0.5098529250723273 & 0.7994134281158405\end{array}$

$\begin{array}{lllll}0.3668655989665669 & 0.7529722324595036 & 0.5971297550597899\end{array}$

$\begin{array}{lllll}0.4843262918396057 & 0.5143696308493503 & 0.6771798337342249\end{array}$

$\begin{array}{lllll}0.0937795020179571 & 0.0550990019561047 & 0.4112530560219270\end{array}$

$\begin{array}{llllll}0.3444173131264189 & 0.0551689724186058 & 0.4109302865450480\end{array}$

$\begin{array}{lllll}0.0518068103207746 & 0.1403455572702450 & 0.1847083472893758\end{array}$

$\begin{array}{lllll}0.1701436763035211 & 0.3079096930596734 & 0.1613868599257479\end{array}$

$\begin{array}{lllll}0.1303747660246634 & 0.2212992886604981 & 0.3358246198528922\end{array}$

$\begin{array}{lllll}0.2443577773723710 & 0.0392940819398921 & 0.3452877385973467\end{array}$

$\begin{array}{llll}0.1713413050521152 & 0.0672495935434729 & 0.1483349241387177\end{array}$ $\begin{array}{lllll}0.3015749897793698 & 0.1399480814104621 & 0.1837123554191016\end{array}$ $\begin{array}{lllll}0.4209278163030771 & 0.3092214750762791 & 0.1640440287891899\end{array}$ $\begin{array}{lllll}0.3802145380695858 & 0.2200392735851040 & 0.3348338183694733\end{array}$ $\begin{array}{lllll}0.4934507838954243 & 0.0391138163282891 & 0.3444438047818900\end{array}$ $\begin{array}{lllll}0.4228143758526986 & 0.0673581792984963 & 0.1484184666945883\end{array}$ $\begin{array}{lllll}0.0581275618701034 & 0.4845706276733154 & 0.1456393423565738\end{array}$ $\begin{array}{llll}0.2491279599392523 & 0.3911717607743835 & 0.3177561381177375\end{array}$ $\begin{array}{lllll}0.1281911115503433 & 0.4622708223722131 & 0.3505458327382406\end{array}$ $\begin{array}{lllll}0.2701587593385550 & 0.4927871815127294 & 0.1880863103941648\end{array}$ $\begin{array}{lllll}0.4968884330825765 & 0.3886616931736626 & 0.3147181366027372\end{array}$ $\begin{array}{lllll}0.3772278984590458 & 0.4612796667994972 & 0.3463375042265816\end{array}$ $\begin{array}{lllll}0.2118363775037957 & 0.4699403295014131 & 0.0837453410511309\end{array}$ $\begin{array}{lllll}0.4526889899755134 & 0.4771240993441536 & 0.0871985134687355\end{array}$ $\begin{array}{lllll}0.1567753209799695 & 0.4380883146745883 & 0.4681267718169910\end{array}$ $\begin{array}{lllll}0.3932918229983715 & 0.4405068681141743 & 0.4635431750337325\end{array}$ $\begin{array}{lllll}0.1460315151538998 & 0.0907601773585565 & 0.0295541417160809\end{array}$ $\begin{array}{llll}0.3984492187813122 & 0.0909214725570452 & 0.0296462369997573\end{array}$ $\begin{array}{lllll}0.0829528206425289 & 0.5021860706261918 & 0.2947698036779224\end{array}$ $\begin{array}{lllll}0.4100134893115647 & 0.4832075319954083 & 0.2810335355350188\end{array}$ $\begin{array}{lllll}0.1368408780030288 & 0.0465177408881462 & 0.2118555200012412\end{array}$ $\begin{array}{lllll}0.3861587093897894 & 0.0476429003567123 & 0.2107406302848363\end{array}$ $\begin{array}{llll}0.2119039519121550 & 0.4182051803232368 & 0.2435424614954705\end{array}$ $\begin{array}{lllll}0.4597526626943650 & 0.4144227468630328 & 0.2392649691875252\end{array}$ $\begin{array}{lllll}0.3387686888745212 & 0.1129160360593783 & 0.2576789532478547\end{array}$ $\begin{array}{lllll}0.0890121518958045 & 0.1124477403093200 & 0.2580065814523326\end{array}$ $\begin{array}{lllll}0.0954856876734792 & 0.0558905381172251 & 0.9099311299812419\end{array}$ $\begin{array}{llll}0.3459000548802920 & 0.0552709746596075 & 0.9104052637105964\end{array}$ $\begin{array}{lllll}0.0523252482392282 & 0.1407779506707198 & 0.6842490141247874\end{array}$ $\begin{array}{llll}0.1684171354534599 & 0.3070965070101930 & 0.6572515637014515\end{array}$ $\begin{array}{lllll}0.1315384065872551 & 0.2222297436120484 & 0.8353103534651388\end{array}$ $\begin{array}{lllll}0.2444017363772810 & 0.0425016022972041 & 0.8474771232927464\end{array}$ $\begin{array}{lllll}0.1721569368038619 & 0.0682836512721981 & 0.6485597598697089\end{array}$ $\begin{array}{lllll}0.3025778903051881 & 0.1397547588436600 & 0.6820127771566101\end{array}$ $\begin{array}{lllll}0.4207957199730098 & 0.3097569434617645 & 0.6615980903554879\end{array}$ $\begin{array}{llll}0.3809049102797186 & 0.2216816312266383 & 0.8345863883115046\end{array}$ $\begin{array}{lllll}0.4942791367657209 & 0.0406786913812751 & 0.8444510405376049\end{array}$ $\begin{array}{lllll}0.4238475155558843 & 0.0687447263218942 & 0.6477181234701003\end{array}$ $\begin{array}{lllll}0.0570638171771437 & 0.4850334801247169 & 0.6457254532898137\end{array}$ $\begin{array}{llllll}0.2480557359422240 & 0.3737186441842514 & 0.7948248491228741\end{array}$ $\begin{array}{lllll}0.1287340626447901 & 0.4625373901236121 & 0.8505426683883245\end{array}$ $\begin{array}{llll}0.2566368187600260 & 0.4914063574468452 & 0.6904206412840935\end{array}$ $\begin{array}{lllll}0.4929346439727597 & 0.3849609528107502 & 0.8048769618583261\end{array}$ $\begin{array}{lllll}0.3653035756260752 & 0.4621704599692479 & 0.8498677590840839\end{array}$ 
$\begin{array}{llll}0.2117895747235607 & 0.4697838341247308 & 0.5824462249722284\end{array}$ $\begin{array}{lllll}0.4243008107162576 & 0.4988496639566707 & 0.5513909853429109\end{array}$ $\begin{array}{lllll}0.1567941456878956 & 0.4385650184640208 & 0.9681323675246322\end{array}$ $\begin{array}{llll}0.4039922378497660 & 0.4394025866664026 & 0.9680590733823695\end{array}$ $\begin{array}{llll}0.1459291442634084 & 0.0913792582224850 & 0.5300815718479200\end{array}$ $\begin{array}{lllll}0.3978402655959968 & 0.0919487967218492 & 0.5298598548275131\end{array}$ $0.0822171183296095 \quad 0.5024959133286790 \quad 0.7962028986469224$ $\begin{array}{lllll}0.3953800803996529 & 0.4975542085399964 & 0.7942833814032885\end{array}$ $\begin{array}{lllll}0.1373139772596173 & 0.0480783854748137 & 0.7120314466238460\end{array}$ $\begin{array}{lllll}0.3874068181790451 & 0.0487942128453479 & 0.7101206732950578\end{array}$ $\begin{array}{lllll}0.2106957750841694 & 0.4166615049010440 & 0.7421770702939339\end{array}$ $\begin{array}{lllll}0.4594764858852112 & 0.4200753657410330 & 0.7376892546826084\end{array}$ $\begin{array}{lllll}0.3397143892620117 & 0.1137133054404878 & 0.7570154182587885\end{array}$ $\begin{array}{lllll}0.0900762545315636 & 0.1137796119997186 & 0.7580248884630172\end{array}$ $\begin{array}{lllll}0.6084397959842403 & 0.5799567713920870 & 0.4847013828847961\end{array}$ $\begin{array}{lllll}0.5916770919900076 & 0.0563922509469287 & 0.4100006144620714\end{array}$ $\begin{array}{lllll}0.8452553856787371 & 0.0541271809895614 & 0.4113466936938170\end{array}$ $\begin{array}{llll}0.5516766469528123 & 0.1406706149100009 & 0.1850266240304403\end{array}$ $\begin{array}{llll}0.6697422930909652 & 0.3073784418254901 & 0.1610206973708299\end{array}$ $\begin{array}{llll}0.6308323494071010 & 0.2218649870852880 & 0.3372644124078242\end{array}$ $\begin{array}{lllll}0.7439326351405157 & 0.0400351876539499 & 0.3468544798460140\end{array}$ $\begin{array}{lllll}0.6711756097060478 & 0.0673606535313094 & 0.1485686953492413\end{array}$ $\begin{array}{llll}0.8014389471015272 & 0.1394967418548440 & 0.1836669333048749\end{array}$ $\begin{array}{lllll}0.9211576537316394 & 0.3093984008920897 & 0.1649578695511476\end{array}$ $\begin{array}{llllll}0.8802845672716327 & 0.2194867324178017 & 0.3348100114603218\end{array}$ $\begin{array}{lllll}0.9941398672977069 & 0.0391043924044227 & 0.3449345230103995\end{array}$ $\begin{array}{lllll}0.9225262127544949 & 0.0671650771597501 & 0.1485832638520285\end{array}$ $\begin{array}{lllll}0.5588423703312475 & 0.4849943048753793 & 0.1475671949038311\end{array}$ $\begin{array}{lllll}0.7497294898203654 & 0.3902324389589450 & 0.3182130974772212\end{array}$ $\begin{array}{llll}0.6276146282079603 & 0.4622346547072252 & 0.3523169475279636\end{array}$ $\begin{array}{llll}0.7714588513141473 & 0.4935601068708300 & 0.1908976951040802\end{array}$ $\begin{array}{lllll}0.9979247933108496 & 0.3878705308739794 & 0.3133113816650719\end{array}$ $\begin{array}{lllll}0.8789347971670086 & 0.4617036861089432 & 0.3485654603519881\end{array}$ $\begin{array}{lllll}0.7132974778050190 & 0.4701108194781755 & 0.0875740437609217\end{array}$ $\begin{array}{lllll}0.9522882494477710 & 0.4772452870113247 & 0.0863730123655111\end{array}$ $\begin{array}{lllll}0.6623864790088827 & 0.4359465102806691 & 0.4698523672306698\end{array}$ $\begin{array}{lllll}0.9035755381794405 & 0.4386802068160293 & 0.4673981776370793\end{array}$ $\begin{array}{lllll}0.6451397491739795 & 0.0909948662023308 & 0.0298458449838979\end{array}$ $\begin{array}{lllll}0.8974230563572767 & 0.0907538264292059 & 0.0298861347456849\end{array}$ $\begin{array}{llll}0.5789599513006628 & 0.5002292393054669 & 0.2972795964239219\end{array}$ $\begin{array}{lllll}0.9125649169548382 & 0.4818107087559779 & 0.2835562582934024\end{array}$ $\begin{array}{lllll}0.6364306979821354 & 0.0467828141073695 & 0.2119722295945072\end{array}$ $\begin{array}{lllll}0.8864367289337544 & 0.0472332538015139 & 0.2112905807421677\end{array}$ $\begin{array}{llll}0.7125766621925390 & 0.4186688672294355 & 0.2453239502136692\end{array}$ $\begin{array}{llllll}0.9602415291422747 & 0.4145055809890004 & 0.2390476902065462\end{array}$ $\begin{array}{lllll}0.8387404141762633 & 0.1127352053622366 & 0.2578502345408808\end{array}$ $\begin{array}{llllll}0.5889783472414455 & 0.1126899090912074 & 0.2582367002766888\end{array}$ $\begin{array}{lllll}0.5610011634290778 & 0.7150574214151713 & 0.6488176731486982\end{array}$ $\begin{array}{lllll}0.5759366623037805 & 0.6665768126766836 & 0.5335699406870884\end{array}$ $\begin{array}{llll}0.6355104969997297 & 0.5251377015296212 & 0.5413365697245095\end{array}$ $\begin{array}{llll}0.5832008870703423 & 0.6668402978098142 & 0.7596239076541909\end{array}$ $\begin{array}{lllll}0.5932036302299702 & 0.0565307925328437 & 0.9098908743675508\end{array}$ $\begin{array}{llll}0.8446259187818721 & 0.0555633265578240 & 0.9106699820105811\end{array}$ $\begin{array}{lllll}0.5525947638555446 & 0.1437708500605120 & 0.6865205308800538\end{array}$ $\begin{array}{llll}0.6700984088012895 & 0.3084728125746458 & 0.6601900341850253\end{array}$ $\begin{array}{lllll}0.6314333091192716 & 0.2236811902531612 & 0.8374843419440677\end{array}$ $\begin{array}{lllll}0.7441997604856166 & 0.0408398045357683 & 0.8456012434541655\end{array}$ $\begin{array}{lllll}0.6702942881265739 & 0.0692451250268643 & 0.6475210247033011\end{array}$ $\begin{array}{lllll}0.8021039780555411 & 0.1410583034437165 & 0.6834450050801929\end{array}$ $\begin{array}{lllll}0.9212368558455658 & 0.3093407296126032 & 0.6641852903063442\end{array}$ $\begin{array}{llll}0.8816848288383412 & 0.2213571267824468 & 0.8369944611142076\end{array}$ $\begin{array}{llll}0.9954132194552965 & 0.0403251929906063 & 0.8463481171228663\end{array}$ $\begin{array}{lllll}0.9233355691856223 & 0.0672406257693594 & 0.6488916367870727\end{array}$ $\begin{array}{lllll}0.5642448512060604 & 0.4889472829208207 & 0.6528708519559470\end{array}$ $\begin{array}{llllll}0.7504798170161523 & 0.3940793581399077 & 0.8210997455873267\end{array}$ $\begin{array}{lllll}0.6322324914554712 & 0.4647475902950382 & 0.8539845453947429\end{array}$ $\begin{array}{lllll}0.7658623551591143 & 0.4924941363571093 & 0.6915018121170450\end{array}$ $\begin{array}{lllll}0.9976798099676747 & 0.3882748834229475 & 0.8135170218501726\end{array}$ $\begin{array}{llll}0.8791461852958860 & 0.4627314114491860 & 0.8481035847808863\end{array}$ $\begin{array}{llll}0.7197959016045028 & 0.4665888474552845 & 0.5917758936531849\end{array}$ $\begin{array}{lllll}0.9521785632084188 & 0.4774767974876131 & 0.5861681397071957\end{array}$ $\begin{array}{llll}0.6586158503911291 & 0.4376784041841884 & 0.9726529707188935\end{array}$ $\begin{array}{llll}0.9031133766107703 & 0.4391070713647286 & 0.9673687642455894\end{array}$ 
$\begin{array}{llll}0.6425648180680896 & 0.0924008762423864 & 0.5295839519768423\end{array}$ $\begin{array}{lllll}0.8979230043764286 & 0.0904879571480507 & 0.5303721961628542\end{array}$ $\begin{array}{lllll}0.6213501836575265 & 0.5130909572897794 & 0.7905437159532823\end{array}$ $\begin{array}{lllll}0.9123254022864932 & 0.4831204432986821 & 0.7828186692506105\end{array}$ $\begin{array}{lllll}0.6371031722329287 & 0.0477726945497640 & 0.7115631424905485\end{array}$ $\begin{array}{lllll}0.8883668459842338 & 0.0467755991571917 & 0.7123118517449123\end{array}$ $\begin{array}{llllll}0.7125437551391487 & 0.4193213492037752 & 0.7454574899826996\end{array}$ $\begin{array}{lllll}0.9600933605491315 & 0.4150890424075888 & 0.7393053883211896\end{array}$ $\begin{array}{llll}0.8390874732319218 & 0.1134534950235935 & 0.7570030696927216\end{array}$ $\begin{array}{llll}0.5898075635313522 & 0.1137656778724600 & 0.7578825639652356\end{array}$ $\begin{array}{lllll}0.3854401766428708 & 0.7141975965079176 & 0.6169572319159559\end{array}$ $\begin{array}{llll}0.4539800011256516 & 0.5735693231310445 & 0.7462078795280787\end{array}$ $\begin{array}{lllll}0.4171262741087871 & 0.6502713292125737 & 0.6964209023525828\end{array}$ $\begin{array}{llll}0.4217839171558860 & 0.6339814272225809 & 0.6252128050861562\end{array}$ $\begin{array}{lllll}0.4445806288281461 & 0.5807707603189696 & 0.6144104774393866\end{array}$ $\begin{array}{llll}0.5784740429693878 & 0.6738589886779032 & 0.6479366379492101\end{array}$ $\begin{array}{llllll}0.5869720647570581 & 0.6473863414561858 & 0.5860992655666746\end{array}$ $\begin{array}{lllll}0.6086382694718782 & 0.5927438807966007 & 0.5931678609770582\end{array}$ $\begin{array}{lllll}0.6105846020797229 & 0.5947132857312847 & 0.7183169938802565\end{array}$ $0.46817873124679910 .5386333732018903 \quad 0.8036565446248457$ $\begin{array}{lllll}0.4327459940342390 & 0.6224723693521517 & 0.7585050696628236\end{array}$ $\begin{array}{lllll}0.3938388174454320 & 0.7019494701877287 & 0.6900955659113986\end{array}$ $\begin{array}{llllll}0.4019795911517592 & 0.6744873402440684 & 0.5765421863796477\end{array}$ $\begin{array}{lllll}0.4597771885799588 & 0.5529485709156399 & 0.6797101239107375\end{array}$ $\begin{array}{lllll}0.5913159936347034 & 0.6486229921794326 & 0.7128559259823768\end{array}$ $\begin{array}{lllll}0.6193717513401391 & 0.5642292331773198 & 0.5349376210202970\end{array}$ $\begin{array}{lllll}0.6190840757777666 & 0.5677486293105328 & 0.6574124776257202\end{array}$ $\begin{array}{lllll}0.4519322431134244 & 0.5581677414205484 & 0.5559624880918155\end{array}$ $\begin{array}{lllll}0.0455837308077435 & 0.3499757824112731 & 0.1847462169455174\end{array}$ $\begin{array}{lllll}0.0302607618930177 & 0.2410015335270427 & 0.1921628131468462\end{array}$ $\begin{array}{lllll}0.1553691201348951 & 0.2820588102225823 & 0.1930640369756940\end{array}$ $\begin{array}{lllll}0.2005420178868322 & 0.2067377840399950 & 0.0703832728553347\end{array}$ $\begin{array}{lllll}0.0332068332719829 & 0.2887407945759473 & 0.0665021389753215\end{array}$ $\begin{array}{lllll}0.0768063291596971 & 0.1872521348052763 & 0.0869737588020992\end{array}$ $\begin{array}{lllll}0.1799119050792954 & 0.1677496440004913 & 0.1955466689025169\end{array}$ 0.18009344794215290 .05491874799129690 .2007636113621841 $\begin{array}{lllll}0.0474838560902980 & 0.1101113189988528 & 0.2157954833923098\end{array}$ $\begin{array}{lllll}0.1572511780652831 & 0.1047856202832080 & 0.0805260236038069\end{array}$ $\begin{array}{llll}0.1495375548737861 & 0.1138831287415396 & 0.3121838708141610\end{array}$ $\begin{array}{llll}0.0214949622083336 & 0.0675204130968992 & 0.3320510371132798\end{array}$ $\begin{array}{llll}0.1380324438522132 & 0.0652103572765489 & 0.4394910819185668\end{array}$ $\begin{array}{lllll}0.1050543346676435 & 0.1667846526772032 & 0.4211451870687079\end{array}$ $\begin{array}{lllll}0.0205292206933661 & 0.2883101721493213 & 0.3055827608317566\end{array}$ $\begin{array}{lllll}0.1450501272332799 & 0.2470338116101563 & 0.3040154532238228\end{array}$ $\begin{array}{lllll}0.1006955555639992 & 0.3217235122728411 & 0.4267903459185415\end{array}$ $\begin{array}{lllll}0.0170982540322595 & 0.2412152482016359 & 0.4312246883361616\end{array}$ $\begin{array}{lllll}0.1200765792052083 & 0.3614634005669644 & 0.3016583417790177\end{array}$ $\begin{array}{lllll}0.2297716498731539 & 0.1403135132296143 & 0.4382364869703916\end{array}$ $\begin{array}{lllll}0.2539108077359767 & 0.1787920881507591 & 0.3126266797966306\end{array}$ $\begin{array}{lllll}0.2238423937810882 & 0.3424271010968349 & 0.4094720290456499\end{array}$ $\begin{array}{lllll}0.2959954080208623 & 0.3487579692322713 & 0.1842952451606497\end{array}$ $\begin{array}{llll}0.2802748570509081 & 0.2399357327957895 & 0.19197582444755523\end{array}$ $\begin{array}{lllll}0.4055337499920828 & 0.2818068799901082 & 0.1929681790587949\end{array}$ $\begin{array}{lllll}0.4503013219969485 & 0.2083995503664312 & 0.0706148827190991\end{array}$ $\begin{array}{llllll}0.2846084894381788 & 0.2867244550399854 & 0.0661776651259367\end{array}$ $\begin{array}{lllll}0.3272341365023078 & 0.1853701894099533 & 0.0874296557313756\end{array}$ $\begin{array}{lllll}0.4310151758838486 & 0.1679569034277117 & 0.1954878748872558\end{array}$ $\begin{array}{lllll}0.4300757203156595 & 0.0552456487049292 & 0.2008714096797897\end{array}$ $\begin{array}{lllll}0.2973333304830630 & 0.1101036364889308 & 0.2154854767050783\end{array}$ $\begin{array}{llll}0.4107491259692910 & 0.1051444141588353 & 0.0803573781900013\end{array}$ $\begin{array}{lllll}0.3994828755004646 & 0.1147645366212436 & 0.3119268746279281\end{array}$ $\begin{array}{lllll}0.2717488328398003 & 0.0673871770008582 & 0.3316278995487724\end{array}$ $\begin{array}{llllll}0.3886818815016048 & 0.0653735463970131 & 0.4390282064323112\end{array}$ $\begin{array}{lllll}0.3547357331789708 & 0.1668271801661401 & 0.4210218409351668\end{array}$ $\begin{array}{lllll}0.2705279331768095 & 0.2874460256379389 & 0.3050762489988421\end{array}$ $\begin{array}{lllll}0.3951076278115915 & 0.2462722062826994 & 0.3038619762292795\end{array}$ $\begin{array}{lllll}0.3504354741541402 & 0.3215057728627408 & 0.4263410914793821\end{array}$ $\begin{array}{lllll}0.2664193319382048 & 0.2410604024679547 & 0.4307704414450166\end{array}$ $\begin{array}{lllll}0.3702576796947197 & 0.3598655657293087 & 0.3004194646286249\end{array}$ $\begin{array}{lllll}0.4797849303624369 & 0.1409589516465571 & 0.4382116083611380\end{array}$ $\begin{array}{lllll}0.0046295401106297 & 0.1790577362635518 & 0.3128832600045829\end{array}$ $\begin{array}{lllll}0.4733408966966654 & 0.3435939542724886 & 0.4093203749968423\end{array}$ $\begin{array}{llll}0.1501476219237285 & 0.4150349071729651 & 0.1857341186884092\end{array}$ 
$\begin{array}{llll}0.0291221285561212 & 0.4642140032999068 & 0.1703314288735427\end{array}$ $\begin{array}{llllll}0.0707342061948032 & 0.3898482763391146 & 0.0603605282424341\end{array}$ $\begin{array}{llllll}0.1658523870111824 & 0.4618113914924091 & 0.0576533383912439\end{array}$ $\begin{array}{llll}0.1949333812590980 & 0.3599133027453765 & 0.0777225301713829\end{array}$ $\begin{array}{llll}0.1165472402711852 & 0.4743775584965204 & 0.2980648848484661\end{array}$ $\begin{array}{lllll}0.0017193569796427 & 0.4177838617452976 & 0.2815692761317255\end{array}$ $\begin{array}{llll}0.1432281853559149 & 0.4241491189067360 & 0.4169272420725340\end{array}$ $\begin{array}{llll}0.4006398265081732 & 0.4130473490493729 & 0.1852951714272473\end{array}$ $\begin{array}{llll}0.2792611133540237 & 0.4597406493329939 & 0.1633971129112946\end{array}$ $\begin{array}{lllll}0.3196953275747827 & 0.3881906618124703 & 0.0597153060228731\end{array}$ $\begin{array}{lllll}0.4100460113796898 & 0.4647730647456730 & 0.0584055844880648\end{array}$ $\begin{array}{llll}0.4458369384512659 & 0.3638303444429970 & 0.0758150453791407\end{array}$ $\begin{array}{lllll}0.3676790209998497 & 0.4739840452406335 & 0.2936485280255798\end{array}$ $\begin{array}{llll}0.2542891487010225 & 0.4188658547597766 & 0.2832371134446196\end{array}$ $\begin{array}{llll}0.3899474670116772 & 0.4245562340597995 & 0.4124945349720970\end{array}$ $\begin{array}{lllll}0.0456172381731416 & 0.3505871163647200 & 0.6846485890968635\end{array}$ $\begin{array}{lllll}0.0307257997290396 & 0.2414388384212756 & 0.6921582226399303\end{array}$ $\begin{array}{lllll}0.1557364128862231 & 0.2831962948570073 & 0.6927596874783132\end{array}$ $\begin{array}{llll}0.2010287621970759 & 0.2083105143510361 & 0.5706845773954997\end{array}$ $\begin{array}{lllll}0.0331246575284793 & 0.2890811089122838 & 0.5664809966792580\end{array}$ $\begin{array}{lllll}0.0774318756584596 & 0.1877805296099052 & 0.5869835981262154\end{array}$ $\begin{array}{llllll}0.1810362191770591 & 0.1689733774767730 & 0.6954302119240573\end{array}$ $\begin{array}{llll}0.1807027286277912 & 0.0561008675393025 & 0.7010118818125530\end{array}$ $\begin{array}{lllll}0.0484989315541022 & 0.1107497144495029 & 0.7158451441694504\end{array}$ $\begin{array}{llllll}0.1586701941456278 & 0.1058626677549771 & 0.5806579160018832\end{array}$ $\begin{array}{lllll}0.1504440354225371 & 0.1158962907444104 & 0.8119861846860551\end{array}$ $\begin{array}{llll}0.0229063973736402 & 0.0681643325579556 & 0.8322234521607982\end{array}$ $\begin{array}{lllll}0.1396168693972786 & 0.0660998396494133 & 0.9385207065934819\end{array}$ $\begin{array}{llll}0.1054815435944875 & 0.1676147570968517 & 0.9215750890730534\end{array}$ $\begin{array}{llllll}0.0209301680771336 & 0.2888554822240587 & 0.8053529954074535\end{array}$ $\begin{array}{lllll}0.1461086816530255 & 0.2482553434641868 & 0.8038261075013619\end{array}$ $\begin{array}{lllll}0.1004913648308968 & 0.3227185460484522 & 0.9265739775378874\end{array}$ $\begin{array}{lllll}0.0170772214597438 & 0.2419213846640782 & 0.9308804392844235\end{array}$ $\begin{array}{lllll}0.1206843483101710 & 0.3620596375375408 & 0.8016615825129700\end{array}$ $\begin{array}{lllll}0.2306304913887506 & 0.1419468381670273 & 0.9377264110069061\end{array}$ $\begin{array}{lllll}0.2554408164956245 & 0.1805831353992927 & 0.8119776311184744\end{array}$ $\begin{array}{lllll}0.2235258585719657 & 0.3418103907948671 & 0.9123715445743613\end{array}$ $\begin{array}{lllll}0.3000195996936511 & 0.3507235275642350 & 0.6843352587930319\end{array}$ $\begin{array}{lllll}0.2810235841856966 & 0.2420920430281099 & 0.6923124412101438\end{array}$ $\begin{array}{lllll}0.4067856140322282 & 0.2833725681813217 & 0.6929978069121127\end{array}$ $\begin{array}{llll}0.4500927001173920 & 0.2101729076413861 & 0.5704966719018290\end{array}$ $\begin{array}{llll}0.2856974178963764 & 0.2877267890980623 & 0.5662583494666316\end{array}$ $\begin{array}{llll}0.3274420622614039 & 0.1861129230616163 & 0.5882962464809002\end{array}$ $\begin{array}{lllll}0.4318628698495093 & 0.1693180800674608 & 0.6954193467736709\end{array}$ $\begin{array}{lllll}0.4312825208516977 & 0.0563159192940322 & 0.7000806411879695\end{array}$ $\begin{array}{lllll}0.2981507356332152 & 0.1107243836524642 & 0.7150121269227890\end{array}$ $\begin{array}{llll}0.4114324571164494 & 0.1065571451160030 & 0.5802224790854608\end{array}$ $\begin{array}{lllll}0.4004865617512441 & 0.1152399785560225 & 0.8112847180793219\end{array}$ $\begin{array}{lllll}0.2724597100547648 & 0.0691858944092941 & 0.8316357498436111\end{array}$ $\begin{array}{llllll}0.3902052151261006 & 0.0654422444926101 & 0.9382334390122385\end{array}$ $\begin{array}{lllll}0.3559422533132826 & 0.1670002871520934 & 0.9206205237394759\end{array}$ $\begin{array}{llll}0.2707707418158470 & 0.2910427128511542 & 0.8048290793082824\end{array}$ $\begin{array}{lllll}0.3959016099711178 & 0.2480087144547923 & 0.8038182972806730\end{array}$ $\begin{array}{lllll}0.3512403646083651 & 0.3232104803922350 & 0.9260294607800073\end{array}$ $\begin{array}{llll}0.2690023588986313 & 0.2420167338613263 & 0.9301825608519080\end{array}$ $\begin{array}{lllll}0.3733486521864035 & 0.3625395492811742 & 0.8010036009955638\end{array}$ $\begin{array}{lllll}0.4808996720435311 & 0.1412223896973477 & 0.9375600842461126\end{array}$ $\begin{array}{lllll}0.0052538700216708 & 0.1796069731696749 & 0.8128673533952498\end{array}$ $\begin{array}{lllll}0.4746704758032209 & 0.3437337870707787 & 0.9114727900729889\end{array}$ $\begin{array}{llll}0.1485708045468400 & 0.4171737977468660 & 0.6847949325718015\end{array}$ $\begin{array}{llll}0.0278242938661909 & 0.4648382218165883 & 0.6703061753740710\end{array}$ $\begin{array}{llll}0.0705864564140722 & 0.3898446655959727 & 0.5597376541402282\end{array}$ $\begin{array}{lllll}0.1661374300121394 & 0.4608902792827735 & 0.5555180791792047\end{array}$ $\begin{array}{lllll}0.1951110662520443 & 0.3591085578189879 & 0.5807967662617598\end{array}$ $\begin{array}{llll}0.1162289810891299 & 0.4751132454972540 & 0.7982394591320677\end{array}$ $\begin{array}{llll}0.0015316090246569 & 0.4181208519245936 & 0.7816712505983225\end{array}$ $\begin{array}{lllll}0.1434579213967448 & 0.4248834171551861 & 0.9169947449214620\end{array}$ $\begin{array}{lllll}0.3979748544198034 & 0.4242154030433291 & 0.6819561013891732\end{array}$ $\begin{array}{lllll}0.2742897506666721 & 0.4625088882049657 & 0.6640289773960372\end{array}$ $\begin{array}{llll}0.3184395443598154 & 0.3894487270737015 & 0.5593108156049129\end{array}$ $\begin{array}{llll}0.3958824329993403 & 0.4656423245391408 & 0.5416869123974656\end{array}$ $\begin{array}{llll}0.4459563611420825 & 0.3702533933283230 & 0.5777452588791340\end{array}$ $\begin{array}{llll}0.3557838597920780 & 0.4758327456799199 & 0.7984898270354966\end{array}$ 
$\begin{array}{llll}0.2522695905422010 & 0.4111246511982085 & 0.7816064365218542\end{array}$ $\begin{array}{llll}0.3929736859765146 & 0.4255001938742788 & 0.9171165535681162\end{array}$ $\begin{array}{llll}0.5459912059332283 & 0.3500312673787205 & 0.1854314808697107\end{array}$ $\begin{array}{llll}0.5304290445639107 & 0.2410295188930051 & 0.1923204632221416\end{array}$ $\begin{array}{lllll}0.6556204190289916 & 0.2821369176995580 & 0.1939357613892787\end{array}$ $\begin{array}{lllll}0.7005850390717793 & 0.2071163457962959 & 0.0710093915930539\end{array}$ $\begin{array}{lllll}0.5341519425301413 & 0.2889969068288800 & 0.0670473400702965\end{array}$ $\begin{array}{lllll}0.5769290881042072 & 0.1873226337682811 & 0.0871343946579336\end{array}$ $\begin{array}{lllll}0.6800380787245386 & 0.1678568397411440 & 0.1959400360836662\end{array}$ $\begin{array}{llll}0.6797636955593225 & 0.0550169496137903 & 0.2009717457841838\end{array}$ $\begin{array}{lllll}0.5474380377721956 & 0.1103433576888499 & 0.2159799920481263\end{array}$ $\begin{array}{lllll}0.6575100524597792 & 0.1050107748916882 & 0.0807544348707887\end{array}$ $\begin{array}{lllll}0.6493223071870765 & 0.1140794742872110 & 0.3123800149872054\end{array}$ $\begin{array}{lllll}0.5211020049253792 & 0.0674639315529134 & 0.3319389290114564\end{array}$ $\begin{array}{lllll}0.6358337092798506 & 0.0660821369847047 & 0.4392948910113306\end{array}$ $\begin{array}{lllll}0.6048697033528911 & 0.1680051265978482 & 0.4206876370058104\end{array}$ $\begin{array}{llllll}0.5205112208240456 & 0.2882534753171507 & 0.3056496561710833\end{array}$ $\begin{array}{lllll}0.6452955518169400 & 0.2472305370682893 & 0.3047939845653390\end{array}$ $\begin{array}{llll}0.5999836067281052 & 0.3217441862049534 & 0.4274726784831219\end{array}$ $\begin{array}{llll}0.5158583488125280 & 0.2418167978526458 & 0.4313121795218411\end{array}$ $\begin{array}{lllll}0.6200454276869668 & 0.3615923980998788 & 0.3028718720519153\end{array}$ $\begin{array}{lllll}0.7293221547043873 & 0.1400535243222449 & 0.4385127406653859\end{array}$ $\begin{array}{lllll}0.7539902440328694 & 0.1787385957412403 & 0.3130815713222021\end{array}$ $\begin{array}{llll}0.7237973498406635 & 0.3416011670118849 & 0.4107551569223296\end{array}$ $\begin{array}{lllll}0.7967498208326268 & 0.3489878809307886 & 0.1858968695174265\end{array}$ $\begin{array}{lllll}0.7804575862061782 & 0.2401273044968757 & 0.1927364308514637\end{array}$ $\begin{array}{lllll}0.9056891733459963 & 0.2820136216881812 & 0.1938661783715756\end{array}$ $\begin{array}{lllll}0.9500867324907619 & 0.2079980537941479 & 0.0706931386880839\end{array}$ $\begin{array}{llll}0.7847995728591252 & 0.2870407980053508 & 0.0672004781358205\end{array}$ $\begin{array}{llllll}0.8271051323740505 & 0.1854818999287297 & 0.0879372866662473\end{array}$ $\begin{array}{llll}0.9309547434476103 & 0.1678885752953605 & 0.1957828984078908\end{array}$ $\begin{array}{lllll}0.9301632743980148 & 0.0550553669087722 & 0.2010618761631559\end{array}$ $\begin{array}{lllll}0.7972066656405316 & 0.1098803767760590 & 0.2157973225626811\end{array}$ $\begin{array}{llll}0.9100167394544367 & 0.1049392548675326 & 0.0806566639805483\end{array}$ $\begin{array}{lllll}0.8995715083913188 & 0.1145817448086636 & 0.3120495647308651\end{array}$ $\begin{array}{lllll}0.7715874908798975 & 0.0674117470004138 & 0.3319125803801589\end{array}$ $\begin{array}{lllll}0.8894774329836669 & 0.0645828312288808 & 0.4391129175240218\end{array}$ $\begin{array}{lllll}0.8546269950555909 & 0.1659340495349554 & 0.4215514144431079\end{array}$ $\begin{array}{llll}0.7709704862880083 & 0.2874880861307093 & 0.3060548922141217\end{array}$ $\begin{array}{lllll}0.8954674310305298 & 0.2461518821589885 & 0.3046415916884169\end{array}$ $\begin{array}{lllll}0.8505028417400884 & 0.3213860861895686 & 0.4276616042665415\end{array}$ $\begin{array}{lllll}0.7669499985065488 & 0.2405136066797299 & 0.4315452563912655\end{array}$ $\begin{array}{lllll}0.8708519251502392 & 0.3604038251585175 & 0.3025668181905697\end{array}$ $\begin{array}{lllll}0.9797882985944094 & 0.1406798029409279 & 0.4382794105209778\end{array}$ $\begin{array}{lllll}0.5042676958711335 & 0.1793229788878732 & 0.3128927977285113\end{array}$ $\begin{array}{lllll}0.9740054481553551 & 0.3424416294977445 & 0.4109417064293968\end{array}$ $\begin{array}{llllll}0.6506663682178374 & 0.4154243499420993 & 0.1880713072507727\end{array}$ $\begin{array}{lllll}0.5296149134061973 & 0.4645097755368270 & 0.1718096216008806\end{array}$ $\begin{array}{lllll}0.5714599385452958 & 0.3904625901216179 & 0.0619769876430446\end{array}$ $\begin{array}{lllll}0.6673671730588516 & 0.4622199175913658 & 0.0609160209894841\end{array}$ $\begin{array}{lllll}0.6951488072389008 & 0.3599332884584275 & 0.0800362039419908\end{array}$ $\begin{array}{llllll}0.6152761132175124 & 0.4747919264552454 & 0.3002772208354823\end{array}$ $\begin{array}{lllll}0.5012212818691572 & 0.4177613838520796 & 0.2818425416368732\end{array}$ $\begin{array}{lllll}0.6446562465304605 & 0.4236520646558298 & 0.4191120712673089\end{array}$ $\begin{array}{lllll}0.9008181178956050 & 0.4132275515578697 & 0.1858787699752629\end{array}$ $\begin{array}{lllll}0.7802664757279744 & 0.4604956652843334 & 0.1660956446683940\end{array}$ $\begin{array}{llll}0.8192794762504070 & 0.3885992212843062 & 0.0608778809525212\end{array}$ $\begin{array}{lllll}0.9094862704993866 & 0.4647410109384671 & 0.0581227134267820\end{array}$ $\begin{array}{lllll}0.9452483598598204 & 0.3638543329191851 & 0.0756424436954169\end{array}$ $\begin{array}{lllll}0.8697729119256282 & 0.4738054728175238 & 0.2960714454978257\end{array}$ $\begin{array}{lllll}0.7550258325449820 & 0.4189039564042363 & 0.2851360634672205\end{array}$ $\begin{array}{llll}0.8921272153726537 & 0.4240337103624618 & 0.4167661209800207\end{array}$ $\begin{array}{lllll}0.61774449301292097 & 0.5747310573733698 & 0.7804558254040029\end{array}$ $\begin{array}{lllll}0.5472458093849117 & 0.3524739768820010 & 0.6857364440497158\end{array}$ $\begin{array}{lllll}0.5308713423279456 & 0.2429687366678537 & 0.6919392392160718\end{array}$ $\begin{array}{lllll}0.6559627209678641 & 0.2835259926264249 & 0.6935083617742505\end{array}$ $\begin{array}{lllll}0.7013268191429877 & 0.2078775340825573 & 0.5703096826967262\end{array}$ $\begin{array}{llll}0.5304070326315483 & 0.2925817749083848 & 0.5664928367503600\end{array}$ $\begin{array}{lllll}0.5772006129628506 & 0.1913341848116352 & 0.5855196939205334\end{array}$ $\begin{array}{lllll}0.6794518703144814 & 0.1692822205876794 & 0.6951869682252627\end{array}$ $\begin{array}{lllll}0.6800305550680673 & 0.0563014721955981 & 0.6997172801327701\end{array}$ $\begin{array}{llll}0.5482959759191381 & 0.1122784959886733 & 0.7153965079261517\end{array}$ $\begin{array}{llll}0.6549033345524912 & 0.1067956266731108 & 0.5802553569890057\end{array}$ 
$\begin{array}{llll}0.6499163771847818 & 0.1144511383045668 & 0.8118922241164461\end{array}$ $\begin{array}{lllll}0.5223006642845732 & 0.0683648208685936 & 0.8308951618289633\end{array}$ $\begin{array}{lllll}0.6373265136489629 & 0.0663819750414914 & 0.9389272275055524\end{array}$ $\begin{array}{llll}0.6057658842563042 & 0.1683663352323175 & 0.9203414947071294\end{array}$ $\begin{array}{lllll}0.5209802652780470 & 0.2898275078975074 & 0.8058280409753442\end{array}$ $\begin{array}{llllll}0.6455326628666161 & 0.2486728622671266 & 0.8043252570896636\end{array}$ $\begin{array}{lllll}0.6014395480897189 & 0.3217262263742717 & 0.9280350334343748\end{array}$ $\begin{array}{lllll}0.5168326557940620 & 0.2421960383885883 & 0.9310295526447483\end{array}$ $\begin{array}{lllll}0.6202901809274357 & 0.3629142993706593 & 0.8039301810926240\end{array}$ $\begin{array}{lllll}0.7302355844540301 & 0.1406834459331960 & 0.9378835369550610\end{array}$ $\begin{array}{lllll}0.7537390422979560 & 0.1796928376991758 & 0.8123229880081362\end{array}$ $\begin{array}{lllll}0.7245582195166045 & 0.3429360681659353 & 0.9102687587875613\end{array}$ $\begin{array}{lllll}0.7971949664557297 & 0.3495391901839277 & 0.6857965536366519\end{array}$ $\begin{array}{llll}0.7807378144776925 & 0.2407623059438161 & 0.6923997166489667\end{array}$ $\begin{array}{lllll}0.9059974236775504 & 0.2822600044081836 & 0.6937143997565575\end{array}$ $\begin{array}{llll}0.9506006481453203 & 0.2079817658804823 & 0.5706524392056105\end{array}$ $\begin{array}{llllll}0.7863747753653191 & 0.2873039173334299 & 0.5670556597966968\end{array}$ $\begin{array}{llllll}0.8276568265828271 & 0.1855222502089754 & 0.5881860656692148\end{array}$ $\begin{array}{lllll}0.9316590101729874 & 0.1680908442289052 & 0.6958215387799697\end{array}$ $0.9316814421298770 \quad 0.0551026343070610 \quad 0.7013591014962235$ $\begin{array}{lllll}0.7975334122882261 & 0.1112340704457460 & 0.7151312187579201\end{array}$ $\begin{array}{lllll}0.9105246599455252 & 0.1049032887797386 & 0.5809568069318864\end{array}$ $\begin{array}{lllll}0.9003770729607171 & 0.1145446305768218 & 0.8117263516957984\end{array}$ $\begin{array}{llll}0.7719848692701853 & 0.0681495569374759 & 0.8307227116665666\end{array}$ 0.88873827251872150 .06590074734706620 .9389529731241004 $\begin{array}{lllll}0.8550895332306427 & 0.1674545979279431 & 0.9200022586055143\end{array}$ $\begin{array}{llll}0.7713713382867564 & 0.2881924506060700 & 0.8056152464509305\end{array}$ $\begin{array}{lllll}0.8959640395726730 & 0.2468310951833648 & 0.8045563930136054\end{array}$ $\begin{array}{lllll}0.8509007369821653 & 0.3217858823577789 & 0.9272190470904026\end{array}$ $\begin{array}{llll}0.7667899929651243 & 0.2414436703410685 & 0.9309485809366299\end{array}$ $\begin{array}{lllll}0.8711480326592880 & 0.3612002143434910 & 0.8022719713147719\end{array}$ $\begin{array}{lllll}0.9800339285984649 & 0.1413128980395737 & 0.9380421628025510\end{array}$ $\begin{array}{lllll}0.5050251071616760 & 0.1804684096975478 & 0.8124858111139566\end{array}$ $\begin{array}{llll}0.9741264409786333 & 0.3432283019496370 & 0.9105036620011859\end{array}$ $\begin{array}{llll}0.6519157756023279 & 0.4163451831102035 & 0.6879255465544282\end{array}$ $\begin{array}{lllll}0.5333381834132976 & 0.4668592689960045 & 0.6731955163756160\end{array}$ $\begin{array}{llll}0.5721779160303583 & 0.3926785398300036 & 0.5616331327416521\end{array}$ $\begin{array}{lllll}0.6737770994908231 & 0.4603796977685658 & 0.5607864192720120\end{array}$ $\begin{array}{lllll}0.6950520700254086 & 0.3586605559061564 & 0.5805189309843478\end{array}$ $0.61634101093668890 .4737194188995776 \quad 0.8019041569403923$ $\begin{array}{lllll}0.5001952685940256 & 0.4176877436186217 & 0.7798356823045478\end{array}$ $\begin{array}{llll}0.6450477367779001 & 0.4242146624615227 & 0.9215317213146620\end{array}$ $\begin{array}{llll}0.9004400489758606 & 0.4142930740672534 & 0.6855054805310591\end{array}$ $\begin{array}{lllll}0.7800336724168198 & 0.4617186235663482 & 0.6654922433645548\end{array}$ $\begin{array}{llllll}0.8187978729585301 & 0.3893025344443494 & 0.5608100122684143\end{array}$ $0.9095538548015143 \quad 0.4649208933904564 \quad 0.5574341972416764$ $\begin{array}{lllll}0.9449427010130217 & 0.3640219493953209 & 0.5758091235588459\end{array}$ $\begin{array}{lllll}0.8697827279897585 & 0.4746950138404415 & 0.7956246531337502\end{array}$ $\begin{array}{lllll}0.7555886916847256 & 0.4203683234814417 & 0.7846946202181898\end{array}$ $\begin{array}{llllll}0.8920336708871681 & 0.4247087311850557 & 0.9165953469770466\end{array}$ $\begin{array}{llll}0.2130415107748203 & 0.2336448229281555 & 0.2501003075108454\end{array}$ $\begin{array}{lllll}0.0874255876241158 & 0.2954130548042301 & 0.2469352376047905\end{array}$ $\begin{array}{lllll}0.2227711401120862 & 0.1125890286281520 & 0.2657893861529247\end{array}$ $\begin{array}{llllll}0.4633034547563735 & 0.2338485874699391 & 0.2502850150228971\end{array}$ $\begin{array}{llll}0.3374297949063038 & 0.2946731161652163 & 0.2469972949248695\end{array}$ $0.47285717620742200 .1130384654932454 \quad 0.2659504859665663$ $\begin{array}{lllll}0.0778956136944869 & 0.4159309787006008 & 0.2321824017805738\end{array}$ $\begin{array}{lllll}0.3270338667989791 & 0.4148706423606882 & 0.2307454160843040\end{array}$ $\begin{array}{lllll}0.2139307320999666 & 0.2353073379508284 & 0.7497420905540871\end{array}$ $\begin{array}{lllll}0.0877693582135712 & 0.2962351806431291 & 0.7468608736113931\end{array}$ 0.22364386070814800 .11418659933943090 .7654063509062456 $\begin{array}{llll}0.4643670484147189 & 0.2353154614452329 & 0.7502373218390621\end{array}$ $\begin{array}{lllll}0.3393916987624864 & 0.2969490274282941 & 0.7472724577735228\end{array}$ $\begin{array}{llll}0.4738509774071223 & 0.1141817547294638 & 0.7653571043361065\end{array}$ $\begin{array}{lllll}0.0773602836801698 & 0.4167475390329879 & 0.7321483517227968\end{array}$ $\begin{array}{lllll}0.3262142669075146 & 0.4188881772384682 & 0.7298912524424374\end{array}$ $\begin{array}{llll}0.7131526157586230 & 0.2336292367870081 & 0.2507321575600760\end{array}$ $\begin{array}{lllll}0.5873859445588998 & 0.2952888351224452 & 0.2474547159643626\end{array}$ $\begin{array}{llll}0.7226391677895192 & 0.1125458594727055 & 0.2661005383988508\end{array}$ $\begin{array}{lllll}0.9635511460912255 & 0.2337147474154815 & 0.2505510078200756\end{array}$ $\begin{array}{lllll}0.8377935383378391 & 0.2945863689628459 & 0.2480660899996608\end{array}$ $\begin{array}{llllll}0.9730167593582020 & 0.1128062143035250 & 0.2660196006502776\end{array}$ $\begin{array}{llll}0.5777362645389358 & 0.4157282954322517 & 0.2333818033232880\end{array}$ 
$\begin{array}{llll}0.8278601927844758 & 0.4149404229656978 & 0.2323906891282694\end{array}$ $\begin{array}{llllll}0.7131147378439396 & 0.2347841523186199 & 0.7501942955609130\end{array}$ $\begin{array}{llll}0.5880791428991016 & 0.2973895062910014 & 0.7473983112599030\end{array}$ $\begin{array}{lllll}0.7229340072781315 & 0.1135829955083776 & 0.7652238964682863\end{array}$ $\begin{array}{lllll}0.9640146497235069 & 0.2341722818536942 & 0.7505895034981622\end{array}$ $\begin{array}{lllll}0.8381921247954913 & 0.2951827481527564 & 0.7480676786803788\end{array}$ $\begin{array}{llll}0.9740082585874256 & 0.1132175244725169 & 0.7661074917153218\end{array}$ $\begin{array}{lllll}0.5799400037377415 & 0.4183006039498480 & 0.7363582073457963\end{array}$ $\begin{array}{lllll}0.8278954651395297 & 0.4157018097416953 & 0.7322728767373762\end{array}$ $\begin{array}{llll}0.0232103775584706 & 0.2319333403344145 & 0.1035928878334386\end{array}$ $\begin{array}{lllll}0.1533927165748352 & 0.1678633967599917 & 0.1073961378726222\end{array}$ $\begin{array}{lllll}0.1470832173323176 & 0.3613659549892147 & 0.3897242001918535\end{array}$ $\begin{array}{lllll}0.1557563452000153 & 0.1204034592530933 & 0.3996163899711189\end{array}$ $\begin{array}{llll}0.0266711754882031 & 0.1804674561236300 & 0.4017342904732719\end{array}$ $\begin{array}{llllll}0.0276013753367935 & 0.2978862138746994 & 0.3940652999845267\end{array}$ $\begin{array}{lllll}0.0234409949118612 & 0.3496038967448876 & 0.0958556141996361\end{array}$ $\begin{array}{lllll}0.2737080408230028 & 0.2302719874004120 & 0.1036181769880103\end{array}$ $\begin{array}{lllll}0.4046126116250897 & 0.1679936498722347 & 0.1073360072489660\end{array}$ $\begin{array}{llll}0.3959946865562951 & 0.3615681038889605 & 0.3886856154837243\end{array}$ $\begin{array}{llll}0.4058535094814320 & 0.1208419641150825 & 0.3994323288643309\end{array}$ $\begin{array}{lllll}0.2762922730869156 & 0.1803193921989476 & 0.4014480056756397\end{array}$ $\begin{array}{llllll}0.2773994982834948 & 0.2976000007997768 & 0.3933580169048194\end{array}$ $\begin{array}{llllll}0.2737301326880336 & 0.3472132021529071 & 0.0956287417298700\end{array}$ $\begin{array}{lllll}0.1459409231937166 & 0.4078323137501935 & 0.0989098028091365\end{array}$ $\begin{array}{lllll}0.3935849624706374 & 0.4090132554157321 & 0.0977953451214739\end{array}$ $\begin{array}{lllll}0.0235420301386674 & 0.2322183150290803 & 0.6036348635008626\end{array}$ $\begin{array}{lllll}0.1542150357284641 & 0.1689109397304110 & 0.6073617186685286\end{array}$ $\begin{array}{lllll}0.1477360372124544 & 0.3620082471558102 & 0.8899217974693955\end{array}$ $\begin{array}{lllll}0.1566474823457983 & 0.1218961853386259 & 0.8994984906570958\end{array}$ $\begin{array}{llllll}0.0270663968288884 & 0.1811618424886551 & 0.9017436021746265\end{array}$ $\begin{array}{lllll}0.0277497223081768 & 0.2986417019181783 & 0.8938052190702024\end{array}$ $\begin{array}{llll}0.0232617046847026 & 0.3499490600056390 & 0.5957641390514131\end{array}$ $\begin{array}{lllll}0.2743663897707449 & 0.2315583896994763 & 0.6040720784483372\end{array}$ $\begin{array}{lllll}0.4051490429464097 & 0.1693344632855548 & 0.6073864094185703\end{array}$ $\begin{array}{llll}0.3980335239802376 & 0.3629275574553000 & 0.8895124254252803\end{array}$ $\begin{array}{lllll}0.4069607423609120 & 0.1211578250054857 & 0.8988268621024816\end{array}$ $\begin{array}{lllll}0.2777881215036948 & 0.1812903542802570 & 0.9008171786043527\end{array}$ $\begin{array}{lllll}0.2782575969407221 & 0.2992746478732390 & 0.8939951058864067\end{array}$ $\begin{array}{lllll}0.2746282902030443 & 0.3480869789675397 & 0.5962096884984726\end{array}$ $0.14571042711488330 .4080093030372723 \quad 0.5985690141182264$ $\begin{array}{lllll}0.3911882974916944 & 0.4152420111635381 & 0.5946435859641325\end{array}$ $\begin{array}{llll}0.5234684700256398 & 0.2321346127465929 & 0.1037065173705170\end{array}$ $\begin{array}{lllll}0.6534905615585755 & 0.1680165291700887 & 0.1077758614933545\end{array}$ $\begin{array}{lllll}0.6471630243547264 & 0.3607637410358521 & 0.3907690554877034\end{array}$ $\begin{array}{lllll}0.6550315629189518 & 0.1209048745835394 & 0.3997545850410271\end{array}$ $\begin{array}{lllll}0.5262532770872752 & 0.1811918614067104 & 0.4017428718328728\end{array}$ $\begin{array}{lllll}0.5266729896593502 & 0.2984148574036934 & 0.3939471777372199\end{array}$ $\begin{array}{lllll}0.5239521951192001 & 0.3497696487294666 & 0.0964578890475893\end{array}$ $\begin{array}{lllll}0.7737985588209373 & 0.2304592792832311 & 0.1043429278479432\end{array}$ $\begin{array}{lllll}0.9043971801394245 & 0.1677952149724014 & 0.1076656740464773\end{array}$ $\begin{array}{llll}0.8970630645058861 & 0.3608984249104443 & 0.3906996254141359\end{array}$ $\begin{array}{lllll}0.9059798910075003 & 0.1202981518611647 & 0.3995895160982545\end{array}$ $\begin{array}{lllll}0.7763215619484453 & 0.1797372008011389 & 0.4019164228459348\end{array}$ $\begin{array}{lllll}0.7776666635553715 & 0.2971271508671259 & 0.3943648540058993\end{array}$ $\begin{array}{lllll}0.7739894247621408 & 0.3473603615327589 & 0.0972085832818840\end{array}$ $\begin{array}{llll}0.6465110788693020 & 0.4082145114857215 & 0.1012098550270520\end{array}$ $\begin{array}{lllll}0.8935044233967571 & 0.4092125731885534 & 0.0982592409849347\end{array}$ $\begin{array}{lllll}0.5228790499876828 & 0.2350659986967316 & 0.6030404913195379\end{array}$ $\begin{array}{llllll}0.6528197153428735 & 0.1700232797597833 & 0.6070288215843554\end{array}$ $\begin{array}{lllll}0.6476865237150662 & 0.3621763643202711 & 0.8920134305667413\end{array}$ $\begin{array}{llllll}0.6559405596267295 & 0.1214031768667784 & 0.8992767087066468\end{array}$ $\begin{array}{llll}0.5272199654118357 & 0.1817223890180232 & 0.9012976488009520\end{array}$ $\begin{array}{lllll}0.5278848289114216 & 0.2990956746793020 & 0.8944952766681015\end{array}$ $\begin{array}{lllll}0.5232353845131803 & 0.3536623022823007 & 0.5972311521642781\end{array}$ $\begin{array}{llll}0.7745539566406470 & 0.2308211700298656 & 0.6041795273124720\end{array}$ $\begin{array}{lllll}0.9049525022303119 & 0.1678326954437086 & 0.6077715972477880\end{array}$ $\begin{array}{llll}0.8971939013814223 & 0.3616218420481809 & 0.8904241302262107\end{array}$ $0.9061807981885122 \quad 0.12119239702855090 .8990613908415434$ $\begin{array}{llll}0.77655776550439045 & 0.1808282589452797 & 0.9010621766520022\end{array}$ $\begin{array}{llllll}0.7777669718225642 & 0.2980886935425021 & 0.8938242095895944\end{array}$ $\begin{array}{lllll}0.7743400727949930 & 0.3473103778278486 & 0.5972443633635809\end{array}$ $\begin{array}{llllll}0.6475871472298963 & 0.4080446559217881 & 0.6014064835219480\end{array}$ $\begin{array}{llll}0.8933229480960402 & 0.4096606876111227 & 0.5980136714335944\end{array}$ 
Cyt/Thy :

H 139 C 9 N 5 O 243 Al 32 Si 6

1.00000000000000

$20.2970019999999991 \quad 0.0000000000000000-3.7471719999999999$

$0.0646860000000000 \quad 24.9946149999999996-0.5148360000000000$

$\begin{array}{llll}0.0000000000000000 & 0.0000000000000000 & 18.6939999999999991\end{array}$

$\mathrm{H}$ C N O Al Si

$\begin{array}{llllll}139 & 9 & 5 & 243 & 32 & 64\end{array}$

Direct

$0.68047864941831750 .5658762768016077 \quad 0.4800189344609646$

$\begin{array}{llll}0.4955126816936703 & 0.6472782094990799 & 0.5612018189270644\end{array}$

$\begin{array}{llll}0.6109308183754680 & 0.5169344365129465 & 0.5371936217463239\end{array}$

$\begin{array}{lllll}0.6499274983734969 & 0.6975665304132981 & 0.4096914650426559\end{array}$

$\begin{array}{llll}0.7163153522328587 & 0.6521491276492352 & 0.4383514332432606\end{array}$ $\begin{array}{llll}0.6999726206022893 & 0.7067622941013086 & 0.4959237932792254\end{array}$ $\begin{array}{llll}0.0926812590278563 & 0.0547071319423578 & 0.4099493907529175\end{array}$ $\begin{array}{llll}0.3422820543331840 & 0.0556593566728645 & 0.4093992778257688\end{array}$ $\begin{array}{llll}0.0494017699800540 & 0.1391145591783522 & 0.1813897206080569\end{array}$ $\begin{array}{lllll}0.1687931194930839 & 0.3087446866554551 & 0.1614282476345200\end{array}$ $\begin{array}{llll}0.1281869958826772 & 0.2204909718736563 & 0.3333357864005285\end{array}$ $\begin{array}{llll}0.2426630640407040 & 0.0391293554120654 & 0.3428288555585046\end{array}$ $\begin{array}{llll}0.1703445546792449 & 0.0671204182999844 & 0.1468240831763258\end{array}$ $\begin{array}{llll}0.2993316131159290 & 0.1396269543401466 & 0.1813642408230163\end{array}$ $\begin{array}{llll}0.4180279757976388 & 0.3085795854057710 & 0.1596677724349414\end{array}$ $\begin{array}{llll}0.3780713281049266 & 0.2211941615384420 & 0.3329495476813840\end{array}$ $\begin{array}{llll}0.4914424031043130 & 0.0391886492722175 & 0.3416219584487112\end{array}$ $\begin{array}{llll}0.4197909084525278 & 0.0677595062059296 & 0.1465158447696473\end{array}$ $\begin{array}{llll}0.0550424790752417 & 0.4889645674107792 & 0.1496812557650887\end{array}$ $\begin{array}{llll}0.2473391720481087 & 0.3903195945157844 & 0.3123171347634132\end{array}$ $\begin{array}{llll}0.1267463317030814 & 0.4618568484484141 & 0.3469414041350407\end{array}$ $\begin{array}{llll}0.3041967233281135 & 0.4892071902119510 & 0.1485093980584270\end{array}$ $\begin{array}{llll}0.4967336463799051 & 0.3900707341907756 & 0.3121506285392291\end{array}$ $\begin{array}{llll}0.3759042306672756 & 0.4619024442257292 & 0.3459552966090628\end{array}$ $\begin{array}{llll}0.2040690287156893 & 0.4740948330334022 & 0.0851107332129476\end{array}$ $\begin{array}{llll}0.4540244650848880 & 0.4750111552541336 & 0.0852111125091136\end{array}$ $\begin{array}{llll}0.1516829456646326 & 0.4388978489213770 & 0.4655036601812223\end{array}$ $\begin{array}{llll}0.3939403816272287 & 0.4403483579863238 & 0.4633579766962566\end{array}$ $\begin{array}{llll}0.1463011573842848 & 0.0907344217250520 & 0.0279901924015938\end{array}$ $\begin{array}{llll}0.3951875473789244 & 0.0915382381934022 & 0.0275334032559705\end{array}$ $\begin{array}{llll}0.1625090096959407 & 0.4819052059158782 & 0.2840267980517123\end{array}$ $\begin{array}{llll}0.4115750200639134 & 0.4826000870681694 & 0.2830063471945508\end{array}$ $\begin{array}{llll}0.1345302599083045 & 0.0469759433832678 & 0.2096118218582066\end{array}$ $\begin{array}{llll}0.3833903451288181 & 0.0480618336948146 & 0.2090251882332806\end{array}$ $\begin{array}{llll}0.2096688293778244 & 0.4163045275384169 & 0.2375701128445299\end{array}$ $\begin{array}{lllll}0.4596023573006883 & 0.4167113075982347 & 0.2376916393958959\end{array}$ $\begin{array}{llll}0.3367504723856095 & 0.1133777289820263 & 0.2560633577027716\end{array}$ $\begin{array}{llll}0.0866445367140473 & 0.1126230442113840 & 0.2558867483142521\end{array}$ $\begin{array}{llll}0.0953712950642061 & 0.0548472390793200 & 0.9088353235802746\end{array}$ $\begin{array}{lllll}0.3430180447806157 & 0.0572959173989197 & 0.9081650616452204\end{array}$ 0.05042375601389790 .13937854146552650 .6814834636555935 $\begin{array}{llll}0.1695786194746710 & 0.3104976569500862 & 0.6615442833254918\end{array}$ $\begin{array}{llll}0.1289328426064545 & 0.2206012915782726 & 0.8313072258479103\end{array}$ $\begin{array}{lllll}0.2436200830910542 & 0.0405845870864581 & 0.8422409665945029\end{array}$ $\begin{array}{lllll}0.1716962623003205 & 0.0687809455126530 & 0.6468301568413147\end{array}$ $0.30039643932208990 .1406976817722367 \quad 0.6805074708233326$ $\begin{array}{llll}0.4189442086864371 & 0.3107064094601623 & 0.6589599481388040\end{array}$ $\begin{array}{llll}0.3784139952957503 & 0.2223305240070566 & 0.8310430145454532\end{array}$ $\begin{array}{llll}0.4922202860986730 & 0.0415407593745980 & 0.8422529525435882\end{array}$ $\begin{array}{llll}0.4208878285662833 & 0.0693299929752413 & 0.6466758494221456\end{array}$ $0.05660991025847190 .4880112489084168 \quad 0.6467841377322014$ $\begin{array}{llll}0.2479203568014830 & 0.3905594200044848 & 0.8117691824457705\end{array}$ $0.1267704605123802 \quad 0.4626302521167456 \quad 0.8462773509166697$ $0.30172255111838380 .4895461406465706 \quad 0.6449082972527139$ $\begin{array}{llll}0.4933440254169031 & 0.3803533996010360 & 0.8008035251997024\end{array}$ $\begin{array}{llll}0.3766374488984943 & 0.4638016929585613 & 0.8468564110420406\end{array}$ $\begin{array}{lllll}0.2023009867313388 & 0.4758003373161527 & 0.5859458543031750\end{array}$ $\begin{array}{llll}0.4393315843178530 & 0.4905286457846677 & 0.5618228411064121\end{array}$ $\begin{array}{lllll}0.1514178786160319 & 0.4391360794811918 & 0.9653084596281811\end{array}$ $\begin{array}{lllll}0.4028996726413352 & 0.4401257521686061 & 0.9652481919654160\end{array}$ $\begin{array}{llll}0.1461023088015607 & 0.0917880356359026 & 0.5285123636783984\end{array}$ $\begin{array}{llll}0.3950223329478364 & 0.0923063779833775 & 0.5284254532486004\end{array}$ $\begin{array}{lllll}0.1635556314069718 & 0.4817780666745750 & 0.7838327422864387\end{array}$ 
$\begin{array}{llll}0.4087159556896870 & 0.4860637418033352 & 0.7821479645140244\end{array}$ $\begin{array}{lllll}0.1352346735269733 & 0.0490231945397175 & 0.7092522252054362\end{array}$ $\begin{array}{lllll}0.3850947989399158 & 0.0492573556885641 & 0.7095030834398998\end{array}$ $\begin{array}{lllll}0.2093830812362032 & 0.4163924480957922 & 0.7373236319681548\end{array}$ $\begin{array}{llll}0.4609392800260767 & 0.4188710025553812 & 0.7381129010173805\end{array}$ $\begin{array}{lllll}0.3378252539651013 & 0.1149129212963672 & 0.7555793722016362\end{array}$ $\begin{array}{lllll}0.0882189118676721 & 0.1136835058039798 & 0.7563028249678582\end{array}$ $\begin{array}{llllll}0.5893621961535778 & 0.0568533921636796 & 0.4080548310420837\end{array}$ $\begin{array}{lllll}0.8421309126662166 & 0.0546014055339722 & 0.4093972311233557\end{array}$ $\begin{array}{lllll}0.5490382864951164 & 0.1401309715080742 & 0.1815798055145795\end{array}$ $\begin{array}{lllll}0.6678689450314876 & 0.3078470549526143 & 0.1592565955862886\end{array}$ $\begin{array}{lllll}0.6285714405822821 & 0.2219820167889765 & 0.3347256223654585\end{array}$ $\begin{array}{lllll}0.7414310802992256 & 0.0401299180603927 & 0.3442076876763427\end{array}$ $\begin{array}{llll}0.6693350734264830 & 0.0675415001371295 & 0.1464794712801621\end{array}$ $\begin{array}{lllll}0.7991101995434529 & 0.1399051864959886 & 0.1818299583014895\end{array}$ $\begin{array}{lllll}0.9183519849656888 & 0.3078372159021708 & 0.1599985632752318\end{array}$ $\begin{array}{lllll}0.8780128581595303 & 0.2203287987650001 & 0.3329882439836959\end{array}$ $\begin{array}{lllll}0.9920718529368107 & 0.0393473627598709 & 0.3436193235631035\end{array}$ $\begin{array}{llll}0.9194170477106043 & 0.0670968149649323 & 0.1467492267914179\end{array}$ $\begin{array}{lllll}0.5551478906494621 & 0.4885751814388894 & 0.1477701742383611\end{array}$ $\begin{array}{lllll}0.7472027307792179 & 0.3885397380422459 & 0.3108476994392539\end{array}$ $\begin{array}{lllll}0.6280297499248818 & 0.4617874254262217 & 0.3474669011475923\end{array}$ $\begin{array}{lllll}0.8060055613235554 & 0.4877372919070580 & 0.1475247453872987\end{array}$ $\begin{array}{llll}0.9975088339991536 & 0.3890251243687839 & 0.3118135953913292\end{array}$ $\begin{array}{lllll}0.8778690339640688 & 0.4615481218606286 & 0.3476552032224992\end{array}$ $\begin{array}{llll}0.7051184958488533 & 0.4748081441652403 & 0.0870616442103182\end{array}$ $\begin{array}{lllll}0.9555702772590463 & 0.4733941518938279 & 0.0859138823903899\end{array}$ $\begin{array}{lllll}0.6570039233482070 & 0.4362640780356380 & 0.4663291135263998\end{array}$ $\begin{array}{lllll}0.9040142276230227 & 0.4381494103770400 & 0.4662220014584663\end{array}$ $\begin{array}{llllll}0.6440526203818777 & 0.0911849145050477 & 0.0277679464370243\end{array}$ $\begin{array}{lllll}0.8936459767476359 & 0.0907404062698234 & 0.0279736723198169\end{array}$ $\begin{array}{lllll}0.6620161238126921 & 0.4823032413049724 & 0.2834508896783220\end{array}$ $\begin{array}{lllll}0.9121108948815568 & 0.4824677669778920 & 0.2839748509529947\end{array}$ $\begin{array}{lllll}0.6341380759285111 & 0.0469909909288696 & 0.2095920136250814\end{array}$ $\begin{array}{lllll}0.8842574486110992 & 0.0467737241862070 & 0.2099928059233348\end{array}$ $\begin{array}{llll}0.7097266855176816 & 0.4166866111349351 & 0.2377801245173715\end{array}$ $\begin{array}{lllll}0.9600513779707381 & 0.4163568508721724 & 0.2381581694903867\end{array}$ $\begin{array}{lllll}0.8363928818897084 & 0.1128381132380602 & 0.2558129905530525\end{array}$ $\begin{array}{lllll}0.5862633461296153 & 0.1130638171570977 & 0.2556148516059466\end{array}$ $\begin{array}{lllll}0.7098581872772451 & 0.7173293868397680 & 0.6649831662485453\end{array}$ $\begin{array}{llll}0.8009720590910441 & 0.6691917029682173 & 0.6162359459122678\end{array}$ $\begin{array}{lllll}0.8150631771453416 & 0.5200636657408391 & 0.6323349365300990\end{array}$ $\begin{array}{llll}0.8483936271272059 & 0.5783662210077100 & 0.5989346091458826\end{array}$ $\begin{array}{lllll}0.6385620202286936 & 0.6656195710667159 & 0.7228692962940684\end{array}$ $\begin{array}{lllll}0.5915136818315663 & 0.0566768346702108 & 0.9089917130373145\end{array}$ $\begin{array}{lllll}0.8413462210393937 & 0.0561347374851611 & 0.9079670940000678\end{array}$ $\begin{array}{llll}0.5504156248937839 & 0.1443173377070940 & 0.6845526199591274\end{array}$ $\begin{array}{lllll}0.6697197247090066 & 0.3084116363286015 & 0.6594045471541077\end{array}$ $\begin{array}{lllll}0.6295867149498440 & 0.2229517716037592 & 0.8348120134271572\end{array}$ $\begin{array}{llllll}0.7421928896424541 & 0.0401016760433854 & 0.8428603121024614\end{array}$ $\begin{array}{lllll}0.6690212704085016 & 0.0691422887318036 & 0.6454694171537781\end{array}$ $\begin{array}{llll}0.8002104388405966 & 0.1404069557094716 & 0.6812667417609650\end{array}$ $\begin{array}{llll}0.9204840614205689 & 0.3088401964222183 & 0.6627517441071504\end{array}$ $\begin{array}{lllll}0.8801555157758859 & 0.2212504129988046 & 0.8355630497478225\end{array}$ $\begin{array}{llllll}0.9938194300874146 & 0.0401570264691707 & 0.8453273964985238\end{array}$ $\begin{array}{lllll}0.9210203365759861 & 0.0666982981144510 & 0.6475152910418845\end{array}$ $\begin{array}{llll}0.5335413525450856 & 0.4892151645263635 & 0.6450740665618772\end{array}$ $\begin{array}{lllll}0.7493032442498297 & 0.3941923728116083 & 0.8193424652882217\end{array}$ $\begin{array}{llllll}0.6289606985472747 & 0.4688368574721341 & 0.8437867485417350\end{array}$ $\begin{array}{lllll}0.7558460336124804 & 0.4888491327964366 & 0.6882782579991185\end{array}$ $\begin{array}{lllll}0.9983157730172925 & 0.3910336544661054 & 0.8127760354599292\end{array}$ $\begin{array}{llll}0.8782315658181410 & 0.4623657197662862 & 0.8475021355833389\end{array}$ $\begin{array}{lllll}0.7135939349873419 & 0.4690199622909652 & 0.5850040494097851\end{array}$ $\begin{array}{llll}0.9563565008702690 & 0.4738486758720865 & 0.5873420022231254\end{array}$ $\begin{array}{llll}0.6555225035848107 & 0.4401324193110923 & 0.9658900002907415\end{array}$ $\begin{array}{llll}0.9034536264926224 & 0.4384306175132478 & 0.9663144604794555\end{array}$ $\begin{array}{lllll}0.6417084629178573 & 0.0924489181235701 & 0.5276035349182725\end{array}$ $\begin{array}{lllll}0.8953749430360977 & 0.0901075799690149 & 0.5287805816388321\end{array}$ $\begin{array}{lllll}0.6277954998289816 & 0.5104027207113668 & 0.7739425388258164\end{array}$ $\begin{array}{llll}0.9119503200515352 & 0.4835394047556975 & 0.7833384642684258\end{array}$ $\begin{array}{lllll}0.6351814146667171 & 0.0477040330008131 & 0.7091269396054097\end{array}$ $\begin{array}{llll}0.8860845757922249 & 0.0466425516921751 & 0.7111349334538124\end{array}$ $\begin{array}{lllll}0.7111171165555145 & 0.4178380450405776 & 0.7426589666053642\end{array}$ 
$\begin{array}{llll}0.9603265634551863 & 0.4170551942813726 & 0.7382336006552275\end{array}$ $\begin{array}{llll}0.8373684793771358 & 0.1133564962617400 & 0.7552866254915606\end{array}$ $\begin{array}{llll}0.5872436353898921 & 0.1140033363248188 & 0.7556375336276286\end{array}$ $\begin{array}{llll}0.6771867390358866 & 0.6759540468646644 & 0.4565283994545021\end{array}$ $\begin{array}{llll}0.6394108765125766 & 0.5876138867441789 & 0.4965871430256509\end{array}$ $\begin{array}{llll}0.6318343037317394 & 0.6413813067443422 & 0.4891968119637214\end{array}$ $\begin{array}{llll}0.5775619891146065 & 0.6668060750841800 & 0.5174041591999486\end{array}$ $\begin{array}{llll}0.5420967990726809 & 0.5760074236280938 & 0.5495023403827248\end{array}$ $\begin{array}{llll}0.7179860154638680 & 0.6743071291269819 & 0.6697257010250731\end{array}$ $\begin{array}{lllll}0.7668566882876545 & 0.6479079763174971 & 0.6434688802900624\end{array}$ $\begin{array}{llll}0.7699562185845842 & 0.5908692559817046 & 0.6515209415217376\end{array}$ $\begin{array}{llll}0.6802425365034823 & 0.5909958638546733 & 0.7126211643295436\end{array}$ $\begin{array}{llll}0.5969853216086149 & 0.5561486283873026 & 0.5259163317478938\end{array}$ $\begin{array}{llll}0.5349651886422570 & 0.6307586536301604 & 0.5429452015822088\end{array}$ $\begin{array}{llll}0.6755406991975224 & 0.6467598091149235 & 0.7028850025154445\end{array}$ $\begin{array}{llll}0.8144654289448287 & 0.5612140402503202 & 0.6240268938196829\end{array}$ $\begin{array}{llll}0.7280177223526900 & 0.5644007287511061 & 0.6856194441462784\end{array}$ $\begin{array}{llll}0.5674630406816891 & 0.7154408003095647 & 0.5209060120438409\end{array}$ $\begin{array}{llll}0.5026933487018235 & 0.5480897062446387 & 0.5750860992181290\end{array}$ $\begin{array}{llll}0.0444109753496404 & 0.3496845522687314 & 0.1831939896421735\end{array}$ $\begin{array}{llll}0.0283378987198045 & 0.2405611286258000 & 0.1906515344922465\end{array}$ $\begin{array}{llll}0.1535089489218684 & 0.2821782683228380 & 0.1916660904652037\end{array}$ $\begin{array}{llll}0.1981536216666982 & 0.2078657498914959 & 0.0688854197285814\end{array}$ $\begin{array}{llll}0.0325044052891132 & 0.2874327084893634 & 0.0651528073615454\end{array}$ $\begin{array}{llll}0.0749434149436819 & 0.1858411854680452 & 0.0859770596457342\end{array}$ $\begin{array}{llll}0.1786466418316978 & 0.1679658885990702 & 0.1938779549444981\end{array}$ $\begin{array}{llll}0.1781453217402698 & 0.0550906858945460 & 0.1993077891021388\end{array}$ $0.04511838568180350 .1096919452908664 \quad 0.2138073883237119$ $\begin{array}{llll}0.1575487709784696 & 0.1049426803086760 & 0.0788514805548760\end{array}$ $\begin{array}{lllll}0.1473524050160015 & 0.1146139428773136 & 0.3101721253654888\end{array}$ $\begin{array}{llll}0.0192933754969016 & 0.0676515861356740 & 0.3301996283445625\end{array}$ $\begin{array}{llll}0.1369537046934722 & 0.0651667997004961 & 0.4375422461969168\end{array}$ $\begin{array}{llll}0.1026067901693651 & 0.1665480994646070 & 0.4194645974671108\end{array}$ $\begin{array}{lllll}0.0184838037314666 & 0.2881131765884628 & 0.3037076527276965\end{array}$ $\begin{array}{llll}0.1431722736125668 & 0.2467687487893487 & 0.3024743565479529\end{array}$ $\begin{array}{llll}0.0986957778702523 & 0.3213536419011355 & 0.4251094683945182\end{array}$ $\begin{array}{lllll}0.0146865351694867 & 0.2410337168547432 & 0.4292251235272140\end{array}$ $\begin{array}{llll}0.1181418923941232 & 0.3610806284497969 & 0.3001811580779865\end{array}$ $\begin{array}{llll}0.2275398108937329 & 0.1409921049201328 & 0.4363693326377019\end{array}$ $\begin{array}{llll}0.2523638443001008 & 0.1792846148313228 & 0.3109979851268879\end{array}$ $\begin{array}{llll}0.2219554162853161 & 0.3432831222737316 & 0.4080914998442291\end{array}$ $\begin{array}{lllll}0.2939197120759906 & 0.3502447777025107 & 0.1825459862312948\end{array}$ $\begin{array}{llll}0.2782252846672359 & 0.2410394402190030 & 0.1903788009267475\end{array}$ $\begin{array}{llll}0.4032724675857398 & 0.2825933549699493 & 0.1910959596409666\end{array}$ $\begin{array}{lllll}0.4481202771497811 & 0.2085216835743273 & 0.0688150056272079\end{array}$ $\begin{array}{llll}0.2822496173248361 & 0.2879877529908345 & 0.0647909603275035\end{array}$ $\begin{array}{llll}0.3247689556807700 & 0.1865117122740023 & 0.0855124071111056\end{array}$ $\begin{array}{llll}0.4283317350163883 & 0.1685352852926239 & 0.1935912853424496\end{array}$ $\begin{array}{lllll}0.4272332390330284 & 0.0557773278693014 & 0.1990239105557288\end{array}$ $\begin{array}{lllll}0.2952751439474282 & 0.1101493941372002 & 0.2137748890841265\end{array}$ $\begin{array}{llll}0.4073345562884642 & 0.1056245878697432 & 0.0784519450785159\end{array}$ $\begin{array}{lllll}0.3970162220801954 & 0.1155239675508697 & 0.3101902890051561\end{array}$ $\begin{array}{llll}0.2695841838115275 & 0.0679425010330600 & 0.3302251456588173\end{array}$ $\begin{array}{lllll}0.3865467318579991 & 0.0659647745818676 & 0.4373748569096609\end{array}$ $\begin{array}{lllll}0.3527187164545786 & 0.1674702186858201 & 0.4196482238023119\end{array}$ $\begin{array}{llll}0.2682687469782590 & 0.2884765601573633 & 0.3033972201775156\end{array}$ $\begin{array}{lllll}0.3930203866639025 & 0.2474796838893096 & 0.3020779269283598\end{array}$ $\begin{array}{llll}0.3485468339202644 & 0.3217433545951654 & 0.4245979263446187\end{array}$ $\begin{array}{llll}0.2641939706381956 & 0.2416909542236870 & 0.4290275032255755\end{array}$ $\begin{array}{llll}0.3677812300070655 & 0.3613317637821520 & 0.2991801841221662\end{array}$ 0.47768235651905680 .14149750992146610 .4362165107221244 $\begin{array}{llll}0.0021964325680048 & 0.1789552088611144 & 0.3109889664016122\end{array}$ $\begin{array}{lllll}0.4714662773135561 & 0.3443385680803360 & 0.4072135531907874\end{array}$ $\begin{array}{llll}0.1492667480327289 & 0.4140212158395846 & 0.1834329981023060\end{array}$ $\begin{array}{lllll}0.0273812217680822 & 0.4612041860639656 & 0.1637041271575604\end{array}$ $0.0688552070478203 \quad 0.38810794779990630 .0574938925756197$ $\begin{array}{llll}0.1597698404177849 & 0.4640218642713516 & 0.0568098314535215\end{array}$ $\begin{array}{llll}0.1938729338143057 & 0.3624140830269550 & 0.0737026893324895\end{array}$ $\begin{array}{lllll}0.1188791370671179 & 0.4739028953091700 & 0.2944213406124880\end{array}$ $\begin{array}{llll}0.5011909463622015 & 0.4195737376297893 & 0.2798912754452792\end{array}$ $\begin{array}{llll}0.1394438665551230 & 0.4243096129254962 & 0.4149445198011216\end{array}$ $\begin{array}{llll}0.3991280593395624 & 0.4148793497658027 & 0.1833514296555791\end{array}$ $\begin{array}{llll}0.2766346184273468 & 0.4614181337221273 & 0.1626506318486823\end{array}$ 
$\begin{array}{llll}0.3191941106938339 & 0.3885702677319003 & 0.0571598551899848\end{array}$ $\begin{array}{lllll}0.4100956205389418 & 0.4644540709591144 & 0.0567555026991812\end{array}$ $\begin{array}{lllll}0.4441858120497643 & 0.3627343614502587 & 0.0744773750977627\end{array}$ $\begin{array}{lllll}0.3680050593350880 & 0.4745775924244998 & 0.2934664629747535\end{array}$ $\begin{array}{llll}0.2512107970938136 & 0.4196655495444611 & 0.2796373101810961\end{array}$ $\begin{array}{llll}0.3877479465251724 & 0.4249461672264964 & 0.4126421901322078\end{array}$ $\begin{array}{llllll}0.0437781633095697 & 0.3502400355945974 & 0.6828687042099719\end{array}$ $\begin{array}{lllll}0.0294223518483123 & 0.2408162434982811 & 0.6905919807025459\end{array}$ $\begin{array}{llll}0.1541021894306146 & 0.2835890893646059 & 0.6911563369437909\end{array}$ $\begin{array}{lllll}0.1991714925914935 & 0.2097785194791832 & 0.5685676855676557\end{array}$ $\begin{array}{lllll}0.0314423274451565 & 0.2880702397990486 & 0.5647153111553960\end{array}$ $\begin{array}{lllll}0.0761154419481431 & 0.1869919047779840 & 0.5853241054680823\end{array}$ $\begin{array}{lllll}0.1799375252780065 & 0.1696086683372151 & 0.6935378744507992\end{array}$ $\begin{array}{lllll}0.1791049086921426 & 0.0566958337294554 & 0.6992992804472862\end{array}$ $\begin{array}{llllll}0.0467245642425711 & 0.1099582055425931 & 0.7141037927453545\end{array}$ $\begin{array}{llllll}0.1593573991389348 & 0.1065248019071417 & 0.5788485583831058\end{array}$ $\begin{array}{lllll}0.1486234943204955 & 0.1165935117931712 & 0.8101616457219415\end{array}$ $\begin{array}{llllll}0.0211885121809610 & 0.0678798330523984 & 0.8307089314908794\end{array}$ $\begin{array}{llll}0.1395430715789541 & 0.0654450241691945 & 0.9366610295432003\end{array}$ $\begin{array}{lllll}0.1035387075947273 & 0.1665324422578121 & 0.9207828037320770\end{array}$ $\begin{array}{lllll}0.0194282829577569 & 0.2882026145986757 & 0.8037836104351930\end{array}$ $\begin{array}{llllll}0.1442803021796907 & 0.2477639302642541 & 0.8020128391409623\end{array}$ $\begin{array}{lllll}0.0995624014889356 & 0.3215568801541274 & 0.9250414878469286\end{array}$ $\begin{array}{lllll}0.0155437939740060 & 0.2412180531189496 & 0.9293433035646386\end{array}$ $\begin{array}{llllll}0.1180664880301243 & 0.3616702318853731 & 0.7998140068000932\end{array}$ $\begin{array}{lllll}0.2288878471862672 & 0.1425674643505137 & 0.9361959195799601\end{array}$ $\begin{array}{lllll}0.2538646860047953 & 0.1808696366158358 & 0.8105736964991213\end{array}$ $\begin{array}{lllll}0.2222385464561922 & 0.3445644819782425 & 0.9077570943815291\end{array}$ $\begin{array}{lllll}0.2951706865080547 & 0.3523105834254976 & 0.6824167664740608\end{array}$ $0.2793270186304003 \quad 0.2430139193264604 \quad 0.6899972022428735$ $\begin{array}{lllll}0.4047633959035761 & 0.2849982741388914 & 0.6911533427988550\end{array}$ $\begin{array}{llll}0.4487575564242442 & 0.2097379174575794 & 0.5685442619921439\end{array}$ $\begin{array}{lllll}0.2839971626992025 & 0.2897973700727988 & 0.5645678914375325\end{array}$ $\begin{array}{llll}0.3255258840883112 & 0.1879420833485338 & 0.5854414681070886\end{array}$ $0.42903217785258090 .1702145999584602 \quad 0.6936174897019096$ $\begin{array}{lllll}0.4287515734086295 & 0.0572528059863110 & 0.6992042766232618\end{array}$ $\begin{array}{llll}0.2963829110645183 & 0.1114866134174692 & 0.7133654862943990\end{array}$ $\begin{array}{lllll}0.4078643425659486 & 0.1068938173926383 & 0.5788859654399631\end{array}$ $\begin{array}{lllll}0.3980587363438344 & 0.1172086814411746 & 0.8100081215297308\end{array}$ $\begin{array}{lllll}0.2706080806302939 & 0.0694979551562162 & 0.8300348395291791\end{array}$ $\begin{array}{llll}0.3872975311328991 & 0.0673193764514901 & 0.9365523595673876\end{array}$ $\begin{array}{llll}0.3538719588662163 & 0.1689872824713226 & 0.9197224048662923\end{array}$ $\begin{array}{lllll}0.2690308668667589 & 0.2903212380247837 & 0.8028447570162418\end{array}$ $\begin{array}{lllll}0.3940169241616636 & 0.2492988834592383 & 0.8016346354184910\end{array}$ $\begin{array}{lllll}0.3487608542510854 & 0.3237914153310238 & 0.9242659894906616\end{array}$ $\begin{array}{lllll}0.2651677621165359 & 0.2432322990891357 & 0.9284075157442884\end{array}$ $\begin{array}{llll}0.3691626596104424 & 0.3632969348448024 & 0.7992990186918614\end{array}$ $\begin{array}{lllll}0.4787730700299159 & 0.1429243553267566 & 0.9360690859666280\end{array}$ $\begin{array}{lllll}0.0038215584186787 & 0.1790179128774284 & 0.8113453932765076\end{array}$ $\begin{array}{lllll}0.4719926063626438 & 0.3435148688672662 & 0.9093061194021874\end{array}$ $\begin{array}{lllll}0.1492143550251256 & 0.4138495181922768 & 0.6830372185151482\end{array}$ $\begin{array}{lllll}0.0277876060999156 & 0.4619988159189391 & 0.6628035576160417\end{array}$ $\begin{array}{lllll}0.0694720880604818 & 0.3880635658028979 & 0.5570910797624916\end{array}$ $\begin{array}{lllll}0.1585482358739924 & 0.4649810648729441 & 0.5567739538820776\end{array}$ $\begin{array}{lllll}0.1952968229717370 & 0.3637279072561996 & 0.5730709400003640\end{array}$ $\begin{array}{lllll}0.1195623128887755 & 0.4744418458906258 & 0.7937355324371960\end{array}$ $\begin{array}{lllll}0.5003893391371985 & 0.4151191484075721 & 0.7805438386417292\end{array}$ $\begin{array}{lllll}0.1387243294105383 & 0.4248541996412663 & 0.9146166025653627\end{array}$ $\begin{array}{lllll}0.3969506610078588 & 0.4216535631069556 & 0.6820147993315097\end{array}$ $\begin{array}{lllll}0.2735724136521637 & 0.4641353681279173 & 0.6632229121152984\end{array}$ $\begin{array}{lllll}0.3207215369034915 & 0.3906638641163772 & 0.5574121174979277\end{array}$ $\begin{array}{lllll}0.3997010910445887 & 0.4683250432852680 & 0.5470944388605931\end{array}$ $\begin{array}{lllll}0.4454812281401439 & 0.3685228501053941 & 0.5743160579061605\end{array}$ $\begin{array}{llll}0.3663646710667524 & 0.4766872783455322 & 0.7947580191579499\end{array}$ $\begin{array}{llll}0.2508187648223733 & 0.4203267508534628 & 0.7794539193048335\end{array}$ $\begin{array}{llll}0.3908190633136178 & 0.4260690911089969 & 0.9144459160840890\end{array}$ $\begin{array}{lllll}0.5445717668744394 & 0.3504339817093252 & 0.1830561534246168\end{array}$ $\begin{array}{lllll}0.5281941259868690 & 0.2412937788627701 & 0.1903750881846660\end{array}$ $\begin{array}{lllll}0.6535201473279906 & 0.2823434207262900 & 0.1916081442474669\end{array}$ $\begin{array}{llllll}0.6981929779808898 & 0.2078646463275951 & 0.0689508420876626\end{array}$ $\begin{array}{lllll}0.5327689168509779 & 0.2884502080948788 & 0.0651604943388020\end{array}$ $\begin{array}{lllll}0.5747394707535745 & 0.1867786505178888 & 0.0856272900364217\end{array}$ $\begin{array}{llll}0.6782394436450213 & 0.1681897503935656 & 0.1938368996416329\end{array}$ 
$\begin{array}{llll}0.6775607206919619 & 0.0553125419840959 & 0.1989197093896501\end{array}$ $\begin{array}{lllll}0.5447084638167282 & 0.1104394845071698 & 0.2135524581359264\end{array}$ $\begin{array}{lllll}0.6563455401076271 & 0.1053055974155667 & 0.0786652917157116\end{array}$ $\begin{array}{llll}0.6468780899153032 & 0.1147237844604186 & 0.3100284457357282\end{array}$ $\begin{array}{lllll}0.5186003420068558 & 0.0681448282735072 & 0.3298640951654522\end{array}$ $\begin{array}{lllll}0.6336413530264539 & 0.0665732657637766 & 0.4369701395721484\end{array}$ $\begin{array}{lllll}0.6026194965475793 & 0.1685776867966714 & 0.4184072360279823\end{array}$ $\begin{array}{lllll}0.5181910229328934 & 0.2889561232713253 & 0.3032764998409607\end{array}$ $\begin{array}{lllll}0.6431635423688121 & 0.2475218826886262 & 0.3025542194981277\end{array}$ $\begin{array}{llll}0.5980635590252603 & 0.3218936435299502 & 0.4247703731806650\end{array}$ $\begin{array}{llllll}0.5134190901552869 & 0.2423333862341846 & 0.4287684139411362\end{array}$ $\begin{array}{lllll}0.6183438652918923 & 0.3615007524585118 & 0.2999188541927059\end{array}$ $\begin{array}{llll}0.7269690066287505 & 0.1405849348729400 & 0.4362131047387794\end{array}$ $\begin{array}{lllll}0.7519804544360038 & 0.1792266731608710 & 0.3109478870145790\end{array}$ $\begin{array}{lllll}0.7218358624355153 & 0.3422722910674131 & 0.4086693075242901\end{array}$ $\begin{array}{lllll}0.7948141113959865 & 0.3499251300298062 & 0.1834049280103698\end{array}$ $\begin{array}{llllll}0.7782640089413914 & 0.2408591939522531 & 0.1906050467702584\end{array}$ $\begin{array}{lllll}0.9036935591221846 & 0.2820753523425580 & 0.1918012921894916\end{array}$ $\begin{array}{lllll}0.9483465963366090 & 0.2073803308305842 & 0.0690703524658745\end{array}$ $\begin{array}{llll}0.7822214115941759 & 0.2880023075715845 & 0.0651648891191355\end{array}$ $\begin{array}{lllll}0.8248506125487932 & 0.1864658854812236 & 0.0857597957720308\end{array}$ $\begin{array}{lllll}0.9283807823562099 & 0.1678167598841850 & 0.1939393932627889\end{array}$ $\begin{array}{lllll}0.9276863779697975 & 0.0549789533382528 & 0.1992300339972794\end{array}$ $\begin{array}{lllll}0.7948553318311034 & 0.1101650330398216 & 0.2137482438247785\end{array}$ $\begin{array}{lllll}0.9062459775516716 & 0.1048579939724924 & 0.0788129096377141\end{array}$ $\begin{array}{lllll}0.8971157524331018 & 0.1144824690911417 & 0.3101897424874183\end{array}$ $\begin{array}{lllll}0.7690635742596321 & 0.0677984227175928 & 0.3299217156704164\end{array}$ $\begin{array}{lllll}0.8863968556072119 & 0.0648757087441416 & 0.4372716512716109\end{array}$ $\begin{array}{lllll}0.8523779926384253 & 0.1664071795377285 & 0.4195113172477450\end{array}$ $\begin{array}{lllll}0.7686641511437954 & 0.2883226633380862 & 0.3037604742412527\end{array}$ $\begin{array}{lllll}0.8932405809235334 & 0.2468058443677767 & 0.3025754749840356\end{array}$ $\begin{array}{lllll}0.8485400584912020 & 0.3214863369198587 & 0.4254681552136454\end{array}$ $\begin{array}{lllll}0.7646627811982250 & 0.2410327537865360 & 0.4292943892880786\end{array}$ $\begin{array}{llll}0.8683984589006081 & 0.3611793092420478 & 0.3005339530736143\end{array}$ $\begin{array}{llll}0.9773201415210759 & 0.1405069919480838 & 0.4363762378889408\end{array}$ $\begin{array}{lllll}0.5019036783904586 & 0.1798472081626308 & 0.3106762340822323\end{array}$ $\begin{array}{llllll}0.9720148149975214 & 0.3424712157401711 & 0.4086247211868944\end{array}$ $\begin{array}{lllll}0.6493643085742947 & 0.4154039961977031 & 0.1841677821380273\end{array}$ $\begin{array}{lllll}0.5271847355360274 & 0.4620782590921757 & 0.1641513175960675\end{array}$ $\begin{array}{lllll}0.5694086352610062 & 0.3892225664975053 & 0.0579464047120865\end{array}$ $\begin{array}{lllll}0.6611819889829627 & 0.4646951474184664 & 0.0580206162347943\end{array}$ $\begin{array}{llll}0.6941048935648124 & 0.3625320002988507 & 0.0754561023334720\end{array}$ $\begin{array}{lllll}0.6188678791546388 & 0.4745640937922143 & 0.2953577428439606\end{array}$ $\begin{array}{lllll}0.0016691211866453 & 0.4189824770655534 & 0.2802122972629042\end{array}$ $\begin{array}{lllll}0.6414281315386003 & 0.4240023454717942 & 0.4152735322258846\end{array}$ $\begin{array}{lllll}0.8994484004283806 & 0.4146877739847976 & 0.1840136551989127\end{array}$ $\begin{array}{lllll}0.7776637928840250 & 0.4617685095082040 & 0.1644074275016585\end{array}$ $\begin{array}{lllll}0.8192299516104312 & 0.3886049998351684 & 0.0579853670641778\end{array}$ $\begin{array}{lllll}0.9113075754905933 & 0.4634931075461233 & 0.0572610963611050\end{array}$ $\begin{array}{llll}0.9439090283666626 & 0.3616189555357721 & 0.0750879130471193\end{array}$ $\begin{array}{lllll}0.8689684025727390 & 0.4740429556057288 & 0.2953626760498078\end{array}$ $\begin{array}{lllll}0.7514683225180995 & 0.4189734417792570 & 0.2800719851296407\end{array}$ $\begin{array}{lllll}0.8905159660988287 & 0.4240258358121299 & 0.4156998597829252\end{array}$ $\begin{array}{lllll}0.6391435060139694 & 0.5700929390212462 & 0.7449999669218202\end{array}$ $\begin{array}{llll}0.5466405991615418 & 0.3504912322412828 & 0.6830533447491844\end{array}$ $\begin{array}{lllll}0.5292126703185465 & 0.2424203691952950 & 0.6901703968569685\end{array}$ $\begin{array}{lllll}0.6549829849566908 & 0.2827171958984959 & 0.6912604307056744\end{array}$ $\begin{array}{lllll}0.6999070380093533 & 0.2084709606192414 & 0.5686436119546309\end{array}$ $\begin{array}{lllll}0.5297113715993219 & 0.2913976111740509 & 0.5641183925366640\end{array}$ $\begin{array}{llll}0.5758636620168863 & 0.1904272421969936 & 0.5840646040028511\end{array}$ $\begin{array}{llll}0.6785746518041477 & 0.1690888391519499 & 0.6933133761158179\end{array}$ $\begin{array}{llll}0.6782512567952630 & 0.0562244851108867 & 0.6976790411837418\end{array}$ $\begin{array}{llll}0.5457211929965882 & 0.1129254090589464 & 0.7134872852516004\end{array}$ $\begin{array}{lllll}0.6548835132685872 & 0.1068447761657238 & 0.5781775123799424\end{array}$ $\begin{array}{lllll}0.6481176724578058 & 0.1144549228011550 & 0.8097841847311804\end{array}$ $\begin{array}{lllll}0.5197727313865235 & 0.0699564206301001 & 0.8297349648363125\end{array}$ $\begin{array}{lllll}0.6359224679430598 & 0.0664148679172295 & 0.9372008568135363\end{array}$ $\begin{array}{llll}0.6039754464253888 & 0.1682474545803609 & 0.9181384252453569\end{array}$ $\begin{array}{lllll}0.5195462380255895 & 0.2908050596709874 & 0.8035749095099108\end{array}$ $\begin{array}{lllll}0.6441526965164013 & 0.2482695189800757 & 0.8023700590754165\end{array}$ $\begin{array}{lllll}0.5995147452226679 & 0.3245998745480684 & 0.9251360433735377\end{array}$ $\begin{array}{llll}0.5172126807209823 & 0.2432337174155979 & 0.9292177343415912\end{array}$ $\begin{array}{llll}0.6201840322892381 & 0.3628400298993678 & 0.7999752063736001\end{array}$ 
$\begin{array}{llll}0.7284487685181662 & 0.1408197402533359 & 0.9359016539412782\end{array}$ $\begin{array}{lllll}0.7524179180961446 & 0.1795290844551942 & 0.8105378847273519\end{array}$ $\begin{array}{lllll}0.7231762642478623 & 0.3437209891564629 & 0.9078206935110349\end{array}$ $\begin{array}{lllll}0.7967016163771710 & 0.3495467590258062 & 0.6839057117369858\end{array}$ $\begin{array}{lllll}0.7797344283169283 & 0.2408247546956014 & 0.6906289228199993\end{array}$ $\begin{array}{llll}0.9049425406265672 & 0.2818465966244972 & 0.6921581283615889\end{array}$ $\begin{array}{llllll}0.9493041909009714 & 0.2070181961363096 & 0.5691052983928965\end{array}$ $\begin{array}{lllll}0.7857441240515695 & 0.2876201625663248 & 0.5653722346774660\end{array}$ $\begin{array}{llll}0.8261166751585286 & 0.1855774374085976 & 0.5863319834918115\end{array}$ $\begin{array}{llllll}0.9299534136559479 & 0.1676606363374144 & 0.6942993862374217\end{array}$ $\begin{array}{lllll}0.9294270217539625 & 0.0547127833246728 & 0.7000361921485020\end{array}$ $\begin{array}{llll}0.7958705277081155 & 0.1107921809941663 & 0.7133701298789120\end{array}$ $\begin{array}{llll}0.9081511314489265 & 0.1043455365401294 & 0.5794644153675450\end{array}$ $\begin{array}{lllll}0.8984773889294908 & 0.1144762586791871 & 0.8101866062188204\end{array}$ $\begin{array}{lllll}0.7699725894423477 & 0.0678208876029950 & 0.8289597372615510\end{array}$ $\begin{array}{lllll}0.8854440129993066 & 0.0660570273916691 & 0.9369754633855073\end{array}$ $\begin{array}{llll}0.8533546099871230 & 0.1678823490365170 & 0.9183828951253058\end{array}$ $\begin{array}{lllll}0.7699992229340435 & 0.2881688062599566 & 0.8036778151016067\end{array}$ $\begin{array}{lllll}0.8945907315333121 & 0.2465595704855381 & 0.8030066593396850\end{array}$ $\begin{array}{llll}0.8494962172081540 & 0.3214450017565209 & 0.9253376859408690\end{array}$ $\begin{array}{lllll}0.7645955428734262 & 0.2417566648738815 & 0.9289348572107600\end{array}$ $\begin{array}{lllll}0.8701969037823152 & 0.3610336330830968 & 0.8007351032290175\end{array}$ $\begin{array}{lllll}0.9779722413312749 & 0.1408146789120471 & 0.9365741446164619\end{array}$ $\begin{array}{llll}0.5029880409463321 & 0.1815902580632980 & 0.8105659375495019\end{array}$ $\begin{array}{llll}0.9731285423448486 & 0.3426640834327326 & 0.9091277466309218\end{array}$ $\begin{array}{lllll}0.6513034050040322 & 0.4155616888979879 & 0.6847952962885542\end{array}$ $\begin{array}{lllll}0.5236925061047527 & 0.4541859105597493 & 0.6617450850541087\end{array}$ $\begin{array}{llll}0.5721953830341893 & 0.3897762624793284 & 0.5567968935532605\end{array}$ $\begin{array}{llll}0.6679518237335451 & 0.4614124828392938 & 0.5569000260546448\end{array}$ $\begin{array}{lllll}0.6955115273809255 & 0.3601718689628274 & 0.5766949406626208\end{array}$ $\begin{array}{lllll}0.6117266992529301 & 0.4760097729525418 & 0.7917158760845683\end{array}$ $\begin{array}{llll}0.0021949472786375 & 0.4203072188738553 & 0.7799994120014619\end{array}$ $\begin{array}{lllll}0.6446233931988625 & 0.4266684759383469 & 0.9148609752140210\end{array}$ $\begin{array}{lllll}0.9000284654225541 & 0.4146387519766772 & 0.6843247002193232\end{array}$ $\begin{array}{lllll}0.7797123929123938 & 0.4615090247476673 & 0.6641409797161471\end{array}$ $\begin{array}{lllll}0.8188968145348056 & 0.3899317382245346 & 0.5590421871474566\end{array}$ $\begin{array}{lllll}0.9115327958579688 & 0.4652355468368886 & 0.5584742968898185\end{array}$ $\begin{array}{lllll}0.9439282000221381 & 0.3633677534046040 & 0.5738656990752456\end{array}$ $\begin{array}{llll}0.8691902110912261 & 0.4743591406003563 & 0.7950446629147444\end{array}$ $\begin{array}{lllll}0.7541103971985379 & 0.4199781170351818 & 0.7822056468228767\end{array}$ $\begin{array}{lllll}0.8914023524216264 & 0.4241482217078830 & 0.9155541113850834\end{array}$ $\begin{array}{lllll}0.2113339099054685 & 0.23387777438338438 & 0.2485895628083078\end{array}$ $\begin{array}{lllll}0.0855308076384538 & 0.2951169400314731 & 0.2456299578783759\end{array}$ $\begin{array}{lllll}0.2208427084488442 & 0.1129659626456262 & 0.2641824047372890\end{array}$ $\begin{array}{lllll}0.4610017363577051 & 0.2344153760286607 & 0.2482118005075671\end{array}$ $\begin{array}{lllll}0.3352391208601603 & 0.2957333107983467 & 0.2451013112262104\end{array}$ $\begin{array}{llll}0.4702405967167322 & 0.1135518793256926 & 0.2639029430805010\end{array}$ $\begin{array}{lllll}0.0760781670469800 & 0.4160134823111671 & 0.2297813122449678\end{array}$ $\begin{array}{lllll}0.3255399978665495 & 0.4166163570717380 & 0.2291093213848190\end{array}$ $\begin{array}{lllll}0.2125310913563190 & 0.2355291009225845 & 0.7480500625988465\end{array}$ $\begin{array}{lllll}0.0860177962572323 & 0.2958160947257361 & 0.7452709203229040\end{array}$ $\begin{array}{lllll}0.2220594800920158 & 0.1146294058504823 & 0.7638907305624699\end{array}$ $\begin{array}{lllll}0.4621890097794741 & 0.2363382143971095 & 0.7480279705704188\end{array}$ $\begin{array}{lllll}0.3363948895900937 & 0.2979476943965884 & 0.7448260288674853\end{array}$ $\begin{array}{llll}0.4712966670796822 & 0.1153432438483687 & 0.7637314837022426\end{array}$ $\begin{array}{lllll}0.0761685719128165 & 0.4165929911668374 & 0.7293414695556930\end{array}$ $\begin{array}{lllll}0.3248493445288760 & 0.4194749138145047 & 0.7284467624413229\end{array}$ $\begin{array}{lllll}0.7110838807159383 & 0.2339522028061715 & 0.2485849899259999\end{array}$ $\begin{array}{lllll}0.5853136590234359 & 0.2956332326255918 & 0.2452726821785369\end{array}$ $\begin{array}{llll}0.7203059616177047 & 0.1129531510429023 & 0.2640077857072892\end{array}$ $\begin{array}{lllll}0.9613132473907749 & 0.2336350274553279 & 0.2486297268981245\end{array}$ $\begin{array}{lllll}0.8356439424291314 & 0.2952035004336904 & 0.2456001849507197\end{array}$ $\begin{array}{lllll}0.9705860854952870 & 0.1126812135477061 & 0.2641350753266685\end{array}$ $\begin{array}{lllll}0.5756805722457364 & 0.4167060759281056 & 0.2298139127651902\end{array}$ $\begin{array}{lllll}0.8261100702648322 & 0.4162851613960329 & 0.2301235323541228\end{array}$ $\begin{array}{lllll}0.7120474208603657 & 0.2344682559048348 & 0.7483710253009198\end{array}$ $\begin{array}{llll}0.5871958475969835 & 0.2969244908100622 & 0.7452363400298753\end{array}$ $\begin{array}{lllll}0.7211724002222831 & 0.1134053491455761 & 0.7633503064889648\end{array}$ $\begin{array}{lllll}0.9625853613604729 & 0.2335683583925818 & 0.7490622537775935\end{array}$ $\begin{array}{llll}0.8370172664055539 & 0.2949260224404627 & 0.7463110086806942\end{array}$ $\begin{array}{llll}0.9721677900337011 & 0.1127118302894623 & 0.7646026736626202\end{array}$ $\begin{array}{lllll}0.5768718323210158 & 0.4176568315309471 & 0.7309124660424671\end{array}$ $\begin{array}{llll}0.8273328785414006 & 0.4155127552055128 & 0.7312648681964429\end{array}$ 
$\begin{array}{llll}0.0215999531977931 & 0.2308491115060285 & 0.1022642111468548\end{array}$ $\begin{array}{lllll}0.1521357793123260 & 0.1678602458516980 & 0.1057344421047775\end{array}$ $\begin{array}{lllll}0.1448402818959126 & 0.3613220538813645 & 0.3883087062284393\end{array}$ $\begin{array}{lllll}0.1537408755191529 & 0.1206273810075740 & 0.3976662985363670\end{array}$ $\begin{array}{llll}0.0242832442488293 & 0.1802232137204673 & 0.3998564498189692\end{array}$ $\begin{array}{lllll}0.0255274890307212 & 0.2976477402315624 & 0.3921701011962464\end{array}$ $\begin{array}{lllll}0.0223990572528031 & 0.3481790185211103 & 0.0943478641131303\end{array}$ $\begin{array}{llllll}0.2713878035793928 & 0.2314482826331371 & 0.1019737938314124\end{array}$ $\begin{array}{lllll}0.4019062991635075 & 0.1684816198673656 & 0.1054369436615137\end{array}$ $\begin{array}{lllll}0.3940741936171669 & 0.3619699702766406 & 0.3874073647160375\end{array}$ $\begin{array}{lllll}0.4036681685277354 & 0.1213993447365632 & 0.3977560642675154\end{array}$ $\begin{array}{llll}0.2743047969687631 & 0.1808924321004913 & 0.3998949836230073\end{array}$ $\begin{array}{lllll}0.2752630644117101 & 0.2982295973178309 & 0.3918114908283230\end{array}$ $\begin{array}{lllll}0.2722422554594763 & 0.3488453179070573 & 0.0936625412306218\end{array}$ $\begin{array}{lllll}0.1428811516724739 & 0.4082422241555114 & 0.0958547698408333\end{array}$ $\begin{array}{lllll}0.3930582272621237 & 0.4087433621911310 & 0.0958681869612238\end{array}$ $\begin{array}{lllll}0.0221612509227228 & 0.2313180554258248 & 0.6021117603060872\end{array}$ $\begin{array}{lllll}0.1534434107412875 & 0.1694916445539491 & 0.6053903522142265\end{array}$ $\begin{array}{lllll}0.1449393165268153 & 0.3619769260231308 & 0.8879179415510823\end{array}$ $\begin{array}{lllll}0.1553023070663179 & 0.1216723818538491 & 0.8978259120628250\end{array}$ $\begin{array}{lllll}0.0253470161838389 & 0.1803521689397459 & 0.9002954987398327\end{array}$ $\begin{array}{llllll}0.0263980167361655 & 0.2978187515972286 & 0.8922024743283328\end{array}$ $\begin{array}{lllll}0.0221715430178002 & 0.3489213332516157 & 0.5939403120312833\end{array}$ $\begin{array}{lllll}0.2725122387064674 & 0.2333341597305236 & 0.6016172543867221\end{array}$ $\begin{array}{llllll}0.4027053543956544 & 0.1699202259484124 & 0.6054685738501141\end{array}$ $\begin{array}{lllll}0.3957514407034982 & 0.3631583846377597 & 0.8874765226358461\end{array}$ $\begin{array}{lllll}0.4047351042991361 & 0.1230083077143530 & 0.8975857926726851\end{array}$ $\begin{array}{lllll}0.2755065510359645 & 0.1824768446554735 & 0.8995147107829672\end{array}$ $\begin{array}{lllll}0.2759231588466770 & 0.2998649917601773 & 0.8912609702813989\end{array}$ $\begin{array}{lllll}0.2740853581646882 & 0.3508528019543479 & 0.5933893518426596\end{array}$ $\begin{array}{lllll}0.1433664411171171 & 0.4085762244443736 & 0.5954546798306384\end{array}$ $\begin{array}{lllll}0.3940969671790322 & 0.4143241661374787 & 0.5954244103448643\end{array}$ $\begin{array}{lllll}0.5214550355531374 & 0.2318326275733595 & 0.1019310664215036\end{array}$ $\begin{array}{llll}0.6516482746072935 & 0.1682122243449602 & 0.1057012568665219\end{array}$ $\begin{array}{lllll}0.6450394284251452 & 0.3608742005350581 & 0.3878345832146466\end{array}$ $\begin{array}{lllll}0.6526751422860602 & 0.1214061254985026 & 0.3974118051294960\end{array}$ $\begin{array}{lllll}0.5239851411609691 & 0.1816608975132847 & 0.3995294809509221\end{array}$ $\begin{array}{lllll}0.5245401984695202 & 0.29887755738402502 & 0.3916318139438996\end{array}$ $\begin{array}{lllll}0.5225418533193336 & 0.3492455243834439 & 0.0941653924716749\end{array}$ $\begin{array}{lllll}0.7714218696184734 & 0.2313451165635201 & 0.1021504738631984\end{array}$ $\begin{array}{lllll}0.9017539801117317 & 0.1678004897983926 & 0.1058297189212501\end{array}$ $\begin{array}{llll}0.8951225952059404 & 0.3609723011208965 & 0.3885858425396975\end{array}$ $\begin{array}{lllll}0.9033960132509811 & 0.1204209117654585 & 0.3976863943808276\end{array}$ $\begin{array}{lllll}0.7740856105012246 & 0.1802238917039048 & 0.3998046417494585\end{array}$ $\begin{array}{llll}0.7755006979573360 & 0.2976281908648603 & 0.3922017578901131\end{array}$ $\begin{array}{lllll}0.7724316785113940 & 0.34877707106823544 & 0.0945736413130172\end{array}$ $\begin{array}{lllll}0.6434340928635616 & 0.4090578821748486 & 0.0965854364098787\end{array}$ $\begin{array}{lllll}0.8934078554127335 & 0.4081378190792490 & 0.0965451556489843\end{array}$ $\begin{array}{lllll}0.5215899582083265 & 0.2343985958027204 & 0.6012898056801327\end{array}$ $\begin{array}{llllll}0.6518403501970845 & 0.1699708719444561 & 0.6051604484726001\end{array}$ $\begin{array}{lllll}0.6464782161797783 & 0.3638270065217457 & 0.8879823410423965\end{array}$ $\begin{array}{llll}0.6542064403142445 & 0.1213690964221708 & 0.8971444339554195\end{array}$ $\begin{array}{lllll}0.5256997510510796 & 0.1825558693632555 & 0.8992767900860980\end{array}$ $\begin{array}{lllll}0.5267366049293903 & 0.3002273156567477 & 0.8922921530247363\end{array}$ $\begin{array}{llllll}0.5230555313339645 & 0.3526996015910314 & 0.5947634733623979\end{array}$ $\begin{array}{lllll}0.7733337286779417 & 0.2311508271073593 & 0.6023553334559757\end{array}$ $\begin{array}{lllll}0.9031911716102798 & 0.1673361679490708 & 0.6062381978072093\end{array}$ $\begin{array}{lllll}0.8961909458339501 & 0.3611420601217122 & 0.8889038903319750\end{array}$ $\begin{array}{lllll}0.9039783196760157 & 0.1211552553572485 & 0.8974978899283617\end{array}$ $\begin{array}{llll}0.7747853530530415 & 0.1811069951666429 & 0.8993277362423333\end{array}$ $\begin{array}{llll}0.7759913807537392 & 0.2983744114979051 & 0.8918203724029709\end{array}$ $\begin{array}{llll}0.7742474905785663 & 0.3478731633303823 & 0.5952975471475412\end{array}$ $\begin{array}{llll}0.6458752646802676 & 0.4073797849670110 & 0.5983972963255395\end{array}$ $\begin{array}{llll}0.8934798253402474 & 0.4093833908091049 & 0.5968447966554187\end{array}$

Gua/Gua :

$\begin{array}{llllll}\mathrm{H} 138 & \mathrm{C} 10 & \mathrm{~N} 10 & \mathrm{O} 242 & \mathrm{Al} 32 & \mathrm{Si}\end{array}$

1.00000000000000

$20.2970019999999991 \quad 0.0000000000000000 \quad-3.7471719999999999$

$\begin{array}{llll}0.0646860000000000 & 24.9946149999999996 & -0.5148360000000000\end{array}$ $\begin{array}{llll}0.0000000000000000 & 0.0000000000000000 & 18.6939999999999991\end{array}$

$\mathrm{H} \quad \mathrm{C} \quad \mathrm{N} O \mathrm{Al} \mathrm{Si}$

$\begin{array}{llllll}138 & 10 & 10 & 242 & 32 & 64\end{array}$ 
Direct

0.26004839036922040 .75407850880645480 .5203112260182247 $\begin{array}{llllll}0.3893712281676008 & 0.5880732493283520 & 0.5175381591398234\end{array}$ $\begin{array}{llll}0.3582012867334842 & 0.5278108365425040 & 0.5370078488796159\end{array}$ $\begin{array}{llll}0.1393230210948570 & 0.7714219551869058 & 0.5337972513496093\end{array}$ $\begin{array}{lllll}0.2392742497569632 & 0.5263487571736165 & 0.5364145754866032\end{array}$ $\begin{array}{llll}0.0958568695618643 & 0.0555365913697412 & 0.4085005180625346\end{array}$ $\begin{array}{llll}0.3442193829614309 & 0.0564890231908803 & 0.4079569533335532\end{array}$ $\begin{array}{lllll}0.0534500319411955 & 0.1402322179520449 & 0.1819786484783587\end{array}$ $\begin{array}{llll}0.1721821930006416 & 0.3082326974703855 & 0.1599821676783248\end{array}$ $\begin{array}{lllll}0.1321671034007589 & 0.2210060226660240 & 0.3335063092738836\end{array}$ $\begin{array}{lllll}0.2456763822319228 & 0.0392771564950557 & 0.3427733952760335\end{array}$ $\begin{array}{lllll}0.1738179753786278 & 0.0672087257908942 & 0.1469020551509540\end{array}$ $\begin{array}{llll}0.3031256142948716 & 0.1398109282939938 & 0.1816600398559993\end{array}$ $\begin{array}{lllll}0.4218028254037416 & 0.3075903768160722 & 0.1592976803693256\end{array}$ $\begin{array}{lllll}0.3824874588028811 & 0.2213395567073551 & 0.3342548939109972\end{array}$ $\begin{array}{lllll}0.4958920159333619 & 0.0397885468336022 & 0.3437961209258786\end{array}$ $\begin{array}{lllll}0.4237271214178876 & 0.0671307261519853 & 0.1467597379896275\end{array}$ $\begin{array}{lllll}0.0578908069884528 & 0.4898959329315393 & 0.1504508943420777\end{array}$ $\begin{array}{lllll}0.2507689738146499 & 0.3900692286729678 & 0.3126036336848440\end{array}$ $\begin{array}{llllll}0.1300838450451655 & 0.4616029362869701 & 0.3464937186204561\end{array}$ $\begin{array}{lllll}0.3092121216768004 & 0.4894007207973370 & 0.1501193693163714\end{array}$ $\begin{array}{lllll}0.0010161221429287 & 0.3898290356553725 & 0.3121687634964684\end{array}$ $\begin{array}{lllll}0.3810957616836008 & 0.4628218488205153 & 0.3474037633432823\end{array}$ $\begin{array}{lllll}0.2090689746766270 & 0.4741456751302787 & 0.0868340956761920\end{array}$ $\begin{array}{lllll}0.4595909659028731 & 0.4731140632637991 & 0.0863403193403142\end{array}$ $\begin{array}{lllll}0.1493768407521033 & 0.4392950306248141 & 0.4647273193200975\end{array}$ $\begin{array}{lllll}0.4113562516594534 & 0.4365593958195969 & 0.4662181690035591\end{array}$ $\begin{array}{llll}0.1479066663080342 & 0.0905687756871903 & 0.0282804438657305\end{array}$ $\begin{array}{lllll}0.3987227169511955 & 0.0907284804771014 & 0.0279891755576785\end{array}$ $\begin{array}{lllll}0.1654130636605541 & 0.4827700906952091 & 0.2834479887308015\end{array}$ $\begin{array}{llll}0.4163955661027491 & 0.4821163387195693 & 0.2840187441180331\end{array}$ $\begin{array}{lllll}0.1381105573326178 & 0.0472867459701363 & 0.2098239418520090\end{array}$ $\begin{array}{lllll}0.3881801554535928 & 0.0470367027783282 & 0.2098143373003095\end{array}$ $\begin{array}{lllll}0.2134798538869305 & 0.4164425811154930 & 0.2377897558000752\end{array}$ $\begin{array}{lllll}0.4639730951327052 & 0.4161721383702606 & 0.2382455450127335\end{array}$ $\begin{array}{lllll}0.3406687260835819 & 0.1130886615205786 & 0.2558587665255243\end{array}$ $\begin{array}{lllll}0.0907134330023841 & 0.1130948662966940 & 0.2558342754120614\end{array}$ $\begin{array}{lllll}0.2580460716551395 & 0.7567414268266702 & 0.7403175134955586\end{array}$ $\begin{array}{lllll}0.4054808263372802 & 0.5998663463402986 & 0.7252960923157631\end{array}$ $\begin{array}{lllll}0.3723618500699775 & 0.5378031329322446 & 0.7352303231869241\end{array}$ $\begin{array}{lllll}0.1350028254344355 & 0.7623346196286898 & 0.7502560167111325\end{array}$ $\begin{array}{lllll}0.2605139699066996 & 0.5271347193452143 & 0.7105547226794584\end{array}$ $\begin{array}{lllll}0.0947061883425458 & 0.0548302196983257 & 0.9094548227580751\end{array}$ $\begin{array}{llll}0.3471355697145053 & 0.0544121828953144 & 0.9073242528553749\end{array}$ $\begin{array}{llll}0.0542953708175451 & 0.1402368226961352 & 0.6817505423721693\end{array}$ $\begin{array}{lllll}0.1754438649660873 & 0.3107550424300446 & 0.6656861551592884\end{array}$ $\begin{array}{lllll}0.1322982212383237 & 0.2210603437203077 & 0.8346449823538895\end{array}$ $\begin{array}{lllll}0.2467695220385787 & 0.0393514721234976 & 0.8437898370924556\end{array}$ $\begin{array}{llll}0.1741789335505599 & 0.0675822282152053 & 0.6454428728157915\end{array}$ 0.30301747281191890 .14172578070838890 .6829587716011033 $\begin{array}{llll}0.4264871769580119 & 0.3149030588994896 & 0.6746717886506314\end{array}$ $\begin{array}{lllll}0.3831231362445534 & 0.2201586789136287 & 0.8349882838507137\end{array}$ $\begin{array}{lllll}0.4963159785045165 & 0.0401895519667953 & 0.8424024388211873\end{array}$ $\begin{array}{lllll}0.4231801078481059 & 0.0690018466346953 & 0.6455117512591357\end{array}$ $\begin{array}{lllll}0.0501152006054116 & 0.49777777070626562 & 0.6648684617579238\end{array}$ $\begin{array}{llll}0.2512217353955034 & 0.3972902974811265 & 0.8171692490654199\end{array}$ $\begin{array}{lllll}0.1315517726352605 & 0.4656040111578563 & 0.8468020074686182\end{array}$ $\begin{array}{lllll}0.3149382825997411 & 0.4783215636493702 & 0.6378934893867698\end{array}$ $\begin{array}{llll}-0.0000833562117057 & 0.3758081880400435 & 0.7954061996795574\end{array}$ $\begin{array}{lllll}0.3862091026277048 & 0.4604536371908963 & 0.8517174024627279\end{array}$ $\begin{array}{lllll}0.1416909008879506 & 0.5028948789554250 & 0.5506583601587434\end{array}$ $\begin{array}{lllll}0.4602158241061413 & 0.4744238467442308 & 0.6005136215277704\end{array}$ $\begin{array}{lllll}0.1603360975447743 & 0.4389306478431614 & 0.9671393736255713\end{array}$ $\begin{array}{llll}0.4053732155508604 & 0.4367046179973433 & 0.9690663515864056\end{array}$ $\begin{array}{lllll}0.1482109986289263 & 0.0910987782351028 & 0.5276774664759513\end{array}$ $\begin{array}{lllll}0.3969213407747749 & 0.0921785732277992 & 0.5275452603097086\end{array}$ $\begin{array}{lllll}0.1336325051341633 & 0.5060037457078141 & 0.7751279353533492\end{array}$ $\begin{array}{lllll}0.4351014196078722 & 0.4646977616984168 & 0.7968641223712845\end{array}$ $\begin{array}{llllll}0.1393111718307787 & 0.0464577876709804 & 0.7085881388490076\end{array}$ $\begin{array}{lllll}0.3879081442089299 & 0.0486121948346475 & 0.7085660255471582\end{array}$ $\begin{array}{lllll}0.2171133386078399 & 0.4237509285613742 & 0.7411794161154444\end{array}$ $\begin{array}{llll}0.4640585334273101 & 0.4115469564671281 & 0.7358492821569430\end{array}$ 
$\begin{array}{llll}0.3410712745978902 & 0.1135995336918830 & 0.7555053446922098\end{array}$ $\begin{array}{lllll}0.0907760359691476 & 0.1121383143506299 & 0.7552918233949967\end{array}$ $\begin{array}{lllll}0.5963397491454304 & 0.0549382764435479 & 0.4091712513422653\end{array}$ $\begin{array}{llll}0.8461194943117583 & 0.0550446917710423 & 0.4095976569739646\end{array}$ $\begin{array}{llll}0.5534608162366829 & 0.1394925543198541 & 0.1814790232754282\end{array}$ $\begin{array}{lllll}0.6725626842151176 & 0.3082280847608519 & 0.1602333673448583\end{array}$ $\begin{array}{llll}0.6320680466735648 & 0.2204590035338843 & 0.3328516681604233\end{array}$ $\begin{array}{llll}0.7462598699382426 & 0.0391730202873525 & 0.3429077584752146\end{array}$ $\begin{array}{llll}0.6740406565055151 & 0.0670936278766459 & 0.1465585619404616\end{array}$ $\begin{array}{llllll}0.8036481445685391 & 0.1398038332930227 & 0.1818930386220571\end{array}$ $\begin{array}{lllll}0.9225190968942668 & 0.3083369932122069 & 0.1603973251312908\end{array}$ $\begin{array}{lllll}0.8823628020291093 & 0.2209407327049745 & 0.3337015996773919\end{array}$ $\begin{array}{lllll}0.9961801709868791 & 0.0393255713948339 & 0.3430213160457740\end{array}$ $\begin{array}{lllll}0.9240184985616888 & 0.0672962688501594 & 0.1464598981246999\end{array}$ $\begin{array}{lllll}0.5585041487265361 & 0.4894619770417656 & 0.1501796225339755\end{array}$ $\begin{array}{llll}0.7513256747061712 & 0.3888509709793363 & 0.3112088941824475\end{array}$ $\begin{array}{lllll}0.6311836717225172 & 0.4619188161735354 & 0.3468562613692724\end{array}$ $\begin{array}{lllll}0.8079695041058180 & 0.4895763306010166 & 0.1498113932050957\end{array}$ $\begin{array}{lllll}0.5017716714803634 & 0.3887200207047184 & 0.3116549682545828\end{array}$ $\begin{array}{lllll}0.8808965477764619 & 0.4619810249300446 & 0.3469448883206013\end{array}$ $\begin{array}{lllll}0.7085945582513166 & 0.4737379683782875 & 0.0842174974424007\end{array}$ $\begin{array}{llllll}0.9588070897190507 & 0.4738487805818372 & 0.0842589145222436\end{array}$ $\begin{array}{lllll}0.6573867195105789 & 0.4388827211243825 & 0.4651677764672729\end{array}$ $\begin{array}{lllll}0.9083944764433090 & 0.4388788346354931 & 0.4654565693352238\end{array}$ $\begin{array}{lllll}0.6491347613650261 & 0.0908644328424191 & 0.0277336309552324\end{array}$ $\begin{array}{lllll}0.8985758251046241 & 0.0909889493406904 & 0.0277346413991858\end{array}$ $\begin{array}{lllll}0.6660941242967944 & 0.4825814051554085 & 0.2836055069418748\end{array}$ $\begin{array}{lllll}0.9152132329893833 & 0.4834338753523553 & 0.2834912992873778\end{array}$ $\begin{array}{lllll}0.6384217831048926 & 0.0468203494447287 & 0.2094360007342267\end{array}$ $\begin{array}{lllll}0.8889332876526778 & 0.0465280229536136 & 0.2095561045092078\end{array}$ $\begin{array}{lllll}0.7140078943378955 & 0.4163677367384512 & 0.2377678792169774\end{array}$ $\begin{array}{lllll}0.9637865513897507 & 0.4165238165883379 & 0.2377871601819876\end{array}$ $\begin{array}{lllll}0.8408182176892521 & 0.1126288490413722 & 0.2558575777848602\end{array}$ $\begin{array}{lllll}0.5907250425459508 & 0.1127875472472369 & 0.2558170467207512\end{array}$ $\begin{array}{llll}0.5971058628639152 & 0.0551360706710325 & 0.9085476850232382\end{array}$ $\begin{array}{lllll}0.8463398676757374 & 0.0565145270891702 & 0.9082480818390380\end{array}$ $\begin{array}{lllll}0.5534630331908029 & 0.1400428892513132 & 0.6798171821112535\end{array}$ $\begin{array}{lllll}0.6725578915265342 & 0.3091150352854586 & 0.6594191916047913\end{array}$ $\begin{array}{lllll}0.6324084245744594 & 0.2217840989427271 & 0.8327481597103686\end{array}$ $\begin{array}{lllll}0.7468261314791247 & 0.0400347946905392 & 0.8422712359089642\end{array}$ $\begin{array}{lllll}0.6744187693475248 & 0.0681699040927673 & 0.6462981992036341\end{array}$ $\begin{array}{lllll}0.8037240255643633 & 0.1407202707169599 & 0.6810722427147086\end{array}$ $\begin{array}{lllll}0.9215542748858231 & 0.3075582247389543 & 0.6564550741013657\end{array}$ $\begin{array}{lllll}0.8827312307800387 & 0.2217037693710924 & 0.8324558737879809\end{array}$ $\begin{array}{llllll}0.9949252242019165 & 0.0409248056318501 & 0.8430390880654690\end{array}$ $\begin{array}{lllll}0.9237470223817866 & 0.0681384328681091 & 0.6467683395632366\end{array}$ $\begin{array}{lllll}0.5597793934425123 & 0.4883881537230978 & 0.6460262501928833\end{array}$ $\begin{array}{lllll}0.7520886619346121 & 0.3896964319884997 & 0.8117856636644332\end{array}$ $\begin{array}{llll}0.6312754441262503 & 0.4625683426223606 & 0.8470876226636443\end{array}$ $\begin{array}{lllll}0.8079674717106234 & 0.4900085389091389 & 0.6492128058901939\end{array}$ $\begin{array}{lllll}0.5047800216150075 & 0.3901637846583668 & 0.8140435946529471\end{array}$ $\begin{array}{llll}0.8822212106974697 & 0.4624698135082955 & 0.8469623572970150\end{array}$ $\begin{array}{lllll}0.7094962919312469 & 0.4755038596335889 & 0.5854334220337136\end{array}$ $\begin{array}{lllll}0.9635006693477443 & 0.4720294226700718 & 0.5828645058115218\end{array}$ $\begin{array}{lllll}0.6558882126474700 & 0.4390579811167326 & 0.9651949392301931\end{array}$ $\begin{array}{llll}0.9070250709329443 & 0.4391727757831152 & 0.9653333866498135\end{array}$ $\begin{array}{lllll}0.6487832812153189 & 0.0914782560665391 & 0.5279747954732670\end{array}$ $\begin{array}{lllll}0.8985409050233866 & 0.0914910565809930 & 0.5282368363926786\end{array}$ $\begin{array}{lllll}0.6655129588803577 & 0.4842250761658254 & 0.7835936558298687\end{array}$ $\begin{array}{lllll}0.9153828905034285 & 0.4843182780128798 & 0.7827173089002349\end{array}$ $\begin{array}{lllll}0.6392345610863043 & 0.0475400072217356 & 0.7093966880316871\end{array}$ $\begin{array}{lllll}0.8880184852418008 & 0.0481832924176196 & 0.7097032433665892\end{array}$ $\begin{array}{llllll}0.7147417826687776 & 0.4173842671021864 & 0.7387256972451101\end{array}$ $\begin{array}{llll}0.9642726800016539 & 0.4157065615721837 & 0.7368230202814985\end{array}$ $\begin{array}{llll}0.8411092158674405 & 0.1143656905089959 & 0.7556756015442794\end{array}$ $\begin{array}{llll}0.5907466613146612 & 0.1138060101730942 & 0.7546238075424199\end{array}$ $\begin{array}{lllll}0.1617770731666109 & 0.7319604056703479 & 0.5336970699307544\end{array}$ $\begin{array}{llll}0.2947165449271123 & 0.5944242142580688 & 0.5255356862806684\end{array}$ $\begin{array}{lllll}0.2395941381761263 & 0.6712425433788743 & 0.5279944667419443\end{array}$ 0.17975423838687420 .64789448793049310 .5364162665759552 $\begin{array}{lllll}0.1773521665132029 & 0.5911190190216238 & 0.5402059961279906\end{array}$ $\begin{array}{lllll}0.1614516580973087 & 0.7251653379953164 & 0.7447432286695590\end{array}$ $\begin{array}{llll}0.3078986363662235 & 0.6005885774472015 & 0.7225532208307691\end{array}$ 
$0.2455375034570556 \quad 0.6721989677232354 \quad 0.7327057182623140$ $\begin{array}{lllll}0.1880171764681458 & 0.6431201224468637 & 0.7350001420461246\end{array}$ $\begin{array}{lllll}0.1911455061021600 & 0.5862055441762848 & 0.7292060203129286\end{array}$ $\begin{array}{lllll}0.3485977392731484 & 0.5652897333746479 & 0.5164809056799601\end{array}$ $\begin{array}{llll}0.2971816557460878 & 0.6472829942250328 & 0.5224748262656957\end{array}$ $\begin{array}{llll}0.2271916125759832 & 0.7252645811202635 & 0.5266438899662625\end{array}$ $\begin{array}{lllll}0.1319140105440899 & 0.6864724910641841 & 0.5397233418128505\end{array}$ $\begin{array}{lllll}0.2387583282543539 & 0.5672930706636982 & 0.5344949592178473\end{array}$ $\begin{array}{lllll}0.3654965397184745 & 0.5755047373495145 & 0.7150220597810546\end{array}$ $0.30604952335262680 .6535478616207863 \quad 0.7275653084467270$ $\begin{array}{lllll}0.2275404110249589 & 0.7248545866629139 & 0.7386468115806365\end{array}$ $\begin{array}{lllll}0.1362887649838667 & 0.6769320904884871 & 0.7428227611641811\end{array}$ $\begin{array}{lllll}0.2543294082567092 & 0.5679992051315942 & 0.7220192064713084\end{array}$ $\begin{array}{lllll}0.1295335593273517 & 0.5625128164923192 & 0.5475436378099386\end{array}$ $\begin{array}{lllll}0.0482133005389147 & 0.3498719225717405 & 0.1827378393038042\end{array}$ $\begin{array}{lllll}0.0322252796235393 & 0.2406978961889650 & 0.1903096453340763\end{array}$ $\begin{array}{lllll}0.1574620223357662 & 0.2820944681306458 & 0.1912463377306447\end{array}$ $\begin{array}{lllll}0.2022328174555237 & 0.2074942360214432 & 0.0688153891451902\end{array}$ $\begin{array}{llll}0.0362151240951989 & 0.2878201361628323 & 0.0647883998918424\end{array}$ $\begin{array}{lllll}0.0787603815498321 & 0.1862661283150306 & 0.0854904525831340\end{array}$ $\begin{array}{lllll}0.1823654915205379 & 0.1679733911876830 & 0.1938050566138067\end{array}$ $\begin{array}{lllll}0.1817660960763585 & 0.0551481095165956 & 0.1994103794645637\end{array}$ $\begin{array}{lllll}0.0492838749877223 & 0.1103113996527563 & 0.2136484154244456\end{array}$ $\begin{array}{lllll}0.1607577342544007 & 0.1049029050814950 & 0.0789101231367414\end{array}$ $\begin{array}{lllll}0.1513444626360414 & 0.1148574624614468 & 0.3102379683976562\end{array}$ $\begin{array}{lllll}0.0236005831329762 & 0.0676006816394781 & 0.3298740016233352\end{array}$ $\begin{array}{lllll}0.1400919424032546 & 0.0655664706796327 & 0.4370114198618678\end{array}$ $\begin{array}{lllll}0.1067387948278771 & 0.1672064771461884 & 0.4193315880700502\end{array}$ $\begin{array}{lllll}0.0224181693301311 & 0.2881300848420316 & 0.3034058366881885\end{array}$ $\begin{array}{llllll}0.1470540682804977 & 0.2469666352068064 & 0.3021466961138093\end{array}$ $\begin{array}{lllll}0.1027245711429897 & 0.3212919221603467 & 0.4246780818695813\end{array}$ $\begin{array}{lllll}0.0181774778548718 & 0.2414008128799927 & 0.4289269648717083\end{array}$ $\begin{array}{lllll}0.1220548893647112 & 0.3608503474690880 & 0.2993244210544312\end{array}$ $\begin{array}{lllll}0.2316942009403619 & 0.1409648665411627 & 0.4363835991931723\end{array}$ $\begin{array}{lllll}0.2560614585349874 & 0.1793686228464146 & 0.3109725045863401\end{array}$ $\begin{array}{lllll}0.2256375594236126 & 0.3437023806291367 & 0.4074947733069851\end{array}$ $\begin{array}{lllll}0.2985486708683932 & 0.3498415972962571 & 0.1833303650527173\end{array}$ $\begin{array}{lllll}0.2822579012865025 & 0.2406876141755097 & 0.1904750811903571\end{array}$ $\begin{array}{lllll}0.4074710071170713 & 0.2821071519344471 & 0.1916895937468819\end{array}$ $\begin{array}{lllll}0.4522211474134674 & 0.2076814962019317 & 0.0688245756587920\end{array}$ $\begin{array}{lllll}0.2864256598053013 & 0.2877256177383723 & 0.0651495109438690\end{array}$ $\begin{array}{lllll}0.3288335722589897 & 0.1861234677580433 & 0.0857167823714603\end{array}$ $\begin{array}{lllll}0.4324929101379136 & 0.1679478245820108 & 0.1937470525287245\end{array}$ $\begin{array}{lllll}0.4317119577335063 & 0.0551163864302667 & 0.1992413575010187\end{array}$ $\begin{array}{lllll}0.2990527930792301 & 0.1101731280740900 & 0.2138077724910879\end{array}$ $\begin{array}{lllll}0.4110435061962809 & 0.1049439190807348 & 0.0786729680372780\end{array}$ $\begin{array}{lllll}0.4011963865018566 & 0.1148286057719102 & 0.3100831525194252\end{array}$ $\begin{array}{lllll}0.2731808821856295 & 0.0676710342062846 & 0.3301008095815486\end{array}$ $\begin{array}{lllll}0.3885274531311535 & 0.0661511345043447 & 0.4368848012361315\end{array}$ $\begin{array}{lllll}0.3567265325180961 & 0.1679563383188308 & 0.4188806832697490\end{array}$ $\begin{array}{lllll}0.2724808670413529 & 0.2882654825608564 & 0.3036078216223874\end{array}$ $\begin{array}{lllll}0.3972897636617729 & 0.2471542848861333 & 0.3026048792635079\end{array}$ $\begin{array}{lllll}0.3521123618512177 & 0.3215830745654670 & 0.4254410310301353\end{array}$ $\begin{array}{lllll}0.2677663164358828 & 0.2418209879529592 & 0.4292924997341727\end{array}$ $\begin{array}{lllll}0.3720518497887028 & 0.3613417959376695 & 0.3006454724582554\end{array}$ $\begin{array}{lllll}0.4813216998779188 & 0.1407514785857688 & 0.4362640804977772\end{array}$ $\begin{array}{llll}0.0061995280141185 & 0.1791049469856437 & 0.3108681724627019\end{array}$ $\begin{array}{lllll}0.4759037756401276 & 0.3424900970798209 & 0.4083962772711829\end{array}$ $\begin{array}{lllll}0.1534152675847685 & 0.4143573818897550 & 0.1838641504470594\end{array}$ $\begin{array}{lllll}0.0310354732217799 & 0.4609951036663391 & 0.1629598863344604\end{array}$ $\begin{array}{lllll}0.0731549145511271 & 0.3884346941092141 & 0.0574671641442054\end{array}$ $\begin{array}{lllll}0.1651292759175109 & 0.4640158202071942 & 0.0578189138977118\end{array}$ $\begin{array}{lllll}0.1979216849921477 & 0.3620279311186524 & 0.0750094405615729\end{array}$ $\begin{array}{llll}0.1220281880870377 & 0.4741847543311765 & 0.2939420708865695\end{array}$ $\begin{array}{lllll}0.0053295683567567 & 0.4192244969606889 & 0.2797479124331085\end{array}$ $\begin{array}{llll}0.1424515485823081 & 0.4244776248007247 & 0.4131041494340579\end{array}$ $\begin{array}{lllll}0.4034422661115371 & 0.4143887022623239 & 0.1846274420862860\end{array}$ $\begin{array}{lllll}0.2817228042931596 & 0.4615213780724637 & 0.1641301621512212\end{array}$ $\begin{array}{lllll}0.3232687685265727 & 0.3883205127101022 & 0.0581808461310074\end{array}$ $\begin{array}{lllll}0.4151491230827770 & 0.4633831879581426 & 0.0578517654052783\end{array}$ $\begin{array}{lllll}0.4478524203772246 & 0.3616777778165391 & 0.0754210332351763\end{array}$ $\begin{array}{lllll}0.3729336266471440 & 0.4743452051022902 & 0.2950317162588925\end{array}$ $\begin{array}{lllll}0.2552538150884590 & 0.4193474359272883 & 0.2800308950088797\end{array}$ 
$\begin{array}{llll}0.3944664184069314 & 0.4238845801322713 & 0.4162678107062980\end{array}$ $\begin{array}{lllll}0.1453754480172486 & 0.5536865336958996 & 0.7292021701671209\end{array}$ $\begin{array}{llllll}0.0524272255104964 & 0.3505160157782071 & 0.6832863836366407\end{array}$ $\begin{array}{llll}0.0330390263686780 & 0.2418922424891595 & 0.6908384617132773\end{array}$ $\begin{array}{lllll}0.1590689864255211 & 0.2822693706570221 & 0.6921066663222696\end{array}$ $\begin{array}{lllll}0.2020406072650293 & 0.2081343557191293 & 0.5689068031961321\end{array}$ $\begin{array}{lllll}0.0377915877885871 & 0.2879455568306133 & 0.5648730851284404\end{array}$ $\begin{array}{lllll}0.0787848493921627 & 0.1861515160312441 & 0.5863631699831917\end{array}$ $\begin{array}{lllll}0.1826550072804984 & 0.1678141529149076 & 0.6938317890743090\end{array}$ $\begin{array}{lllll}0.1826576045121799 & 0.0547700371239281 & 0.6977017796372847\end{array}$ $\begin{array}{lllll}0.0491992912763887 & 0.1104965521255028 & 0.7133865991565527\end{array}$ $\begin{array}{lllll}0.1611181475235431 & 0.1053541843401220 & 0.5783708799042496\end{array}$ $\begin{array}{lllll}0.1518924760476388 & 0.1124241956027197 & 0.8097600745051390\end{array}$ $\begin{array}{llll}0.0227435483257892 & 0.0689345824711304 & 0.8299400175433591\end{array}$ $\begin{array}{lllll}0.1391735724012340 & 0.0645619481257574 & 0.9373599421675615\end{array}$ $\begin{array}{lllll}0.1075343449554207 & 0.1664788881967396 & 0.9179547914504613\end{array}$ $\begin{array}{lllll}0.0229941309955318 & 0.2906355207707104 & 0.8037666377677126\end{array}$ $\begin{array}{lllll}0.1471342282050798 & 0.2465134688184287 & 0.8025620996497568\end{array}$ $\begin{array}{llll}0.1033381254398150 & 0.3236299068697360 & 0.9252432699810996\end{array}$ $\begin{array}{llll}0.0215609678506126 & 0.2420520203541375 & 0.9290860161623566\end{array}$ $\begin{array}{lllll}0.1256296051504790 & 0.3617000512136172 & 0.8003000820417645\end{array}$ $\begin{array}{llllll}0.2318763672917797 & 0.1384467703516764 & 0.9359685109821188\end{array}$ $\begin{array}{llllll}0.2559342164871634 & 0.1781350148980167 & 0.8110581901920765\end{array}$ $\begin{array}{llll}0.2273757991952099 & 0.3420941319728765 & 0.9094676795423304\end{array}$ $\begin{array}{llllll}0.2976104711698544 & 0.3503718987539087 & 0.6852282654260538\end{array}$ $\begin{array}{lllll}0.2828029579858629 & 0.2405259421169264 & 0.6905059710850465\end{array}$ $\begin{array}{llll}0.4074804734676184 & 0.2835271292150422 & 0.6925997284740053\end{array}$ $\begin{array}{lllll}0.4527523958464215 & 0.2089749259678753 & 0.5680835443489544\end{array}$ $\begin{array}{lllll}0.2832650008449193 & 0.2901717166499417 & 0.5654951176515135\end{array}$ $\begin{array}{llll}0.3291296454454652 & 0.1891040402880459 & 0.5837773476779078\end{array}$ $\begin{array}{lllll}0.4321763275426726 & 0.1694487705880298 & 0.6929407229786109\end{array}$ $\begin{array}{llll}0.4314764815042448 & 0.0563841118246182 & 0.6978354112696261\end{array}$ $\begin{array}{lllll}0.2995936834987085 & 0.1108061304627875 & 0.7131720078009086\end{array}$ $\begin{array}{lllll}0.4099805820013609 & 0.1067012254579434 & 0.5780776520654199\end{array}$ $\begin{array}{lllll}0.4015539411021841 & 0.1154618276566150 & 0.8094201094416741\end{array}$ $\begin{array}{lllll}0.2745838207679013 & 0.0664713548635149 & 0.8285159593771744\end{array}$ $\begin{array}{llll}0.3911021218685744 & 0.0649384915327420 & 0.9359288823174515\end{array}$ $\begin{array}{lllll}0.3566169120484339 & 0.1664465613119146 & 0.9193696783625148\end{array}$ $\begin{array}{lllll}0.2728139623651158 & 0.2865374796663025 & 0.8046622629839352\end{array}$ $\begin{array}{lllll}0.3972046492271791 & 0.2460295064727126 & 0.8029331106397918\end{array}$ $\begin{array}{lllll}0.3534026559175012 & 0.3179001388151006 & 0.9267775153093877\end{array}$ $\begin{array}{lllll}0.2669133738536855 & 0.2396750093276456 & 0.9296519749135388\end{array}$ $\begin{array}{lllll}0.3719883635014866 & 0.3595977516809644 & 0.8029221522901275\end{array}$ $\begin{array}{lllll}0.4816758850336693 & 0.1410882083943372 & 0.9356200961149792\end{array}$ $\begin{array}{llll}0.0068662177810845 & 0.1804952040933400 & 0.8103931404381366\end{array}$ $\begin{array}{lllll}0.4762506227677183 & 0.3427980567584184 & 0.9097836008429119\end{array}$ $\begin{array}{lllll}0.1546401385878576 & 0.4198263984990641 & 0.6830996785000030\end{array}$ $\begin{array}{lllll}0.0308149798471718 & 0.4621800778083722 & 0.6626673302882998\end{array}$ $\begin{array}{lllll}0.0733177011119761 & 0.3889950057920211 & 0.5581729503202219\end{array}$ $\begin{array}{llllll}0.1596972570810508 & 0.4645293801577847 & 0.5464346319735328\end{array}$ $\begin{array}{llll}0.1988093906258993 & 0.3681970176355521 & 0.5742815799927313\end{array}$ $\begin{array}{lllll}0.1133848485375001 & 0.4740052201225955 & 0.7952280786047443\end{array}$ $\begin{array}{lllll}0.0046949466234338 & 0.4121596155358248 & 0.7785840780606486\end{array}$ $\begin{array}{llll}0.1497906981205437 & 0.4252167463425805 & 0.9161484396153832\end{array}$ $\begin{array}{lllll}0.4074691737135362 & 0.4063921535781538 & 0.6861128612565944\end{array}$ $\begin{array}{lllll}0.2926005533963341 & 0.4658141405883727 & 0.6756620320931662\end{array}$ $\begin{array}{lllll}0.3239658779274582 & 0.3904060619388379 & 0.5609979260711295\end{array}$ $\begin{array}{lllll}0.4156254119441891 & 0.4663855813368546 & 0.5685484486613697\end{array}$ $\begin{array}{lllll}0.4497606791136118 & 0.3643048259538264 & 0.5687761858421649\end{array}$ $\begin{array}{lllll}0.3879638903402738 & 0.4697902767110549 & 0.7994385498098342\end{array}$ $\begin{array}{lllll}0.2591937251123203 & 0.4237334603033666 & 0.7821123677178344\end{array}$ $\begin{array}{llll}0.3914579620050042 & 0.4218275631880284 & 0.9187484303605610\end{array}$ $\begin{array}{llll}0.5487177327005893 & 0.3498487904189092 & 0.1830960918516887\end{array}$ $\begin{array}{lllll}0.5322545931237631 & 0.2407928637884859 & 0.1904015087567908\end{array}$ $\begin{array}{lllll}0.6576172639270116 & 0.2821351445993265 & 0.1913980495947064\end{array}$ $\begin{array}{lllll}0.7021540630452187 & 0.2077680205834582 & 0.0687920574721100\end{array}$ $\begin{array}{lllll}0.5364409296288304 & 0.2876608582663526 & 0.0648432251923567\end{array}$ $\begin{array}{lllll}0.5788462046822133 & 0.1860670501075567 & 0.0857017869241956\end{array}$ $\begin{array}{llllll}0.6825378359215498 & 0.1679208765538610 & 0.1937147875577428\end{array}$ $\begin{array}{lllll}0.6819749395178880 & 0.0550661739809727 & 0.1990544479697114\end{array}$ $\begin{array}{llll}0.5491384852459145 & 0.1099667567001083 & 0.2137155161874398\end{array}$ $\begin{array}{lllll}0.6610920763000430 & 0.1049680827903719 & 0.0785732922574546\end{array}$ $\begin{array}{lllll}0.6513038801094154 & 0.1146956418044288 & 0.3100446240630582\end{array}$ 
$\begin{array}{llll}0.5233101687572013 & 0.0678219134870512 & 0.3300325615419747\end{array}$ $\begin{array}{lllll}0.6405646089504915 & 0.0652391971867866 & 0.4371899583300624\end{array}$ $\begin{array}{lllll}0.6066540734447803 & 0.1666931103748002 & 0.4193631262115711\end{array}$ $\begin{array}{llll}0.5225843786717111 & 0.2883558747110183 & 0.3034533990056935\end{array}$ $\begin{array}{llll}0.6471729407752059 & 0.2468557396098600 & 0.3022404614074252\end{array}$ $\begin{array}{lllll}0.6025879365074508 & 0.3216637100088438 & 0.4249457505344698\end{array}$ $\begin{array}{lllll}0.5187514658213170 & 0.2412388083927276 & 0.4290520219482518\end{array}$ $\begin{array}{lllll}0.6225684930136359 & 0.3611288048139371 & 0.2999795619688926\end{array}$ $\begin{array}{lllll}0.7315686844925610 & 0.1408692995903162 & 0.4362746839393707\end{array}$ $\begin{array}{lllll}0.7562361088124434 & 0.1792151257045906 & 0.3108377430709309\end{array}$ $\begin{array}{lllll}0.7260429806686810 & 0.3428271880012528 & 0.4084892323496279\end{array}$ $\begin{array}{lllll}0.7984058333041153 & 0.3499384082114020 & 0.1828956169772473\end{array}$ $\begin{array}{llll}0.7822731457401925 & 0.2408581807431293 & 0.1904169838286241\end{array}$ $\begin{array}{lllll}0.9075037281046786 & 0.2821530919507399 & 0.1913923954393676\end{array}$ $\begin{array}{lllll}0.9521273617063140 & 0.2076831625344499 & 0.0687811889540263\end{array}$ $\begin{array}{lllll}0.7862461013713874 & 0.2878171490050621 & 0.0648275819817964\end{array}$ $\begin{array}{llllll}0.8287621647360247 & 0.1862616857527802 & 0.0856809802691857\end{array}$ $\begin{array}{lllll}0.9323831472933214 & 0.1679327549841028 & 0.1937881758135960\end{array}$ $\begin{array}{llll}0.9322922016091613 & 0.0550975960936472 & 0.1989193436363953\end{array}$ $\begin{array}{lllll}0.7992601581438157 & 0.1100537720873268 & 0.2137482737948784\end{array}$ $\begin{array}{lllll}0.9107375833313974 & 0.1050346392099583 & 0.0786335556821918\end{array}$ $\begin{array}{llllll}0.9014551646864978 & 0.1143197449619594 & 0.3101651852507845\end{array}$ $\begin{array}{lllll}0.7733253236952956 & 0.0678048030415667 & 0.3300673755619264\end{array}$ $\begin{array}{llll}0.8903722067265335 & 0.0653547862473753 & 0.4375162324740917\end{array}$ $\begin{array}{lllll}0.8568044636551176 & 0.1668974048066516 & 0.4191416408878839\end{array}$ $\begin{array}{lllll}0.7724168377810835 & 0.2884257308477373 & 0.3035022566609785\end{array}$ $\begin{array}{lllll}0.8970818471776607 & 0.2468880934527165 & 0.3022036305673648\end{array}$ $\begin{array}{lllll}0.8528003498267474 & 0.3216243319191237 & 0.4247993479505632\end{array}$ $\begin{array}{llll}0.7687769676598625 & 0.2413941460834073 & 0.4290851849794077\end{array}$ $\begin{array}{lllll}0.8722605561612639 & 0.3611840580868601 & 0.2998133315239828\end{array}$ $\begin{array}{lllll}0.9816521459864584 & 0.1406645988850640 & 0.4362863634500319\end{array}$ $\begin{array}{lllll}0.5062296396893224 & 0.1792148958992363 & 0.3108483878357129\end{array}$ $\begin{array}{llll}0.9760263952276079 & 0.3429614716917412 & 0.4079699955024411\end{array}$ $\begin{array}{llll}0.6531147763670135 & 0.4147461984669523 & 0.1833063363453451\end{array}$ $\begin{array}{lllll}0.5310643537498099 & 0.4613607490540144 & 0.1637540477755161\end{array}$ $\begin{array}{lllll}0.5728276896981336 & 0.3883379147372517 & 0.0573906089462093\end{array}$ $\begin{array}{llllll}0.6643396454792541 & 0.4635413722691711 & 0.0560019227895527\end{array}$ $\begin{array}{lllll}0.6977788304165771 & 0.3619644476852713 & 0.0745210986695770\end{array}$ $\begin{array}{llll}0.6227575723993859 & 0.4741029237130741 & 0.2944031584406096\end{array}$ $\begin{array}{llll}0.5057492788029323 & 0.4188474276627665 & 0.2802790084383876\end{array}$ $\begin{array}{lllll}0.6440056704105495 & 0.4242781674514369 & 0.4147881994878084\end{array}$ $\begin{array}{llll}0.9030089423623958 & 0.4147812226525990 & 0.1832766005541085\end{array}$ $\begin{array}{lllll}0.7807550610370764 & 0.4612844349815564 & 0.1633014197010517\end{array}$ $\begin{array}{lllll}0.8228754324928026 & 0.3884952012324635 & 0.0572708028194889\end{array}$ $\begin{array}{lllll}0.9144666293058846 & 0.4637898185231014 & 0.0561753119113848\end{array}$ $\begin{array}{lllll}0.9477623725280550 & 0.3621146443894520 & 0.0745421338503211\end{array}$ $\begin{array}{llll}0.8721738552038409 & 0.4741562956263093 & 0.2944087401818351\end{array}$ $\begin{array}{lllll}0.7554110125096196 & 0.4189599915047714 & 0.2798455803140806\end{array}$ $\begin{array}{llll}0.8943147802899108 & 0.4243486687382533 & 0.4147027787004837\end{array}$ $\begin{array}{llll}0.5473771839464189 & 0.3513474091222337 & 0.6817822737258814\end{array}$ $\begin{array}{llll}0.5320963857476525 & 0.2421760623375445 & 0.6900670584079632\end{array}$ $\begin{array}{lllll}0.6574911551805894 & 0.2833864753799599 & 0.6909911500399758\end{array}$ $\begin{array}{lllll}0.7023873451969378 & 0.2089428832192496 & 0.5685091963427751\end{array}$ $\begin{array}{lllll}0.5380069083349741 & 0.2885858963126357 & 0.5644828638038677\end{array}$ $\begin{array}{llll}0.5790930304226795 & 0.1869349246707730 & 0.5859445556651952\end{array}$ $\begin{array}{lllll}0.6829224178640984 & 0.1690527517278142 & 0.6934352130805980\end{array}$ $\begin{array}{lllll}0.6826311071056761 & 0.0559674910403528 & 0.6987379970908885\end{array}$ $\begin{array}{lllll}0.5491934133552081 & 0.1108600776570239 & 0.7126189344426923\end{array}$ $\begin{array}{lllll}0.6614393326453724 & 0.1060137801658707 & 0.5785179024312430\end{array}$ $\begin{array}{lllll}0.6515276867378129 & 0.1154577368280977 & 0.8093518028973912\end{array}$ $\begin{array}{lllll}0.5235377954749071 & 0.0684318386979840 & 0.8288172161734652\end{array}$ $\begin{array}{lllll}0.6412465210008466 & 0.0656239319095767 & 0.9364165694971759\end{array}$ $\begin{array}{lllll}0.6069287045300387 & 0.1670425223815478 & 0.9189902047607406\end{array}$ $\begin{array}{lllll}0.5221001293862143 & 0.2893930776639079 & 0.8032440470812222\end{array}$ $\begin{array}{llll}0.6471182170356342 & 0.2480614194177224 & 0.8016907016501416\end{array}$ $\begin{array}{llll}0.6027457074225189 & 0.3220945445763940 & 0.9242966945460676\end{array}$ $\begin{array}{llll}0.5191397660061179 & 0.2414877591298424 & 0.9286209745832215\end{array}$ $\begin{array}{llll}0.6218677869175494 & 0.3620628657889998 & 0.7991240797271340\end{array}$ $\begin{array}{lllll}0.7317840718699081 & 0.1416486020744754 & 0.9357329529027466\end{array}$ $\begin{array}{lllll}0.7564196555806839 & 0.1802779046701661 & 0.8105114114891232\end{array}$ $\begin{array}{llll}0.7257523588289736 & 0.3440164901238199 & 0.9072873700318472\end{array}$ $\begin{array}{llll}0.7992305366829170 & 0.3510641957795105 & 0.6826665726405763\end{array}$ $\begin{array}{lllll}0.7825193529582727 & 0.2419951595779706 & 0.6900476496045503\end{array}$ 
$\begin{array}{llll}0.9082181470866487 & 0.2832795940511327 & 0.6911106318227053\end{array}$ $\begin{array}{lllll}0.9525476814731406 & 0.2088139224829592 & 0.5690962390654986\end{array}$ $\begin{array}{llllll}0.7870318960975727 & 0.2888143625510601 & 0.5646033101841126\end{array}$ $\begin{array}{llll}0.8290543372742256 & 0.1871173814131495 & 0.5855824429960674\end{array}$ $\begin{array}{llll}0.9328380762404409 & 0.1690121065087438 & 0.6937028507243409\end{array}$ $\begin{array}{lllll}0.9316329104759465 & 0.0561178849234464 & 0.6992529422231684\end{array}$ $\begin{array}{lllll}0.7996018353820451 & 0.1112084536843189 & 0.7134090940959985\end{array}$ $\begin{array}{lllll}0.9111442057146366 & 0.1059640157340085 & 0.5788121174140253\end{array}$ $\begin{array}{llll}0.9013285891403474 & 0.1165043675504002 & 0.8098860970368585\end{array}$ $\begin{array}{llllll}0.7737952964056506 & 0.0688320771876321 & 0.8297025304670643\end{array}$ $\begin{array}{lllll}0.8905420703644019 & 0.0667237133875162 & 0.9365723642613023\end{array}$ $\begin{array}{lllll}0.8568588475887335 & 0.1683704934978161 & 0.9192986008710410\end{array}$ $\begin{array}{lllll}0.7725663873610139 & 0.2894153095730108 & 0.8028880042829472\end{array}$ $\begin{array}{lllll}0.8978424079608266 & 0.2482065389592596 & 0.8020096151417966\end{array}$ $\begin{array}{llll}0.8522041261629603 & 0.3227074186834139 & 0.9245416884848560\end{array}$ $\begin{array}{lllll}0.7679564419989146 & 0.2424955487842011 & 0.9283692650986396\end{array}$ $\begin{array}{lllll}0.8731001612754538 & 0.3617890572625615 & 0.7998069250926623\end{array}$ $\begin{array}{lllll}0.9819070879803097 & 0.1422393672781338 & 0.9359373852837626\end{array}$ $\begin{array}{llll}0.5067908783057382 & 0.1797029948429999 & 0.8101662244804186\end{array}$ $\begin{array}{llll}0.9755752310678016 & 0.3419760699354203 & 0.9107080951920743\end{array}$ $\begin{array}{lllll}0.6528730084851772 & 0.4161661545210748 & 0.6831213507506254\end{array}$ $\begin{array}{llllll}0.5307133509555614 & 0.4638454555334241 & 0.6645245185296279\end{array}$ $\begin{array}{lllll}0.5745849346218819 & 0.3897142222061986 & 0.5569890849112884\end{array}$ $\begin{array}{lllll}0.6654503360818045 & 0.4652184161547906 & 0.5565491923562876\end{array}$ $\begin{array}{llllll}0.6991599639106485 & 0.3633794150036141 & 0.5745792490051861\end{array}$ $\begin{array}{lllll}0.6225027119498494 & 0.4750136747162292 & 0.7946532591250122\end{array}$ $\begin{array}{llll}0.5056046189332672 & 0.4193627244013430 & 0.7801844075253178\end{array}$ $\begin{array}{lllll}0.6431376364248186 & 0.4248873291298989 & 0.9143475928678608\end{array}$ $\begin{array}{lllll}0.9021417778195685 & 0.4174662591204358 & 0.6823501282621057\end{array}$ $\begin{array}{llll}0.7800501650882410 & 0.4626093087462745 & 0.6637167674933400\end{array}$ $\begin{array}{llll}0.8243763128140272 & 0.3892976550870526 & 0.5567226658438216\end{array}$ $\begin{array}{lllll}0.9187928180512256 & 0.4624004603665490 & 0.5542123898732012\end{array}$ $\begin{array}{lllll}0.9490045728457162 & 0.3607519610149987 & 0.5783681113024953\end{array}$ $\begin{array}{lllll}0.8727448555447106 & 0.4749754575994044 & 0.7946162221445956\end{array}$ $\begin{array}{lllll}0.7559857080329416 & 0.4197552980643413 & 0.7802760433122213\end{array}$ $\begin{array}{llll}0.8945418653863815 & 0.4248969709378803 & 0.9146082045194222\end{array}$ $\begin{array}{lllll}0.2150004612954914 & 0.2339151213922532 & 0.2483734264153700\end{array}$ $\begin{array}{lllll}0.0893729639288116 & 0.2954381644303395 & 0.2451875538198103\end{array}$ $\begin{array}{lllll}0.2245805063819094 & 0.1130465713188748 & 0.2641094071254149\end{array}$ $\begin{array}{llllll}0.4652116334343511 & 0.2338504145569389 & 0.2484583773918659\end{array}$ $\begin{array}{lllll}0.3393020390633743 & 0.2952031398302376 & 0.2454762311500832\end{array}$ $\begin{array}{lllll}0.4746326707425036 & 0.1128991948192019 & 0.2640074591059170\end{array}$ $\begin{array}{lllll}0.0797537732066015 & 0.4162821119379813 & 0.2295019650758711\end{array}$ $\begin{array}{lllll}0.3296535901700006 & 0.4162478678558146 & 0.2301766365601272\end{array}$ $\begin{array}{llllll}0.2152824995577377 & 0.2331885378634607 & 0.7490254306145634\end{array}$ $\begin{array}{lllll}0.0915058116469873 & 0.2965443195291774 & 0.7462584421445335\end{array}$ $\begin{array}{llll}0.2251417226279903 & 0.1120505520716000 & 0.7636091061133612\end{array}$ $\begin{array}{lllll}0.4650411135274371 & 0.2343419048644805 & 0.7485399011261322\end{array}$ $\begin{array}{lllll}0.3387927102879940 & 0.2942901165057524 & 0.7466605863093672\end{array}$ $\begin{array}{llll}0.4748164203656529 & 0.1136322699308258 & 0.7630599844308330\end{array}$ $\begin{array}{lllll}0.0810164468711334 & 0.4183502271423334 & 0.7298907542299997\end{array}$ $\begin{array}{lllll}0.3342873024816475 & 0.4143402320052901 & 0.7348083065012284\end{array}$ $\begin{array}{llllll}0.7153852448096568 & 0.2338713862849261 & 0.2484271898736347\end{array}$ $\begin{array}{lllll}0.5896860484355195 & 0.2952571973377812 & 0.2453290469110725\end{array}$ $\begin{array}{llllll}0.7247594759442474 & 0.1128790104766704 & 0.2639639323872350\end{array}$ $\begin{array}{lllll}0.9652383795572385 & 0.2338678608021681 & 0.2484401568851366\end{array}$ $\begin{array}{lllll}0.8396102734014667 & 0.2954414479709722 & 0.2453906313355682\end{array}$ $\begin{array}{lllll}0.9747780568496494 & 0.1128620023538518 & 0.2639827534436899\end{array}$ $\begin{array}{lllll}0.5801089563738262 & 0.4162606631255218 & 0.2297108650063116\end{array}$ $\begin{array}{lllll}0.8299363205434027 & 0.4164539999126706 & 0.2294946520781640\end{array}$ $\begin{array}{lllll}0.7155874548724303 & 0.2350161956683509 & 0.7480282317292353\end{array}$ $\begin{array}{lllll}0.5892868891100144 & 0.2961886357465461 & 0.7447165323872817\end{array}$ $\begin{array}{lllll}0.7251477026816778 & 0.1139277605595326 & 0.7636043433873604\end{array}$ $\begin{array}{lllll}0.9659968755939500 & 0.2353311656711191 & 0.7482646010026720\end{array}$ $\begin{array}{llll}0.8402160964125699 & 0.2966034911150693 & 0.7450745339266169\end{array}$ $\begin{array}{llll}0.9746443119492075 & 0.1142389471470483 & 0.7636351389625901\end{array}$ $\begin{array}{lllll}0.5803184557526869 & 0.4174189184549251 & 0.7294724777324234\end{array}$ $\begin{array}{lllll}0.8304122678001951 & 0.4177273213093476 & 0.7293206666995722\end{array}$ $\begin{array}{lllll}0.0253596360185524 & 0.2312581736891763 & 0.1018599289296771\end{array}$ $\begin{array}{lllll}0.1557213516457119 & 0.1678811008188629 & 0.1056855846139656\end{array}$ $\begin{array}{lllll}0.1482516437646156 & 0.3616588902700053 & 0.3875912752217586\end{array}$ $\begin{array}{lllll}0.1576448231399326 & 0.1210614045042711 & 0.3976982269457419\end{array}$ $\begin{array}{lllll}0.0282678850472559 & 0.1806786537600742 & 0.3997130697891951\end{array}$ 
$\begin{array}{llll}0.0293800477426115 & 0.2979790025515201 & 0.3918308677065965\end{array}$ $\begin{array}{lllll}0.0262038289829667 & 0.3486629614318673 & 0.0938854958081518\end{array}$ $\begin{array}{lllll}0.2753920030366620 & 0.2310757833711463 & 0.1020354883067162\end{array}$ $\begin{array}{llll}0.4058820629169769 & 0.1678886723588526 & 0.1056335264775006\end{array}$ $\begin{array}{lllll}0.3989362908509419 & 0.3607174321673349 & 0.3884879704678895\end{array}$ $\begin{array}{lllll}0.4071562001658417 & 0.1212332547713578 & 0.3975092628691421\end{array}$ $\begin{array}{lllll}0.2781289586683304 & 0.1811342431723277 & 0.3998079237891914\end{array}$ $\begin{array}{lllll}0.2786754415965831 & 0.2983414551159133 & 0.3918673738307065\end{array}$ $\begin{array}{lllll}0.2762250506770973 & 0.3484874088818944 & 0.0944364031192024\end{array}$ $\begin{array}{llll}0.1470252126246880 & 0.4084576184729938 & 0.0962787509847694\end{array}$ $\begin{array}{lllll}0.3971682701854105 & 0.4080860639510229 & 0.0971909037491932\end{array}$ $\begin{array}{llllll}0.0260803457739704 & 0.2318576914977347 & 0.6024583094523646\end{array}$ $\begin{array}{lllll}0.1559516515473278 & 0.1681973643654912 & 0.6057214388054675\end{array}$ $\begin{array}{llll}0.1509140842091335 & 0.3625552988486324 & 0.8885317585178663\end{array}$ 0.15749855629758820 .11937344913130790 .8970883119009176 $\begin{array}{lllll}0.0294111534710258 & 0.1813380602446557 & 0.8991535644858081\end{array}$ $\begin{array}{lllll}0.0305449311863073 & 0.2993746090455456 & 0.8927706806445818\end{array}$ $\begin{array}{lllll}0.0281979559137149 & 0.3486261853213393 & 0.5949932102821422\end{array}$ $\begin{array}{llll}0.2747890925669463 & 0.2327209850267042 & 0.6016052248059285\end{array}$ $\begin{array}{llll}0.4056154913176289 & 0.1697881587634915 & 0.6047217807323059\end{array}$ $\begin{array}{lllll}0.3985264832746115 & 0.3592960596344340 & 0.8912240113047808\end{array}$ $\begin{array}{lllll}0.4078313120463507 & 0.1207971484356678 & 0.8970235749058449\end{array}$ $\begin{array}{lllll}0.2779575414868570 & 0.1792338447445382 & 0.8999051877770552\end{array}$ $\begin{array}{lllll}0.2791666256909044 & 0.2962752315469328 & 0.8929870733795849\end{array}$ $\begin{array}{llll}0.2751995046625800 & 0.3508491256825183 & 0.5962788552370465\end{array}$ $\begin{array}{lllll}0.1463306380105612 & 0.4135164386457166 & 0.5956074521312594\end{array}$ $\begin{array}{lllll}0.3992761409881037 & 0.4067557080474876 & 0.5979579374042627\end{array}$ $\begin{array}{lllll}0.5255225670517680 & 0.2310758349977051 & 0.1020245663814427\end{array}$ $\begin{array}{lllll}0.6559821389893703 & 0.1678702497152023 & 0.1056008497431222\end{array}$ $\begin{array}{llll}0.6491526253986832 & 0.3612725737943306 & 0.3880989951856929\end{array}$ $\begin{array}{lllll}0.6577028974031159 & 0.1206892424182087 & 0.3975455333622169\end{array}$ $\begin{array}{llll}0.5283275959611545 & 0.1804060957791530 & 0.3996870956163229\end{array}$ $\begin{array}{lllll}0.5295681721153833 & 0.2977949385663262 & 0.3918410665847095\end{array}$ $\begin{array}{lllll}0.5264004144337012 & 0.3483728686407032 & 0.0943104344028169\end{array}$ $\begin{array}{lllll}0.7754324049637858 & 0.2312544774705988 & 0.1020008873688165\end{array}$ $\begin{array}{lllll}0.9058154119646521 & 0.1679524725158597 & 0.1056699437837378\end{array}$ $\begin{array}{llllll}0.8990989861239058 & 0.3615230149875487 & 0.3879268996776651\end{array}$ $\begin{array}{lllll}0.9076788990750654 & 0.1206491803115192 & 0.3976166739877647\end{array}$ $\begin{array}{lllll}0.7784457038357113 & 0.1806174584836826 & 0.3996700205982927\end{array}$ $\begin{array}{lllll}0.7796231960249269 & 0.2980398578201383 & 0.3919596377117872\end{array}$ $\begin{array}{lllll}0.7763384264839688 & 0.3486233928908315 & 0.0940560284492254\end{array}$ $\begin{array}{lllll}0.6469658881907675 & 0.4082665182755489 & 0.0958900127856011\end{array}$ $\begin{array}{lllll}0.8969715114820499 & 0.4084901740137186 & 0.0958324403329954\end{array}$ $\begin{array}{lllll}0.5258626445522909 & 0.2322335543501220 & 0.6017700973000383\end{array}$ $\begin{array}{lllll}0.6563231852187061 & 0.1689418024784571 & 0.6053684897154556\end{array}$ $\begin{array}{lllll}0.6487974862726671 & 0.3619981687440694 & 0.8873544835081961\end{array}$ $\begin{array}{lllll}0.6580519050287161 & 0.1212527296415762 & 0.8969015121343928\end{array}$ $\begin{array}{lllll}0.5286778965653046 & 0.1807102552705906 & 0.8990818996999814\end{array}$ $\begin{array}{lllll}0.5295868083117486 & 0.2982493518965370 & 0.8919317386521386\end{array}$ $\begin{array}{lllll}0.5276468198008759 & 0.3494832347424551 & 0.5925042116222679\end{array}$ $\begin{array}{llll}0.7758016719716435 & 0.2322784571122805 & 0.6016948076434810\end{array}$ $\begin{array}{llll}0.9061960346438276 & 0.1689202673049412 & 0.6056436149474448\end{array}$ $\begin{array}{lllll}0.8996655047826627 & 0.3619400119955145 & 0.8880526746376836\end{array}$ $\begin{array}{lllll}0.9078664896264581 & 0.1223562237515709 & 0.8974356530981588\end{array}$ $\begin{array}{lllll}0.7784336841483811 & 0.1817399527084603 & 0.8993706456777559\end{array}$ $\begin{array}{lllll}0.7793882529669787 & 0.2990374958409160 & 0.8912962155200609\end{array}$ $\begin{array}{lllll}0.7777101698921837 & 0.3497743909606189 & 0.5937878877479400\end{array}$ $\begin{array}{lllll}0.6485515658900650 & 0.4096468868227821 & 0.5959126757669463\end{array}$ $\begin{array}{lllll}0.8986979055056251 & 0.4089478488289964 & 0.5957897068631192\end{array}$

Gua/Thy :

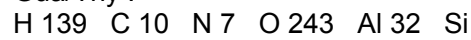

1.00000000000000

$20.2970019999999991 \quad 0.0000000000000000 \quad-3.7471719999999999$

$\begin{array}{llll}0.0646860000000000 & 24.9946149999999996 & -0.5148360000000000\end{array}$

$\begin{array}{llll}0.0000000000000000 & 0.0000000000000000 & 18.6939999999999991\end{array}$ $\mathrm{H} \quad \mathrm{C} \quad \mathrm{N} \quad \mathrm{O}$ Al $\mathrm{Si}$

$\begin{array}{llllll}139 & 10 & 7 & 243 & 32 & 64\end{array}$

Direct

$0.6993625302389768 \quad 0.70261113007366750 .7168687241715493$ $\begin{array}{lllll}0.7716069329117697 & 0.5180251836008859 & 0.6844121812527980\end{array}$ $0.6742166259264899 \quad 0.65445183268878350 .6046497687874264$ $\begin{array}{llll}0.7506485963647347 & 0.6402167087500349 & 0.8859000051369154\end{array}$ 
$\begin{array}{llll}0.7526161866002262 & 0.7044574326710089 & 0.8464631370043623\end{array}$ $\begin{array}{lllll}0.8254090680886328 & 0.6639135218580333 & 0.8653894336679674\end{array}$ $\begin{array}{lllll}0.1027041802702363 & 0.0532492212634095 & 0.4048483986306706\end{array}$ $\begin{array}{lllll}0.3556593912206647 & 0.0519248615139198 & 0.4059194034832880\end{array}$ $\begin{array}{llll}0.0610998340985086 & 0.1393537313071594 & 0.1791454245466395\end{array}$ $\begin{array}{lllll}0.1798601949881830 & 0.3064421107735231 & 0.1562805124907051\end{array}$ $\begin{array}{lllll}0.1404410879917329 & 0.2188632699685463 & 0.3301331035485473\end{array}$ $\begin{array}{lllll}0.2539647994250342 & 0.0381390797957392 & 0.3406657354384829\end{array}$ $\begin{array}{lllll}0.1810101690052260 & 0.0656406304130230 & 0.1424235642807989\end{array}$ $\begin{array}{lllll}0.3115248734243757 & 0.1381529887121738 & 0.1782415317610948\end{array}$ $\begin{array}{lllll}0.4297092323538187 & 0.3058148377560392 & 0.1549392935027296\end{array}$ $\begin{array}{lllll}0.3896491312863314 & 0.2178514770731461 & 0.3276497457428349\end{array}$ $\begin{array}{lllll}0.5044815062281421 & 0.0375630291805471 & 0.3394016672180870\end{array}$ $\begin{array}{llll}0.4325378491176100 & 0.0656817766338143 & 0.1430243969233382\end{array}$ $\begin{array}{lllll}0.0057119935319567 & 0.4789110244931343 & 0.1861236795577865\end{array}$ $\begin{array}{lllll}0.2583241980056420 & 0.3855376652000833 & 0.3079739364216007\end{array}$ $\begin{array}{lllll}0.1400047361033662 & 0.4597646123912015 & 0.3480842647541725\end{array}$ $\begin{array}{lllll}0.2738012906653166 & 0.4898095524470123 & 0.1837196882489907\end{array}$ $\begin{array}{lllll}0.0095055523938213 & 0.3894811987518771 & 0.3179076784181191\end{array}$ $\begin{array}{lllll}0.38427555528774668 & 0.4585469668639419 & 0.3420948037748457\end{array}$ $\begin{array}{lllll}0.2211010737625511 & 0.4688022962761960 & 0.0763858336189413\end{array}$ $\begin{array}{lllll}0.4669329551972108 & 0.4712485097342442 & 0.0672529705064978\end{array}$ $\begin{array}{llllll}0.1674096157628906 & 0.4354729783819736 & 0.4648853898111541\end{array}$ $\begin{array}{llll}0.4007339853547777 & 0.4357651422492076 & 0.4588617518854076\end{array}$ $\begin{array}{lllll}0.1549920295948455 & 0.0889370566206629 & 0.0240631954776584\end{array}$ $\begin{array}{lllll}0.4078729717015572 & 0.0885858028917133 & 0.0248057665795597\end{array}$ $\begin{array}{lllll}0.1066556222897683 & 0.5042406415585039 & 0.2888336698528073\end{array}$ $\begin{array}{llll}0.4194093466983002 & 0.4800050103992899 & 0.2790323584263792\end{array}$ $\begin{array}{lllll}0.1466389565028844 & 0.0446632302226939 & 0.2059875774180971\end{array}$ $\begin{array}{lllll}0.3970011202670031 & 0.0451792309541896 & 0.2059023366060125\end{array}$ $\begin{array}{lllll}0.2210738159398206 & 0.4162226093838813 & 0.2372652947382541\end{array}$ $\begin{array}{lllll}0.4704054705887266 & 0.4103326234531708 & 0.2378915027767821\end{array}$ $\begin{array}{lllll}0.3486328761732221 & 0.1107729309380382 & 0.2519048382247178\end{array}$ $\begin{array}{lllll}0.0982815593886051 & 0.1109143461318622 & 0.2518770372113602\end{array}$ $\begin{array}{llll}0.1028942564489948 & 0.0534034906631928 & 0.9049424160770416\end{array}$ $\begin{array}{llll}0.3569507180704402 & 0.0508251196170228 & 0.9065920717980019\end{array}$ $\begin{array}{lllll}0.0620990726182911 & 0.1397288056696619 & 0.6784632795717719\end{array}$ $\begin{array}{lllll}0.1801883330994546 & 0.3042110509673751 & 0.6545287453570411\end{array}$ $\begin{array}{llll}0.1425201550963073 & 0.2190506916501853 & 0.8321265304517693\end{array}$ $\begin{array}{lllll}0.2551828343866533 & 0.0378441236745809 & 0.8419375177082131\end{array}$ $\begin{array}{lllll}0.1825562971717335 & 0.0643599715042446 & 0.6429584728278822\end{array}$ $\begin{array}{lllll}0.3129255364818155 & 0.1369418937293289 & 0.6784817036991869\end{array}$ $\begin{array}{lllll}0.4308259183505538 & 0.3051382845366584 & 0.6558535470002071\end{array}$ $\begin{array}{lllll}0.3911863141618957 & 0.2175813108154639 & 0.8291919904186854\end{array}$ $\begin{array}{lllll}0.5060830086455773 & 0.0369299967045503 & 0.8410227613458471\end{array}$ $\begin{array}{lllll}0.4333567886841033 & 0.0643094078311418 & 0.6436366983828476\end{array}$ $\begin{array}{lllll}0.0063269812563877 & 0.4781935329953772 & 0.6895625561225850\end{array}$ $\begin{array}{lllll}0.2594944153828553 & 0.3816777749985844 & 0.8042620408769436\end{array}$ $\begin{array}{lllll}0.1413106117177779 & 0.4594711811019272 & 0.8478585376756059\end{array}$ $\begin{array}{llll}0.2709491750004306 & 0.4885648772603525 & 0.6848371907502460\end{array}$ $\begin{array}{lllll}0.0124849577541415 & 0.3893398098390100 & 0.8205871549487803\end{array}$ $\begin{array}{lllll}0.3863200095970768 & 0.4591627543463794 & 0.8434920688373783\end{array}$ $\begin{array}{lllll}0.2239268291012533 & 0.4672761861478889 & 0.5768754488983754\end{array}$ $\begin{array}{lllll}0.4514382461292596 & 0.4811635650532932 & 0.5441480832767467\end{array}$ $\begin{array}{llll}0.1672397032903812 & 0.4354498173628537 & 0.9646036271086820\end{array}$ $\begin{array}{lllll}0.4117168439963500 & 0.4360341012450700 & 0.9610172499809847\end{array}$ $\begin{array}{llll}0.1561231740506965 & 0.0880735529724582 & 0.5245000118636096\end{array}$ $\begin{array}{lllll}0.4077756987472143 & 0.0878758553791328 & 0.5249939359820051\end{array}$ $\begin{array}{lllll}0.1029356449920366 & 0.5028531737594834 & 0.7914926276565999\end{array}$ $\begin{array}{lllll}0.4179807160288761 & 0.4843431578492944 & 0.7798497261740227\end{array}$ $\begin{array}{lllll}0.1485704089186980 & 0.0433327484410672 & 0.7068804463787671\end{array}$ $\begin{array}{llll}0.3984871808500760 & 0.0439116008896270 & 0.7070821178429784\end{array}$ $\begin{array}{lllll}0.2223712471188751 & 0.4160271662694960 & 0.7377890177654962\end{array}$ $\begin{array}{lllll}0.4725962335524477 & 0.4057066943645052 & 0.7459456046872469\end{array}$ $\begin{array}{lllll}0.3500460070906087 & 0.1097698121634364 & 0.7524208994898486\end{array}$ $\begin{array}{lllll}0.0987405234301446 & 0.1107988814388106 & 0.7510063430543303\end{array}$ $\begin{array}{llll}0.5069458947291745 & 0.5375908768664382 & 0.4694246007601308\end{array}$ $\begin{array}{lllll}0.6036693362580440 & 0.0545523184028684 & 0.4045462253623519\end{array}$ $\begin{array}{lllll}0.8513893978158611 & 0.0550403991146382 & 0.4039862404447490\end{array}$ $\begin{array}{lllll}0.5613848606780115 & 0.1388123804956184 & 0.1786077061929112\end{array}$ $\begin{array}{lllll}0.6799730994729746 & 0.3079084605723987 & 0.1567696106369050\end{array}$ $\begin{array}{lllll}0.6402751686066357 & 0.2197220657024600 & 0.3295661118353524\end{array}$ $\begin{array}{lllll}0.7529136914598277 & 0.0382438694312809 & 0.3386112305817665\end{array}$ 
$\begin{array}{llll}0.6817994019478256 & 0.0666503197636643 & 0.1428466651973232\end{array}$ $\begin{array}{lllll}0.8111947184810985 & 0.1391663463514159 & 0.1784841529981753\end{array}$ $\begin{array}{lllll}0.9295036211633351 & 0.3061622111929668 & 0.1548624052202534\end{array}$ $\begin{array}{lllll}0.8904406386164788 & 0.2200334563049778 & 0.3305021785262633\end{array}$ $\begin{array}{llll}0.0031129635563085 & 0.0378635824522640 & 0.3390477238939975\end{array}$ $\begin{array}{lllll}0.9312646663205572 & 0.0663049520069499 & 0.1422841307234984\end{array}$ $\begin{array}{lllll}0.5039691124660960 & 0.4784832579081231 & 0.1829551275263109\end{array}$ $\begin{array}{llll}0.7580436373714835 & 0.3882815136775561 & 0.3118370998578963\end{array}$ $\begin{array}{lllll}0.6373805928327311 & 0.4616379514271803 & 0.3457194158382496\end{array}$ $\begin{array}{llllll}0.7733901112507114 & 0.4907723162387247 & 0.1860665116016373\end{array}$ $\begin{array}{lllll}0.5081834977053303 & 0.3895449396523083 & 0.3160679644765986\end{array}$ $\begin{array}{lllll}0.8863084987477282 & 0.4603802865705228 & 0.3455935513763947\end{array}$ $\begin{array}{lllll}0.7203629988341909 & 0.4704423810872911 & 0.0768390300839981\end{array}$ $\begin{array}{llll}0.9702764288664981 & 0.4712993225670548 & 0.0722945634842506\end{array}$ $\begin{array}{lllll}0.6554499135314487 & 0.4378335716342939 & 0.4618501959458506\end{array}$ $\begin{array}{lllll}0.9166867923048626 & 0.4363729093749241 & 0.4634901086912212\end{array}$ $\begin{array}{llll}0.6572018308931890 & 0.0898254924831382 & 0.0243010748490138\end{array}$ $\begin{array}{llll}0.9055802553861548 & 0.0898246600421635 & 0.0238758665819520\end{array}$ $\begin{array}{llll}0.6064700688496159 & 0.5058103285498429 & 0.2841271983852175\end{array}$ $\begin{array}{lllll}0.9185687902583107 & 0.4831349819173573 & 0.2813665921023453\end{array}$ $\begin{array}{lllll}0.6452282424495331 & 0.0470798664973432 & 0.2052435584527802\end{array}$ $\begin{array}{llllll}0.8956199050815129 & 0.0459710452037557 & 0.2051307268892735\end{array}$ $\begin{array}{lllll}0.7210732090622691 & 0.4166219735729570 & 0.2387996938015001\end{array}$ $\begin{array}{lllll}0.9713248423364167 & 0.4109023118893666 & 0.2401636144521264\end{array}$ $\begin{array}{lllll}0.8483824597538976 & 0.1116333210044859 & 0.2521980462249045\end{array}$ $\begin{array}{llll}0.5990047437138910 & 0.1119854924091335 & 0.2526698655664038\end{array}$ $\begin{array}{llll}0.4938019555760484 & 0.7263920690916862 & 0.5548330908200074\end{array}$ $\begin{array}{lllll}0.5584079866652073 & 0.4974354388896109 & 0.5267684043978069\end{array}$ $\begin{array}{lllll}0.5308169370574417 & 0.7708023101770862 & 0.6813236209274828\end{array}$ $\begin{array}{llll}0.5561519250488552 & 0.5116409891483621 & 0.6532734832082906\end{array}$ $\begin{array}{lllll}0.6058129118216257 & 0.0533715931902651 & 0.9044421579606500\end{array}$ $\begin{array}{lllll}0.8529985758817048 & 0.0544184518213599 & 0.9041413618257134\end{array}$ $\begin{array}{lllll}0.5621800829811339 & 0.1384917054651687 & 0.6796229538942735\end{array}$ $\begin{array}{llll}0.6850258816137885 & 0.3136319765672706 & 0.6687626545343490\end{array}$ $\begin{array}{llllll}0.6415578514601796 & 0.2184800001782956 & 0.8295870677016062\end{array}$ $\begin{array}{lllll}0.7540595253880533 & 0.0381447630576779 & 0.8373218922974992\end{array}$ $\begin{array}{lllll}0.6829986529024281 & 0.0677740150870723 & 0.6419943101317337\end{array}$ $\begin{array}{llll}0.8119861252810556 & 0.1421826830560246 & 0.6805038010107365\end{array}$ $\begin{array}{lllll}0.9313561510021314 & 0.3075379551897277 & 0.6559899073868504\end{array}$ $\begin{array}{lllll}0.8914219784615435 & 0.2209433438486479 & 0.8306362363240601\end{array}$ $\begin{array}{lllll}0.0034928563588414 & 0.0380723192109539 & 0.8380469635226785\end{array}$ $\begin{array}{lllll}0.9301346311224898 & 0.0680547222406593 & 0.6410862577755961\end{array}$ $\begin{array}{lllll}0.4928999053940338 & 0.4602950492935092 & 0.6770333482813494\end{array}$ $\begin{array}{lllll}0.7584598398464690 & 0.3930204783884514 & 0.8118241065697746\end{array}$ $\begin{array}{llll}0.6380267349649369 & 0.4653297969977490 & 0.8454616867674510\end{array}$ $\begin{array}{lllll}0.8086191835338870 & 0.4683856486283740 & 0.6200539706761548\end{array}$ $\begin{array}{lllll}0.5111224053425759 & 0.3892947469339011 & 0.8247144142977418\end{array}$ $\begin{array}{llll}0.8525642274561520 & 0.5057444166567939 & 0.7960752701482930\end{array}$ $\begin{array}{lllll}0.6780692047104907 & 0.4995914895595190 & 0.5618845851966884\end{array}$ $\begin{array}{llll}0.9755388794146537 & 0.4696781483748020 & 0.5772542065189691\end{array}$ $\begin{array}{llll}0.6653490339927032 & 0.4364552756629430 & 0.9648209937644435\end{array}$ $\begin{array}{lllll}0.9154392009180730 & 0.4363827205644328 & 0.9643606430459114\end{array}$ $\begin{array}{lllll}0.6569026659537425 & 0.0907030092740140 & 0.5241910899664868\end{array}$ $\begin{array}{lllll}0.9029918139549856 & 0.0913465254201408 & 0.5234270012964404\end{array}$ $\begin{array}{lllll}0.6135956338121310 & 0.5108127502209419 & 0.7811246174538200\end{array}$ $\begin{array}{lllll}0.8946527107989615 & 0.4606193349815252 & 0.8443910019774730\end{array}$ $\begin{array}{lllll}0.6459609640602015 & 0.0481736080254405 & 0.7039595633443292\end{array}$ $\begin{array}{lllll}0.8963183543409637 & 0.0463981902533616 & 0.7046095809708017\end{array}$ $\begin{array}{lllll}0.7226875747410910 & 0.4202155579318511 & 0.7377479439218659\end{array}$ $\begin{array}{llll}0.9738543215718000 & 0.4118932900091215 & 0.7437335832826267\end{array}$ $\begin{array}{lllll}0.8490639725415764 & 0.1120853308973937 & 0.7517585096275657\end{array}$ $\begin{array}{llll}0.6002629135446221 & 0.1114921368229396 & 0.7532441206309625\end{array}$ $\begin{array}{lllll}0.7717399836235930 & 0.6633893439638248 & 0.8462793965786424\end{array}$ $\begin{array}{lllll}0.7190088918798944 & 0.6622286425886861 & 0.7139064615825126\end{array}$ $\begin{array}{lllll}0.7549174558099154 & 0.6378449269918678 & 0.7723586663186182\end{array}$ $\begin{array}{lllll}0.7776574648782733 & 0.5835919023724508 & 0.7615127922833346\end{array}$ $\begin{array}{llll}0.7197118895242802 & 0.5839574135631206 & 0.6354654302972876\end{array}$ $\begin{array}{lllll}0.5350932599616570 & 0.7282663962809476 & 0.6696999779168803\end{array}$ $\begin{array}{lllll}0.5316630733095331 & 0.5676657350578659 & 0.5705399092837040\end{array}$ $\begin{array}{llll}0.5264715918048195 & 0.6526656364434857 & 0.6045634450682694\end{array}$ $\begin{array}{lllll}0.5537235689183633 & 0.6441654814259117 & 0.6780246703154342\end{array}$ $\begin{array}{lllll}0.5709530258310940 & 0.5902825703633784 & 0.6992882901689195\end{array}$ $\begin{array}{lllll}0.7036930533955424 & 0.6366576546889601 & 0.6470982738312580\end{array}$ 
$\begin{array}{llll}0.7567284706394642 & 0.5596464552916282 & 0.6936969095243940\end{array}$ $\begin{array}{lllll}0.5200471117407475 & 0.5246637750494471 & 0.5214795522719456\end{array}$ $\begin{array}{lllll}0.5152600062959262 & 0.6167640949566970 & 0.5485538567535703\end{array}$ $\begin{array}{llll}0.5148937948488692 & 0.7068487586425903 & 0.6003683232663730\end{array}$ $\begin{array}{llll}0.5589636104239005 & 0.6919285078033947 & 0.7175021445501811\end{array}$ $\begin{array}{lllll}0.5575094526475645 & 0.5543654748870540 & 0.6401600800429034\end{array}$ $\begin{array}{lllll}0.8146640098236200 & 0.5588499263651248 & 0.8103667624085482\end{array}$ $\begin{array}{llll}0.7010019914196520 & 0.5614528677433196 & 0.5749146129373606\end{array}$ $0.0567311674136548 \quad 0.34816899014802050 .1811351130087233$ $\begin{array}{lllll}0.0403548917999978 & 0.2390917495277589 & 0.1867553788128071\end{array}$ $\begin{array}{lllll}0.1655604860275985 & 0.2804163537003630 & 0.1880569454516288\end{array}$ $\begin{array}{llllll}0.2103473979460064 & 0.2052854854756707 & 0.0645665038859062\end{array}$ $\begin{array}{lllll}0.0436707355297376 & 0.2873464902559144 & 0.0617460899867012\end{array}$ $\begin{array}{lllll}0.0868605634188695 & 0.1856301911096151 & 0.0812143255561977\end{array}$ $\begin{array}{lllll}0.1900977631001018 & 0.1661229820588253 & 0.1897902070116899\end{array}$ $\begin{array}{lllll}0.1898081741226312 & 0.0532248029789586 & 0.1948280121498744\end{array}$ $\begin{array}{lllll}0.0567167737850860 & 0.1088946084270463 & 0.2098219004364872\end{array}$ $\begin{array}{llll}0.1673953128217920 & 0.1032067306922954 & 0.0748195163384541\end{array}$ $\begin{array}{llll}0.1591165707721869 & 0.1121590579135081 & 0.3061226138803679\end{array}$ $\begin{array}{lllll}0.0306853163617792 & 0.0659550622241944 & 0.3257139739298315\end{array}$ $\begin{array}{lllll}0.1470142312999784 & 0.0632242480184298 & 0.4330972597657868\end{array}$ $\begin{array}{llll}0.1142247870604005 & 0.1649538263414382 & 0.4150014295710122\end{array}$ $\begin{array}{lllll}0.0310208672926195 & 0.2860524925688905 & 0.3003837052959226\end{array}$ $\begin{array}{llllll}0.1553541954957764 & 0.2448351347825494 & 0.2988024335905888\end{array}$ $\begin{array}{lllll}0.1105892458491882 & 0.3193068049437180 & 0.4220294038021484\end{array}$ $\begin{array}{lllll}0.0260474297031201 & 0.2392855115625713 & 0.4257822679722548\end{array}$ $\begin{array}{llll}0.1312640333585767 & 0.3593868527724127 & 0.2976166146760090\end{array}$ $\begin{array}{lllll}0.2389337989036810 & 0.1381621627230358 & 0.4322794809165078\end{array}$ $\begin{array}{lllll}0.2641549168088069 & 0.1768558246279137 & 0.3068691682110957\end{array}$ $\begin{array}{llll}0.2342763965127766 & 0.3396268673241312 & 0.4056921960975676\end{array}$ $\begin{array}{lllll}0.3064257895785149 & 0.3473770453101801 & 0.1783295703315383\end{array}$ $\begin{array}{lllll}0.2901142311613079 & 0.2386095996196640 & 0.1860347776202635\end{array}$ $\begin{array}{lllll}0.4155166130107051 & 0.2799719363583937 & 0.1869714695495683\end{array}$ $\begin{array}{lllll}0.4604012406931485 & 0.2062682716160364 & 0.0649716341075782\end{array}$ $\begin{array}{lllll}0.2936025997286615 & 0.2857656507747391 & 0.0601837785843749\end{array}$ $\begin{array}{lllll}0.3371716532537086 & 0.1843706967112652 & 0.0814804150249526\end{array}$ $\begin{array}{lllll}0.4407296313746781 & 0.1662421527343636 & 0.1899362941924507\end{array}$ $\begin{array}{lllll}0.4405388778539898 & 0.0533685579099551 & 0.1954470306670401\end{array}$ $\begin{array}{llll}0.3072444558345034 & 0.1081582918199570 & 0.2097494059227340\end{array}$ $\begin{array}{llll}0.4197435595190365 & 0.1032961100099388 & 0.0752983050488427\end{array}$ $\begin{array}{lllll}0.4095844799102321 & 0.1125451727891225 & 0.3063685140482906\end{array}$ $\begin{array}{llll}0.2815362933166589 & 0.0656455786876118 & 0.3258838483699587\end{array}$ $\begin{array}{lllll}0.3998668364753352 & 0.0624976869105281 & 0.4334766689140074\end{array}$ $\begin{array}{llllll}0.3643121234607486 & 0.1636306016277714 & 0.4158892575429497\end{array}$ $\begin{array}{lllll}0.2805102294722246 & 0.2858182273666495 & 0.2995972560187746\end{array}$ $\begin{array}{lllll}0.4050597380997252 & 0.2446884469708896 & 0.2979180593890458\end{array}$ $\begin{array}{lllll}0.3609124739955724 & 0.3187137444017689 & 0.4206750972543842\end{array}$ $\begin{array}{lllll}0.2769327906054281 & 0.2384570148440355 & 0.4252904263068155\end{array}$ $\begin{array}{lllll}0.3803202075951070 & 0.3581563907226357 & 0.2944428083421153\end{array}$ $\begin{array}{lllll}0.4896049912194481 & 0.1390613532482273 & 0.4326126895422889\end{array}$ $\begin{array}{lllll}0.0135845695856815 & 0.1774971023676952 & 0.3071267260470708\end{array}$ $\begin{array}{llll}0.4836932536738736 & 0.3420649768871225 & 0.4029048283912180\end{array}$ $\begin{array}{lllll}0.1599073384136169 & 0.4136073684252942 & 0.1807285043796557\end{array}$ $\begin{array}{lllll}0.0408554891691240 & 0.4614259227731344 & 0.1663250201966896\end{array}$ $\begin{array}{llllll}0.0794195944312674 & 0.3888210065891018 & 0.0561907010243017\end{array}$ $\begin{array}{lllll}0.1751092673883798 & 0.4604685769193245 & 0.0510878578693462\end{array}$ $\begin{array}{lllll}0.2040573145955176 & 0.3592486890207452 & 0.0726222675988279\end{array}$ $\begin{array}{lllll}0.1307109118678417 & 0.4706802131218778 & 0.2949474470471444\end{array}$ $\begin{array}{lllll}0.0126649113414878 & 0.4158847341162124 & 0.2808543069766429\end{array}$ $\begin{array}{llll}0.1535282722724727 & 0.4215027416131020 & 0.4136151090531067\end{array}$ $\begin{array}{lllll}0.4100537005538317 & 0.4141615044254386 & 0.1792417665202464\end{array}$ $\begin{array}{lllll}0.2881863496330831 & 0.4588724974664303 & 0.1583480083118145\end{array}$ $\begin{array}{lllll}0.3291562555229919 & 0.3873231568868941 & 0.0542752767839460\end{array}$ $\begin{array}{llll}0.4207450883815360 & 0.4611011022034504 & 0.0480233507938868\end{array}$ $\begin{array}{llll}0.4548981150975824 & 0.3608174445977109 & 0.0714609844540065\end{array}$ $\begin{array}{lllll}0.3760424777007044 & 0.4721526201672168 & 0.2899063266112833\end{array}$ $\begin{array}{lllll}0.2636527147697865 & 0.4156722908958219 & 0.2772478189669234\end{array}$ $\begin{array}{lllll}0.3993023350903816 & 0.4217370096142374 & 0.4077561832270151\end{array}$ $\begin{array}{lllll}0.0592317764586194 & 0.3475715794705986 & 0.6812407353298972\end{array}$ $\begin{array}{llllll}0.0413671935478485 & 0.2389662781828198 & 0.6869631862807596\end{array}$ $\begin{array}{lllll}0.1671611509676277 & 0.2793366255520922 & 0.6886581068954015\end{array}$ $\begin{array}{llll}0.2110848596717653 & 0.2046222527120705 & 0.5656125279743005\end{array}$ $\begin{array}{llll}0.0484762392954574 & 0.2861217305424459 & 0.5622554498555533\end{array}$ 
$\begin{array}{llll}0.0878364901781228 & 0.1837824367460596 & 0.5826198565768612\end{array}$ $\begin{array}{lllll}0.1917270920871278 & 0.1650378339347580 & 0.6905280548467665\end{array}$ $\begin{array}{llllll}0.1915462305559422 & 0.0520905487849391 & 0.6953834898241505\end{array}$ $\begin{array}{llll}0.0572230847264565 & 0.1094080644172748 & 0.7092147962146426\end{array}$ $\begin{array}{llll}0.1691919196481953 & 0.1021129782088369 & 0.5752757216413708\end{array}$ $\begin{array}{lllll}0.1602449382998884 & 0.1111417849848393 & 0.8060333881067553\end{array}$ $\begin{array}{llll}0.0311256852731564 & 0.0660404529081152 & 0.8245152174419156\end{array}$ $\begin{array}{lllll}0.1470710368795412 & 0.0634036390766597 & 0.9334197167242821\end{array}$ $\begin{array}{lllll}0.1151608461487888 & 0.1651371435690396 & 0.9137427084070348\end{array}$ $\begin{array}{lllll}0.0325162534074985 & 0.2858076086692284 & 0.8004360146156951\end{array}$ $\begin{array}{lllll}0.1569590962102331 & 0.2442760581438728 & 0.7994671671412230\end{array}$ $\begin{array}{llll}0.1121233710103298 & 0.3188512554076397 & 0.9221497490687991\end{array}$ $\begin{array}{lllll}0.0270885861377716 & 0.2391206500829788 & 0.9257262370824985\end{array}$ $\begin{array}{llll}0.1338006145349531 & 0.3586969962243761 & 0.7978956906250075\end{array}$ $\begin{array}{llll}0.2397152602244856 & 0.1377719026412770 & 0.9324352801488018\end{array}$ $\begin{array}{llllll}0.2655921025352872 & 0.1762489651941114 & 0.8074174183261505\end{array}$ $\begin{array}{lllll}0.2359562907595391 & 0.3387834404992716 & 0.9071832370805564\end{array}$ $\begin{array}{llllll}0.3093894591113406 & 0.3470851433192167 & 0.6794922460203798\end{array}$ $\begin{array}{llll}0.2915030796754627 & 0.2380764684099005 & 0.6872153678248384\end{array}$ $\begin{array}{llll}0.4170635149809059 & 0.2791901679723653 & 0.6879991501422889\end{array}$ $\begin{array}{lllll}0.4605938385020835 & 0.2047271568934612 & 0.5659020519784890\end{array}$ $\begin{array}{lllll}0.2946592049140868 & 0.2845706631109441 & 0.5613228653564195\end{array}$ $\begin{array}{lllll}0.3375885822489163 & 0.1828990359703424 & 0.5828696124003994\end{array}$ $\begin{array}{lllll}0.4416339167580737 & 0.1649988272118592 & 0.6909160185748813\end{array}$ $\begin{array}{lllll}0.4418433974393710 & 0.0520617498554136 & 0.6960738005344629\end{array}$ $\begin{array}{llll}0.3086462085398443 & 0.1071804650394308 & 0.7103579612170715\end{array}$ $\begin{array}{lllll}0.4198958070436082 & 0.1019102148695942 & 0.5758400632018293\end{array}$ $\begin{array}{llllll}0.4109950953363906 & 0.1111549020041717 & 0.8070154475461387\end{array}$ $\begin{array}{lllll}0.2828801268578110 & 0.0650340844672648 & 0.8266324725602425\end{array}$ $\begin{array}{llllll}0.4010473947684147 & 0.0615211911915214 & 0.9342747408943097\end{array}$ $\begin{array}{lllll}0.3656346283492738 & 0.1626319980658057 & 0.9163245748453570\end{array}$ $\begin{array}{llll}0.2821601550273082 & 0.2857384436022639 & 0.8005012072156732\end{array}$ $\begin{array}{lllll}0.4065176478757340 & 0.2440638322637294 & 0.7988850156480060\end{array}$ $\begin{array}{llll}0.3628674942755815 & 0.3185035341279223 & 0.9212847673430200\end{array}$ $\begin{array}{llll}0.2791925014580444 & 0.2378559481544460 & 0.9259064380627505\end{array}$ $\begin{array}{lllll}0.3826271655974700 & 0.3584282873265372 & 0.7955110016010354\end{array}$ $\begin{array}{lllll}0.4908102484227107 & 0.1380097332551076 & 0.9332299068820887\end{array}$ $\begin{array}{llllll}0.0137180006820326 & 0.1776349720538438 & 0.8066257788235470\end{array}$ $\begin{array}{lllll}0.4859072698601248 & 0.3413465546213977 & 0.9038845322777047\end{array}$ $\begin{array}{llll}0.1607711762608827 & 0.4148544708092682 & 0.6813736346497119\end{array}$ $\begin{array}{lllll}0.0399901274776196 & 0.4606821991896790 & 0.6670840736463397\end{array}$ $\begin{array}{lllll}0.0810646431657414 & 0.3886860763709374 & 0.5565470391111027\end{array}$ $\begin{array}{lllll}0.1780305160014384 & 0.4588478345069523 & 0.5509181320159993\end{array}$ $\begin{array}{llll}0.2046142116963265 & 0.3571285680543055 & 0.5759957878832999\end{array}$ $\begin{array}{lllll}0.1311983661914005 & 0.4714078043565843 & 0.7952277242022753\end{array}$ $\begin{array}{llll}0.0162320100184621 & 0.4147155110824212 & 0.7825855654044104\end{array}$ $\begin{array}{llll}0.1551978059389720 & 0.4210616279316539 & 0.9135000462468933\end{array}$ $\begin{array}{lllll}0.4105307062765237 & 0.4174930472401922 & 0.6776620204020208\end{array}$ $\begin{array}{lllll}0.2882731127353650 & 0.4590672147919600 & 0.6592351712428968\end{array}$ $\begin{array}{llll}0.3280313827389105 & 0.3860860048087054 & 0.5542661416267882\end{array}$ $0.4089196029457344 \quad 0.4618526184345886 \quad 0.5408579344954516$ $\begin{array}{llll}0.4552520037158072 & 0.3635640784810921 & 0.5720563377060716\end{array}$ $\begin{array}{lllll}0.3763048619484856 & 0.4722056178249089 & 0.7912759074827898\end{array}$ $\begin{array}{lllll}0.2649129173288853 & 0.4139883993004351 & 0.7775614611837401\end{array}$ $\begin{array}{llll}0.4026201191796422 & 0.4215917939332340 & 0.9099127521110530\end{array}$ $\begin{array}{lllll}0.5553626264310443 & 0.3483353532022948 & 0.1800840456431593\end{array}$ $\begin{array}{lllll}0.5405552685149803 & 0.2391203180299718 & 0.1864548430605875\end{array}$ $\begin{array}{llllll}0.6654229406311296 & 0.2815698580399871 & 0.1879430315426225\end{array}$ $\begin{array}{lllll}0.7104019249639428 & 0.2071281317688670 & 0.0649075251083241\end{array}$ $\begin{array}{llll}0.5437490940918528 & 0.2871682727581120 & 0.0612512546799647\end{array}$ $\begin{array}{llll}0.5870712905141413 & 0.1856077935439550 & 0.0809489531310536\end{array}$ $\begin{array}{lllll}0.6902546871057383 & 0.1673483840531559 & 0.1897938846996637\end{array}$ $\begin{array}{lllll}0.6891619416717719 & 0.0545620502722586 & 0.1953281004657297\end{array}$ $\begin{array}{lllll}0.5575356622348537 & 0.1087874182829275 & 0.2102131275277080\end{array}$ $\begin{array}{lllll}0.6693408605154992 & 0.1042919433096656 & 0.0749017244626753\end{array}$ $\begin{array}{lllll}0.6590313159652622 & 0.1142513472372440 & 0.3064763099850357\end{array}$ $\begin{array}{lllll}0.5317957130815893 & 0.0659785546708454 & 0.3264466281012744\end{array}$ $\begin{array}{llll}0.6479182806836290 & 0.0645521575809123 & 0.4331359371899182\end{array}$ $\begin{array}{lllll}0.6146063293618989 & 0.1662164380834768 & 0.4158354960488742\end{array}$ $\begin{array}{llll}0.5305471363275985 & 0.2861840872766593 & 0.2997934238075751\end{array}$ $\begin{array}{lllll}0.6552163708622460 & 0.2460201120840293 & 0.2987016973208144\end{array}$ $\begin{array}{llllll}0.6101385546321992 & 0.3200436009148001 & 0.4216357354611677\end{array}$ $\begin{array}{llll}0.5255297511945326 & 0.2400571354873317 & 0.4254491894600583\end{array}$ 
$\begin{array}{llll}0.6296209155935517 & 0.3602020404447248 & 0.2967837253887578\end{array}$ $\begin{array}{lllll}0.7395826816718887 & 0.1399121824399633 & 0.4326146134196064\end{array}$ $\begin{array}{lllll}0.7638982533645057 & 0.1783925143866670 & 0.3071234897055964\end{array}$ $\begin{array}{lllll}0.7333695657450165 & 0.3422788871693870 & 0.4041462762076258\end{array}$ $0.80711648469838790 .3487930508850824 \quad 0.1799340614916438$ $\begin{array}{lllll}0.7902583796345211 & 0.2399990449532371 & 0.1865902338668099\end{array}$ $\begin{array}{llll}0.9157476891881058 & 0.2810021157546145 & 0.1881123414819661\end{array}$ $\begin{array}{llllll}0.9602232336801678 & 0.2066525790541667 & 0.0650212474934354\end{array}$ $\begin{array}{lllll}0.7946252271022360 & 0.2874032767487706 & 0.0612418224482605\end{array}$ $\begin{array}{lllll}0.8368418182553236 & 0.1856937141319729 & 0.0815444574953798\end{array}$ $\begin{array}{lllll}0.9400847396937090 & 0.1667542996739480 & 0.1898523020410608\end{array}$ $\begin{array}{lllll}0.9392141598771926 & 0.0539870526977351 & 0.1946943165603100\end{array}$ $\begin{array}{lllll}0.8067454569486910 & 0.1092163235606464 & 0.2099906256643364\end{array}$ $\begin{array}{llll}0.9183756028335582 & 0.1040609014928619 & 0.0745709108598715\end{array}$ $\begin{array}{llll}0.9087539808774800 & 0.1130429093975793 & 0.3061514563078139\end{array}$ $\begin{array}{lllll}0.7804730457831404 & 0.0666715138381969 & 0.3261540906336676\end{array}$ $\begin{array}{lllll}0.8956644577061464 & 0.0646284731143220 & 0.4330105719996546\end{array}$ $\begin{array}{llll}0.8646329178609728 & 0.1666062021453044 & 0.4147722442986376\end{array}$ $\begin{array}{llll}0.7806252586194785 & 0.2871894070210059 & 0.3000984352053994\end{array}$ $\begin{array}{lllll}0.9052286276221729 & 0.2458321640093437 & 0.2988290579267325\end{array}$ $\begin{array}{lllll}0.8600938480387198 & 0.3206377790616395 & 0.4219800338311771\end{array}$ $\begin{array}{lllllll}0.7759175354918317 & 0.2406226134125441 & 0.4256554660096222\end{array}$ $\begin{array}{llll}0.8805775104048369 & 0.3599666753329950 & 0.2969555635606829\end{array}$ $\begin{array}{llll}0.9890306432504726 & 0.1386665073855261 & 0.4323998934693629\end{array}$ $\begin{array}{lllll}0.5142169322294095 & 0.1774968245814899 & 0.3072938413304389\end{array}$ $\begin{array}{lllll}0.9840651039589923 & 0.3409741569971743 & 0.4047098339079709\end{array}$ $\begin{array}{lllll}0.6594767739432738 & 0.4134871773043067 & 0.1808555446719551\end{array}$ $\begin{array}{lllll}0.5397615978731163 & 0.4612039731318167 & 0.1640995533773540\end{array}$ $\begin{array}{lllll}0.5800692358369618 & 0.3886005053593088 & 0.0556385660890745\end{array}$ $\begin{array}{llll}0.6744842869816579 & 0.4616432150936642 & 0.0521388135209914\end{array}$ $\begin{array}{lllll}0.7051279302710587 & 0.3605108220820062 & 0.0730792048171111\end{array}$ $\begin{array}{lllll}0.6289682410309504 & 0.4716955174907944 & 0.2923855125515321\end{array}$ $\begin{array}{llll}0.5110408957384944 & 0.4162449753486595 & 0.2792396605648243\end{array}$ $\begin{array}{llll}0.6500206138748006 & 0.4231089510900460 & 0.4111247415374810\end{array}$ $\begin{array}{lllll}0.9107041235997847 & 0.4156958410524526 & 0.1820002159234957\end{array}$ $\begin{array}{lllll}0.7887253796236737 & 0.4601320649072991 & 0.1609181052244577\end{array}$ $\begin{array}{lllll}0.8302718167466699 & 0.3891614355399530 & 0.0563312381558833\end{array}$ $\begin{array}{lllll}0.9240382033590687 & 0.4618877462834030 & 0.0516071030677714\end{array}$ $\begin{array}{lllll}0.9549093376730088 & 0.3607936382160798 & 0.0747110644492070\end{array}$ $\begin{array}{llllll}0.8762400475114197 & 0.4733758808745768 & 0.2934188091045882\end{array}$ $\begin{array}{lllll}0.7633159979741035 & 0.4169992927767027 & 0.2788202955712090\end{array}$ $\begin{array}{llll}0.9033765987947149 & 0.4226497147900440 & 0.4124319564532966\end{array}$ $\begin{array}{lllll}0.5949538761075079 & 0.5736597532587897 & 0.7613154080739963\end{array}$ $\begin{array}{lllll}0.5562067808704476 & 0.3477687519566990 & 0.6820674042898462\end{array}$ $\begin{array}{lllll}0.5417991461090620 & 0.2378936454523377 & 0.6873226078909720\end{array}$ $\begin{array}{lllll}0.6666438763189935 & 0.2827820798723631 & 0.6886813248520685\end{array}$ $\begin{array}{lllll}0.7105845867106479 & 0.2083255361338587 & 0.5644441142996601\end{array}$ $\begin{array}{llllll}0.5403454602510869 & 0.2869074158168197 & 0.5621567588222569\end{array}$ $\begin{array}{lllll}0.5876092751287958 & 0.1861190552907270 & 0.5804058830364854\end{array}$ $\begin{array}{lllll}0.6909582689660307 & 0.1683061981763158 & 0.6895369462217621\end{array}$ $\begin{array}{lllll}0.6901073739991052 & 0.0553243703312009 & 0.6943920897368360\end{array}$ $\begin{array}{llll}0.5587244625458107 & 0.1080477274634509 & 0.7106602919882093\end{array}$ $\begin{array}{lllll}0.6703742992194365 & 0.1052925020379341 & 0.5745942298874076\end{array}$ $\begin{array}{llllll}0.6601666421177369 & 0.1142590339017694 & 0.8064201979714820\end{array}$ $\begin{array}{llll}0.5334183573327336 & 0.0648817576561730 & 0.8268867865609673\end{array}$ $\begin{array}{lllll}0.6500134371814842 & 0.0635919647365849 & 0.9329209578326478\end{array}$ $\begin{array}{llll}0.6158209422908386 & 0.1650655902693449 & 0.9166757167600893\end{array}$ $\begin{array}{lllll}0.5319922333853320 & 0.2851269358337152 & 0.8006677369909838\end{array}$ $\begin{array}{lllll}0.6561736175689006 & 0.2450888845406709 & 0.7989367478159943\end{array}$ $\begin{array}{llll}0.6122443653843921 & 0.3187239251077735 & 0.9217714411009734\end{array}$ $\begin{array}{lllll}0.5268686084538685 & 0.2390240724565180 & 0.9260663421981645\end{array}$ $\begin{array}{llll}0.6323572571740910 & 0.3596949001905319 & 0.7980544268617011\end{array}$ $\begin{array}{lllll}0.7409771304903877 & 0.1394681277155035 & 0.9323799671357580\end{array}$ $\begin{array}{lllll}0.7651355442982873 & 0.1784237357621041 & 0.8068736803690685\end{array}$ $\begin{array}{llll}0.7355828376765026 & 0.3418111465348438 & 0.9059376902914136\end{array}$ $\begin{array}{llll}0.8064859392872500 & 0.3504430037122018 & 0.6802417175988460\end{array}$ $\begin{array}{lllll}0.7907825080973319 & 0.2412449345863751 & 0.6860927890901483\end{array}$ $\begin{array}{lllll}0.9164165073804114 & 0.2819546500862385 & 0.6879157041690009\end{array}$ $\begin{array}{lllll}0.9618128152628118 & 0.2071817383317268 & 0.5646450558615149\end{array}$ $\begin{array}{lllll}0.7904114188814630 & 0.2909693866600647 & 0.5605324394799805\end{array}$ $\begin{array}{lllll}0.8377190686622012 & 0.1901397677392816 & 0.5796031206931587\end{array}$ $\begin{array}{lllll}0.9397551270995932 & 0.1678269553835794 & 0.6892146659329738\end{array}$ $\begin{array}{lllll}0.9394007980185837 & 0.0549678490955381 & 0.6932236944977560\end{array}$ 
$\begin{array}{llll}0.8075469081356296 & 0.1106884391961649 & 0.7093202042195490\end{array}$ $\begin{array}{lllll}0.9156440713807522 & 0.1057966363672275 & 0.5740023261932000\end{array}$ $\begin{array}{lllll}0.9094227617732212 & 0.1128746524527082 & 0.8057416270028303\end{array}$ $\begin{array}{lllll}0.7815725107515833 & 0.0666661330952364 & 0.8250220779668521\end{array}$ $\begin{array}{llll}0.8972531934442604 & 0.0642036313551762 & 0.9327912230550329\end{array}$ $\begin{array}{llll}0.8658196678276130 & 0.1661678317874832 & 0.9148045502652536\end{array}$ $\begin{array}{llllll}0.7814163901020885 & 0.2874374504354641 & 0.8002281075342949\end{array}$ $\begin{array}{llllll}0.9058026645726304 & 0.2464742811046718 & 0.7983529964146573\end{array}$ $\begin{array}{llll}0.8622117372124487 & 0.3194863071970238 & 0.9220556470850111\end{array}$ $\begin{array}{llll}0.7772096089112012 & 0.2401844653535293 & 0.9254513066965597\end{array}$ $\begin{array}{lllll}0.8813697823974115 & 0.3603141372819936 & 0.7975687509654024\end{array}$ $\begin{array}{lllll}0.9902073821072226 & 0.1383856551684961 & 0.9319569579037188\end{array}$ $\begin{array}{lllll}0.5154261031170072 & 0.1764391252250338 & 0.8079910052743620\end{array}$ $\begin{array}{lllll}0.9856302612108004 & 0.3408517469778927 & 0.9043092971029713\end{array}$ $\begin{array}{llll}0.6590261646682001 & 0.4164612223803313 & 0.6798508220117572\end{array}$ $\begin{array}{lllll}0.5376652357342159 & 0.4568630984138496 & 0.6674040916392396\end{array}$ $\begin{array}{lllll}0.5803195521252066 & 0.3858526598408039 & 0.5559256461330866\end{array}$ $\begin{array}{lllll}0.6510208717095516 & 0.4666829525165704 & 0.5451258850881004\end{array}$ $\begin{array}{llll}0.7083827489078840 & 0.3716140380328162 & 0.5686878849001473\end{array}$ $\begin{array}{lllll}0.6268923410963655 & 0.4723722021878621 & 0.7923977479751453\end{array}$ $\begin{array}{lllll}0.5129042512980313 & 0.4132215476635130 & 0.7843269435316281\end{array}$ $\begin{array}{llll}0.6522380629654074 & 0.4220713795584553 & 0.9140009741131018\end{array}$ $\begin{array}{lllll}0.9136049857579891 & 0.4128796822687765 & 0.6822234973100625\end{array}$ $\begin{array}{llll}0.7924486250672661 & 0.4626463269507452 & 0.6649217864796149\end{array}$ $\begin{array}{llll}0.8341091952829103 & 0.3908098581176009 & 0.5568525163426975\end{array}$ $\begin{array}{lllll}0.9297446568993023 & 0.4600959127183538 & 0.5534281264151559\end{array}$ $\begin{array}{llll}0.9576327505390378 & 0.3577391256292350 & 0.5739757346486551\end{array}$ $\begin{array}{lllll}0.8815104866850335 & 0.4728779749085110 & 0.7929925893612726\end{array}$ $\begin{array}{llll}0.7646973945034518 & 0.4201482711895425 & 0.7770450694724756\end{array}$ $\begin{array}{lllll}0.9051067587666897 & 0.4218714908819403 & 0.9135186234561653\end{array}$ $\begin{array}{lllll}0.2230924454052974 & 0.2317688849095192 & 0.2444961649509546\end{array}$ $\begin{array}{lllll}0.0977613228977563 & 0.2933957767775024 & 0.2422692962940581\end{array}$ $\begin{array}{lllll}0.2325414519501364 & 0.1107172319385806 & 0.2599746893573120\end{array}$ $\begin{array}{lllll}0.4734368692229588 & 0.2320280200757113 & 0.2443762728064922\end{array}$ $\begin{array}{lllll}0.3474002177935570 & 0.2929316299592144 & 0.2407728549314500\end{array}$ $\begin{array}{lllll}0.4830321290459719 & 0.1111880406258455 & 0.2604097568678068\end{array}$ $\begin{array}{lllll}0.0890352097892842 & 0.4140410169367588 & 0.2286493195403992\end{array}$ $\begin{array}{lllll}0.3360795531365242 & 0.4136259047601386 & 0.2245123181947914\end{array}$ $\begin{array}{llll}0.2246485650529763 & 0.2311309769799820 & 0.7453563699337901\end{array}$ $\begin{array}{llll}0.0995551476842719 & 0.2929733260744308 & 0.7428918015151256\end{array}$ $\begin{array}{llllll}0.2338885363074268 & 0.1099311390614874 & 0.7604695266235036\end{array}$ $\begin{array}{llll}0.4747782046168670 & 0.2310095373316286 & 0.7453580773792710\end{array}$ $\begin{array}{lllll}0.3491840182890952 & 0.2926300934269687 & 0.7420317038375775\end{array}$ $\begin{array}{lllll}0.4843277812192799 & 0.1100379823556267 & 0.7610924535103888\end{array}$ $\begin{array}{llllll}0.0900803114871325 & 0.4138726754948072 & 0.7293914714223244\end{array}$ $\begin{array}{lllll}0.3376633017250982 & 0.4142996131380124 & 0.7250235702737264\end{array}$ $\begin{array}{llll}0.7231757067258268 & 0.2331620648155320 & 0.2446059921244571\end{array}$ $\begin{array}{lllll}0.5972294096313576 & 0.2939439799002467 & 0.2416657231536531\end{array}$ $\begin{array}{lllll}0.7322925424649814 & 0.1122345943688293 & 0.2601856565639222\end{array}$ $\begin{array}{llll}0.9731584924630508 & 0.2324536271658290 & 0.2447617665724061\end{array}$ $\begin{array}{lllll}0.8477580306406951 & 0.2942629546581539 & 0.2419975642066065\end{array}$ $\begin{array}{llll}0.9821142972980270 & 0.1114265877868255 & 0.2599814506623620\end{array}$ $\begin{array}{lllll}0.5878402048770544 & 0.4143010344689385 & 0.2271856546588982\end{array}$ $\begin{array}{lllll}0.8366018060407614 & 0.4150117956696013 & 0.2270240648357746\end{array}$ $\begin{array}{lllll}0.7243074143796718 & 0.2334935268770874 & 0.7449317131180609\end{array}$ $\begin{array}{lllll}0.5981360761122070 & 0.2931681953826422 & 0.7427298268626548\end{array}$ $\begin{array}{lllll}0.7332912651221629 & 0.1125736156193513 & 0.7597452614302482\end{array}$ $\begin{array}{lllll}0.9736874101149141 & 0.2329997424085165 & 0.7445306621652754\end{array}$ $\begin{array}{lllll}0.8482577864459732 & 0.2952898892293776 & 0.7417159842182750\end{array}$ $\begin{array}{lllll}0.9825120594372169 & 0.1118150465584386 & 0.7591635064219792\end{array}$ $\begin{array}{llll}0.5904962707103473 & 0.4135016020731784 & 0.7322331538621806\end{array}$ $\begin{array}{lllll}0.8415677018573979 & 0.4162969393770459 & 0.7302455536683782\end{array}$ $\begin{array}{lllll}0.0333926968672685 & 0.2303065333859795 & 0.0981672354944735\end{array}$ $\begin{array}{lllll}0.1634057817351480 & 0.1662557208602912 & 0.1016480470909267\end{array}$ $\begin{array}{lllll}0.1576367882711199 & 0.3589071301454414 & 0.3857604392672546\end{array}$ $\begin{array}{lllll}0.1648587893535579 & 0.1185067812891011 & 0.3935291525023453\end{array}$ $\begin{array}{llll}0.0358075574270398 & 0.1786104443618615 & 0.3959333223005067\end{array}$ $\begin{array}{lllll}0.0374436709412133 & 0.2957967547842385 & 0.3886325609792736\end{array}$ $\begin{array}{lllll}0.0336455102954276 & 0.3478099410663206 & 0.0924281092768388\end{array}$ $\begin{array}{llll}0.2833988740931308 & 0.2290843551919325 & 0.0975920973434515\end{array}$ 0.41428373615391590 .16630489999597690 .1017772003943058 $\begin{array}{lllll}0.4059135045083517 & 0.3587314949037121 & 0.3825721073650978\end{array}$ $\begin{array}{lllll}0.4159353492633032 & 0.1183618716842502 & 0.3938845595532277\end{array}$ 
$\begin{array}{llll}0.2861375349014698 & 0.1776673172177803 & 0.3957464298606727\end{array}$ $\begin{array}{llllll}0.2875063667796934 & 0.2950531053315364 & 0.3881082882576679\end{array}$ $\begin{array}{lllll}0.2827362379837460 & 0.3462930675827349 & 0.08977799991906384\end{array}$ $\begin{array}{lllll}0.1546832862776007 & 0.4074498691302447 & 0.0937724083543762\end{array}$ $\begin{array}{lllll}0.4036064333087125 & 0.4072968849876036 & 0.0918659648859841\end{array}$ $\begin{array}{llll}0.0353297572666749 & 0.2296035244783978 & 0.5986684491418540\end{array}$ 0.16467624794031190 .16501478524582340 .6025365426043792 $\begin{array}{lllll}0.1595260014423960 & 0.3583970973294687 & 0.8861708680868230\end{array}$ $\begin{array}{llll}0.1656613504233612 & 0.1182108183413842 & 0.8932518706853031\end{array}$ $\begin{array}{lllll}0.0366183280021285 & 0.1785825679960806 & 0.8953263531651223\end{array}$ $\begin{array}{lllll}0.0388866250697192 & 0.2956206084936708 & 0.8886627886611180\end{array}$ $\begin{array}{llllll}0.0368285641176674 & 0.3463431081257681 & 0.5926082694386076\end{array}$ $\begin{array}{lllll}0.2842870623494740 & 0.2280245194982941 & 0.5988649951073254\end{array}$ $\begin{array}{lllll}0.4147352084850793 & 0.1648313463706934 & 0.6028692034040333\end{array}$ $\begin{array}{llll}0.4081625152856153 & 0.3586919204796945 & 0.8837697461730197\end{array}$ $\begin{array}{lllll}0.4171615474131908 & 0.1172407000673864 & 0.8944595744634312\end{array}$ $\begin{array}{lllll}0.2875326001432015 & 0.1769889696608924 & 0.8962670676241774\end{array}$ $\begin{array}{lllll}0.2894352446676196 & 0.2947100324789792 & 0.8891747655981775\end{array}$ $\begin{array}{llll}0.2835271186048354 & 0.3448757390909519 & 0.5913631744997192\end{array}$ $\begin{array}{llllll}0.1563417437260027 & 0.4069479401449171 & 0.5948641916482684\end{array}$ $\begin{array}{lllll}0.4016991923171887 & 0.4087679750442172 & 0.5901105623229933\end{array}$ $\begin{array}{lllll}0.5335178775992635 & 0.2302048534544164 & 0.0978934270781789\end{array}$ $\begin{array}{lllll}0.6640550020868418 & 0.1672939983532032 & 0.1015742585996399\end{array}$ $\begin{array}{llll}0.6561291885470955 & 0.3601868582943554 & 0.3848880765312462\end{array}$ 0.66552505300235120 .12008003956105330 .3940333638130098 $\begin{array}{lllll}0.5360601086222510 & 0.1793067817138257 & 0.3961893529400582\end{array}$ $\begin{array}{lllll}0.5369962896708393 & 0.2964146948215762 & 0.3879942569078924\end{array}$ $\begin{array}{llllll}0.5335916973660998 & 0.3478272550478908 & 0.0911909068569350\end{array}$ $\begin{array}{lllll}0.7836019935556816 & 0.2306788314103470 & 0.0981192234652311\end{array}$ $\begin{array}{llll}0.9136959909575991 & 0.1669788300061942 & 0.1016952759355075\end{array}$ $\begin{array}{lllll}0.9070344646267020 & 0.3597498507426147 & 0.3849571611778371\end{array}$ $\begin{array}{llll}0.9147308647976158 & 0.1195251351508625 & 0.3935979550535285\end{array}$ $\begin{array}{llll}0.7861016981241603 & 0.1799726790165599 & 0.3959694015690780\end{array}$ $\begin{array}{lllll}0.7868749105516185 & 0.2971716924518804 & 0.3883592927228715\end{array}$ $\begin{array}{llllll}0.7838261323930547 & 0.3478827445497597 & 0.0912572003278303\end{array}$ $\begin{array}{lllll}0.6548876169883671 & 0.4080170119126617 & 0.0939887018118636\end{array}$ $\begin{array}{llllll}0.9047991136024064 & 0.4085318882274376 & 0.0946601286189499\end{array}$ $\begin{array}{lllll}0.5332520443914066 & 0.2295409594795174 & 0.5985069097276113\end{array}$ $\begin{array}{lllll}0.6647320670909591 & 0.1682831489524024 & 0.6012881232987046\end{array}$ $\begin{array}{lllll}0.6584234810752444 & 0.3599558826015308 & 0.8865901821999786\end{array}$ $\begin{array}{lllll}0.6669684319393234 & 0.1194183497519173 & 0.8941387249035807\end{array}$ $\begin{array}{lllll}0.5373194906814529 & 0.1782282216702798 & 0.8968796683206595\end{array}$ $\begin{array}{lllll}0.5389655989180328 & 0.2953800156833419 & 0.8888787296280903\end{array}$ $\begin{array}{llllll}0.5330659678542784 & 0.3476061158655434 & 0.5932621679940207\end{array}$ $\begin{array}{llll}0.7830232841821924 & 0.2334373255193622 & 0.5972481963754122\end{array}$ $\begin{array}{lllll}0.9133964379538138 & 0.1689616579813490 & 0.6010307448611395\end{array}$ $\begin{array}{lllll}0.9085237178686936 & 0.3596331871192504 & 0.8857023994060229\end{array}$ $\begin{array}{lllll}0.9158604104218233 & 0.1192397904848751 & 0.8932409692204236\end{array}$ $0.78735815297930090 .1796562936638752 \quad 0.8957276940288410$ $\begin{array}{lllll}0.7885847032724608 & 0.2968995803220515 & 0.8887128231814934\end{array}$ $\begin{array}{lllll}0.7843782172402297 & 0.3519338711354454 & 0.5915336104323160\end{array}$ $\begin{array}{llll}0.6522385597475907 & 0.4116114499984195 & 0.5927929649651975\end{array}$ $\begin{array}{llll}0.9100731399025981 & 0.4063599216779125 & 0.5956127447534977\end{array}$

Thy/Thy :

H $140 \quad$ C $10 \quad \mathrm{~N} 4 \quad$ O $244 \quad$ Al $32 \quad \mathrm{Si}$

1.00000000000000

$20.2970019999999991 \quad 0.0000000000000000 \quad-3.7471719999999999$

$0.0646860000000000 \quad 24.9946149999999996 \quad-0.5148360000000000$

$\begin{array}{llll}0.0000000000000000 & 0.0000000000000000 & 18.6939999999999991\end{array}$

$\mathrm{H} \quad \mathrm{C} \quad \mathrm{N} \quad \mathrm{O}$ Al $\mathrm{S}$

$\begin{array}{llllll}140 & 10 & 4 & 244 & 32 & 64\end{array}$

Direct

0.44824036151854450 .71124110844643730 .5569406272338734

$\begin{array}{lllll}0.43335568874442856 & 0.5164456770522030 & 0.5553243120342749\end{array}$

$\begin{array}{lllll}0.4259876867556686 & 0.6589333806841712 & 0.4478605198148131\end{array}$

$\begin{array}{lllll}0.4356859481459177 & 0.6646864570426255 & 0.7302450494648358\end{array}$

$\begin{array}{lllll}0.4773704335692686 & 0.7154828436528643 & 0.6907039331386191\end{array}$

$\begin{array}{lllll}0.5208248615156088 & 0.6581240289103025 & 0.7306862989558180\end{array}$

$\begin{array}{llll}0.0930297294157296 & 0.0558619447596295 & 0.4083688192540634\end{array}$

$\begin{array}{lllll}0.3421632012595728 & 0.0560528884682400 & 0.4079619682650849\end{array}$

$\begin{array}{llllll}0.0505870595952217 & 0.1398038629558613 & 0.1817386031906364\end{array}$

$\begin{array}{llll}0.1698441867852723 & 0.3091743410471404 & 0.1608806372325813\end{array}$ 
$\begin{array}{llll}0.1297213169410799 & 0.2212964667418060 & 0.3334530088611327\end{array}$ $\begin{array}{lllll}0.2425496371581247 & 0.0401357675475893 & 0.3432272841165314\end{array}$ $\begin{array}{lllll}0.1713442447626619 & 0.0678783261922645 & 0.1464412442477555\end{array}$ $\begin{array}{llll}0.3005671823361070 & 0.1402660375444026 & 0.1816972734324623\end{array}$ $\begin{array}{lllll}0.4196001249064951 & 0.3085249268923926 & 0.1595460463557247\end{array}$ $\begin{array}{llll}0.3797528396203819 & 0.2219572694188298 & 0.3339179067088217\end{array}$ $\begin{array}{lllll}0.4926628648432718 & 0.0400184386994513 & 0.3433983715186620\end{array}$ $\begin{array}{llll}0.4207082425434363 & 0.0677611464605445 & 0.1462445249305243\end{array}$ $\begin{array}{llll}0.0562182454414915 & 0.4891784308967880 & 0.1487221444918496\end{array}$ $\begin{array}{llllll}0.2484004680373198 & 0.3894214952672301 & 0.3117895151338688\end{array}$ $\begin{array}{lllll}0.1281201859215560 & 0.4621089057941072 & 0.3460132699296327\end{array}$ $\begin{array}{lllll}0.3047702578112178 & 0.4896926714311325 & 0.1487951321613533\end{array}$ $\begin{array}{lllll}0.4979214339924515 & 0.3917572637251530 & 0.3128681137339659\end{array}$ $\begin{array}{lllll}0.3762732117143721 & 0.4629676987557892 & 0.3490630269316078\end{array}$ $\begin{array}{lllll}0.2050020531950707 & 0.4744342151143220 & 0.0845052614673706\end{array}$ $\begin{array}{lllll}0.4567107845920294 & 0.4744283223760943 & 0.0825348109877130\end{array}$ $\begin{array}{lllll}0.1523553833527427 & 0.4389880784211519 & 0.4648287598443069\end{array}$ $\begin{array}{lllll}0.4063023896806027 & 0.4389754940489107 & 0.4678921735246820\end{array}$ $\begin{array}{lllll}0.1468397867974931 & 0.0913531669624686 & 0.0278188915396664\end{array}$ $\begin{array}{lllll}0.3944250099867340 & 0.0912729255648670 & 0.0276815190731368\end{array}$ $\begin{array}{lllll}0.1647137508541885 & 0.4812748179058022 & 0.2834685036185008\end{array}$ $\begin{array}{llllll}0.4074498316227330 & 0.4898067307486665 & 0.2864614835483753\end{array}$ $\begin{array}{llllll}0.1350176342173267 & 0.0480514935505058 & 0.2089587531992411\end{array}$ $\begin{array}{llll}0.3852125611912156 & 0.0476828261007240 & 0.2092660759073779\end{array}$ $\begin{array}{llll}0.2109385843495623 & 0.4155423852130042 & 0.2372301710690375\end{array}$ $\begin{array}{lllll}0.4615447727969129 & 0.4191073292250266 & 0.2387107566667394\end{array}$ $\begin{array}{lllll}0.3378647752274778 & 0.1131660922479633 & 0.2556529214780869\end{array}$ $\begin{array}{lllll}0.0879953562003807 & 0.1132283341677226 & 0.2560732004625658\end{array}$ $\begin{array}{llll}0.0947136075034510 & 0.0559830583912383 & 0.9089348281663188\end{array}$ $\begin{array}{lllll}0.3413812513616137 & 0.0571088366130570 & 0.9081812114165385\end{array}$ $\begin{array}{llllll}0.0503552234530128 & 0.1401305685387304 & 0.6822310064898491\end{array}$ $\begin{array}{lllll}0.1685407920108374 & 0.3092667275041304 & 0.6587621910906719\end{array}$ $\begin{array}{lllll}0.1295225812918493 & 0.2217290108127384 & 0.8321664560682268\end{array}$ $\begin{array}{lllll}0.2423590983373658 & 0.0410164640176898 & 0.8432580212338936\end{array}$ $\begin{array}{lllll}0.1705502826815570 & 0.0681920152252697 & 0.6467833595987076\end{array}$ $\begin{array}{lllll}0.3005115520167660 & 0.1412806742240568 & 0.6821762892635665\end{array}$ $\begin{array}{lllll}0.4173980028606624 & 0.3060011398296554 & 0.6539474561505985\end{array}$ $\begin{array}{lllll}0.3796682432305951 & 0.2234374134912902 & 0.8341266956532100\end{array}$ $\begin{array}{lllll}0.4917088966465301 & 0.0408547891165554 & 0.8451183555990728\end{array}$ $0.4200426748868200 \quad 0.06768513670264490 .6463337833844305$ $\begin{array}{lllll}0.0536222159825360 & 0.4885107011438041 & 0.6464921395649134\end{array}$ $\begin{array}{lllll}0.2486472442680570 & 0.3905255406465663 & 0.8085110806078442\end{array}$ $\begin{array}{llll}0.1264250784060110 & 0.4628450237499490 & 0.8453500111011647\end{array}$ $\begin{array}{lllll}0.3048568354075958 & 0.4857920743653188 & 0.6383898862300328\end{array}$ $\begin{array}{lllll}0.4936714167956263 & 0.3869225969170318 & 0.8039509151745162\end{array}$ $\begin{array}{lllll}0.3793238675867147 & 0.4643134931495386 & 0.8426092681886433\end{array}$ $\begin{array}{llll}0.2033710022003155 & 0.4743284407731916 & 0.5838807624661109\end{array}$ $\begin{array}{lllll}0.5027673329764623 & 0.4578906214959377 & 0.6139288235107621\end{array}$ $\begin{array}{llll}0.1520848032541680 & 0.4393682116477878 & 0.9645288940470452\end{array}$ $\begin{array}{llll}0.4039186758805736 & 0.4402934689678893 & 0.9629118930134353\end{array}$ $\begin{array}{llll}0.1449624186016342 & 0.0912127812389177 & 0.5279346834065186\end{array}$ $\begin{array}{llll}0.3945548415773535 & 0.0910375029910912 & 0.5275642790662162\end{array}$ $\begin{array}{llll}0.1620344302364315 & 0.4826208478864559 & 0.7822291105264810\end{array}$ $\begin{array}{llllll}0.4071708330721356 & 0.4899758590633310 & 0.7761899796580443\end{array}$ $\begin{array}{lllll}0.1352537693396773 & 0.0481746687077961 & 0.7099987866949764\end{array}$ $\begin{array}{llll}0.3849998560465963 & 0.0474835531201224 & 0.7097041797278325\end{array}$ $\begin{array}{lllll}0.2099973268224979 & 0.4170268412773888 & 0.7348781640806181\end{array}$ $\begin{array}{lllll}0.4573983465478399 & 0.4166545650975695 & 0.7309060100890790\end{array}$ $\begin{array}{lllll}0.3376307467806153 & 0.1135284744398590 & 0.7556679010764312\end{array}$ $\begin{array}{llll}0.0881802335819845 & 0.1136946075072406 & 0.7563521097702660\end{array}$ $\begin{array}{llll}0.5926523425387568 & 0.0556660257265389 & 0.4085648184420076\end{array}$ $\begin{array}{lllll}0.8431658191505961 & 0.0553935309090475 & 0.4091529856779240\end{array}$ $\begin{array}{lllll}0.5505220029772377 & 0.1404723522043671 & 0.1820744671113431\end{array}$ $\begin{array}{lllll}0.6699017931903872 & 0.3088593968964768 & 0.1610268899671521\end{array}$ $\begin{array}{lllll}0.6295512030179841 & 0.2213627624673325 & 0.3339158478331737\end{array}$ $\begin{array}{lllll}0.7429002455399982 & 0.0400682777912326 & 0.3441956527336221\end{array}$ $\begin{array}{lllll}0.6708744382468262 & 0.0675345145239357 & 0.1469159553499316\end{array}$ $\begin{array}{lllll}0.8006668473473650 & 0.1399443536046109 & 0.1820779658441899\end{array}$ $\begin{array}{lllll}0.9197795931922724 & 0.3085812201279103 & 0.1606062908146871\end{array}$ $\begin{array}{llll}0.8794057521232013 & 0.2210786580921565 & 0.3334798025275782\end{array}$ $\begin{array}{lllll}0.9933724249083989 & 0.0397186947777948 & 0.3437327277297419\end{array}$ $\begin{array}{lllll}0.9209145220193697 & 0.0672621501218540 & 0.1471955795040724\end{array}$ $\begin{array}{lllll}0.5563516178457407 & 0.4906634155611673 & 0.1515272494795983\end{array}$ 
$\begin{array}{llll}0.7486252320047107 & 0.3900297666584510 & 0.3116468966769658\end{array}$ $\begin{array}{lllll}0.6271674616982474 & 0.4632965710806267 & 0.3468505517508524\end{array}$ $\begin{array}{lllll}0.8063744976461572 & 0.4892310448597834 & 0.1487266192604129\end{array}$ $\begin{array}{llll}0.9987727029921024 & 0.3905197030840484 & 0.3121629839409287\end{array}$ $\begin{array}{lllll}0.8779499910148980 & 0.4621400236942502 & 0.3464919965041820\end{array}$ $\begin{array}{lllll}0.7060552232233753 & 0.4741416521826926 & 0.0850927006583476\end{array}$ $\begin{array}{llllll}0.9560181158401594 & 0.4739644505974861 & 0.0844355085353163\end{array}$ $\begin{array}{lllll}0.6560309457881682 & 0.4376859542908066 & 0.4661166152443288\end{array}$ $\begin{array}{llll}0.9016686491993631 & 0.4389247829004028 & 0.4652978822180062\end{array}$ $\begin{array}{lllll}0.6445748349586883 & 0.0908413672848544 & 0.0284172053025000\end{array}$ $\begin{array}{lllll}0.8957526394458100 & 0.0904520359010731 & 0.0285907005283545\end{array}$ $\begin{array}{llllll}0.6650714901232807 & 0.4815993382420856 & 0.2851877766358218\end{array}$ $\begin{array}{lllll}0.9136836922575167 & 0.4823460349874950 & 0.2835907057196373\end{array}$ $\begin{array}{lllll}0.6354477727348188 & 0.0474070764308141 & 0.2099873546436477\end{array}$ $\begin{array}{lllll}0.8856808560725254 & 0.0470994689060005 & 0.2104718222245538\end{array}$ $\begin{array}{lllll}0.7110872244686196 & 0.4164452427342117 & 0.2370779478512468\end{array}$ $\begin{array}{lllll}0.9612674738648960 & 0.4166144231727082 & 0.2374574265614800\end{array}$ $\begin{array}{lllll}0.8379268960182839 & 0.1129904008477347 & 0.2561730685625386\end{array}$ $\begin{array}{llll}0.5877754678729982 & 0.1131656919054298 & 0.2557926269532333\end{array}$ $\begin{array}{llll}0.6365046590470427 & 0.7103200058588425 & 0.6711206236268547\end{array}$ $\begin{array}{lllll}0.6452467198714755 & 0.5270433395321921 & 0.5855796763459490\end{array}$ $\begin{array}{lllll}0.6109295582083818 & 0.6856551992646027 & 0.5460966292928391\end{array}$ $\begin{array}{lllll}0.6388362435219663 & 0.6193261674602558 & 0.8145389435395080\end{array}$ $\begin{array}{lllll}0.6702796086611909 & 0.6835770992892758 & 0.7989541536289323\end{array}$ $\begin{array}{llll}0.7229568740730856 & 0.6261104739384223 & 0.8094518062772832\end{array}$ $\begin{array}{llll}0.5916006474371380 & 0.0558734038950820 & 0.9087350557342178\end{array}$ $\begin{array}{llll}0.8441518578060713 & 0.0546466247886173 & 0.9093160645517785\end{array}$ $\begin{array}{lllll}0.5508003244058731 & 0.1388861213236643 & 0.6813972794065605\end{array}$ $\begin{array}{lllll}0.6683827023061536 & 0.3055231803066784 & 0.6580235602090498\end{array}$ $\begin{array}{lllll}0.6305415897218748 & 0.2214478103711916 & 0.8362781979894107\end{array}$ $\begin{array}{lllll}0.7430627731090369 & 0.0398916505967511 & 0.8466266516287346\end{array}$ $\begin{array}{lllll}0.6714872531925948 & 0.0658776943642055 & 0.6477043567204588\end{array}$ $\begin{array}{lllll}0.8007699720475011 & 0.1381335998764143 & 0.6822578621722416\end{array}$ $\begin{array}{lllll}0.9189652019279757 & 0.3081788074666758 & 0.6604419649927823\end{array}$ $\begin{array}{lllll}0.8796858963497259 & 0.2203248544617661 & 0.8334926613293716\end{array}$ $\begin{array}{llll}0.9941223243780055 & 0.0398988344538329 & 0.8447854123498936\end{array}$ $\begin{array}{lllll}0.9218188360949872 & 0.0666314958319625 & 0.6477088734939351\end{array}$ $\begin{array}{lllll}0.5107383864879679 & 0.4999816781076043 & 0.6780998571071362\end{array}$ $\begin{array}{lllll}0.7487596032533486 & 0.3897705481994807 & 0.8175247446674525\end{array}$ $\begin{array}{llllll}0.6283145976908664 & 0.4650521271604871 & 0.8430872681445828\end{array}$ $\begin{array}{lllll}0.7535765091684665 & 0.4873326959605657 & 0.6894425891700794\end{array}$ $\begin{array}{llll}0.9969871368466153 & 0.3900039199958885 & 0.8109294197930464\end{array}$ $\begin{array}{lllll}0.8767892963122227 & 0.4623296591586928 & 0.8456986568642250\end{array}$ $\begin{array}{llll}0.7162522963834098 & 0.4638421545821655 & 0.5894681037471717\end{array}$ $\begin{array}{llll}0.9523080684204073 & 0.4745470108468554 & 0.5818025007228409\end{array}$ $\begin{array}{lllll}0.6543446580976349 & 0.4391820856666368 & 0.9649587455728965\end{array}$ $\begin{array}{lllll}0.9035172501696006 & 0.4387465219305787 & 0.9647296541369991\end{array}$ $\begin{array}{lllll}0.6462593062606112 & 0.0896104829272140 & 0.5284673517913459\end{array}$ $\begin{array}{lllll}0.8962623417316971 & 0.0899180143504935 & 0.5286789063944024\end{array}$ $\begin{array}{llll}0.6433381079643253 & 0.4973848609134615 & 0.7711593188246140\end{array}$ $\begin{array}{llllll}0.9058672971118036 & 0.4863661105965144 & 0.7792470150042364\end{array}$ $\begin{array}{lllll}0.6357034691506153 & 0.0463677058347670 & 0.7108732162967628\end{array}$ $\begin{array}{lllll}0.8859260741216657 & 0.0470228283128140 & 0.7106847401907910\end{array}$ $\begin{array}{lllll}0.7104831994698575 & 0.4150587836950716 & 0.7427862531525248\end{array}$ $\begin{array}{lllll}0.9595207939005392 & 0.4175598593423532 & 0.7377013641831678\end{array}$ $\begin{array}{lllll}0.8384479403087836 & 0.1122243902053400 & 0.7570363672407617\end{array}$ $\begin{array}{lllll}0.5878228189056637 & 0.1124823840925136 & 0.7561098604536712\end{array}$ $\begin{array}{lllll}0.4742724473130576 & 0.6721076787222926 & 0.6979996325146223\end{array}$ $\begin{array}{lllll}0.4477482057386226 & 0.6677320666615685 & 0.5610171680256871\end{array}$ $\begin{array}{llll}0.4598634767316828 & 0.6428731916713160 & 0.6262595713630014\end{array}$ $\begin{array}{lllll}0.4589615849148354 & 0.5845848541144739 & 0.6248759984281602\end{array}$ $\begin{array}{lllll}0.4288928352827427 & 0.5841315695772492 & 0.4909217218635115\end{array}$ $\begin{array}{lllll}0.6732391482178105 & 0.6406011462046071 & 0.7864643203424477\end{array}$ $\begin{array}{lllll}0.6395139260619608 & 0.6681017660435802 & 0.6559754346754649\end{array}$ $\begin{array}{lllll}0.6573211932488721 & 0.6296343171203964 & 0.7062665895944324\end{array}$ $\begin{array}{lllll}0.6618284545569263 & 0.5749333471547138 & 0.6796373713286232\end{array}$ $\begin{array}{lllll}0.6228845330150707 & 0.6046432165058210 & 0.5532608485495266\end{array}$ $\begin{array}{lllll}0.4341856767669663 & 0.6399049948342029 & 0.4962575309906644\end{array}$ $\begin{array}{lllll}0.4413418273656641 & 0.5594826946420743 & 0.5577560814637075\end{array}$ $\begin{array}{llll}0.6241515211521249 & 0.6564117952926218 & 0.5829304124204198\end{array}$ $\begin{array}{lllll}0.6428410961213573 & 0.5662137182455023 & 0.6052675993265754\end{array}$ $\begin{array}{lllll}0.4737124004626542 & 0.5578228840833741 & 0.6819983476840553\end{array}$ $\begin{array}{llll}0.4142745193148681 & 0.5604722813593472 & 0.4320849603947302\end{array}$ 
$\begin{array}{llll}0.0451820074570988 & 0.3499962576242723 & 0.1825775581352042\end{array}$ $\begin{array}{lllll}0.0294481320429516 & 0.2407709430177647 & 0.1903672378512905\end{array}$ $\begin{array}{lllll}0.1545485155573534 & 0.2825410198466640 & 0.1910269549475122\end{array}$ $\begin{array}{lllll}0.1993712009692561 & 0.2086623124461627 & 0.0685849080344090\end{array}$ $\begin{array}{llll}0.0333538740374109 & 0.2876382065964459 & 0.0647098573866343\end{array}$ $\begin{array}{lllll}0.0761468655312125 & 0.1862689373992489 & 0.0855510602039265\end{array}$ $\begin{array}{llllll}0.1798899153398977 & 0.1685254363239655 & 0.1935336439285483\end{array}$ $\begin{array}{llllll}0.1788483746506717 & 0.0557104422936286 & 0.1988969510833733\end{array}$ $\begin{array}{lllll}0.0464356461252446 & 0.1101852008139757 & 0.2138984520588177\end{array}$ $\begin{array}{lllll}0.1591023331495628 & 0.1056528676915998 & 0.0785526015831807\end{array}$ $\begin{array}{lllll}0.1485040334460717 & 0.1152693455859107 & 0.3100356078712044\end{array}$ $\begin{array}{llll}0.0207437008520500 & 0.0679269566579886 & 0.3303319702717691\end{array}$ $\begin{array}{llllll}0.1372756268912234 & 0.0659021189481755 & 0.4368633906866026\end{array}$ $\begin{array}{llll}0.1038972534507891 & 0.1674757232384768 & 0.4193115029878448\end{array}$ $\begin{array}{lllll}0.0194869333276390 & 0.2884721618768556 & 0.3032050245226432\end{array}$ $\begin{array}{lllll}0.1443846465293195 & 0.2472750944017237 & 0.3019682784016735\end{array}$ $\begin{array}{lllll}0.0995723172249054 & 0.3217340090753278 & 0.4245386757640207\end{array}$ $\begin{array}{lllll}0.0153550508809594 & 0.2417242401820852 & 0.4288315857323211\end{array}$ $\begin{array}{llll}0.1191585378852604 & 0.3612797450621156 & 0.2994974389041752\end{array}$ $\begin{array}{llll}0.2288454258621553 & 0.1413476843292113 & 0.4360187803194709\end{array}$ $\begin{array}{lllll}0.2535465263211428 & 0.1796359116254529 & 0.3104601633801377\end{array}$ $\begin{array}{lllll}0.2228933559572293 & 0.3433217804443178 & 0.4077245564693027\end{array}$ $\begin{array}{lllll}0.2960611450545574 & 0.3507611753585628 & 0.1823686151359915\end{array}$ $\begin{array}{llll}0.2795392545045859 & 0.2415243470211843 & 0.1900478136233250\end{array}$ $\begin{array}{llll}0.4047520151345116 & 0.2824445810915626 & 0.1908138700424324\end{array}$ $\begin{array}{lllll}0.4494025771131159 & 0.2078494829780670 & 0.0685499964480744\end{array}$ $\begin{array}{lllll}0.2840277844368299 & 0.2885453065129796 & 0.0644621314467576\end{array}$ $\begin{array}{lllll}0.3258805083194400 & 0.1868975406883542 & 0.0852106172626606\end{array}$ $\begin{array}{lllll}0.4294783955679368 & 0.1682578686768360 & 0.1935252049153108\end{array}$ $\begin{array}{llllll}0.4288290885935734 & 0.0554699259196933 & 0.1986901570937512\end{array}$ $\begin{array}{lllll}0.2963656161211659 & 0.1104183704417183 & 0.2134788547284856\end{array}$ $\begin{array}{lllll}0.4074134100682424 & 0.1053877693716357 & 0.0784618148699786\end{array}$ $\begin{array}{lllll}0.3984249392363899 & 0.1147217144358439 & 0.3099087941896780\end{array}$ $\begin{array}{lllll}0.2703171631144209 & 0.0680356656615631 & 0.3297510885825952\end{array}$ $\begin{array}{llll}0.3864490946540243 & 0.0658440289160633 & 0.4366813592091587\end{array}$ $\begin{array}{lllll}0.3539693332478975 & 0.1676046923240974 & 0.4188927651023315\end{array}$ $\begin{array}{lllll}0.2694843469903154 & 0.2889637637317087 & 0.3028174266342140\end{array}$ $\begin{array}{lllll}0.3943234102342529 & 0.2474423131755256 & 0.3017267541718821\end{array}$ $\begin{array}{lllll}0.3498903930051490 & 0.3218859997153569 & 0.4238272993026511\end{array}$ $\begin{array}{lllll}0.2655474457590412 & 0.2419767378875406 & 0.4282969861180522\end{array}$ $\begin{array}{lllll}0.3696663460409608 & 0.3616279165897396 & 0.2991020393574072\end{array}$ $\begin{array}{lllll}0.4786531866724486 & 0.1408458937166189 & 0.4359810926061707\end{array}$ $\begin{array}{lllll}0.0033440900162471 & 0.1793424328307680 & 0.3109237982669342\end{array}$ $\begin{array}{lllll}0.4729136025853620 & 0.3432220129451030 & 0.4077746561228213\end{array}$ $\begin{array}{lllll}0.1503040738066562 & 0.4138740983955026 & 0.1827108801827079\end{array}$ $\begin{array}{llll}0.0285849065056300 & 0.4613823501873752 & 0.1627115343826325\end{array}$ $\begin{array}{lllll}0.0703642796225975 & 0.3879944021153168 & 0.0566901400505766\end{array}$ $\begin{array}{lllll}0.1608213880472582 & 0.4641643215717033 & 0.0561212398291907\end{array}$ $\begin{array}{lllll}0.1956201082541270 & 0.3627154273472294 & 0.0733125424685514\end{array}$ $\begin{array}{lllll}0.1207277553676049 & 0.4740852216804349 & 0.2935086294960553\end{array}$ $\begin{array}{lllll}0.5034469483732421 & 0.4204836144792848 & 0.2798960661502309\end{array}$ $\begin{array}{llll}0.1403356161207606 & 0.4244684855872406 & 0.4141322296221349\end{array}$ $\begin{array}{lllll}0.4003046565857086 & 0.4169211449575259 & 0.1831927882798521\end{array}$ $\begin{array}{lllll}0.2774220320320226 & 0.4617780157245426 & 0.1630203279334741\end{array}$ $\begin{array}{llllll}0.3207848113847141 & 0.3892769858103343 & 0.0568935577249066\end{array}$ $\begin{array}{lllll}0.4123666836054458 & 0.4641925305333190 & 0.0548650845981598\end{array}$ $\begin{array}{llll}0.4452945509175052 & 0.3626909632140345 & 0.0753400810944029\end{array}$ $\begin{array}{lllll}0.3665032478058705 & 0.4747086890753809 & 0.2959990624665788\end{array}$ $\begin{array}{lllll}0.2520880017368811 & 0.4189740957174526 & 0.2794161858056205\end{array}$ $\begin{array}{llll}0.3914049602019745 & 0.4246984081113973 & 0.4150340012459507\end{array}$ $\begin{array}{lllll}0.0438849568827215 & 0.3501784697301588 & 0.6822278610670678\end{array}$ $\begin{array}{lllll}0.0292157786609958 & 0.2407989275508120 & 0.6900756666100917\end{array}$ $\begin{array}{llll}0.1539735647446750 & 0.2831544939185648 & 0.6901791380905280\end{array}$ $\begin{array}{lllll}0.1992534467719848 & 0.2079313534329240 & 0.5678855516168276\end{array}$ $\begin{array}{llll}0.0306582844310827 & 0.2882863176666399 & 0.5641720621974491\end{array}$ $\begin{array}{lllll}0.0757716091517868 & 0.1872503404895843 & 0.5846861243283515\end{array}$ $\begin{array}{llllll}0.1792476612388697 & 0.1690408078313321 & 0.6931825549888877\end{array}$ $\begin{array}{lllll}0.1787855042172767 & 0.0561563902428777 & 0.6993294301060323\end{array}$ $\begin{array}{lllll}0.0466538681585100 & 0.1102776118039340 & 0.7141564262146032\end{array}$ $\begin{array}{lllll}0.1572578932832850 & 0.1055637683768123 & 0.5786459819560361\end{array}$ $\begin{array}{lllll}0.1485293552613246 & 0.1163783289307085 & 0.8101374727329261\end{array}$ $\begin{array}{llllll}0.0213000875813142 & 0.0680407752977101 & 0.8308604782380633\end{array}$ $\begin{array}{lllll}0.1389988400271349 & 0.0663442017305187 & 0.9368828242506473\end{array}$ 
$\begin{array}{llll}0.1040161096774580 & 0.1676647659318358 & 0.9200444194408219\end{array}$ $\begin{array}{lllll}0.0193525684561058 & 0.2884545009722175 & 0.8030372234231752\end{array}$ $\begin{array}{lllll}0.1443968360185412 & 0.2481669873629787 & 0.8014671901933744\end{array}$ $\begin{array}{llll}0.0996362776157945 & 0.3220110445514318 & 0.9240601737956714\end{array}$ $\begin{array}{lllll}0.0154843338733419 & 0.2418567775478992 & 0.9288213317226446\end{array}$ $\begin{array}{lllll}0.1181561979858388 & 0.3619654507156244 & 0.7988610871365407\end{array}$ $\begin{array}{lllll}0.2291893634386064 & 0.1429288781971094 & 0.9358164289167072\end{array}$ $\begin{array}{lllll}0.2533840283723707 & 0.1808040062154256 & 0.8099358397046820\end{array}$ $\begin{array}{lllll}0.2224400390450257 & 0.3446302391138992 & 0.9064927381365978\end{array}$ $\begin{array}{lllll}0.2961333133749159 & 0.3509273770269264 & 0.6806796229783700\end{array}$ $\begin{array}{lllll}0.2791281000474545 & 0.2417346281355447 & 0.6892009833646359\end{array}$ $\begin{array}{lllll}0.4048012387737190 & 0.2824001000762790 & 0.6899846628597346\end{array}$ $\begin{array}{lllll}0.4499707953749776 & 0.2075577354535948 & 0.5685725034367513\end{array}$ $\begin{array}{llll}0.2827132559307129 & 0.2883439874236581 & 0.5633589351567486\end{array}$ 0.32605592715334360 .18713453001086290 .5847534924630429 $\begin{array}{lllll}0.4292530255987569 & 0.1681315833459290 & 0.6932876465418092\end{array}$ $\begin{array}{lllll}0.4284444019862406 & 0.0553600061732897 & 0.6987407894891547\end{array}$ $\begin{array}{lllll}0.2960759418721046 & 0.1111874595273619 & 0.7134665808711994\end{array}$ $\begin{array}{llll}0.4073064111624293 & 0.1052377088006107 & 0.5783198016491615\end{array}$ $\begin{array}{llll}0.3981500514054960 & 0.1147599026473885 & 0.8098380142920059\end{array}$ $\begin{array}{lllll}0.2700457619304183 & 0.0690366592448394 & 0.8299297987623248\end{array}$ $\begin{array}{llll}0.3856836094244780 & 0.0668663979705685 & 0.9369017018849751\end{array}$ $\begin{array}{llll}0.3541274695129051 & 0.1689508216419583 & 0.9180035978965910\end{array}$ $\begin{array}{llll}0.2694433140498335 & 0.2902169621858067 & 0.8015679478097525\end{array}$ $\begin{array}{lllll}0.3941535388180463 & 0.2485293307586752 & 0.8013419816725585\end{array}$ $\begin{array}{llllll}0.3491223088375440 & 0.3245387305555744 & 0.9224703597800974\end{array}$ $\begin{array}{lllll}0.2662512695971709 & 0.2435946617865410 & 0.9275617155549812\end{array}$ $\begin{array}{lllll}0.3701272391576356 & 0.3630476583764140 & 0.7970447841487945\end{array}$ $\begin{array}{llll}0.4785216149884661 & 0.1412436949202725 & 0.9358421230885136\end{array}$ $\begin{array}{lllll}0.0037446020942611 & 0.1792866681839017 & 0.8111386687004868\end{array}$ $\begin{array}{lllll}0.4726319615025560 & 0.3437772949492097 & 0.9065055321464420\end{array}$ $\begin{array}{lllll}0.1488793219598847 & 0.4146935962972070 & 0.6820634279290626\end{array}$ $\begin{array}{llllll}0.0263704428510336 & 0.4610846423395383 & 0.6620030434935398\end{array}$ $\begin{array}{lllll}0.0685489344213828 & 0.3881232305137077 & 0.5564008859182022\end{array}$ $\begin{array}{lllll}0.1593047613141814 & 0.4639587949540019 & 0.5551088436436261\end{array}$ $\begin{array}{lllll}0.1943716918124643 & 0.3625928689382157 & 0.5733411468029802\end{array}$ $\begin{array}{lllll}0.1184549886215467 & 0.4747186213473008 & 0.7928157237764398\end{array}$ $\begin{array}{lllll}0.4998627559462614 & 0.4173467832039894 & 0.7739517338987021\end{array}$ $\begin{array}{lllll}0.1394327251582996 & 0.4251586616648958 & 0.9137769443047361\end{array}$ $\begin{array}{lllll}0.3993260192296208 & 0.4183413913108614 & 0.6782047981798068\end{array}$ $\begin{array}{lllll}0.2761404169060069 & 0.4624733544768757 & 0.6593888748024489\end{array}$ $\begin{array}{llll}0.3198660055022893 & 0.3885492486244790 & 0.5546642144935813\end{array}$ $\begin{array}{lllll}0.4224350707591705 & 0.4564731493892295 & 0.5491758020664822\end{array}$ $\begin{array}{lllll}0.4435826059766076 & 0.3557034574931326 & 0.5783942747970808\end{array}$ $\begin{array}{lllll}0.3672331672207192 & 0.4763026605919650 & 0.7903353281374833\end{array}$ $\begin{array}{llll}0.2520881569194541 & 0.4202610014641862 & 0.7763479398416264\end{array}$ $\begin{array}{llll}0.3925858445052003 & 0.4266319181444670 & 0.9117305792611463\end{array}$ $\begin{array}{lllll}0.6057752077620764 & 0.5950835645979091 & 0.4879862788279702\end{array}$ $\begin{array}{lllll}0.5459810480331537 & 0.3501763105798086 & 0.1829805421987615\end{array}$ $\begin{array}{lllll}0.5295450326681388 & 0.2409041252368710 & 0.1901722245337281\end{array}$ $\begin{array}{lllll}0.6547190194706393 & 0.2823075696514030 & 0.1913677862761242\end{array}$ $\begin{array}{lllll}0.6994936893691993 & 0.2076826433047548 & 0.0688692493801626\end{array}$ $\begin{array}{lllll}0.5331590071304424 & 0.2881633400307058 & 0.0646421147798000\end{array}$ $\begin{array}{llll}0.5760327584007520 & 0.1866638984804510 & 0.0852510778320332\end{array}$ $\begin{array}{lllll}0.6795441437760146 & 0.1681923640576024 & 0.1938847134369854\end{array}$ $\begin{array}{lllll}0.6790155839347083 & 0.0553013125387701 & 0.1993703516858745\end{array}$ $\begin{array}{lllll}0.5463589412337918 & 0.1104484071591649 & 0.2135754483275444\end{array}$ $\begin{array}{lllll}0.65771102641298019 & 0.1051454448886573 & 0.0790535155810650\end{array}$ $\begin{array}{lllll}0.6484050985085003 & 0.1148084767385260 & 0.3102320171830427\end{array}$ $\begin{array}{llll}0.5204526102094807 & 0.0677725075800283 & 0.3296020123024895\end{array}$ $\begin{array}{llll}0.6368777456009070 & 0.0657019896770952 & 0.4370574855290986\end{array}$ $\begin{array}{lllll}0.6036553904925440 & 0.1674071107085840 & 0.4191657289746472\end{array}$ $\begin{array}{lllll}0.5196855641368823 & 0.2884084522750736 & 0.3030632007952849\end{array}$ $\begin{array}{lllll}0.6443825512419237 & 0.2471798291573555 & 0.3023240488530168\end{array}$ $\begin{array}{lllll}0.5993694819782590 & 0.3215881604418692 & 0.4247990784710783\end{array}$ $\begin{array}{lllll}0.5150360744085347 & 0.2416393511837484 & 0.4284792193785452\end{array}$ $\begin{array}{llll}0.6195579261613888 & 0.3613219535241015 & 0.2999963654761073\end{array}$ $\begin{array}{lllll}0.7285091800921851 & 0.1410461806877718 & 0.4363560773378042\end{array}$ $\begin{array}{lllll}0.7534107533318437 & 0.1793723951964035 & 0.3108904581743835\end{array}$ $\begin{array}{lllll}0.7228203066981566 & 0.3428410874846372 & 0.4086291329764823\end{array}$ $\begin{array}{lllll}0.7955377199385317 & 0.3500716274368599 & 0.1826502615139518\end{array}$ $\begin{array}{llll}0.7794689995436987 & 0.2409357290196826 & 0.1904376859121170\end{array}$ $\begin{array}{llll}0.9046118958432230 & 0.2822338162901303 & 0.1912548997673333\end{array}$ 
$\begin{array}{llll}0.9494784592813261 & 0.2074053279844544 & 0.0689046939808660\end{array}$ $\begin{array}{llllll}0.7834610905987730 & 0.2878497342274374 & 0.0647774499189310\end{array}$ $\begin{array}{lllll}0.8260300787939371 & 0.1862881849370696 & 0.0857723139038954\end{array}$ $\begin{array}{llll}0.9295449911967646 & 0.1680314604468681 & 0.1939953318348385\end{array}$ $\begin{array}{llll}0.9291459462110189 & 0.0551358207580102 & 0.1996862257263114\end{array}$ $\begin{array}{lllll}0.7963978962313183 & 0.1102188044385538 & 0.2140237806842137\end{array}$ $\begin{array}{lllll}0.9078543379332988 & 0.1048266294013739 & 0.0792858652063786\end{array}$ $\begin{array}{lllll}0.8985454193831638 & 0.1146998167588312 & 0.3104349167768884\end{array}$ $\begin{array}{llll}0.7705064680733056 & 0.0679124025831268 & 0.3302939195488309\end{array}$ $\begin{array}{lllll}0.8874195743713913 & 0.0654940970168364 & 0.4374109110939296\end{array}$ $\begin{array}{lllll}0.8538032633909064 & 0.1670537900784477 & 0.4194861466391200\end{array}$ $\begin{array}{lllll}0.7695333072473708 & 0.2886175757316961 & 0.3033705949124327\end{array}$ $\begin{array}{lllll}0.8942907281413034 & 0.2471358821690599 & 0.3022520731873042\end{array}$ $\begin{array}{llll}0.8494925749697971 & 0.3219225216793623 & 0.4247996592568452\end{array}$ $\begin{array}{llll}0.7656973285573361 & 0.2415562022455422 & 0.4289313536326291\end{array}$ $\begin{array}{lllll}0.8693829006746598 & 0.3612963540915119 & 0.2996067557619475\end{array}$ $\begin{array}{llll}0.9787628128830861 & 0.1410595633655365 & 0.4364751536601682\end{array}$ $\begin{array}{lllll}0.5032325593400703 & 0.1793765336256398 & 0.3105118935175128\end{array}$ $\begin{array}{lllll}0.9728666141342328 & 0.3432419524906836 & 0.4078769471911240\end{array}$ $\begin{array}{llll}0.6510976869765561 & 0.4141836423184301 & 0.1835009235092580\end{array}$ $\begin{array}{lllll}0.5295256871531030 & 0.4613108005063284 & 0.1627366649052172\end{array}$ $\begin{array}{lllll}0.5704975403375482 & 0.3884388540609955 & 0.0571973621764289\end{array}$ $\begin{array}{lllll}0.6617937022076387 & 0.4641401493340773 & 0.0566854890442873\end{array}$ $\begin{array}{llll}0.6952844853202380 & 0.3624701012172089 & 0.0737861274175696\end{array}$ $\begin{array}{lllll}0.6208076887835315 & 0.4742993805866708 & 0.2942050013671199\end{array}$ $\begin{array}{lllll}0.0028259640971474 & 0.4198218255950568 & 0.2794847596490726\end{array}$ $\begin{array}{lllll}0.6410299798671639 & 0.4241529999004717 & 0.4155475750861926\end{array}$ $\begin{array}{lllll}0.9006606039574082 & 0.4144324727374940 & 0.1831348417523615\end{array}$ $\begin{array}{lllll}0.7787359047470011 & 0.4614887488329080 & 0.1628482416547081\end{array}$ $\begin{array}{llllll}0.8204614012177212 & 0.3883443341390574 & 0.0570120995418055\end{array}$ $\begin{array}{lllll}0.9117472942736154 & 0.4638417354383982 & 0.0561723568659430\end{array}$ $\begin{array}{lllll}0.9451772371626133 & 0.3621555427061416 & 0.0737916012593597\end{array}$ $\begin{array}{lllll}0.8700906704747774 & 0.4742555906156656 & 0.2939762301601689\end{array}$ $\begin{array}{lllll}0.7527777393080520 & 0.4195813796398569 & 0.2794064172247248\end{array}$ $\begin{array}{llll}0.8906725374650624 & 0.4245201437632929 & 0.4143190729077874\end{array}$ $\begin{array}{llll}0.6824074854901532 & 0.5367665515960841 & 0.7200337735479727\end{array}$ $\begin{array}{lllll}0.5464867900838735 & 0.3489561009662450 & 0.6828740474315158\end{array}$ $\begin{array}{lllll}0.5296494613935417 & 0.2402294251187202 & 0.6903278374448590\end{array}$ $\begin{array}{lllll}0.6549468505594396 & 0.2809052771807389 & 0.6921339400902846\end{array}$ $\begin{array}{llll}0.6993452434383740 & 0.2066160556573325 & 0.5695513960550513\end{array}$ $\begin{array}{lllll}0.5371784731354458 & 0.2860257783208313 & 0.5650606516735512\end{array}$ $\begin{array}{llll}0.5762556840308496 & 0.1839978390482628 & 0.5867651255246539\end{array}$ $\begin{array}{llll}0.6802409154238727 & 0.1666900263033606 & 0.6945393924685651\end{array}$ $\begin{array}{lllll}0.6793500626058401 & 0.0539224258533645 & 0.7002251118153571\end{array}$ $\begin{array}{lllll}0.5462575125154741 & 0.1096921433333595 & 0.7140820317646662\end{array}$ $\begin{array}{lllll}0.6591878151905526 & 0.1035491594837785 & 0.5793694219983357\end{array}$ $\begin{array}{llll}0.6485405921321538 & 0.1138135401680505 & 0.8105224739201909\end{array}$ $\begin{array}{llll}0.5199391629119604 & 0.0677051373105502 & 0.8299489813189140\end{array}$ $\begin{array}{lllll}0.6357146496249462 & 0.0659067527892517 & 0.9375603634212039\end{array}$ $\begin{array}{lllll}0.6035553109076565 & 0.1678278128025842 & 0.9182223255375983\end{array}$ $\begin{array}{lllll}0.5198720485499676 & 0.2884749382667799 & 0.8029929918951973\end{array}$ $\begin{array}{lllll}0.6446765448556021 & 0.2463714623203443 & 0.8030294237941832\end{array}$ $\begin{array}{lllll}0.5993115045835423 & 0.3221154104551108 & 0.9251606021097237\end{array}$ $\begin{array}{lllll}0.5150188109195633 & 0.2420379376755390 & 0.9283353985123748\end{array}$ $\begin{array}{lllll}0.6191230010597868 & 0.3609516165274007 & 0.8006671359064798\end{array}$ $\begin{array}{lllll}0.7281838070868660 & 0.1405073152214866 & 0.9368385077758760\end{array}$ $\begin{array}{lllll}0.7536226398595832 & 0.1783904356258559 & 0.8116124905428643\end{array}$ $\begin{array}{lllll}0.7229062858082574 & 0.3424258717584673 & 0.9078220709744658\end{array}$ $\begin{array}{lllll}0.7948462117812910 & 0.3486963242595321 & 0.6833805980632941\end{array}$ $\begin{array}{llll}0.7796585345247488 & 0.2396906861239179 & 0.6909211233381911\end{array}$ $\begin{array}{lllll}0.9043953313928934 & 0.2817743322253142 & 0.6914580435895309\end{array}$ $\begin{array}{llllll}0.9488435153968855 & 0.2070633985611907 & 0.5689631484721458\end{array}$ $\begin{array}{lllll}0.7838565594190458 & 0.2863584676559936 & 0.5652465092119782\end{array}$ $\begin{array}{lllll}0.8258955333802811 & 0.1847050062696369 & 0.5862353457920664\end{array}$ $\begin{array}{llll}0.9298450711564479 & 0.1675627461368125 & 0.6942604881031527\end{array}$ $\begin{array}{llll}0.9296124629219052 & 0.0547918126810656 & 0.7003016465162532\end{array}$ $\begin{array}{llll}0.7968354494973298 & 0.1087920908988657 & 0.7149258618162884\end{array}$ $\begin{array}{lllll}0.9088322641857245 & 0.1041114257669139 & 0.5794710925188854\end{array}$ $\begin{array}{lllll}0.8988559594159361 & 0.1146650617254441 & 0.8108805655090507\end{array}$ $\begin{array}{llll}0.7708951580631425 & 0.0669861969089060 & 0.8313397971250658\end{array}$ $\begin{array}{lllll}0.8883023049221211 & 0.0649774125219701 & 0.9376347103859211\end{array}$ $\begin{array}{lllll}0.8537930213024413 & 0.1663555234516877 & 0.9203775786968637\end{array}$ $\begin{array}{llll}0.7699501217999690 & 0.2873022850915651 & 0.8038564491624350\end{array}$ 
$\begin{array}{llll}0.8946060985821589 & 0.2465914714099958 & 0.8025935968262661\end{array}$ $\begin{array}{llllll}0.8495759937929930 & 0.3216365625300791 & 0.9248967413748077\end{array}$ $\begin{array}{llll}0.7657485100632839 & 0.2409895979966565 & 0.9295194298884929\end{array}$ $\begin{array}{llll}0.8693142445566737 & 0.3608391119632703 & 0.7996988617372939\end{array}$ $\begin{array}{lllll}0.9789313573697687 & 0.1411926715652610 & 0.9367824706671013\end{array}$ $\begin{array}{llll}0.5025779658439415 & 0.1795783907241783 & 0.8103256046103195\end{array}$ $\begin{array}{lllll}0.9729331546061599 & 0.3431321260479974 & 0.9079385733395131\end{array}$ $\begin{array}{llll}0.6486663939027449 & 0.4130947938332034 & 0.6846352210708256\end{array}$ $\begin{array}{llll}0.5319474175929710 & 0.4662437995826988 & 0.6616636495620900\end{array}$ $\begin{array}{lllll}0.5676481454980794 & 0.3896061086539750 & 0.5600618062464635\end{array}$ $\begin{array}{lllll}0.6688827498596421 & 0.4599683752793644 & 0.5597902078522990\end{array}$ $\begin{array}{llllll}0.6924313274238943 & 0.3577943813813037 & 0.5767791081794857\end{array}$ $\begin{array}{lllll}0.6116289158824229 & 0.4744836838723867 & 0.7910563304867690\end{array}$ $\begin{array}{llll}0.0012754141681228 & 0.4197779803015811 & 0.7790934682423090\end{array}$ $\begin{array}{lllll}0.6427599235878345 & 0.4249989309133848 & 0.9141244103482287\end{array}$ $\begin{array}{lllll}0.8977962039079643 & 0.4151984246938412 & 0.6830757743343218\end{array}$ $\begin{array}{lllll}0.7751058317830467 & 0.4601505049273257 & 0.6633523224867653\end{array}$ $\begin{array}{lllll}0.8165865640937129 & 0.3885770529021788 & 0.5586170911679740\end{array}$ $0.90829838679666090 .4636539015629742 \quad 0.5541679770061918$ $\begin{array}{llll}0.9425231794362372 & 0.3628302986140119 & 0.5744795241145247\end{array}$ $\begin{array}{lllll}0.8651430230905601 & 0.4744440283398038 & 0.7934071530397981\end{array}$ $\begin{array}{lllll}0.7524445761285571 & 0.4176092871851467 & 0.7826493923985265\end{array}$ $\begin{array}{lllll}0.8909855744758951 & 0.4244203156458391 & 0.9140387771203913\end{array}$ $\begin{array}{lllll}0.2126288590358743 & 0.2345128530021800 & 0.2481412772574746\end{array}$ $\begin{array}{lllll}0.0865844890070998 & 0.2955378904034497 & 0.2451014545855079\end{array}$ $\begin{array}{lllll}0.2218288741024540 & 0.1134839827780159 & 0.2637511949654754\end{array}$ $\begin{array}{lllll}0.4622633214018060 & 0.2342584327018588 & 0.2480816909262747\end{array}$ $\begin{array}{lllll}0.3369293521965157 & 0.2963164908597382 & 0.2450578584510461\end{array}$ $\begin{array}{lllll}0.4717341003300584 & 0.1131901844329067 & 0.2637081667885925\end{array}$ $\begin{array}{lllll}0.0772583977720817 & 0.4163856667624936 & 0.2290043488165797\end{array}$ $\begin{array}{lllll}0.3273569846210325 & 0.4175536689960733 & 0.2299247719526903\end{array}$ $\begin{array}{lllll}0.2122557584113857 & 0.2352764319734246 & 0.7472412588748499\end{array}$ $\begin{array}{lllll}0.0859873398273868 & 0.2960009045447273 & 0.7444600420944510\end{array}$ $\begin{array}{lllll}0.2216240527050699 & 0.1143482743262372 & 0.7636732513149371\end{array}$ $\begin{array}{lllll}0.4618599836230034 & 0.2342669222770105 & 0.7476162686339743\end{array}$ $\begin{array}{lllll}0.3367860766344335 & 0.2967003275121420 & 0.7433557663440675\end{array}$ $\begin{array}{lllll}0.4714780681120921 & 0.1131054952018634 & 0.7637980334879000\end{array}$ $\begin{array}{lllll}0.0755221035445740 & 0.4167049171793737 & 0.7284063455995812\end{array}$ $\begin{array}{llll}0.3264033139273350 & 0.4180768250364543 & 0.7254380236502918\end{array}$ $\begin{array}{lllll}0.7123626477460938 & 0.2340790204630688 & 0.2484766456871361\end{array}$ $\begin{array}{llll}0.5866606158754183 & 0.2955847361553362 & 0.2453136636437862\end{array}$ $\begin{array}{llll}0.7218427607919068 & 0.1130912056906682 & 0.2641889015751640\end{array}$ $\begin{array}{lllll}0.9623894460753490 & 0.2340258196605705 & 0.2484290871696327\end{array}$ $\begin{array}{lllll}0.8366465412405525 & 0.2955330738912819 & 0.2452449914204472\end{array}$ $\begin{array}{lllll}0.9719559861416907 & 0.1130654737182966 & 0.2642781693666156\end{array}$ $\begin{array}{lllll}0.5774869597345577 & 0.4165507676231976 & 0.2296718529535751\end{array}$ $\begin{array}{lllll}0.8272409943756667 & 0.4164898345014328 & 0.2292354296290024\end{array}$ $\begin{array}{lllll}0.7128280878680486 & 0.2329350715729052 & 0.7491380719748453\end{array}$ $\begin{array}{lllll}0.5870202647000061 & 0.2941265243905811 & 0.7456444315498000\end{array}$ $\begin{array}{llllll}0.7222539501771127 & 0.1119651887100064 & 0.7650066670103307\end{array}$ $\begin{array}{lllll}0.9625241330401829 & 0.2337762757337523 & 0.7484836956406234\end{array}$ $\begin{array}{lllll}0.8366938335177837 & 0.2947443040880712 & 0.7457720963035845\end{array}$ $\begin{array}{lllll}0.9723192051721316 & 0.1129303414880025 & 0.7647369757550209\end{array}$ $\begin{array}{lllll}0.5784474553215213 & 0.4134794628797210 & 0.7325525245864749\end{array}$ $\begin{array}{llll}0.8253084332651558 & 0.4154578207654939 & 0.7293694116261771\end{array}$ $\begin{array}{lllll}0.0226580212718377 & 0.2311252215374201 & 0.1019871559458048\end{array}$ $\begin{array}{lllll}0.1534246147597373 & 0.1685678414762394 & 0.1054232327171804\end{array}$ $\begin{array}{lllll}0.1458702230489971 & 0.3615384253556363 & 0.3876134348300377\end{array}$ $\begin{array}{lllll}0.1548233582379851 & 0.1213812469715943 & 0.3975435240683391\end{array}$ $\begin{array}{llll}0.0254563151944000 & 0.1809421947383822 & 0.3997635031946655\end{array}$ $\begin{array}{lllll}0.0262955494880948 & 0.2982964177010169 & 0.3915815784617739\end{array}$ $\begin{array}{lllll}0.0235445825439653 & 0.3485081741855724 & 0.0936794248827138\end{array}$ $\begin{array}{lllll}0.2727535936626120 & 0.2321003718027138 & 0.1016895789460934\end{array}$ $\begin{array}{lllll}0.4027949377777341 & 0.1683680660940816 & 0.1054521456834200\end{array}$ $\begin{array}{llll}0.3959593430948385 & 0.3626467611145054 & 0.3875304383510243\end{array}$ $\begin{array}{lllll}0.4045309582997303 & 0.1211748961081330 & 0.3973199632018245\end{array}$ $\begin{array}{llll}0.2755386437129078 & 0.1812333035980065 & 0.3992804707171104\end{array}$ $\begin{array}{lllll}0.2766421661328766 & 0.2986586941497278 & 0.3912769147857769\end{array}$ $\begin{array}{lllll}0.2740917066343374 & 0.3494510931764478 & 0.0936128193232814\end{array}$ $\begin{array}{lllll}0.1442600412976069 & 0.4083109535038385 & 0.0952544832198971\end{array}$ $\begin{array}{lllll}0.3946157174398260 & 0.4095018238683675 & 0.0959908091122444\end{array}$ $\begin{array}{llll}0.0217581317167686 & 0.2314797090069309 & 0.6015250288652096\end{array}$ $\begin{array}{llll}0.1526447709822179 & 0.1686782309464859 & 0.6050569778703029\end{array}$ 
$\begin{array}{llll}0.1451943033865070 & 0.3623013538113158 & 0.8869225954925921\end{array}$ $\begin{array}{lllll}0.1553854116278100 & 0.1222438888802856 & 0.8977254477673904\end{array}$ $\begin{array}{lllll}0.0256253779334880 & 0.1810355874812366 & 0.9000324032247974\end{array}$ $\begin{array}{lllll}0.0264043193254679 & 0.2983451200601491 & 0.8914268685364308\end{array}$ $\begin{array}{lllll}0.0210239901966568 & 0.3491588441976001 & 0.5934302676604861\end{array}$ $\begin{array}{lllll}0.2722814822284518 & 0.2319137005258911 & 0.6008260215716734\end{array}$ $\begin{array}{llllll}0.4027710109594933 & 0.1682559212852071 & 0.6051588137481987\end{array}$ $\begin{array}{lllll}0.3961564936124361 & 0.3634892670923245 & 0.8853226983293415\end{array}$ $\begin{array}{llll}0.4042330894926337 & 0.1218695384664473 & 0.8972166975782023\end{array}$ $\begin{array}{llll}0.2757123843044878 & 0.1826911609950099 & 0.8987321875902592\end{array}$ $\begin{array}{lllll}0.2762598565086494 & 0.3001999262491810 & 0.8899325785447892\end{array}$ $\begin{array}{lllll}0.2731243347160046 & 0.3494532271300305 & 0.5918596614529659\end{array}$ $\begin{array}{lllll}0.1426428740787969 & 0.4082756105741188 & 0.5945630934909011\end{array}$ $\begin{array}{llll}0.3954165062412318 & 0.4081351054260354 & 0.5903825235754719\end{array}$ $\begin{array}{lllll}0.5225449983648794 & 0.2315327511117637 & 0.1017445926257746\end{array}$ $\begin{array}{lllll}0.6529407529581949 & 0.1681776173178634 & 0.1057837806057343\end{array}$ $\begin{array}{llll}0.6458309085311565 & 0.3613806043786800 & 0.3880834106185211\end{array}$ $\begin{array}{llllll}0.6544952342161028 & 0.1211773917800107 & 0.3976563928286650\end{array}$ $\begin{array}{llll}0.5252061395370287 & 0.1809205057437572 & 0.3993201105552240\end{array}$ $\begin{array}{llll}0.5259798951865408 & 0.2983298989759313 & 0.3913200517897003\end{array}$ $\begin{array}{lllll}0.5236021181840320 & 0.3489657045883732 & 0.0942072399585931\end{array}$ $\begin{array}{llllll}0.7726625078050258 & 0.2313092137277875 & 0.1020502209930013\end{array}$ $\begin{array}{lllll}0.9030205776278882 & 0.1678793253609468 & 0.1058856350939050\end{array}$ $\begin{array}{lllll}0.8958364162308579 & 0.3615994676155458 & 0.3877618244655038\end{array}$ $\begin{array}{lllll}0.9048086544101507 & 0.1209772476736294 & 0.3978988241490425\end{array}$ $\begin{array}{lllll}0.7754590729552444 & 0.1807614852247490 & 0.3997279799085703\end{array}$ $\begin{array}{lllll}0.7763321881813968 & 0.2982085094013641 & 0.3917728806319943\end{array}$ $\begin{array}{lllll}0.7735753957432935 & 0.3487318582903133 & 0.0937875193076805\end{array}$ $\begin{array}{llll}0.6443750620180578 & 0.4085660417208496 & 0.0958815717708654\end{array}$ $\begin{array}{llll}0.8944412843743073 & 0.4083425274666879 & 0.0956292930488674\end{array}$ $\begin{array}{lllll}0.5236371435777183 & 0.2298333572855449 & 0.6021806981225745\end{array}$ $\begin{array}{lllll}0.6536337603048921 & 0.1663884213814760 & 0.6064908435263368\end{array}$ $\begin{array}{lllll}0.6461620851211849 & 0.3617465208162796 & 0.8888482077095045\end{array}$ $\begin{array}{lllll}0.6541321613480511 & 0.1209152979358200 & 0.8978215270524570\end{array}$ $\begin{array}{llllll}0.5250125468894046 & 0.1812640589147913 & 0.8990961474679693\end{array}$ $\begin{array}{lllll}0.5260893973579284 & 0.2987022151492588 & 0.8913134428487391\end{array}$ $\begin{array}{lllll}0.5228295672618235 & 0.3458485256076749 & 0.5941712678329818\end{array}$ $\begin{array}{lllll}0.7728018592599275 & 0.2298422672857925 & 0.6026195729036445\end{array}$ $\begin{array}{llll}0.9032105196143247 & 0.1670443425268758 & 0.6061744669344344\end{array}$ $\begin{array}{llllll}0.8959051089203637 & 0.3613405519390624 & 0.8877847273276567\end{array}$ $\begin{array}{llll}0.9051452171979976 & 0.1207135425207999 & 0.8983987355485546\end{array}$ $\begin{array}{lllll}0.7754837633449411 & 0.1800846822081838 & 0.9004923667382050\end{array}$ $\begin{array}{lllll}0.7765944692811567 & 0.2975006891838017 & 0.8920750949879990\end{array}$ $\begin{array}{lllll}0.7719245446008574 & 0.3466269673001771 & 0.5947747151194387\end{array}$ $\begin{array}{lllll}0.6438421427976257 & 0.4060862365083414 & 0.5978545890944107\end{array}$ $\begin{array}{llll}0.8911511222614729 & 0.4091219953408530 & 0.5957148977884900\end{array}$

TpT :

H 155 C $20 \quad$ N $4 \quad 0254$ Al $32 \quad \mathrm{Si}$

1.00000000000000

$20.2970019999999991 \quad 0.0000000000000000 \quad-3.7471719999999999$

$0.0905600000000000 \quad 34.9924600000000012-0.7207700000000000$

$\begin{array}{llll}0.0000000000000000 & 0.0000000000000000 & 18.6939999999999991\end{array}$

$\mathrm{H} \quad \mathrm{C} \quad \mathrm{N} \quad \mathrm{O}$ Al $\mathrm{Si} \mathrm{P}$

$\begin{array}{lllllll}155 & 20 & 4 & 254 & 32 & 64 & 1\end{array}$

Direct

$\begin{array}{llll}0.5780599639334379 & 0.5306123929486046 & 0.7504850432000878\end{array}$ $\begin{array}{lllll}0.7130265269889196 & 0.5468846073963632 & 0.7590483340312939\end{array}$ $\begin{array}{llll}0.6470271308409129 & 0.5793854599557083 & 0.7624179695426714\end{array}$ $\begin{array}{llll}0.6756780814668638 & 0.5891153524090964 & 0.6482854530830736\end{array}$ $\begin{array}{lllll}0.6885968572454153 & 0.5206953754565237 & 0.5102899719408838\end{array}$ $\begin{array}{lllll}0.6735983242683371 & 0.4776954531091288 & 0.6663117553802941\end{array}$ $\begin{array}{lllll}0.5487840832866540 & 0.4140649639144184 & 0.4576967128041283\end{array}$ $\begin{array}{llll}0.5609805810828132 & 0.5324343531776250 & 0.6272385135407595\end{array}$ $\begin{array}{lllll}0.5574441800561989 & 0.5370603275205400 & 0.4957186258551236\end{array}$ $\begin{array}{lllll}0.5928986577771823 & 0.6019663153935546 & 0.4988579715553805\end{array}$ $\begin{array}{llll}0.4556602960571866 & 0.6002188008939165 & 0.5141861476405103\end{array}$ $\begin{array}{lllll}0.3703808118842463 & 0.6061120000134567 & 0.5195626527009654\end{array}$ $\begin{array}{llll}0.3914800045602731 & 0.5616052881540853 & 0.4207989715256302\end{array}$ $\begin{array}{lllll}0.4410537882882053 & 0.4719965480182429 & 0.4313471938918933\end{array}$ $\begin{array}{lllll}0.4402273892494810 & 0.5052486088921190 & 0.6140526879212560\end{array}$ $\begin{array}{lllll}0.4176159674661227 & 0.3663623711584477 & 0.5634610155964230\end{array}$ $\begin{array}{llll}0.3251072080409881 & 0.5261253762362141 & 0.5371033288250820\end{array}$ 
$\begin{array}{llll}0.3131377296191983 & 0.4684334396529035 & 0.4554486258486867\end{array}$ $\begin{array}{lllll}0.2980383826790338 & 0.5141977432422515 & 0.3520047870097381\end{array}$ $\begin{array}{lllll}0.2361214162924161 & 0.5440133026458417 & 0.4574483561454362\end{array}$ $\begin{array}{lllll}0.4571187292873482 & 0.4966632023682205 & 0.7449505795185396\end{array}$ $\begin{array}{lllll}0.4187443412263439 & 0.4551857601039171 & 0.7728741825533347\end{array}$ $\begin{array}{llll}0.5039011566180712 & 0.4545052453262562 & 0.7711582544320785\end{array}$ $\begin{array}{llll}0.6708726352409305 & 0.4236898210864685 & 0.7413729183577524\end{array}$ $\begin{array}{lllll}0.5966027006264188 & 0.3969746382609615 & 0.7194456943349689\end{array}$ $\begin{array}{lllll}0.6696498609755888 & 0.3788889572987466 & 0.6930505462501579\end{array}$ $\begin{array}{llllll}0.4990801390379949 & 0.6450418027343314 & 0.7108913259540502\end{array}$ $\begin{array}{lllll}0.0954568271385631 & 0.0394893560338572 & 0.4087815729598384\end{array}$ $\begin{array}{lllll}0.3443357666360936 & 0.0402141482885124 & 0.4076286260021898\end{array}$ $\begin{array}{lllll}0.0517615237522111 & 0.0998188124289633 & 0.1816892700281913\end{array}$ $\begin{array}{llll}0.1711958024295203 & 0.2215887059384096 & 0.1618840105845548\end{array}$ $\begin{array}{llll}0.1305396334435019 & 0.1578280839140969 & 0.3325231702859107\end{array}$ $\begin{array}{lllll}0.2442407071290459 & 0.0288880380029320 & 0.3428016458756786\end{array}$ $\begin{array}{llllll}0.1724419218265653 & 0.0487426508947198 & 0.1464495762350682\end{array}$ $\begin{array}{lllll}0.3013671570444408 & 0.1005842333614760 & 0.1811035761888131\end{array}$ $\begin{array}{llll}0.4205864055111932 & 0.2213682648233338 & 0.1595071272847743\end{array}$ $\begin{array}{lllll}0.3805777554654661 & 0.1589055588878031 & 0.3328180159233949\end{array}$ $\begin{array}{lllll}0.4932818104492118 & 0.0287990341317336 & 0.3412807853505473\end{array}$ $\begin{array}{lllll}0.4218397415904268 & 0.0491031531488538 & 0.1453606150201482\end{array}$ $\begin{array}{lllll}0.0576760330374903 & 0.3495105720520552 & 0.1487256677774927\end{array}$ $\begin{array}{lllll}0.2496131329101784 & 0.2791852656871636 & 0.3126522530904371\end{array}$ $\begin{array}{llllll}0.1296690735328979 & 0.3300613025082487 & 0.3461434123665433\end{array}$ $\begin{array}{lllll}0.3046751497678281 & 0.3510679084113498 & 0.1505819964224526\end{array}$ $\begin{array}{lllll}0.4983284238121485 & 0.2803254165975826 & 0.3133468233755286\end{array}$ $\begin{array}{llll}0.3770812106995728 & 0.3300057451053351 & 0.3481141513595181\end{array}$ $\begin{array}{lllll}0.2058067967795814 & 0.3389858416568089 & 0.0843176969275199\end{array}$ $\begin{array}{llllll}0.4563312861975711 & 0.3400805401051164 & 0.0825835399773861\end{array}$ $\begin{array}{lllll}0.1544839925136142 & 0.3133772733023754 & 0.4650625553670951\end{array}$ $\begin{array}{lllll}0.3974840199763521 & 0.3162938327441499 & 0.4678848496040468\end{array}$ $\begin{array}{lllll}0.1485483185850521 & 0.0653287448690446 & 0.0279283139790990\end{array}$ $\begin{array}{lllll}0.3956078463576948 & 0.0657338005318156 & 0.0268892427995927\end{array}$ $\begin{array}{lllll}0.1669183772697554 & 0.3431965524141962 & 0.2838297006187602\end{array}$ $\begin{array}{lllll}0.4099268420497015 & 0.3482130799141139 & 0.2849049549624742\end{array}$ $\begin{array}{lllll}0.1361037198502978 & 0.0346555141591664 & 0.2090328513303526\end{array}$ $\begin{array}{lllll}0.3861104525147627 & 0.0347736918173873 & 0.2082170329610560\end{array}$ $\begin{array}{llll}0.2118584641553754 & 0.2968866660445953 & 0.2371073818326279\end{array}$ $\begin{array}{lllll}0.4620366531489687 & 0.2991260495995309 & 0.2380949842095770\end{array}$ $0.3388782135639754 \quad 0.08135611157103750 .2550768061661540$ $\begin{array}{lllll}0.0893581024649796 & 0.0810092118294682 & 0.2562168187997806\end{array}$ $\begin{array}{lllll}0.0976210871876546 & 0.0393861071721094 & 0.9094787143878234\end{array}$ $\begin{array}{lllll}0.3424208971277361 & 0.0409327005284626 & 0.9072152358001527\end{array}$ $\begin{array}{lllll}0.0517813141031385 & 0.0994107132611528 & 0.6816214630363638\end{array}$ $\begin{array}{lllll}0.1691072714571953 & 0.2200485625445406 & 0.6573510692461778\end{array}$ $\begin{array}{lllll}0.1302821869784724 & 0.1576615719374364 & 0.8309843265353326\end{array}$ $\begin{array}{lllll}0.2438595099114932 & 0.0289399134743413 & 0.8429863716248536\end{array}$ $\begin{array}{lllll}0.1717408448848566 & 0.0477731403094190 & 0.6478278402746235\end{array}$ $\begin{array}{lllll}0.3016531083815984 & 0.0999580992680123 & 0.6811591753587868\end{array}$ $\begin{array}{lllll}0.4184898530018579 & 0.2200423755719676 & 0.6552111894644452\end{array}$ $\begin{array}{llll}0.3807635460494081 & 0.1595191658110455 & 0.8327480036442404\end{array}$ $\begin{array}{lllll}0.4926075205967281 & 0.0286309555975031 & 0.8415359005421591\end{array}$ $\begin{array}{lllll}0.4224086899503137 & 0.0489638455255125 & 0.6448673759132982\end{array}$ $\begin{array}{llll}0.0561021674234058 & 0.3485668414251712 & 0.6462979115393594\end{array}$ $\begin{array}{lllll}0.2510036054515642 & 0.2797451921812723 & 0.8076921541662081\end{array}$ $\begin{array}{lllll}0.1287176542498589 & 0.3302932873929834 & 0.8457393441510285\end{array}$ $\begin{array}{lllll}0.3064889283818321 & 0.3454926657042617 & 0.6328400752959948\end{array}$ $\begin{array}{lllll}0.4952114426681422 & 0.2769505738816459 & 0.8075408658722264\end{array}$ $\begin{array}{llllll}0.3854705684513943 & 0.3348835260539510 & 0.8368807031185570\end{array}$ $0.2067506803774312 \quad 0.3373045242217634 \quad 0.5849714755018242$ $\begin{array}{llll}0.5470600325811240 & 0.3468324545293815 & 0.6303601815332424\end{array}$ $\begin{array}{llll}0.1531885526785896 & 0.3136502501508045 & 0.9645921462590261\end{array}$ $\begin{array}{llll}0.4053705813577856 & 0.3153751824896932 & 0.9612291359839866\end{array}$ $\begin{array}{lllll}0.1465666297209511 & 0.0643875042629478 & 0.5283032212411948\end{array}$ $\begin{array}{lllll}0.3977618972118270 & 0.0655611347921544 & 0.5265237778292448\end{array}$ $\begin{array}{llll}0.1653067420847932 & 0.3438736354260805 & 0.7830392083433997\end{array}$ $\begin{array}{llllll}0.3993155173826844 & 0.3570560681277264 & 0.7643827232164070\end{array}$ $\begin{array}{lllll}0.1363395528677444 & 0.0341420769623838 & 0.7112887898121086\end{array}$ 0.38513887881963290 .03501562678284240 .7067931927689983 $\begin{array}{lllll}0.2114932180036079 & 0.2979825576414131 & 0.7339882245298984\end{array}$ $\begin{array}{llllll}0.4591169274109101 & 0.2966524720036578 & 0.7326338121167438\end{array}$ $\begin{array}{llll}0.3389269329278525 & 0.0809788996496536 & 0.7556693015068360\end{array}$ 
$\begin{array}{llll}0.0898181705642736 & 0.0812425944334545 & 0.7566684653274789\end{array}$ $\begin{array}{llll}0.5912348360516008 & 0.0410564108601668 & 0.4074332380299345\end{array}$ $\begin{array}{lllll}0.8444034749769445 & 0.0392834592148399 & 0.4095443083622993\end{array}$ $\begin{array}{lllll}0.5516252047792414 & 0.1014707288609544 & 0.1822402744375146\end{array}$ $\begin{array}{llll}0.6702193167450369 & 0.2201824918038528 & 0.1587800583238862\end{array}$ $\begin{array}{lllll}0.6307737849447700 & 0.1591008399669289 & 0.3347182921984508\end{array}$ $\begin{array}{lllll}0.7434714779801731 & 0.0294122493239236 & 0.3449497483497400\end{array}$ $\begin{array}{lllll}0.6712353816178389 & 0.0486912050225044 & 0.1461929012079425\end{array}$ $\begin{array}{llll}0.8017243644650388 & 0.1004047617861943 & 0.1821813143559488\end{array}$ $\begin{array}{lllll}0.9212436399486607 & 0.2206264580438488 & 0.1616364867764686\end{array}$ $\begin{array}{lllll}0.8805880688993788 & 0.1578230862127262 & 0.3339888349635311\end{array}$ $\begin{array}{lllll}0.9946544894942059 & 0.0287549093335910 & 0.3448836691338294\end{array}$ $\begin{array}{lllll}0.9221563802269184 & 0.0480392462459117 & 0.1470881515950625\end{array}$ $\begin{array}{lllll}0.5573716368639062 & 0.3505038798673098 & 0.1503077194096943\end{array}$ $\begin{array}{lllll}0.7496450825216014 & 0.2781610767285446 & 0.3116521002636584\end{array}$ $\begin{array}{lllll}0.6299878608798378 & 0.3302957721429551 & 0.3478650548946898\end{array}$ $\begin{array}{lllll}0.8080252385758648 & 0.3503162728129868 & 0.1520010458558014\end{array}$ $\begin{array}{lllll}0.0003537996422777 & 0.2793069236315505 & 0.3127704916514391\end{array}$ $\begin{array}{lllll}0.8793974646170889 & 0.3299816015322884 & 0.3476971722225380\end{array}$ $\begin{array}{lllll}0.7094251531959883 & 0.3381449743881755 & 0.0854897377657647\end{array}$ $\begin{array}{lllll}0.9574422965051068 & 0.3384823596005163 & 0.0857676806338992\end{array}$ $\begin{array}{lllll}0.6600640023843440 & 0.3128699843688014 & 0.4664189547060821\end{array}$ $\begin{array}{lllll}0.9036502791551735 & 0.3130095188693240 & 0.4665200710550370\end{array}$ $\begin{array}{lllll}0.6442258016422377 & 0.0653586569798736 & 0.0278436723920074\end{array}$ $\begin{array}{lllll}0.8971475022972669 & 0.0646414012819266 & 0.0286168562442842\end{array}$ $\begin{array}{llllll}0.6642919143355074 & 0.3453957433731317 & 0.2843823234947557\end{array}$ $\begin{array}{lllll}0.9161949824430302 & 0.3437294042309742 & 0.2853522540177468\end{array}$ $\begin{array}{llllll}0.6364842674782655 & 0.0338909596535891 & 0.2095448474192445\end{array}$ $\begin{array}{lllll}0.8872080939884950 & 0.0334429690355659 & 0.2104659320574659\end{array}$ $\begin{array}{llll}0.7125257897665283 & 0.2980726889825885 & 0.2382161186355221\end{array}$ $\begin{array}{llll}0.9625575330020829 & 0.2976535649274238 & 0.2378389190949476\end{array}$ $\begin{array}{lllll}0.8387851991466876 & 0.0807132114351232 & 0.2557883089922706\end{array}$ $\begin{array}{lllll}0.5886493525948676 & 0.0812337336999187 & 0.2551910924924821\end{array}$ $\begin{array}{lllll}0.5907241766301624 & 0.0405001491382828 & 0.9079029380090171\end{array}$ $\begin{array}{llll}0.8456067314520569 & 0.0382772868002707 & 0.9100161177433376\end{array}$ $\begin{array}{lllll}0.5517921597233408 & 0.1008417118282578 & 0.6822004256569159\end{array}$ $\begin{array}{lllll}0.6687120438731070 & 0.2178993516476180 & 0.6553521245544645\end{array}$ $\begin{array}{lllll}0.6310830906590980 & 0.1584785093223473 & 0.8353312096723173\end{array}$ $\begin{array}{lllll}0.7439853465437017 & 0.0286726270942484 & 0.8457238557036024\end{array}$ $\begin{array}{lllll}0.6711236464312742 & 0.0476827038053331 & 0.6464171108883249\end{array}$ $\begin{array}{lllll}0.8020437288954142 & 0.0991475717141234 & 0.6819606179029063\end{array}$ $\begin{array}{lllll}0.9214975241920969 & 0.2204953476688294 & 0.6635604170826129\end{array}$ $\begin{array}{lllll}0.8813649633159455 & 0.1571038502018179 & 0.8351539643613207\end{array}$ $\begin{array}{lllll}0.9960558455438228 & 0.0285303142998441 & 0.8460730095326532\end{array}$ $\begin{array}{lllll}0.9234654202376942 & 0.0474931072983454 & 0.6473737474444500\end{array}$ $\begin{array}{lllll}0.4948079328093913 & 0.3489755123549932 & 0.6841366059807512\end{array}$ $\begin{array}{lllll}0.7482087134888253 & 0.2764697466740245 & 0.8097607098654801\end{array}$ $\begin{array}{lllll}0.6280498773654652 & 0.3279131218147443 & 0.8528618545276603\end{array}$ $\begin{array}{lllll}0.8090801364096637 & 0.3511215973818013 & 0.6577764099635522\end{array}$ $\begin{array}{llll}0.0003248461909667 & 0.2792154860924985 & 0.8132031015260812\end{array}$ $\begin{array}{lllll}0.8796950841623907 & 0.3292195634017955 & 0.8487856424735341\end{array}$ $\begin{array}{lllll}0.7188606524429120 & 0.3335179790525648 & 0.5878936834985077\end{array}$ $\begin{array}{lllll}0.9545850788223067 & 0.3381912279189168 & 0.5862480601612975\end{array}$ $\begin{array}{llll}0.6568454609248163 & 0.3124866833582732 & 0.9683133545383302\end{array}$ $\begin{array}{llll}0.9049161242605908 & 0.3128985900130890 & 0.9671086600356080\end{array}$ $\begin{array}{lllll}0.6443410993054121 & 0.0647884527025246 & 0.5275213651190083\end{array}$ $\begin{array}{llll}0.8975789804977549 & 0.0643711795882431 & 0.5286120360312492\end{array}$ $\begin{array}{lllll}0.6581515020262305 & 0.3467122360688363 & 0.7885865573354691\end{array}$ $\begin{array}{lllll}0.9172774992621022 & 0.3424756910357963 & 0.7868214617390870\end{array}$ $\begin{array}{llll}0.6370123443472042 & 0.0327623884617252 & 0.7101903184894176\end{array}$ $\begin{array}{lllll}0.8884083105941225 & 0.0327457669100416 & 0.7105778125766780\end{array}$ $\begin{array}{lllll}0.7131789605419113 & 0.2995576569143636 & 0.7401359695335121\end{array}$ $\begin{array}{lllll}0.9620807775965660 & 0.2969560227010541 & 0.7377879204881733\end{array}$ $\begin{array}{lllll}0.8391135481949049 & 0.0798872980697699 & 0.7560452022296629\end{array}$ $\begin{array}{llll}0.5886019488886169 & 0.0804466941842021 & 0.7551725624911928\end{array}$ $\begin{array}{lllll}0.6610778692980298 & 0.5528222478814917 & 0.7359446240828498\end{array}$ $\begin{array}{lllll}0.6556719206156187 & 0.5604541013867222 & 0.6549654557377372\end{array}$ $\begin{array}{llll}0.6568053963226599 & 0.5207224362243017 & 0.5515370477940603\end{array}$ $\begin{array}{lllll}0.6457310478126507 & 0.4614698873057345 & 0.6208564571605630\end{array}$ $\begin{array}{lllll}0.6253384417814453 & 0.4252053434280686 & 0.6289027935208359\end{array}$ $\begin{array}{lllll}0.5860808035824624 & 0.4061546936898593 & 0.5665908153543213\end{array}$ $\begin{array}{lllll}0.5987841540063223 & 0.4634035230461207 & 0.4925843002062003\end{array}$ $\begin{array}{llll}0.5864497292880994 & 0.5571923612223064 & 0.6097214775975245\end{array}$ 
$\begin{array}{llll}0.5997999916587478 & 0.5492452559846127 & 0.5330713341645328\end{array}$ $\begin{array}{lllll}0.4120279722439711 & 0.5859106028217286 & 0.5270285322089516\end{array}$ $\begin{array}{lllll}0.3946760776515457 & 0.5515158422087771 & 0.4771549202983844\end{array}$ $\begin{array}{lllll}0.4167741542907871 & 0.4858277117708140 & 0.4727380925974198\end{array}$ $\begin{array}{llll}0.4362850814067462 & 0.4743013164587513 & 0.6072776663158453\end{array}$ $\begin{array}{lllll}0.4422602277357228 & 0.4507328855222701 & 0.6654174035566548\end{array}$ $\begin{array}{llllll}0.4370982978256757 & 0.4098938094962868 & 0.6503876574111965\end{array}$ $\begin{array}{lllll}0.4210906997677188 & 0.4214267050063035 & 0.5199068973108908\end{array}$ $\begin{array}{lllll}0.3304560053718389 & 0.5305996923927817 & 0.4802102228523061\end{array}$ $\begin{array}{llllll}0.3432527963170188 & 0.4923686275592731 & 0.4423533949368732\end{array}$ $\begin{array}{lllll}0.4561051247801854 & 0.4652842599905541 & 0.7425528407775525\end{array}$ $\begin{array}{lllll}0.6414901277036617 & 0.4051949924356156 & 0.6997638474384098\end{array}$ $\begin{array}{lllll}0.6322721277764796 & 0.4807954765177663 & 0.5565772659611357\end{array}$ $\begin{array}{llll}0.5770562937684227 & 0.4267994169165105 & 0.5023812056044757\end{array}$ $\begin{array}{llll}0.4266547713481377 & 0.4608857229725550 & 0.5366528787934323\end{array}$ $\begin{array}{lllll}0.4247861607732688 & 0.3981956402034668 & 0.5788201979883705\end{array}$ $\begin{array}{lllll}0.6232293539140851 & 0.5212590558141004 & 0.7515466978799271\end{array}$ $\begin{array}{lllll}0.6930764250717624 & 0.5325907590266624 & 0.6200123588558448\end{array}$ $\begin{array}{llll}0.5597695559347109 & 0.3742042055345231 & 0.5655419116601631\end{array}$ $\begin{array}{llll}0.5885894076444879 & 0.4793622648739810 & 0.4335039560258708\end{array}$ $\begin{array}{llll}0.6260660772932737 & 0.5818044480490323 & 0.5038274959726919\end{array}$ $\begin{array}{lllll}0.5478092455961601 & 0.5915915875502883 & 0.6147824007398605\end{array}$ $\begin{array}{lllll}0.4707537679879987 & 0.6327798248763172 & 0.6694574581622759\end{array}$ $\begin{array}{llll}0.5011563762308626 & 0.5649980601707930 & 0.7238255338058365\end{array}$ $\begin{array}{llll}0.4251666200348360 & 0.5743724985821851 & 0.6024331471296087\end{array}$ $\begin{array}{lllll}0.4448915825936922 & 0.5226667851959629 & 0.4925945481087882\end{array}$ $\begin{array}{llll}0.4449774530355832 & 0.3853494879626504 & 0.7003893868081272\end{array}$ $\begin{array}{lllll}0.4133480034527280 & 0.4096327991626295 & 0.4562411468327258\end{array}$ $\begin{array}{lllll}0.3359048088705193 & 0.4972525692308201 & 0.3661366796143492\end{array}$ $\begin{array}{llllll}0.2763232976574789 & 0.5517086678621141 & 0.4413936741328907\end{array}$ $\begin{array}{lllll}0.0458354793507329 & 0.2501758933019595 & 0.1826699451666499\end{array}$ $\begin{array}{llllll}0.0304396277621053 & 0.1721264977920191 & 0.1902884302900781\end{array}$ $\begin{array}{lllll}0.1554169861305928 & 0.2021917615803265 & 0.1909011293830760\end{array}$ $\begin{array}{lllll}0.2002199734286114 & 0.1492684527375575 & 0.0681810243089566\end{array}$ $\begin{array}{lllll}0.0340766273586021 & 0.2056539614663077 & 0.0646275716438198\end{array}$ $\begin{array}{lllll}0.0770849189587626 & 0.1332481637971560 & 0.0853478859085174\end{array}$ $\begin{array}{llll}0.1808291343226967 & 0.1206735193455545 & 0.1933136226939151\end{array}$ $\begin{array}{lllll}0.1799457104496727 & 0.0400419223196079 & 0.1989045037369490\end{array}$ $\begin{array}{llll}0.0477593536649099 & 0.0786973571065409 & 0.2139800182603114\end{array}$ $\begin{array}{llllll}0.1601274718373932 & 0.0756569891039347 & 0.0786145765226065\end{array}$ $\begin{array}{lllll}0.1496940815288616 & 0.0825657939492154 & 0.3100032798215129\end{array}$ $\begin{array}{lllll}0.0221596928553880 & 0.0485373438561699 & 0.3304297700376421\end{array}$ $\begin{array}{lllll}0.1396444046872480 & 0.0469003466105898 & 0.4369136263319718\end{array}$ $\begin{array}{lllll}0.1048165147593348 & 0.1192631935017649 & 0.4197549623884137\end{array}$ $\begin{array}{lllll}0.0205437572581852 & 0.2059521541270120 & 0.3033714662470963\end{array}$ $\begin{array}{lllll}0.1453318179424684 & 0.1766562882769673 & 0.3016654029693948\end{array}$ $\begin{array}{llllll}0.1008862888127861 & 0.2296633941281347 & 0.4244738422670741\end{array}$ $\begin{array}{lllll}0.0166020230949354 & 0.1724860456734207 & 0.4289930272140118\end{array}$ $\begin{array}{lllll}0.1199470248763031 & 0.2581436236023510 & 0.2994471622532833\end{array}$ $\begin{array}{llllll}0.2300921270793864 & 0.1013294522175094 & 0.4357894936916382\end{array}$ $\begin{array}{lllll}0.2547064039932566 & 0.1285967543901944 & 0.3101239354032745\end{array}$ $\begin{array}{lllll}0.2240396589725862 & 0.2454262265539070 & 0.4072144366964242\end{array}$ $\begin{array}{lllll}0.2962319453270730 & 0.2509732705488429 & 0.1816358645132962\end{array}$ $\begin{array}{lllll}0.2803874392872458 & 0.1729045223465365 & 0.1894427759113423\end{array}$ $\begin{array}{lllll}0.4054909797600511 & 0.2024656105856830 & 0.1900579288527963\end{array}$ $\begin{array}{lllll}0.4502710776946171 & 0.1492780908745236 & 0.0676789264726373\end{array}$ $\begin{array}{lllll}0.2845943193867534 & 0.2064532003847183 & 0.0637699833226229\end{array}$ $\begin{array}{lllll}0.3267898362534113 & 0.1339639514393168 & 0.0844139684976759\end{array}$ $\begin{array}{lllll}0.4304391880697409 & 0.1208735641095733 & 0.1925698622502518\end{array}$ $\begin{array}{llll}0.4297842472743552 & 0.0403466194736936 & 0.1978109019771156\end{array}$ $\begin{array}{lllll}0.2973405209069102 & 0.0792469858436552 & 0.2129058843324854\end{array}$ $\begin{array}{lllll}0.4088240492178310 & 0.0759596631882655 & 0.0775027406004003\end{array}$ $\begin{array}{lllll}0.3993559974258818 & 0.0826943750645656 & 0.3090709213607328\end{array}$ $\begin{array}{lllll}0.2717149513162547 & 0.0490085442267906 & 0.3294792459871622\end{array}$ $\begin{array}{llll}0.3886985169354773 & 0.0473439160337249 & 0.4358351108331056\end{array}$ $0.3551397467452456 \quad 0.11993035561606490 .4186885726299657$ $\begin{array}{lllll}0.2704223610284406 & 0.2066258224947669 & 0.3023528052820343\end{array}$ $\begin{array}{lllll}0.3951162733223380 & 0.1772151734362617 & 0.3008077513503859\end{array}$ $\begin{array}{lllll}0.3511813277016054 & 0.2304000456397382 & 0.4230761548597221\end{array}$ $\begin{array}{llll}0.2670000121692132 & 0.1731703321724998 & 0.4278813180303485\end{array}$ $\begin{array}{lllll}0.3700855094238135 & 0.2587047158041404 & 0.2981135717750519\end{array}$ $\begin{array}{llll}0.4799574244247865 & 0.1014029196517374 & 0.4351372111515722\end{array}$ $\begin{array}{llll}0.0045226979900035 & 0.1280359501119045 & 0.3109884688755133\end{array}$ 
$\begin{array}{llll}0.4735816323407966 & 0.2465343870250055 & 0.4059632804814651\end{array}$ $\begin{array}{lllll}0.1511347249181812 & 0.2955415964310408 & 0.1823915046909936\end{array}$ $\begin{array}{lllll}0.0297667198242709 & 0.3299115402851551 & 0.1631067372328519\end{array}$ $\begin{array}{lllll}0.0708521764337557 & 0.2773882063998730 & 0.0566351312704488\end{array}$ $\begin{array}{lllll}0.1613911468890554 & 0.3318589018081806 & 0.0560828820714573\end{array}$ $\begin{array}{lllll}0.1962396930922488 & 0.2594677002396301 & 0.0722756895754057\end{array}$ $\begin{array}{llllll}0.1226352269647636 & 0.3386392461303980 & 0.2936410661247862\end{array}$ $\begin{array}{llllll}0.5037732860763703 & 0.3005417259652051 & 0.2796603214779471\end{array}$ $\begin{array}{lllll}0.1412685348745380 & 0.3031429921668602 & 0.4143090992436558\end{array}$ $\begin{array}{lllll}0.4011477778147615 & 0.2977906406823184 & 0.1825571079470241\end{array}$ $\begin{array}{lllll}0.2781063231046236 & 0.3300626712633464 & 0.1621879784383100\end{array}$ $\begin{array}{lllll}0.3216089753390462 & 0.2783126609835234 & 0.0562162217627925\end{array}$ $\begin{array}{llllll}0.4123481693557887 & 0.3324311771169901 & 0.0547150724219636\end{array}$ $\begin{array}{lllll}0.4465299181423459 & 0.2600152607469066 & 0.0742683134485091\end{array}$ $\begin{array}{lllll}0.3678291463378553 & 0.3393140683345548 & 0.2948275696546839\end{array}$ $\begin{array}{lllll}0.2528362411413991 & 0.2998807313900413 & 0.2792151475841340\end{array}$ $\begin{array}{lllll}0.3904694016588874 & 0.3044587366051170 & 0.4131168425653580\end{array}$ $\begin{array}{llllll}0.0450393292996013 & 0.2501128546377981 & 0.6824233225122518\end{array}$ $\begin{array}{lllll}0.0304435275379870 & 0.1719782378858619 & 0.6905741587420711\end{array}$ $\begin{array}{llll}0.1551759742184085 & 0.2020118391720165 & 0.6903778826615808\end{array}$ $\begin{array}{lllll}0.2001273397097854 & 0.1472801811021855 & 0.5683413419665004\end{array}$ $\begin{array}{lllll}0.0334803926922859 & 0.2055051519147533 & 0.5648250263060871\end{array}$ $\begin{array}{llll}0.0766019100233321 & 0.1329800409483044 & 0.5856589395117150\end{array}$ $\begin{array}{llll}0.1802310110950483 & 0.1201254330634430 & 0.6937327991717973\end{array}$ $\begin{array}{llll}0.1799584303025454 & 0.0395578171143691 & 0.7005428111126559\end{array}$ $\begin{array}{lllll}0.0483343479626088 & 0.0784354312360280 & 0.7144128153321164\end{array}$ $\begin{array}{llll}0.1576769449047161 & 0.0744113938565360 & 0.5792826596932412\end{array}$ $\begin{array}{lllll}0.1498954238936961 & 0.0832518351513005 & 0.8106066806499874\end{array}$ $\begin{array}{llll}0.0232523851534533 & 0.0483117758591170 & 0.8310825006341555\end{array}$ $\begin{array}{lllll}0.1417239306119386 & 0.0470810501151985 & 0.9373179866616068\end{array}$ $\begin{array}{lllll}0.1051076965060110 & 0.1191710422548761 & 0.9209945769693818\end{array}$ $\begin{array}{llllll}0.0207131777172292 & 0.2057435608944727 & 0.8036200671728787\end{array}$ $\begin{array}{lllll}0.1457309263373079 & 0.1770321833235091 & 0.8017020503248653\end{array}$ $\begin{array}{lllll}0.1012471798589385 & 0.2296093466077777 & 0.9243425959999704\end{array}$ $\begin{array}{lllll}0.0168322281692936 & 0.1723007695821087 & 0.9292244165889540\end{array}$ $\begin{array}{lllll}0.1195190827981333 & 0.2583080839093654 & 0.7990696285471888\end{array}$ $\begin{array}{llll}0.2306496366205002 & 0.1025071626849526 & 0.9361323653522500\end{array}$ $\begin{array}{lllll}0.2548961310646032 & 0.1290611466339822 & 0.8100885903349666\end{array}$ $\begin{array}{lllll}0.2239008562892059 & 0.2460966967105877 & 0.9067116785504609\end{array}$ $\begin{array}{lllll}0.2962507454602690 & 0.2503508887067138 & 0.6809519219300029\end{array}$ $\begin{array}{lllll}0.2802588974344677 & 0.1720965029337369 & 0.6895603962079770\end{array}$ $\begin{array}{llll}0.4055565652774692 & 0.2023007316962674 & 0.6894221917256315\end{array}$ $\begin{array}{lllll}0.4503004306266310 & 0.1497833181372979 & 0.5676582655129666\end{array}$ $\begin{array}{lllll}0.2826257830936649 & 0.2046869657479140 & 0.5634924912552852\end{array}$ $\begin{array}{llll}0.3270293740863520 & 0.1328381060324978 & 0.5847501429598456\end{array}$ $\begin{array}{lllll}0.4308592832069539 & 0.1207054577903761 & 0.6922793525689429\end{array}$ $\begin{array}{lllll}0.4292561378623374 & 0.0402311785100809 & 0.6972962446101278\end{array}$ $\begin{array}{lllll}0.2972243288412746 & 0.0788859064327948 & 0.7134218576845146\end{array}$ $\begin{array}{lllll}0.4110186276427689 & 0.0759120464680382 & 0.5770649209332975\end{array}$ $\begin{array}{llll}0.3991520368891893 & 0.0823802306129033 & 0.8090224160302413\end{array}$ $\begin{array}{lllll}0.2712730064734937 & 0.0493509799551275 & 0.8306302387066449\end{array}$ $\begin{array}{lllll}0.3869284299723651 & 0.0476694201849736 & 0.9359059413770402\end{array}$ $\begin{array}{llll}0.3557499824720339 & 0.1205861542850966 & 0.9179933735024470\end{array}$ $\begin{array}{lllll}0.2707914387908629 & 0.2073426261339391 & 0.8016071478497858\end{array}$ $\begin{array}{lllll}0.3953941271929830 & 0.1778062564289010 & 0.8007116817183767\end{array}$ $\begin{array}{lllll}0.3506694872772347 & 0.2328182500126143 & 0.9218369357343563\end{array}$ $\begin{array}{llll}0.2688632404640162 & 0.1742370136783408 & 0.9276956105696863\end{array}$ $\begin{array}{lllll}0.3712451667120397 & 0.2599686524252090 & 0.7961625755085804\end{array}$ $\begin{array}{lllll}0.4800216875248375 & 0.1007161053942860 & 0.9349957551617541\end{array}$ $\begin{array}{llll}0.0052239145592830 & 0.1276792214842894 & 0.8114917941396419\end{array}$ $\begin{array}{lllll}0.4740571024059076 & 0.2461676114909478 & 0.9050291067671317\end{array}$ $\begin{array}{lllll}0.1500540476940534 & 0.2961868100673601 & 0.6817364713499110\end{array}$ $\begin{array}{lllll}0.0279922479459231 & 0.3297873115729478 & 0.6629025984167092\end{array}$ $\begin{array}{lllll}0.0695176502967220 & 0.2773173411282938 & 0.5564194913973569\end{array}$ $\begin{array}{llll}0.1623437075046987 & 0.3305400259258278 & 0.5551556000489251\end{array}$ $\begin{array}{lllll}0.1943199831489404 & 0.2574723119790206 & 0.5739840434513676\end{array}$ $\begin{array}{lllll}0.1213369486253796 & 0.3388237846910941 & 0.7932148549393538\end{array}$ $\begin{array}{lllll}0.5012363343028632 & 0.2977018527856667 & 0.7752755586778584\end{array}$ $\begin{array}{lllll}0.1403616978298664 & 0.3033925403883667 & 0.9139470331774266\end{array}$ $\begin{array}{lllll}0.4003581621714268 & 0.2980801658052603 & 0.6763381393061632\end{array}$ $\begin{array}{lllll}0.2775509848455865 & 0.3302510209091739 & 0.6564338922532817\end{array}$ $\begin{array}{llll}0.3197870011160445 & 0.2751701165927178 & 0.5540949196043864\end{array}$ $\begin{array}{lllll}0.4040505621870767 & 0.3292398477724862 & 0.5410399770867645\end{array}$ 
$\begin{array}{llll}0.4467977248313408 & 0.2586550484658457 & 0.5737609561901230\end{array}$ $\begin{array}{lllll}0.3677704041220045 & 0.3416362053945454 & 0.7852927225361238\end{array}$ $\begin{array}{llll}0.2535890236310587 & 0.3009094345241992 & 0.7751240823770902\end{array}$ $\begin{array}{lllll}0.3951928794534652 & 0.3058511961739132 & 0.9099826015909686\end{array}$ $\begin{array}{lllll}0.5471808388207042 & 0.2507552215827598 & 0.1826118536761939\end{array}$ $\begin{array}{lllll}0.5303633659899587 & 0.1727896039694034 & 0.1894004837634488\end{array}$ $\begin{array}{lllll}0.6558155369229081 & 0.2020217828546780 & 0.1912161511999748\end{array}$ $\begin{array}{lllll}0.7006200469621767 & 0.1486861733046764 & 0.0686593731625901\end{array}$ $\begin{array}{lllll}0.5342531277961344 & 0.2066403519669968 & 0.0640259428027199\end{array}$ $\begin{array}{lllll}0.5769829244458381 & 0.1341385082138796 & 0.0844777928913182\end{array}$ $\begin{array}{lllll}0.6802109317514432 & 0.1204529653302203 & 0.1934543367733342\end{array}$ $\begin{array}{lllll}0.6798055921548708 & 0.0398136464207916 & 0.1985668371881292\end{array}$ $\begin{array}{lllll}0.5472327025866270 & 0.0797119603197162 & 0.2129191311298104\end{array}$ $\begin{array}{llll}0.6580505173301110 & 0.0755848058410041 & 0.0783923391663340\end{array}$ $\begin{array}{lllll}0.6491629821035964 & 0.0820139165515662 & 0.3097543665216729\end{array}$ $\begin{array}{lllll}0.5209582227536125 & 0.0490403474023718 & 0.3287811584084285\end{array}$ $\begin{array}{lllll}0.6354456743000516 & 0.0479434032384044 & 0.4366227290999440\end{array}$ $\begin{array}{llllll}0.6046920517754394 & 0.1209185634512611 & 0.4176281883826193\end{array}$ $\begin{array}{lllll}0.5206045618779502 & 0.2066384723811241 & 0.3023938676827883\end{array}$ $\begin{array}{llll}0.6452557870420355 & 0.1770858855173338 & 0.3020181332683654\end{array}$ $\begin{array}{lllll}0.5999293148891568 & 0.2304502778219988 & 0.4244027197787366\end{array}$ $\begin{array}{lllll}0.5152942290882427 & 0.1735933311383313 & 0.4277797769508446\end{array}$ $\begin{array}{lllll}0.6208031510235978 & 0.2586280133820328 & 0.2997911670085933\end{array}$ $\begin{array}{lllll}0.7290303029962953 & 0.1006574286282736 & 0.4359701069356444\end{array}$ $\begin{array}{lllll}0.7539129937669310 & 0.1283053173319411 & 0.3106375107291404\end{array}$ $\begin{array}{lllll}0.7238544105733510 & 0.2446593069168709 & 0.4088527414083747\end{array}$ $\begin{array}{lllll}0.7972669274493449 & 0.2501112853101361 & 0.1834377934463960\end{array}$ $\begin{array}{lllll}0.7804890785420541 & 0.1722427696072125 & 0.1904711897049077\end{array}$ $\begin{array}{llll}0.9057994909527590 & 0.2016281191875008 & 0.1917280044484688\end{array}$ $\begin{array}{llll}0.9504228380670565 & 0.1482813259029638 & 0.0688613962833449\end{array}$ $\begin{array}{lllll}0.7849909164654171 & 0.2057514423087425 & 0.0650318007067271\end{array}$ $\begin{array}{llll}0.8271660252750243 & 0.1331107693866828 & 0.0859441485054561\end{array}$ $\begin{array}{lllll}0.9307690963500044 & 0.1199988968752062 & 0.1940282631081878\end{array}$ $\begin{array}{llll}0.9305670825263856 & 0.0392906529031432 & 0.1995125373077976\end{array}$ $\begin{array}{lllll}0.7972756181535510 & 0.0790263601870478 & 0.2137308305755460\end{array}$ $\begin{array}{lllll}0.9091283014072639 & 0.0749588423877978 & 0.0792496589132372\end{array}$ $\begin{array}{lllll}0.8997011160358259 & 0.0816318469806521 & 0.3102182980867056\end{array}$ $\begin{array}{lllll}0.7713928165538085 & 0.0487479853364155 & 0.3297492566185961\end{array}$ $\begin{array}{llll}0.8885787263444626 & 0.0467029963099288 & 0.4375177416529699\end{array}$ $\begin{array}{lllll}0.8544634900297773 & 0.1191700078714854 & 0.4189906638990397\end{array}$ $\begin{array}{lllll}0.7707740407491400 & 0.2061072358781043 & 0.3036639295834762\end{array}$ $\begin{array}{llll}0.8953686146312333 & 0.1763299526053406 & 0.3024792439668486\end{array}$ $\begin{array}{lllll}0.8506732604073538 & 0.2295966588751553 & 0.4252863264392553\end{array}$ $\begin{array}{lllll}0.7666176712200066 & 0.1723627981351332 & 0.4291878076145967\end{array}$ $\begin{array}{llll}0.8707613875704138 & 0.2580534561627645 & 0.3004666075357705\end{array}$ $\begin{array}{llll}0.9794669618974816 & 0.1006959642887823 & 0.4363770413535008\end{array}$ $\begin{array}{llllll}0.5039652503198860 & 0.1289175172976261 & 0.3096840927923560\end{array}$ $\begin{array}{lllll}0.9742027705231837 & 0.2449381229896130 & 0.4084891192554847\end{array}$ $\begin{array}{lllll}0.6520555880907394 & 0.2971433782736816 & 0.1841694025166837\end{array}$ $\begin{array}{llll}0.5301309744131165 & 0.3302217417242310 & 0.1634224201477628\end{array}$ $\begin{array}{lllll}0.5720656116855491 & 0.2782699404569927 & 0.0574131174918782\end{array}$ $\begin{array}{llll}0.6651246191414939 & 0.3311937041122232 & 0.0567427154982700\end{array}$ $\begin{array}{lllll}0.6963100934252500 & 0.2585230397195085 & 0.0759449747647042\end{array}$ $\begin{array}{lllll}0.6211274654583286 & 0.3392840727500093 & 0.2955535111578988\end{array}$ $\begin{array}{lllll}0.0041248434348065 & 0.3002051899292685 & 0.2799188681698193\end{array}$ $\begin{array}{lllll}0.6439543171216692 & 0.3033276882484447 & 0.4155872946937563\end{array}$ $\begin{array}{lllll}0.9020706860676654 & 0.2960178039576748 & 0.1839740515516852\end{array}$ $\begin{array}{lllll}0.7805573566000792 & 0.3299204571038035 & 0.1642957490270723\end{array}$ $\begin{array}{lllll}0.8211659398290532 & 0.2777304322891643 & 0.0578814133806928\end{array}$ $\begin{array}{lllll}0.9130452404988815 & 0.3314795776566845 & 0.0572920928867418\end{array}$ $\begin{array}{llll}0.9458249720187005 & 0.2588106682804308 & 0.0740194068966863\end{array}$ $\begin{array}{lllll}0.8721603581104694 & 0.3386262279444683 & 0.2952546054526878\end{array}$ $\begin{array}{lllll}0.7542555260521510 & 0.2996037899227351 & 0.2803256693869625\end{array}$ $\begin{array}{lllll}0.8919007445965669 & 0.3029205666728881 & 0.4156708813241515\end{array}$ $\begin{array}{llll}0.5492685029942849 & 0.2505490245293407 & 0.6822189211508801\end{array}$ $\begin{array}{llll}0.5304652887430350 & 0.1726591591419375 & 0.6890830671386855\end{array}$ $\begin{array}{llll}0.6565383199121440 & 0.2012361482988936 & 0.6918056087650194\end{array}$ $\begin{array}{lllll}0.7008130674721249 & 0.1476925037163390 & 0.5694340836789268\end{array}$ $\begin{array}{lllll}0.5368851491441544 & 0.2065375706312866 & 0.5644098663352872\end{array}$ $\begin{array}{llll}0.5769758396049744 & 0.1335653598445418 & 0.5849526852570853\end{array}$ $\begin{array}{lllll}0.6802234826668283 & 0.1194938884200129 & 0.6938567691313698\end{array}$ $\begin{array}{lllll}0.6801079234271248 & 0.0388558864600744 & 0.6988000389787598\end{array}$ $\begin{array}{llll}0.5471546650835450 & 0.0791787546214493 & 0.7130120808569550\end{array}$ 
$\begin{array}{llll}0.6573168892654819 & 0.0746835080959652 & 0.5785063744374876\end{array}$ $\begin{array}{lllll}0.6492490192773236 & 0.0809279876985690 & 0.8098969453400741\end{array}$ $\begin{array}{llll}0.5205719000280199 & 0.0485739923555405 & 0.8284681832129903\end{array}$ $\begin{array}{llll}0.6348662605653864 & 0.0474429542440253 & 0.9370979939087700\end{array}$ $\begin{array}{llll}0.6045784041877067 & 0.1204490906262219 & 0.9169127563030268\end{array}$ $\begin{array}{llll}0.5209735777976998 & 0.2063053975444833 & 0.8021442270222352\end{array}$ $\begin{array}{lllll}0.6454210166268962 & 0.1763289900949295 & 0.8022700664873894\end{array}$ $\begin{array}{lllll}0.6003765855882511 & 0.2289865218774847 & 0.9248664487955387\end{array}$ $\begin{array}{llll}0.5146635578304632 & 0.1729794763255963 & 0.9275144944047992\end{array}$ $\begin{array}{llll}0.6217644323378300 & 0.2578218497228294 & 0.8011431472684961\end{array}$ $\begin{array}{lllll}0.7288163510892333 & 0.0998283950053931 & 0.9362430443944141\end{array}$ $\begin{array}{lllll}0.7538669970912136 & 0.1274450122585524 & 0.8110744971637172\end{array}$ $\begin{array}{lllll}0.7245696667369375 & 0.2437930234825008 & 0.9096822237247385\end{array}$ $\begin{array}{llll}0.7976499374564880 & 0.2492631865982969 & 0.6851640882711885\end{array}$ $\begin{array}{llll}0.7808728097188539 & 0.1712235260916337 & 0.6914387677904305\end{array}$ $\begin{array}{llll}0.9058837242528470 & 0.2010732718350849 & 0.6926413818327712\end{array}$ $\begin{array}{llllll}0.9500472178909720 & 0.1481168993305507 & 0.5692950304913336\end{array}$ $\begin{array}{llll}0.7851807335481242 & 0.2046424513557310 & 0.5662096822906421\end{array}$ $\begin{array}{llll}0.8272865510104266 & 0.1319739660580095 & 0.5867830376509865\end{array}$ $\begin{array}{llll}0.9314006014596423 & 0.1194970009940158 & 0.6944914267332103\end{array}$ $\begin{array}{lllll}0.9317537011495678 & 0.0387963054302596 & 0.6998232316036371\end{array}$ $\begin{array}{lllll}0.7975708602438726 & 0.0780631455483275 & 0.7141590136688563\end{array}$ $\begin{array}{lllll}0.9101647719113853 & 0.0744657415932205 & 0.5794159577162671\end{array}$ $\begin{array}{llll}0.9003247578369052 & 0.0810217883060608 & 0.8104842647489561\end{array}$ $\begin{array}{lllll}0.7717394916427710 & 0.0479780613479000 & 0.8301421915478834\end{array}$ $\begin{array}{lllll}0.8896570381186198 & 0.0459429920292476 & 0.9378305254215651\end{array}$ $\begin{array}{llll}0.8544901265867695 & 0.1182182552223174 & 0.9192697191549308\end{array}$ $\begin{array}{lllll}0.7711737987399465 & 0.2052900786383802 & 0.8046116505634052\end{array}$ $\begin{array}{lllll}0.8958501106430217 & 0.1755860215663635 & 0.8033963344798085\end{array}$ $\begin{array}{lllll}0.8514116520145840 & 0.2287114099317474 & 0.9262685632072535\end{array}$ $\begin{array}{lllll}0.7669600800626382 & 0.1714358696248010 & 0.9297974977585325\end{array}$ $\begin{array}{llll}0.8708646573639335 & 0.2574488728335900 & 0.8017024487662047\end{array}$ $\begin{array}{lllll}0.9796606445491588 & 0.1004394458364076 & 0.9367210867265462\end{array}$ $\begin{array}{lllll}0.5035189197200143 & 0.1286370940614792 & 0.8094826120979648\end{array}$ $\begin{array}{lllll}0.9746820175441520 & 0.2447174091461294 & 0.9090836921959154\end{array}$ $\begin{array}{lllll}0.6498318241916241 & 0.2998495960933641 & 0.6844061969836481\end{array}$ $\begin{array}{lllll}0.5306384818829479 & 0.3324006555618771 & 0.6697838376405316\end{array}$ $\begin{array}{lllll}0.5716831580607675 & 0.2799604834803276 & 0.5594800483598892\end{array}$ $\begin{array}{llll}0.6743821473374859 & 0.3281152268743506 & 0.5567889029755286\end{array}$ 0.69455327905282630 .25550268549866690 .5819238330771256 $0.61703837261379990 .3373756542765474 \quad 0.8004676366807221$ $\begin{array}{llll}0.0035163524498757 & 0.3000399190495402 & 0.7800019185522228\end{array}$ $\begin{array}{lllll}0.6437687470773095 & 0.3019876116780512 & 0.9176551608313895\end{array}$ $\begin{array}{lllll}0.9018036252552859 & 0.2948736908048963 & 0.6843438841539777\end{array}$ $\begin{array}{llll}0.7817856189830589 & 0.3298451193041922 & 0.6665895928107097\end{array}$ $\begin{array}{lllll}0.8187762709691842 & 0.2769435987593338 & 0.5593763211363272\end{array}$ $\begin{array}{llll}0.9102920151267312 & 0.3310022144083977 & 0.5577470146989036\end{array}$ $\begin{array}{lllll}0.9443890964070246 & 0.2584427167361408 & 0.5735148641466116\end{array}$ $\begin{array}{lllll}0.8729020637801295 & 0.3378131874762416 & 0.7962304264308822\end{array}$ $\begin{array}{llllll}0.7544058363069688 & 0.2989281984532611 & 0.7815112442954260\end{array}$ $\begin{array}{lllll}0.8919283058245618 & 0.3024039818097348 & 0.9166007175053374\end{array}$ $\begin{array}{lllll}0.2137097129667890 & 0.1678053290444180 & 0.2478491790551261\end{array}$ $\begin{array}{lllll}0.0875166005098679 & 0.2111976341330098 & 0.2450931725135566\end{array}$ $\begin{array}{llll}0.2230287489504786 & 0.0813665929978045 & 0.2635615507578830\end{array}$ $\begin{array}{lllll}0.4630414501466827 & 0.1680346745089029 & 0.2472530284737356\end{array}$ $\begin{array}{lllll}0.3376766685760071 & 0.2121819582988625 & 0.2445043393978271\end{array}$ $\begin{array}{lllll}0.4725713265512633 & 0.0816183893641347 & 0.2628443505842467\end{array}$ $\begin{array}{llllll}0.0784535779481547 & 0.2975464251511754 & 0.2291177876703862\end{array}$ $\begin{array}{lllll}0.3280589580364802 & 0.2985369084685178 & 0.2292718954880313\end{array}$ $\begin{array}{lllll}0.2136066530150171 & 0.1678849003222762 & 0.7474611535960981\end{array}$ $\begin{array}{lllll}0.0873573070140724 & 0.2112743949837646 & 0.7447609060928206\end{array}$ $\begin{array}{llll}0.2229621949987975 & 0.0815472000220680 & 0.7640142884585697\end{array}$ $\begin{array}{lllll}0.4629541513420327 & 0.1678184703909056 & 0.7466739920019672\end{array}$ $\begin{array}{lllll}0.3376696033509127 & 0.2122114281741500 & 0.7432470257847414\end{array}$ $\begin{array}{lllll}0.4723663243435819 & 0.0812524856892683 & 0.7627077762676311\end{array}$ $\begin{array}{lllll}0.0775539318520307 & 0.2976438893151426 & 0.7286769003697569\end{array}$ $\begin{array}{lllll}0.3280746672501946 & 0.2990328751566644 & 0.7238854935779144\end{array}$ $\begin{array}{llll}0.7131215769433932 & 0.1674449595458242 & 0.2482882860325490\end{array}$ $\begin{array}{lllll}0.5876309378563602 & 0.2116592249670122 & 0.2448906549420192\end{array}$ $\begin{array}{llllll}0.7225505886865783 & 0.0809621705406862 & 0.2637249710263270\end{array}$ $\begin{array}{llll}0.9635491927033074 & 0.1670754318464160 & 0.2486100550231568\end{array}$ $\begin{array}{lllll}0.8378967090766689 & 0.2109965280262570 & 0.2457696009093203\end{array}$ $\begin{array}{llll}0.9732494352389913 & 0.0806495437333032 & 0.2642926948336292\end{array}$ 
$\begin{array}{llll}0.5781397758341721 & 0.2981464486765247 & 0.2299956710765148\end{array}$ $\begin{array}{lllll}0.8287899391435198 & 0.2974213427758037 & 0.2303010956894699\end{array}$ $\begin{array}{llll}0.7134741139735359 & 0.1665506393654822 & 0.7487784880606321\end{array}$ $\begin{array}{llll}0.5880880159163819 & 0.2105484176202690 & 0.7444736423223878\end{array}$ $\begin{array}{llll}0.7227223727098810 & 0.0800377778061750 & 0.7640308587298451\end{array}$ $\begin{array}{lllll}0.9639944920284268 & 0.1666092768687110 & 0.7492101041667999\end{array}$ $\begin{array}{llll}0.8382167736794455 & 0.2101161590488056 & 0.7469675909233956\end{array}$ $\begin{array}{lllll}0.9740082811622064 & 0.0802030444749691 & 0.7648521751749430\end{array}$ $\begin{array}{llll}0.5800547294391003 & 0.2959786699633824 & 0.7340464826677757\end{array}$ $\begin{array}{llll}0.8296093775016432 & 0.2964452177705100 & 0.7317746934262236\end{array}$ $\begin{array}{lllll}0.0236070564857278 & 0.1652454897688225 & 0.1019035683125060\end{array}$ $\begin{array}{lllll}0.1544147119532477 & 0.1206416615028813 & 0.1052009747945049\end{array}$ $\begin{array}{lllll}0.1469891179779377 & 0.2582408203311210 & 0.3875368481492710\end{array}$ $\begin{array}{llll}0.1562380161851426 & 0.0867598477672782 & 0.3975851919936650\end{array}$ $\begin{array}{lllll}0.0265079039799507 & 0.1290480272897338 & 0.3998551942096498\end{array}$ $\begin{array}{lllll}0.0276089825047492 & 0.2128879210425401 & 0.3917714826517127\end{array}$ $\begin{array}{lllll}0.0242725190583215 & 0.2490889179009564 & 0.0937746884514499\end{array}$ $\begin{array}{lllll}0.2735502444422820 & 0.1661479032889628 & 0.1011069286916454\end{array}$ $\begin{array}{llll}0.4038100996562126 & 0.1209234827698345 & 0.1044892273859065\end{array}$ $\begin{array}{lllll}0.3962580741898143 & 0.2601937395141091 & 0.3868006622801619\end{array}$ $\begin{array}{lllll}0.4059458741637367 & 0.0870714887017220 & 0.3965781578410554\end{array}$ $\begin{array}{lllll}0.2767789269502168 & 0.1297764388075879 & 0.3989553609459335\end{array}$ $\begin{array}{lllll}0.2780486660156027 & 0.2136565106097278 & 0.3908639182796608\end{array}$ $\begin{array}{llll}0.2747179198451229 & 0.2499635180381076 & 0.0928408948997808\end{array}$ $\begin{array}{lllll}0.1449159545177498 & 0.2918435067843079 & 0.0948786450994098\end{array}$ $\begin{array}{lllll}0.3952607228552878 & 0.2930665923018302 & 0.0952328831680095\end{array}$ $\begin{array}{llllll}0.0232648192688493 & 0.1650671561982411 & 0.6021430194204532\end{array}$ $\begin{array}{llllll}0.1534320662942877 & 0.1195040799336358 & 0.6056860395633368\end{array}$ $\begin{array}{lllll}0.1466530396098542 & 0.2584876130391592 & 0.8871619184068128\end{array}$ $\begin{array}{lllll}0.1571230954668951 & 0.0872542663134043 & 0.8982263188760142\end{array}$ $\begin{array}{lllll}0.0268317395735510 & 0.1288051695971817 & 0.9004451155055392\end{array}$ $\begin{array}{lllll}0.0279969995180880 & 0.2126769683508090 & 0.8920480704920483\end{array}$ $\begin{array}{lllll}0.0229606927585566 & 0.2489695567215098 & 0.5934902318498356\end{array}$ $\begin{array}{lllll}0.2730166816542745 & 0.1645658559265132 & 0.6012965223163017\end{array}$ $\begin{array}{lllll}0.4045208508967471 & 0.1207388546284241 & 0.6041052831317322\end{array}$ $\begin{array}{lllll}0.3975335067780082 & 0.2606906631504015 & 0.8843535361646957\end{array}$ $\begin{array}{llllll}0.4056111787443708 & 0.0869906340380777 & 0.8965712939366661\end{array}$ $\begin{array}{lllll}0.2774499210328700 & 0.1306510556921114 & 0.8988679948924680\end{array}$ $\begin{array}{lllll}0.2782079497893306 & 0.2147263919719598 & 0.8899383469502276\end{array}$ $\begin{array}{llllll}0.2732977503273037 & 0.2482363151707684 & 0.5922331974495897\end{array}$ $\begin{array}{lllll}0.1440882132058304 & 0.2910813879066061 & 0.5943150353885625\end{array}$ $\begin{array}{lllll}0.3936146497999148 & 0.2927865599761110 & 0.5878283448183740\end{array}$ $\begin{array}{lllll}0.5234263383825959 & 0.1661514828906174 & 0.1009667904223616\end{array}$ $\begin{array}{lllll}0.6536619174892460 & 0.1205731203982723 & 0.1053281164850656\end{array}$ $\begin{array}{lllll}0.6471479725694649 & 0.2584303548707665 & 0.3878723635495490\end{array}$ $\begin{array}{lllll}0.6546844082959641 & 0.0871078169500338 & 0.3970453219217142\end{array}$ $\begin{array}{llll}0.5260193406850449 & 0.1303118535606703 & 0.3984651707816174\end{array}$ $\begin{array}{llll}0.5263596694219892 & 0.2140995935160959 & 0.3905529028329511\end{array}$ $\begin{array}{lllll}0.5246769261868239 & 0.2500543024361540 & 0.0938413994319601\end{array}$ $\begin{array}{llll}0.7738715365189712 & 0.1653238818172896 & 0.1020997012198421\end{array}$ $\begin{array}{lllll}0.9041800462784816 & 0.1199673452919150 & 0.1059319853638023\end{array}$ $\begin{array}{llll}0.8971549500794809 & 0.2579853949928260 & 0.3886019510280526\end{array}$ $\begin{array}{llll}0.9056124891282059 & 0.0862905846451881 & 0.3976038199977934\end{array}$ $\begin{array}{lllll}0.7761085131012623 & 0.1289960149882515 & 0.3994464757038726\end{array}$ $\begin{array}{lllll}0.7773983336771018 & 0.2128190819848512 & 0.3920804305219268\end{array}$ $\begin{array}{lllll}0.7748656783348129 & 0.2490647005597195 & 0.0946984147883119\end{array}$ $\begin{array}{lllll}0.6459694880452571 & 0.2921346415386137 & 0.0968857300022910\end{array}$ $\begin{array}{lllll}0.8953472897324762 & 0.2918165457064593 & 0.0964052622717741\end{array}$ $\begin{array}{lllll}0.5239851064174873 & 0.1660881689477318 & 0.6005782415218255\end{array}$ $\begin{array}{llll}0.6535240054522019 & 0.1196353205116766 & 0.6057372801465779\end{array}$ $\begin{array}{llll}0.6479048555071912 & 0.2571708388956163 & 0.8896606474353108\end{array}$ $\begin{array}{llll}0.6544791693168057 & 0.0863466695587637 & 0.8970804008714052\end{array}$ $\begin{array}{llll}0.5258376596043800 & 0.1297025996852019 & 0.8982468275211106\end{array}$ $\begin{array}{lllll}0.5265725414018826 & 0.2133012929968160 & 0.8904404451157452\end{array}$ $\begin{array}{llll}0.5249626379751120 & 0.2499142601628867 & 0.5932190090942976\end{array}$ $\begin{array}{lllll}0.7741048612555093 & 0.1641546039231165 & 0.6030736607580316\end{array}$ $\begin{array}{llllll}0.9046103276026491 & 0.1193500656615736 & 0.6064395244013752\end{array}$ $\begin{array}{lllll}0.8975223918838631 & 0.2574724279713276 & 0.8898294376691834\end{array}$ $\begin{array}{lllll}0.9061039287675159 & 0.0856220668505567 & 0.8978500764876508\end{array}$ $\begin{array}{lllll}0.7761595104218174 & 0.1280442677095717 & 0.8998813601703283\end{array}$ $\begin{array}{lllll}0.7781885626388851 & 0.2119043264477118 & 0.8931033475007351\end{array}$ $\begin{array}{llll}0.7739459347425601 & 0.2475404864520320 & 0.5966739191898125\end{array}$ $\begin{array}{llll}0.6470288780499680 & 0.2918435391783896 & 0.5977887938018479\end{array}$ 
$0.8935007331656095 \quad 0.29106082212735390 .5965088631688393$

$0.4877028479733540 \quad 0.58881943107496090 .6584883081119401$

INCAR :

SYSTEM $=$ TSUB_MNT-Na+

Wavefunction and Charge Density files

ISTART $=1$

LWAVE $=$.TRUE

LCHARG $=$. FALSE

Electronic minimization

ENCUT $=400$

PREC $=$ NORMAL

NELMIN $=6$

EDIFF $=1.0 \mathrm{E}-06$

ISMEAR $=0$

SIGMA $=0.1$

$\mathrm{ALGO}=\mathrm{FAST}$

LREAL = AUTO

lonic minimization

EDIFFG $=-1.0 \mathrm{E}-02$

$\mathrm{NSW}=1000$

IBRION = 3

POTIM $=0$

IOPT $=1$

ISIF $=2$

Forces

IVDW = 11

LPLANE $=$. TRUE

$\mathrm{NPAR}=4$

LSCALU $=$. FALSE

$\mathrm{NSIM}=4$ 